Division of Geological \& Geophysical Surveys

RAW-DATA FILE 2011-4

Version 2

GEOCHEMICAL, MAJOR-OXIDE, MINOR-OXIDE, TRACE-ELEMENT, CARBON, AND RARE-EARTH-ELEMENT DATA FROM ROCKS COLLECTED IN 2011 IN THE MORAN AREA,

TANANA AND MELOZITNA QUADRANGLES, ALASKA

by

Trevelyn A. Lough, Larry K. Freeman, Rainer J. Newberry, Brent A. Elliot, Gerry A. Griesel, and David J. Szumigala

$\$ 18.00$

February 2012

THIS REPORT HAS NOT BEEN REVIEWED FOR TECHNICAL CONTENT OR FOR CONFORMITY TO THE EDITORIAL STANDARDS OF DGGS

Released by

STATE OF ALASKA

DEPARTMENT OF NATURAL RESOURCES

Division of Geological \& Geophysical Surveys 3354 College Road

Fairbanks, Alaska 99709-3707 



\section{CONTENTS}

PAGE

INTRODUCTION 1

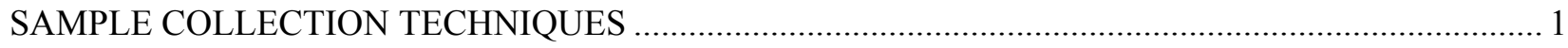

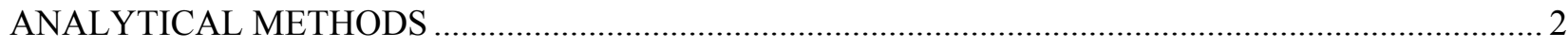

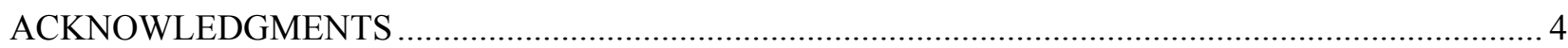

\section{TABLES}

TABLE 1. LOCATION AND DESCRIPTION OF ROCK SAMPLES COLLECTED FOR TRACE-ELEMENT ANALYSES IN THE MORAN AREA, TANANA AND MELOZITNA QUADRANGLES, ALASKA .

TABLE 2. TRACE-ELEMENT RESULTS FOR ROCKS COLLECTED IN THE MORAN AREA, TANANA AND MELOZITNA QUADRANGLES, ALASKA.

TABLE 3. DETECTION LIMITS AND ANALYTICAL METHODS FOR TRACE-ELEMENT GEOCHEMICAL ANALYSES OF ROCK SAMPLES.

TABLE 4. LOCATION AND DESCRIPTION OF ROCKS COLLECTED FOR MAJOR-OXIDE, MINOR-OXIDE, AND TRACE-ELEMENT ANALYSES IN THE MORAN AREA, TANANA AND MELOZITNA QUADRANGLES, ALASKA.

TABLE 5. MAJOR-OXIDE, MINOR-OXIDE, AND TRACE-ELEMENT RESULTS FOR ROCKS COLLECTED IN THE MORAN AREA, TANANA AND MELOZITNA QUADRANGLES, ALASKA

TABLE 6. DETECTION LIMITS AND ANALYTICAL METHODS FOR MAJOR- AND MINOR-OXIDE AND TRACE-METAL ANALYSES OF WHOLE-ROCK SAMPLES ...... 83

TABLE 7. LOCATION, DESCRIPTION, AND RESULTS OF ROCKS COLLECTED FOR NONCARBONATE-CARBON ANALYSES IN THE MORAN AREA, TANANA AND MELOZITNA QUADRANGLES, ALASKA .

TABLE 8: LOCATION, DESCRIPTION, AND RESULTS FOR ROCKS COLLECTED FOR MAJOR-OXIDE, MINOR-OXIDE, AND TRACE-ELEMENT ANALYSES ON POLISHED ROCK SLABS IN THE MORAN AREA, TANANA AND MELOZITNA QUADRANGLES, ALASKA

TABLE 9: DETECTION LIMITS AND ANALYTICAL METHODS FOR MAJOR-OXIDE, MINOR-OXIDE, AND TRACE-ELEMENT ANALYSES OF POLISHED ROCK SLAB SAMPLES

TABLE 10: LOCATION AND DESCRIPTION OF ROCKS COLLECTED FOR RARE-EARTHAND TRACE-ELEMENT ANALYSES IN THE MORAN AREA, TANANA AND MELOZITNA QUADRANGLES, ALASKA 170

TABLE 11: RARE-EARTH- AND TRACE-ELEMENT RESULTS FOR ROCKS COLLECTED IN THE MORAN AREA, TANANA AND MELOZITNA QUADRANGLES, ALASKA ....... 173

TABLE 12: DETECTION LIMITS AND GEOCHEMICAL MEHODS FOR RARE-EARTH- AND TRACE-ELEMENT ANALYSES OF ROCK SAMPLES

Note: This report (including all analytical data and tables) is available in digital format from the DGGS website (www.dggs.alaska.gov) at no charge. The digital data are available as PDF files and .csv files. 



\title{
GEOCHEMICAL, MAJOR-OXIDE, MINOR-OXIDE, TRACE-ELEMENT, CARBON, AND RARE-EARTH-ELEMENT DATA FROM ROCKS COLLECTED IN 2011 IN THE MORAN AREA, TANANA AND MELOZITNA QUADRANGLES, ALASKA
}

\author{
by \\ Trevelyn A. Lough ${ }^{1}$, Larry K. Freeman ${ }^{1}$, Rainer J. Newberry ${ }^{2}$, Brent A. Elliot ${ }^{1}$, Gerry A. Griesel ${ }^{1}$, \\ and David J. Szumigala ${ }^{1}$
}

\section{INTRODUCTION}

Mineral-resources personnel from the Alaska Division of Geological \& Geophysical Surveys carried out a geologic field survey, including mapping and sampling in the Moran area in the Tanana A-6 and B-6 quadrangles, and the Melozitna A-1, A-2, B-1, and B-2 quadrangles, Alaska, from June 17 to August 15, 2011. The fieldwork provides basic information critical to building an understanding of Alaska's geology and is part of an integrated program of airborne geophysical surveys followed by geologic mapping. During 2011, 212 rock samples were collected for geochemical trace-element analysis (tables 1-3), 58 rock samples were collected for whole-rock (major- and minor-oxide and petrogenetically important trace elements) analyses (tables 4-6), two samples were collected for analysis of non-carbonate carbon content (table 7), 439 polished rock slabs were analyzed for whole rock and petrogenetically important trace elements (tables 8 and 9), and 26 samples were analyzed for rare earth elements (tables 10-12). Location were collected as Universal Transverse Mercator (UTM) coordinates based on the Clark 1866 spheroid, NAD 27, zone 5N projection using hand-held, Garmin eTrex Legend HCx, GPS units (no differential correction was applied). Estimated position error calculated by the GPS units had a minimum of 1 meter, a maximum of 9 meters, and an average of approximately 3 meters. Coordinates were converted to latitude and longitude based on the North American Datum of 1927 (NAD 27) for Alaska using the "Calculate Geometry" tool in ArcMap 10.0. Some accuracy may be lost in this conversion. Additional details about the sampling program can be found in the metadata file associated with the digital version of this report. Sample numbers, location data, descriptions, and analytical results for each sample are tabulated in tables 1-12.

\section{SAMPLE COLLECTION TECHNIQUES}

Samples of visibly mineralized rock, or rocks exhibiting features associated with mineralization, were preferentially collected and analyzed for geochemical trace elements. Most samples are "grab" samples, which were randomly collected at a location; however, a few samples are "select" samples, which were deliberately collected from a specific feature. These instances are noted in the sample's description. Samples of visibly carbonaceous or graphite-bearing metamorphic rock were preferentially collected and analyzed for their non-carbonate carbon content. We collected rock samples for major-oxide, minor-oxide, and trace-element analyses to determine bulk rock composition of igneous and meta-igneous lithologies, or to identify the tectonic setting of a sample's protolith from petrogenetically important trace elements. These rocks were collected in a random distribution across the study area. Of the 58 samples sent to

\footnotetext{
${ }^{1}$ Alaska Division of Geological \& Geophysical Surveys, 3354 College Rd., Fairbanks, AK 99709-3707;

${ }^{2}$ Department of Geology \& Geophysics, University of Alaska, P.O. Box 755780, Fairbanks, AK 997755780
} 
ALS Chemex for whole-rock analyses, 26 plutonic and metamorphic rocks were also analyzed for rare earth elements. These samples were selected based on sample quality (unweathered) and location (random distribution across study area).

\section{ANALYTICAL METHODS}

\section{Sample Preparation}

Tables 1-7 and 10-12: Trace-element geochemical analyses (table 2), whole-rock analyses (table 4), non-carbonate carbon analysis (table 7), and rare-earth-element analyses (tables 11) were performed by ALS Chemex. Rock samples were crushed with a Terminator oscillating-jaw crusher with chrome steel alloy plates so that at least 70 percent of the material passed through a 10 mesh $(2 \mathrm{~mm})$ screen. Representative aliquots of 250 grams $(\mathrm{g})$ each were taken using a stainless steel riffle splitter. These samples were then pulverized with an Essa ring mill bowl and pucks made of Essa's Standard Steel alloy so that 85 percent of the sample passed through a -200 mesh (75 micron) screen. Trace-element analyses, whole-rock analyses, gold analyses, and rareearth-element analyses were performed using representative splits of each $250 \mathrm{~g}$ pulp.

Tables 8-9: Major- and minor-oxide and trace-element compositions were performed by X-ray fluorescence (XRF) spectroscopy at the University of Alaska Fairbanks (UAF) on polished rock slabs and are necessarily approximations to the true compositions. Rocks with a grain size of 3 $\mathrm{mm}$ or less were cut to fit in 37-mm-diameter sample holders. Slabs of potentially lower-thannormal quality are noted in the table 8 "Comments" column with any combination of the following descriptors:

- $\quad$ Altered $=$ altered sample

- Inhomogeneous $=$ sample contains crystals or clasts $>3 \mathrm{~mm}$ and/or a texture such as banding or boxwork

- $\quad$ Poor polish = poorly polished sample

- $\quad$ Porous $=$ porous sample

- $\quad$ Small $=$ sample is smaller than the $37-\mathrm{mm}$-diameter sample holder and a $27-\mathrm{mm}$ sample holder was used instead

\section{Analytical Methods for DGGS Samples Analyzed by ALS Chemex}

Tables 1-3: All potentially mineralized samples were assayed for gold by inductively coupled plasma-atomic emission spectroscopy following a $30 \mathrm{~g}$ fire assay fusion (FA-ICP-AES). Selected samples were assayed for platinum and palladium by FA-ICP-AES. Trace-element geochemical analyses were performed by inductively coupled plasma-atomic emission spectroscopy (ICPAES) methods after four-acid, near-total digestion. This method of digestion is possibly incomplete for some elements and may result in lower analytical results. Sample locations and descriptions are tabulated in table 1, analytical results are tabulated in table 2 and, analytical methods as well as lower and upper reporting limits are tabulated in table 3 . The "Lithology_Description" column in table 1 begins with the rock name derived from field observations, followed by a semi-colon and a sample description. Rock samples with traceelement values above the detection limit in the initial trace-element geochemical analysis were reanalyzed for those elements by the following methods:

- gold - fire assay with a gravimetric finish using a $30 \mathrm{~g}$ sub-sample (FA-GRAV)

- phosphorus - high-grade ICP-AES methods after four-acid, near-total digestion; and

- arsenic and/or zinc - high-grade ICP-AES methods after four-acid, near-total digestion. 
Results and detection limits for the reanalysis of gold, arsenic, phosphorus, and zinc are listed in tables 2 and 3, respectively.

Tables 4-6: Major- and minor-element oxides were determined at ALS Chemex by XRF spectrometry following a lithium borate fusion (LBM-XRF). Trace element values for $\mathrm{Cr}, \mathrm{Nb}, \mathrm{Ni}, \mathrm{Rb}$, $\mathrm{Sr}, \mathrm{Y}$, and $\mathrm{Zr}$ were determined for mafic rocks by XRF on a pressed pellet. Trace element values for $\mathrm{Ba}, \mathrm{Nb}, \mathrm{Rb}, \mathrm{Sr}, \mathrm{Th}, \mathrm{U}, \mathrm{Y}$, and $\mathrm{Zr}$ were determined for felsic rocks by XRF on a pressed pellet. Sample locations and descriptions are tabulated in table 4, analytical results are tabulated in table 5, and analytical methods and detection limits are tabulated in table 6. The "Lithology Description" column in table 4 consists of three components: 1) the rock name derived from field observations; 2) the rock name-enclosed in parentheses-derived from geochemical data and hand-sample textures; and 3) a sample description.

Table 7: Non-carbonate carbon analyses were performed by ALS Chemex. Non-carbonate carbon content was determined using a LECO furnace after a dilute acid digestion. Sample location, description, and analytical results are listed in table 7 . The lower detection limit is $0.01 \%$, and the upper detection limit is 50\% carbon. The "Lithology_Description" column in table 7 begins with the rock name derived from field observations, followed by a sample description.

Tables 10-12: Rare earth and trace elements were determined at ALS Chemex by inductively coupled plasma-mass spectroscopy (ICP-MS) after a lithium metaborate fusion. This method of digestion is possibly incomplete for some elements and may result in lower analytical results. Sample location and description are tabulated in table 10, analytical results are tabulated in table 11 , and analytical methods as well as lower and upper reporting limits are tabulated in table 12. The "Lithology_Description" column in table 10 consists of three components: 1) the rock name derived from field observations; 2) the rock name-enclosed in parentheses-derived from geochemical data and hand-sample textures; and 3) a sample description.

Quality control: In addition to ALS Chemex's own internal quality control program, DGGS monitored analysis quality by inserting one sample of known composition into the sample roster for every 20-sample batch. For the trace-element geochemical analyses, the results of one DGGSstandard sample differs from its known composition by more than one standard deviation; all samples in this 20 -sample batch are noted in table 2 by a carat next to the Sample_ID, and mineralized samples were re-run for gold by FA-ICP-AES (results in table 2); repeat values of the standard are within acceptable range.

\section{Analytical Methods for DGGS Samples Analyzed at UAF}

Tables 8-9: XRF spectroscopic analyses performed at UAF were run on a PANalytical Axios spectrometer using SuperQ software. $\mathrm{Nb}, \mathrm{Rb}, \mathrm{Sr}, \mathrm{Y}$, and $\mathrm{Zr}$ were measured using SuperQ's $37 \mathrm{mmRbSrYZr}$ routine software program; other elements were measured with the IQ $+37 \mathrm{mmVac}$ software program.

- $\quad 37 \mathrm{mmRBSRYZr}$ routine - uses specific pre-determined peak and background positions for which x-ray intensities are measured for 2-800 seconds (depending on the element). The intensity of the Rh Compton peak is used to estimate mass-absorption coefficients (MACs) for both unknowns and well-characterized natural standards. Peak intensities are computed and converted to concentrations using calibration curves employing at least 10 natural rock standards.

- IQ37mm Vac - scans over a series of energies corresponding to a range from $\mathrm{Ce}-\mathrm{K} \alpha$ to $\mathrm{O}-\mathrm{K} \alpha$. Peak heights and backgrounds, and X-Ray elemental interferences are picked 
with the software and checked manually to ensure quality control. Elemental abundances for all elements with atomic numbers between 8 and 92 are estimated from artificial standards and these estimations are used to calculate MACs for each element present above the detection limit. Revised concentrations are employed to calculate revised MACs until a stable solution is determined. Elemental abundances are then normalized to $100 \%$.

Quality Control:

- $37 \mathrm{mmRBSRYZr}$ routine-These procedures are routinely checked by analysis of secondary natural standards that were not employed in making the calibration curves. Elemental abundances are typically within $2-5 \%$ of the amount present for concentrations $>10$ times the detection limit, within $5-10 \%$ of the amount present for concentrations 4-10 times the detection limit and within 30\% of the amount present for concentrations near the detection limit.

- IQ37mm Vac-The software is routinely checked using pressed pellets of wellcharacterized natural rock standards. Elemental abundances are within 1-2\% of the amount present for major elements, $2-5 \%$ of the amount present for minor elements, and $5-10 \%$ of the amount present for trace elements.

\section{ACKNOWLEDGMENTS}

This project is part of the Alaska Airborne Geophysical/Geological Mineral Inventory program funded by the Alaska State Legislature and managed by the State of Alaska, Department of Natural Resources, Division of Geological \& Geophysical Surveys. Partial funding for the geologic mapping and geochemical analyses was also provided through the State of Alaska General Fund and the U.S. Geological Survey STATEMAP Program under award number G11AC20203. 
Table 1. Location and description of rocks collected for trace-element analyses in the Moran area, Tanana and Melozitna Quadrangles, Alaska.

NOTE: Coordinates are based on the North American Datum of 1927 (NAD 27)

\begin{tabular}{|c|c|c|c|c|c|c|}
\hline Sample_ID & Latitude & Longitude & Easting & Northing & $\begin{array}{l}\text { UTM_- } \\
\text { Zone }\end{array}$ & Lithology_Description \\
\hline 2011BAE021A & 65.282036 & -152.847237 & 507128 & 7239704 & $5 \mathrm{~N}$ & $\begin{array}{l}\text { Quartzite; fine grained, white to light gray with areas of oxidation and fractures. } \\
\text { Comprises } 20 \% \text { of exposure. Geochemistry sample for possible sulfide assay. }\end{array}$ \\
\hline 2011BAE055A & 65.337448 & -152.716683 & 513192 & 7245901 & $5 \mathrm{~N}$ & $\begin{array}{l}\text { Quartz vein; very-fine-grained, massive float with dark-gray to brown oxidation } \\
\text { and veinlets. } 100 \% \text { of exposure. }\end{array}$ \\
\hline 2011BAE104A & 65.487497 & -152.847442 & 507063 & 7262604 & $5 \mathrm{~N}$ & $\begin{array}{l}\text { Paragneiss; medium grained, light pink to pale pinkish gray, equigranular, and } \\
\text { massive. Occasional pink, euhedral potassium feldspar megacrysts }(\sim 1.5 \mathrm{~cm}) \text {. } \\
\text { Quartz crystals are subhedral. } 100 \% \text { of exposure. }\end{array}$ \\
\hline 2011BAE123B & 65.409953 & -152.831526 & 507823 & 7253963 & $5 \mathrm{~N}$ & Quartz vein; very fine grained. $1 \%$ of exposure. \\
\hline 2011BAE128C & 65.411646 & -152.854732 & 506745 & 7254149 & $5 \mathrm{~N}$ & Quartz vein; trace. \\
\hline 2011BAE133B & 65.414397 & -152.882330 & 505463 & 7254453 & $5 \mathrm{~N}$ & Quartz vein; very fine grained with some iron oxidation. $1 \%$ of exposure. \\
\hline 2011BAE135B & 65.416252 & -152.890508 & 505083 & 7254659 & $5 \mathrm{~N}$ & Quartz vein; very fine grained with dark vein-fill. $1 \%$ of exposure. \\
\hline 2011BAE138B & 65.442911 & -152.883345 & 505410 & 7257631 & $5 \mathrm{~N}$ & $\begin{array}{l}\text { Quartz vein; sulfides and/or micas may be present inside veins. Rock has visible } \\
\text { shear planes. } 1 \% \text { of exposure. }\end{array}$ \\
\hline 2011BAE153B & 65.250603 & -152.663285 & 515730 & 7236234 & $5 \mathrm{~N}$ & Quartz vein; cuts quartz schist. Vein is 2-cm-thick. Trace. \\
\hline 2011BAE156C & 65.257774 & -152.731348 & 512547 & 7237018 & $5 \mathrm{~N}$ & Quartz vein; very-fine-grained float with some vugs. $1 \%$ of exposure. \\
\hline 2011BAE187A & 65.452629 & -152.567396 & 520055 & 7258778 & $5 \mathrm{~N}$ & Quartz vein; very-fine-grained float. $1 \%$ of exposure. \\
\hline 2011BAE205B & 65.450331 & -152.576429 & 519638 & 7258519 & $5 \mathrm{~N}$ & Quartz vein; $1 \%$ of exposure. \\
\hline 2011BAE226C & 65.410063 & -152.557459 & 520549 & 7254037 & $5 \mathrm{~N}$ & $\begin{array}{l}\text { Volcanic breccia(?); possibly brecciated altered volcaniclastics or greenstone. } \\
\text { Rock is fine grained. } 25 \% \text { of exposure. }\end{array}$ \\
\hline 2011BAE226D & 65.410063 & -152.557459 & 520549 & 7254037 & $5 \mathrm{~N}$ & Quartz vein; $1 \%$ of exposure. \\
\hline 2011BAE234B & 65.332655 & -152.874300 & 505854 & 7245343 & $5 \mathrm{~N}$ & stain. $1 \%$ of exposure. \\
\hline 2011BAE247B & 65.325527 & -152.905396 & 504407 & 7244546 & $5 \mathrm{~N}$ & Quartz vein; orangish stain. Trace. \\
\hline 2011BAE250B & 65.325292 & -152.831230 & 507862 & 7244527 & $5 \mathrm{~N}$ & $\begin{array}{l}\text { Quartz vein; fine-grained, white, massive bull quartz with minor iron-oxide } \\
\text { staining. Epidote and schistose mica fill fractures and veins. } 1 \% \text { of exposure. }\end{array}$ \\
\hline 2011BAE262B & 65.337936 & -152.975280 & 501151 & 7245926 & $5 \mathrm{~N}$ & $\begin{array}{l}\text { Quartz schist; quartz occurs in bands and pinch/swirls and contains both sulfides } \\
\text { and iron oxide. Selected sample of quartz-rich material. } 15 \% \text { of exposure. }\end{array}$ \\
\hline
\end{tabular}


Table 1. (continued)

\begin{tabular}{|c|c|c|c|c|c|c|}
\hline Sample_ID & Latitude & Longitude & Easting & Northing & $\begin{array}{l}\text { UTM_- } \\
\text { Zone }\end{array}$ & Lithology_Description \\
\hline 2011BAE298C & 65.184843 & -153.826282 & 461304 & 7229116 & $5 \mathrm{~N}$ & $\begin{array}{l}\text { Tonalite(?) dike; possibly quartz diorite. Rock is fine to medium grained, dark } \\
\text { gray, silicified, and foliated or sheared. It contains quartz and possibly sulfides. } \\
5 \% \text { of exposure. }\end{array}$ \\
\hline 2011BAE321B & 65.338140 & -153.440128 & 479507 & 7246020 & $5 \mathrm{~N}$ & Quartz vein; very-fine-grained, white, and massive bull quartz. $10 \%$ of exposure. \\
\hline 2011BAE332B & 65.328973 & -153.092619 & 495686 & 7244930 & $5 \mathrm{~N}$ & $\begin{array}{l}\text { Metagabbro; very fine to coarse grained. Sulfides and white plagioclase are } \\
\text { scattered throughout the rock. Visible sulfides include euhedral pyrite, some } \\
\text { chalcopyrite, and possible pyrrhotite or arsenopyrite. The rock has a green, } \\
\text { micaceous matrix, which }\end{array}$ \\
\hline 2011BAE342B & 65.314608 & -153.107953 & 494969 & 7243330 & $5 \mathrm{~N}$ & Quartz vein; orangish stain. $1 \%$ of exposure. \\
\hline 2011BAE351B & 65.462650 & -152.805055 & 509034 & 7259840 & $5 \mathrm{~N}$ & Quartz vein; very fine grained with some iron oxide in cracks. $1 \%$ of exposure. \\
\hline 2011BAE394A & 65.383185 & -152.531600 & 521772 & 7251050 & $5 \mathrm{~N}$ & $\begin{array}{l}\text { Serpentinite(?); possibly pyroxenite. This dark green rock is medium to coarse } \\
\text { grained, massive, undeformed, and homogenous with minimal weathering. } \\
100 \% \text { of exposure. }\end{array}$ \\
\hline 2011BAE410B & 65.369379 & -152.865394 & 506260 & 7249437 & $5 \mathrm{~N}$ & Quartz vein; very fine grained. $1 \%$ of exposure. \\
\hline 2011BAE429B & 65.426604 & -152.822390 & 508242 & 7255820 & $5 \mathrm{~N}$ & Quartz vein; bull quartz. $1 \%$ of exposure. \\
\hline 2011BAE432B & 65.255887 & -152.871625 & 505996 & 7236787 & $5 \mathrm{~N}$ & $\begin{array}{l}\text { Quartz vein; very fine grained with orangish staining and black and goldish colors } \\
\text { (micas?) on fractures. } 1 \% \text { of exposure. }\end{array}$ \\
\hline 2011BAE468B & 65.199067 & -152.828851 & 508011 & 7230459 & $5 \mathrm{~N}$ & $\begin{array}{l}\text { Quartz vein; quartz and sericite with turquoise staining (copper?). Selected } \\
\text { sample from a foliation-parallel quartz vein. Trace. }\end{array}$ \\
\hline 2011BAE470B & 65.199775 & -152.812652 & 508769 & 7230540 & $5 \mathrm{~N}$ & $\begin{array}{l}\text { Micaceous quartzite/quartz schist; fine to medium grained, tan to gray with } \\
\text { small brown specks in fold hinges. Occasional, very small sulfide specks are } \\
\text { visible. Epidote, quartz, and sericite veining parallels foliation. } 20 \% \text { of exposure. }\end{array}$ \\
\hline 2011BAE491B & 65.234865 & -152.994395 & 500262 & 7234438 & $5 \mathrm{~N}$ & $\begin{array}{l}\text { Quartz vein; white and massive. Locally strongly fractured with open space and } \\
\text { moderate iron-oxide staining. } 1 \% \text { of exposure. }\end{array}$ \\
\hline 2011BAE537B & 65.471914 & -152.520946 & 522192 & 7260943 & $5 \mathrm{~N}$ & Quartz vein; large, white vein. $1 \%$ of exposure. \\
\hline 2011GG010C & 65.299309 & -152.868175 & 506147 & 7241627 & $5 \mathrm{~N}$ & Quartz vein; milky-white to clear. Trace. \\
\hline
\end{tabular}


Table 1. (continued)

\begin{tabular}{|c|c|c|c|c|c|c|}
\hline Sample_ID & Latitude & Longitude & Easting & Northing & $\begin{array}{l}\text { UTM_- } \\
\text { Zone }\end{array}$ & Lithology_Description \\
\hline 2011GG016B & 65.299024 & -152.881666 & 505518 & 7241594 & $5 \mathrm{~N}$ & $\begin{array}{l}\text { Gabbroic dike; mostly plagioclase with chloritized amphiboles. Moderately } \\
\text { altered with mineralized vugs. } 5 \% \text { of exposure. }\end{array}$ \\
\hline 2011GG033A & 65.328167 & -152.805106 & 509078 & 7244851 & $5 \mathrm{~N}$ & $\begin{array}{l}\text { Schist; black and white with abundant orange, oxidized pits (possibly former } \\
\text { calcite or ankerite). These oxidized pits encompass very-fine-grained minerals } \\
\text { with square cleavage planes. The pinched ends of quartz boudins contain fine- } \\
\text { grained, prismatic quartz crystals that are typically stained with orange iron } \\
\text { oxide. } 100 \% \text { of exposure. }\end{array}$ \\
\hline 2011GG034A & 65.330640 & -152.802296 & 509208 & 7245127 & $5 \mathrm{~N}$ & $\begin{array}{l}\text { Schist; orange iron-oxidized phyllitic schist with foliation-parallel quartz-vein } \\
\text { boudins. Very-fine-grained oxides fill vugs. } 100 \% \text { of exposure. }\end{array}$ \\
\hline 2011GG035B & 65.331724 & -152.801107 & 509263 & 7245248 & $5 \mathrm{~N}$ & $\begin{array}{l}\text { Quartz boudin; vuggy. Strong oxidation and abundant prismatic quartz crystals } \\
\text { within vugs. Square, } 1-2 \mathrm{~mm} \text { black flecks disseminated throughout milky white } \\
\text { quartz. Pervasive malachite staining throughout the outcrop. } 1 \% \text { of exposure. }\end{array}$ \\
\hline 2011GG037B & 65.335465 & -152.794508 & 509569 & 7245666 & $5 \mathrm{~N}$ & $\begin{array}{l}\text { Dike; medium grained with quartz, biotite, and feldspar. Contains both coarse, } \\
\text { prismatic quartz and euhedral quartz. Fine-grained, yellowish gold and red } \\
\text { oxides occur within cavities and fractures. } 1 \% \text { of exposure. }\end{array}$ \\
\hline 2011GG037C & 65.335465 & -152.794508 & 509569 & 7245666 & $5 \mathrm{~N}$ & $\begin{array}{l}\text { Quartz vein; } 2.5 \text {-cm-thick vein cuts foliation at a right angle. Vein contains } \\
\text { possible sulfides as well as a red, rhombic mineral with perfect cleavage. } 1 \% \text { of } \\
\text { exposure. }\end{array}$ \\
\hline 2011GG041C & 65.402597 & -152.776974 & 510359 & 7253151 & $5 \mathrm{~N}$ & Quartz vein; oxidized with black specks. Trace. \\
\hline 2011GG044B & 65.408391 & -152.758254 & 511226 & 7253800 & $5 \mathrm{~N}$ & $\begin{array}{l}\text { Fault breccia; selected sample of highly oxidized, orangish brown, welded, and } \\
\text { resilicified fault material with boxwork structure and gossan. Contains sericite } \\
\text { and quartz crystals within cavities. Represents less than } 1 \% \text { of the rock at the } \\
\text { station, which is dominantly quartz-mica schist. } 1 \% \text { of exposure. }\end{array}$ \\
\hline 2011GG059B & 65.323668 & -152.816237 & 508561 & 7244348 & $5 \mathrm{~N}$ & $\begin{array}{l}\text { Quartz-plagioclase dike(?); possibly vein. Visible minerals include euhedral } \\
\text { plagioclase crystals with perfect cleavage and striations, prismatic quartz } \\
\text { crystals, milky white quartz grains, clusters of very-fine-grained books of aligned } \\
\text { sericite, and localized clusters of a translucent yellow, anhedral, drusy mineral. } \\
\text { Rock is highly iron-oxidized with orange- and-brown-oxidation-coated cavities. } \\
\text { Trace. }\end{array}$ \\
\hline
\end{tabular}

RDF 2011-4 Version 2 
Table 1. (continued)

\begin{tabular}{|c|c|c|c|c|c|c|}
\hline Sample_ID & Latitude & Longitude & Easting & Northing & $\begin{array}{l}\text { UTM_- } \\
\text { Zone }\end{array}$ & Lithology_Description \\
\hline 2011GG062A & 65.408754 & -152.755387 & 511359 & 7253841 & $5 \mathrm{~N}$ & $\begin{array}{l}\text { Mica quartz schist; selected sample of moderately altered schist with about } 30 \% \\
\text { of the same fault gouge material as } 2011 \mathrm{GG} 044 \mathrm{~B} \text {, with boxwork, gossan, and } \\
\text { hematite. } 100 \% \text { of exposure. }\end{array}$ \\
\hline 2011GG063A & 65.408620 & -152.755582 & 511350 & 7253826 & $5 \mathrm{~N}$ & $\begin{array}{l}\text { Quartz vein; massive, milky white, quartz boulders that are heavily oxidized } \\
\text { when broken. } 10 \% \text { of exposure. }\end{array}$ \\
\hline 2011GG064A & 65.408621 & -152.756099 & 511326 & 7253826 & $5 \mathrm{~N}$ & $\begin{array}{l}\text { Quartz-mica schist; boxwork with sericite alteration in 1-6 cm spaces } \\
\text { (weathered-out boudins?). Contains hematite. Selected sample. 100\% of } \\
\text { exposure. }\end{array}$ \\
\hline 2011GG065A & 65.408310 & -152.758018 & 511237 & 7253791 & $5 \mathrm{~N}$ & $\begin{array}{l}\text { Schist; with zones of fault gouge and gossan, abundant hematite and boxwork. } \\
\text { Selected sample. } 100 \% \text { of exposure. }\end{array}$ \\
\hline 2011GG069A & 65.409127 & -152.747308 & 511734 & 7253884 & $5 \mathrm{~N}$ & $\begin{array}{l}\text { Quartz vein; highly oxidized, fractured, and resilicified vein with hematite and } \\
\text { other fine-grained oxides. } 5 \% \text { of exposure. }\end{array}$ \\
\hline 2011GG094B & 65.454078 & -152.840324 & 507402 & 7258880 & $5 \mathrm{~N}$ & Quartz vein; massive milky white. Trace. \\
\hline 2011GG097A & 65.455349 & -152.855223 & 506711 & 7259020 & $5 \mathrm{~N}$ & $\begin{array}{l}\text { Skarn; black weathering. Fresh rock is black and green with areas of pink. } 20 \% \text { of } \\
\text { exposure. }\end{array}$ \\
\hline 2011GG097C & 65.455349 & -152.855223 & 506711 & 7259020 & $5 \mathrm{~N}$ & Skarn; fine-grained, dark green rock with a 2 -mm-wide vein. $60 \%$ of exposure. \\
\hline 2011GG098B & 65.455682 & -152.856538 & 506650 & 7259057 & $5 \mathrm{~N}$ & Hornfels; dark brown to black weathering, no fresh faces. $33 \%$ of exposure. \\
\hline 2011GG102B & 65.452317 & -152.837768 & 507521 & 7258684 & $5 \mathrm{~N}$ & $\begin{array}{l}\text { Hornfelsed quartzite(?); white to gray, recrystallized quartz with a sparkly, } \\
\text { brown, powdery, and soft mineral on fractures. Rock also has biotite and very- } \\
\text { fine-grained sericite. } 1 \% \text { of exposure. }\end{array}$ \\
\hline 2011GG105A & 65.449944 & -152.842398 & 507307 & 7258419 & $5 \mathrm{~N}$ & $\begin{array}{l}\text { Skarn (?); possibly hornfels. Maroon weathering with abundant iron oxide, } \\
\text { hematite, and limonite. Rock contains very-fine-grained and possibly cubic } \\
\text { minerals, micas, and several hematite-lined cavities. } 5 \% \text { of exposure. }\end{array}$ \\
\hline 2011GG106A & 65.449628 & -152.849237 & 506990 & 7258383 & $5 \mathrm{~N}$ & $\begin{array}{l}\text { Hornfelsed quartz schist; dark gray to black rock with a sugary texture and } \\
\text { whitish highlights. Weathered surfaces show a relict schistose layer, and } \\
\text { foliation-parallel quartz veins. Most rocks have minor to moderate iron-oxide } \\
\text { staining, but some have intense staining with hematite. Selected sample of } \\
\text { veined pieces. } 100 \% \text { of exposure. }\end{array}$ \\
\hline
\end{tabular}


Table 1. (continued)

\begin{tabular}{|c|c|c|c|c|c|c|}
\hline Sample_ID & Latitude & Longitude & Easting & Northing & $\begin{array}{l}\text { UTM_- } \\
\text { Zone }\end{array}$ & Lithology_Description \\
\hline 2011GG110A & 65.447450 & -152.861219 & 506435 & 7258139 & $5 \mathrm{~N}$ & Marble; white with 5-10 cm calcite crystals. Trace. \\
\hline 2011GG111A & 65.446639 & -152.866745 & 506179 & 7258048 & $5 \mathrm{~N}$ & $\begin{array}{l}\text { Marble; small }(1 \mathrm{~cm}) \text {, white to gray chips. Rock has rillenstein weathering and a } \\
\text { strong reaction to } \mathrm{HCl} .60 \% \text { of exposure. }\end{array}$ \\
\hline 2011GG111D & 65.446639 & -152.866745 & 506179 & 7258048 & $5 \mathrm{~N}$ & $\begin{array}{l}\text { Dike; very fine grained, quartz rich with some feldspar. Rock is oxidized with a } \\
\text { very-fine-grained, disseminated black mineral, and zones of sericite. } 10 \% \text { of } \\
\text { exposure. }\end{array}$ \\
\hline 2011GG138B & 65.252358 & -152.623788 & 517574 & 7236440 & $5 \mathrm{~N}$ & $\begin{array}{l}\text { Quartz vein; milky white with orange iron-oxide and black to dark blue staining. } \\
\text { Rock is locally vuggy with very-fine-grained orange, prismatic minerals as well as } \\
\text { a small amount of sericite. } 20 \% \text { of exposure. }\end{array}$ \\
\hline 2011GG167A & 65.296763 & -152.728678 & 512653 & 7241364 & $5 \mathrm{~N}$ & $\begin{array}{l}\text { Quartz vein; milky white with chloritic schist inclusions. Cavities are coated with } \\
\text { a dull brown powder, iron-oxide staining, and minor hematite. 50\% of exposure. }\end{array}$ \\
\hline 2011GG168A & 65.295792 & -152.727509 & 512708 & 7241256 & $5 \mathrm{~N}$ & $\begin{array}{l}\text { Quartz vein; brecciated and oxidized. Rock has zones of fine-grained, black } \\
\text { sericite and disseminated pyrite. } 100 \% \text { of exposure. }\end{array}$ \\
\hline 2011GG170A & 65.292632 & -152.716971 & 513201 & 7240906 & $5 \mathrm{~N}$ & Quartz vein; quartz, calcite, and sericite. $2 \%$ of exposure. \\
\hline 2011GG179A & 65.462444 & -152.599689 & 518551 & 7259862 & $5 \mathrm{~N}$ & $\begin{array}{l}\text { Quartz vein; peach-orange oxide. Vein contains epidote as well as a light, key- } \\
\text { lime-green mineral. } 1 \% \text { of exposure. }\end{array}$ \\
\hline 2011GG179C & 65.462444 & -152.599689 & 518551 & 7259862 & $5 \mathrm{~N}$ & $\begin{array}{l}\text { Quartz-epidote vein; green, black, and white crystalline vein scattered } \\
\text { throughout outcrop area. Trace. }\end{array}$ \\
\hline 2011GG185A & 65.461117 & -152.590172 & 518993 & 7259717 & $5 \mathrm{~N}$ & $\begin{array}{l}\text { Quartz-epidote vein breccia; cuts aphanitic greenstone. Brecciated texture with } \\
\text { clasts of greenstone. Quartz vein fill is mixed with an aphanitic, white (felsic?) } \\
\text { rock. Trace. }\end{array}$ \\
\hline 2011GG186B & 65.461044 & -152.589440 & 519027 & 7259709 & $5 \mathrm{~N}$ & $\begin{array}{l}\text { Quartz vein; vuggy, milky white to crystalline quartz vein that cuts an aphanitic } \\
\text { greenstone. Vein is possibly slightly brecciated. Vugs are filled with subhedral } \\
\text { prismatic quartz crystals, iron oxides, and a very-fine-grained, sparkly, black } \\
\text { mineral that is associated with very-fine-grained, disseminated pyrite. Trace. }\end{array}$ \\
\hline 2011GG194A & 65.442144 & -152.594566 & 518803 & 7257601 & $5 \mathrm{~N}$ & $\begin{array}{l}\text { Metasandstone; well sorted, weakly metamorphosed sandstone or metawacke } \\
\text { cut by veins of iron oxide. } 50 \% \text { of exposure. }\end{array}$ \\
\hline
\end{tabular}


Table 1. (continued)

\begin{tabular}{|c|c|c|c|c|c|c|}
\hline Sample_ID & Latitude & Longitude & Easting & Northing & $\begin{array}{l}\text { UTM_- } \\
\text { Zone }\end{array}$ & Lithology_Description \\
\hline 2011GG209A & 65.301805 & -152.881053 & 505546 & 7241904 & $5 N$ & $\begin{array}{l}\text { Quartz schist; highly oxidized with abundant muscovite on foliation planes. Dark, } \\
\text { mm-thick stacks of sericite are perpendicular to foliation. Rock is generally } 60 \% \\
\text { quartz and } 40 \% \text { mica, but grades into graphite-rich layers with only } 30-40 \% \\
\text { quartz. Rectangular porphyroclasts occur on the foliation plane. Oxidation colors } \\
\text { range locally from red/orange to a full-spectrum, metallic sheen. }\end{array}$ \\
\hline 2011GG210C & 65.302085 & -152.883496 & 505432 & 7241935 & $5 \mathrm{~N}$ & $\begin{array}{l}\text { Quartz vein; milky white with brown to orange oxidation throughout. One pyrite } \\
\text { crystal is visible within a black, oxidized streak. Rock contains localized epidote } \\
\text { and hematite. Trace. }\end{array}$ \\
\hline 2011GG217B & 65.313360 & -152.891471 & 505058 & 7243191 & $5 \mathrm{~N}$ & $\begin{array}{l}\text { Quartz vein; milky-white, massive, weakly brecciated quartz with lots of red and } \\
\text { orange oxides. Visible minerals include patches of sericite, mm-size quartz } \\
\text { crystals, minor epidote, and some very-fine-grained sulfides(?). }\end{array}$ \\
\hline 2011GG300B & 65.316064 & -153.009935 & 499537 & 7243488 & $5 \mathrm{~N}$ & $\begin{array}{l}\text { Quartz vein; } 90 \% \text { massive, milky white quartz in vein center. Vein margins } \\
\text { contain subhedral, elongate quartz crystals, a white, carbonate powder, and } \\
\text { calcite crystals. Sericite, occasional hematite, and minor copper-green staining } \\
\text { fill cavities. } 1 \% \text { of exposure. }\end{array}$ \\
\hline 2011GG304B & 65.311192 & -153.012808 & 499403 & 7242945 & $5 \mathrm{~N}$ & $\begin{array}{l}\text { Quartz vein; highly oxidized, foliation-parallel quartz vein that contains sericite } \\
\text { and traces of hematite. Trace. }\end{array}$ \\
\hline 2011GG323A & 65.439310 & -152.776338 & 510374 & 7257243 & $5 \mathrm{~N}$ & $\begin{array}{l}\text { Plagioclase-porphyroclastic greenschist; fine-grained, massive, turquoise-green } \\
\text { rock with moderate foliation. Rock has a sugary texture and subtle layers of } \\
\text { darker and lighter material. Visible minerals include plagioclase and }<1 \% \\
\text { disseminated chalcopyrite. Rock is hard to break and forms sharp conchoidal } \\
\text { edges. } 100 \% \text { of exposure. }\end{array}$ \\
\hline 2011GG335A & 65.429489 & -152.819612 & 508370 & 7256142 & $5 \mathrm{~N}$ & $\begin{array}{l}\text { Quartz vein; highly oxidized and fractures easily. Contains occasional vugs, which } \\
\text { are filled with oxidized material. } 1 \% \text { of exposure. }\end{array}$ \\
\hline 2011GG344A & 65.489523 & -152.813646 & 508627 & 7262834 & $5 \mathrm{~N}$ & $\begin{array}{l}\text { Paragneiss(?) and/or quartzite (?); rock is dark black and hornfelsed. It appears } \\
\text { to be quartz-rich with mica-rich layers. Rock contains }<5 \% \text { very-fine-grained, } \\
\text { oxidized, disseminated pyrite. } 50 \% \text { of exposure. }\end{array}$ \\
\hline
\end{tabular}


Table 1. (continued)

\begin{tabular}{|c|c|c|c|c|c|c|}
\hline Sample_ID & Latitude & Longitude & Easting & Northing & $\begin{array}{l}\text { UTM_- } \\
\text { Zone }\end{array}$ & Lithology_Description \\
\hline 2011GG349A & 65.495693 & -152.798024 & 509348 & 7263524 & $5 \mathrm{~N}$ & $\begin{array}{l}\text { Paragneiss; strongly foliated with lots of oxidation. Rock contains pits and vugs } \\
\text { filled with red, yellow, and orange oxides. Very-fine-grained sulfides are } \\
\text { disseminated in black, mica-rich layers and one quartz-rich layer. A mm-scale } \\
\text { quartz veinlet and remnants of a cm-scale dike are visible. Rock is strongly } \\
\text { oxidized. } 100 \% \text { of exposure. }\end{array}$ \\
\hline 2011GG353A & 65.507439 & -152.785677 & 509915 & 7264835 & $5 \mathrm{~N}$ & $\begin{array}{l}\text { Quartzite; gray to white and strongly foliated. Rock has very distinct parting on } \\
\text { both the outcrop scale as well as } \mathrm{cm} \text { scale }(1-4 \mathrm{~cm}) \text {. Rock has green impurities, } \\
\text { vugs filled with prismatic quartz and sericite, and local oxidation. Selected } \\
\text { sample of highly oxidized and vuggy material. } 100 \% \text { of exposure. }\end{array}$ \\
\hline 2011GG356A & 65.405536 & -152.821650 & 508283 & 7253472 & $5 \mathrm{~N}$ & $\begin{array}{l}\text { Quartz vein; massive, milky white quartz vein boulders }(0.25-0.5 \mathrm{~m} \text { in diameter). } \\
\text { Rock has abundant orange iron-oxide staining as well as occasional vugs, which } \\
\text { are lined with brown, yellow and red oxides. Micas from schist wall rock line } \\
\text { some quartz-vein boulders. Selected sample of the most oxidized pieces. } 5 \% \text { of } \\
\text { exposure. }\end{array}$ \\
\hline 2011GG359A & 65.404071 & -152.819011 & 508406 & 7253309 & $5 \mathrm{~N}$ & $\begin{array}{l}\text { Quartz schist; fine-grained and white with disseminated purplish brown spots. } \\
\text { Visible minerals include } 30-60 \% \text { quartz, 30-40\% mica, 5-30\% purplish brown } \\
\text { unknown mineral, trace tourmaline(?), and trace magnetite(?). Layer is } \\
\text { approximately } 50 \mathrm{~cm} \text { thick and } 10 \% \text { of exposure. }\end{array}$ \\
\hline 2011GG365A & 65.396665 & -152.816027 & 508547 & 7252484 & $5 \mathrm{~N}$ & $\begin{array}{l}\text { Quartzite and quartz schist; } 60-70 \% \text { of this rock is highly oxidized ( } 90 \% \text { altered) } \\
\text { and slightly gossanous. Oxidation colors vary from red to orange to brown. } \\
\text { Selected sample of highly oxidized/gossanous rocks. } 100 \% \text { of exposure. }\end{array}$ \\
\hline 2011GG366A & 65.394048 & -152.818133 & 508450 & 7252192 & $5 \mathrm{~N}$ & $\begin{array}{l}\text { Muscovite-schist breccia; dark brown to maroon and highly (90\%) altered and } \\
\text { oxidized with abundant gossan. The unaltered } 10 \% \text { of the rock contains } \\
\text { muscovite and white mica. Rock is intact and fairly hard to break. } 100 \% \text { of } \\
\text { exposure. }\end{array}$ \\
\hline
\end{tabular}


Table 1. (continued)

\begin{tabular}{|c|c|c|c|c|c|c|}
\hline Sample_ID & Latitude & Longitude & Easting & Northing & $\begin{array}{l}\text { UTM_- } \\
\text { Zone }\end{array}$ & Lithology_Description \\
\hline 2011GG376A & 65.377683 & -152.906951 & 504326 & 7250359 & $5 \mathrm{~N}$ & $\begin{array}{l}\text { Quartz vein; mostly quartz with occasional weathered feldspars. Vein cuts a } \\
\text { greenish schistose rock. Vein margin is heavily oxidized with red, yellow, and } \\
\text { orange colors as well as patches of sericite. Subhedral to euhedral, prismatic } \\
\text { quartz crystals fill vugs. Localized boxwork around feldspars. }<1 \% \text { of exposure. }\end{array}$ \\
\hline 2011GG376C & 65.377683 & -152.906951 & 504326 & 7250359 & $5 \mathrm{~N}$ & $\begin{array}{l}\text { Schist; } 1.5 \times 3 \text { m zone of highly oxidized, easily broken rock with remnant mica } \\
\text { and cleavage planes barely recognizable because of alteration. Broken rock } \\
\text { faces are oxidized red, yellow, and orange with hematite and dark red veinlets. } \\
2 \% \text { of exposure. }\end{array}$ \\
\hline $2011 G G 382 C$ & 65.379733 & -152.887110 & 505248 & 7250589 & $5 N$ & $\begin{array}{l}\text { Quartz vein; milky white, highly oxidized, and slightly brecciated. Vein is } \\
\text { weathered out from within foliation at a large fracture with lots of iron-oxide } \\
\text { staining. Contains occasional single grains of pyrite. } 2 \% \text { of exposure. }\end{array}$ \\
\hline 2011GG393A & 65.284543 & -152.922861 & 503599 & 7239977 & $5 \mathrm{~N}$ & $\begin{array}{l}\text { Schist, quartz schist, and quartzite; highly weathered and oxidized. High-angle, } \\
0.5 \text {-cm-wide veins cut foliation, one of which contains rusted pyrite. Veins have } \\
\text { been fractured and possibly resilicified. } 100 \% \text { of exposure. }\end{array}$ \\
\hline 2011GG395A & 65.280794 & -152.924865 & 503506 & 7239559 & $5 \mathrm{~N}$ & $\begin{array}{l}\text { Felsic dike(?); white and black with oxidized pits and gossan throughout. } 50 \% \text { of } \\
\text { exposure. }\end{array}$ \\
\hline 2011GG403A & 65.264316 & -152.839373 & 507500 & 7237730 & $5 \mathrm{~N}$ & $\begin{array}{l}\text { Quartzite; gray to dark gray, foliated, and locally altered. Rock has }<15 \% \\
\text { oxidized pits and moderate iron-oxide staining. Occasional very-fine-grained } \\
\text { pyrite, partially to fully filled pits. Unaltered rock pieces have }<1 \% \text { disseminated } \\
\text { sulfides (chalcopyrite?) perpendicular to foliation. Some fresh faces have bright, } \\
\text { metallic, colorful oxidation. Minerals include } 85 \% \text { quartz and } 15 \% \text { foliation- } \\
\text { perpendicular mica. } 100 \% \text { of exposure. }\end{array}$ \\
\hline 2011GG422B & 65.198982 & -152.832612 & 507835 & 7230449 & $5 \mathrm{~N}$ & $\begin{array}{l}\text { Aplite dike(?); possibly sill. Rock is rusty and crumbled with occasional small, } \\
\text { rusty, oxidized, prismatic, elongate, and subhedral quartz crystals with sericite. } \\
2 \% \text { of exposure. }\end{array}$ \\
\hline 2011GG456C & 65.226260 & -152.944845 & 502579 & 7233480 & $5 \mathrm{~N}$ & Quartz vein; milky white and oxidized. $30 \%$ of exposure. \\
\hline 2011GG464A & 65.441757 & -152.636294 & 516868 & 7257546 & $5 \mathrm{~N}$ & $\begin{array}{l}\text { Schist; very fine grained with various alteration and oxidation colors. Rock } \\
\text { contains weathered pyrite pits. } 60 \% \text { of exposure. }\end{array}$ \\
\hline
\end{tabular}


Table 1. (continued)

\begin{tabular}{|c|c|c|c|c|c|c|}
\hline Sample_ID & Latitude & Longitude & Easting & Northing & $\begin{array}{l}\text { UTM_- } \\
\text { Zone }\end{array}$ & Lithology_Description \\
\hline 2011GG470B & 65.439449 & -152.620954 & 517581 & 7257293 & $5 \mathrm{~N}$ & $\begin{array}{l}\text { Quartz vein; milky white with very-fine-grained, subhedral, prismatic, elongate } \\
\text { quartz and minor calcite and sericite. } 1 \% \text { of exposure. }\end{array}$ \\
\hline 2011GG480A & 65.200676 & -152.794848 & 509602 & 7230643 & $5 \mathrm{~N}$ & $\begin{array}{l}\text { Metagrit; dark gray with white, sugary zones. } 10-20 \% \text { mica defines foliation in } \\
\text { the rock. Contains } 5-10 \% \text { black to bluish grit and is slightly vuggy. } 1-3-\mathrm{mm} \text {-wide } \\
\text { veinlets cut foliation. } 70 \% \text { of exposure. }\end{array}$ \\
\hline 2011GG481B & 65.200665 & -152.786879 & 509975 & 7230643 & $5 \mathrm{~N}$ & $\begin{array}{l}\text { Quartz vein; milky white, massive, and slightly oxidized. } 2 \mathrm{~cm} \text { thick. Selected } \\
\text { sample from three different veins. }\end{array}$ \\
\hline 2011GG484A & 65.202328 & -152.759751 & 511244 & 7230833 & $5 \mathrm{~N}$ & $\begin{array}{l}\text { Quartzite; white and mostly unaltered. Rock has moderately spaced foliation } \\
\text { and is locally vuggy and oxidized. Selected sample of oxidized and vuggy pieces. } \\
100 \% \text { of exposure. }\end{array}$ \\
\hline 2011GG491A & 65.384066 & -152.932358 & 503144 & 7251069 & $5 \mathrm{~N}$ & $\begin{array}{l}\text { Quartz schist; black and white with phyllitic foliation planes. Rock is heavily iron- } \\
\text { oxide stained with local, }<1 \text {-mm-thick, oxidized veinlets that cut foliation at high } \\
\text { angles. Rock contains zones of secondary epidote mineralization as well as } \\
\text { graphite-rich layers. } 100 \% \text { of exposure. }\end{array}$ \\
\hline 2011GG523B & 65.474621 & -152.604058 & 518340 & 7261218 & $5 \mathrm{~N}$ & $\begin{array}{l}\text { Greenstone(?); black, blue, green, conchoidal, and aphanitic with foliation. A } \\
\text { localized, folded, vuggy, oxidized quartz vein that contains epidote. Selected } \\
\text { sample of vein-bearing material. } 10 \% \text { of exposure. }\end{array}$ \\
\hline 2011GG529A & 65.384380 & -153.822997 & 461748 & 7251352 & $5 \mathrm{~N}$ & Granite; cut by multiple 2 - to 5 -mm-thick veins. $100 \%$ of exposure. \\
\hline $2011 G G 551 A$ & 65.368019 & -152.971446 & 501328 & 7249279 & $5 \mathrm{~N}$ & $\begin{array}{l}\text { Quartz vein; highly oxidized vein material of variable thickness. Parallel and } \\
\text { perpendicular to foliation. } 1 \% \text { of exposure. }\end{array}$ \\
\hline 2011GG554B & 65.371887 & -152.977334 & 501054 & 7249710 & $5 \mathrm{~N}$ & $\begin{array}{l}\text { Quartz vein; perpendicular to foliation. Highly oxidized with red, yellow, and } \\
\text { orange oxides that fill vugs. Trace. }\end{array}$ \\
\hline 2011GG564A & 65.388016 & -152.962559 & 501740 & 7251508 & $5 \mathrm{~N}$ & $\begin{array}{l}\text { Schist; dark black with biotite, porphyroblasts or porphyroclasts of plagioclase, } \\
\text { and possible magnetite. } 10 \% \text { of exposure. }\end{array}$ \\
\hline 2011LF025B & 65.297826 & -153.124954 & 494173 & 7241461 & $5 \mathrm{~N}$ & $\begin{array}{l}\text { Clay gouge(?); gray, possibly weathered bedrock, which contains pyrite clasts } \\
\text { and crushed quartz. Representative grab sample of } 2 \text {-m-wide zone from bedrock } \\
\text { in Nugget Creek placer cut. } 95 \% \text { of exposure. }\end{array}$ \\
\hline 2011LF027B & 65.299307 & -153.124275 & 494205 & 7241626 & $5 \mathrm{~N}$ & $\begin{array}{l}\text { Quartz vein; white, sheared, and broken quartz vein with } 5 \% \text { micaceous } \\
\text { fragments. Most micas are foliated, but some are in boxwork and look } \\
\text { recrystallized. Chip sample is } 3 \mathrm{~m} \text { wide and oblique to vein. Vein width is } \\
\text { concealed. } 30 \% \text { of exposure. }\end{array}$ \\
\hline
\end{tabular}


Table 1. (continued)

\begin{tabular}{|c|c|c|c|c|c|c|}
\hline Sample_ID & Latitude & Longitude & Easting & Northing & $\begin{array}{l}\text { UTM_- } \\
\text { Zone }\end{array}$ & Lithology_Description \\
\hline 2011LF039B & 65.321290 & -152.717458 & 513164 & 7244100 & $5 \mathrm{~N}$ & $\begin{array}{l}\text { Gossan; } 15 \% \text { elongate slabs of gray, very-fine-grained quartz in an orange to } \\
\text { brown, fine-grained boxwork of iron oxide and altered carbonate(?). Grab } \\
\text { sample of single, } 0.5-\mathrm{m}^{3} \text { block. } 5 \% \text { of exposure. }\end{array}$ \\
\hline 2011LF040B & 65.319644 & -152.714921 & 513283 & 7243917 & $5 \mathrm{~N}$ & Graphite quartzite; $10 \%$ of exposure. \\
\hline 2011LF040C & 65.319644 & -152.714921 & 513283 & 7243917 & $5 \mathrm{~N}$ & $\begin{array}{l}\text { Graphite-mica quartzite; } 1-5 \% \text { cavities with pale iron oxide, either after pyrite } \\
\text { or carbonate. } 10 \% \text { of exposure. }\end{array}$ \\
\hline 2011LF070C & 65.451621 & -152.727938 & 512613 & 7258624 & $5 \mathrm{~N}$ & $\begin{array}{l}\text { Fault breccia; } 60-70 \% \text { quartzite and quartz phyllite clasts and strongly fractured } \\
\text { wall rock with } 30-40 \% \text { punky, yellow matrix. Clasts are displaced, angular, and } \\
\text { rotated in matrix. } 5 \% \text { of exposure. }\end{array}$ \\
\hline 2011LF081A & 65.441548 & -152.691883 & 514290 & 7257509 & $5 \mathrm{~N}$ & $\begin{array}{l}\text { Breccia; gossan-filled breccia with } 60 \% \text { angular, siliceous clasts (quartzite, quartz } \\
\text { schist, or silicified greenstone?) in a yellow to orange, limonitic gossan matrix. } \\
100 \% \text { of exposure. }\end{array}$ \\
\hline 2011LF100A & 65.500642 & -152.868155 & 506101 & 7264067 & $5 \mathrm{~N}$ & $\begin{array}{l}\text { Calc-silicate hornfels; pale to dark green with mineral alignment and fractures } \\
\text { that may be relict foliation. Minerals include } 50 \% 0.2-\mathrm{mm} \text {-long amphibole, } 20 \% \\
0.1-\mathrm{mm} \text {-wide diopside, and } 30 \% 0.1 \text {-mm plagioclase. Grab sample selected from } \\
\text { three boulders with strong iron oxide on fractures, local drusy quartz veins, and } \\
1 \% \text { gossanous patches. } 5 \% \text { of exposure. }\end{array}$ \\
\hline 2011LF101A & 65.502403 & -152.870783 & 505979 & 7264263 & $5 \mathrm{~N}$ & $\begin{array}{l}\text { Epidote-quartz-actinolite granofels; possibly skarn. Minerals include } 10 \% \\
\text { epidote, } 30 \% 0.5-\mathrm{mm} \text {, sugary quartz, } 60 \% 0.5-\mathrm{mm} \text {-long, acicular actinolite, and } \\
\text { trace scheelite on fractures. Rock also has strong, irregular veins of drusy quartz } \\
\text { and iron oxide. } 100 \% \text { of exposure. }\end{array}$ \\
\hline 2011LF119A & 65.431399 & -152.851411 & 506894 & 7256351 & $5 \mathrm{~N}$ & $\begin{array}{l}\text { Mica quartz schist; strong silicified tourmaline replacement along open, } \\
\text { moderately gossanous, drusy-quartz-filled gash fractures. Gash fractures are } \\
\text { oblique to foliation. } 5 \% \text { of exposure. }\end{array}$ \\
\hline 2011LF123A & 65.428775 & -152.866103 & 506213 & 7256057 & $5 \mathrm{~N}$ & $\begin{array}{l}\text { Hornfels(?); pale green to dark brown, banded rock. Bands are } 2-8 \mathrm{~mm} \text { thick. } \\
100 \% \text { of exposure. }\end{array}$ \\
\hline 2011LF128A & 65.427137 & -152.881454 & 505501 & 7255873 & $5 \mathrm{~N}$ & $\begin{array}{l}\text { Quartzite; sugary. Rock has micaceous partings, } 0.1 \% \text { tourmaline prisms, and } \\
\text { trace, } 5 \text {-cm-wide, foliation-parallel, open lenses filled with biotite and iron- } \\
\text { oxide. Selected sample of rocks with iron-oxide lenses. } 100 \% \text { of exposure. }\end{array}$ \\
\hline
\end{tabular}


Table 1. (continued)

\begin{tabular}{|c|c|c|c|c|c|c|}
\hline Sample_ID & Latitude & Longitude & Easting & Northing & $\begin{array}{l}\text { UTM_- } \\
\text { Zone }\end{array}$ & Lithology_Description \\
\hline 2011LF129A & 65.427301 & -152.884212 & 505373 & 7255891 & $5 N$ & $\begin{array}{l}\text { Mica quartz schist; light iron-oxide coating and local } 1 \mathrm{~cm} \times 2 \mathrm{~mm} \text { lenses of } \\
\text { goethite. Semi-random grab of pieces that are more stained with iron-oxide. } \\
100 \% \text { of exposure. }\end{array}$ \\
\hline 2011LF180B & 65.345109 & -152.702100 & 513867 & 7246758 & $5 \mathrm{~N}$ & $\begin{array}{l}\text { Quartz schist(?); strongly fractured and stained orange; iron oxide fills fractures. } \\
\text { Rock chips in overturned tree roots. } 20 \% \text { of exposure. }\end{array}$ \\
\hline 2011LF223A & 65.424623 & -152.524266 & 522078 & 7255671 & $5 \mathrm{~N}$ & $\begin{array}{l}\text { Greenstone; very fine grained, light green, bleached, and aphanitic. Rock has } 1 \% \\
\text { ragged pyrite or non-magnetic pyrrhotite and is possibly silicified. Random grab } \\
\text { sample of rubble. } 50 \% \text { of exposure. }\end{array}$ \\
\hline 2011LF261C & 65.330765 & -152.917852 & 503826 & 7245129 & $5 N$ & $\begin{array}{l}\text { Quartz vein; white and massive. Locally strongly fractured with open space and } \\
\text { moderate iron-oxide staining. } 30 \% \text { of exposure. }\end{array}$ \\
\hline 2011LF268A & 65.235238 & -152.765122 & 510979 & 7234500 & $5 \mathrm{~N}$ & $\begin{array}{l}\text { Quartzite; } 100 \% \text { gray, vitreous, } 0.5 \mathrm{~mm} \text { quartz. A } 1 \text {-cm-thick quartz vein cuts the } \\
\text { rock. Grab sample of vein and wall rock. } 50 \% \text { of exposure. }\end{array}$ \\
\hline 2011LF271A & 65.222230 & -152.718964 & 513143 & 7233059 & $5 N$ & $\begin{array}{l}\text { Schist; fine-grained with a color that varies from tan to a rusty, pale green. Rock } \\
\text { has } 10 \% 1 \mathrm{~mm} \text { to } 2 \mathrm{~cm} \text { cavities after carbonate. } 100 \% \text { of exposure. }\end{array}$ \\
\hline 2011LF276B & 65.341591 & -153.136439 & 493648 & 7246340 & $5 \mathrm{~N}$ & $\begin{array}{l}\text { Quartzite; white; strongly fracutred with } 1 \% \text { druzy vugs, } 1 \% 3-\mathrm{cm} \text {-wide oxidized } \\
\text { carbonate, and } 1 \% \text { colored sericite. Moderately stained with iron oxide on } \\
\text { fractures. Random grab sample. } 50 \% \text { of exposure. }\end{array}$ \\
\hline 2011LF297A & 65.455291 & -152.743670 & 511882 & 7259030 & $5 \mathrm{~N}$ & $\begin{array}{l}\text { Quartzite; }<15 \% \text { phyllitic mica in very-fine-grained partings. Rock is strongly } \\
\text { fractured with brown iron-oxide fracture coatings. Fractures contain localized } \\
\text { vugs filled with drusy, fine-grained quartz and localized pyrite pseudomorphs, } \\
\text { now brown oxide. Random sample of } 2 \times 2 \mathrm{~m} \text { area. } 100 \% \text { of exposure. }\end{array}$ \\
\hline 2011LF300A & 65.456970 & -152.749824 & 511596 & 7259216 & $5 \mathrm{~N}$ & $\begin{array}{l}\text { Quartz schist; strongly hornfelsed. Strong, 0.5- to } 2 \text {-cm-wide tourmaline veinlets } \\
\text { cut the rock, and strong tourmaline flooding occurs parallel to foliation. } \\
\text { Tourmaline color includes black, yellow, green, and red-brown. Rock contains } \\
\text { acicular, } 1-2-\mathrm{mm} \text {-long, moderately iron-oxide-filled cavities. Random sample of } \\
1 \times 1 \mathrm{~m} \text { area. } 5 \% \text { of exposure. }\end{array}$ \\
\hline 2011LF314A & 65.466000 & -152.663820 & 515577 & 7260241 & $5 N$ & $\begin{array}{l}\text { Quartz phyllite; very-fine-grained, gray to black chert-like quartz with white } \\
\text { phyllitic partings. Rock has minor localized green-blue stain. Selected sample of } \\
\text { stained rocks. } 100 \% \text { of exposure. }\end{array}$ \\
\hline
\end{tabular}


Table 1. (continued)

\begin{tabular}{|c|c|c|c|c|c|c|}
\hline Sample_ID & Latitude & Longitude & Easting & Northing & $\begin{array}{l}\text { UTM_- } \\
\text { Zone }\end{array}$ & Lithology_Description \\
\hline 2011LF317A & 65.469164 & -152.666391 & 515456 & 7260593 & $5 N$ & $\begin{array}{l}\text { Phyllitic quartzite; gray. Green copper mineral occurs extensively on fractures } \\
\text { and foliation within a } 20+m \text { area. Rock has rusty blotches on some fractures. } \\
100 \% \text { of exposure. }\end{array}$ \\
\hline 2011LF332A & 65.493425 & -152.720114 & 512955 & 7263285 & $5 \mathrm{~N}$ & $\begin{array}{l}\text { Biotite-quartz monzonite; coarse grained. Visible minerals include } 15 \% 1.5 \mathrm{~mm} \\
\text { quartz, } 20 \% 1.5 \mathrm{~cm} \text { potassium feldspar, } 20 \% 2 \mathrm{~mm} \text { biotite, and } 45 \% \text { feldspars in } \\
\text { groundmass. Rock contains minor quartz and aplite veinlets. Selected sample of } \\
\text { vein material. } 100 \% \text { of exposure. }\end{array}$ \\
\hline 2011LF345A & 65.452993 & -152.595282 & 518762 & 7258810 & $5 \mathrm{~N}$ & $\begin{array}{l}\text { Phyllitic greenstone; texture varies. Rock has abundant pale- and dark-green } \\
\text { chloritic and serpentine sheers. Contains moderate albite, quartz, and chlorite } \\
\text { veins, which are locally vuggy. Selected sample of vein material. } 10 \% \text { of } \\
\text { exposure. }\end{array}$ \\
\hline 2011LF365A & 65.299591 & -153.116513 & 494567 & 7241657 & $5 \mathrm{~N}$ & $\begin{array}{l}\text { Micaceous quartzite; light green. Rock has } 15 \% \text { mica, which is well foliated. A 5- } \\
\mathrm{cm} \text {-thick, wedge-shaped, irregular quartz vein with graphite/carbon and iron- } \\
\text { oxide selvage cuts rock. Selected sample of vein selvage and adjacent wall rock. } \\
100 \% \text { of exposure. }\end{array}$ \\
\hline 2011LF369B & 65.378777 & -152.870315 & 506029 & 7250484 & $5 \mathrm{~N}$ & $\begin{array}{l}\text { Quartz vein; white and elongate. Vein is up to } 3 \mathrm{~m} \text { thick and } 10 \mathrm{~m} \text { long. } 5 \% \text { of } \\
\text { exposure. }\end{array}$ \\
\hline 2011LF394A & 65.254317 & -153.015435 & 499279 & 7236606 & $5 \mathrm{~N}$ & $\begin{array}{l}\text { Carbonate-mica quartz schist; light gray to white. Rock contains quartz veins } \\
\text { parallel to } \mathrm{S} 1 \text {, which are isoclinally folded on } \mathrm{S} 2 \text {. The veins have random sericite } \\
\text { and } 10 \% \text { cavities (probably after carbonate). Fractures are slightly stained with } \\
\text { iron oxide. } 10 \% \text { of exposure. }\end{array}$ \\
\hline 2011LF401A & 65.246978 & -153.009246 & 499568 & 7235788 & $5 \mathrm{~N}$ & $\begin{array}{l}\text { Carbonate-mica quartz schist; gray. Visible minerals include } 5-30 \% 1 \mathrm{~mm} \text {, rusty, } \\
\text { orange- to brown-filled pits in irregular, wispy bands, } 20-50 \% \text { gray mica, } 50 \% \\
\text { quartz in some segregations and lensoidal veins. Selected sample of more pitted } \\
\text { rocks. } 100 \% \text { of exposure }\end{array}$ \\
\hline 2011LF408A & 65.253262 & -152.934705 & 503050 & 7236490 & $5 \mathrm{~N}$ & $\begin{array}{l}\text { Quartzite; strongly fractured. A 1-mm-thick iron-oxide/gossan coating covers } \\
\text { irregular fractures. Quartzite is moderately to locally-strongly bleached with a } \\
\text { black, } 30-\mathrm{cm} \text {-wide, sooty zone near the alteration. Representative gram sample } \\
\text { of } 30-\mathrm{cm} \text {-wide fractured, bleached material. } 100 \% \text { of exposure. }\end{array}$ \\
\hline 2011LF411B & 65.245959 & -152.917152 & 503871 & 7235677 & $5 \mathrm{~N}$ & Calc schist(?); punky, buff weathering rock. $90 \%$ of exposure. \\
\hline
\end{tabular}


Table 1. (continued)

\begin{tabular}{|c|c|c|c|c|c|c|}
\hline Sample_ID & Latitude & Longitude & Easting & Northing & $\begin{array}{l}\text { UTM_- } \\
\text { Zone }\end{array}$ & Lithology_Description \\
\hline 2011LF412B & 65.263746 & -152.834579 & 507724 & 7237667 & $5 \mathrm{~N}$ & $\begin{array}{l}\text { Quartz vein; strongly fractured with strong yellow, red, and orange gossan. Vein } \\
\text { is } 5-15 \mathrm{~cm} \text { wide and foliation-parallel with gossan on one vein margin. Wall rock } \\
\text { is bleached white. Selected sample of vein and gossan. } 5 \% \text { of exposure. }\end{array}$ \\
\hline 2011LF485A & 65.398017 & -152.635953 & 516912 & 7252671 & $5 \mathrm{~N}$ & $\begin{array}{l}\text { Micaceous quartzite; strongly oxidized with } 5 \% \text { gossanous (iron oxide), irregular, } \\
1-2 \mathrm{~mm} \text {, foliation-parallel cavities, } 15 \% \text { mica in } 1-3 \mathrm{~mm} \text { foliation planes, and } 1 \% \\
1 \mathrm{~mm}^{3} \text {, iron-oxide-filled cavities after pyrite. } 10 \% \text { of exposure. }\end{array}$ \\
\hline 2011LF498A & 65.414902 & -152.953625 & 502153 & 7254505 & $5 \mathrm{~N}$ & $\begin{array}{l}\text { Biotite-mica quartzite; visible minerals include } 10 \% 1 \mathrm{~mm} \text { muscovite in foliation } \\
\text { planes, } 5 \% 0.1 \mathrm{~mm} \text { disseminated biotite, } 1 \% \text { tourmaline on micaceous foliation } \\
\text { planes, and } 85 \% 0.2 \mathrm{~mm} \text { sugary quartz. A } 5 \mathrm{~mm} \text { feldspar-tourmaline vein cuts } \\
\text { the rock. } 100 \% \text { of exposure }\end{array}$ \\
\hline 2011LF502A & 65.208837 & -152.740436 & 512145 & 7231562 & $5 \mathrm{~N}$ & $\begin{array}{l}\text { Breccia; very strong iron oxide and weathering. Rock is a breccia of subangular } \\
\text { to subrounded quartzite and quartz schist clasts that are cemented with iron } \\
\text { oxide. Some clasts are completely leached of all quartz. } 100 \% \text { of exposure. }\end{array}$ \\
\hline 2011LF507A & 65.352262 & -152.681261 & 514833 & 7247560 & $5 \mathrm{~N}$ & $\begin{array}{l}\text { Quartzite; gray with } 5 \% \text { micaceous partings. Rock is strongly brecciated and } \\
\text { fractured. Yellow, orange, and red oxides from ground-up rock fill open spaces. } \\
100 \% \text { of exposure. }\end{array}$ \\
\hline 2011LF528A & 65.390001 & -152.661608 & 515725 & 7251771 & $5 \mathrm{~N}$ & $\begin{array}{l}\text { Quartzite; fine grained, gray and strongly fractured. Rock is moderately } \\
\text { brecciated and clast supported. Iron oxide fills fractures and makes up boxwork } \\
\text { matrix. } 100 \% \text { of exposure. }\end{array}$ \\
\hline 2011LF557B & 65.336904 & -152.484123 & 524021 & 7245909 & $5 \mathrm{~N}$ & Fault gouge; graphitic. $20 \%$ of exposure. \\
\hline 2011LF587B & 65.107451 & -154.735482 & 418494 & 7221357 & $5 \mathrm{~N}$ & $\begin{array}{l}\text { Granodiorite; visible minerals include } 30 \% \text { biotite, } 20 \% \text { quartz, } 50 \% \text { feldspar. } \\
\text { Pale-green, irregular, and anastomosing epidote veins up to } 3 \mathrm{~cm} \text { wide cut rock. } \\
\text { Strong dark-green chlorite, epidote, and quartz alteration. } 5 \% \text { of exposure. }\end{array}$ \\
\hline 2011RN153A & 65.246770 & -152.923806 & 503560 & 7235767 & $5 \mathrm{~N}$ & $\begin{array}{l}\text { Graphite-pyrite-muscovite quartzite; very fine to fine grained. Rock is }>95 \% \\
\text { quartz with spots weathered out to calcite (former pyrite?) along foliation } \\
\text { surfaces. Contains }<1 \% \text { graphite and } \sim 1 \% \text { former pyrite. } 100 \% \text { of exposure. }\end{array}$ \\
\hline
\end{tabular}


Table 1. (continued)

\begin{tabular}{|c|c|c|c|c|c|c|}
\hline Sample_ID & Latitude & Longitude & Easting & Northing & $\begin{array}{l}\text { UTM_- } \\
\text { Zone }\end{array}$ & Lithology_Description \\
\hline 2011RN177B & 65.404843 & -152.990224 & 500454 & 7253383 & $5 \mathrm{~N}$ & Quartz vein; $2-5 \mathrm{~mm}$ biotite. Vein is $1-2 \mathrm{~cm}$ thick. $<1 \%$ of exposure. \\
\hline 2011RN180B & 65.409032 & -152.981436 & 500862 & 7253850 & $5 \mathrm{~N}$ & $\begin{array}{l}\text { Skarn(?); possibly hornfels. Rock is dark green with some residual layering. Rock } \\
\text { is pyroxene rich. A high magnetic susceptibility suggests magnetite or pyrrhotite. } \\
2 \% \text { of exposure. }\end{array}$ \\
\hline 2011RN180C & 65.409032 & -152.981436 & 500862 & 7253850 & $5 \mathrm{~N}$ & $\begin{array}{l}\text { Quartz vein; } \pm \text { tourmaline. Vein has tourmaline sprays in massive quartz and is } \\
5-10 \mathrm{~cm} \text { thick. }<1 \% \text { of exposure. }\end{array}$ \\
\hline 2011Z005B & 65.390349 & -153.081709 & 496203 & 7251770 & $5 \mathrm{~N}$ & $\begin{array}{l}\text { Quartz-tourmaline-veined, biotite granite; medium-grained ( } 2-5 \mathrm{~mm} \text { ) and } \\
\text { weathered white to light gray. Broken rock is mottled white, gray, and pale } \\
\text { yellow with black biotite. Minerals include } 12-15 \% \text { black biotite, } 20 \% \text { gray, } \\
\text { irregular splotches and grains of quartz, and } \sim 0.5 \times 2 \mathrm{~cm} \text { white to slightly yellow- } \\
\text { stained feldspar phenocrysts. Tourmaline-bearing quartz veins and } \\
\text { tourmaline(?)-quartz-feldspar-pegmatitic veins cut rock and are up to } 4 \mathrm{~cm} \\
\text { wide. One pegmatite vein has elongate to equant, pale-rose crystals with black } \\
\text { tourmaline crystals. One pegmatite vein has black tourmaline crystals elongate } \\
\text { perpendicular to the granite/vein contact. Veins have rare vugs. Tourmaline } \\
\text { crystals are up to } 1 \mathrm{~mm} \times 1 \mathrm{~cm} \text {. Selected sample of veined material includes wall } \\
\text { rock. }\end{array}$ \\
\hline $2011 Z 012 E$ & 65.273176 & -152.987145 & 500600 & 7238708 & $5 \mathrm{~N}$ & $\begin{array}{l}\text { Quartz vein; } 1 \% \text { clear to white quartz vein fragments, some with orange stains } \\
\text { of iron oxide. } 1 \% \text { of exposure. }\end{array}$ \\
\hline 2011Z024A & 65.299073 & -153.124252 & 494206 & 7241600 & $5 \mathrm{~N}$ & $\begin{array}{l}\text { Quartz-white-mica schist; black, almost sooty, and sheared. Weak orange stain } \\
\text { of iron oxide on some fractures, and occasional open vugs in quartz-rich areas. } \\
\text { Rock is intermixed with some rounded cobbles. } 100 \% \text { of exposure. }\end{array}$ \\
\hline 2011Z026B & 65.354411 & -152.993488 & 500303 & 7247762 & $5 \mathrm{~N}$ & $\begin{array}{l}\text { Quartz vein; massive, milky-white quartz vein about } 60 \mathrm{~cm} \text { thick. Gray-white } \\
\text { mica and sometimes very weak iron-oxide stains fill occasional vugs. Minor } \\
\text { chlorite fracture fillings and chlorite occurs along the vein margin. Manganese } \\
\text { dendrites occur on some surfaces. } 5 \% \text { of exposure. }\end{array}$ \\
\hline
\end{tabular}


Table 1. (continued)

\begin{tabular}{|c|c|c|c|c|c|c|}
\hline Sample_ID & Latitude & Longitude & Easting & Northing & $\begin{array}{l}\text { UTM_- } \\
\text { Zone }\end{array}$ & Lithology_Description \\
\hline 2011Z028A & 65.350122 & -152.992716 & 500339 & 7247284 & $5 \mathrm{~N}$ & $\begin{array}{l}\text { White-mica-bearing quartzite; fine grained, slightly punky, possibly calcareous, } \\
\text { and weathers tan, yellow, and gray. Broken rock is light gray in places, but } \\
\text { mostly strongly stained with yellow, orange, and brown iron oxides. White mica } \\
\text { is gray and fine grained. Composition is between micaceous quartzite and } \\
\text { paragneiss. } 100 \% \text { of exposure. }\end{array}$ \\
\hline 2011Z029A & 65.348893 & -152.989815 & 500474 & 7247147 & $5 \mathrm{~N}$ & $\begin{array}{l}\text { Biotite-feldspar-white-mica quartz schist; fine grained and dark gray } \\
\text { weathering. Broken rock is light orange and does not react with } \mathrm{HCl} \text {. Contains } \\
\text { indistinct and/or contorted foliation. Minerals include fine-grained white } \\
\text { feldspar grains(?), up to } 5 \% \text { black biotite flakes in micaceous layers, and } \\
\text { occasional, well-formed, } 0.5 \text {-mm pyrite cubes that weather to vugs with orange } \\
\text { iron-oxide staining. } 100 \% \text { of exposure. }\end{array}$ \\
\hline 2011Z044A & 65.320560 & -152.751248 & 511590 & 7244012 & $5 \mathrm{~N}$ & $\begin{array}{l}\text { Quartz-veined, quartz-white-mica paragneiss; very fine grained and black with } \\
\text { minor iron-oxide staining. Rock is } 30 \% \text { white mica and has orangestains of iron } \\
\text { oxide on many foliation surfaces. Contains several white, sugary, very-fine- } \\
\text { grained, foliation-parallel quartz veins. A somewhat vuggy quartz vein with } \\
\text { anhedral to subhedral, white to clear quartz crystals and localized brown iron- } \\
\text { oxide earths cuts foliation. Random sample of vein material. } 90 \% \text { of exposure. }\end{array}$ \\
\hline 2011Z050A & 65.340941 & -152.840882 & 507408 & 7246270 & $5 \mathrm{~N}$ & $\begin{array}{l}\text { Quartz vein; very-fine- to fine-grained, angular, milky white boulders up to } 1 \mathrm{~m} \\
\text { in diameter. No obvious mineralization. Contains trace amounts of iron oxide } \\
\text { (orange limonite) as spots and streaks that generally (always?) have white mica } \\
\text { clots/inclusions. 95\% of exposure. }\end{array}$ \\
\hline 2011Z059A & 65.353086 & -152.822542 & 508258 & 7247626 & $5 \mathrm{~N}$ & $\begin{array}{l}\text { Quartz vein; milky-white, massive bull quartz cobbles and boulders up to } 70 \mathrm{~cm} \\
\text { in diameter. Contains very minor iron-oxide staining on some fractures and very } \\
\text { minor schist wall rock. Random sample over } \sim 10 \mathrm{~m} \text { area. } 20 \% \text { of exposure. }\end{array}$ \\
\hline 2011Z061A & 65.357539 & -152.817182 & 508506 & 7248123 & $5 \mathrm{~N}$ & $\begin{array}{l}\text { Quartz vein; milky-white, massive bull quartz vein approximately } 5 \mathrm{~m} \text { wide. No } \\
\text { iron-oxide staining and no obvious mineralization. Random sample over vein } \\
\text { area. } 100 \% \text { of exposure. }\end{array}$ \\
\hline
\end{tabular}


Table 1. (continued)

\begin{tabular}{|c|c|c|c|c|c|c|}
\hline Sample_ID & Latitude & Longitude & Easting & Northing & $\begin{array}{l}\text { UTM_- } \\
\text { Zone }\end{array}$ & Lithology_Description \\
\hline 2011Z072B & 65.446691 & -152.751324 & 511531 & 7258070 & $5 \mathrm{~N}$ & $\begin{array}{l}\text { Fault breccia; quartzite. Brown and orange iron-oxide-stained, matrix-supported } \\
\text { breccia with angular, gray quartzite clasts. Some cobbles have slickensides. } \\
\text { Clasts are subrounded to angular and vary from }<1 \mathrm{~mm} \text { to } 2 \mathrm{~cm} \text { in diameter. } \\
\text { Some pieces contain white quartz (shattered vein?) and vuggy spaces with } \\
\text { anhedral quartz. Abundant iron oxides in matrix. Rock does not react with } \mathrm{HCl} \text {. } \\
\text { Trace. }\end{array}$ \\
\hline 2011Z073A & 65.447313 & -152.747867 & 511691 & 7258140 & $5 \mathrm{~N}$ & $\begin{array}{l}\text { Graphite-white-mica quartzite; very fine grained, dark gray, and platy to flaggy. } \\
\text { Contains } 1 \% \text { graphite and } 12-15 \% \text { white mica. Outcrop extends over a } 10 \mathrm{~m}^{2} \\
\text { area. Occasional blue to sky-blue chrysocolla or turquoise crusts coat fracture } \\
\text { surfaces perpendicular to foliation planes, but never on foliation surfaces. These } \\
\text { crusts are sometimes found on fractures within rocks and occasionally have } \\
\text { cauliform and botryoidal shapes. Coatings aren't soluble in } \mathrm{HCl} \text {, with no copper } \\
\text { plating on steel. Selected sample of mineralized fractures and host quartzite. } \\
100 \% \text { of exposure. }\end{array}$ \\
\hline 2011Z075A & 65.450035 & -152.744907 & 511827 & 7258444 & $5 \mathrm{~N}$ & $\begin{array}{l}\text { White-mica quartzite; fine grained, gray, and flaggy. Many joint surfaces are } \\
\text { strongly iron-oxide stained dark brown, orange, and black (manganese oxide?). } \\
\text { Orange iron oxide is also on foliation surfaces. Contains } 15 \% \text { dark gray white } \\
\text { mica, possible graphite, and } 1-2 \% \text { foliation-parallel white quartz veins. Selected } \\
\text { sample of most iron-oxide stained rock. } 100 \% \text { of exposure. }\end{array}$ \\
\hline 2011Z078B & 65.453010 & -152.732238 & 512413 & 7258778 & $5 \mathrm{~N}$ & $\begin{array}{l}\text { Quartzite breccia; very-fine-grained, matrix-supported breccia consisting of } \\
\text { subrounded to angular quartzite clasts in an iron-oxide matrix with possible } \\
\text { crushed quartzite. Clasts are mostly } 0.5 \mathrm{~cm} \text { in diameter. } 1 \% \text { of exposure. }\end{array}$ \\
\hline 2011Z082B & 65.459858 & -152.723472 & 512816 & 7259543 & $5 \mathrm{~N}$ & $\begin{array}{l}\text { Quartzite; fine-grained, light gray, mottled rock with gray weathering. Contains } \\
\text { abundant open vugs partially filled with euhedral quartz crystals. Vugs and } \\
\text { fractures are stained tan and orange with iron oxide. One vug is filled with soft, } \\
\text { purple fluorite. No HCL reaction. } 1 \% \text { of exposure. }\end{array}$ \\
\hline
\end{tabular}


Table 1. (continued)

\begin{tabular}{|c|c|c|c|c|c|c|}
\hline Sample_ID & Latitude & Longitude & Easting & Northing & $\begin{array}{l}\text { UTM_- } \\
\text { Zone }\end{array}$ & Lithology_Description \\
\hline 2011Z088B & 65.462192 & -152.805253 & 509025 & 7259789 & $5 \mathrm{~N}$ & $\begin{array}{l}\text { Quartz vein; milky white, massive quartz-vein cobbles and boulders }<40 \mathrm{~cm} \times 20 \\
\mathrm{~cm} \times 30 \mathrm{~cm} \text {. Occasional black manganese coatings on fracture surfaces. } \\
\text { Common vugs with anhedral quartz coatings } \pm \text { black manganese masses and } \\
\text { black goethite. Weak iron-oxide staining on fractures. Trace. }\end{array}$ \\
\hline 2011Z093A & 65.466367 & -152.814653 & 508588 & 7260253 & $5 \mathrm{~N}$ & $\begin{array}{l}\text { Biotite granite; medium grained }(1-2 \mathrm{~mm}) \text { and equigranular. Broken surface is } \\
\text { cream to light orange with a common weathering rind stained with iron oxide. } \\
\text { Minerals include } 15 \% \text { black biotite, } 25 \% \text { gray quartz, and white feldspar. Rock } \\
\text { contains } 2 \text {-mm-wide quartz veinlets, one of which has a black, metallic, non- } \\
\text { magnetic mineral. } 100 \% \text { of exposure. }\end{array}$ \\
\hline 2011Z095A & 65.469621 & -152.819141 & 508379 & 7260615 & $5 \mathrm{~N}$ & $\begin{array}{l}\text { Biotite granite; coarse grained, pink, and porphyritic. Large feldspar and biotite } \\
\text { porphyritic phenocrysts set in a 1-2 mm matrix. Rock contains common black, } \\
\text { razor-thin }(<1 \mathrm{~mm}) \text { joints (probably) or fractures. Black, very-fine-grained } \\
\text { mineral (tourmaline } \pm \text { ?) fills fractures. Selected sample of granite with black } \\
\text { fracture fills collected over } 15 \mathrm{~m} \text { x } 5 \mathrm{~m} \text { area. } 100 \% \text { of exposure. }\end{array}$ \\
\hline 2011Z097A & 65.472232 & -152.826894 & 508019 & 7260905 & $5 \mathrm{~N}$ & $\begin{array}{l}\text { Quartz-tourmaline-fluorite-veined, biotite granite; medium grained ( } 1 \mathrm{~mm}) \text {; } \\
\text { tan, orange, and gray altered boulders with rough weathering. Broken rock is } \\
\text { light gray color with equigranular texture. Minerals include } 15 \% \text { biotite, } 20-25 \% \\
\text { quartz, rare purple fluorite masses with goethite and } \pm \text { quartz, and fine-grained } \\
\text { mica with greisen alteration. Rock is commonly iron-oxide-stained orange, } \\
\text { brown, and black (limonite, goethite } \pm \text { manganese oxide). Rock contains black, } \\
\text { tourmaline-filled (possibly), hairline fractures and some vuggy quartz veinlets (< } \\
1 \mathrm{~mm} \text { wide) with euhedral quartz crystal linings. No visible scheelite. Selected } \\
\text { sample of veined and strongly iron-oxide-stained granite. }\end{array}$ \\
\hline
\end{tabular}


Table 1. (continued)

\begin{tabular}{|c|c|c|c|c|c|c|}
\hline Sample_ID & Latitude & Longitude & Easting & Northing & $\begin{array}{l}\text { UTM_ } \\
\text { Zone }\end{array}$ & Lithology_Description \\
\hline 2011Z098A & 65.472829 & -152.823933 & 508156 & 7260972 & $5 \mathrm{~N}$ & $\begin{array}{l}\text { Altered and veined biotite granite; orange and black stained with abundant iron } \\
\text { oxide and manganese oxide in places. Some specimens have very-fine-grained, } \\
\text { almost web-like tourmaline veinlets. Occasional purple fluorite fillings with } \\
\text { tourmaline and and unknown mineral. No visible scheelite. Tourmaline almost } \\
\text { completely replaces the matrix in some cobbles, which have almost brecciated } \\
\text { appearances. } 100 \% \text { of exposure. }\end{array}$ \\
\hline 2011Z104A & 65.390872 & -152.942756 & 502660 & 7251827 & $5 \mathrm{~N}$ & $\begin{array}{l}\text { Quartz-veined, white-mica quartz schist; fine-grained, gray to dark gray rock } \\
\text { that weathers brownish to gray. Minerals include } 20 \% \text { dark-gray white mica, } \\
75-80 \% \text { quartz, and trace amounts of very-fine-grained, disseminated pyrite. } \\
\text { Rock does not react with } \mathrm{HCl} \text {. Contains } 2-3 \% 1 \text {-mm- to } 1 \text {-cm-thick, foliation- } \\
\text { parallel, white quartz veins. Some pieces have vuggy, open quartz veins with } \\
\text { orange iron-oxide (limonite) staining as well as euhedral to subhedral quartz } \\
\text { crystals. Selected sample of veined and iron-oxide-stained schist. } 90 \% \text { of } \\
\text { exposure. }\end{array}$ \\
\hline 2011Z107B & 65.398229 & -152.942762 & 502659 & 7252647 & $5 \mathrm{~N}$ & $\begin{array}{l}\text { Quartz vein; milky white quartz containing biotite inclusions with weak iron- } \\
\text { oxide staining and occasional vugs with subhedral to euhedral quartz crystals as } \\
\text { well as black and orange iron oxide and manganese oxide. One vug with } \\
\text { boxworks. Random sample over } 5 \text {-m-diameter area. Largest quartz cobble is } 10 \\
\mathrm{~cm} \times 6 \mathrm{~cm} \times 8 \mathrm{~cm} \text {. Trace. }\end{array}$ \\
\hline 2011Z111A & 65.405480 & -152.926231 & 503426 & 7253456 & $5 \mathrm{~N}$ & $\begin{array}{l}\text { Quartz-tourmaline vein; medium to coarse grained. White and black boulders } \\
\text { up to } 10 \mathrm{~cm} \times 25 \mathrm{~cm} \text {. Black tourmaline crystals up to } 15 \mathrm{~mm} \times 2 \mathrm{~mm} \text { comprise } \\
50-60 \% \text { of rock, and are commonly in sprays and rosettes. Later, thin }(1-2 \mathrm{~mm}) \\
\text { quartz veinlets cut quartz-tourmaline veins at } 90 \text { degrees in one boulder. Trace. }\end{array}$ \\
\hline
\end{tabular}


Table 1. (continued)

\begin{tabular}{|c|c|c|c|c|c|c|}
\hline Sample_ID & Latitude & Longitude & Easting & Northing & $\begin{array}{l}\text { UTM_- } \\
\text { Zone }\end{array}$ & Lithology_Description \\
\hline 2011Z116A & 65.413436 & -152.921772 & 503632 & 7254343 & $5 \mathrm{~N}$ & $\begin{array}{l}\text { Biotite-quartz-feldspar gneiss; possibly orthogneiss. Rock is medium grained } \\
\text { with a rough, light brownish-gray weathering surface that shows foliation. } \\
\text { Broken rock is dark gray and white with localized gneissic habit and is possibly } \\
\text { recrystallized. Rock contains } 25 \% \text { biotite, gray to brownish-gray feldspar and } \\
\text { quartz, and rare, milky white, foliation-parallel quartz veinlets. Selected sample } \\
\text { of most iron-oxide-stained rock. No vein material, but several pieces with open } \\
\text { vugs ( } 1-3 \mathrm{~mm} \text { across) with iron-oxide earths. } 100 \% \text { of exposure. }\end{array}$ \\
\hline 2011Z120A & 65.258635 & -152.752088 & 511578 & 7237110 & $5 \mathrm{~N}$ & $\begin{array}{l}\text { White-mica quartzite; fine grained and light orange to tan, slightly stained with } \\
\text { iron oxide (limonite). } 5 \% \text { white mica defines foliation. Selected sample of } \\
\text { material with foliation-parallel vuggy quartz vein }(0.5 \mathrm{~cm} \text { thick) with iron-oxide } \\
\text { staining and some orange iron-oxide earths in vugs. } 50 \% \text { of exposure. }\end{array}$ \\
\hline 2011Z121B & 65.261300 & -152.752277 & 511568 & 7237407 & $5 \mathrm{~N}$ & $\begin{array}{l}\text { Calc schist; fine grained and weathered brown. Broken rock is punky with } 60 \% \\
\text { dark-gray white mica and minor quartz. Grab sample. } 40 \% \text { of exposure. }\end{array}$ \\
\hline 2011Z130A & 65.250679 & -152.653288 & 516197 & 7236245 & $5 \mathrm{~N}$ & $\begin{array}{l}\text { White-mica-bearing quartzite; very fine grained and a light gray color to a } \\
\text { slightly iron-oxide-stained bright orange. } 5-7 \% \text { light gray white mica defines } \\
\text { faint foliation. Contains localized open spaces lined with drusy quartz crystals } \\
\text { and weak staining from iron oxide. Select sample. } 100 \% \text { of exposure. }\end{array}$ \\
\hline 2011Z135C & 65.250036 & -152.610378 & 518202 & 7236185 & $5 \mathrm{~N}$ & $\begin{array}{l}\text { Quartz vein; several cobbles up to } 3 \mathrm{~mm} \text { in diameter of light gray to white, } \\
\text { massive quartz vein with brown vugs filled with iron oxide. Several vugs have } \\
\text { square outlines. Trace. }\end{array}$ \\
\hline
\end{tabular}


Table 1. (continued)

\begin{tabular}{|c|c|c|c|c|c|c|}
\hline Sample_ID & Latitude & Longitude & Easting & Northing & $\begin{array}{l}\text { UTM_- } \\
\text { Zone }\end{array}$ & Lithology_Description \\
\hline 2011Z149A & 65.298478 & -152.586974 & 519260 & 7241591 & $5 \mathrm{~N}$ & $\begin{array}{l}\text { Quartz-veined, white-mica-bearing quartzite; very-fine- to fine-grained, orange- } \\
\text { brown to brown slabs and thin plates of rocks stained with iron oxide. } \mathrm{No} \mathrm{HCl} \\
\text { reaction. Broken rock and foliation surfaces contain } 20 \% \text { irregularly shaped, } \\
\text { orange pits and vugs stained with iron oxide. Broken rock is dark gray with } \\
\text { white, very-fine-grained, elongate mineral that flashes in the sun. Contains }<10 \% \\
\text { very-fine-grained, silvery gray white mica. Rock has vuggy open spaces } \\
\text { (veinlets?) with anhedral to subhedral fine-grained quartz-crystal linings. One } \\
\text { cobble has a slightly vuggy, 1-cm-wide, foliation-parallel, white quartz vein. } \\
100 \% \text { of exposure. }\end{array}$ \\
\hline 2011Z161B & 65.244856 & -153.598211 & 472048 & 7235684 & $5 \mathrm{~N}$ & $\begin{array}{l}\text { Skarn; skarnoid. Very-fine- and coarse-grained rock with greenish weathering. } \\
\text { Broken rock is dark green with locally folded light green, dark green, and gray } \\
\text { bands (strong). Rock has localized moderate reactions to } \mathrm{HCl} \text {. Minerals include } \\
\text { epidote, carbonate, amphibole, clinopyroxene(?), and iron oxide } \pm \text { garnet. Large, } \\
2 \text {-cm-long, folded, fibrous amphibole appears to be in secondary veins. Selected } \\
\text { sample of skarn with quartz-amphibole-sulfide veins and orange iron oxide. } \\
\text { Veins have open spaces. Sulfide is black, resinous sphalerite with very-fine- } \\
\text { grained pyrite. Rock contains } 1 \% \text { brownish garnet and } 0.5 \% \text { manganese. } 40 \% \text { of } \\
\text { exposure. }\end{array}$ \\
\hline 2011Z162B & 65.246636 & -153.597459 & 472085 & 7235882 & $5 \mathrm{~N}$ & $\begin{array}{l}\text { Quartz vein; } 0.5 \mathrm{~m} \text { wide, orange, brown, and white. Vein is locally shattered with } \\
\text { moderately steep shearing. Broken rock is milky white with some included white } \\
\text { mica near host contact. Some fracture surfaces contain brown and orange iron- } \\
\text { oxide earths. Grab sample. Trace. }\end{array}$ \\
\hline $2011 Z 162 C$ & 65.246636 & -153.597459 & 472085 & 7235882 & $5 \mathrm{~N}$ & $\begin{array}{l}\text { Quartz vein; white, orange, and brown. Vein has a maximum width of } 8 \mathrm{~cm} \text {. } \\
\text { Contains subangular quartz crystals up to } 3 \mathrm{~cm} \text { long. Brown iron-oxide earths } \\
\text { completely fill some very abundant open spaces. Occasional boxworks. Trace. }\end{array}$ \\
\hline
\end{tabular}


Table 1. (continued)

\begin{tabular}{|c|c|c|c|c|c|c|}
\hline Sample_ID & Latitude & Longitude & Easting & Northing & $\begin{array}{l}\text { UTM_- } \\
\text { Zone }\end{array}$ & Lithology_Description \\
\hline 2011Z163B & 65.217375 & -153.496507 & 476776 & 7232580 & $5 N$ & $\begin{array}{l}\text { Quartz vein; white and orange, vuggy quartz veins, } 1 \text { to } 5 \mathrm{~cm} \text { thick, that cut } \\
\text { foliation at a high angle. Iron-oxide earths commonly fill vugs. Rock contains up } \\
\text { to } 1 \% \text { black oxide earths with small, brassy sulfide specks (chalcopyrite?). No } \\
\text { plating of copper on steel knife blade when soaked with } \mathrm{HCl} \text {. One quartz vein } \\
\text { has vein-parallel growth banding. Selected sample of quartz vein stained with } \\
\text { iron oxide. Trace. }\end{array}$ \\
\hline 2011Z166B & 65.452720 & -152.571084 & 519884 & 7258787 & $5 \mathrm{~N}$ & $\begin{array}{l}\text { Quartz vein; milky white, occasionally vuggy quartz veins up to } 4 \mathrm{~cm} \text { wide. Veins } \\
\text { incorporate pieces of greenstone wall rock. Occasionally iron oxides weakly } \\
\text { stain vein margins and partially to completely fill vugs with orange material. } \\
\text { Random sample over } 5 \mathrm{~m} \times 30 \mathrm{~m} \text { area. }<1 \% \text { of exposure. }\end{array}$ \\
\hline 2011Z174C & 65.441179 & -152.566744 & 520094 & 7257502 & $5 N$ & $\begin{array}{l}\text { Quartz vein; vuggy, milky white quartz veins up to } 5 \mathrm{~cm} \text { thick. Anhedral quartz } \\
\text { crystals line some vugs. Random sample collected over } 15 \text {-m-diameter area. } \\
50 \% \text { of exposure. }\end{array}$ \\
\hline 2011Z205B & 65.341598 & -152.981871 & 500844 & 7246334 & $5 \mathrm{~N}$ & $\begin{array}{l}\text { Quartz vein; milky white, and up to } 20 \mathrm{~cm} \text { wide. Mostly massive, anhedral } \\
\text { quartz with occasional vugs stained by orange and brown iron oxides. Subhedral } \\
\text { to anhedral, } \mathrm{mm} \text {-size quartz crystals sometime line vugs. Random sample. Trace. }\end{array}$ \\
\hline 2011Z245A & 65.495795 & -152.899336 & 504659 & 7263524 & $5 \mathrm{~N}$ & $\begin{array}{l}\text { Quartzite; gray-brown, banded, and possibly hornfels. Broken rock has mottled } \\
\text { appearance with a green cast in places, but mostly dark gray and gray with some } \\
\text { orange bands. Rock is moderately stained orange, black, and brown from iron } \\
\text { oxide. It contains occasional } 2-4 \text { mm patches of fine-grained black biotite, } 1 \% \\
\text { milky white quartz veins, and probably localized magnetite (inferred from high } \\
\text { magnetic susceptibility in some spots). Selected sample of quartz-veined } \\
\text { quartzite, and at least one piece of quartz vein that cuts foliation. } 90 \% \text { of } \\
\text { exposure. }\end{array}$ \\
\hline 2011Z246A & 65.495106 & -152.902471 & 504514 & 7263447 & $5 \mathrm{~N}$ & $\begin{array}{l}\text { Mica quartzite; fine grained, and stained orange with iron oxides. Broken rock is } \\
\text { somewhat banded dark gray to black. Possible biotite development and changes } \\
\text { in quartz colors suggest possible contact metamorphism. Rock contains some } \\
\text { open vugs with iron-oxide earths (limonite and goethite). }<1 \% \text { milky white } \\
\text { quartz vein is visible. Random sample over } 7 \text {-m-diameter area excludes quartz } \\
\text { vein. } 100 \% \text { of exposure. }\end{array}$ \\
\hline
\end{tabular}


Table 1. (continued)

\begin{tabular}{|c|c|c|c|c|c|c|}
\hline Sample_ID & Latitude & Longitude & Easting & Northing & $\begin{array}{l}\text { UTM_- } \\
\text { Zone }\end{array}$ & Lithology_Description \\
\hline $2011 Z 260 C$ & 65.186690 & -153.892286 & 458216 & 7229364 & $5 \mathrm{~N}$ & $\begin{array}{l}\text { Quartz vein; gray and somewhat glassy, 1-cm-wide quartz vein. Quartz is } \\
\text { aphanitic with no vugs. Sample is a mix of quartz vein and quartz monzonite wall } \\
\text { rock. Trace. }\end{array}$ \\
\hline 2011Z271B & 65.319730 & -153.516055 & 475955 & 7243995 & $5 \mathrm{~N}$ & $\begin{array}{l}\text { Quartz vein; white and clear with strong orange, brown, and black stains of iron } \\
\text { oxide on fractures. Contains abundant vugs and open spaces with iron-oxide } \\
\text { earths and subhedral to euhedral, fine-grained, quartz-crystal linings. Rock has } \\
\text { minor boxworks and occasional manganese dendrites. Rock is highly fractured } \\
\text { and does not react with } \mathrm{HCl} \text {. No visible scheelite when lamped. Grab sample. } \\
\text { Trace. }\end{array}$ \\
\hline 2011Z302A & 65.470603 & -152.693527 & 514198 & 7260747 & $5 N$ & $\begin{array}{l}\text { Micaceous quartzite; very-fine-grained, blocky, black and white rock. Broken } \\
\text { rock is also black and white with abundant orange and black stains from iron } \\
\text { oxide on fractures. Minerals include } 10 \% \text { mica and } 90 \% \text { very-fine-grained, sugary } \\
\text { quartz. Contains occasional, very-fine-grained, sometimes square vugs (pyrite } \\
\text { casts?). Rock does not react with } \mathrm{HCl} \text {. Random sample of limited rock at surface. } \\
100 \% \text { of exposure. }\end{array}$ \\
\hline 2011Z309A & 65.480956 & -152.671726 & 515202 & 7261906 & $5 \mathrm{~N}$ & $\begin{array}{l}\text { Quartzite; fine grained and sugary with light orange, brown, and gray } \\
\text { weathering. Broken rock is generally vuggy ( } \leq 2 \mathrm{~mm} \text { ) with moderate to strong } \\
\text { orange and brown stains from iron oxide. Some very-fine-grained vugs have } \\
\text { square outlines (after pyrite?). Locally, rock is banded with minor white mica on } \\
\text { band surfaces. Bands are locally folded. One boulder contains a 1-cm-wide, } \\
\text { translucent, white quartz vein. Selected sample of most iron-oxide-stained } \\
\text { quartzite. } 100 \% \text { of exposure. }\end{array}$ \\
\hline 2011Z312A & 65.485950 & -152.666220 & 515454 & 7262464 & $5 \mathrm{~N}$ & $\begin{array}{l}\text { Quartzite; very-fine-grained, blocky rock that weathers tan, brown, and gray } \\
\text { with iron-oxide staining as well as localized light gray to bluish-green copper } \\
\text { staining. Broken rock is massive with faint gray and light gray bands. Light, pale- } \\
\text { green turquoise with staining coats of iron oxide on some surfaces. Some } \\
\text { weathered surfaces have caliche-like coatings. Rock does not react with } \mathrm{HCl} \text {. } \\
\text { Contains occasional fine-grained vugs and one } 0.5-\mathrm{cm} \text {-wide quartz vein. There is } \\
\text { a 3-m-diameter area of copper-stained rocks. Selected sample of quartzite with } \\
\text { copper staining. } 100 \% \text { of exposure. }\end{array}$ \\
\hline
\end{tabular}


Table 1. (continued)

\begin{tabular}{|c|c|c|c|c|c|c|}
\hline Sample_ID & Latitude & Longitude & Easting & Northing & $\begin{array}{l}\text { UTM_- } \\
\text { Zone }\end{array}$ & Lithology_Description \\
\hline 2011Z314A & 65.487987 & -152.662414 & 515629 & 7262692 & $5 \mathrm{~N}$ & $\begin{array}{l}\text { Quartzite; very-fine-grained, pale green to gray, massive, highly fractured, } \\
\text { angular boulders that are pale gray to gray. A soft, brown and orange iron-oxide- } \\
\text { stained, occasionally pale green mineral (wavellite) coats fractures. A soft, white } \\
\text { to cream-tan, fibrous mineral (not calcite) fills some fractures. This fibrous } \\
\text { mineral has crystal fibers perpendicular to the vein wall, and may be scapolite or } \\
\text { wollastonite. Random grab sample over } 2 \text {-m area. } 100 \% \text { of exposure. }\end{array}$ \\
\hline 2011Z316A & 65.491543 & -152.659581 & 515758 & 7263089 & $5 \mathrm{~N}$ & $\begin{array}{l}\text { Quartzite; very-fine-grained, sugary, platy rock that weathers light tan to cream. } \\
\text { Light orange and orange iron oxides (limonite) commonly stain rock. Contains } \\
5 \% \text { dark gray to black phyllitic partings and one thin quartz veinlet. Sky blue to } \\
\text { bluish-green turquoise thinly coats some fracture surfaces. Random sample. } \\
100 \% \text { of exposure. }\end{array}$ \\
\hline 2011Z345B & 65.358387 & -152.853866 & 506799 & 7248213 & $5 \mathrm{~N}$ & $\begin{array}{l}\text { Cataclasite; medium-grained, subrounded cobbles that are strongly stained } \\
\text { orange and brown from iron oxides (limonite } \pm \text { unkown mineral). Contains } 40 \% \\
\text { orange and brown iron oxides and } 60 \% \text { pale green to greenish gray, } \sim 1 \mathrm{~mm} \\
\text { chlorite grains. Rock does not react with } \mathrm{HCl} \text {. One cobble has a foliation-parallel } \\
\text { quartz vein. Cut rock has cataclastic texture with white, } \leq 1 \mathrm{~cm} \times 6 \mathrm{~mm} \text {, } \\
\text { subrounded quartz clasts in a schistose matrix extremely stained with iron } \\
\text { oxide. } 20 \% \text { of exposure. }\end{array}$ \\
\hline 2011Z349A & 65.360504 & -152.844933 & 507214 & 7248450 & $5 \mathrm{~N}$ & $\begin{array}{l}\text { Gabbro dike(?); fine-grained, non-foliated, granular, blocky, green rock with a } \\
\text { brown weathering rind. Broken rock is green to dark green with pale brown } \\
\text { splotches. Rock has a weak reaction to } \mathrm{HCl} \text {. Contains } 1 \% \text { pyrrhotite as } \leq 1 \mathrm{~mm} \text { in } \\
\text { diameter, disseminated grains and discontinuous veinlets. Minerals include } \\
40-50 \% \text { white to light tan, irregularly shaped feldspar(?) and } 50-60 \% \text { green } \\
\text { chlorite + an unkown mineral. Possible hairline white quartz veinlets. } 100 \% \text { of } \\
\text { exposure. }\end{array}$ \\
\hline $2011 Z 362 A$ & 65.300994 & -153.112380 & 494760 & 7241813 & $5 \mathrm{~N}$ & $\begin{array}{l}\text { Quartz vein; white, foliation-parallel quartz veins in micaceous quartzite with } \\
\text { some euhedral, clear quartz crystals. Select sample. } 100 \% \text { of exposure. }\end{array}$ \\
\hline
\end{tabular}


Table 1. (continued)

\begin{tabular}{|c|c|c|c|c|c|c|}
\hline Sample_ID & Latitude & Longitude & Easting & Northing & $\begin{array}{l}\text { UTM_- } \\
\text { Zone }\end{array}$ & Lithology_Description \\
\hline 2011Z366B & 65.373707 & -152.891480 & 505046 & 7249917 & $5 \mathrm{~N}$ & $\begin{array}{l}\text { Quartz vein; white vein with common yellow, light brown, and brown iron-oxide } \\
\text { staining. Commonly vuggy with clear, euhedral quartz crystals partially filling } \\
\text { vugs. Occasional boxworks and } \leq 1-\mathrm{mm} \text {-thick patches of brown iron oxide. } \\
\text { Contains rare, pale green chlorite included in quartz, and more commonly along } \\
\text { vein margin. Trace. }\end{array}$ \\
\hline 2011Z380A & 65.258591 & -152.957411 & 501989 & 7237083 & $5 \mathrm{~N}$ & $\begin{array}{l}\text { Chlorite-feldspar schist; platy cobbles. Broken rock is strongly weathered } \pm \\
\text { altered. Iron oxide moderately to strongly stains rock. Grab sample. } 100 \% \text { of } \\
\text { exposure. }\end{array}$ \\
\hline 2011Z382A & 65.255934 & -152.953689 & 502163 & 7236787 & $5 \mathrm{~N}$ & $\begin{array}{l}\text { Carbonate-white-mica quartz schist; strongly stained with iron } \\
\text { oxide(weathering?) with } 15-20 \% \text { porphyroblastic, orange spots of iron oxide. } \\
\text { Contains one foliation-parallel, white quartz vein. Grab sample of all pebbles and } \\
\text { cobbles found. } 100 \% \text { of exposure. }\end{array}$ \\
\hline 2011Z383B & 65.254238 & -152.952279 & 502229 & 7236598 & $5 \mathrm{~N}$ & $\begin{array}{l}\text { Quartz-veined, chlorite schist; iron oxide weakly to moderately stains } \\
\text { weathered surfaces. Broken rock is dull greenish-gray with pale gray and green } \\
\text { mica. Possibly contains subequal amounts of quartz and chlorite or white mica. } \\
\text { Rock has both foliation-parallel and crosscutting milky white to slightly } \\
\text { translucent quartz veins } \leq 2 \mathrm{~cm} \text { wide. Minor iron oxide along vein margins. Select } \\
\text { sample of quartz-veined material. } 30 \% \text { of exposure. }\end{array}$ \\
\hline 2011Z384A & 65.253806 & -152.947570 & 502449 & 7236550 & $5 \mathrm{~N}$ & $\begin{array}{l}\text { Quartz-veined quartzite; fine-grained, isoclinally folded, dark gray to gray, } \\
\text { pitted, micaceous quartzite with iron oxide weakly staining foliation surfaces } \\
\text { and moderately staining fracture surfaces orange and brown (limonite). } \\
\text { Contains foliation-parallel white quartz veins } \leq 3 \mathrm{~mm} \text { thick. Quartz is massive to } \\
\text { anhedral and generally slightly stained with iron oxide, but occasionally } \\
\text { translucent to clear. Selected sample of quartz veins and quartz-veined } \\
\text { quartzite. } 100 \% \text { of exposure. }\end{array}$ \\
\hline
\end{tabular}


Table 1. (continued)

\begin{tabular}{|c|c|c|c|c|c|c|}
\hline Sample_ID & Latitude & Longitude & Easting & Northing & $\begin{array}{l}\text { UTM_- } \\
\text { Zone }\end{array}$ & Lithology_Description \\
\hline 2011Z385A & 65.254046 & -152.942860 & 502669 & 7236577 & $5 N$ & $\begin{array}{l}\text { Greenstone; possibly chloritized gabbro (?). Very angular, fine-grained, and dark- } \\
\text { brown weathering rock. Broken rock has a mottled texture ranging in color from } \\
\text { green to dark green with orange spots of iron oxide. Brown and reddish-orange } \\
\text { iron oxides moderately to strongly stain abundant fracture surfaces. Locally, } \\
\text { rock has a green, waxy to fibrous habit (talc or serpentine?). Spots of iron oxide } \\
\text { are sometimes vuggy with occasional fine-grained, disseminated pyrrhotite in } \\
\text { the center. Rock has no } \mathrm{HCl} \text { reaction. } 100 \% \text { of exposure. }\end{array}$ \\
\hline 2011Z389A & 65.254056 & -152.871505 & 506002 & 7236583 & $5 \mathrm{~N}$ & $\begin{array}{l}\text { Quartz-calcite-veined, graphite-carbonate-mica quartzite; very fine grained. } \\
\text { Rock contains } 5 \% \text { orange spots of iron oxide (limonite) after carbonate, } 1 \% \\
\text { brown to orange, cubic casts of iron oxide (after pyrite?), and occasional (1\%) } \\
\text { layers of graphite. Contains two types of veins, both } \leq 1 \mathrm{~cm} \text { thick : (1) milky } \\
\text { white, foliation-parallel quartz veins with } \leq 2-\mathrm{mm} \text {-in-diameter spots of iron oxide } \\
\text { that make up } 1 \% \text { of rock; and ( } 2 \text { ) vuggy, orange and white quartz-carbonate (?) } \\
\text { veins that cross-cut foliation. Subhedral, white to translucent quartz crystals } \\
\text { occur in vugs. Rock does not react with } \mathrm{HCl} \text {. Selected sample of veined quartzite. } \\
100 \% \text { of exposure. }\end{array}$ \\
\hline 2011Z393A & 65.247940 & -152.864536 & 506329 & 7235902 & $5 \mathrm{~N}$ & $\begin{array}{l}\text { Quartz-veined, chlorite-carbonate-white-mica quartz schist; medium-grained } \\
\text { and tan to light brown, platy to angular rock. Iron oxides moderatly stain } \\
\text { fracture, weathering, and foliation surfaces orange, brown, and black. White to } \\
\text { silvery gray white mica comprises } 35-40 \% \text { of rock and is relatively coarse } \\
\text { grained. Minerals include almost pure white quartz, } 2-4 \% \text { orange spots of iron } \\
\text { oxide (limonite?), and irregular patches of dark green, locally aligned chlorite on } \\
\text { foliation surfaces. Rock does not react with } \mathrm{HCl} \text {. Iron oxide stains irregularly } \\
\text { shaped vugs with drusy quartz crystals. Rock looks recrystallized. Several areas } \\
\text { of drill pad contain milky white to slightly iron-oxide-stained quartz veins that } \\
\text { are broken into pieces } \leq 4 \mathrm{~cm} \text { in shortest dimension. Several cobbles have } \leq 1-\mathrm{cm} \text { - } \\
\text { wide foliation-crosscutting quartz veins. Random sample of rocks stained with } \\
\text { iron oxide over a } 3 \mathrm{~m} \text { by } 6 \mathrm{~m} \text { area. } 100 \% \text { of exposure. }\end{array}$ \\
\hline
\end{tabular}


Table 1. (continued)

\begin{tabular}{|c|c|c|c|c|c|c|}
\hline Sample_ID & Latitude & Longitude & Easting & Northing & $\begin{array}{l}\text { UTM_- } \\
\text { Zone }\end{array}$ & Lithology_Description \\
\hline 2011Z395C & 65.244636 & -152.862584 & 506421 & 7235534 & $5 \mathrm{~N}$ & $\begin{array}{l}\text { Quartz-veined quartzite; selected sample of iron-oxide-stained quartzite and } \\
\text { schist. One piece with } 2 \text {-cm-thick glassy quartz vein containing a black, oxidized } \\
\text { pyrite(?) grain. Trace. }\end{array}$ \\
\hline 2011Z399A & 65.243818 & -152.860855 & 506502 & 7235443 & $5 \mathrm{~N}$ & $\begin{array}{l}\text { Carbonate(?)-white-mica-quartz paragneiss; blocky to semischistose with light } \\
\text { tan weathering and crenulated foliation. Contains subequal amounts of white } \\
\text { quartz and gray white mica as well as } 20 \% \text { orange spots of iron oxide (after } \\
\text { carbonate or pyrite?). Rock is moderately to strongly stained by iron oxide. } \\
\text { Ragged vugs occur on foliation surfaces (after carbonate or sulfides?). One } \\
\text { quartz vein present. } 100 \% \text { of exposure. }\end{array}$ \\
\hline 2011Z403A & 65.246659 & -152.866383 & 506243 & 7235759 & $5 \mathrm{~N}$ & $\begin{array}{l}\text { Quartz-veined quartzite; fine to medium grained with tan to light-brown } \\
\text { weathering. Broken rock is light gray and tan. Flaky white mica varies from light } \\
\text { gray to silvery gray in color and } 3-15 \% \text { in abundance. Rock varies from very } \\
\text { blocky to semischistose. Rock commonly has squarish to rhombic(?), } 1 \mathrm{~mm} \text { vugs } \\
\text { that comprise up to } 15 \% \text { of foliation surfaces. Some vugs contain remnant } \\
\text { orange and brown iron oxide (limonite } \pm \text { goethite). Some vugs may be a result of } \\
\text { altered or replaced carbonate. White quartz veins up to } 1 \mathrm{~cm} \text { wide comprise } \\
1-2 \% \text { of rock. Quartz veins cross-cut rock and vary from massive to slightly } \\
\text { vuggy. Subhedral white to translucent quartz crystals and weak iron-oxide } \\
\text { staining line vugs. Random sample that includes quartz-veined and non-quartz- } \\
\text { veined rocks. } 100 \% \text { of exposure. }\end{array}$ \\
\hline 2011Z407A & 65.242873 & -152.867215 & 506205 & 7235337 & $5 \mathrm{~N}$ & $\begin{array}{l}\text { Carbonate(?)-white-mica quartz schist and micaceous quartzite; fine grained, } \\
\text { moderately stained with iron oxide, and platy with light tan to light brown } \\
\text { weathering. Broken rock is white to light gray in color with } 30 \% \text { orange, vuggy } \\
\text { spots of iron oxide (limonite) and lenses (carbonate or oxidized sulfides?). Rock } \\
\text { does not react with } \mathrm{HCl} \text {. Rock is schistose and possibly sericitized and/or } \\
\text { bleached. Contains more quartz than white mica. Random sample of quartz } \\
\text { schist and quartzite. Quartzite is fine grained with tan to light brown } \\
\text { weathering. Broken quartzite is white to light gray with } 5-10 \% \text { white to silver- } \\
\text { gray white mica. Iron oxide stains the overall rock. One } 0.5-\mathrm{cm} \text {-thick, white, } \\
\text { foliation-parallel quartz vein is visible. } 90 \% \text { of exposure. }\end{array}$ \\
\hline
\end{tabular}


Table 1. (continued)

\begin{tabular}{|c|c|c|c|c|c|c|}
\hline Sample_ID & Latitude & Longitude & Easting & Northing & $\begin{array}{l}\text { UTM_- } \\
\text { Zone }\end{array}$ & Lithology_Description \\
\hline 2011Z408A & 65.242817 & -152.864840 & 506316 & 7235331 & $5 N$ & $\begin{array}{l}\text { Quartz-veined quartzite; fine grained with tan and light yellowish-brown } \\
\text { weathering. Weak iron oxide stains on foliation surfaces. Broken rock is white to } \\
\text { light gray with }<10 \% \text { silvery-gray to gray-white mica. Some rock is strongly } \\
\text { stained orange and dark brown from iron oxide (limonite and goethite?). } \\
\text { Contains square pyrite (?) molds } \leq 15 \mathrm{~mm} \text { across. Several pieces contain white, } \\
\text { slightly vuggy quartz veins. Vugs contain sericite. Selected sample of most iron- } \\
\text { oxide-stained quartzite. } 100 \% \text { of exposure. }\end{array}$ \\
\hline 2011Z408B & 65.242817 & -152.864840 & 506316 & 7235331 & $5 N$ & $\begin{array}{l}\text { Fault(?) breccia; dark cobble with angular quartz } \pm \text { quartzite in clasts } \leq 3 \mathrm{~cm} \text { long } \\
\text { and as small as sand grains. A somewhat vuggy iron-oxide matrix that contains } \\
\text { some orange sericite. Euhedral quartz crystals partially fill some vugs in matrix. } \\
\text { Trace. }\end{array}$ \\
\hline 2011Z425C & 65.458074 & -152.635054 & 516915 & 7259365 & $5 N$ & $\begin{array}{l}\text { Quartz vein; vuggy white quartz-vein pebbles with vugs weakly stained by iron } \\
\text { oxide and filled with subhedral quartz crystals. Trace. }\end{array}$ \\
\hline 2011Z426A & 65.456474 & -152.637535 & 516801 & 7259186 & $5 \mathrm{~N}$ & $\begin{array}{l}\text { Quartzite; dark gray and white phyllitic quartzite that sometimes has } \\
\text { disseminated, iron-oxidized to slightly oxidized pyrite cubes } \leq 9 \mathrm{~mm}^{3} \text {. Selected } \\
\text { sample of pyritic quartzite. }\end{array}$ \\
\hline 2011Z437B & 65.376905 & -152.928828 & 503309 & 7250271 & $5 \mathrm{~N}$ & $\begin{array}{l}\text { Quartz vein; occasionally vuggy quartz vein weakly stained with iron oxide. } \\
\text { Trace. }\end{array}$ \\
\hline 2011Z441B & 65.372953 & -152.939892 & 502795 & 7249830 & $5 \mathrm{~N}$ & $\begin{array}{l}\text { Quartz vein; isoclinally folded, } 0.5-\mathrm{cm} \text {-thick vein with small vugs and abundant } \\
\text { staining from iron oxide. Vugs have } 1-2 \mathrm{~mm} \text { anhedral to subhedral white quartz } \\
\text { crystals. Orange and brown iron oxide also moderately to strongly stains } \\
\text { fractures through and around the vein. Selected sample of iron-oxide-stained } \\
\text { quartz vein and wall rock. Trace. }\end{array}$ \\
\hline 2011Z448B & 65.347432 & -152.675647 & 515097 & 7247023 & $5 \mathrm{~N}$ & $\begin{array}{l}\text { Fault breccia; black and orange, angular cobble. Strongly stained brown, orange, } \\
\text { and black with iron oxide. Contains angular quartzite clasts from } 0.05-\mathrm{mm} \text { to } 2- \\
\mathrm{cm} \text { in diameter. The vuggy matrix contains iron oxide } \pm \text { manganese oxide and } \\
\text { fine-grained silica. Vugs are abundant and small. } 10 \% \text { of exposure. }\end{array}$ \\
\hline 2011Z455C & 65.340905 & -152.653733 & 516121 & 7246301 & $5 \mathrm{~N}$ & $\begin{array}{l}\text { Quartz vein; white and varying from massive to almost chalcedonic. Contains 1 } \\
\mathrm{cm} \text { boxwork vugs. Overall, iron oxide weakly stains rock. Trace. }\end{array}$ \\
\hline
\end{tabular}


Table 1. (continued)

\begin{tabular}{|c|c|c|c|c|c|c|}
\hline Sample_ID & Latitude & Longitude & Easting & Northing & $\begin{array}{l}\text { UTM_- } \\
\text { Zone }\end{array}$ & Lithology_Description \\
\hline 2011Z480A & 65.376349 & -152.806426 & 509000 & 7250221 & $5 \mathrm{~N}$ & $\begin{array}{l}\text { Quartz-carbonate-white-mica-pyrite-cassiterite(?) vein; heavy, dark brown } \\
\text { and gray pebbles of quartz, sparry carbonate, silvery white mica, brassy pyrite } \\
\text { cubes } \leq 2 \mathrm{~mm} \text {, and a silvery, greasy, irregular mineral (cassiterite?). Yellow-green } \\
\text { scorodite coats one pebble. Possible tourmaline (black, elongate mineral sprays) } \\
\text { is sometimes in vugs and sometimes in host rock. Selected sample of pyrite- } \\
\text { bearing rock over a } 4 \mathrm{~m} \times 10 \mathrm{~m} \text { area. Trace. }\end{array}$ \\
\hline $2011 Z 482 A$ & 65.375371 & -152.799444 & 509325 & 7250113 & $5 \mathrm{~N}$ & $\begin{array}{l}\text { Graphite-quartz-white-mica schist; dark gray, slightly punky, strongly folded, } \\
\text { and dominated by phyllitic white mica with some graphitic soot. White quartz } \\
\text { occurs in ribbons and layers. Orange iron oxide commonly stains foliation } \\
\text { surfaces. Contains } 5 \% \text { orange spots of iron oxide. } 100 \% \text { of exposure. }\end{array}$ \\
\hline 2011Z488B & 65.358993 & -152.971971 & 501304 & 7248273 & $5 \mathrm{~N}$ & Quartz vein; grab sample of vuggy veins. $1 \%$ of exposure. \\
\hline
\end{tabular}


Table 2. Trace-element results for rocks collected in the Moran area, Tanana and Melozitna quadrangles, Alaska.

NOTE: ${ }^{\wedge}=$ sample from batch with inconsistend standard (see report text for details); "---" = element not analyzed

\begin{tabular}{|c|c|c|c|c|c|c|c|c|c|c|c|}
\hline Sample_ID & Au_ppm & Au_ppm_2 & Au_ppm_3 & Au_ppm_4 & Ag_ppm & Al_pct & As_ppm & As_pct & Ba_ppm & Be_ppm & Bi_ppm \\
\hline 2011BAE021A & $<0.001$ & --- & --- & --- & $<0.5$ & 1.12 & $<5$ & --- & 20 & $<0.5$ & $<2$ \\
\hline 2011BAE055A & 0.002 & --- & -- & --- & $<0.5$ & 7.88 & 5 & --- & 130 & $<0.5$ & $<2$ \\
\hline 2011BAE104A & $<0.001$ & --- & --- & --- & $<0.5$ & 0.03 & $<5$ & --- & $<10$ & $<0.5$ & 4 \\
\hline 2011BAE123B & $<0.001$ & --- & --- & --- & $<0.5$ & 1.05 & $<5$ & --- & 50 & $<0.5$ & $<2$ \\
\hline 2011BAE128C & $<0.001$ & --- & --- & --- & $<0.5$ & 0.44 & 6 & --- & 10 & $<0.5$ & $<2$ \\
\hline 2011BAE133B & 0.001 & --- & --- & --- & $<0.5$ & 0.12 & $<5$ & --- & 10 & $<0.5$ & $<2$ \\
\hline 2011BAE135B & $<0.001$ & --- & --- & --- & $<0.5$ & 2.66 & $<5$ & --- & 10 & 0.6 & $<2$ \\
\hline 2011BAE138B & $<0.001$ & --- & --- & --- & $<0.5$ & 2.28 & $<5$ & --- & 120 & $<0.5$ & $<2$ \\
\hline 2011BAE153B & $<0.001$ & --- & --- & --- & $<0.5$ & 2 & $<5$ & --- & 180 & $<0.5$ & $<2$ \\
\hline 2011BAE156C & $<0.001$ & --- & --- & --- & $<0.5$ & 1.94 & 24 & --- & 60 & $<0.5$ & $<2$ \\
\hline 2011BAE187A & $<0.001$ & --- & --- & --- & $<0.5$ & 1.4 & 6 & --- & 150 & $<0.5$ & $<2$ \\
\hline 2011BAE205B & 0.006 & --- & --- & --- & $<0.5$ & 1.17 & 8 & --- & 20 & $<0.5$ & $<2$ \\
\hline 2011BAE226C & --- & 0.003 & --- & --- & 1.1 & 7.3 & 6 & --- & 720 & 0.7 & 3 \\
\hline 2011BAE226D & $<0.001$ & --- & --- & --- & $<0.5$ & 8.18 & $<5$ & --- & 460 & $<0.5$ & $<2$ \\
\hline 2011BAE234B & $<0.001$ & --- & --- & --- & $<0.5$ & 0.06 & $<5$ & --- & $<10$ & $<0.5$ & $<2$ \\
\hline 2011BAE247B & $<0.001$ & --- & --- & --- & $<0.5$ & 0.05 & $<5$ & --- & 10 & $<0.5$ & $<2$ \\
\hline 2011BAE250B & $<0.001$ & --- & --- & --- & $<0.5$ & 0.23 & $<5$ & --- & 30 & $<0.5$ & $<2$ \\
\hline 2011BAE262B & 0.002 & --- & --- & --- & $<0.5$ & 2.77 & 7 & --- & 220 & 0.8 & $<2$ \\
\hline 2011BAE298C & $<0.001$ & --- & --- & --- & 0.8 & 8.55 & 10 & --- & 1050 & 1.3 & $<2$ \\
\hline 2011BAE321B & $<0.001$ & --- & --- & --- & $<0.5$ & 0.38 & $<5$ & --- & 40 & $<0.5$ & $<2$ \\
\hline 2011BAE332B & $<0.001$ & --- & --- & --- & 0.9 & 7.32 & $<5$ & --- & 40 & 0.7 & $<2$ \\
\hline 2011BAE342B & $<0.001$ & --- & --- & --- & $<0.5$ & 0.34 & $<5$ & --- & 20 & $<0.5$ & $<2$ \\
\hline 2011BAE351B & $<0.001$ & --- & --- & --- & $<0.5$ & 0.06 & $<5$ & --- & 10 & $<0.5$ & 2 \\
\hline 2011BAE394A & --- & 0.004 & --- & --- & $<0.5$ & 2.88 & $<5$ & --- & 90 & $<0.5$ & 2 \\
\hline 2011BAE410B & 0.004 & --- & --- & --- & $<0.5$ & 0.06 & 17 & --- & 10 & $<0.5$ & 2 \\
\hline 2011BAE429B & $<0.001$ & --- & --- & --- & $<0.5$ & 0.06 & $<5$ & --- & $<10$ & $<0.5$ & $<2$ \\
\hline 2011BAE432B & 0.004 & --- & --- & --- & $<0.5$ & 0.83 & 27 & --- & 60 & $<0.5$ & $<2$ \\
\hline 2011BAE468B & 0.001 & --- & --- & --- & $<0.5$ & 0.55 & 6 & --- & 50 & $<0.5$ & $<2$ \\
\hline 2011BAE470B & $<0.001$ & --- & --- & --- & $<0.5$ & 3.53 & $<5$ & --- & 300 & 1 & 2 \\
\hline 2011BAE491B & $<0.001$ & --- & --- & --- & $<0.5$ & 0.07 & $<5$ & --- & 10 & $<0.5$ & $<2$ \\
\hline
\end{tabular}




\begin{tabular}{|c|c|c|c|c|c|c|c|c|c|c|c|}
\hline Sample_ID & Ca_pct & Cd_ppm & Co_ppm & Cr_ppm & Cu_ppm & Fe_pct & Ga_ppm & K_pct & La_ppm & Mg_pct & Mn_ppm \\
\hline 2011BAE021A & 0.03 & $<0.5$ & 4 & 32 & 3 & 1.62 & $<10$ & 0.02 & 10 & 0.96 & 397 \\
\hline 2011BAE055A & 3.32 & $<0.5$ & 9 & 18 & 3 & 2.71 & 10 & 0.18 & $<10$ & 0.8 & 509 \\
\hline 2011BAE104A & 0.01 & $<0.5$ & $<1$ & 28 & 2 & 0.55 & $<10$ & 0.01 & $<10$ & $<0.01$ & 58 \\
\hline 2011BAE123B & 0.05 & $<0.5$ & 2 & 32 & 9 & 1.41 & $<10$ & 0.27 & 10 & 0.16 & 174 \\
\hline 2011BAE128C & 0.01 & $<0.5$ & 1 & 28 & 10 & 1.8 & $<10$ & 0.09 & $<10$ & 0.06 & 151 \\
\hline 2011BAE133B & $<0.01$ & $<0.5$ & 9 & 32 & 23 & 1.75 & $<10$ & 0.04 & $<10$ & 0.01 & 79 \\
\hline 2011BAE135B & 0.13 & $<0.5$ & 1 & 34 & 2 & 1.5 & 10 & 0.01 & $<10$ & 0.79 & 125 \\
\hline 2011BAE138B & 0.01 & $<0.5$ & 6 & 25 & 6 & 2.33 & 10 & 0.33 & 10 & 0.21 & 225 \\
\hline 2011BAE153B & 0.08 & $<0.5$ & 3 & 25 & 4 & 1.71 & 10 & 0.58 & 10 & 0.18 & 407 \\
\hline 2011BAE156C & 0.03 & $<0.5$ & 4 & 30 & 7 & 1.17 & $<10$ & 0.13 & $<10$ & 0.17 & 187 \\
\hline 2011BAE187A & 0.11 & $<0.5$ & 4 & 25 & 9 & 1.36 & $<10$ & 0.03 & $<10$ & 0.44 & 737 \\
\hline 2011BAE205B & 0.17 & $<0.5$ & 1 & 36 & 15 & 0.91 & $<10$ & 0.01 & $<10$ & 0.11 & 109 \\
\hline 2011BAE226C & 9.47 & $<0.5$ & 34 & 96 & 563 & 7.27 & 40 & 0.12 & $<10$ & 2.07 & 28300 \\
\hline 2011BAE226D & 1.48 & $<0.5$ & 12 & 34 & 77 & 2.74 & 10 & 0.1 & $<10$ & 0.66 & 5100 \\
\hline 2011BAE234B & 0.01 & $<0.5$ & 1 & 24 & 12 & 0.92 & $<10$ & $<0.01$ & $<10$ & 0.02 & 84 \\
\hline 2011BAE247B & 0.01 & $<0.5$ & $<1$ & 26 & 3 & 0.72 & $<10$ & 0.01 & $<10$ & 0.01 & 57 \\
\hline 2011BAE250B & 0.01 & $<0.5$ & $<1$ & 37 & 7 & 1.12 & $<10$ & 0.03 & $<10$ & 0.03 & 84 \\
\hline 2011BAE262B & 0.11 & $<0.5$ & 8 & 45 & 19 & 2.81 & 10 & 0.79 & 10 & 0.42 & 232 \\
\hline 2011BAE298C & 7.32 & 0.8 & 36 & 278 & 86 & 6.79 & 20 & 0.7 & 10 & 4.18 & 1875 \\
\hline 2011BAE321B & 0.03 & $<0.5$ & 2 & 17 & 11 & 1.24 & $<10$ & 0.08 & $<10$ & 0.09 & 117 \\
\hline 2011BAE332B & 4.75 & 0.7 & 38 & 4 & 23 & 10.05 & 20 & 0.01 & 10 & 3.09 & 1230 \\
\hline 2011BAE342B & 0.04 & $<0.5$ & 1 & 21 & 8 & 1.37 & $<10$ & 0.04 & $<10$ & 0.16 & 160 \\
\hline 2011BAE351B & 0.01 & $<0.5$ & 1 & 29 & 9 & 1.13 & $<10$ & 0.01 & $<10$ & 0.01 & 788 \\
\hline 2011BAE394A & 4.75 & $<0.5$ & 101 & 1320 & 107 & 8.39 & $<10$ & 0.03 & $<10$ & 15.3 & 1380 \\
\hline 2011BAE410B & $<0.01$ & $<0.5$ & 1 & 24 & 12 & 0.95 & $<10$ & 0.02 & $<10$ & 0.01 & 82 \\
\hline 2011BAE429B & 0.04 & $<0.5$ & 6 & 25 & 5 & 0.96 & $<10$ & $<0.01$ & $<10$ & 0.01 & 121 \\
\hline 2011BAE432B & $<0.01$ & $<0.5$ & 1 & 46 & 100 & 2.24 & $<10$ & 0.26 & $<10$ & 0.04 & 98 \\
\hline 2011BAE468B & 5.6 & 0.8 & 7 & 18 & 5 & 3.11 & $<10$ & 0.15 & $<10$ & 1.79 & 1935 \\
\hline 2011BAE470B & 0.26 & $<0.5$ & 11 & 30 & 35 & 3.28 & 10 & 1.32 & 20 & 0.3 & 2210 \\
\hline 2011BAE491B & $<0.01$ & $<0.5$ & $<1$ & 22 & 6 & 0.8 & $<10$ & 0.01 & $<10$ & 0.01 & 60 \\
\hline
\end{tabular}




\begin{tabular}{|c|c|c|c|c|c|c|c|c|c|c|c|}
\hline Sample_ID & Mo_ppm & $\mathrm{Na}$ _pct & Ni_ppm & P_ppm & P_ppm_2 & Pb_ppm & Pd_ppm & Pt_ppm & S_pct & Sb_ppm & Sc_ppm \\
\hline 2011BAE021A & $<1$ & 0.07 & 13 & 170 & --- & $<2$ & -- & -- & $<0.01$ & $<5$ & 2 \\
\hline 2011BAE055A & $<1$ & 4.07 & 20 & 240 & --- & 3 & --- & --- & $<0.01$ & $<5$ & 7 \\
\hline 2011BAE104A & $<1$ & $<0.01$ & $<1$ & 10 & --- & $<2$ & --- & --- & $<0.01$ & $<5$ & $<1$ \\
\hline 2011BAE123B & $<1$ & 0.06 & 4 & 200 & --- & 2 & --- & --- & $<0.01$ & $<5$ & 2 \\
\hline 2011BAE128C & $<1$ & 0.03 & 4 & 50 & --- & 3 & --- & --- & $<0.01$ & $<5$ & 1 \\
\hline 2011BAE133B & $<1$ & $<0.01$ & 38 & 20 & --- & $<2$ & --- & --- & $<0.01$ & 5 & $<1$ \\
\hline 2011BAE135B & $<1$ & 0.24 & 8 & 20 & --- & $<2$ & --- & --- & $<0.01$ & $<5$ & 6 \\
\hline 2011BAE138B & $<1$ & 0.28 & 14 & 100 & --- & 13 & --- & --- & $<0.01$ & $<5$ & 4 \\
\hline 2011BAE153B & $<1$ & 0.36 & 8 & 370 & --- & 15 & --- & --- & $<0.01$ & $<5$ & 2 \\
\hline 2011BAE156C & $<1$ & 1.13 & 9 & 30 & --- & 4 & --- & --- & $<0.01$ & $<5$ & 1 \\
\hline 2011BAE187A & $<1$ & 0.83 & 10 & 140 & --- & 11 & --- & --- & $<0.01$ & $<5$ & 3 \\
\hline 2011BAE205B & $<1$ & 0.88 & 3 & 20 & --- & $<2$ & --- & --- & $<0.01$ & $<5$ & 1 \\
\hline 2011BAE226C & 1 & 1.02 & 89 & 740 & --- & 3 & 0.005 & 0.01 & 0.02 & 5 & 28 \\
\hline 2011BAE226D & 2 & 6.4 & 26 & 240 & --- & 5 & --- & --- & $<0.01$ & $<5$ & 8 \\
\hline 2011BAE234B & $<1$ & 0.01 & 3 & 20 & --- & 4 & --- & --- & $<0.01$ & $<5$ & $<1$ \\
\hline 2011BAE247B & $<1$ & $<0.01$ & 2 & 20 & --- & 2 & --- & --- & $<0.01$ & $<5$ & $<1$ \\
\hline 2011BAE250B & 1 & 0.04 & 5 & 30 & --- & $<2$ & --- & --- & $<0.01$ & $<5$ & $<1$ \\
\hline 2011BAE262B & 1 & 0.09 & 19 & 310 & --- & 5 & --- & --- & $<0.01$ & $<5$ & 7 \\
\hline 2011BAE298C & 3 & 1.43 & 128 & 920 & --- & 17 & --- & --- & 0.29 & 6 & 27 \\
\hline 2011BAE321B & $<1$ & 0.03 & 5 & 30 & --- & $<2$ & --- & --- & $<0.01$ & $<5$ & 1 \\
\hline 2011BAE332B & 4 & 1.53 & 17 & 960 & $\begin{array}{l}-- \\
\end{array}$ & 14 & $\begin{array}{l}-- \\
\end{array}$ & $\begin{array}{l}-- \\
\end{array}$ & 0.09 & 5 & 28 \\
\hline 2011BAE342B & 1 & 0.03 & 4 & 240 & --- & $<2$ & --- & --- & $<0.01$ & $<5$ & 1 \\
\hline 2011BAE351B & 1 & 0.01 & 3 & 30 & --- & 2 & --- & --- & $<0.01$ & $<5$ & $<1$ \\
\hline 2011BAE394A & $<1$ & 0.09 & 714 & 120 & --- & $<2$ & 0.025 & 0.037 & 0.02 & 5 & 23 \\
\hline 2011BAE410B & $<1$ & $<0.01$ & 2 & 20 & --- & $<2$ & --- & --- & $<0.01$ & $<5$ & $<1$ \\
\hline 2011BAE429B & $<1$ & $<0.01$ & 10 & 160 & --- & $<2$ & --- & -- & $<0.01$ & $<5$ & $<1$ \\
\hline 2011BAE432B & 2 & 0.02 & 4 & 130 & --- & 10 & --- & --- & $<0.01$ & $<5$ & 1 \\
\hline 2011BAE468B & $<1$ & 0.09 & 17 & 160 & --- & 2 & --- & --- & $<0.01$ & $<5$ & 3 \\
\hline 2011BAE470B & 3 & 0.23 & 13 & 1060 & --- & 3 & --- & --- & 0.02 & $<5$ & 4 \\
\hline 2011BAE491B & 1 & 0.01 & 1 & 50 & --- & $<2$ & --- & --- & $<0.01$ & $<5$ & $<1$ \\
\hline
\end{tabular}




\begin{tabular}{|c|c|c|c|c|c|c|c|c|c|c|c|}
\hline Sample_ID & Sn_ppm & Sr_ppm & Th_ppm & Ti_pct & TI_ppm & U_ppm & V_ppm & W_ppm & W_ppm_2 & Zn_ppm & Zn_pct \\
\hline 2011BAE021A & --- & 13 & $<20$ & 0.05 & $<10$ & $<10$ & 25 & $<10$ & -- & 15 & -- \\
\hline 2011BAE055A & --- & 418 & $<20$ & 0.1 & $<10$ & 20 & 184 & $<10$ & --- & 14 & --- \\
\hline 2011BAE104A & --- & 1 & $<20$ & $<0.01$ & $<10$ & $<10$ & $<1$ & $<10$ & -- & $<2$ & --- \\
\hline 2011BAE123B & --- & 8 & $<20$ & 0.16 & $<10$ & $<10$ & 13 & $<10$ & -- & 12 & --- \\
\hline 2011BAE128C & --- & 3 & $<20$ & 0.01 & $<10$ & $<10$ & 4 & $<10$ & -- & 11 & -- \\
\hline 2011BAE133B & --- & 1 & $<20$ & 0.01 & $<10$ & $<10$ & 2 & $<10$ & --- & $<2$ & --- \\
\hline 2011BAE135B & --- & 45 & $<20$ & 0.08 & $<10$ & $<10$ & 40 & $<10$ & -- & 8 & --- \\
\hline 2011BAE138B & --- & 21 & $<20$ & 0.07 & $<10$ & $<10$ & 23 & $<10$ & --- & 38 & --- \\
\hline 2011BAE153B & --- & 26 & $<20$ & 0.06 & $<10$ & $<10$ & 17 & $<10$ & --- & 13 & --- \\
\hline 2011BAE156C & --- & 16 & $<20$ & 0.04 & $<10$ & $<10$ & 9 & $<10$ & --- & 12 & --- \\
\hline 2011BAE187A & --- & 16 & $<20$ & 0.04 & $<10$ & 10 & 11 & $<10$ & --- & 22 & --- \\
\hline 2011BAE205B & --- & 18 & $<20$ & 0.02 & $<10$ & 10 & 8 & $<10$ & -- & 3 & --- \\
\hline 2011BAE226C & --- & 145 & $<20$ & 0.75 & $<10$ & $<10$ & 300 & $<10$ & --- & 104 & --- \\
\hline 2011BAE226D & --- & 204 & $<20$ & 0.24 & $<10$ & 30 & 80 & $<10$ & --- & 32 & --- \\
\hline 2011BAE234B & $\begin{array}{l}-- \\
\end{array}$ & 1 & $<20$ & $<0.01$ & $<10$ & $<10$ & 2 & $<10$ & --- & 7 & $\begin{array}{l}-- \\
\end{array}$ \\
\hline 2011BAE247B & --- & $<1$ & $<20$ & $<0.01$ & $<10$ & $<10$ & 1 & $<10$ & --- & 5 & --- \\
\hline 2011BAE250B & --- & 4 & $<20$ & 0.01 & $<10$ & $<10$ & 4 & $<10$ & --- & 6 & --- \\
\hline 2011BAE262B & --- & 10 & $<20$ & 0.08 & $<10$ & $<10$ & 54 & $<10$ & --- & 39 & --- \\
\hline 2011BAE298C & --- & 423 & $<20$ & 0.95 & $<10$ & $<10$ & 239 & $<10$ & --- & 149 & --- \\
\hline 2011BAE321B & --- & 6 & $<20$ & 0.02 & $<10$ & $<10$ & 8 & $<10$ & --- & 11 & --- \\
\hline 2011BAE332B & $\begin{array}{l}-- \\
\end{array}$ & 308 & $<20$ & 1.25 & $<10$ & $<10$ & 362 & $<10$ & --- & 160 & $\begin{array}{l}-- \\
\end{array}$ \\
\hline 2011BAE342B & --- & 2 & $<20$ & 0.01 & $<10$ & $<10$ & 7 & $<10$ & --- & 8 & --- \\
\hline 2011BAE351B & --- & 3 & $<20$ & 0.01 & $<10$ & $<10$ & 4 & $<10$ & --- & 10 & --- \\
\hline 2011BAE394A & $\begin{array}{l}-- \\
\end{array}$ & 43 & $<20$ & 0.19 & $<10$ & $<10$ & 104 & $<10$ & --- & 92 & $\begin{array}{l}-- \\
\end{array}$ \\
\hline 2011BAE410B & $<5$ & $<1$ & $<20$ & $<0.01$ & $<10$ & $<10$ & 3 & $<10$ & $<10$ & 4 & --- \\
\hline 2011BAE429B & --- & 1 & $<20$ & 0.01 & $<10$ & $<10$ & 2 & $<10$ & --- & 9 & --- \\
\hline 2011BAE432B & --- & 7 & $<20$ & 0.04 & $<10$ & $<10$ & 36 & $<10$ & --- & 7 & --- \\
\hline 2011BAE468B & --- & 109 & $<20$ & 0.02 & $<10$ & $<10$ & 18 & $<10$ & --- & 47 & --- \\
\hline 2011BAE470B & --- & 45 & $<20$ & 0.12 & $<10$ & $<10$ & 27 & $<10$ & --- & 45 & --- \\
\hline 2011BAE491B & --- & 2 & $<20$ & $<0.01$ & $<10$ & $<10$ & 2 & $<10$ & --- & 5 & --- \\
\hline
\end{tabular}




\begin{tabular}{|c|c|c|c|c|c|c|c|c|c|c|c|}
\hline Sample_ID & Au_ppm & Au_ppm_2 & Au_ppm_3 & Au_ppm_4 & Ag_ppm & Al_pct & As_ppm & As_pct & Ba_ppm & Be_ppm & Bi_ppm \\
\hline 2011BAE537B & $<0.001$ & -- & -- & -- & $<0.5$ & 1.23 & $<5$ & -- & 110 & $<0.5$ & $<2$ \\
\hline 2011GG010C & 0.001 & --- & -- & --- & $<0.5$ & 0.87 & 5 & --- & $<10$ & $<0.5$ & $<2$ \\
\hline 2011GG016B & 0.001 & --- & -- & -- & $<0.5$ & 8.99 & 28 & -- & 190 & 0.7 & $<2$ \\
\hline 2011GG033A & $<0.001$ & --- & -- & -- & $<0.5$ & 7.18 & 10 & --- & 500 & 2 & $<2$ \\
\hline 2011GG034A & 0.002 & -- & -- & -- & $<0.5$ & 4.37 & 8 & -- & 430 & 1.2 & $<2$ \\
\hline 2011GG035B & $<0.001$ & --- & -- & -- & $<0.5$ & 6.85 & 9 & -- & 160 & 0.5 & $<2$ \\
\hline 2011GG037B & $<0.001$ & --- & -- & -- & 0.5 & 8.11 & 6 & --- & 60 & 0.8 & $<2$ \\
\hline 2011GG037C & 0.008 & --- & --- & --- & $<0.5$ & 6 & 5 & --- & 20 & 0.5 & $<2$ \\
\hline 2011GG041C & 0.002 & --- & --- & --- & $<0.5$ & 0.09 & 32 & --- & $<10$ & $<0.5$ & $<2$ \\
\hline 2011GG044B & $>10.0$ & --- & --- & 22.3 & 9.6 & 3.2 & $>10000$ & 1.805 & 210 & 0.8 & 63 \\
\hline 2011GG059B & 0.008 & --- & --- & --- & $<0.5$ & 4.96 & 20 & --- & 110 & $<0.5$ & $<2$ \\
\hline 2011GG062A^ & 0.045 & --- & 0.044 & --- & $<0.5$ & 6.12 & 1285 & --- & 760 & 1.6 & $<2$ \\
\hline 2011GG063A^ & 0.001 & -- & 0.007 & -- & $<0.5$ & 0.22 & 91 & -- & 10 & $<0.5$ & $<2$ \\
\hline 2011GG064A^ & 0.006 & --- & 0.006 & --- & $<0.5$ & 5.69 & 1360 & --- & 230 & 1.4 & $<2$ \\
\hline 2011GG065A^ & 9.29 & -- & 9.29 & -- & 22.3 & 4.95 & $>10000$ & 0.985 & 590 & 1.4 & 54 \\
\hline 2011GG069A^ & 0.009 & --- & 0.009 & --- & 3.3 & 2.1 & 287 & --- & 200 & $<0.5$ & $<2$ \\
\hline 2011GG094B^ & 0.021 & --- & 0.023 & --- & $<0.5$ & 0.02 & 39 & --- & $<10$ & $<0.5$ & $<2$ \\
\hline 2011GG097A^ & 0.001 & --- & 0.002 & --- & $<0.5$ & 3.19 & $<5$ & --- & 30 & 25.6 & $<2$ \\
\hline $2011 \mathrm{GG097C}^{\wedge}$ & 0.002 & --- & 0.003 & --- & $<0.5$ & 7.39 & 8 & --- & 380 & 142.5 & $<2$ \\
\hline 2011GG098B & 0.002 & --- & & --- & $<0.5$ & 4.16 & 8 & --- & $<10$ & 13.5 & 5 \\
\hline 2011GG102B & $<0.001$ & --- & -- & --- & 0.5 & 5.6 & 29 & -- & 300 & 2.8 & $<2$ \\
\hline 2011GG105A & 0.004 & -- & -- & --- & 2.3 & 6.82 & 30 & --- & 60 & 2.7 & 996 \\
\hline 2011GG106A & 0.003 & -- & -- & --- & $<0.5$ & 6.05 & 7 & -- & 1470 & 1.8 & 3 \\
\hline 2011GG110A & $<0.001$ & -- & -- & -- & 0.6 & 0.67 & 111 & -- & 50 & $<0.5$ & 14 \\
\hline 2011GG111A & $<0.001$ & --- & -- & --- & $<0.5$ & 2.04 & $<5$ & --- & 200 & 0.7 & $<2$ \\
\hline 2011GG111D & $<0.001$ & --- & --- & --- & $<0.5$ & 7.08 & $<5$ & --- & 440 & 28.1 & $<2$ \\
\hline 2011GG138B & $<0.001$ & --- & --- & --- & $<0.5$ & 0.51 & $<5$ & --- & 50 & $<0.5$ & $<2$ \\
\hline 2011GG167A & $<0.001$ & --- & --- & --- & $<0.5$ & 0.49 & $<5$ & --- & 20 & $<0.5$ & $<2$ \\
\hline 2011GG168A & $<0.001$ & --- & --- & --- & $<0.5$ & 3.96 & $<5$ & --- & 30 & $<0.5$ & 3 \\
\hline 2011GG170A & $<0.001$ & --- & --- & --- & $<0.5$ & 5.17 & 24 & --- & 70 & $<0.5$ & 5 \\
\hline
\end{tabular}




\begin{tabular}{|c|c|c|c|c|c|c|c|c|c|c|c|}
\hline Sample_ID & Ca_pct & Cd_ppm & Co_ppm & Cr_ppm & Cu_ppm & Fe_pct & Ga_ppm & K_pct & La_ppm & Mg_pct & Mn_ppm \\
\hline 2011BAE537B & 0.55 & $<0.5$ & 2 & 18 & 6 & 1.27 & $<10$ & 0.06 & $<10$ & 0.17 & 166 \\
\hline 2011GG010C & 1.23 & $<0.5$ & 4 & 33 & 21 & 1.67 & $<10$ & 0.02 & $<10$ & 0.3 & 256 \\
\hline 2011GG016B & 0.39 & 0.5 & 24 & 12 & 51 & 7.7 & 20 & 0.15 & 10 & 2.53 & 1380 \\
\hline 2011GG033A & 0.23 & $<0.5$ & 20 & 79 & 39 & 5.43 & 20 & 1.97 & 30 & 1.04 & 404 \\
\hline 2011GG034A & 0.13 & $<0.5$ & 6 & 59 & 26 & 3.65 & 10 & 1.42 & 10 & 0.63 & 187 \\
\hline 2011GG035B & 2.89 & $<0.5$ & 21 & 56 & 21 & 3.31 & 10 & 0.25 & 30 & 1.41 & 372 \\
\hline 2011GG037B & 0.3 & $<0.5$ & 11 & 52 & 23 & 2.71 & 10 & 0.07 & 10 & 1.2 & 313 \\
\hline 2011GG037C & 0.35 & $<0.5$ & 7 & 21 & 24 & 1.48 & 10 & 0.03 & 10 & 0.41 & 167 \\
\hline 2011GG041C & $<0.01$ & $<0.5$ & 3 & 19 & 8 & 1.24 & $<10$ & 0.01 & $<10$ & $<0.01$ & 65 \\
\hline 2011GG044B & 0.01 & 1.9 & $<1$ & 29 & 407 & 25.2 & 10 & 1.04 & 20 & 0.18 & 108 \\
\hline 2011GG059B & 0.15 & $<0.5$ & 2 & 27 & 44 & 1.83 & 10 & 0.08 & 10 & 0.2 & 231 \\
\hline 2011GG062A^ & 0.02 & 0.6 & 1 & 82 & 113 & 6.25 & 20 & 1.55 & 30 & 0.22 & 60 \\
\hline 2011GG063A^ & 0.01 & $<0.5$ & $<1$ & 22 & 11 & 1.1 & $<10$ & 0.06 & $<10$ & 0.01 & 55 \\
\hline 2011GG064A^ & 0.02 & 1.5 & 9 & 91 & 43 & 9.27 & 10 & 1.3 & 30 & 1.2 & 321 \\
\hline 2011GG065A^ & 0.03 & 3.3 & $<1$ & 53 & 234 & 20 & 10 & 1.41 & 80 & 0.48 & 56 \\
\hline 2011GG069A^ & 0.01 & 1.7 & 1 & 38 & 163 & 6.76 & $<10$ & 0.55 & 20 & 0.05 & 68 \\
\hline 2011GG094B^ & 0.01 & $<0.5$ & $<1$ & 20 & 4 & 0.64 & $<10$ & 0.01 & $<10$ & $<0.01$ & 46 \\
\hline 2011GG097A^ & 18.2 & $<0.5$ & 13 & 20 & 3 & 15.85 & 20 & 0.06 & $<10$ & 0.67 & 13600 \\
\hline 2011GG097C^ & 8.47 & $<0.5$ & 6 & 56 & 4 & 5.78 & 30 & 2.35 & 20 & 0.75 & 5190 \\
\hline 2011GG098B & 21.4 & $<0.5$ & $<1$ & 23 & 2 & 15.4 & 30 & 0.05 & $<10$ & 0.22 & 8750 \\
\hline 2011GG102B & 0.73 & $<0.5$ & 24 & 75 & 26 & 3.25 & 20 & 0.55 & 60 & 1.1 & 605 \\
\hline 2011GG105A & 0.6 & 0.6 & 30 & 156 & 119 & 11.25 & 30 & 0.12 & 10 & 1.84 & 2160 \\
\hline 2011GG106A & 1.74 & $<0.5$ & 8 & 74 & 37 & 1.98 & 10 & 3.04 & 30 & 1.46 & 227 \\
\hline 2011GG110A & 11.95 & $<0.5$ & 3 & 12 & 16 & 0.95 & $<10$ & 0.19 & $<10$ & 0.21 & 2200 \\
\hline 2011GG111A & 27.3 & $<0.5$ & 2 & 19 & 6 & 1.01 & $<10$ & 0.71 & 10 & 0.43 & 231 \\
\hline 2011GG111D & 0.43 & $<0.5$ & 2 & 8 & 10 & 0.71 & 30 & 5.57 & 10 & 0.15 & 228 \\
\hline 2011GG138B & 0.01 & $<0.5$ & 3 & 30 & 4 & 1.13 & $<10$ & 0.16 & $<10$ & 0.07 & 136 \\
\hline 2011GG167A & 0.12 & $<0.5$ & 4 & 35 & 7 & 1.5 & $<10$ & 0.02 & $<10$ & 0.31 & 215 \\
\hline 2011GG168A & 0.23 & $<0.5$ & 10 & 89 & 6 & 2.83 & 10 & 0.02 & 10 & 0.68 & 343 \\
\hline 2011GG170A & 1.13 & $<0.5$ & 30 & 150 & 6 & 7.3 & 20 & 0.08 & $<10$ & 2.88 & 1095 \\
\hline
\end{tabular}




\begin{tabular}{|c|c|c|c|c|c|c|c|c|c|c|c|}
\hline Sample_ID & Mo_ppm & $\mathrm{Na}$ _pct & Ni_ppm & P_ppm & P_ppm_2 & Pb_ppm & Pd_ppm & Pt_ppm & S_pct & Sb_ppm & Sc_ppm \\
\hline 2011BAE537B & $<1$ & 0.43 & 2 & 130 & -- & $<2$ & -- & -- & $<0.01$ & $<5$ & 1 \\
\hline 2011GG010C & $<1$ & 0.05 & 9 & 150 & --- & 2 & --- & --- & $<0.01$ & $<5$ & 4 \\
\hline 2011GG016B & $<1$ & 4.78 & 26 & 1750 & -- & 5 & --- & -- & $<0.01$ & $<5$ & 18 \\
\hline 2011GG033A & $<1$ & 0.29 & 45 & 710 & --- & 13 & --- & --- & 0.01 & $<5$ & 18 \\
\hline 2011GG034A & $<1$ & 0.19 & 24 & 680 & -- & 16 & -- & -- & 0.01 & $<5$ & 11 \\
\hline 2011GG035B & $<1$ & 4.04 & 44 & 140 & --- & 13 & --- & --- & $<0.01$ & $<5$ & 12 \\
\hline 2011GG037B & $<1$ & 5.9 & 24 & 40 & --- & 8 & --- & --- & $<0.01$ & $<5$ & 10 \\
\hline 2011GG037C & $<1$ & 4.6 & 13 & 100 & --- & 6 & --- & --- & $<0.01$ & $<5$ & 4 \\
\hline 2011GG041C & $<1$ & 0.01 & 7 & 40 & --- & 2 & --- & --- & $<0.01$ & 5 & $<1$ \\
\hline 2011GG044B & $<1$ & 0.07 & 2 & 730 & --- & 98 & --- & --- & 0.08 & 94 & 7 \\
\hline 2011GG059B & 2 & 3.78 & 10 & 800 & --- & 40 & --- & --- & 0.02 & $<5$ & 2 \\
\hline 2011GG062A^ & $<1$ & 0.38 & 13 & 1080 & --- & 95 & -- & --- & 0.01 & 5 & 12 \\
\hline 2011GG063A^ & $<1$ & 0.01 & 5 & 70 & -- & 23 & -- & -- & $<0.01$ & $<5$ & $<1$ \\
\hline 2011GG064A^ & 2 & 0.47 & 46 & 580 & --- & 39 & --- & --- & 0.02 & 5 & 15 \\
\hline 2011GG065A^ & 1 & 0.21 & $<1$ & 1710 & -- & 483 & -- & -- & 0.07 & 55 & 13 \\
\hline 2011GG069A^ & $<1$ & 0.04 & 8 & 940 & --- & 2300 & --- & --- & 0.09 & 41 & 6 \\
\hline 2011GG094B^ & $<1$ & 0.01 & $<1$ & 10 & --- & 7 & --- & --- & $<0.01$ & $<5$ & $<1$ \\
\hline 2011GG097A^ & 853 & 0.15 & 36 & 520 & --- & 17 & --- & --- & 0.03 & $<5$ & 5 \\
\hline $2011 \mathrm{GG097C}^{\wedge}$ & 9 & 1.82 & 14 & 310 & --- & 12 & --- & --- & 0.01 & $<5$ & 12 \\
\hline 2011GG098B & $<1$ & 0.04 & $<1$ & 400 & --- & 15 & --- & --- & $<0.01$ & $<5$ & 6 \\
\hline 2011GG102B & $<1$ & 0.48 & 39 & 840 & --- & 62 & --- & --- & 0.02 & $<5$ & 12 \\
\hline 2011GG105A & $<1$ & 0.61 & 78 & 380 & --- & 71 & -- & --- & 0.05 & $<5$ & 22 \\
\hline 2011GG106A & 2 & 0.8 & 47 & 700 & -- & 4 & -- & -- & 0.1 & $<5$ & 10 \\
\hline 2011GG110A & $<1$ & 0.02 & 7 & 110 & -- & 46 & -- & -- & 0.01 & $<5$ & 2 \\
\hline 2011GG111A & $<1$ & 0.23 & 5 & 220 & --- & 4 & --- & --- & 0.06 & 5 & 3 \\
\hline 2011GG111D & $<1$ & 1.56 & 2 & 20 & --- & 23 & --- & --- & 0.01 & $<5$ & 5 \\
\hline 2011GG138B & $<1$ & 0.02 & 6 & 20 & --- & $<2$ & --- & --- & $<0.01$ & $<5$ & 1 \\
\hline 2011GG167A & $<1$ & 0.05 & 11 & 410 & --- & $<2$ & --- & --- & $<0.01$ & $<5$ & 2 \\
\hline 2011GG168A & $<1$ & 2.59 & 25 & 870 & --- & $<2$ & --- & --- & $<0.01$ & $<5$ & 6 \\
\hline 2011GG170A & $<1$ & 1.43 & 65 & 510 & --- & 6 & --- & --- & $<0.01$ & 7 & 14 \\
\hline
\end{tabular}




\begin{tabular}{|c|c|c|c|c|c|c|c|c|c|c|c|}
\hline Sample_ID & Sn_ppm & Sr_ppm & Th_ppm & Ti_pct & TI_ppm & U_ppm & V_ppm & W_ppm & W_ppm_2 & Zn_ppm & Zn_pct \\
\hline 2011BAE537B & --- & 122 & $<20$ & 0.03 & $<10$ & $<10$ & 46 & $<10$ & -- & 8 & -- \\
\hline 2011GG010C & --- & 106 & $<20$ & 0.1 & $<10$ & $<10$ & 48 & $<10$ & --- & 13 & --- \\
\hline 2011GG016B & --- & 62 & $<20$ & 1.07 & $<10$ & $<10$ & 236 & 20 & -- & 154 & --- \\
\hline 2011GG033A & -- & 40 & $<20$ & 0.18 & $<10$ & $<10$ & 137 & $<10$ & -- & 102 & -- \\
\hline 2011GG034A & -- & 21 & $<20$ & 0.17 & $<10$ & $<10$ & 104 & $<10$ & -- & 62 & --- \\
\hline 2011GG035B & --- & 66 & $<20$ & 0.21 & $<10$ & $<10$ & 82 & $<10$ & --- & 42 & --- \\
\hline 2011GG037B & --- & 28 & $<20$ & 0.3 & $<10$ & $<10$ & 78 & $<10$ & --- & 34 & --- \\
\hline 2011GG037C & --- & 28 & $<20$ & 0.11 & $<10$ & $<10$ & 42 & $<10$ & --- & 16 & --- \\
\hline 2011GG041C & --- & 2 & $<20$ & 0.02 & $<10$ & $<10$ & 3 & $<10$ & --- & 13 & --- \\
\hline 2011GG044B & --- & 9 & $<20$ & 0.05 & $<10$ & $<10$ & 62 & $<10$ & --- & 95 & --- \\
\hline 2011GG059B & --- & 30 & $<20$ & 0.07 & $<10$ & $<10$ & 26 & $<10$ & --- & 34 & --- \\
\hline 2011GG062A^ & --- & 66 & $<20$ & 0.19 & $<10$ & $<10$ & 135 & $<10$ & -- & 103 & --- \\
\hline 2011GG063A^ & --- & 1 & $<20$ & 0.01 & $<10$ & $<10$ & 4 & $<10$ & -- & 17 & -- \\
\hline 2011GG064A^ & --- & 53 & $<20$ & 0.22 & $<10$ & $<10$ & 141 & $<10$ & --- & 290 & --- \\
\hline 2011GG065A^ & -- & 33 & $<20$ & 0.09 & $<10$ & $<10$ & 111 & $<10$ & -- & 39 & -- \\
\hline 2011GG069A^ & --- & 37 & $<20$ & 0.06 & $<10$ & $<10$ & 68 & $<10$ & --- & 191 & --- \\
\hline 2011GG094B^ & --- & $<1$ & $<20$ & $<0.01$ & $<10$ & $<10$ & 1 & $<10$ & --- & 4 & --- \\
\hline 2011GG097A^ & 178 & 24 & $<20$ & 0.15 & $<10$ & $<10$ & 31 & 10 & 70 & 233 & --- \\
\hline $2011 \mathrm{GG097C}^{\wedge}$ & 139 & 406 & 20 & 0.37 & $<10$ & $<10$ & 67 & 280 & 290 & 101 & --- \\
\hline 2011GG098B & 743 & 3 & $<20$ & 0.15 & $<10$ & $<10$ & 33 & $<10$ & 50 & 56 & --- \\
\hline 2011GG102B & -- & 70 & $<20$ & 0.44 & $<10$ & $<10$ & 112 & 10 & -- & 63 & --- \\
\hline 2011GG105A & 32 & 57 & $<20$ & 0.28 & $<10$ & $<10$ & 178 & 2690 & 2690 & 363 & --- \\
\hline 2011GG106A & $<5$ & 215 & $<20$ & 0.23 & $<10$ & $<10$ & 97 & 4590 & 8960 & 21 & --- \\
\hline 2011GG110A & --- & 71 & $<20$ & 0.03 & $<10$ & $<10$ & 14 & 40 & -- & 36 & --- \\
\hline 2011GG111A & --- & 1745 & 20 & 0.13 & $<10$ & $<10$ & 20 & 10 & --- & 25 & --- \\
\hline 2011GG111D & --- & 404 & $<20$ & $<0.01$ & $<10$ & 20 & $<1$ & 30 & --- & 2 & --- \\
\hline 2011GG138B & --- & 3 & $<20$ & 0.03 & $<10$ & $<10$ & 6 & $<10$ & --- & 8 & --- \\
\hline 2011GG167A & --- & 6 & $<20$ & 0.03 & $<10$ & $<10$ & 20 & $<10$ & --- & 13 & --- \\
\hline 2011GG168A & --- & 65 & $<20$ & 0.5 & $<10$ & 10 & 61 & $<10$ & --- & 33 & --- \\
\hline 2011GG170A & --- & 76 & $<20$ & 0.61 & $<10$ & 10 & 210 & $<10$ & --- & 89 & --- \\
\hline
\end{tabular}




\begin{tabular}{|c|c|c|c|c|c|c|c|c|c|c|c|}
\hline Sample_ID & Au_ppm & Au_ppm_2 & Au_ppm_3 & Au_ppm_4 & Ag_ppm & Al_pct & As_ppm & As_pct & Ba_ppm & Be_ppm & Bi_ppm \\
\hline 2011GG179A & $<0.001$ & -- & -- & -- & $<0.5$ & 2.45 & $<5$ & --- & 20 & $<0.5$ & $<2$ \\
\hline 2011GG179C & 0.001 & --- & -- & --- & $<0.5$ & 7.71 & 6 & --- & 160 & $<0.5$ & 6 \\
\hline 2011GG185A & -- & $<0.001$ & -- & -- & 3.2 & 8.11 & $<5$ & -- & 120 & $<0.5$ & 2 \\
\hline 2011GG186B & -- & $<0.001$ & -- & -- & 0.7 & 6.63 & $<5$ & --- & 140 & 0.6 & $<2$ \\
\hline 2011GG194A & $<0.001$ & --- & -- & --- & 1 & 5.94 & 17 & --- & 350 & 0.8 & $<2$ \\
\hline 2011GG209A & 0.026 & --- & -- & -- & 0.6 & 5.74 & 759 & -- & 720 & 1.4 & $<2$ \\
\hline 2011GG210C & $<0.001$ & --- & -- & -- & $<0.5$ & 2.9 & 17 & --- & 30 & $<0.5$ & $<2$ \\
\hline 2011GG217B & 0.001 & --- & --- & --- & $<0.5$ & 7.79 & 5 & --- & 70 & 0.6 & $<2$ \\
\hline 2011GG300B & $<0.001$ & --- & --- & --- & $<0.5$ & 2.11 & $<5$ & --- & 30 & $<0.5$ & $<2$ \\
\hline 2011GG304B & $<0.001$ & --- & --- & --- & $<0.5$ & 1.76 & $<5$ & --- & 120 & 0.5 & 2 \\
\hline 2011GG323A & $<0.001$ & --- & --- & --- & 0.7 & 7.6 & $<5$ & --- & 110 & $<0.5$ & $<2$ \\
\hline 2011GG335A & $<0.001$ & --- & --- & --- & $<0.5$ & 1 & 24 & --- & 100 & $<0.5$ & $<2$ \\
\hline 2011GG344A & 0.001 & -- & -- & -- & $<0.5$ & 5.17 & 6 & -- & 170 & 2.3 & 3 \\
\hline 2011GG349A & $<0.001$ & --- & --- & --- & 0.5 & 7.68 & 34 & --- & 1800 & 1.9 & 2 \\
\hline 2011GG353A & 0.004 & -- & -- & --- & 9.9 & 0.56 & 18 & -- & 30 & 0.7 & 6 \\
\hline 2011GG356A & $<0.001$ & --- & --- & --- & $<0.5$ & 0.38 & 17 & --- & 50 & $<0.5$ & 2 \\
\hline 2011GG359A & $<0.001$ & --- & -- & --- & $<0.5$ & 8.12 & 6 & --- & 600 & 2.4 & $<2$ \\
\hline 2011GG365A & $<0.001$ & --- & --- & --- & 0.8 & 3.47 & 37 & --- & 240 & 1.6 & $<2$ \\
\hline 2011GG366A & $<0.001$ & --- & --- & --- & 0.8 & 1.32 & 202 & --- & 90 & 0.6 & $<2$ \\
\hline 2011GG376A & 0.001 & --- & --- & --- & 0.5 & 3.28 & 68 & --- & 340 & 0.8 & $<2$ \\
\hline 2011GG376C & 0.008 & -- & --- & --- & 0.7 & 6.22 & 53 & -- & 190 & 0.7 & $<2$ \\
\hline 2011GG382C & $<0.001$ & -- & --- & --- & $<0.5$ & 2.48 & 19 & --- & 140 & 0.8 & $<2$ \\
\hline 2011GG393A & 0.001 & -- & -- & --- & $<0.5$ & 8 & 15 & -- & 240 & 0.8 & $<2$ \\
\hline 2011GG395A & 0.001 & -- & -- & -- & $<0.5$ & 9.6 & 31 & -- & 240 & 1.7 & $<2$ \\
\hline 2011GG403A & 0.002 & --- & --- & --- & $<0.5$ & 3.37 & 30 & --- & 160 & 0.8 & $<2$ \\
\hline 2011GG422B & 0.002 & --- & --- & --- & $<0.5$ & 7.59 & 7 & --- & 20 & $<0.5$ & $<2$ \\
\hline 2011GG456C & $<0.001$ & --- & --- & --- & $<0.5$ & 0.39 & 7 & --- & 60 & $<0.5$ & 2 \\
\hline 2011GG464A & 0.009 & --- & --- & --- & $<0.5$ & 4.3 & 15 & --- & 340 & 0.5 & $<2$ \\
\hline 2011GG470B & 0.008 & --- & --- & --- & $<0.5$ & 0.82 & 180 & --- & 260 & $<0.5$ & 2 \\
\hline 2011GG480A & $<0.001$ & --- & --- & --- & $<0.5$ & 1.67 & $<5$ & --- & 150 & $<0.5$ & $<2$ \\
\hline
\end{tabular}




\begin{tabular}{|c|c|c|c|c|c|c|c|c|c|c|c|}
\hline Sample_ID & Ca_pct & Cd_ppm & Co_ppm & Cr_ppm & Cu_ppm & Fe_pct & Ga_ppm & K_pct & La_ppm & Mg_pct & Mn_ppm \\
\hline 2011GG179A & 3.25 & $<0.5$ & $<1$ & 18 & 1 & 2.42 & 10 & 0.01 & $<10$ & 0.02 & 249 \\
\hline 2011GG179C & 9.8 & $<0.5$ & 34 & 146 & 92 & 8.33 & 20 & 0.06 & $<10$ & 2.58 & 2170 \\
\hline 2011GG185A & 5.34 & $<0.5$ & 14 & 42 & 24 & 4.47 & 20 & 0.03 & 10 & 0.67 & 1075 \\
\hline 2011GG186B & 2.86 & $<0.5$ & 25 & 57 & 124 & 5.63 & 10 & 0.1 & $<10$ & 2.29 & 1235 \\
\hline 2011GG194A & 2.06 & 1.3 & 36 & 23 & 45 & 8.77 & 20 & 0.3 & 20 & 3.15 & 987 \\
\hline 2011GG209A & 0.07 & 0.6 & 3 & 83 & 59 & 7.09 & 20 & 1.29 & 40 & 0.59 & 318 \\
\hline 2011GG210C & 1.82 & $<0.5$ & 6 & 31 & 13 & 3.87 & 10 & 0.04 & 10 & 0.67 & 438 \\
\hline 2011GG217B & 1.25 & $<0.5$ & 6 & 20 & 46 & 4.16 & 10 & 0.12 & 10 & 1.1 & 475 \\
\hline 2011GG300B & 2.99 & $<0.5$ & 13 & 66 & 6 & 2.69 & $<10$ & 0.01 & $<10$ & 1.55 & 576 \\
\hline 2011GG304B & 0.03 & $<0.5$ & 2 & 40 & 16 & 2.65 & 10 & 0.4 & $<10$ & 0.47 & 164 \\
\hline 2011GG323A & 5.66 & $<0.5$ & 43 & 127 & 79 & 8.96 & 20 & 0.05 & $<10$ & 4.62 & 1300 \\
\hline 2011GG335A & 0.01 & $<0.5$ & 3 & 28 & 18 & 1.54 & $<10$ & 0.23 & $<10$ & 0.05 & 83 \\
\hline 2011GG344A & 1.98 & $<0.5$ & 18 & 78 & 25 & 5.21 & 20 & 1.36 & 30 & 1.14 & 747 \\
\hline 2011GG349A & 0.28 & $<0.5$ & 15 & 100 & 33 & 5.07 & 20 & 2.18 & 30 & 0.96 & 484 \\
\hline 2011GG353A & 0.12 & 19.1 & 16 & 22 & 35 & 2.36 & 10 & 0.05 & $<10$ & 0.21 & 1800 \\
\hline 2011GG356A & 0.01 & $<0.5$ & 1 & 22 & 11 & 1.24 & $<10$ & 0.07 & $<10$ & 0.09 & 95 \\
\hline 2011GG359A & 0.19 & $<0.5$ & 27 & 71 & 4 & 5.13 & 20 & 2.69 & 30 & 0.75 & 2190 \\
\hline 2011GG365A & 0.04 & 1.3 & 166 & 36 & 192 & 25.5 & $<10$ & 0.74 & 50 & 0.13 & 4580 \\
\hline 2011GG366A & 0.01 & 0.5 & 30 & 15 & 20 & 48.4 & $<10$ & 0.45 & 10 & 0.06 & 1030 \\
\hline 2011GG376A & 0.65 & $<0.5$ & 4 & 59 & 43 & 3.27 & 10 & 0.74 & 20 & 0.42 & 160 \\
\hline 2011GG376C & 0.01 & 0.9 & 22 & 92 & 124 & 8.01 & 10 & 0.73 & 10 & 1.04 & 527 \\
\hline 2011GG382C & 0.08 & $<0.5$ & 9 & 41 & 33 & 3.16 & $<10$ & 0.3 & 10 & 0.42 & 259 \\
\hline 2011GG393A & 0.13 & $<0.5$ & 24 & 56 & 72 & 5.86 & 10 & 0.51 & 10 & 0.5 & 1125 \\
\hline 2011GG395A & 0.1 & $<0.5$ & 56 & 71 & 91 & 5.75 & 20 & 0.71 & 20 & 0.5 & 6440 \\
\hline 2011GG403A & 0.07 & $<0.5$ & 1 & 95 & 18 & 2.38 & 10 & 0.79 & 10 & 0.65 & 159 \\
\hline 2011GG422B & 0.05 & $<0.5$ & 5 & 14 & 12 & 1.07 & 10 & 0.04 & 10 & 0.05 & 746 \\
\hline 2011GG456C & 0.02 & $<0.5$ & 1 & 16 & 6 & 0.93 & $<10$ & 0.05 & $<10$ & 0.02 & 205 \\
\hline 2011GG464A & 0.02 & $<0.5$ & 19 & 118 & 30 & 4.69 & 10 & 0.4 & 10 & 0.7 & 298 \\
\hline 2011GG470B & 0.01 & $<0.5$ & 2 & 36 & 62 & 1.95 & $<10$ & 0.15 & $<10$ & 0.03 & 59 \\
\hline 2011GG480A & 0.07 & $<0.5$ & 4 & 24 & 4 & 1.69 & $<10$ & 0.59 & 10 & 0.31 & 409 \\
\hline
\end{tabular}




\begin{tabular}{|c|c|c|c|c|c|c|c|c|c|c|c|}
\hline Sample_ID & Mo_ppm & $\mathrm{Na}$ _pct & Ni_ppm & P_ppm & P_ppm_2 & Pb_ppm & Pd_ppm & Pt_ppm & S_pct & Sb_ppm & Sc_ppm \\
\hline 2011GG179A & $<1$ & 0.01 & 1 & 20 & -- & 4 & -- & -- & $<0.01$ & $<5$ & $<1$ \\
\hline 2011GG179C & $<1$ & 1.84 & 101 & 590 & --- & 14 & --- & --- & $<0.01$ & 5 & 39 \\
\hline 2011GG185A & $<1$ & 3.34 & 25 & 230 & -- & 9 & 0.002 & $<0.005$ & $<0.01$ & $<5$ & 11 \\
\hline 2011GG186B & $<1$ & 4.21 & 33 & 1120 & -- & $<2$ & 0.004 & $<0.005$ & $<0.01$ & $<5$ & 15 \\
\hline 2011GG194A & 4 & 0.9 & 50 & 1860 & --- & 5 & --- & -- & 0.01 & $<5$ & 27 \\
\hline 2011GG209A & 3 & 0.36 & 22 & 790 & --- & 14 & --- & -- & 0.13 & 7 & 13 \\
\hline 2011GG210C & 1 & 0.21 & 17 & 280 & --- & 11 & --- & --- & $<0.01$ & $<5$ & 11 \\
\hline 2011GG217B & 3 & 4.58 & 6 & 1010 & --- & 7 & --- & --- & 0.01 & $<5$ & 12 \\
\hline 2011GG300B & 1 & 0.37 & 39 & 50 & --- & 3 & --- & --- & $<0.01$ & 5 & 8 \\
\hline 2011GG304B & 1 & 0.09 & 12 & 130 & --- & 10 & --- & --- & 0.01 & $<5$ & 4 \\
\hline 2011GG323A & 3 & 2.43 & 59 & 470 & --- & 8 & --- & --- & 0.07 & 6 & 42 \\
\hline 2011GG335A & 1 & 0.03 & 9 & 220 & --- & 14 & -- & --- & 0.02 & $<5$ & 2 \\
\hline 2011GG344A & 6 & 0.35 & 37 & 690 & -- & 10 & -- & -- & 0.19 & 5 & 14 \\
\hline 2011GG349A & 3 & 0.42 & 79 & 950 & --- & 13 & --- & --- & 0.01 & $<5$ & 17 \\
\hline 2011GG353A & 1 & $<0.01$ & 4 & 230 & -- & 8760 & -- & -- & 0.02 & 12 & 1 \\
\hline 2011GG356A & $<1$ & 0.01 & 3 & 60 & --- & 13 & --- & --- & $<0.01$ & $<5$ & 1 \\
\hline 2011GG359A & $<1$ & 1.03 & 39 & 340 & --- & 27 & --- & --- & $<0.01$ & $<5$ & 15 \\
\hline 2011GG365A & $<1$ & 0.1 & 206 & 800 & --- & 23 & --- & --- & 0.03 & 8 & 7 \\
\hline 2011GG366A & $<1$ & 0.03 & 96 & 3350 & --- & 15 & --- & --- & 0.07 & 73 & 2 \\
\hline 2011GG376A & $<1$ & 0.2 & 15 & 3220 & --- & 36 & --- & --- & 0.01 & $<5$ & 9 \\
\hline 2011GG376C & $<1$ & 0.15 & 35 & 700 & --- & 245 & -- & -- & 0.02 & 8 & 26 \\
\hline 2011GG382C & $<1$ & 0.36 & 22 & 210 & --- & 29 & -- & --- & 0.01 & $<5$ & 5 \\
\hline 2011GG393A & $<1$ & 1.05 & 24 & 550 & -- & 9 & -- & -- & $<0.01$ & $<5$ & 19 \\
\hline 2011GG395A & $<1$ & 1.48 & 77 & 520 & -- & 40 & -- & -- & 0.01 & $<5$ & 17 \\
\hline 2011GG403A & 2 & 0.23 & 37 & 660 & --- & 6 & --- & --- & 0.02 & $<5$ & 6 \\
\hline 2011GG422B & $<1$ & 7.4 & 15 & 180 & --- & 4 & --- & --- & 0.03 & $<5$ & 2 \\
\hline 2011GG456C & 1 & 0.14 & 3 & 40 & --- & $<2$ & --- & --- & $<0.01$ & $<5$ & $<1$ \\
\hline 2011GG464A & 2 & 0.04 & 65 & 250 & --- & 10 & --- & --- & 0.01 & $<5$ & 14 \\
\hline 2011GG470B & 2 & 0.05 & 28 & 400 & --- & 4 & --- & --- & 0.01 & 43 & 2 \\
\hline 2011GG480A & $<1$ & 0.05 & 11 & 320 & --- & $<2$ & --- & --- & $<0.01$ & $<5$ & 2 \\
\hline
\end{tabular}




\begin{tabular}{|c|c|c|c|c|c|c|c|c|c|c|c|}
\hline Sample_ID & Sn_ppm & Sr_ppm & Th_ppm & Ti_pct & TI_ppm & U_ppm & V_ppm & W_ppm & W_ppm_2 & Zn_ppm & Zn_pct \\
\hline 2011GG179A & --- & 558 & $<20$ & 0.01 & $<10$ & $<10$ & 139 & $<10$ & -- & 11 & -- \\
\hline 2011GG179C & --- & 1350 & $<20$ & 0.98 & $<10$ & 10 & 440 & $<10$ & --- & 141 & --- \\
\hline 2011GG185A & --- & 3160 & 20 & 0.28 & $<10$ & $<10$ & 258 & $<10$ & -- & 25 & --- \\
\hline 2011GG186B & -- & 90 & $<20$ & 0.36 & $<10$ & $<10$ & 169 & $<10$ & -- & 62 & -- \\
\hline 2011GG194A & -- & 55 & $<20$ & 1.93 & $<10$ & $<10$ & 348 & $<10$ & -- & 222 & --- \\
\hline 2011GG209A & --- & 80 & $<20$ & 0.29 & $<10$ & $<10$ & 158 & $<10$ & --- & 152 & --- \\
\hline 2011GG210C & --- & 163 & $<20$ & 0.17 & $<10$ & $<10$ & 156 & $<10$ & --- & 57 & --- \\
\hline 2011GG217B & --- & 99 & $<20$ & 0.5 & $<10$ & 20 & 127 & $<10$ & --- & 49 & --- \\
\hline 2011GG300B & --- & 20 & $<20$ & 0.07 & $<10$ & $<10$ & 47 & $<10$ & --- & 23 & --- \\
\hline 2011GG304B & --- & 7 & $<20$ & 0.06 & $<10$ & $<10$ & 38 & $<10$ & --- & 36 & --- \\
\hline 2011GG323A & --- & 140 & $<20$ & 0.76 & $<10$ & 10 & 330 & $<10$ & --- & 112 & --- \\
\hline 2011GG335A & --- & 8 & $<20$ & 0.03 & $<10$ & $<10$ & 19 & $<10$ & -- & 25 & --- \\
\hline 2011GG344A & $<5$ & 87 & $<20$ & 0.77 & $<10$ & $<10$ & 144 & $<10$ & 20 & 109 & --- \\
\hline 2011GG349A & $<5$ & 70 & $<20$ & 0.49 & $<10$ & $<10$ & 176 & $<10$ & 20 & 113 & --- \\
\hline 2011GG353A & -- & 6 & $<20$ & 0.01 & $<10$ & $<10$ & 32 & 10 & -- & $>10000$ & 4.97 \\
\hline 2011GG356A & $<5$ & 4 & $<20$ & 0.02 & $<10$ & $<10$ & 10 & $<10$ & $<10$ & 19 & --- \\
\hline 2011GG359A & $<5$ & 114 & $<20$ & 0.42 & $<10$ & $<10$ & 76 & $<10$ & 10 & 76 & --- \\
\hline 2011GG365A & $<5$ & 80 & $<20$ & 0.08 & 10 & $<10$ & 45 & $<10$ & 100 & 761 & --- \\
\hline 2011GG366A & --- & 44 & $<20$ & 0.07 & $<10$ & $<10$ & 18 & $<10$ & --- & 277 & --- \\
\hline 2011GG376A & --- & 38 & $<20$ & 0.14 & $<10$ & $<10$ & 95 & $<10$ & --- & 81 & --- \\
\hline 2011GG376C & --- & 14 & $<20$ & 0.4 & $<10$ & $<10$ & 238 & $<10$ & --- & 145 & --- \\
\hline 2011GG382C & --- & 34 & $<20$ & 0.11 & $<10$ & $<10$ & 42 & $<10$ & -- & 104 & --- \\
\hline 2011GG393A & --- & 104 & $<20$ & 0.17 & $<10$ & $<10$ & 198 & $<10$ & -- & 76 & --- \\
\hline 2011GG395A & -- & 352 & $<20$ & 0.14 & $<10$ & $<10$ & 112 & $<10$ & -- & 80 & --- \\
\hline 2011GG403A & --- & 35 & $<20$ & 0.12 & $<10$ & $<10$ & 95 & $<10$ & --- & 70 & --- \\
\hline 2011GG422B & --- & 133 & $<20$ & 0.06 & $<10$ & $<10$ & 4 & $<10$ & --- & 21 & --- \\
\hline 2011GG456C & --- & 5 & $<20$ & 0.01 & $<10$ & $<10$ & 5 & $<10$ & --- & 4 & --- \\
\hline 2011GG464A & --- & 27 & $<20$ & 0.2 & $<10$ & $<10$ & 155 & $<10$ & --- & 79 & --- \\
\hline 2011GG470B & --- & 20 & $<20$ & 0.03 & $<10$ & $<10$ & 51 & $<10$ & --- & 108 & --- \\
\hline 2011GG480A & --- & 12 & $<20$ & 0.09 & $<10$ & $<10$ & 15 & $<10$ & --- & 22 & --- \\
\hline
\end{tabular}




\begin{tabular}{|c|c|c|c|c|c|c|c|c|c|c|c|}
\hline Sample_ID & Au_ppm & Au_ppm_2 & Au_ppm_3 & Au_ppm_4 & Ag_ppm & Al_pct & As_ppm & As_pct & Ba_ppm & Be_ppm & Bi_ppm \\
\hline 2011GG481B & $<0.001$ & -- & -- & -- & $<0.5$ & 1.47 & 6 & --- & 110 & $<0.5$ & $<2$ \\
\hline 2011GG484A & $<0.001$ & --- & -- & --- & $<0.5$ & 0.73 & $<5$ & --- & 210 & $<0.5$ & $<2$ \\
\hline 2011GG491A & 0.005 & --- & -- & -- & $<0.5$ & 8.37 & 21 & -- & 1100 & 2.3 & $<2$ \\
\hline 2011GG523B & $<0.001$ & --- & -- & -- & $<0.5$ & 0.94 & 6 & --- & 290 & $<0.5$ & $<2$ \\
\hline 2011GG529A & $<0.001$ & -- & -- & -- & $<0.5$ & 7.56 & $<5$ & -- & 750 & 4.2 & $<2$ \\
\hline 2011GG551A & $<0.001$ & --- & -- & -- & $<0.5$ & 0.19 & $<5$ & -- & 120 & $<0.5$ & 2 \\
\hline 2011GG554B & 0.001 & --- & -- & -- & $<0.5$ & 0.8 & 20 & --- & 70 & $<0.5$ & $<2$ \\
\hline 2011GG564A & $<0.001$ & --- & --- & --- & $<0.5$ & 8.01 & $<5$ & --- & 480 & 2 & 2 \\
\hline 2011LF025B & 0.004 & --- & --- & --- & $<0.5$ & 5.95 & 9 & --- & 700 & 1.1 & 2 \\
\hline 2011LF027B & $<0.001$ & --- & --- & --- & $<0.5$ & 0.23 & $<5$ & --- & 20 & $<0.5$ & $<2$ \\
\hline 2011LF039B & 0.002 & --- & --- & --- & $<0.5$ & 2.56 & 27 & --- & 190 & 0.8 & $<2$ \\
\hline 2011LF040B & 0.02 & --- & --- & --- & $<0.5$ & 1.55 & $<5$ & --- & 230 & 0.6 & $<2$ \\
\hline 2011LF040C & 0.01 & -- & -- & -- & $<0.5$ & 1.61 & $<5$ & -- & 190 & 0.5 & $<2$ \\
\hline 2011LF070C^ & 0.025 & --- & --- & --- & 0.6 & 2.03 & 36 & --- & 1090 & 1 & $<2$ \\
\hline 2011LF081A^ & 0.002 & -- & -- & -- & $<0.5$ & 1.55 & 22 & -- & 90 & 0.8 & $<2$ \\
\hline 2011LF100A^ & 0.003 & --- & --- & --- & $<0.5$ & 5.18 & 28 & --- & 100 & 1.9 & $<2$ \\
\hline 2011LF101A^ & 0.001 & --- & -- & --- & 0.6 & 4.78 & 14 & --- & 150 & 32.5 & $<2$ \\
\hline 2011LF119A & $<0.001$ & --- & --- & --- & $<0.5$ & 7.87 & 32 & --- & 460 & 3 & 3 \\
\hline 2011LF123A & $<0.001$ & --- & --- & --- & $<0.5$ & 7.5 & 116 & --- & 980 & 2.5 & $<2$ \\
\hline 2011LF128A & $<0.001$ & --- & --- & --- & $<0.5$ & 1.37 & $<5$ & --- & 100 & 0.5 & $<2$ \\
\hline 2011LF129A & 0.001 & -- & --- & --- & $<0.5$ & 2.79 & 10 & -- & 160 & 1.2 & $<2$ \\
\hline 2011LF180B & $<0.001$ & -- & --- & --- & $<0.5$ & 7.58 & 37 & -- & 100 & 1 & 7 \\
\hline 2011LF223A & $<0.001$ & -- & -- & --- & 0.8 & 7.26 & $<5$ & -- & 390 & 0.5 & 2 \\
\hline 2011LF261C & 1.11 & -- & -- & -- & 2.1 & 0.33 & 10 & -- & 40 & $<0.5$ & 18 \\
\hline 2011LF268A & $<0.001$ & --- & -- & --- & $<0.5$ & 1.89 & 5 & --- & 90 & $<0.5$ & 2 \\
\hline 2011LF271A & 0.003 & --- & --- & --- & $<0.5$ & 2.5 & 13 & --- & 240 & 0.7 & $<2$ \\
\hline 2011LF276B & $<0.001$ & --- & --- & --- & $<0.5$ & 0.16 & $<5$ & --- & 10 & $<0.5$ & $<2$ \\
\hline 2011LF297A & $<0.001$ & --- & --- & --- & $<0.5$ & 1.67 & 21 & --- & 60 & 0.5 & $<2$ \\
\hline 2011LF300A & $<0.001$ & --- & --- & --- & $<0.5$ & 6.18 & 13 & --- & 530 & 32.8 & 7 \\
\hline 2011LF314A & 0.001 & --- & --- & --- & $<0.5$ & 1.24 & 6 & --- & 150 & 0.5 & $<2$ \\
\hline
\end{tabular}




\begin{tabular}{|c|c|c|c|c|c|c|c|c|c|c|c|}
\hline Sample_ID & Ca_pct & Cd_ppm & Co_ppm & Cr_ppm & Cu_ppm & Fe_pct & Ga_ppm & K_pct & La_ppm & Mg_pct & Mn_ppm \\
\hline 2011GG481B & 0.17 & $<0.5$ & 2 & 20 & 7 & 1.41 & $<10$ & 0.33 & 10 & 0.06 & 820 \\
\hline 2011GG484A & 0.01 & $<0.5$ & 2 & 19 & 21 & 1.4 & $<10$ & 0.34 & $<10$ & 0.08 & 99 \\
\hline 2011GG491A & 0.11 & $<0.5$ & 18 & 91 & 47 & 5.21 & 20 & 2.69 & 40 & 0.92 & 313 \\
\hline 2011GG523B & 0.19 & $<0.5$ & 8 & 12 & 15 & 1.72 & $<10$ & 0.2 & $<10$ & 0.47 & 249 \\
\hline 2011GG529A & 1.93 & $<0.5$ & 7 & 22 & 7 & 3.07 & 20 & 3.57 & 50 & 0.79 & 529 \\
\hline 2011GG551A & 0.01 & $<0.5$ & 2 & 16 & 29 & 0.91 & $<10$ & 0.06 & $<10$ & 0.01 & 80 \\
\hline 2011GG554B & 0.01 & $<0.5$ & 10 & 36 & 12 & 1.66 & $<10$ & 0.17 & 10 & 0.2 & 181 \\
\hline 2011GG564A & 0.28 & $<0.5$ & 19 & 72 & 43 & 4.7 & 20 & 2.76 & 40 & 0.99 & 443 \\
\hline 2011LF025B & 4.56 & $<0.5$ & 23 & 193 & 108 & 5.72 & 10 & 1.13 & 10 & 3.04 & 915 \\
\hline 2011LF027B & 0.05 & $<0.5$ & 1 & 18 & 13 & 0.74 & $<10$ & 0.07 & $<10$ & 0.02 & 62 \\
\hline 2011LF039B & 0.04 & 1.6 & 10 & 86 & 91 & 12.5 & $<10$ & 0.31 & 10 & 0.11 & 169 \\
\hline 2011LF040B & 0.03 & $<0.5$ & $<1$ & 41 & 4 & 0.68 & 10 & 0.65 & $<10$ & 0.21 & 57 \\
\hline 2011LF040C & 0.01 & $<0.5$ & $<1$ & 37 & 11 & 0.98 & 10 & 0.62 & $<10$ & 0.18 & 48 \\
\hline 2011LF070C^ & 1.24 & 0.8 & 2 & 49 & 138 & 3.66 & $<10$ & 0.83 & 10 & 0.3 & 77 \\
\hline 2011LF081A^ & 0.01 & $<0.5$ & 6 & 31 & 135 & 5.52 & $<10$ & 0.21 & $<10$ & 0.06 & 195 \\
\hline 2011LF100A^ & 1.06 & $<0.5$ & 16 & 94 & 113 & 11.9 & 20 & 0.28 & 10 & 0.96 & 2710 \\
\hline 2011LF101A^ & 0.08 & 1.5 & 16 & 44 & 14 & 11.8 & 30 & 0.31 & 30 & 1.61 & 3160 \\
\hline 2011LF119A & 0.15 & $<0.5$ & 3 & 76 & 34 & 3.64 & 20 & 1.97 & 30 & 0.92 & 183 \\
\hline 2011LF123A & 9.5 & $<0.5$ & 12 & 71 & 17 & 4.33 & 20 & 2.24 & 30 & 2.85 & 1285 \\
\hline 2011LF128A & 0.02 & $<0.5$ & 1 & 27 & 8 & 1.45 & $<10$ & 0.5 & 10 & 0.1 & 123 \\
\hline 2011LF129A & 0.01 & $<0.5$ & 12 & 31 & 18 & 3.61 & 10 & 0.74 & 10 & 0.11 & 507 \\
\hline 2011LF180B & 0.02 & 0.5 & 7 & 111 & 138 & 13.95 & 30 & 0.02 & $<10$ & 0.02 & 205 \\
\hline 2011LF223A & 7.95 & 1 & 38 & 150 & 109 & 7.85 & 20 & 0.17 & 10 & 3.48 & 6060 \\
\hline 2011LF261C & 0.01 & $<0.5$ & 3 & 28 & 19 & 1.43 & $<10$ & 0.11 & $<10$ & 0.04 & 131 \\
\hline 2011LF268A & 0.06 & $<0.5$ & 6 & 41 & 12 & 2.93 & $<10$ & 0.27 & 10 & 0.22 & 1615 \\
\hline 2011LF271A & 0.01 & $<0.5$ & 6 & 30 & 8 & 1.85 & 10 & 0.83 & 20 & 0.1 & 120 \\
\hline 2011LF276B & 0.02 & $<0.5$ & $<1$ & 27 & 8 & 1.39 & $<10$ & 0.02 & $<10$ & 0.04 & 84 \\
\hline 2011LF297A & 0.07 & $<0.5$ & 7 & 41 & 85 & 5.9 & 10 & 0.06 & 10 & 0.6 & 895 \\
\hline 2011LF300A & 0.11 & $<0.5$ & 7 & 134 & 62 & 6.22 & 20 & 1.28 & 30 & 0.53 & 305 \\
\hline 2011LF314A & 4.68 & $<0.5$ & 1 & 30 & 265 & 1.06 & $<10$ & 0.28 & $<10$ & 0.25 & 116 \\
\hline
\end{tabular}




\begin{tabular}{|c|c|c|c|c|c|c|c|c|c|c|c|}
\hline Sample_ID & Mo_ppm & $\mathrm{Na}$ _pct & Ni_ppm & P_ppm & P_ppm_2 & Pb_ppm & Pd_ppm & Pt_ppm & S_pct & Sb_ppm & Sc_ppm \\
\hline 2011GG481B & 1 & 0.19 & 7 & 620 & -- & 28 & -- & -- & 0.01 & $<5$ & 2 \\
\hline 2011GG484A & $<1$ & 0.02 & 11 & 80 & --- & 7 & --- & --- & $<0.01$ & 5 & 2 \\
\hline 2011GG491A & $<1$ & 0.33 & 48 & 840 & -- & 23 & --- & -- & 0.01 & $<5$ & 17 \\
\hline 2011GG523B & $<1$ & 0.01 & 21 & 150 & -- & $<2$ & -- & -- & $<0.01$ & $<5$ & 2 \\
\hline 2011GG529A & $<1$ & 1.67 & 5 & 760 & --- & 26 & --- & -- & 0.01 & $<5$ & 10 \\
\hline 2011GG551A & $<1$ & 0.01 & 4 & 30 & --- & $<2$ & --- & -- & $<0.01$ & $<5$ & 1 \\
\hline 2011GG554B & $<1$ & 0.03 & 23 & 30 & --- & $<2$ & --- & --- & 0.01 & $<5$ & 2 \\
\hline 2011GG564A & $<1$ & 0.77 & 36 & 330 & --- & 14 & --- & --- & $<0.01$ & $<5$ & 15 \\
\hline 2011LF025B & $<1$ & 0.08 & 106 & 1120 & --- & 5 & --- & --- & 0.64 & $<5$ & 20 \\
\hline 2011LF027B & $<1$ & 0.01 & 4 & 170 & --- & $<2$ & --- & --- & $<0.01$ & $<5$ & $<1$ \\
\hline 2011LF039B & 1 & 0.21 & 135 & 2730 & --- & 43 & --- & --- & 0.01 & 11 & 9 \\
\hline 2011LF040B & $<1$ & 0.02 & 14 & 200 & --- & 2 & -- & --- & 0.01 & $<5$ & 4 \\
\hline 2011LF040C & 2 & 0.02 & 9 & 150 & -- & 2 & -- & -- & 0.01 & $<5$ & 4 \\
\hline 2011LF070C^ & 4 & 0.01 & 38 & $>10000$ & 9990 & 14 & --- & --- & 0.01 & $<5$ & 6 \\
\hline 2011LF081A^ & 4 & 0.01 & 16 & 1760 & -- & 6 & -- & -- & 0.01 & $<5$ & 3 \\
\hline 2011LF100A^ & 16 & 0.72 & 58 & 710 & --- & 21 & --- & --- & 0.45 & $<5$ & 12 \\
\hline 2011LF101A^ & 2 & 0.02 & 72 & 490 & --- & 504 & --- & --- & $<0.01$ & $<5$ & 10 \\
\hline 2011LF119A & $<1$ & 0.35 & 5 & 230 & --- & 11 & --- & --- & $<0.01$ & $<5$ & 13 \\
\hline 2011LF123A & $<1$ & 0.45 & 41 & 640 & --- & 16 & --- & --- & 0.02 & $<5$ & 13 \\
\hline 2011LF128A & $<1$ & 0.02 & 4 & 100 & --- & 4 & --- & --- & $<0.01$ & $<5$ & 2 \\
\hline 2011LF129A & $<1$ & 0.02 & 17 & 260 & --- & 5 & -- & -- & $<0.01$ & $<5$ & 5 \\
\hline 2011LF180B & 1 & 0.02 & 37 & 3560 & --- & 4 & -- & -- & 0.01 & 10 & 40 \\
\hline 2011LF223A & 3 & 1.32 & 72 & 800 & -- & 7 & -- & -- & 0.09 & 6 & 34 \\
\hline 2011LF261C & 3 & 0.02 & 5 & 30 & -- & 42 & -- & -- & $<0.01$ & $<5$ & 1 \\
\hline 2011LF268A & 1 & 0.27 & 9 & 290 & --- & 55 & --- & --- & $<0.01$ & $<5$ & 3 \\
\hline 2011LF271A & 1 & 0.17 & 10 & 110 & --- & 6 & --- & --- & $<0.01$ & $<5$ & 3 \\
\hline 2011LF276B & $<1$ & 0.01 & 4 & 70 & --- & $<2$ & --- & --- & $<0.01$ & $<5$ & $<1$ \\
\hline 2011LF297A & 2 & $<0.01$ & 17 & 420 & --- & 7 & --- & --- & 0.02 & $<5$ & 4 \\
\hline 2011LF300A & 4 & 0.3 & 48 & 1060 & --- & 6 & --- & --- & 0.01 & 6 & 12 \\
\hline 2011LF314A & 1 & 0.01 & 7 & $>10000$ & 22800 & 9 & --- & --- & 0.01 & $<5$ & 3 \\
\hline
\end{tabular}




\begin{tabular}{|c|c|c|c|c|c|c|c|c|c|c|c|}
\hline Sample_ID & Sn_ppm & Sr_ppm & Th_ppm & Ti_pct & TI_ppm & U_ppm & V_ppm & W_ppm & W_ppm_2 & Zn_ppm & Zn_pct \\
\hline 2011GG481B & --- & 35 & $<20$ & 0.05 & $<10$ & $<10$ & 13 & $<10$ & -- & 8 & -- \\
\hline 2011GG484A & --- & 24 & $<20$ & 0.04 & $<10$ & $<10$ & 14 & $<10$ & --- & 37 & --- \\
\hline 2011GG491A & --- & 78 & $<20$ & 0.29 & $<10$ & $<10$ & 173 & $<10$ & -- & 162 & --- \\
\hline 2011GG523B & -- & 16 & $<20$ & 0.05 & $<10$ & $<10$ & 20 & $<10$ & -- & 5 & -- \\
\hline 2011GG529A & 5 & 338 & 20 & 0.4 & $<10$ & $<10$ & 51 & $<10$ & 10 & 62 & --- \\
\hline 2011GG551A & --- & 3 & $<20$ & 0.01 & $<10$ & $<10$ & 4 & $<10$ & --- & 9 & --- \\
\hline 2011GG554B & --- & 4 & $<20$ & 0.04 & $<10$ & $<10$ & 20 & $<10$ & --- & 40 & --- \\
\hline 2011GG564A & --- & 110 & 20 & 0.36 & $<10$ & $<10$ & 80 & $<10$ & --- & 87 & --- \\
\hline 2011LF025B & --- & 94 & $<20$ & 0.79 & 10 & $<10$ & 178 & $<10$ & --- & 89 & --- \\
\hline 2011LF027B & --- & 4 & $<20$ & 0.01 & $<10$ & $<10$ & 10 & $<10$ & --- & $<2$ & --- \\
\hline 2011LF039B & --- & 41 & $<20$ & 0.11 & 10 & $<10$ & 176 & $<10$ & --- & 508 & --- \\
\hline 2011LF040B & --- & 10 & $<20$ & 0.09 & $<10$ & $<10$ & 234 & $<10$ & -- & 44 & --- \\
\hline 2011LF040C & -- & 6 & $<20$ & 0.09 & $<10$ & $<10$ & 245 & $<10$ & -- & 37 & --- \\
\hline 2011LF070C^ & --- & 94 & $<20$ & 0.1 & $<10$ & $<10$ & 328 & $<10$ & --- & 80 & --- \\
\hline 2011LF081A^ & -- & 7 & $<20$ & 0.06 & $<10$ & 10 & 129 & $<10$ & -- & 24 & -- \\
\hline 2011LF100A^ & 7 & 57 & $<20$ & 0.37 & $<10$ & $<10$ & 170 & 20 & 50 & 199 & --- \\
\hline 2011LF101A^ & 15 & 5 & $<20$ & 0.18 & $<10$ & $<10$ & 117 & 20 & 60 & 932 & --- \\
\hline 2011LF119A & 67 & 76 & $<20$ & 0.32 & $<10$ & $<10$ & 77 & 10 & 10 & 15 & --- \\
\hline 2011LF123A & 7 & 294 & $<20$ & 0.41 & $<10$ & $<10$ & 108 & $<10$ & 20 & 159 & --- \\
\hline 2011LF128A & --- & 6 & $<20$ & 0.11 & $<10$ & $<10$ & 16 & $<10$ & --- & 10 & --- \\
\hline 2011LF129A & --- & 12 & $<20$ & 0.19 & $<10$ & $<10$ & 33 & $<10$ & -- & 40 & --- \\
\hline 2011LF180B & --- & 11 & $<20$ & 1.79 & $<10$ & 20 & 448 & $<10$ & -- & 142 & --- \\
\hline 2011LF223A & -- & 75 & $<20$ & 0.94 & $<10$ & $<10$ & 306 & $<10$ & -- & 106 & --- \\
\hline 2011LF261C & --- & 4 & $<20$ & 0.02 & $<10$ & $<10$ & 8 & $<10$ & --- & 22 & --- \\
\hline 2011LF268A & --- & 36 & $<20$ & 0.1 & $<10$ & $<10$ & 28 & $<10$ & --- & 26 & --- \\
\hline 2011LF271A & --- & 29 & $<20$ & 0.08 & $<10$ & $<10$ & 21 & $<10$ & --- & 25 & --- \\
\hline 2011LF276B & --- & 2 & $<20$ & 0.01 & $<10$ & $<10$ & 6 & $<10$ & --- & 9 & --- \\
\hline 2011LF297A & 17 & 13 & $<20$ & 0.1 & $<10$ & $<10$ & 56 & 20 & 30 & 95 & --- \\
\hline 2011LF300A & 16 & 32 & $<20$ & 0.29 & $<10$ & $<10$ & 148 & 110 & 150 & 53 & --- \\
\hline 2011LF314A & --- & 166 & $<20$ & 0.04 & $<10$ & 10 & 123 & $<10$ & --- & 52 & --- \\
\hline
\end{tabular}




\begin{tabular}{|c|c|c|c|c|c|c|c|c|c|c|c|}
\hline Sample_ID & Au_ppm & Au_ppm_2 & Au_ppm_3 & Au_ppm_4 & Ag_ppm & Al_pct & As_ppm & As_pct & Ba_ppm & Be_ppm & Bi_ppm \\
\hline 2011LF317A & 0.01 & -- & -- & -- & $<0.5$ & 1.4 & 13 & -- & 400 & 0.6 & $<2$ \\
\hline 2011LF332A & $<0.001$ & --- & -- & --- & $<0.5$ & 6.12 & $<5$ & --- & 140 & 15.3 & 17 \\
\hline 2011LF345A & $<0.001$ & -- & -- & -- & $<0.5$ & 7.1 & $<5$ & -- & 40 & $<0.5$ & $<2$ \\
\hline 2011LF365A & 0.001 & --- & -- & -- & $<0.5$ & 0.86 & 11 & -- & 60 & $<0.5$ & $<2$ \\
\hline 2011LF369B & $<0.001$ & --- & -- & -- & $<0.5$ & 0.41 & $<5$ & -- & 40 & $<0.5$ & $<2$ \\
\hline 2011LF394A & 0.001 & --- & -- & -- & $<0.5$ & 1.23 & 6 & -- & 80 & 0.6 & $<2$ \\
\hline 2011LF401A & 0.002 & --- & -- & -- & $<0.5$ & 4.94 & 9 & -- & 190 & 1.2 & $<2$ \\
\hline 2011LF408A & 0.007 & --- & --- & --- & 0.6 & 1.56 & 27 & --- & 80 & $<0.5$ & $<2$ \\
\hline 2011LF411B & 0.003 & --- & --- & --- & $<0.5$ & 7.74 & 41 & --- & 530 & 1.2 & $<2$ \\
\hline 2011LF412B & 0.005 & --- & --- & --- & $<0.5$ & 4.04 & 121 & --- & 450 & 1 & $<2$ \\
\hline 2011LF485A & 0.001 & --- & --- & --- & $<0.5$ & 0.74 & 35 & --- & 70 & 0.5 & $<2$ \\
\hline 2011LF498A & $<0.001$ & --- & --- & --- & $<0.5$ & 1.85 & 12 & -- & 130 & 0.5 & $<2$ \\
\hline 2011LF502A & 0.019 & -- & -- & -- & $<0.5$ & 4.08 & $<5$ & -- & 390 & 1.1 & $<2$ \\
\hline 2011LF507A & 0.006 & --- & --- & --- & $<0.5$ & 0.95 & 16 & --- & 100 & $<0.5$ & $<2$ \\
\hline 2011LF528A & 0.002 & -- & -- & --- & $<0.5$ & 1.59 & 11 & -- & 80 & $<0.5$ & $<2$ \\
\hline 2011LF557B & 0.035 & --- & --- & --- & 1.3 & 5.49 & 88 & --- & 230 & 1.1 & $<2$ \\
\hline 2011LF587B & 0.003 & --- & -- & --- & 0.8 & 9.69 & 5 & --- & 90 & $<0.5$ & $<2$ \\
\hline 2011RN153A & 0.002 & --- & --- & --- & 0.5 & 1.51 & 10 & --- & 340 & 0.5 & $<2$ \\
\hline 2011RN177B & 0.001 & --- & --- & --- & $<0.5$ & 5.74 & $<5$ & --- & 790 & 4.9 & 3 \\
\hline 2011RN180B & $<0.001$ & --- & --- & --- & $<0.5$ & 9.43 & $<5$ & --- & 670 & 2.7 & $<2$ \\
\hline 2011RN180C & $<0.001$ & --- & --- & --- & $<0.5$ & 1.78 & $<5$ & --- & 110 & 0.6 & $<2$ \\
\hline 2011Z005B & 0.004 & -- & --- & --- & $<0.5$ & 6.35 & 5 & --- & 250 & 3.8 & 5 \\
\hline $2011 Z 012 \mathrm{E}$ & 0.001 & -- & --- & --- & $<0.5$ & 3.35 & 11 & -- & 280 & 0.9 & $<2$ \\
\hline $2011 Z 024 \mathrm{~A}$ & 0.031 & -- & -- & -- & 1.5 & 4.04 & 56 & -- & 400 & 1.7 & $<2$ \\
\hline 2011Z026B & $<0.001$ & --- & --- & --- & $<0.5$ & 0.88 & $<5$ & --- & 50 & $<0.5$ & $<2$ \\
\hline $2011 Z 028 \mathrm{~A}$ & 0.03 & --- & --- & --- & $<0.5$ & 2.59 & 10 & --- & 1510 & 0.6 & $<2$ \\
\hline 2011Z029A & $<0.001$ & --- & --- & --- & $<0.5$ & 4.91 & $<5$ & --- & 6710 & 1.3 & $<2$ \\
\hline 2011Z044A & 0.001 & --- & --- & --- & $<0.5$ & 5.9 & 16 & --- & 510 & 1.2 & $<2$ \\
\hline 2011Z050A & $<0.001$ & --- & --- & --- & $<0.5$ & 0.05 & $<5$ & --- & 10 & $<0.5$ & $<2$ \\
\hline 2011Z059A & $<0.001$ & --- & --- & --- & $<0.5$ & 0.17 & 12 & --- & 10 & $<0.5$ & $<2$ \\
\hline
\end{tabular}




\begin{tabular}{|c|c|c|c|c|c|c|c|c|c|c|c|}
\hline Sample_ID & Ca_pct & Cd_ppm & Co_ppm & Cr_ppm & Cu_ppm & Fe_pct & Ga_ppm & K_pct & La_ppm & Mg_pct & Mn_ppm \\
\hline 2011LF317A & 0.09 & $<0.5$ & $<1$ & 47 & 1090 & 1.43 & $<10$ & 0.35 & $<10$ & 0.13 & 88 \\
\hline 2011LF332A & 0.34 & $<0.5$ & 1 & 8 & 6 & 2.09 & 20 & 4.09 & 40 & 0.08 & 407 \\
\hline 2011LF345A & 0.08 & $<0.5$ & 3 & 11 & 7 & 0.74 & 10 & 0.03 & $<10$ & 0.14 & 86 \\
\hline 2011LF365A & 0.03 & $<0.5$ & 2 & 20 & 31 & 0.95 & $<10$ & 0.37 & $<10$ & 0.12 & 64 \\
\hline 2011LF369B & 0.06 & $<0.5$ & 1 & 23 & 8 & 1 & $<10$ & 0.09 & $<10$ & 0.07 & 139 \\
\hline 2011LF394A & 0.12 & $<0.5$ & 1 & 28 & 12 & 0.86 & $<10$ & 0.54 & 10 & 0.13 & 114 \\
\hline 2011LF401A & 0.05 & $<0.5$ & 6 & 58 & 33 & 2.95 & 10 & 0.79 & 10 & 0.54 & 721 \\
\hline 2011LF408A & 0.01 & $<0.5$ & 1 & 31 & 19 & 2.63 & $<10$ & 0.41 & 10 & 0.09 & 95 \\
\hline 2011LF411B & 0.37 & 0.8 & 51 & 566 & 118 & 10.35 & 20 & 0.82 & 10 & 2.66 & 746 \\
\hline 2011LF412B & 0.23 & 3.7 & 34 & 194 & 825 & 15.4 & 10 & 0.6 & 10 & 0.72 & 588 \\
\hline 2011LF485A & $<0.01$ & $<0.5$ & 4 & 21 & 83 & 3.43 & $<10$ & 0.16 & $<10$ & 0.04 & 134 \\
\hline 2011LF498A & 0.04 & $<0.5$ & 1 & 20 & 13 & 1.39 & $<10$ & 0.95 & 10 & 0.15 & 106 \\
\hline 2011LF502A & 0.03 & $<0.5$ & 7 & 37 & 20 & 3.89 & 10 & 0.99 & 20 & 0.14 & 141 \\
\hline 2011LF507A & 0.02 & $<0.5$ & 3 & 28 & 27 & 4.53 & $<10$ & 0.19 & $<10$ & 0.07 & 126 \\
\hline 2011LF528A & 16.1 & $<0.5$ & 11 & 127 & 27 & 4.22 & $<10$ & 0.15 & $<10$ & 6.71 & 1025 \\
\hline 2011LF557B & 1.32 & 1 & $<1$ & 93 & 218 & 4.16 & 10 & 0.93 & 40 & 0.18 & 82 \\
\hline 2011LF587B & 9.32 & $<0.5$ & 17 & 103 & 38 & 3.06 & 20 & 0.13 & $<10$ & 1.97 & 563 \\
\hline 2011RN153A & 0.09 & $<0.5$ & 1 & 86 & 39 & 1.3 & 10 & 0.59 & $<10$ & 0.17 & 49 \\
\hline 2011RN177B & 1.58 & $<0.5$ & 2 & 15 & 14 & 2.73 & 10 & 3.71 & 50 & 0.31 & 445 \\
\hline 2011RN180B & 0.14 & $<0.5$ & 27 & 94 & 2 & 6.41 & 30 & 2.51 & 20 & 1.33 & 1475 \\
\hline 2011RN180C & 0.04 & $<0.5$ & 1 & 17 & 7 & 1.05 & $<10$ & 0.78 & $<10$ & 0.14 & 124 \\
\hline 2011Z005B & 0.38 & $<0.5$ & 2 & 11 & 3 & 1.41 & 20 & 4.36 & 50 & 0.1 & 230 \\
\hline $2011 Z 012 \mathrm{E}$ & 0.04 & $<0.5$ & 7 & 57 & 13 & 2.54 & 10 & 0.55 & 10 & 0.25 & 513 \\
\hline $2011 Z 024 \mathrm{~A}$ & 0.76 & $<0.5$ & 2 & 73 & 31 & 2.93 & 10 & 2.07 & 30 & 0.51 & 145 \\
\hline 2011Z026B & 0.01 & $<0.5$ & 3 & 33 & 8 & 1.37 & $<10$ & 0.18 & 10 & 0.21 & 102 \\
\hline $2011 Z 028 \mathrm{~A}$ & 0.02 & $<0.5$ & 2 & 40 & 76 & 3.54 & 10 & 0.56 & 10 & 0.2 & 96 \\
\hline 2011Z029A & 0.08 & $<0.5$ & 12 & 52 & 68 & 3.25 & 10 & 1.34 & 20 & 0.68 & 2180 \\
\hline 2011Z044A & 0.16 & $<0.5$ & 7 & 92 & 44 & 4.72 & 10 & 1.05 & 10 & 1.02 & 355 \\
\hline $2011 Z 050 \mathrm{~A}$ & $<0.01$ & $<0.5$ & 1 & 24 & 5 & 0.77 & $<10$ & 0.01 & $<10$ & $<0.01$ & 70 \\
\hline 2011Z059A & 0.01 & $<0.5$ & 1 & 22 & 2 & 0.86 & $<10$ & 0.02 & $<10$ & 0.05 & 74 \\
\hline
\end{tabular}




\begin{tabular}{|c|c|c|c|c|c|c|c|c|c|c|c|}
\hline Sample_ID & Mo_ppm & $\mathrm{Na}$ _pct & Ni_ppm & P_ppm & P_ppm_2 & Pb_ppm & Pd_ppm & Pt_ppm & S_pct & Sb_ppm & Sc_ppm \\
\hline 2011LF317A & 2 & 0.01 & 6 & 4480 & -- & 25 & -- & -- & $<0.01$ & 6 & 3 \\
\hline 2011LF332A & $<1$ & 1.74 & 1 & 290 & --- & 20 & --- & --- & $<0.01$ & $<5$ & 3 \\
\hline 2011LF345A & $<1$ & 7.1 & 4 & 70 & -- & $<2$ & --- & -- & $<0.01$ & $<5$ & 1 \\
\hline 2011LF365A & 1 & 0.03 & 11 & 180 & --- & 4 & --- & -- & $<0.01$ & $<5$ & 3 \\
\hline 2011LF369B & 1 & 0.03 & 6 & 60 & --- & $<2$ & --- & -- & $<0.01$ & $<5$ & 1 \\
\hline 2011LF394A & 1 & 0.06 & 4 & 590 & -- & 4 & --- & -- & $<0.01$ & $<5$ & 2 \\
\hline 2011LF401A & $<1$ & 0.71 & 26 & 300 & --- & 8 & --- & -- & 0.01 & $<5$ & 6 \\
\hline 2011LF408A & 10 & 0.03 & 16 & 1300 & --- & 12 & --- & --- & 0.01 & $<5$ & 5 \\
\hline 2011LF411B & 2 & 0.1 & 288 & 1910 & --- & 5 & --- & --- & 0.01 & 11 & 36 \\
\hline 2011LF412B & 11 & 0.01 & 304 & 8900 & --- & 9 & --- & --- & 0.02 & 33 & 12 \\
\hline 2011LF485A & 5 & 0.05 & 10 & 830 & --- & $<2$ & --- & --- & $<0.01$ & $<5$ & 3 \\
\hline 2011LF498A & $<1$ & 0.3 & 5 & 180 & --- & 6 & -- & -- & $<0.01$ & $<5$ & 2 \\
\hline 2011LF502A & $<1$ & 0.12 & 24 & 290 & -- & 15 & -- & -- & $<0.01$ & $<5$ & 6 \\
\hline 2011LF507A & 3 & 0.02 & 26 & 1440 & --- & 2 & --- & --- & 0.01 & 5 & 3 \\
\hline 2011LF528A & $<1$ & 0.02 & 74 & 300 & -- & $<2$ & -- & -- & $<0.01$ & 16 & 6 \\
\hline 2011LF557B & 14 & 0.01 & 9 & 4530 & --- & 30 & --- & --- & 1.36 & 8 & 12 \\
\hline 2011LF587B & $<1$ & 1.28 & 31 & 60 & --- & 9 & --- & --- & 0.01 & $<5$ & 12 \\
\hline 2011RN153A & $<1$ & 0.03 & 31 & 340 & --- & $<2$ & --- & --- & 0.01 & $<5$ & 6 \\
\hline 2011RN177B & 2 & 1.3 & $<1$ & 550 & --- & 14 & --- & --- & 0.01 & $<5$ & 14 \\
\hline 2011RN180B & $<1$ & 0.81 & 58 & 310 & --- & 15 & --- & --- & $<0.01$ & $<5$ & 15 \\
\hline 2011RN180C & $<1$ & 0.24 & 3 & 110 & --- & 5 & --- & --- & $<0.01$ & $<5$ & 1 \\
\hline 2011Z005B & $<1$ & 1.7 & 3 & 150 & --- & 39 & -- & --- & $<0.01$ & $<5$ & 5 \\
\hline $2011 Z 012 \mathrm{E}$ & $<1$ & 0.3 & 20 & 320 & -- & 2 & -- & -- & $<0.01$ & $<5$ & 6 \\
\hline $2011 Z 024 \mathrm{~A}$ & 9 & 0.04 & 25 & 6260 & -- & 35 & -- & -- & 0.01 & 11 & 9 \\
\hline 2011Z026B & $<1$ & 0.05 & 8 & 60 & --- & $<2$ & --- & --- & $<0.01$ & $<5$ & 2 \\
\hline $2011 Z 028 \mathrm{~A}$ & $<1$ & 0.31 & 9 & 320 & --- & 6 & --- & --- & 0.03 & $<5$ & 7 \\
\hline 2011Z029A & $<1$ & 0.43 & 45 & 470 & --- & 11 & --- & --- & 0.05 & $<5$ & 12 \\
\hline 2011Z044A & $<1$ & 0.55 & 41 & 580 & --- & 6 & --- & --- & 0.01 & $<5$ & 13 \\
\hline $2011 Z 050 \mathrm{~A}$ & $<1$ & 0.01 & 4 & 10 & --- & $<2$ & --- & --- & $<0.01$ & $<5$ & $<1$ \\
\hline 2011Z059A & $<1$ & 0.01 & 2 & 20 & --- & $<2$ & --- & --- & $<0.01$ & $<5$ & $<1$ \\
\hline
\end{tabular}




\begin{tabular}{|c|c|c|c|c|c|c|c|c|c|c|c|}
\hline Sample_ID & Sn_ppm & Sr_ppm & Th_ppm & Ti_pct & TI_ppm & U_ppm & V_ppm & W_ppm & W_ppm_2 & Zn_ppm & Zn_pct \\
\hline 2011LF317A & -- & 20 & $<20$ & 0.05 & $<10$ & 10 & 99 & $<10$ & -- & 180 & -- \\
\hline 2011LF332A & 5 & 37 & 40 & 0.08 & $<10$ & 10 & 7 & 30 & 30 & 14 & --- \\
\hline 2011LF345A & -- & 30 & $<20$ & 0.04 & $<10$ & 30 & 18 & $<10$ & -- & 6 & --- \\
\hline 2011LF365A & -- & 6 & $<20$ & 0.03 & $<10$ & $<10$ & 34 & $<10$ & --- & 20 & --- \\
\hline 2011LF369B & $<5$ & 6 & $<20$ & 0.01 & $<10$ & $<10$ & 8 & $<10$ & $<10$ & 9 & -- \\
\hline 2011LF394A & $\begin{array}{l}-- \\
\end{array}$ & 19 & $<20$ & 0.08 & $<10$ & $<10$ & 33 & $<10$ & --- & 10 & --- \\
\hline 2011LF401A & -- & 44 & $<20$ & 0.17 & $<10$ & $<10$ & 79 & $<10$ & --- & 43 & --- \\
\hline 2011LF408A & --- & 30 & $<20$ & 0.07 & $<10$ & $<10$ & 123 & $<10$ & --- & 120 & --- \\
\hline 2011LF411B & --- & 23 & $<20$ & 1.04 & $<10$ & $<10$ & 281 & $<10$ & --- & 207 & --- \\
\hline 2011LF412B & --- & 150 & $<20$ & 0.35 & $<10$ & 10 & 135 & 10 & --- & 1550 & --- \\
\hline 2011LF485A & --- & 8 & $<20$ & 0.03 & $<10$ & 10 & 88 & $<10$ & --- & 15 & --- \\
\hline 2011LF498A & --- & 21 & $<20$ & 0.11 & $<10$ & $<10$ & 17 & 10 & -- & 12 & --- \\
\hline 2011LF502A & -- & 109 & $<20$ & 0.15 & $<10$ & $<10$ & 47 & 10 & -- & 65 & -- \\
\hline 2011LF507A & --- & 10 & $<20$ & 0.04 & $<10$ & $<10$ & 89 & $<10$ & --- & 106 & --- \\
\hline 2011LF528A & -- & 57 & $<20$ & 0.1 & $<10$ & $<10$ & 64 & $<10$ & -- & 37 & -- \\
\hline 2011LF557B & --- & 170 & $<20$ & 0.28 & $<10$ & $<10$ & 538 & $<10$ & --- & 116 & --- \\
\hline 2011LF587B & --- & 569 & $<20$ & 0.12 & $<10$ & 10 & 88 & $<10$ & --- & 59 & --- \\
\hline 2011RN153A & --- & 23 & $<20$ & 0.13 & $<10$ & $<10$ & 55 & $<10$ & --- & 89 & --- \\
\hline 2011RN177B & 8 & 176 & 20 & 0.22 & $<10$ & $<10$ & 38 & 220 & 220 & 39 & --- \\
\hline 2011RN180B & $<5$ & 92 & $<20$ & 0.38 & $<10$ & $<10$ & 123 & $<10$ & 20 & 130 & --- \\
\hline 2011RN180C & $<5$ & 20 & $<20$ & 0.03 & $<10$ & $<10$ & 5 & $<10$ & $<10$ & 8 & --- \\
\hline 2011Z005B & 5 & 68 & 40 & 0.1 & $<10$ & $<10$ & 9 & 20 & 10 & 19 & --- \\
\hline $2011 Z 012 \mathrm{E}$ & -- & 15 & $<20$ & 0.21 & $<10$ & $<10$ & 54 & $<10$ & -- & 36 & --- \\
\hline $2011 Z 024 \mathrm{~A}$ & -- & 111 & $<20$ & 0.17 & $<10$ & $<10$ & 552 & $<10$ & -- & 158 & --- \\
\hline 2011Z026B & --- & 3 & $<20$ & 0.06 & $<10$ & $<10$ & 19 & $<10$ & --- & 13 & --- \\
\hline $2011 Z 028 \mathrm{~A}$ & --- & 123 & $<20$ & 0.16 & $<10$ & $<10$ & 64 & $<10$ & --- & 42 & --- \\
\hline 2011Z029A & --- & 70 & $<20$ & 0.17 & $<10$ & $<10$ & 106 & $<10$ & --- & 100 & --- \\
\hline 2011Z044A & --- & 78 & $<20$ & 0.42 & $<10$ & $<10$ & 127 & $<10$ & --- & 130 & --- \\
\hline $2011 Z 050 \mathrm{~A}$ & --- & 1 & $<20$ & $<0.01$ & $<10$ & $<10$ & 1 & $<10$ & --- & $<2$ & --- \\
\hline 2011Z059A & --- & $<1$ & $<20$ & $<0.01$ & $<10$ & $<10$ & 4 & $<10$ & --- & 5 & --- \\
\hline
\end{tabular}




\begin{tabular}{|c|c|c|c|c|c|c|c|c|c|c|c|}
\hline Sample_ID & Au_ppm & Au_ppm_2 & Au_ppm_3 & Au_ppm_4 & Ag_ppm & Al_pct & As_ppm & As_pct & Ba_ppm & Be_ppm & Bi_ppm \\
\hline 2011Z061A & $<0.001$ & -- & -- & -- & $<0.5$ & 0.01 & 6 & --- & $<10$ & $<0.5$ & $<2$ \\
\hline $2011 Z 072 B^{\wedge}$ & 0.006 & --- & -- & --- & $<0.5$ & 0.87 & 31 & --- & 90 & $<0.5$ & $<2$ \\
\hline $2011 Z 073 A^{\wedge}$ & 0.008 & -- & -- & -- & $<0.5$ & 1.32 & 14 & -- & 120 & 0.8 & $<2$ \\
\hline $2011 Z 075 A^{\wedge}$ & 0.012 & --- & -- & -- & $<0.5$ & 1.28 & 14 & --- & 90 & 0.8 & $<2$ \\
\hline $2011 Z 078 B^{\wedge}$ & 0.009 & -- & -- & -- & 0.7 & 1.15 & 67 & --- & 280 & 0.8 & $<2$ \\
\hline $2011 Z 082 B^{\wedge}$ & 0.001 & --- & -- & -- & 0.5 & 2.37 & 6 & -- & 90 & 4.7 & 145 \\
\hline $2011 Z^{2} 888 B^{\wedge}$ & $<0.001$ & --- & -- & -- & $<0.5$ & 0.05 & 11 & -- & 10 & $<0.5$ & $<2$ \\
\hline 2011Z093A & $<0.001$ & --- & --- & --- & 0.6 & 6.12 & 8 & --- & 30 & 7.8 & $<2$ \\
\hline $2011 Z 095 A^{\wedge}$ & $<0.001$ & --- & --- & --- & $<0.5$ & 6.03 & 6 & --- & 20 & 18 & $<2$ \\
\hline 2011Z097A & $<0.001$ & --- & --- & --- & 1.3 & 6.05 & $<5$ & --- & 130 & 44.4 & $<2$ \\
\hline 2011Z098A & $<0.001$ & --- & --- & --- & 6.8 & 6.73 & 50 & --- & 90 & 90.1 & 15 \\
\hline $2011 Z 104 \mathrm{~A}$ & 0.001 & --- & --- & --- & 0.6 & 4.75 & 20 & -- & 530 & 1.6 & $<2$ \\
\hline 2011Z107B & $<0.001$ & -- & -- & -- & $<0.5$ & 0.72 & $<5$ & -- & 40 & $<0.5$ & $<2$ \\
\hline $2011 Z 111 \mathrm{~A}$ & $<0.001$ & --- & -- & --- & $<0.5$ & 6 & 14 & --- & 240 & 1.3 & $<2$ \\
\hline 2011Z116A & $<0.001$ & -- & -- & -- & $<0.5$ & 8.01 & 62 & -- & 790 & 2.9 & $<2$ \\
\hline $2011 Z 120 \mathrm{~A}$ & $<0.001$ & --- & -- & --- & $<0.5$ & 2.15 & 6 & --- & 320 & 0.6 & $<2$ \\
\hline 2011Z121B & $<0.001$ & --- & -- & --- & $<0.5$ & 7.3 & 14 & --- & 540 & 2 & $<2$ \\
\hline $2011 Z 130 A$ & $<0.001$ & --- & --- & --- & $<0.5$ & 1.04 & 5 & --- & 180 & 0.5 & $<2$ \\
\hline $2011 Z 135 C$ & $<0.001$ & --- & --- & --- & $<0.5$ & 0.39 & $<5$ & --- & 40 & $<0.5$ & $<2$ \\
\hline 2011Z149A & --- & 0.001 & --- & --- & $<0.5$ & 6.26 & 13 & --- & 200 & 0.5 & 2 \\
\hline 2011Z161B & $<0.001$ & --- & --- & --- & 11.9 & 6.72 & 17 & --- & 410 & 4.7 & 35 \\
\hline 2011Z162B & $<0.001$ & -- & --- & --- & $<0.5$ & 0.27 & 163 & -- & 10 & $<0.5$ & 2 \\
\hline $2011 Z 162 C$ & 0.01 & -- & -- & --- & $<0.5$ & 0.32 & 1710 & -- & 10 & $<0.5$ & 5 \\
\hline 2011Z163B & 0.009 & -- & -- & -- & $<0.5$ & 1.3 & 18 & -- & 20 & $<0.5$ & $<2$ \\
\hline 2011Z166B & $<0.001$ & --- & -- & --- & $<0.5$ & 2.32 & 9 & --- & 70 & $<0.5$ & $<2$ \\
\hline $2011 Z 174 C$ & 0.004 & --- & --- & --- & $<0.5$ & 1.94 & $<5$ & --- & 300 & $<0.5$ & $<2$ \\
\hline 2011Z205B & $<0.001$ & --- & --- & --- & $<0.5$ & 0.3 & $<5$ & --- & 30 & $<0.5$ & $<2$ \\
\hline $2011 Z 245 A$ & $<0.001$ & --- & --- & --- & $<0.5$ & 8.58 & $<5$ & --- & 440 & 2.8 & $<2$ \\
\hline $2011 Z 246 A$ & 0.001 & --- & --- & --- & $<0.5$ & 8.77 & 10 & -- & 720 & 2.3 & $<2$ \\
\hline $2011 Z 260 \mathrm{C}$ & $<0.001$ & --- & --- & --- & $<0.5$ & 5.56 & $<5$ & --- & 580 & 2.6 & $<2$ \\
\hline
\end{tabular}




\begin{tabular}{|c|c|c|c|c|c|c|c|c|c|c|c|}
\hline Sample_ID & Ca_pct & Cd_ppm & Co_ppm & Cr_ppm & Cu_ppm & Fe_pct & Ga_ppm & K_pct & La_ppm & Mg_pct & Mn_ppm \\
\hline 2011Z061A & $<0.01$ & $<0.5$ & $<1$ & 23 & 2 & 0.73 & $<10$ & $<0.01$ & $<10$ & $<0.01$ & 73 \\
\hline $2011 Z 072 B^{\wedge}$ & 0.01 & $<0.5$ & $<1$ & 23 & 96 & 6.98 & 10 & 0.24 & $<10$ & 0.06 & 58 \\
\hline $2011 Z 073 A^{\wedge}$ & 0.01 & $<0.5$ & 1 & 32 & 614 & 0.84 & $<10$ & 0.38 & $<10$ & 0.15 & 57 \\
\hline $2011 Z 075 A^{\wedge}$ & 0.02 & $<0.5$ & 1 & 35 & 109 & 1.79 & $<10$ & 0.42 & 10 & 0.2 & 88 \\
\hline $2011 Z^{2} 078 B^{\wedge}$ & 0.27 & 0.5 & 5 & 46 & 54 & 7.15 & $<10$ & 0.3 & 10 & 0.16 & 261 \\
\hline $2011 Z 082 B^{\wedge}$ & 0.13 & $<0.5$ & 2 & 14 & 10 & 2.74 & 10 & 0.98 & 50 & 0.05 & 545 \\
\hline $2011 Z^{2} 888 B^{\wedge}$ & $<0.01$ & $<0.5$ & 1 & 22 & 3 & 0.85 & $<10$ & 0.01 & $<10$ & 0.01 & 1195 \\
\hline 2011Z093A & 0.19 & $<0.5$ & $<1$ & 6 & 3 & 1.73 & 20 & 3.84 & 10 & 0.04 & 376 \\
\hline $2011 Z 095 A^{\wedge}$ & 0.3 & $<0.5$ & 1 & 7 & 4 & 1.63 & 20 & 3.61 & 20 & 0.03 & 569 \\
\hline 2011Z097A & 0.23 & 0.5 & 2 & 4 & 6 & 5.44 & 20 & 2.23 & 30 & 0.06 & 3730 \\
\hline 2011Z098A & 0.88 & $<0.5$ & 5 & 3 & 15 & 8.22 & 30 & 2.24 & 20 & 0.04 & 4640 \\
\hline $2011 Z 104 \mathrm{~A}$ & 0.02 & $<0.5$ & 6 & 62 & 43 & 4.1 & 10 & 1.2 & 30 & 0.57 & 236 \\
\hline 2011Z107B & 0.05 & $<0.5$ & 3 & 28 & 7 & 1.35 & $<10$ & 0.17 & $<10$ & 0.07 & 154 \\
\hline $2011 Z 111 \mathrm{~A}$ & 0.39 & $<0.5$ & 6 & 53 & 3 & 2.75 & 20 & 0.42 & 30 & 1.09 & 279 \\
\hline 2011Z116A & 2.75 & $<0.5$ & 11 & 23 & 45 & 3.26 & 20 & 2.08 & 50 & 0.74 & 420 \\
\hline $2011 Z 120 \mathrm{~A}$ & 0.05 & $<0.5$ & 3 & 25 & 12 & 2.36 & $<10$ & 0.81 & 10 & 0.18 & 204 \\
\hline 2011Z121B & 0.23 & $<0.5$ & 19 & 180 & 66 & 5.16 & 10 & 1.73 & 30 & 1.47 & 1445 \\
\hline $2011 Z 130 A$ & 0.01 & $<0.5$ & 5 & 26 & 20 & 1.46 & $<10$ & 0.55 & $<10$ & 0.13 & 1330 \\
\hline $2011 Z 135 C$ & 0.02 & $<0.5$ & $<1$ & 16 & 2 & 1.04 & $<10$ & 0.16 & $<10$ & 0.03 & 107 \\
\hline 2011Z149A & 0.17 & $<0.5$ & 12 & 291 & 36 & 4.08 & 10 & 0.31 & 20 & 2.55 & 329 \\
\hline 2011Z161B & 8.06 & 12.9 & 11 & 39 & 60 & 6.92 & 20 & 0.83 & 30 & 3.83 & 2890 \\
\hline 2011Z162B & 0.03 & $<0.5$ & 13 & 19 & 9 & 1.62 & $<10$ & 0.1 & $<10$ & 0.02 & 355 \\
\hline $2011 Z 162 C$ & 0.03 & $<0.5$ & 28 & 19 & 69 & 3.52 & $<10$ & 0.1 & 10 & 0.02 & 499 \\
\hline 2011Z163B & 2.43 & $<0.5$ & 14 & 53 & 52 & 2.77 & $<10$ & 0.1 & $<10$ & 0.52 & 416 \\
\hline 2011Z166B & 2.41 & $<0.5$ & 13 & 96 & 28 & 2.96 & $<10$ & 0.02 & $<10$ & 1.17 & 498 \\
\hline $2011 Z 174 C$ & 0.66 & $<0.5$ & 3 & 27 & 13 & 1.22 & $<10$ & 0.11 & $<10$ & 0.26 & 232 \\
\hline 2011Z205B & 0.01 & $<0.5$ & $<1$ & 30 & 5 & 1.07 & $<10$ & 0.05 & $<10$ & 0.06 & 104 \\
\hline $2011 Z 245 A$ & 0.15 & $<0.5$ & 19 & 89 & 23 & 5.72 & 20 & 2.37 & 30 & 1.11 & 2400 \\
\hline $2011 Z 246 A$ & 1.8 & $<0.5$ & 9 & 93 & 49 & 4.07 & 30 & 3.91 & 20 & 1.43 & 681 \\
\hline $2011 Z 260 \mathrm{C}$ & 0.55 & $<0.5$ & 2 & 17 & 6 & 1.03 & 10 & 4.63 & 20 & 0.08 & 110 \\
\hline
\end{tabular}




\begin{tabular}{|c|c|c|c|c|c|c|c|c|c|c|c|}
\hline Sample_ID & Mo_ppm & $\mathrm{Na}$ _pct & Ni_ppm & P_ppm & P_ppm_2 & Pb_ppm & Pd_ppm & Pt_ppm & S_pct & Sb_ppm & Sc_ppm \\
\hline 2011Z061A & $<1$ & $<0.01$ & 2 & 10 & -- & $<2$ & -- & -- & $<0.01$ & $<5$ & $<1$ \\
\hline $2011 Z 072 B^{\wedge}$ & 5 & 0.01 & 2 & 2110 & --- & 8 & --- & --- & 0.02 & $<5$ & 2 \\
\hline $2011 Z 073 A^{\wedge}$ & 3 & 0.04 & 4 & 2180 & -- & 5 & --- & -- & 0.01 & $<5$ & 3 \\
\hline $2011 Z 075 A^{\wedge}$ & 4 & 0.01 & 13 & 1000 & --- & 10 & --- & -- & 0.01 & $<5$ & 3 \\
\hline $2011 Z 078 B^{\wedge}$ & 13 & 0.01 & 48 & 4900 & -- & 12 & -- & -- & 0.05 & 18 & 4 \\
\hline $2011 Z 082 B^{\wedge}$ & 12 & 0.03 & 5 & 120 & --- & 56 & --- & --- & 0.01 & $<5$ & 2 \\
\hline $2011 Z^{2} 888 B^{\wedge}$ & $<1$ & $<0.01$ & 5 & 40 & --- & 3 & --- & -- & $<0.01$ & $<5$ & $<1$ \\
\hline 2011Z093A & $<1$ & 2.14 & 1 & 70 & --- & 39 & --- & --- & $<0.01$ & 5 & 3 \\
\hline $2011 Z 095 A^{\wedge}$ & $<1$ & 2.07 & $<1$ & 280 & --- & 23 & --- & --- & $<0.01$ & $<5$ & 4 \\
\hline 2011Z097A & 2 & 1.51 & $<1$ & 60 & --- & 136 & --- & --- & $<0.01$ & $<5$ & 4 \\
\hline 2011Z098A & 5 & 1.98 & $<1$ & 40 & --- & 380 & --- & --- & $<0.01$ & $<5$ & 4 \\
\hline $2011 Z 104 \mathrm{~A}$ & $<1$ & 0.31 & 25 & 420 & --- & 98 & -- & -- & 0.01 & $<5$ & 10 \\
\hline 2011Z107B & 3 & 0.04 & 4 & 190 & -- & 3 & -- & -- & $<0.01$ & $<5$ & 1 \\
\hline $2011 Z 111 \mathrm{~A}$ & $<1$ & 0.48 & 17 & 570 & --- & 10 & --- & --- & $<0.01$ & $<5$ & 16 \\
\hline 2011Z116A & $<1$ & 2.31 & 13 & 790 & -- & 17 & -- & -- & 0.06 & $<5$ & 10 \\
\hline $2011 Z 120 \mathrm{~A}$ & $<1$ & 0.09 & 9 & 270 & --- & 2 & --- & --- & $<0.01$ & 5 & 3 \\
\hline 2011Z121B & $<1$ & 0.86 & 75 & 1170 & --- & 29 & --- & --- & $<0.01$ & 6 & 13 \\
\hline $2011 Z 130 A$ & 9 & 0.02 & 11 & 180 & --- & 15 & --- & --- & $<0.01$ & $<5$ & 3 \\
\hline $2011 Z 135 C$ & $<1$ & 0.02 & 1 & 150 & --- & 6 & --- & --- & $<0.01$ & $<5$ & 1 \\
\hline 2011Z149A & $<1$ & 2.72 & 137 & 760 & --- & 13 & 0.002 & $<0.005$ & 0.01 & $<5$ & 10 \\
\hline 2011Z161B & $<1$ & 1.34 & 20 & 570 & --- & 1900 & --- & --- & 0.06 & 5 & 12 \\
\hline 2011Z162B & $<1$ & 0.01 & 11 & 40 & --- & 7 & --- & --- & $<0.01$ & $<5$ & 1 \\
\hline $2011 Z 162 C$ & $<1$ & 0.01 & 49 & 60 & --- & 4 & --- & --- & 0.02 & 11 & 1 \\
\hline 2011Z163B & $<1$ & 0.11 & 36 & 160 & -- & 3 & -- & -- & $<0.01$ & $<5$ & 7 \\
\hline 2011Z166B & $<1$ & 0.33 & 35 & 200 & --- & 4 & --- & --- & $<0.01$ & $<5$ & 9 \\
\hline $2011 Z 174 C$ & $<1$ & 1.25 & 14 & 230 & --- & 4 & --- & --- & $<0.01$ & $<5$ & 2 \\
\hline 2011Z205B & $<1$ & 0.02 & 4 & 40 & --- & $<2$ & --- & --- & $<0.01$ & $<5$ & 1 \\
\hline $2011 Z 245 A$ & 3 & 0.63 & 40 & 400 & --- & 64 & --- & --- & $<0.01$ & 5 & 15 \\
\hline $2011 Z 246 A$ & 5 & 0.98 & 22 & 260 & --- & 22 & --- & --- & 0.24 & $<5$ & 16 \\
\hline $2011 Z 260 \mathrm{C}$ & 1 & 1.29 & 1 & 400 & --- & 22 & --- & --- & $<0.01$ & $<5$ & 1 \\
\hline
\end{tabular}




\begin{tabular}{|c|c|c|c|c|c|c|c|c|c|c|c|}
\hline Sample_ID & Sn_ppm & Sr_ppm & Th_ppm & Ti_pct & TI_ppm & U_ppm & V_ppm & W_ppm & W_ppm_2 & Zn_ppm & Zn_pct \\
\hline 2011Z061A & -- & $<1$ & $<20$ & $<0.01$ & $<10$ & $<10$ & 1 & $<10$ & -- & 2 & -- \\
\hline $2011 Z^{2072 B^{\wedge}}$ & --- & 5 & $<20$ & 0.04 & $<10$ & $<10$ & 135 & $<10$ & --- & 12 & --- \\
\hline $2011 Z 073 A^{\wedge}$ & -- & 9 & $<20$ & 0.07 & $<10$ & $<10$ & 52 & $<10$ & -- & 26 & -- \\
\hline $20112075 A^{\wedge}$ & --- & 9 & $<20$ & 0.06 & $<10$ & 10 & 159 & $<10$ & --- & 47 & --- \\
\hline $2011 Z 078 B^{\wedge}$ & --- & 31 & $<20$ & 0.05 & $<10$ & $<10$ & 307 & $<10$ & --- & 384 & --- \\
\hline $2011 Z^{2082 B^{\wedge}}$ & 62 & 5 & 70 & 0.05 & $<10$ & 20 & 6 & 10 & 10 & 104 & --- \\
\hline $2011 Z 088 B^{\wedge}$ & --- & 2 & $<20$ & $<0.01$ & $<10$ & $<10$ & 4 & $<10$ & -- & 10 & --- \\
\hline 2011Z093A & $<5$ & 20 & 40 & 0.03 & $<10$ & 30 & 3 & $<10$ & 10 & 199 & --- \\
\hline $2011 Z 095 A^{\wedge}$ & 29 & 11 & 50 & 0.04 & $<10$ & 20 & 2 & 760 & 620 & 16 & --- \\
\hline 2011Z097A & 80 & 45 & 60 & 0.04 & $<10$ & 20 & 2 & $<10$ & 20 & 534 & --- \\
\hline 2011Z098A & 473 & 59 & 50 & 0.03 & $<10$ & 20 & 1 & $<10$ & 30 & 523 & --- \\
\hline $2011 Z 104 A$ & --- & 45 & $<20$ & 0.22 & $<10$ & $<10$ & 104 & $<10$ & --- & 129 & --- \\
\hline 2011Z107B & --- & 13 & $<20$ & 0.02 & $<10$ & $<10$ & 5 & $<10$ & --- & 12 & --- \\
\hline $2011 Z 111 A$ & --- & 70 & 20 & 0.35 & $<10$ & $<10$ & 129 & $<10$ & --- & 39 & --- \\
\hline 2011Z116A & --- & 586 & 20 & 0.27 & $<10$ & $<10$ & 70 & $<10$ & --- & 35 & --- \\
\hline $2011 Z 120 A$ & --- & 17 & $<20$ & 0.07 & $<10$ & $<10$ & 21 & $<10$ & --- & 16 & --- \\
\hline 2011Z121B & --- & 91 & $<20$ & 0.26 & $<10$ & $<10$ & 131 & $<10$ & --- & 111 & --- \\
\hline $2011 Z 130 A$ & --- & 14 & $<20$ & 0.05 & $<10$ & $<10$ & 143 & $<10$ & --- & 34 & --- \\
\hline $2011 Z 135 C$ & --- & 6 & $<20$ & 0.01 & $<10$ & $<10$ & 5 & $<10$ & --- & 11 & --- \\
\hline 2011Z149A & --- & 21 & $<20$ & 0.19 & $<10$ & $<10$ & 119 & $<10$ & --- & 96 & --- \\
\hline 2011Z161B & 187 & 139 & $<20$ & 0.35 & $<10$ & $<10$ & 64 & $<10$ & 40 & 2630 & --- \\
\hline 2011Z162B & $<5$ & 5 & $<20$ & 0.01 & $<10$ & $<10$ & 4 & $<10$ & $<10$ & 12 & --- \\
\hline $2011 Z 162 C$ & $<5$ & 15 & $<20$ & 0.01 & $<10$ & $<10$ & 3 & $<10$ & 10 & 7 & --- \\
\hline 2011Z163B & --- & 40 & $<20$ & 0.13 & $<10$ & 10 & 44 & $<10$ & --- & 39 & --- \\
\hline 2011Z166B & --- & 105 & $<20$ & 0.22 & $<10$ & $<10$ & 91 & $<10$ & --- & 27 & --- \\
\hline $2011 Z 174 C$ & --- & 125 & $<20$ & 0.06 & $<10$ & 10 & 22 & $<10$ & --- & 20 & --- \\
\hline 2011Z205B & --- & 3 & $<20$ & 0.01 & $<10$ & $<10$ & 7 & $<10$ & --- & 13 & --- \\
\hline $2011 Z 245 A$ & 19 & 69 & $<20$ & 0.4 & $<10$ & $<10$ & 100 & 10 & 30 & 160 & --- \\
\hline $2011 Z 246 A$ & $<5$ & 283 & $<20$ & 0.32 & $<10$ & $<10$ & 128 & $<10$ & 10 & 58 & --- \\
\hline $2011 Z 260 C$ & $<5$ & 175 & 20 & 0.06 & $<10$ & 10 & 6 & $<10$ & $<10$ & 15 & --- \\
\hline
\end{tabular}




\begin{tabular}{|c|c|c|c|c|c|c|c|c|c|c|c|}
\hline Sample_ID & Au_ppm & Au_ppm_2 & Au_ppm_3 & Au_ppm_4 & Ag_ppm & Al_pct & As_ppm & As_pct & Ba_ppm & Be_ppm & Bi_ppm \\
\hline 2011Z271B & $<0.001$ & --- & --- & --- & $<0.5$ & 6.06 & $<5$ & --- & 20 & 0.6 & $<2$ \\
\hline $2011 Z 302 \mathrm{~A}$ & 0.007 & --- & --- & --- & $<0.5$ & 1.21 & 18 & --- & 120 & $<0.5$ & $<2$ \\
\hline 2011Z309A & 0.003 & -- & -- & -- & $<0.5$ & 1.47 & 13 & --- & 100 & 0.5 & $<2$ \\
\hline $2011 Z 312 A$ & 0.001 & --- & --- & --- & $<0.5$ & 0.87 & $<5$ & --- & 320 & $<0.5$ & $<2$ \\
\hline $2011 Z 314 \mathrm{~A}$ & 0.01 & -- & -- & -- & $<0.5$ & 2.1 & 6 & --- & 170 & 0.9 & $<2$ \\
\hline 2011Z316A & 0.005 & --- & --- & --- & $<0.5$ & 1.03 & 7 & --- & 270 & $<0.5$ & $<2$ \\
\hline 2011Z345B & 0.002 & --- & --- & --- & 0.6 & 6.06 & 3340 & --- & 550 & 1.7 & $<2$ \\
\hline 2011Z349A & $<0.001$ & --- & --- & --- & $<0.5$ & 8.25 & 23 & --- & 110 & $<0.5$ & 2 \\
\hline $2011 Z 362 A$ & $<0.001$ & --- & --- & --- & $<0.5$ & 0.55 & 12 & --- & 80 & $<0.5$ & $<2$ \\
\hline 2011Z366B & 0.004 & --- & --- & --- & $<0.5$ & 1.1 & 7 & --- & 90 & $<0.5$ & 2 \\
\hline 2011Z380A & 0.091 & --- & --- & --- & $<0.5$ & 8.57 & 148 & --- & 560 & 1 & $<2$ \\
\hline $2011 Z 382 A$ & 0.002 & -- & -- & --- & $<0.5$ & 6.2 & 50 & --- & 630 & 1.2 & $<2$ \\
\hline 2011Z383B & 0.008 & -- & -- & -- & $<0.5$ & 6.88 & 23 & -- & 960 & 0.9 & $<2$ \\
\hline 2011Z384A & 0.001 & --- & --- & --- & $<0.5$ & 1.62 & 6 & --- & 110 & 0.5 & $<2$ \\
\hline $2011 Z 385 A$ & $<0.001$ & -- & -- & --- & $<0.5$ & 1.08 & 16 & -- & 10 & $<0.5$ & $<2$ \\
\hline 2011Z389A & 0.008 & --- & --- & --- & $<0.5$ & 1.72 & 6 & --- & 140 & 0.5 & $<2$ \\
\hline 2011Z393A & 0.003 & --- & --- & --- & $<0.5$ & 4 & 53 & --- & 490 & 1 & $<2$ \\
\hline $2011 Z 395 C$ & 0.005 & --- & --- & --- & $<0.5$ & 2.61 & 285 & --- & 160 & 0.8 & 2 \\
\hline 2011Z399A & 0.004 & --- & --- & --- & $<0.5$ & 7.52 & 143 & --- & 980 & 1.6 & $<2$ \\
\hline $2011 Z 403 \mathrm{~A}$ & 0.002 & --- & --- & --- & $<0.5$ & 2.34 & 83 & --- & 210 & 0.7 & 2 \\
\hline 2011Z407A & 0.005 & -- & -- & --- & $<0.5$ & 3.13 & 96 & -- & 300 & 0.9 & $<2$ \\
\hline $2011 Z 408 \mathrm{~A}$ & 0.006 & -- & -- & --- & 0.8 & 2.68 & 542 & --- & 810 & 0.8 & $<2$ \\
\hline 2011Z408B & 0.002 & --- & --- & --- & $<0.5$ & 0.43 & 428 & -- & 50 & 0.6 & $<2$ \\
\hline $2011 Z 425 C$ & 0.002 & --- & --- & --- & $<0.5$ & 0.63 & 5 & -- & 190 & $<0.5$ & $<2$ \\
\hline $2011 Z 426 A$ & 0.151 & --- & --- & --- & $<0.5$ & 0.86 & 5 & --- & 4010 & $<0.5$ & $<2$ \\
\hline 2011Z437B & $<0.001$ & --- & --- & --- & $<0.5$ & 1.17 & 6 & --- & 160 & $<0.5$ & $<2$ \\
\hline 2011Z441B & 0.002 & --- & --- & --- & $<0.5$ & 5.74 & 11 & --- & 610 & 1.5 & $<2$ \\
\hline 2011Z448B & 0.01 & --- & --- & --- & $<0.5$ & 1.51 & 28 & --- & 80 & $<0.5$ & 3 \\
\hline $2011 Z 455 C$ & $<0.001$ & --- & --- & --- & $<0.5$ & 0.06 & $<5$ & --- & 20 & $<0.5$ & $<2$ \\
\hline 2011Z480A & 0.067 & --- & --- & --- & 9 & 2.55 & 448 & --- & 520 & 0.9 & 2 \\
\hline
\end{tabular}




\begin{tabular}{|c|c|c|c|c|c|c|c|c|c|c|c|}
\hline Sample_ID & Ca_pct & Cd_ppm & Co_ppm & Cr_ppm & Cu_ppm & Fe_pct & Ga_ppm & K_pct & La_ppm & Mg_pct & Mn_ppm \\
\hline 2011Z271B & 0.09 & $<0.5$ & 10 & 67 & 49 & 3.28 & 10 & 0.05 & 20 & 0.96 & 197 \\
\hline $2011 Z 302 \mathrm{~A}$ & 0.04 & $<0.5$ & 2 & 37 & 48 & 1.65 & $<10$ & 0.48 & 10 & 0.11 & 118 \\
\hline 2011Z309A & 0.01 & $<0.5$ & 1 & 27 & 50 & 3.02 & $<10$ & 0.54 & 10 & 0.12 & 86 \\
\hline $2011 Z 312 \mathrm{~A}$ & 0.01 & $<0.5$ & 1 & 29 & 329 & 0.76 & $<10$ & 0.27 & $<10$ & 0.13 & 67 \\
\hline $2011 Z 314 \mathrm{~A}$ & 0.01 & $<0.5$ & $<1$ & 32 & 37 & 1.47 & $<10$ & 0.2 & $<10$ & 0.06 & 56 \\
\hline $2011 Z 316 A$ & 0.02 & $<0.5$ & 1 & 31 & 61 & 1.58 & $<10$ & 0.38 & $<10$ & 0.11 & 109 \\
\hline 2011Z345B & 0.28 & 1.4 & 14 & 83 & 317 & 24.2 & 10 & 1.27 & 30 & 0.82 & 511 \\
\hline 2011Z349A & 5.61 & 0.5 & 49 & 75 & 167 & 9.14 & 20 & 0.05 & $<10$ & 4.26 & 1650 \\
\hline $2011 Z 362 A$ & 0.03 & $<0.5$ & 2 & 25 & 10 & 1.18 & $<10$ & 0.13 & $<10$ & 0.06 & 152 \\
\hline 2011Z366B & 0.03 & $<0.5$ & 2 & 45 & 9 & 1.92 & $<10$ & 0.16 & $<10$ & 0.33 & 132 \\
\hline 2011Z380A & 0.07 & $<0.5$ & 19 & 44 & 65 & 6.32 & 20 & 1.37 & 10 & 1.9 & 675 \\
\hline $2011 Z 382 A$ & 0.03 & 0.5 & 23 & 146 & 129 & 12.4 & 10 & 1.21 & 20 & 0.92 & 1115 \\
\hline 2011Z383B & 0.03 & 0.5 & 9 & 17 & 9 & 4.13 & 20 & 1.12 & 20 & 1.04 & 252 \\
\hline $2011 Z 384 A$ & 0.01 & $<0.5$ & 1 & 29 & 20 & 1.3 & $<10$ & 0.34 & $<10$ & 0.23 & 72 \\
\hline $2011 Z 385 A$ & 0.01 & 0.5 & 91 & 1680 & 10 & 6.01 & $<10$ & 0.01 & 10 & 22.4 & 658 \\
\hline 2011Z389A & 0.04 & $<0.5$ & 1 & 41 & 17 & 1.19 & $<10$ & 0.68 & 10 & 0.22 & 54 \\
\hline $2011 Z 393 A$ & 0.04 & $<0.5$ & 7 & 50 & 17 & 2.78 & 10 & 1.14 & 20 & 0.41 & 358 \\
\hline $2011 Z 395 C$ & 0.01 & 1 & 4 & 31 & 38 & 3.13 & 10 & 0.53 & 10 & 0.15 & 143 \\
\hline 2011Z399A & 0.02 & $<0.5$ & 14 & 67 & 31 & 5.29 & 20 & 2.54 & 30 & 0.59 & 803 \\
\hline 2011Z403A & 0.01 & $<0.5$ & 3 & 29 & 20 & 1.61 & 10 & 0.72 & 10 & 0.13 & 123 \\
\hline 2011Z407A & 0.02 & $<0.5$ & 3 & 37 & 13 & 1.7 & 10 & 0.85 & 10 & 0.14 & 75 \\
\hline $2011 Z 408 \mathrm{~A}$ & 0.02 & 1 & 24 & 30 & 62 & 5.11 & $<10$ & 0.93 & 20 & 0.13 & 11200 \\
\hline 2011Z408B & 0.02 & $<0.5$ & 19 & 30 & 102 & 11.45 & $<10$ & 0.04 & $<10$ & 0.03 & 434 \\
\hline $2011 Z 425 C$ & 0.06 & $<0.5$ & 1 & 19 & 10 & 1.13 & $<10$ & 0.17 & $<10$ & 0.11 & 97 \\
\hline $2011 Z 426 A$ & $<0.01$ & $<0.5$ & $<1$ & 21 & 8 & 0.71 & $<10$ & 0.37 & $<10$ & 0.11 & 27 \\
\hline 2011Z437B & 0.05 & $<0.5$ & 3 & 25 & 22 & 2.17 & $<10$ & 0.31 & 10 & 0.04 & 106 \\
\hline 2011Z441B & 0.13 & $<0.5$ & 7 & 67 & 36 & 3.55 & 10 & 1.4 & 40 & 0.55 & 198 \\
\hline 2011Z448B & 0.01 & $<0.5$ & 6 & 28 & 68 & 10.05 & $<10$ & 0.24 & 10 & 0.05 & 205 \\
\hline $2011 Z 455 C$ & 0.44 & $<0.5$ & 1 & 10 & 2 & 0.85 & $<10$ & 0.01 & $<10$ & 0.21 & 101 \\
\hline 2011Z480A & 8.84 & 0.7 & 12 & 17 & 39 & 7.39 & 10 & 0.55 & 10 & 3.41 & 11750 \\
\hline
\end{tabular}




\begin{tabular}{|c|c|c|c|c|c|c|c|c|c|c|c|}
\hline Sample_ID & Mo_ppm & $\mathrm{Na}$ _pct & Ni_ppm & P_ppm & P_ppm_2 & Pb_ppm & Pd_ppm & Pt_ppm & S_pct & Sb_ppm & Sc_ppm \\
\hline 2011Z271B & 2 & 4.13 & 39 & 410 & -- & 28 & -- & -- & 0.01 & 5 & 4 \\
\hline $2011 Z 302 \mathrm{~A}$ & 4 & 0.01 & 8 & 700 & --- & 20 & --- & --- & 0.03 & $<5$ & 3 \\
\hline 2011Z309A & 5 & 0.02 & 9 & 760 & -- & 6 & --- & -- & 0.02 & $<5$ & 5 \\
\hline $2011 Z 312 \mathrm{~A}$ & 2 & 0.01 & 4 & 1290 & --- & 4 & --- & -- & $<0.01$ & $<5$ & 2 \\
\hline $2011 Z 314 \mathrm{~A}$ & 4 & 0.01 & 4 & $>10000$ & 10450 & 4 & -- & -- & $<0.01$ & $<5$ & 2 \\
\hline $2011 Z 316 A$ & 1 & 0.01 & 7 & 250 & -- & 4 & --- & -- & $<0.01$ & $<5$ & 3 \\
\hline 2011Z345B & 3 & 0.2 & 68 & 3280 & --- & 17 & --- & -- & 0.04 & 6 & 15 \\
\hline 2011Z349A & $<1$ & 2.57 & 60 & 390 & --- & 2 & --- & --- & 0.15 & 6 & 50 \\
\hline $2011 Z 362 A$ & $<1$ & 0.03 & 3 & 100 & --- & 3 & --- & --- & $<0.01$ & $<5$ & 1 \\
\hline 2011Z366B & $<1$ & 0.06 & 11 & 150 & --- & 12 & --- & --- & 0.01 & $<5$ & 3 \\
\hline $2011 Z 380 A$ & $<1$ & 1.04 & 24 & 340 & --- & 15 & --- & --- & 0.01 & 5 & 24 \\
\hline $2011 Z 382 A$ & 4 & 0.63 & 104 & 860 & --- & 13 & -- & -- & 0.01 & $<5$ & 22 \\
\hline 2011Z383B & $<1$ & 0.79 & 11 & 270 & -- & $<2$ & -- & -- & $<0.01$ & $<5$ & 13 \\
\hline $2011 Z 384 A$ & $<1$ & 0.11 & 7 & 160 & --- & 2 & --- & --- & $<0.01$ & $<5$ & 4 \\
\hline $2011 Z 385 A$ & $<1$ & 0.01 & 1810 & 50 & -- & 2 & -- & -- & $<0.01$ & 11 & 10 \\
\hline 2011Z389A & 1 & 0.01 & 12 & 400 & --- & 6 & --- & --- & $<0.01$ & $<5$ & 4 \\
\hline $2011 Z 393 A$ & $<1$ & 0.3 & 22 & 200 & --- & 6 & --- & --- & $<0.01$ & $<5$ & 6 \\
\hline $2011 Z 395 C$ & $<1$ & 0.32 & 14 & 420 & --- & 17 & --- & --- & $<0.01$ & $<5$ & 3 \\
\hline 2011Z399A & $<1$ & 0.31 & 34 & 490 & --- & 29 & --- & --- & $<0.01$ & $<5$ & 14 \\
\hline 2011Z403A & $<1$ & 0.17 & 9 & 250 & --- & 7 & --- & --- & $<0.01$ & $<5$ & 3 \\
\hline 2011Z407A & $<1$ & 0.29 & 9 & 120 & --- & 5 & --- & --- & $<0.01$ & $<5$ & 5 \\
\hline $2011 Z 408 \mathrm{~A}$ & 3 & 0.11 & 24 & 690 & --- & 16 & -- & -- & $<0.01$ & $<5$ & 4 \\
\hline 2011Z408B & 4 & 0.02 & 40 & 1570 & -- & 2 & -- & -- & $<0.01$ & 6 & 2 \\
\hline $2011 Z 425 C$ & 1 & 0.03 & 10 & 270 & -- & $<2$ & -- & -- & $<0.01$ & $<5$ & 1 \\
\hline $2011 Z 426 A$ & 1 & 0.01 & 2 & 40 & --- & 5 & --- & --- & 0.05 & $<5$ & 2 \\
\hline 2011Z437B & 1 & 0.08 & 13 & 250 & --- & 7 & --- & --- & 0.01 & $<5$ & 3 \\
\hline 2011Z441B & $<1$ & 0.44 & 23 & 920 & --- & 12 & --- & --- & 0.01 & $<5$ & 11 \\
\hline 2011Z448B & 4 & 0.01 & 27 & 1840 & --- & 14 & --- & --- & 0.01 & 7 & 3 \\
\hline $2011 Z 455 C$ & $<1$ & 0.01 & 2 & 100 & --- & $<2$ & --- & --- & $<0.01$ & $<5$ & $<1$ \\
\hline 2011Z480A & 2 & 0.12 & 19 & 280 & --- & 833 & --- & --- & 3.75 & 15 & 5 \\
\hline
\end{tabular}




\begin{tabular}{|c|c|c|c|c|c|c|c|c|c|c|c|}
\hline Sample_ID & Sn_ppm & Sr_ppm & Th_ppm & Ti_pct & TI_ppm & U_ppm & V_ppm & W_ppm & W_ppm_2 & Zn_ppm & Zn_pct \\
\hline 2011Z271B & -- & 26 & $<20$ & 0.14 & $<10$ & 10 & 44 & $<10$ & -- & 103 & -- \\
\hline $2011 Z 302 \mathrm{~A}$ & --- & 81 & $<20$ & 0.06 & $<10$ & $<10$ & 102 & $<10$ & -- & 29 & -- \\
\hline 2011Z309A & -- & 10 & $<20$ & 0.06 & $<10$ & $<10$ & 85 & $<10$ & -- & 34 & --- \\
\hline $2011 Z 312 A$ & --- & 6 & $<20$ & 0.04 & $<10$ & $<10$ & 168 & $<10$ & --- & 24 & -- \\
\hline $2011 Z 314 \mathrm{~A}$ & -- & 9 & $<20$ & 0.03 & $<10$ & $<10$ & 205 & $<10$ & -- & 10 & -- \\
\hline $2011 Z 316 A$ & --- & 4 & $<20$ & 0.05 & $<10$ & $<10$ & 65 & $<10$ & --- & 19 & --- \\
\hline 2011Z345B & $<5$ & 59 & 20 & 0.1 & $<10$ & $<10$ & 147 & $<10$ & 70 & 488 & --- \\
\hline 2011Z349A & --- & 152 & $<20$ & 0.77 & $<10$ & 10 & 361 & $<10$ & --- & 108 & --- \\
\hline $2011 Z 362 A$ & --- & 4 & $<20$ & 0.02 & $<10$ & $<10$ & 7 & $<10$ & --- & 12 & --- \\
\hline 2011Z366B & --- & 7 & $<20$ & 0.06 & $<10$ & $<10$ & 24 & $<10$ & --- & 38 & --- \\
\hline 2011Z380A & --- & 48 & $<20$ & 0.19 & $<10$ & $<10$ & 171 & $<10$ & --- & 217 & --- \\
\hline $2011 Z 382 A$ & --- & 50 & $<20$ & 0.14 & $<10$ & $<10$ & 193 & $<10$ & -- & 220 & --- \\
\hline 2011Z383B & -- & 59 & $<20$ & 0.22 & $<10$ & $<10$ & 57 & $<10$ & -- & 87 & -- \\
\hline $2011 Z 384 A$ & --- & 16 & $<20$ & 0.08 & $<10$ & $<10$ & 46 & $<10$ & --- & 26 & --- \\
\hline $2011 Z 385 A$ & -- & 1 & $<20$ & 0.01 & $<10$ & $<10$ & 52 & $<10$ & -- & 52 & -- \\
\hline 2011Z389A & --- & 10 & $<20$ & 0.08 & $<10$ & $<10$ & 193 & $<10$ & --- & 39 & --- \\
\hline $2011 Z 393 A$ & --- & 38 & $<20$ & 0.12 & $<10$ & $<10$ & 38 & $<10$ & --- & 38 & --- \\
\hline $2011 Z 395 C$ & --- & 45 & $<20$ & 0.06 & $<10$ & $<10$ & 22 & $<10$ & --- & 50 & --- \\
\hline 2011Z399A & --- & 47 & $<20$ & 0.16 & $<10$ & $<10$ & 76 & $<10$ & --- & 87 & --- \\
\hline 2011Z403A & --- & 28 & $<20$ & 0.07 & $<10$ & $<10$ & 18 & $<10$ & --- & 26 & --- \\
\hline 2011Z407A & --- & 34 & $<20$ & 0.11 & $<10$ & $<10$ & 31 & $<10$ & --- & 22 & --- \\
\hline 2011Z408A & --- & 67 & $<20$ & 0.06 & $<10$ & $<10$ & 57 & $<10$ & --- & 71 & --- \\
\hline 2011Z408B & --- & 5 & $<20$ & 0.01 & $<10$ & $<10$ & 22 & $<10$ & --- & 116 & --- \\
\hline $2011 Z 425 C$ & -- & 7 & $<20$ & 0.03 & $<10$ & $<10$ & 22 & $<10$ & -- & 15 & -- \\
\hline $2011 Z 426 A$ & --- & 6 & $<20$ & 0.04 & $<10$ & $<10$ & 72 & $<10$ & --- & 13 & --- \\
\hline 2011Z437B & --- & 11 & $<20$ & 0.07 & $<10$ & $<10$ & 34 & $<10$ & --- & 27 & --- \\
\hline 2011Z441B & --- & 64 & $<20$ & 0.17 & $<10$ & $<10$ & 112 & $<10$ & --- & 83 & --- \\
\hline 2011Z448B & --- & 26 & $<20$ & 0.06 & $<10$ & $<10$ & 92 & $<10$ & --- & 235 & --- \\
\hline $2011 Z 455 C$ & --- & 4 & $<20$ & $<0.01$ & $<10$ & $<10$ & 2 & $<10$ & --- & 13 & --- \\
\hline 2011Z480A & 27 & 150 & $<20$ & 0.08 & $<10$ & $<10$ & 55 & $<10$ & 20 & 247 & --- \\
\hline
\end{tabular}




\begin{tabular}{|c|c|c|c|c|c|c|c|c|c|c|c|}
\hline Sample_ID & Au_ppm & Au_ppm_2 & Au_ppm_3 & Au_ppm_4 & Ag_ppm & Al_pct & As_ppm & As_pct & Ba_ppm & Be_ppm & Bi_ppm \\
\hline 2011Z482A & 0.004 & --- & --- & --- & $<0.5$ & 8.28 & 30 & --- & 1350 & 2.5 & $<2$ \\
\hline 2011Z488B & $<0.001$ & --- & --- & --- & $<0.5$ & 1.36 & $<5$ & --- & 180 & $<0.5$ & $<2$ \\
\hline
\end{tabular}


Table 2. (continued)

\begin{tabular}{|c|c|c|c|c|c|c|c|c|c|c|c|}
\hline Sample_ID & Ca_pct & Cd_ppm & Co_ppm & Cr_ppm & Cu_ppm & Fe_pct & Ga_ppm & K_pct & La_ppm & Mg_pct & Mn_ppm \\
\hline 2011Z482A & 0.08 & 0.5 & 15 & 95 & 49 & 5.09 & 20 & 2.47 & 10 & 1.22 & 259 \\
\hline 2011Z488B & 0.1 & $<0.5$ & 5 & 28 & 34 & 1.91 & $<10$ & 0.3 & 10 & 0.18 & 135 \\
\hline
\end{tabular}


Table 2. (continued)

\begin{tabular}{|c|c|c|c|c|c|c|c|c|c|c|c|}
\hline Sample_ID & Mo_ppm & $\mathrm{Na}$ _pct & Ni_ppm & P_ppm & P_ppm_2 & Pb_ppm & Pd_ppm & Pt_ppm & S_pct & Sb_ppm & Sc_ppm \\
\hline 2011Z482A & 3 & 0.56 & 47 & 510 & --- & 14 & --- & --- & 0.01 & $<5$ & 21 \\
\hline 2011Z488B & 1 & 0.11 & 18 & 450 & --- & 6 & --- & --- & 0.01 & $<5$ & 3 \\
\hline
\end{tabular}


Table 2. (continued)

\begin{tabular}{|c|c|c|c|c|c|c|c|c|c|c|c|}
\hline Sample_ID & Sn_ppm & Sr_ppm & Th_ppm & Ti_pct & Tl_ppm & U_ppm & V_ppm & W_ppm & W_ppm_2 & Zn_ppm & Zn_pct \\
\hline $2011 Z 482 A$ & --- & 74 & $<20$ & 0.11 & $<10$ & $<10$ & 257 & $<10$ & --- & 153 & --- \\
\hline 2011Z488B & --- & 15 & $<20$ & 0.03 & $<10$ & $<10$ & 34 & $<10$ & --- & 53 & --- \\
\hline
\end{tabular}


Table 3. Detection limits and geochemical methods for trace-element analyses of rock samples.

\section{Chemex codes include:}

Au-ICP21 = fire assay-inductively coupled plasma-atomic emission spectroscopy

PGM-ICP23 = fire assay-inductively coupled plasma-atomic emission spectroscopy

AU-GRA21 = fire assay with gravimetric determination

ME-ICP61= inductively coupled plasma-atomic emission spectroscopy after four-acid digestion

As-0G62 = high-grade inductively coupled plasma-atomic emission spectroscopy after four-acid digestion

P-ICP61a = high-grade inductively coupled plasma-atomic emission spectroscopy after four-acid digestion

ME-XRF05 = pressed pellet wavelength dispersive X-ray fluorescence;

Zn-0G62 = high-grade inductively coupled plasma-atomic emission spectroscopy after four-acid digestion

Analytical methods include:

FA-ICP-AES = fire assay-inductively coupled plasma-atomic emission spectroscopy

FA-GRAV = fire assay with gravimetric determination

ICP-AES = inductively coupled plasma-atomic emission spectroscopy

pressed pellet-XRF $=$ pressed pellet X-ray fluorescence

\section{Digestions include:}

4 acid $=\mathrm{HNO}_{3}-\mathrm{HClO}_{4}-\mathrm{HF}+\mathrm{HCl}$

FA = Fire assay fusion

--- = digestion not applicable

NOTE: ${ }^{*}=$ possible incomplete digestion of this element dependent on sample matrix; ppm = parts per million; pct $=$ percent

\begin{tabular}{|c|c|c|c|c|c|c|}
\hline \multirow[b]{2}{*}{ Header } & \multirow[b]{2}{*}{ Element } & \multicolumn{5}{|c|}{ ALS Chemex } \\
\hline & & $\begin{array}{c}\text { Lower } \\
\text { Detection } \\
\text { Limit }\end{array}$ & $\begin{array}{c}\text { Upper } \\
\text { Detection } \\
\text { Limit }\end{array}$ & $\begin{array}{l}\text { Chemex } \\
\text { Code }\end{array}$ & $\begin{array}{c}\text { Analytical } \\
\text { Method }\end{array}$ & Digestion \\
\hline$A u \_p p m$ & Gold & 0.001 & 10 & Au-ICP21 & FA-ICP-AES & $\mathrm{FA}$ \\
\hline Au_ppm_2 & Gold & 0.001 & 10 & PGM-ICP23 & ICP-AES & FA \\
\hline Au_ppm_3 & Gold & 0.001 & 10 & Au-ICP21 & FA-ICP-AES & FA \\
\hline Au_ppm_4 & Gold & 0.05 & 1,000 & Au-GRA21 & FA-GRAV & FA \\
\hline Ag_ppm & Silver & 0.5 & 100 & ME-ICP61 & ICP-AES & 4 Acid \\
\hline Al_pct & Aluminum & 0.01 & 50 & ME-ICP61 & ICP-AES & 4 Acid \\
\hline As_ppm & Arsenic & 5 & 10,000 & ME-ICP61 & ICP-AES & 4 Acid \\
\hline As_pct & Arsenic & 0.01 & 30 & As-OG62 & ICP-AES & 4 Acid \\
\hline Ba_ppm & Barium* & 10 & 10,000 & ME-ICP61 & ICP-AES & 4 Acid \\
\hline Be_ppm & Beryllium* & 0.5 & 1,000 & ME-ICP61 & ICP-AES & 4 Acid \\
\hline Bi_ppm & Bismuth & 2 & 10,000 & ME-ICP61 & ICP-AES & 4 Acid \\
\hline Ca_pct & Calcium & 0.01 & 50 & ME-ICP61 & ICP-AES & 4 Acid \\
\hline Cd_ppm & Cadmium & 0.5 & 1,000 & ME-ICP61 & ICP-AES & 4 Acid \\
\hline Co_ppm & Cobalt & 1 & 10,000 & ME-ICP61 & ICP-AES & 4 Acid \\
\hline Cr_ppm & Chromium* & 1 & 10,000 & ME-ICP61 & ICP-AES & 4 Acid \\
\hline Cu_ppm & Copper & 1 & 10,000 & ME-ICP61 & ICP-AES & 4 Acid \\
\hline Fe_pct & Iron & 0.01 & 50 & ME-ICP61 & ICP-AES & 4 Acid \\
\hline Ga_ppm & Gallium & 10 & 10,000 & ME-ICP61 & ICP-AES & 4 Acid \\
\hline K_pct & Potassium & 0.01 & 10 & ME-ICP61 & ICP-AES & 4 Acid \\
\hline La_ppm & Lanthanum & 10 & 10,000 & ME-ICP61 & ICP-AES & 4 Acid \\
\hline Mg_pct & Magnesium & 0.01 & 50 & ME-ICP61 & ICP-AES & 4 Acid \\
\hline Mn_ppm & Manganese & 5 & 100,000 & ME-ICP61 & ICP-AES & 4 Acid \\
\hline
\end{tabular}


Table 3. (continued)

\begin{tabular}{|c|c|c|c|c|c|c|}
\hline \multirow[b]{2}{*}{ Header } & \multirow[b]{2}{*}{ Element } & \multicolumn{5}{|c|}{ ALS Chemex } \\
\hline & & $\begin{array}{c}\text { Lower } \\
\text { Detection } \\
\text { Limit } \\
\end{array}$ & $\begin{array}{c}\text { Upper } \\
\text { Detection } \\
\text { Limit } \\
\end{array}$ & $\begin{array}{c}\text { Chemex } \\
\text { Code }\end{array}$ & $\begin{array}{c}\text { Analytical } \\
\text { Method }\end{array}$ & Digestion \\
\hline Mo_ppm & Molybdenum & 1 & 10,000 & ME-ICP61 & ICP-AES & 4 Acid \\
\hline Na_pct & Sodium & 0.01 & 10 & ME-ICP61 & ICP-AES & 4 Acid \\
\hline Ni_ppm & Nickle & 1 & 10,000 & ME-ICP61 & ICP-AES & 4 Acid \\
\hline P_ppm & Phosphorous & 10 & 10,000 & ME-ICP61 & ICP-AES & 4 Acid \\
\hline P_ppm_2 & Phosphorous & 50 & 100,000 & P-ICP61a & ICP-AES & 4 Acid \\
\hline Pb_ppm & Lead & 2 & 10,000 & ME-ICP61 & ICP-AES & 4 Acid \\
\hline Pd_ppm & Palladium & 0.001 & 10 & PGM-ICP23 & FA-ICP-AES & $\mathrm{FA}$ \\
\hline Pt_ppm & Platinum & 0.005 & 10 & PGM-ICP23 & FA-ICP-AES & FA \\
\hline S_pct & Sulfur & 0.01 & 10 & ME-ICP61 & ICP-AES & 4 Acid \\
\hline Sb_ppm & Antimony & 5 & 10,000 & ME-ICP61 & ICP-AES & 4 Acid \\
\hline Sc_ppm & Scandium & 1 & 10,000 & ME-ICP61 & ICP-AES & 4 Acid \\
\hline Sn_ppm & Tin & 5 & 10,000 & ME-XRF05 & $\begin{array}{c}\text { Pressed } \\
\text { pellet-XRF }\end{array}$ & --- \\
\hline Sr_ppm & Strontium & 1 & 10,000 & ME-ICP61 & ICP-AES & 4 Acid \\
\hline Th_ppm & Thorium & 20 & 10,000 & ME-ICP61 & ICP-AES & 4 Acid \\
\hline Ti_pct & Titanium* & 0.01 & 10 & ME-ICP61 & ICP-AES & 4 Acid \\
\hline TI_ppm & Thallium & 10 & 10,000 & ME-ICP61 & ICP-AES & 4 Acid \\
\hline U_ppm & Uranium & 10 & 10,000 & ME-ICP61 & ICP-AES & 4 Acid \\
\hline V_ppm & Vanadium & 1 & 10,000 & ME-ICP61 & ICP-AES & 4 Acid \\
\hline W_ppm & Tungston* & 10 & 10,000 & ME-ICP61 & ICP-AES & 4 Acid \\
\hline W_ppm_2 & Tungston & 10 & 10,000 & ME-XRF05 & $\begin{array}{c}\text { Pressed } \\
\text { pellet-XRF }\end{array}$ & --- \\
\hline Zn_ppm & Zinc & 2 & 10,000 & ME-ICP61 & ICP-AES & 4 Acid \\
\hline Zn_pct & Zinc & 0.001 & 30 & Zn-OG62 & ICP-AES & 4 Acid \\
\hline
\end{tabular}


Table 4. Location and description of rocks collected for major-oxide, minor-oxide, and trace-element analyses in the Moran area, Tanana and Melozitna quadrangles, Alaska.

NOTE: Coordinates are based on NAD 27

\begin{tabular}{|c|c|c|c|c|c|c|}
\hline Sample_ID & Latitude & Longitude & Easting & Northing & \begin{tabular}{|l|} 
UTM_ \\
Zone
\end{tabular} & Lithology_Description \\
\hline 2011BAE096A & 65.484811 & -152.812298 & 508691 & 7262309 & $5 \mathrm{~N}$ & $\begin{array}{l}\text { Biotite-muscovite granite (Alkali-feldspar granite); medium grained, light pink to } \\
\text { pale pinkish gray, equigranular, and massive. Rock is } 12 \% \text { biotite and contains } \\
\text { subhedral quartz, euhedral potassium feldspar, and occasional pink megacrysts } \\
\text { up to } 1.5 \mathrm{~cm} \text { long. } 100 \% \text { of exposure. }\end{array}$ \\
\hline 2011BAE100A & 65.487364 & -152.831978 & 507779 & 7262591 & $5 \mathrm{~N}$ & $\begin{array}{l}\text { Biotite-muscovite monzogranite (Syenogranite); medium grained, equigranular, } \\
\text { and massive. Minerals include minor muscovite, } 10-12 \% 1-3 \mathrm{~cm} \text { clean biotite } \\
\text { books, occasional euhedral potassium feldspar megacrysts that are up to } 1.5 \mathrm{~cm} \\
\text { long and have clean, straight matrix contacts, and smoky, subhedral quartz } \\
\text { phenocrysts that can be rounded. } 100 \% \text { of exposure. }\end{array}$ \\
\hline 2011BAE107C & 65.484924 & -152.858386 & 506557 & 7262316 & $5 \mathrm{~N}$ & $\begin{array}{l}\text { Aplite dike (Syenogranitic aplite dike); fine grained, pink and aphanitic. } \\
\text { Occasional visible quartz or quartz and feldspar crystals near granite margins. } \\
5 \% \text { of exposure. }\end{array}$ \\
\hline 2011BAE108A & 65.483016 & -152.861506 & 506413 & 7262103 & $5 \mathrm{~N}$ & $\begin{array}{l}\text { Biotite granite (Syenogranite); equigranular, homogenous, and massive. } \\
\text { Minerals include } 10-12 \% \text { biotite, occasional } 0.5-1.5-\mathrm{cm} \text { potassium feldspar } \\
\text { megacrysts, and subhedral smoky quartz. Selected sample of unweathered } \\
\text { material. } 100 \% \text { of exposure. }\end{array}$ \\
\hline 2011BAE112A & 65.478624 & -152.877227 & 505686 & 7261612 & $5 \mathrm{~N}$ & $\begin{array}{l}\text { Biotite granite (Syenogranite); coarse-grained, equigranular, homogenous, and } \\
\text { light pink to white. Minerals include 3-5 mm (slightly larger than matrix) } \\
\text { potassium feldspar, dark gray to smoky quartz, and light gray, interstitial late } \\
\text { quartz. Rock contains } 10-12 \% 1-3 \mathrm{~mm} \text { biotite crystals and possibly minor } \\
\text { muscovite. Quartz and mica crystals define very subtle alignment planes. } 100 \% \\
\text { of exposure. }\end{array}$ \\
\hline 2011BAE202A & 65.456879 & -152.502041 & 523081 & 7259274 & $5 \mathrm{~N}$ & $\begin{array}{l}\text { Metagabbro (Metagabbro); rock is medium grained }(1-2 \mathrm{~mm}) \text {, pale green } \\
\text { (epidotized feldspar?) to dark, olive green, massive, homogenous, and mafic. } \\
100 \% \text { of exposure. }\end{array}$ \\
\hline
\end{tabular}


Table 4. (continued)

\begin{tabular}{|c|c|c|c|c|c|c|}
\hline Sample_ID & Latitude & Longitude & Easting & Northing & $\begin{array}{l}\text { UTM_ } \\
\text { Zone }\end{array}$ & Lithology_Description \\
\hline 2011BAE246B & 65.326011 & -152.904815 & 504434 & 7244600 & $5 \mathrm{~N}$ & $\begin{array}{l}\text { Gabbro (Gabbro); medium grained, massive, and homogenous. Rock color is a } \\
\text { mottled gray, possibly with some lighter colors and/or spots of iron oxide. Rock } \\
\text { cleaves into tabular chunks. } 25 \% \text { of exposure. }\end{array}$ \\
\hline 2011BAE247A & 65.325527 & -152.905396 & 504407 & 7244546 & $5 \mathrm{~N}$ & $\begin{array}{l}\text { Metagabbro (Metagabbro); medium grained and crumbly. Fresh rock color is a } \\
\text { mottled gray with green, unoriented actinolite crystals and possibly some } \\
\text { lighter colors and/or spots of iron oxide. Rock weathers brown. } 100 \% \text { of } \\
\text { exposure. }\end{array}$ \\
\hline 2011BAE272A & 65.446956 & -152.980072 & 500924 & 7258077 & $5 \mathrm{~N}$ & $\begin{array}{l}\text { Monzogranite (Monzogranite); coarse grained, massive, and homogenous. } \\
\text { Minerals include } 5 \% \text { grayish biotite, gray quartz, slightly porphyritic potassium } \\
\text { feldspar, and white plagioclase. Grus weathering covers outcrop. Selected } \\
\text { sample of fresh material. } 100 \% \text { of exposure. }\end{array}$ \\
\hline 2011BAE290A & 65.461131 & -152.941999 & 502688 & 7259658 & $5 \mathrm{~N}$ & $\begin{array}{l}\text { Monzogranite (Syenogranite); coarse grained, pink to grayish pink, equigranular, } \\
\text { massive, and homogenous. Minerals are equally distributed, and include slightly } \\
\text { porphyritic (?) potassium feldspar and } 10 \% \text { biotite. } 90 \% \text { of exposure. }\end{array}$ \\
\hline 2011BAE290B & 65.461131 & -152.941999 & 502688 & 7259658 & $5 \mathrm{~N}$ & $\begin{array}{l}\text { Granite aplite (Syenogranitic aplite); fine to medium grained with occasional } \\
\text { larger } 1-5 \mathrm{~mm} \text { biotite, } 2-5 \mathrm{~mm} \text { quartz, and } 2 \mathrm{~mm}-1 \mathrm{~cm} \text { potassium feldspar. Fresh } \\
\text { rock is pink, but has a white weathering rind. } 10 \% \text { of exposure. }\end{array}$ \\
\hline 2011BAE337A & 65.325190 & -153.102222 & 495238 & 7244509 & $5 \mathrm{~N}$ & $\begin{array}{l}\text { Mica-feldspar-quartz porphyroclastic schist (Metagabbro); gray, fine to coarse } \\
\text { grained, and granular with platy schistosity. Minerals include quartz or feldspar } \\
\text { in foliation planes and a gray mica (biotite/chlorite?). } 100 \% \text { of exposure. }\end{array}$ \\
\hline 2011BAE351C & 65.462650 & -152.805055 & 509034 & 7259840 & $5 \mathrm{~N}$ & $\begin{array}{l}\text { Basalt (Basalt); fine grained, black, massive, homogenous, and undeformed. } \\
\text { Rock has blocky, angular parting and sooty surfaces. Visible minerals include } \\
\text { amphibole and plagioclase. } 49 \% \text { of exposure. Trace. }\end{array}$ \\
\hline
\end{tabular}


Table 4. (continued)

\begin{tabular}{|c|c|c|c|c|c|c|}
\hline Sample_ID & Latitude & Longitude & Easting & Northing & $\begin{array}{l}\text { UTM_ } \\
\text { Zone }\end{array}$ & Lithology_Description \\
\hline 2011BAE357A & 65.463469 & -152.787267 & 509858 & 7259934 & $5 \mathrm{~N}$ & $\begin{array}{l}\text { Biotite-muscovite granite (Alkali-feldspar granite); fine to medium grained (1-2 } \\
\text { mm), massive, homogenous, equigranular, and pink. Minerals include } 5 \% \\
\text { muscovite, } 5-10 \% \text { biotite, and occasional 3-5 mm potassium feldspar } \\
\text { megacrysts. } 50 \% \text { of exposure. }\end{array}$ \\
\hline 2011BAE357B & 65.463469 & -152.787267 & 509858 & 7259934 & $5 \mathrm{~N}$ & $\begin{array}{l}\text { Biotite granite (Syenogranite); medium grained (3-4 mm), pink, granular, } \\
\text { homogenous, and massive. Minerals include } 1 \% \text { muscovite, } 5-10 \% \text { biotite, } \\
\text { slightly porphyritic (5-8 mm) potassium feldspar crystals, 3-6 mm drusy quartz, } \\
\text { and occasional iridescent, smoky quartz. } 50 \% \text { of exposure. }\end{array}$ \\
\hline 2011BAE359B & 65.462807 & -152.782460 & 510081 & 7259861 & $5 \mathrm{~N}$ & $\begin{array}{l}\text { Dacite dike (Rhyolite dike); very fine to fine grained, light gray, massive, } \\
\text { homogenous, and porphyritic. Minerals include euhedral feldspars and quartz } \\
\text { phenocrysts as well spots of iron oxide. } 10 \% \text { of exposure. }\end{array}$ \\
\hline 2011BAE359C & 65.462807 & -152.782460 & 510081 & 7259861 & $5 \mathrm{~N}$ & $\begin{array}{l}\text { Dacite dike (Rhyolite dike); very fine grained, light gray, massive, homogenous, } \\
\text { and aphanitic. Rock contains small, 1-2 mm brown spots (biotite clots after } \\
\text { clinopyroxene?) and occasional small, euhedral quartz or light feldspar. } 10 \% \text { of } \\
\text { exposure. }\end{array}$ \\
\hline 2011BAE363A & 65.467260 & -152.771848 & 510571 & 7260359 & $5 \mathrm{~N}$ & $\begin{array}{l}\text { Biotite granite (Syenogranite); coarse-grained, pink, massive, homogenous, and } \\
\text { aphanitic. Rock contains large, } 2-3 \mathrm{~cm} \text { potassium feldspar megacrysts and } 15 \% 2- \\
4 \mathrm{~mm} \text { biotites in a coarse interstitial granitic material. The rock's weathered } \\
\text { surface shows aligned, brittle shear. } 75 \% \text { of exposure. }\end{array}$ \\
\hline 2011GG007A & 65.297564 & -152.862566 & 506409 & 7241433 & $5 \mathrm{~N}$ & $\begin{array}{l}\text { Chlorite-amphibole schist (Metagabbro); gray-green weathering, medium- } \\
\text { grained, light green, and granular rock with moderately well-developed } \\
\text { schistose foliation defined by aligned chlorite (20-30\%) and amphiboles (20- } \\
30 \%) \text {. Plagioclase (50\%) comprises remainder of rock. } 100 \% \text { of exposure. }\end{array}$ \\
\hline
\end{tabular}


Table 4. (continued)

\begin{tabular}{|c|c|c|c|c|c|c|}
\hline Sample_ID & Latitude & Longitude & Easting & Northing & $\begin{array}{l}\text { UTM_ } \\
\text { Zone }\end{array}$ & Lithology_Description \\
\hline 2011GG084A & 65.462452 & -152.819471 & 508366 & 7259816 & $5 \mathrm{~N}$ & $\begin{array}{l}\text { Granite (Syenogranite); white to tan with black specks. Rock weathers orange to } \\
\text { brown. Minerals include } 55 \% 1 \mathrm{~mm} \text { subhedral quartz, } 35 \% 1 \mathrm{~mm} \text { subhedral } \\
\text { feldspar, } 10 \% 1-4 \mathrm{~mm} \text { euhedral biotite, and } 5 \% \text { localized muscovite. } 50 \% \text { of } \\
\text { exposure. }\end{array}$ \\
\hline 2011GG426A & 65.392661 & -153.402776 & 481285 & 7252085 & $5 \mathrm{~N}$ & $\begin{array}{l}\text { Microgabbro (Microgabbro); fine-grained, black, slightly foliated. Minerals } \\
\text { include approximately equal amounts of plagioclase, amphibole, and pyroxene. } \\
\text { Plagioclase and amphibole crystals are locally aligned. } 100 \% \text { of exposure. }\end{array}$ \\
\hline 2011GG434A & 65.387237 & -153.376764 & 482490 & 7251473 & $5 \mathrm{~N}$ & $\begin{array}{l}\text { Granite (Syenogranite); contact between a fine-grained and medium-grained } \\
\text { granite. Both rocks appear to have the same composition: } 20 \% \text { anhedral, smoky } \\
\text { quartz; } 70-75 \% \text { sub to anhedral feldspar; and } 5-10 \% \text { subhedral biotite. The fine- } \\
\text { grained rock has mostly equant grains ( } 3-5 \mathrm{~mm} \text { ) with some feldspars up to } 25 \\
\text { mm long. Selected sample of fine-grained material. } 100 \% \text { of exposure. }\end{array}$ \\
\hline 2011GG527A & 65.384860 & -153.830285 & 461410 & 7251410 & $5 \mathrm{~N}$ & $\begin{array}{l}\text { Granite (Monzogranite); black and white rock. } 20 \% 1-3 \mathrm{~mm} \text { anhedral clear and } \\
\text { smokey quartz, } 10-15 \% \text { subhedral } 2-3 \mathrm{~mm} \text { biotite, and } 65-70 \% \text { feldspars, mostly } \\
2-5 \mathrm{~mm} \text { anhedral to subhedral crystals with minor 5-7 } \mathrm{mm} \text { subhedral crystals. } \\
100 \% \text { of exposure. }\end{array}$ \\
\hline 2011GG534A & 65.385003 & -153.800596 & 462790 & 7251408 & $5 \mathrm{~N}$ & $\begin{array}{l}\text { Granite (Monzogranite); Monzogranite; mostly white rock, with minor black } \\
\text { specs. } 25 \% 1-2 \mathrm{~mm} \text { anhedral quartz, } 5 \% \text { subhedral } 2 \mathrm{~mm} \text { biotite, and } 70 \% \\
\text { feldspars, mostly 2-3 mm anhedral crystals a few } 5 \mathrm{~mm} \text { anhedral crystals. } 100 \% \\
\text { of exposure. }\end{array}$ \\
\hline 2011LF078A & 65.441124 & -152.704480 & 513706 & 7257459 & $5 \mathrm{~N}$ & $\begin{array}{l}\text { Greenstone (Metabasalt); greenish gray. Rock contains } 2 \% 0.5 \mathrm{~mm} \text { relict } \\
\text { amphiboles and } 3 \% \text { white to gray mica in a fine-grained, sugary groundmass. } \\
100 \% \text { of exposure. }\end{array}$ \\
\hline
\end{tabular}




\begin{tabular}{|c|c|c|c|c|c|c|}
\hline Sample_ID & Latitude & Longitude & Easting & Northing & $\begin{array}{l}\text { UTM_- } \\
\text { Zone }\end{array}$ & Lithology_Description \\
\hline 2011LF194A & 65.454031 & -152.498255 & 523259 & 7258958 & $5 \mathrm{~N}$ & $\begin{array}{l}\text { Gabbro (Gabbro); relict diabasic texture with } 30 \% 1 \mathrm{~mm} \text { plagioclase, } 25 \% 1 \mathrm{~mm} \\
\text { epidote, } 35 \% 1 \mathrm{~mm} \text {, elongate amphibole, and } 10 \% 2-3 \mathrm{~mm} \text { relict pyroxene } \\
\text { altered to amphibole. Rock contains minor albite } \pm \text { quartz veins. } 100 \% \text { of } \\
\text { exposure. }\end{array}$ \\
\hline 2011LF282A & 65.336063 & -153.146976 & 493156 & 7245725 & $5 \mathrm{~N}$ & $\begin{array}{l}\text { Biotite amphibolite (Metagabbro); dark green color. Rock contains } 25 \% 5 \mathrm{~mm} \\
\text { brown biotite, } 75 \% 1 \mathrm{~mm} \text { prismatic amphibole. } 100 \% \text { of exposure. }\end{array}$ \\
\hline 2011LF302A & 65.458714 & -152.751835 & 511502 & 7259410 & $5 \mathrm{~N}$ & $\begin{array}{l}\text { Porphyritic biotite granite (Syenogranite); rock contains } 25 \% 5 \mathrm{~mm} \text { biotite, } 10 \% \\
2 \mathrm{~cm} \text { potassium feldspar, } 20 \% 3 \mathrm{~mm} \text { gray, smoky quartz in } 35 \% 1-2 \mathrm{~mm} \text { quartz } \\
\text { and feldspar groundmass. } 100 \% \text { of exposure. }\end{array}$ \\
\hline 2011LF304A & 65.461891 & -152.752344 & 511477 & 7259764 & $5 \mathrm{~N}$ & $\begin{array}{l}\text { Leucogranite (Syenogranite); coarse-grained seriate texture. Rock contains } 25 \% \\
1 \mathrm{~cm} \text { smoky quartz, } 1 \% 10 \mathrm{~cm} \text { potassium feldspar megacrysts, } 40 \% 1.5 \mathrm{~cm} \text {, } \\
\text { tabular potassium feldspar phenocrysts and } 5 \% \text { biotite in } 30 \% \text { feldspar matrix. } \\
100 \% \text { of exposure. }\end{array}$ \\
\hline 2011LF326A & 65.495858 & -152.741003 & 511987 & 7263552 & $5 \mathrm{~N}$ & $\begin{array}{l}\text { Syenogranite (Syenogranite); coarse-grained seriate texture. Rock contains } 5 \% 3 \\
\mathrm{~cm} \text { potassium feldspar megacrystals, } 30 \% 1 \mathrm{~cm} \text { quartz, } 20 \% 1 \mathrm{~cm} \text { potassium } \\
\text { feldspar, and } 15 \% 2 \mathrm{~mm} \text { biotite in a } 2 \mathrm{~mm} \text { feldspar groundmass. } 100 \% \text { of } \\
\text { exposure. }\end{array}$ \\
\hline 2011LF329A & 65.494491 & -152.728659 & 512559 & 7263402 & $5 \mathrm{~N}$ & $\begin{array}{l}\text { Muscovite-biotite-quartz granite (Alkali-feldspar granite); medium grained. Rock } \\
\text { contains } 2 \% 2 \mathrm{~cm} \text { potassium feldspar phenocrysts, } 15 \% 1-2 \mathrm{~mm} \text { quartz grains, } \\
15 \% 1 \mathrm{~mm} \text { biotite, } 10 \% \text { muscovite, and } 48 \% \text { mixed } 1 \mathrm{~mm} \text { feldspars. } 100 \% \text { of } \\
\text { exposure. }\end{array}$ \\
\hline 2011LF336A & 65.495562 & -152.697556 & 513998 & 7263528 & $5 \mathrm{~N}$ & $\begin{array}{l}\text { Biotite-quartz monzonite (Alkali-feldspar granite); porphyritic. Rock contains } \\
15 \% 5 \mathrm{~mm} \text { smoky, subhedral quartz phenocrysts, } 10 \% 1 \mathrm{~cm} \text { potassium feldspar } \\
\text { phenocrysts, and } 10 \% 3 \mathrm{~mm} \text { biotite in a } 0.5 \mathrm{~mm} \text { quartz and feldspar } \\
\text { groundmass. } 100 \% \text { of exposure. }\end{array}$ \\
\hline 2011LF486C & 65.425908 & -152.983752 & 500754 & 7255731 & $5 \mathrm{~N}$ & $\begin{array}{l}\text { Monzogranite (Syenogranite); fine grained. Rock contains } 10 \% 0.5 \mathrm{~mm} \text { biotite, } \\
3 \% 5 \mathrm{~mm} \text { clots of biotite, and } 5 \% 1 \mathrm{~mm} \text { quartz in a } 0.1 \mathrm{~mm} \text {, mixed-feldspar } \\
\text { groundmass ( } 82 \% \text { of rock). } 25 \% \text { of exposure. }\end{array}$ \\
\hline
\end{tabular}




\begin{tabular}{|c|c|c|c|c|c|c|}
\hline Sample_ID & Latitude & Longitude & Easting & Northing & $\begin{array}{l}\text { UTM_- }_{-} \\
\text {Zone }\end{array}$ & Lithology_Description \\
\hline 2011LF497A & 65.415845 & -152.953795 & 502145 & 7254610 & $5 N$ & $\begin{array}{l}\text { Leucocratic monzonite (Syenogranite); contains } 5 \% 1 \mathrm{~mm} \text { quartz, } 5 \% 0.5 \mathrm{~mm} \\
\text { biotite, } 10 \% 1 \mathrm{~cm} \text { xenoliths with quartz, biotite, and pyrite, and } 80 \% 1-2 \mathrm{~mm} \\
\text { interlocking feldspars. } 10 \% \text { of exposure. }\end{array}$ \\
\hline 2011LF536A & 65.470654 & -152.610335 & 518052 & 7260774 & $5 \mathrm{~N}$ & $\begin{array}{l}\text { Hornblende metagabbro (Metagabbro); } 20 \% 4 \mathrm{~mm} \text { relict hornblende } \\
\text { phenocrysts, } 45 \% \text { clinozoisite after } 1 \mathrm{~mm} \text { plagioclase laths, and } 2 \% 0.5 \mathrm{~mm} \\
\text { sphene in } 35 \% \text { green matrix. } 100 \% \text { of exposure. }\end{array}$ \\
\hline 2011LF573A & 65.291505 & -152.973351 & 501243 & 7240751 & $5 \mathrm{~N}$ & $\begin{array}{l}\text { Metadiorite (Metagabbro); } 60 \% 1 \mathrm{~mm} \text { feldspars, } 35 \% \text { chlorite, and } 0.5 \mathrm{~mm} \\
\text { hornblende pseudomorphs in an interlocking, subhedral texture. } 100 \% \text { of } \\
\text { exposure. }\end{array}$ \\
\hline 2011RN221A & 65.495344 & -152.599618 & 518531 & 7263529 & $5 N$ & $\begin{array}{l}\text { Greenstone (Metabasalt); fine-grained, green, massive and blocky. } 100 \% \text { of } \\
\text { exposure. }\end{array}$ \\
\hline 2011RN260A & 65.359713 & -152.894805 & 504894 & 7248357 & $5 \mathrm{~N}$ & $\begin{array}{l}\text { Greenstone (Metabasalt); fine-grained ( } 1-2 \mathrm{~mm}) \text {, massive, blocky, and foliated } \\
\text { metamafic rock. Contains } 40-60 \% \text { albite } \pm \text { clinozoisite (?) and } 40-60 \% \text { chlorite. } \\
100 \% \text { of exposure. }\end{array}$ \\
\hline 2011RN319A & 65.200204 & -153.687453 & 467824 & 7230750 & $5 \mathrm{~N}$ & $\begin{array}{l}\text { Biotite-granite (Monzogranite); medium-grained }(2-5 \mathrm{~mm}) \text {, equigranular and } \\
\text { contains } 20 \% \text { light gray quartz and possible garnet. Here is a fault contact with } \\
\text { metamorphic rocks. } 100 \% \text { of exposure. }\end{array}$ \\
\hline 2011RN325A & 65.370436 & -152.525978 & 522044 & 7249631 & $5 \mathrm{~N}$ & $\begin{array}{l}\text { Greenstone (Metabasalt); massive, unfoliated, blocky }\left(<3 \mathrm{~m}^{3}\right) \text {, and locally } \\
\text { derived Tozitna greenstone. } 100 \% \text { of exposure. }\end{array}$ \\
\hline 2011TL001A & 65.237525 & -153.582362 & 472781 & 7234860 & $5 \mathrm{~N}$ & $\begin{array}{l}\text { Orthogneiss (Monzogranite); pinkish-tan weathering rind. Contains } 15 \% \text { fine- } \\
\text { grained biotite, } 35 \% \text { clear, } 2-4 \mathrm{~mm} \text { quartz, and } 50 \% \text { white feldspars. Micas are } \\
\text { weakly foliated and form } 0.5 \text {-mm-thick bands. } 100 \% \text { of exposure. }\end{array}$ \\
\hline 2011Z004A & 65.400751 & -153.010419 & 499516 & 7252927 & $5 \mathrm{~N}$ & $\begin{array}{l}\text { Biotite granite (Metamonzogranite); coarse to very coarse grained. Contains } \\
35 \% \text { porphyritic, pinkish potassium feldspar (=3 cm in diameter), } 20 \% \text { gray, } \\
\text { interstitial quartz, } 10 \% \text { fine-grained ( } 1-2 \mathrm{~mm} \text { in diameter), brownish to brownish } \\
\text { black biotite, and } 35 \% \text { white plagioclase. } 100 \% \text { of exposure. }\end{array}$ \\
\hline
\end{tabular}


Table 4. (continued)

\begin{tabular}{|c|c|c|c|c|c|c|}
\hline Sample_ID & Latitude & Longitude & Easting & Northing & $\begin{array}{l}\text { UTM } \\
\text { Zone }\end{array}$ & Lithology_Description \\
\hline 2011Z045A & 65.322647 & -152.748674 & 511709 & 7244245 & $5 \mathrm{~N}$ & $\begin{array}{l}\text { Epidote-feldspar-chlorite sheared metagabbro (Metagabbro); medium-grained, } \\
\text { blocky, and greenish cobbles make up the subcrop. Fresh rock is spotted green } \\
\text { and light gray to white with poorly defined foliation. Minerals include } 50 \% \\
\text { green to dark green chlorite, } 40-45 \% \text { white to slightly tan feldspar, and trace } \\
\text { epidote. Rock does not react to } \mathrm{HCl} \text {. } 95 \% \text { of exposure. }\end{array}$ \\
\hline $2011 Z 082 A$ & 65.459858 & -152.723472 & 512816 & 7259543 & $5 \mathrm{~N}$ & $\begin{array}{l}\text { Biotite granite (Syenogranite); coarse- to very coarse-grained, porphyritic } \\
\text { granite dike that has orange to tan weathering on both external and broken } \\
\text { surfaces. Phenocrysts include } 10 \%=2 \mathrm{~cm} \text { long, white feldspar, } 10 \% 2-3 \mathrm{~mm} \text {, } \\
\text { dark gray, rounded quartz, and } 10 \% \text { black, irregular biotite. } 99 \% \text { of exposure. }\end{array}$ \\
\hline $2011 Z 083 A$ & 65.462248 & -152.725044 & 512742 & 7259809 & $5 \mathrm{~N}$ & $\begin{array}{l}\text { Biotite granite (Syenogranite); medium to very coarse grained. Rock contains } \\
\text { porphyritic pink feldspar phenocrysts }=3 \mathrm{~cm} \text { long (average maximum } \\
\text { dimensions are } 5 \mathrm{~cm} \times 2 \mathrm{~cm} \text { ), } 30 \% \text { biotite crystals =2 mm in diameter, and } 20 \% 2 \\
\mathrm{~mm} \text {, rounded, gray quartz phenocrysts in amedium-grained granitic matrix. } \\
100 \% \text { of exposure. }\end{array}$ \\
\hline 2011Z093A & 65.466367 & -152.814653 & 508588 & 7260253 & $5 \mathrm{~N}$ & $\begin{array}{l}\text { Biotite granite (Alkali-feldspar granite); medium-grained }(1-2 \mathrm{~mm}) \text { and } \\
\text { equigranular. Broken surface is cream to light orange with a common } \\
\text { weathering rind stained with iron oxide. Minerals include } 15 \% \text { black biotite, } 25 \% \\
\text { gray quartz, and white feldspar. Rock contains } 2 \text {-mm-wide quartz veinlets, one } \\
\text { of which has a black, metallic, non-magnetic mineral. } 100 \% \text { of exposure. }\end{array}$ \\
\hline
\end{tabular}


Table 4. (continued)

\begin{tabular}{|c|c|c|c|c|c|c|}
\hline Sample_ID & Latitude & Longitude & Easting & Northing & $\begin{array}{l}\text { UTM_ } \\
\text { Zone }\end{array}$ & Lithology_Description \\
\hline 2011Z116A & 65.413436 & -152.921772 & 503632 & 7254343 & $5 N$ & $\begin{array}{l}\text { Biotite-quartz-feldspar gneiss (Quartz-rich granitoid); possibly orthogneiss. } \\
\text { Rock is medium grained with a rough, light brownish-gray weathering surface } \\
\text { that shows foliation. Broken rock is dark gray and white with localized gneissic } \\
\text { habit and is possibly recrystallized. Rock contains } 25 \% \text { biotite, gray to brownish- } \\
\text { gray feldspar and quartz, and rare, milky white, foliation-parallel quartz veinlets. } \\
\text { Selected sample of most iron-oxide-stained rock. No vein material, but several } \\
\text { pieces with open vugs (1-3 mm across) with iron-oxide earths. } 100 \% \text { of } \\
\text { exposure. }\end{array}$ \\
\hline 2011Z135A & 65.250036 & -152.610378 & 518202 & 7236185 & $5 N$ & $\begin{array}{l}\text { Quartz-amphibole-chlorite-feldspar(?) aragneiss (Metagranite); fine grained, } \\
\text { blocky to platy with light brown weathering. Broken rock is light gray with black } \\
\text { and orange specks. White mica and chlorite define foliation. Contains } 10 \% \text { dark } \\
\text { green and black amphibole (altered to chlorite?) and } 2 \mathrm{~mm} \text {, glassy, rounded, } \\
\text { slightly porphyroblastic, bluish-gray quartz grains in a feldspar or quartz matrix } \\
\text { with } 10 \% \text { chlorite. } 70 \% \text { of exposure. }\end{array}$ \\
\hline 2011Z250A & 65.490276 & -152.912599 & 504046 & 7262908 & $5 \mathrm{~N}$ & $\begin{array}{l}\text { Biotite granite (Alkali-felsdpar granite); porphyritic, coarse to very coarse } \\
\text { grained. Rock contains quartz phenocrysts ranging in color from gray to light, } \\
\text { smoky gray to dark, smoky, grayish-black, } 5 \%=1.5-\mathrm{cm} \text {-long, white feldspar } \\
\text { (potassium?) phenocrysts, and about } 10 \% \text { black biotite clots in a cream and gray } \\
\text { colored matrix. } 100 \% \text { of exposure. }\end{array}$ \\
\hline 2011Z256A & 65.180341 & -153.888678 & 458375 & 7228654 & $5 \mathrm{~N}$ & $\begin{array}{l}\text { Biotite-quartz monzonite (Monzogranite); extremely coarse-grained and } \\
\text { porphyritic. Contains } 40-45 \%=5 \times 2 \mathrm{~cm} \text { porphyritic white feldspar (potassium?) } \\
15 \%=1 \mathrm{~cm} \text { gray, irregular quartz, } 15-25 \% \text { black, irregular masses of biotite in an } \\
\text { orange and black matrix with cream feldspars. Many feldspar phenocrysts are } \\
\text { aligned parallel to long axis. } 100 \% \text { of exposure. }\end{array}$ \\
\hline
\end{tabular}


Table 4. (continued)

\begin{tabular}{|c|c|c|c|c|c|c|}
\hline Sample_ID & Latitude & Longitude & Easting & Northing & $\begin{array}{l}\text { UTM_- } \\
\text { Zone }\end{array}$ & Lithology_Description \\
\hline 2011Z260B & 65.186690 & -153.892286 & 458216 & 7229364 & $5 N$ & $\begin{array}{l}\text { Granitic aplite dike (Alkali-feldspar-granitic aplite dike); fine grained ( } 2 \mathrm{~mm} \text { ), } 10 \text { - } \\
\mathrm{cm} \text {-wide dike. Contains } 10 \% 1 \mathrm{~mm} \text { biotite that are aligned with dike margin. } 1 \% \\
\text { of exposure. }\end{array}$ \\
\hline $2011 Z 265 A$ & 65.185046 & -153.865046 & 459489 & 7229163 & $5 \mathrm{~N}$ & $\begin{array}{l}\text { Quartz monzonite (Monzogranite); porphyritic and very coarse grained. } \\
\text { Contains white to slightly pink, occasionally locally aligned potassium feldspar } \\
\text { phenocrysts up to } 4 \mathrm{~cm} \text { long. Wormy, irregularly shaped, gray quartz is } \\
\text { interstitial to } 50 \% \text { volume of potassium feldspar megacrysts. Black biotite. } 99 \% \\
\text { of exposure. }\end{array}$ \\
\hline 2011Z265B & 65.185046 & -153.865046 & 459489 & 7229163 & $5 \mathrm{~N}$ & $\begin{array}{l}\text { Aplite dike (Alkali-feldspar-granitic aplite dike); fine grained and creamy white } \\
\text { to light gray. Contains biotite grains that are aligned parallel to dike strike. The } \\
\text { immediate area contains several dikes and dikelets; the widest piece is } 25 \mathrm{~cm} \text { in } \\
\text { float and } 20 \mathrm{~cm} \text { in outcrop. }\end{array}$ \\
\hline 2011Z323A & 65.504351 & -152.646101 & 516374 & 7264520 & $5 \mathrm{~N}$ & $\begin{array}{l}\text { Biotite granite (Syenogranite); porphyritic, with medium-grained matrix with } \\
\text { light-orange to buff weathering and equigranular plagioclase and potassium } \\
\text { feldspar. Broken rock is pink and white. Contains }=2 \mathrm{~cm} \text { long, pink potassium } \\
\text { feldspar phenocrysts, } 20 \% 2-3 \mathrm{~mm} \text { gray quartz phenocrysts, and } 15-20 \% \text { black } \\
\text { biotite in a medium-grained (1-2 mm) matrix. } 100 \% \text { of exposure. }\end{array}$ \\
\hline 2011Z336A & 65.389356 & -152.513801 & 522594 & 7251744 & $5 \mathrm{~N}$ & $\begin{array}{l}\text { Metagabbro (Serpentinite); fine-grained with light brown to dull green } \\
\text { weathering. Broken rock looks very fresh and is black and green with possible } \\
\text { relict olivine. } 100 \% \text { of exposure. }\end{array}$ \\
\hline 2011Z371A & 65.264114 & -152.984494 & 500724 & 7237698 & $5 \mathrm{~N}$ & $\begin{array}{l}\text { Altered metagabbro (Metagabbro); fine-grained with a mottled texture ranging } \\
\text { in color from pale green to greenish gray with fine-grained, tan to white grains } \\
\text { of feldspar. Pale-green- and green-colored epidote and chlorite. Rock forms } \\
\text { subrounded cobbles and has weak or no foliation. Some pieces look } \\
\text { semischistose. }\end{array}$ \\
\hline
\end{tabular}


Table 4. (continued)

\begin{tabular}{|l|l|l|l|l|l|l|}
\hline Sample_ID & Latitude & Longitude & Easting & Northing & $\begin{array}{l}\text { UTM_ } \\
\text { Zone }\end{array}$ & \multicolumn{1}{c|}{ Lithology_Description } \\
\hline \hline 2011Z385A & 65.254046 & -152.942860 & 502669 & 7236577 & $5 \mathrm{~N}$ & $\begin{array}{l}\text { Greenstone (Metabasalt); possibly chloritized gabbro (?). Very angular, fine- } \\
\text { grained, and dark-brown weathering rock. Broken rock has a mottled texture } \\
\text { ranging in color from green to dark green with orange spots of iron oxide. } \\
\text { Brown and reddish-orange iron oxides moderately to strongly stain abundant } \\
\text { fracture surfaces. Locally, rock has a green, waxy to fibrous habit (talc or } \\
\text { serpentine?). Spots of iron oxide are sometimes vuggy with occasional fine- } \\
\text { grained, disseminated pyrrhotite in the center. } \text { Rock has no HCl reaction. 100\% } \\
\text { of exposure. }\end{array}$ \\
\hline $2011 Z 490 \mathrm{~A}$ & 65.361513 & -152.967412 & 501516 & 7248554 & $5 \mathrm{~N}$ & $\begin{array}{l}\text { Amphibole-chlorite-feldspar metagabbro (Metagabbro); fine- to medium- } \\
\text { grained, brown and dark brown, blocky foliated outcrop. Rock breaks easily } \\
\text { along planes and has moderate to strong orange and brown stains of iron oxide. } \\
\text { Fine-grained clumps of black amphibole occur on foliation surfaces and are }=1 \\
\text { mm across with halos of iron oxide. Rock contains dark green chlorite, black } \\
\text { mica (biotite or chlorite?) and 15\% amphibole (?). Rock has no } \mathrm{HCl} \text { reaction. } \\
\text { Rock has no HCl reaction. 100\% of exposure. }\end{array}$ \\
\hline
\end{tabular}


Table 5. Major-oxide, minor-oxide, and trace-element results for rocks collected in the Moran area, Tanana and Melozitna quadrangles, Alaska.

NOTE: ppm = parts per million; pct = percent; "---" = element not analyzed

\begin{tabular}{|c|c|c|c|c|c|c|c|c|c|c|c|}
\hline SAMPLE_ID & $\mathrm{SiO}_{2} \_\mathrm{pct}$ & $\mathrm{Al}_{2} \mathrm{O}_{3} \_\mathrm{pct}$ & $\mathrm{Fe}_{2} \mathrm{O}_{3} \_\mathrm{pct}$ & $\mathrm{CaO} \_$pct & $\mathrm{MgO} \_$pct & $\mathrm{Na}_{2} \mathrm{O}_{\text {_ }} \mathrm{pct}$ & $\mathrm{K}_{2} \mathrm{O} \_\mathrm{pct}$ & $\mathrm{Cr}_{2} \mathrm{O}_{3}$ & $\mathrm{TiO}_{2} \_\mathrm{pct}$ & $\mathrm{MnO} \_\mathrm{pct}$ & $\mathrm{P}_{2} \mathrm{O}_{5} \_\mathrm{pct}$ \\
\hline 2011BAE096A & 72.37 & 14.8 & 1.78 & 0.76 & 0.29 & 3.39 & 4.96 & $<0.01$ & 0.1 & 0.05 & 0.171 \\
\hline 2011BAE100A & 75.77 & 12.9 & 2.13 & 0.53 & 0.18 & 3.21 & 4.67 & $<0.01$ & 0.04 & 0.06 & 0.036 \\
\hline 2011BAE107C & 73.45 & 13.76 & 0.82 & 1.35 & 0.06 & 3.24 & 6.23 & $<0.01$ & $<0.01$ & $<0.01$ & 0.01 \\
\hline 2011BAE112A & 75.3 & 13.22 & 2.03 & 0.63 & 0.08 & 3.48 & 4.64 & $<0.01$ & $<0.01$ & 0.06 & 0.014 \\
\hline 2011BAE202A & 48.52 & 7.45 & 10.34 & 15.11 & 13.23 & 1.42 & 0.12 & 0.22 & 0.92 & 0.17 & 0.068 \\
\hline 2011BAE272A & 77.89 & 11.47 & 1.76 & 0.97 & 0.2 & 2.53 & 4.09 & 0.01 & 0.08 & 0.03 & 0.03 \\
\hline 2011BAE290A & 75.87 & 12.87 & 1.79 & 0.56 & 0.13 & 3.17 & 4.87 & 0.01 & 0.05 & 0.04 & 0.024 \\
\hline 2011BAE290B & 76.07 & 13.37 & 1.19 & 0.75 & 0.11 & 3.62 & 4.57 & $<0.01$ & 0.04 & 0.02 & 0.031 \\
\hline 2011BAE337A & 47.1 & 15.74 & 9.56 & 10.73 & 7.91 & 2.44 & 0.25 & 0.02 & 1.01 & 0.15 & 0.094 \\
\hline 2011BAE351C & 50.09 & 13.92 & 12.96 & 9.35 & 6.03 & 2.21 & 0.43 & 0.01 & 1.96 & 0.19 & 0.178 \\
\hline 2011BAE357A & 73.49 & 14.68 & 1.68 & 0.47 & 0.27 & 3.38 & 4.73 & $<0.01$ & 0.09 & 0.05 & 0.101 \\
\hline 2011BAE363A & 75.34 & 12.7 & 2.36 & 0.72 & 0.27 & 2.88 & 4.74 & $<0.01$ & 0.14 & 0.05 & 0.052 \\
\hline 2011GG007A & 49.23 & 14.5 & 11.2 & 10.39 & 6.7 & 2.69 & 0.53 & 0.02 & 1.51 & 0.17 & 0.15 \\
\hline 2011GG084A & 76.58 & 13.29 & 0.71 & 0.54 & 0.11 & 3.27 & 4.77 & $<0.01$ & $<0.01$ & 0.02 & 0.012 \\
\hline 2011GG426A & 46.33 & 13.44 & 15.69 & 11.7 & 6.81 & 2.14 & 0.31 & 0.02 & 2.28 & 0.28 & 0.166 \\
\hline 2011 GG434A & 76.17 & 12.7 & 1.66 & 0.67 & 0.18 & 2.68 & 5.48 & $<0.01$ & 0.07 & 0.02 & 0.023 \\
\hline 2011GG527A & 67.35 & 15.05 & 4.2 & 2.64 & 1.29 & 2.32 & 4.65 & 0.01 & 0.67 & 0.05 & 0.159 \\
\hline 2011GG534A & 75.81 & 13.19 & 1.37 & 0.99 & 0.14 & 2.9 & 4.91 & $<0.01$ & 0.05 & 0.01 & 0.015 \\
\hline 2011LF078A & 48.46 & 14.95 & 10.02 & 9.44 & 7.35 & 3.62 & 0.1 & 0.01 & 1.38 & 0.16 & 0.411 \\
\hline 2011LF194A & 47.04 & 8.2 & 10.96 & 12.42 & 14.95 & 1.14 & 0.34 & 0.18 & 0.92 & 0.19 & 0.079 \\
\hline 2011LF282A & 43.99 & 11.98 & 18.89 & 9.18 & 5.51 & 2.32 & 0.19 & $<0.01$ & 3.65 & 0.22 & 0.135 \\
\hline 2011LF302A & 75.08 & 12.87 & 2.22 & 0.59 & 0.2 & 3.12 & 4.63 & $<0.01$ & 0.07 & 0.04 & 0.027 \\
\hline
\end{tabular}




\begin{tabular}{|c|c|c|c|c|c|c|c|c|c|c|c|}
\hline SAMPLE_ID & SrO__pct & $\mathrm{BaO} \_$pct & LOI_pct & Total_pct & Ba_ppm & Cr_ppm & Nb_ppm & Ni_ppm & Rb_ppm & Sr_ppm & Th_ppm \\
\hline 2011BAE096A & 0.01 & 0.03 & 0.73 & 99.45 & 250 & $\begin{array}{c}-- \\
\end{array}$ & 16 & $\begin{array}{c}-- \\
\end{array}$ & 559 & 71 & 16 \\
\hline 2011BAE100A & 0.01 & 0.01 & 0.38 & 99.92 & 60 & --- & 62 & --- & 492 & 22 & 65 \\
\hline 2011BAE107C & $<0.01$ & $<0.01$ & 0.73 & 99.66 & 10 & --- & 48 & --- & 588 & 5 & 31 \\
\hline 2011BAE112A & 0.01 & $<0.01$ & 0.59 & 100.05 & 20 & --- & 79 & --- & 797 & 6 & 52 \\
\hline 2011BAE202A & 0.02 & 0.02 & 2.45 & 100.05 & --- & 1455 & 4 & 180 & 4 & 97 & --- \\
\hline 2011BAE272A & 0.01 & 0.01 & 0.33 & 99.41 & 120 & --- & 16 & --- & 232 & 85 & 74 \\
\hline 2011BAE290A & 0.01 & 0.01 & 0.33 & 99.72 & 60 & --- & 62 & --- & 544 & 27 & 63 \\
\hline 2011BAE290B & 0.01 & $<0.01$ & 0.31 & 100.05 & 40 & --- & 52 & --- & 361 & 20 & 33 \\
\hline 2011BAE337A & 0.04 & 0.03 & 4.6 & 99.66 & --- & 103 & 5 & 60 & 6 & 294 & --- \\
\hline 2011BAE351C & 0.03 & 0.03 & 1.15 & 98.53 & --- & 56 & 10 & 50 & 42 & 235 & --- \\
\hline 2011BAE357A & 0.01 & 0.02 & 0.88 & 99.85 & 210 & --- & 37 & --- & 514 & 65 & 25 \\
\hline 2011BAE363A & 0.01 & $<0.01$ & 0.43 & 99.7 & 180 & --- & 44 & --- & 409 & 64 & 75 \\
\hline 2011GG007A & 0.04 & 0.03 & 2.36 & 99.53 & --- & 165 & 8 & 50 & 15 & 324 & --- \\
\hline 2011GG084A & 0.01 & 0.01 & 0.58 & 99.9 & 110 & --- & 76 & --- & 448 & 40 & 48 \\
\hline 2011GG426A & 0.03 & 0.01 & 0.5 & 99.7 & --- & 142 & 6 & 50 & 7 & 192 & --- \\
\hline 2011 GG434A & 0.01 & 0.01 & 0.34 & 100 & 60 & --- & 13 & --- & 383 & 46 & 69 \\
\hline 2011GG527A & 0.04 & 0.08 & 0.55 & 99.06 & 780 & --- & 14 & --- & 245 & 326 & 42 \\
\hline 2011GG534A & 0.01 & 0.01 & 0.35 & 99.76 & 40 & --- & 11 & --- & 345 & 45 & 72 \\
\hline 2011LF078A & 0.01 & 0.01 & 2.92 & 98.85 & --- & 105 & 2 & 40 & $<2$ & 114 & --- \\
\hline 2011LF194A & 0.01 & 0.05 & 3.27 & 99.74 & --- & 1210 & 4 & 260 & 8 & 58 & --- \\
\hline 2011LF282A & 0.04 & 0.02 & 3.3 & 99.42 & --- & 7 & 8 & $<10$ & 9 & 341 & --- \\
\hline 2011LF302A & 0.01 & 0.01 & 0.6 & 99.46 & 70 & --- & 80 & --- & 457 & 30 & 84 \\
\hline
\end{tabular}




\begin{tabular}{|l|c|c|c|}
\hline SAMPLE_ID & U_ppm & Y_ppm & Zr_ppm \\
\hline \hline 2011BAE096A & 5 & 129 & 57 \\
\hline 2011BAE100A & 36 & 170 & 96 \\
\hline 2011BAE107C & 17 & 276 & 40 \\
\hline 2011BAE108A & 42 & 249 & 90 \\
\hline 2011BAE112A & 37 & 257 & 78 \\
\hline 2011BAE202A & --- & 16 & 45 \\
\hline 2011BAE246B & --- & 15 & 63 \\
\hline 2011BAE247A & --- & 11 & 25 \\
\hline 2011BAE272A & 16 & 68 & 90 \\
\hline 2011BAE290A & 34 & 161 & 90 \\
\hline 2011BAE290B & 34 & 125 & 46 \\
\hline 2011BAE337A & --- & 16 & 73 \\
\hline 2011BAE351C & --- & 34 & 125 \\
\hline 2011BAE357A & 7 & 137 & 58 \\
\hline 2011BAE357B & 12 & 149 & 73 \\
\hline 2011BAE359B & 34 & 298 & 60 \\
\hline 2011BAE359C & 5 & 253 & 47 \\
\hline 2011BAE363A & 22 & 127 & 136 \\
\hline 2011GG007A & --- & 23 & 113 \\
\hline 2011GG084A & 28 & 176 & 69 \\
\hline 2011GG426A & --- & 41 & 128 \\
\hline 2011GG434A & 7 & 102 & 18 \\
\hline 2011GG527A & 4 & 65 & 211 \\
\hline 2011GG534A & 13 & 97 & 80 \\
\hline 2011LF078A & --- & 61 & 216 \\
\hline 2011LF194A & --- & 17 & 49 \\
\hline 2011LF282A & --- & 23 & 106 \\
\hline 2011LF302A & 49 & 119 & 88 \\
\hline 2011LF304A & 18 & 108 & 113 \\
\hline 2011LF326A & 18 & 106 & 139 \\
\hline
\end{tabular}




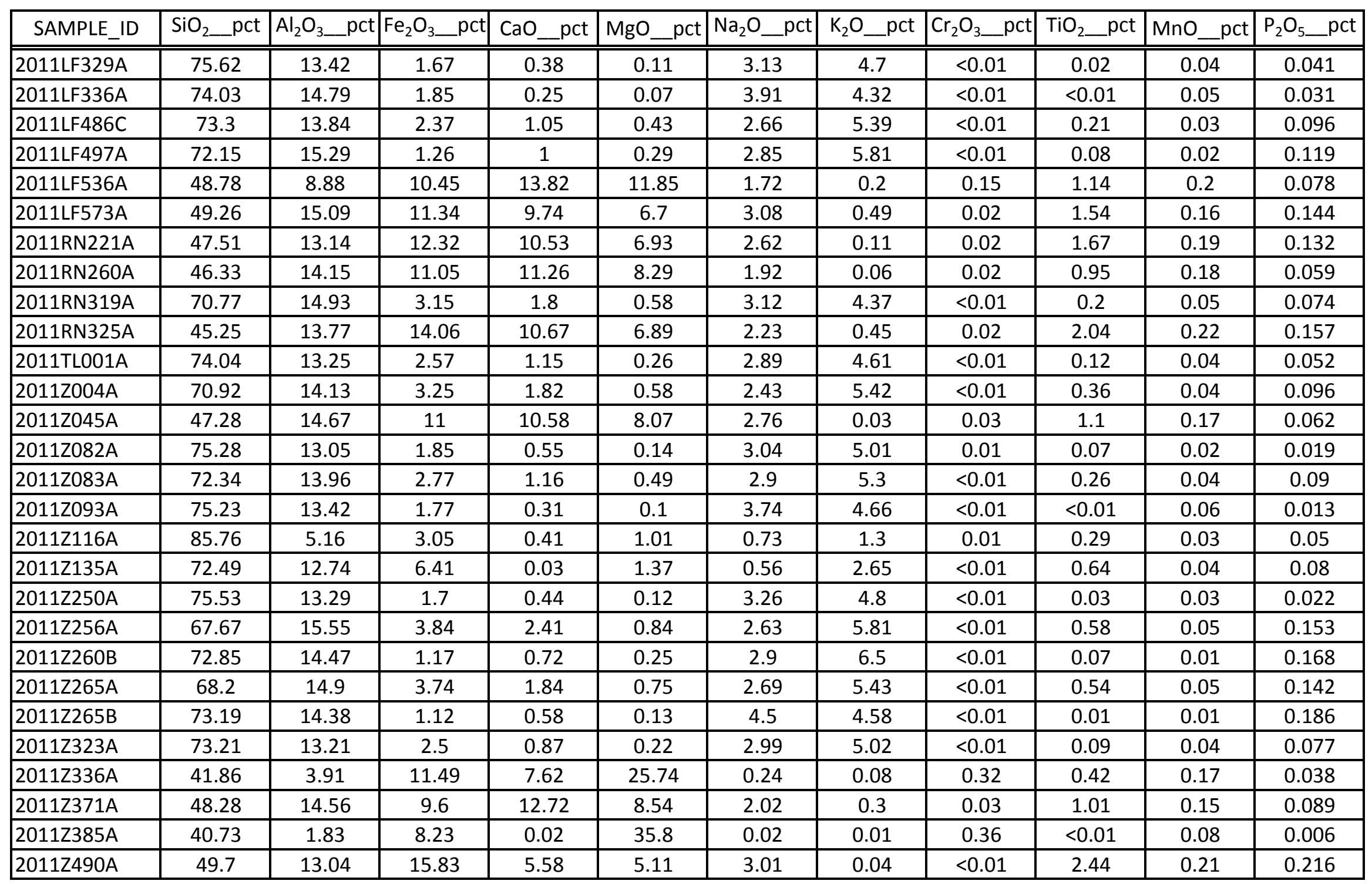




\begin{tabular}{|c|c|c|c|c|c|c|c|c|c|c|c|}
\hline SAMPLE_ID & SrO_pct & $\mathrm{BaO} \_$pct & LOI_pct & Total_pct & Ba_ppm & Cr_ppm & Nb_ppm & Ni_ppm & Rb_ppm & Sr_ppm & Th_ppm \\
\hline 2011LF329A & 0.01 & 0.01 & 0.72 & 99.87 & 60 & --- & 57 & --- & 826 & 14 & 45 \\
\hline 2011LF336A & $<0.01$ & 0.01 & 0.67 & 99.98 & 40 & $\overline{---}$ & 67 & $\overline{---}$ & 961 & 7 & 51 \\
\hline 2011LF486C & 0.02 & 0.04 & 0.41 & 99.85 & 380 & --- & 13 & --- & 291 & 128 & 35 \\
\hline 2011LF497A & 0.01 & 0.04 & 0.73 & 99.66 & 390 & --- & 9 & --- & 244 & 97 & 8 \\
\hline 2011LF536A & 0.02 & 0.01 & 2.65 & 99.95 & --- & 1000 & 4 & 150 & 5 & 106 & --- \\
\hline 2011LF573A & 0.04 & 0.05 & 2.27 & 99.92 & --- & 118 & 8 & 60 & 14 & 305 & --- \\
\hline 2011RN319A & 0.02 & 0.05 & 0.76 & 99.88 & 470 & --- & 12 & --- & 197 & 144 & 21 \\
\hline 2011RN325A & 0.03 & 0.01 & 2.86 & 98.65 & --- & 122 & 6 & 50 & 9 & 217 & --- \\
\hline 2011TL001A & 0.02 & 0.09 & 0.88 & 99.97 & 820 & --- & 14 & -- & 205 & 101 & 28 \\
\hline 2011Z004A & 0.03 & 0.06 & 0.55 & 99.69 & 620 & --- & 10 & --- & 218 & 197 & 26 \\
\hline $2011 Z 045 A$ & 0.02 & 0.02 & 2.8 & 98.6 & --- & 256 & 2 & 80 & $<2$ & 169 & --- \\
\hline 2011Z135A & 0.01 & 0.07 & 2.92 & 100 & 630 & --- & 11 & --- & 100 & 36 & 18 \\
\hline $2011 Z 250 A$ & $<0.01$ & 0.01 & 0.88 & 100.1 & 50 & --- & 69 & --- & 615 & 20 & 68 \\
\hline $2011 Z 256 A$ & 0.03 & 0.14 & 0.51 & 100.2 & 1180 & -- & 14 & --- & 210 & 296 & 24 \\
\hline 2011Z260B & 0.02 & 0.05 & 0.55 & 99.73 & 480 & -- & 7 & -- & 215 & 150 & 8 \\
\hline $2011 Z 265 A$ & 0.03 & 0.12 & 0.8 & 99.24 & 1080 & --- & 15 & --- & 218 & 255 & 33 \\
\hline 2011Z265B & 0.01 & 0.01 & 0.38 & 99.08 & 70 & --- & 11 & -- & 237 & 39 & 5 \\
\hline 2011Z323A & 0.01 & 0.01 & 0.55 & 98.8 & 110 & --- & 34 & --- & 346 & 34 & 58 \\
\hline $2011 Z 336 \mathrm{~A}$ & 0.01 & 0.01 & 6.27 & 98.17 & --- & 2120 & 2 & 660 & 2 & 65 & --- \\
\hline $2011 Z 371 \mathrm{~A}$ & 0.04 & 0.03 & 2.38 & 99.75 & 270 & --- & 4 & --- & 7 & 256 & $<4$ \\
\hline $2011 Z 385 A$ & $<0.01$ & $<0.01$ & 11.55 & 98.64 & --- & 2440 & $<2$ & 2050 & $<2$ & 2 & --- \\
\hline 2011Z490A & 0.02 & 0.05 & 3.38 & 98.63 & --- & 19 & 14 & 50 & 3 & 135 & --- \\
\hline
\end{tabular}




\begin{tabular}{|l|c|c|c|}
\hline SAMPLE_ID & U_ppm & Y_ppm & Zr_ppm \\
\hline \hline 2011LF329A & 19 & 217 & 60 \\
\hline 2011LF336A & 30 & 244 & 66 \\
\hline 2011LF486C & 7 & 81 & 145 \\
\hline 2011LF497A & 5 & 62 & 44 \\
\hline 2011LF536A & --- & 19 & 53 \\
\hline 2011LF573A & --- & 21 & 106 \\
\hline 2011RN221A & --- & 27 & 89 \\
\hline 2011RN260A & --- & 29 & 48 \\
\hline 2011RN319A & 4 & 58 & 115 \\
\hline 2011RN325A & --- & 31 & 117 \\
\hline 2011TL001A & 5 & 68 & 135 \\
\hline 2011Z004A & $<4$ & 57 & 222 \\
\hline 2011Z045A & --- & 30 & 53 \\
\hline 2011Z082A & 13 & 101 & 119 \\
\hline 2011Z083A & 13 & 89 & 185 \\
\hline 2011Z093A & 39 & 175 & 82 \\
\hline 2011Z116A & --- & 22 & 93 \\
\hline 2011Z135A & $<4$ & 25 & 210 \\
\hline 2011Z250A & 29 & 183 & 94 \\
\hline $2011 Z 256 A$ & 4 & 56 & 382 \\
\hline 2011Z260B & 4 & 65 & 43 \\
\hline $2011 Z 265 A$ & 4 & 61 & 345 \\
\hline $2011 Z 265 B$ & 5 & 58 & 20 \\
\hline $2011 Z 323 A$ & 13 & 117 & 98 \\
\hline $2011 Z 336 A$ & --- & 8 & 16 \\
\hline $2011 Z 371 A$ & $<4$ & 16 & 63 \\
\hline $2011 Z 385 A$ & --- & 2 & $<2$ \\
\hline 2011Z490A & --- & 38 & 152 \\
\hline
\end{tabular}


Table 6. Detection limits and analytical methods for major- and minor-oxide and trace-element analyses of whole-rock samples.

\section{Chemex codes include:}

ME-XRF06 = Lithium borate fusion and X-ray fluorescence spectroscopy;

ME-XRF05 = X-ray fluorescence spectroscopy on a pressed pellet;

\section{Analytical methods include :}

LBF-XRF = Lithium borate fusion and X-ray fluorescence spectroscopy.

Note: $\mathrm{Fe}_{2} \mathrm{O}_{3}=$ total iron as $\mathrm{Fe}_{2} \mathrm{O}_{3} ; \mathrm{LOI}^{*}=$ loss on ignition; "---" = not applicable.

\begin{tabular}{|c|c|c|c|c|c|}
\hline \multirow[b]{2}{*}{ Header } & \multirow[b]{2}{*}{ Element } & \multicolumn{4}{|c|}{ ALS Chemex } \\
\hline & & $\begin{array}{c}\text { Lower } \\
\text { Detection } \\
\text { Limit } \\
\end{array}$ & $\begin{array}{c}\text { Upper } \\
\text { Detection } \\
\text { Limit } \\
\end{array}$ & $\begin{array}{c}\text { Chemex } \\
\text { Code }\end{array}$ & $\begin{array}{c}\text { Analytical } \\
\text { Method }\end{array}$ \\
\hline $\mathrm{SiO}_{2} \_\mathrm{pct}$ & $\mathrm{SiO}_{2}$ & 0.01 & 100 & "ME-XRF06 & 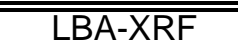 \\
\hline $\mathrm{Al}_{2} \mathrm{O}_{3} \_$pct & $\mathrm{Al}_{2} \mathrm{O}_{3}$ & 0.01 & 100 & ME-XRF06 & LBA-XRF \\
\hline $\mathrm{Fe}_{2} \mathrm{O}_{3} \_\mathrm{pct}$ & $\mathrm{Fe}_{2} \mathrm{O}_{3}$ & 0.01 & 100 & ME-XRF06 & LBA-XRF \\
\hline $\mathrm{CaO} \_$pct & $\mathrm{CaO}$ & 0.01 & 100 & ME-XRF06 & LBA-XRF \\
\hline $\mathrm{MgO} \_$pct & $\mathrm{MgO}$ & 0.01 & 100 & ME-XRF06 & LBA-XRF \\
\hline $\mathrm{Na}_{2} \mathrm{O}$ _pct & $\mathrm{Na}_{2} \mathrm{O}$ & 0.01 & 100 & ME-XRF06 & LBA-XRF \\
\hline $\mathrm{K}_{2} \mathrm{O} \_$pct & $\mathrm{K}_{2} \mathrm{O}$ & 0.01 & 100 & ME-XRF06 & LBA-XRF \\
\hline $\mathrm{Cr}_{2} \mathrm{O}_{3}$ _pct & $\mathrm{Cr}_{2} \mathrm{O}_{3}$ & 0.01 & 100 & ME-XRF06 & LBA-XRF \\
\hline $\mathrm{TiO}_{2} \_\mathrm{pct}$ & $\mathrm{TiO}_{2}$ & 0.01 & 100 & ME-XRF06 & LBA-XRF \\
\hline $\mathrm{MnO} \_$_pct & $\mathrm{MnO}$ & 0.01 & 100 & ME-XRF06 & LBA-XRF \\
\hline $\mathrm{P}_{2} \mathrm{O}_{5} \_\mathrm{pct}$ & $\mathrm{P}_{2} \mathrm{O}_{5}$ & 0.01 & 100 & ME-XRF06 & LBA-XRF \\
\hline SrO__pct & $\mathrm{SrO}$ & 0.01 & 100 & ME-XRF06 & LBA-XRF \\
\hline $\mathrm{BaO} \_$pct & $\mathrm{BaO}$ & 0.01 & 100 & ME-XRF06 & LBA-XRF \\
\hline LOI_pct & LOI* & 0.01 & 100 & -- & Gravimetric \\
\hline Total_pct & Total & --- & --- & -- & Calculation \\
\hline Ba_ppm & Barium & 10 & 10,000 & ME-XRF05 & Pressed pellet- \\
\hline Cr_ppm & Chromium & 5 & 10,000 & ME-XRF05 & Pressed pellet- \\
\hline Nb_ppm & Niobium & 2 & 10,000 & ME-XRF05 & Pressed pellet- \\
\hline Ni_ppm & Nickle & 10 & 15,000 & ME-XRF05 & Pressed pellet- \\
\hline Rb_ppm & Rubidium & 2 & 10,000 & ME-XRF05 & Pressed pellet- \\
\hline Sr_ppm & Strontium & 2 & 10,000 & ME-XRF05 & Pressed pellet- \\
\hline Th_ppm & Thorium & 4 & 10,000 & ME-XRF05 & Pressed pellet- \\
\hline U_ppm & Uranium & 4 & 10,000 & ME-XRF05 & Pressed pellet- \\
\hline Y_ppm & Yttrium & 2 & 10,000 & ME-XRF05 & Pressed pellet- \\
\hline Zr_ppm & Zirconium & 2 & 10,000 & ME-XRF05 & Pressed pellet- \\
\hline
\end{tabular}


Table 7. Location, description, and results for rocks collected for non-carbonate-carbon analyses in the Moran area, Tanana and Melozitna Quadrangles, Alaska.

NOTE: Coordinates are based on the North Amercian Datum of 1927; carbon (non-carbonate) was analyzed by induction furnace pyrolysis following dilute acid digestion with a lower detection limit of $0.01 \%$ and an upper detection limit of $50 \%$ carbon.

\begin{tabular}{|l|c|c|c|c|c|c|c|}
\hline Sample_ID & Latitude & Longitude & Easting & Northing & $\begin{array}{l}\text { UTM_ } \\
\text { Zone }\end{array}$ & Lithology_Description \\
\hline \hline 2011LF557B & 65.336904 & -152.484123 & 524021 & 7245909 & $5 \mathrm{~N}$ & Fault gouge; graphitic. 20\% of exposure. & C_pct \\
\hline 2011Z482A & 65.375371 & -152.799444 & 509325 & 7250113 & $5 \mathrm{~N}$ & $\begin{array}{l}\text { Graphite-quartz-white-mica schist; dark gray, slightly punky, } \\
\text { strongly folded, and dominated by phyllitic white mica with some } \\
\text { graphitic soot. White quartz occurs in ribbons and layers. Orange } \\
\text { iron oxide commonly stains foliation surfaces. Contains 5\% orange } \\
\text { spots of iron oxide. 100\% of exposure. }\end{array}$ & 0.03 \\
\hline
\end{tabular}


Table 8. Location, description, and results for rocks collected for major-oxide, minor-oxide, and trace-element analyses on rock slabs in the Moran area, Tanana and Melozitna quadrangles, Alaska.

NOTE: Coordinates are based on NAD 27

\begin{tabular}{|c|c|c|c|c|c|c|c|}
\hline Sample_ID & Latitude & Longitude & Easting & Northing & $\begin{array}{l}\text { UTM_ } \\
\text { Zone }\end{array}$ & Root_name & Comments \\
\hline 2011BAE010A & 65.297071 & -152.863083 & 506385 & 7241378 & $5 \mathrm{~N}$ & Metabasite & 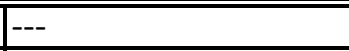 \\
\hline 2011BAE013A & 65.293646 & -152.856047 & 506714 & 7240997 & $5 \mathrm{~N}$ & Metagabbro & --- \\
\hline 2011BAE013B & 65.293646 & -152.856047 & 506714 & 7240997 & $5 \mathrm{~N}$ & Mafic schist & --- \\
\hline 2011BAE014A & 65.291725 & -152.855543 & 506738 & 7240783 & $5 \mathrm{~N}$ & Metagabbro & --- \\
\hline 2011BAE027A & 65.278115 & -152.830203 & 507924 & 7239269 & $5 \mathrm{~N}$ & Mafic gneiss & --- \\
\hline 2011BAE028A & 65.276875 & -152.828519 & 508003 & 7239131 & $5 \mathrm{~N}$ & Metagabbro(?) & --- \\
\hline 2011BAE036A & 65.351827 & \begin{tabular}{|l|}
-152.895847 \\
\end{tabular} & 504847 & 7247478 & $5 \mathrm{~N}$ & Metagabbro & $\overline{---}$ \\
\hline 2011BAE037A & 65.351431 & -152.894710 & 504900 & 7247434 & $5 \mathrm{~N}$ & Mafic schist & --- \\
\hline 2011BAE039A & 65.349813 & -152.890161 & 505112 & 7247254 & $5 \mathrm{~N}$ & Metagabbro(?) & --- \\
\hline 2011BAE041A & 65.347989 & -152.886108 & 505301 & 7247051 & $5 \mathrm{~N}$ & Metagabbro(?) & --- \\
\hline 2011BAE045A & 65.346594 & -152.870194 & 506042 & 7246897 & $5 \mathrm{~N}$ & Metagabbro & --- \\
\hline 2011BAE048A & 65.326453 & -152.714461 & 513301 & 7244676 & $5 \mathrm{~N}$ & Diorite(?) & --- \\
\hline 2011BAE050A & 65.330766 & -152.713212 & 513357 & 7245157 & $5 \mathrm{~N}$ & Diorite(?) or Gabbro(?) & Altered \\
\hline 2011BAE051B & 65.332777 & \begin{tabular}{|l|}
-152.713899 \\
\end{tabular} & 513324 & 7245381 & $5 \mathrm{~N}$ & Gabbro & --- \\
\hline 2011BAE053A & 65.335250 & -152.717115 & 513173 & 7245656 & $5 \mathrm{~N}$ & Metagabbro & --- \\
\hline 2011BAE054A & 65.336322 & -152.718972 & 513086 & 7245775 & $5 \mathrm{~N}$ & Gabbro & --- \\
\hline 2011BAE056A & 65.337695 & -152.714425 & 513297 & 7245929 & $5 \mathrm{~N}$ & Metagabbro & --- \\
\hline 2011BAE065A & 65.398282 & -152.759768 & 511160 & 7252673 & $5 \mathrm{~N}$ & Gabbro(?) & Altered \\
\hline 2011BAE085A & 65.394102 & -152.733613 & 512377 & 7252212 & $5 \mathrm{~N}$ & Mafic schist(?) & --- \\
\hline 2011BAE085B & 65.394102 & -152.733613 & 512377 & 7252212 & $5 \mathrm{~N}$ & Amphibolite(?) & Altered \\
\hline 2011BAE105A & 65.486748 & -152.852393 & 506834 & 7262520 & $5 \mathrm{~N}$ & Andalusite(?) schist & --- \\
\hline 2011BAE127A & 65.411200 & -152.848812 & 507020 & 7254100 & $5 \mathrm{~N}$ & Marble & --- \\
\hline 2011BAE235A & 65.331554 & -152.877526 & 505704 & 7245220 & $5 \mathrm{~N}$ & Mafic schist & --- \\
\hline 2011BAE264A & 65.444624 & -153.013024 & 499396 & 7257817 & $5 \mathrm{~N}$ & Granite & Inhomogenous \\
\hline 2011BAE264B & 65.444624 & -153.013024 & 499396 & 7257817 & $5 \mathrm{~N}$ & Granite & Altered \\
\hline 2011BAE287B & 65.459349 & \begin{tabular}{|l|}
-152.950072 \\
\end{tabular} & 502314 & 7259459 & $5 \mathrm{~N}$ & Fine-grained granite & --- \\
\hline 2011BAE292A & 65.185604 & -153.845866 & 460388 & 7229213 & $5 \mathrm{~N}$ & Diorite orthogneiss & --- \\
\hline 2011BAE292A & 65.185604 & -153.845866 & 460388 & 7229213 & $5 \mathrm{~N}$ & Fine-grained intermediate rock & --- \\
\hline 2011BAE297C & 65.184588 & -153.831719 & 461049 & 7229091 & $5 \mathrm{~N}$ & Metamafic & --- \\
\hline 2011BAE297C & 65.184588 & -153.831719 & 461049 & 7229091 & $5 \mathrm{~N}$ & Biotite amphibolite & --- \\
\hline 2011BAE298C & 65.184843 & -153.826282 & 461304 & 7229116 & $5 \mathrm{~N}$ & Metamafic with dikelet & Inhomogenous \\
\hline 2011BAE305A & 65.184578 & -153.800606 & 462506 & 7229071 & $5 \mathrm{~N}$ & Mafic schist & Porous \\
\hline
\end{tabular}


Table 8. (continued)

\begin{tabular}{|c|c|c|c|c|c|c|c|c|c|}
\hline Sample_ID & $\mathrm{SiO}_{2 \_} \mathrm{pct}$ & Al203_pct & BaO_pct & CaO_pct & FeO_pct & K2O_pct & MgO_pct & MnO_pct & Na2O_pct \\
\hline 2011BAE010A & $\overline{52.7}$ & 14.9 & 0.0621 & 8 & 12 & 0.408 & 4.49 & 0.164 & 5.24 \\
\hline 2011BAE013A & 53.3 & 15.4 & $<0.03$ & 6.85 & 10.9 & 0.0608 & 4.92 & 0.183 & 6.6 \\
\hline 2011BAE013B & 51.7 & 15.8 & 0.0671 & 10.5 & 9.54 & 0.419 & 6.35 & 0.171 & 4.12 \\
\hline 2011BAE014A & 52.8 & 15.4 & 0.0486 & 8.72 & 10.4 & 0.348 & 5.67 & 0.181 & 4.72 \\
\hline 2011BAE027A & 66 & 14.1 & 0.0315 & 1.77 & 6.35 & 0.217 & 1.75 & 0.0551 & 7.42 \\
\hline 2011BAE028A & 51.2 & 15.5 & 0.065 & 10.8 & 10.4 & 0.464 & 6.26 & 0.183 & 3.44 \\
\hline 2011BAE036A & 53.5 & 14.3 & $<0.03$ & 8.91 & 12.3 & 0.0376 & 4.98 & 0.194 & 3.5 \\
\hline 2011BAE037A & 55.1 & 13.2 & $<0.03$ & 5.87 & 15.1 & 0.0416 & 5.04 & 0.247 & 3.61 \\
\hline 2011BAE039A & 55.3 & 14 & $<0.03$ & 3.99 & 15.1 & $<.02$ & 5.03 & 0.257 & 4.27 \\
\hline 2011BAE041A & 52.2 & 16.3 & $<0.03$ & 11.2 & 9.5 & $<.02$ & 8.07 & 0.194 & 1.73 \\
\hline 2011BAE045A & 49.2 & 14.3 & $<0.03$ & 19.1 & 8.66 & 0.0273 & 6.31 & 0.223 & 1.64 \\
\hline 2011BAE048A & 55.7 & 17 & $<0.03$ & 5.14 & 10.7 & 0.232 & 4.08 & 0.182 & 5.35 \\
\hline 2011BAE050A & 59.8 & 15.5 & $<0.03$ & 0.772 & 11.9 & 0.121 & 4.12 & 0.131 & 6.48 \\
\hline 2011BAE051B & 52.8 & 15.7 & $<0.03$ & 6.13 & 11.6 & 0.123 & 4.61 & 0.158 & 7.15 \\
\hline 2011BAE053A & 53 & 12.2 & $<0.03$ & 11.3 & 13.6 & 0.0334 & 6.12 & 0.222 & 1.17 \\
\hline 2011BAE054A & 51.2 & 13.9 & $<0.03$ & 8.49 & 11.6 & 0.0553 & 7.1 & 0.187 & 5.09 \\
\hline 2011BAE056A & 52.6 & 14.2 & 0.0899 & 8.36 & 12.1 & 0.349 & 4.21 & 0.218 & 5.08 \\
\hline 2011BAE065A & 51.2 & 18 & $<0.03$ & 9.13 & 8.68 & 0.104 & 7.3 & 0.256 & 4.68 \\
\hline 2011BAE085A & 56.1 & 17.2 & 0.056 & 5 & 5.61 & 0.438 & 1.69 & 0.102 & 8.28 \\
\hline 2011BAE085B & 55 & 14.3 & 0.032 & 6.67 & 12 & 0.138 & 4.17 & 0.144 & 5.74 \\
\hline 2011BAE105A & 45.2 & 27.9 & 0.233 & 2.46 & 11.1 & 4.72 & 3.38 & 0.241 & 2.16 \\
\hline 2011BAE127A & 13.6 & 1.19 & $<0.03$ & 46.6 & 0.898 & 0.138 & 0.314 & $<0.03$ & 0.16 \\
\hline 2011BAE235A & 53.2 & 14.3 & 0.0738 & 8.75 & 12 & 0.342 & 4.89 & 0.197 & 4.15 \\
\hline 2011BAE264A & 77.4 & 11.4 & $<0.03$ & 1.55 & 1.05 & 4 & 0.171 & 0.206 & 3.88 \\
\hline 2011BAE264B & 76.5 & 10.8 & $<0.03$ & 0.219 & 5.04 & 3.65 & 0.266 & 0.203 & 2.99 \\
\hline 2011BAE287B & 75.1 & 14 & $<0.03$ & 0.489 & 0.418 & 6.05 & 0.0599 & $<0.03$ & 3.61 \\
\hline 2011BAE292A & 61 & 15.8 & 0.132 & 5.92 & 6.63 & 2.73 & 2.55 & 0.099 & 3.06 \\
\hline 2011BAE292A & 60.2 & 16.3 & 0.137 & 6.21 & 6.22 & 2.66 & 2.22 & 0.097 & 3.31 \\
\hline 2011BAE297C & 48.8 & 15.3 & 0.0799 & 12.8 & 8.79 & 1.82 & 10 & 0.227 & 0.958 \\
\hline 2011BAE297C & 48.7 & 16.2 & 0.05 & 13.5 & 7.85 & 1.56 & 9.72 & 0.18 & 1.07 \\
\hline 2011BAE298C & 57.2 & 13.2 & 0.106 & 10.1 & 8.75 & 0.695 & 6.11 & 0.287 & 1.75 \\
\hline 2011BAE305A & 57.3 & 15.3 & $<0.03$ & 2.61 & 12.5 & 0.122 & 6.77 & 0.178 & 3.33 \\
\hline
\end{tabular}


Table 8. (continued)

\begin{tabular}{|c|c|c|c|c|c|c|c|c|c|}
\hline Sample_ID & P2O5_pct & TiO2_pct & CO2_pct & Total_pct & As_ppm & Bi_ppm & Br_ppm & Ce_ppm & Cl_ppm \\
\hline 2011BAE010A & 0.286 & 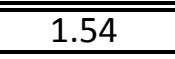 & $2<0.05$ & 99.7901 & $2<4$ & 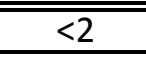 & $<<5$ & $\angle 60$ & 185 \\
\hline 2011BAE013A & 0.25 & 1.37 & $<0.05$ & 99.8495 & 10 & $<2$ & $<5$ & $<60$ & 131 \\
\hline 2011BAE013B & 0.175 & 0.902 & $<0.05$ & 99.7441 & $<4$ & $<2$ & $<5$ & $<60$ & 347 \\
\hline 2011BAE014A & 0.29 & 1.3 & $<0.05$ & 99.8776 & 21 & $<2$ & $<5$ & $<60$ & 71 \\
\hline 2011BAE027A & 0.401 & 1.71 & $<0.05$ & 99.8046 & 36 & $<2$ & $<5$ & $<60$ & 78 \\
\hline 2011BAE028A & 0.345 & 1.17 & $<0.05$ & 99.827 & 5 & $<2$ & $<5$ & $<60$ & 82 \\
\hline 2011BAE036A & 0.323 & 1.75 & $<0.05$ & 99.7946 & 9 & $<2$ & $<5$ & $<60$ & 87 \\
\hline 2011BAE037A & 0.283 & 1.37 & $<0.05$ & 99.8723 & $<4$ & $<2$ & $<5$ & $<60$ & 181 \\
\hline 2011BAE039A & 0.328 & 1.41 & $<0.05$ & 99.7178 & $<4$ & $<2$ & $<5$ & $<60$ & 85 \\
\hline 2011BAE041A & $<0.03$ & 0.582 & $<0.05$ & 99.8342 & 65 & $<2$ & $<5$ & $<60$ & 113 \\
\hline 2011BAE045A & $<0.03$ & 0.372 & $<0.05$ & 99.865 & 4 & $<2$ & $<5$ & $<60$ & 220 \\
\hline 2011BAE048A & 0.226 & 1.16 & $<0.05$ & 99.77 & $<4$ & $<2$ & $<5$ & $<60$ & 57 \\
\hline 2011BAE050A & 0.168 & 0.883 & $<0.05$ & 99.9028 & $<4$ & $<2$ & $<5$ & $<60$ & 173 \\
\hline 2011BAE051B & 0.124 & 1.47 & $<0.05$ & 99.8812 & $<4$ & $<2$ & $<5$ & $<60$ & $<50$ \\
\hline 2011BAE053A & 0.285 & 1.81 & $<0.05$ & 99.757 & 4 & $<2$ & $<5$ & $<60$ & 89 \\
\hline 2011BAE054A & 0.196 & 1.98 & $<0.05$ & 99.821 & $<4$ & $<2$ & $<5$ & $<60$ & 147 \\
\hline 2011BAE056A & 0.352 & 2.21 & $<0.05$ & 99.7689 & $<4$ & $<2$ & $<5$ & $<60$ & 147 \\
\hline 2011BAE065A & 0.0753 & 0.454 & $<0.05$ & 99.9068 & $<4$ & $<2$ & $<5$ & $<60$ & 100 \\
\hline 2011BAE085A & 0.326 & 1.04 & 4 & 99.842 & 16 & $<2$ & $<5$ & $<60$ & 230 \\
\hline 2011BAE085B & 0.244 & 1.37 & $<0.05$ & 99.808 & $<4$ & $<2$ & $<5$ & $<60$ & 163 \\
\hline 2011BAE105A & 0.429 & 1.5 & $<0.05$ & 99.323 & $<4$ & $<2$ & $<5$ & $<60$ & 1240 \\
\hline 2011BAE127A & $<0.03$ & 0.072 & 37 & 99.7307 & $<4$ & $<2$ & $<5$ & $<60$ & 184 \\
\hline 2011BAE235A & 0.268 & 1.57 & $<0.05$ & 99.7408 & $<4$ & $<2$ & $<5$ & $<60$ & 250 \\
\hline 2011BAE264A & $<0.03$ & 0.0581 & $<0.05$ & 99.7923 & 8 & $<2$ & $<5$ & $<60$ & 314 \\
\hline 2011BAE264B & $<0.03$ & 0.0549 & $<0.05$ & 99.7676 & $<4$ & $<2$ & $<5$ & $<60$ & 183 \\
\hline 2011BAE287B & 0.0489 & 0.0441 & $<0.05$ & 99.8491 & $<4$ & $<2$ & $<5$ & $<60$ & 240 \\
\hline 2011BAE292A & 0.402 & 1.35 & $<0.05$ & 99.673 & 7 & $<2$ & $<5$ & $<60$ & 1580 \\
\hline 2011BAE292A & 0.386 & 1.41 & $<0.05$ & 99.15 & $<4$ & $<2$ & $<5$ & 300 & 1420 \\
\hline 2011BAE297C & 0.061 & 0.683 & $<0.05$ & 99.5189 & $<4$ & $<2$ & $<5$ & $<60$ & 804 \\
\hline 2011BAE297C & 0.08 & 0.73 & $<0.05$ & 99.6326 & $<4$ & $<2$ & $<5$ & $<60$ & 882 \\
\hline 2011BAE298C & 0.215 & 1.12 & $<0.05$ & 99.533 & $<4$ & $<2$ & $<5$ & $<60$ & 1020 \\
\hline 2011BAE305A & 0.261 & 1.17 & $<0.05$ & 99.5674 & 49 & $<2$ & $<5$ & $<60$ & $<50$ \\
\hline
\end{tabular}


Table 8. (continued)

\begin{tabular}{|c|c|c|c|c|c|c|c|c|c|}
\hline Sample_ID & Co_ppm & Cr_ppm & Cu_ppm & F_ppm & La_ppm & Mo_ppm & Nb_ppm & Nb_ppm_2 & Ni_ppm \\
\hline 2011BAE010A & 55 & 86 & 37 & $<<110$ & $<50$ & $2<1$ & $<1$ & 9 & 102 \\
\hline 2011BAE013A & 51 & 75 & 10 & $<110$ & $<50$ & $<1$ & $<1$ & 9 & 20 \\
\hline 2011BAE013B & 67 & 165 & 47 & $<110$ & $<50$ & $<1$ & $<1$ & 6 & 69 \\
\hline 2011BAE014A & 48 & 194 & 25 & $<110$ & $<50$ & $<1$ & $<1$ & 8 & 17 \\
\hline 2011BAE027A & $<10$ & 170 & 60 & $<110$ & $<50$ & $<1$ & 29 & $\begin{array}{ll}-- \\
--\end{array}$ & 48 \\
\hline 2011BAE028A & 44 & 145 & 63 & $<110$ & $<50$ & $<1$ & $<1$ & 6 & 54 \\
\hline 2011BAE036A & 69 & 68 & 53 & $<110$ & $<50$ & $<1$ & $<1$ & 12 & 36 \\
\hline 2011BAE037A & 101 & 50 & 59 & $<110$ & $<50$ & $<1$ & $<1$ & 8 & 63 \\
\hline 2011BAE039A & 130 & 98 & 197 & $<110$ & $<50$ & $<1$ & $<1$ & 11 & 30 \\
\hline 2011BAE041A & $<10$ & 394 & 89 & $<110$ & $<50$ & $<1$ & $<1$ & 1 & 159 \\
\hline 2011BAE045A & 37 & 428 & 28 & $<110$ & $<50$ & $<1$ & $<1$ & 2 & 97 \\
\hline 2011BAE048A & 19 & 48 & 53 & $<110$ & $<50$ & $<1$ & $<1$ & 2 & 16 \\
\hline 2011BAE050A & 59 & 67 & 22 & $<110$ & $<50$ & $<1$ & $<1$ & 2 & $<7$ \\
\hline 2011BAE051B & $<10$ & 84 & 47 & $<110$ & $<50$ & $<1$ & $<1$ & 2 & $<7$ \\
\hline 2011BAE053A & 24 & 210 & 98 & $<110$ & $<50$ & $<1$ & $<1$ & 11 & 94 \\
\hline 2011BAE054A & 50 & 356 & 103 & $<110$ & $<50$ & $<1$ & $<1$ & 18 & 93 \\
\hline 2011BAE056A & 42 & 133 & 74 & $<110$ & $<50$ & $<1$ & $<1$ & 12 & 63 \\
\hline 2011BAE065A & 38 & 169 & 45 & $<110$ & $<50$ & $<1$ & $<1$ & 1 & 130 \\
\hline 2011BAE085A & 36 & 172 & 22 & $<110$ & $<50$ & $<1$ & $<1$ & 70 & 49 \\
\hline 2011BAE085B & $<10$ & 62 & 14 & $<110$ & $<50$ & $<1$ & $<1$ & 10 & 30 \\
\hline 2011BAE105A & 35 & 190 & 102 & 2120 & $<50$ & $<1$ & $<1$ & 20 & 88 \\
\hline 2011BAE127A & $<10$ & $<30$ & 36 & $<110$ & $<50$ & $<1$ & $<1$ & $<1$ & $<7$ \\
\hline 2011BAE235A & 69 & 299 & 51 & $<110$ & $<50$ & $<1$ & $<1$ & 10 & 54 \\
\hline 2011BAE264A & $<10$ & 40 & 32 & $<110$ & $<50$ & $<1$ & $<1$ & 35 & 17 \\
\hline 2011BAE264B & 33 & $<30$ & 34 & 365 & $<50$ & $<1$ & $<1$ & 34 & $<7$ \\
\hline 2011BAE287B & $<10$ & $<30$ & 30 & $<110$ & $<50$ & $<1$ & $<1$ & 34 & $<7$ \\
\hline 2011BAE292A & 102 & $<30$ & 40 & $<110$ & $<50$ & $<1$ & $<1$ & 14 & 13 \\
\hline 2011BAE292A & 29 & 41 & $<7$ & 320 & $<50$ & $<1$ & $<1$ & 14 & 31 \\
\hline 2011BAE297C & 72 & 790 & 45 & 1700 & $<50$ & $<1$ & $<1$ & 3 & 225 \\
\hline 2011BAE297C & 88 & 850 & 46 & $<110$ & $<50$ & $<1$ & $<1$ & 3 & 208 \\
\hline 2011BAE298C & 69 & 491 & 37 & $<110$ & $<50$ & $<1$ & $<1$ & 8 & 97 \\
\hline 2011BAE305A & 38 & 389 & 57 & 1970 & $<50$ & $<1$ & $<1$ & 10 & 145 \\
\hline
\end{tabular}


Table 8. (continued)

\begin{tabular}{|c|c|c|c|c|c|c|c|c|c|}
\hline Sample_ID & Pb_ppm & $\mathrm{Rb} \_p p m$ & Rb_ppm_2 & S_ppm & Sb_ppm & Sn_ppm & Sr_ppm & Sr_ppm_2 & Th_ppm \\
\hline 2011BAE010A & $2<4$ & 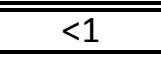 & 15 & 130 & 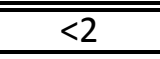 & $2<4$ & 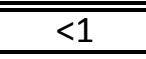 & 291 & $2<2$ \\
\hline 2011BAE013A & $<4$ & $<1$ & 1 & 30 & $<2$ & $<4$ & $<1$ & 249 & $<2$ \\
\hline 2011BAE013B & $<4$ & $<1$ & 14 & 99 & $<2$ & $<4$ & $<1$ & 348 & $<2$ \\
\hline 2011BAE014A & 9 & $<1$ & 14 & 59 & $<2$ & $<4$ & $<1$ & 295 & $<2$ \\
\hline 2011BAE027A & 17 & 6 & --- & 467 & $<2$ & $<4$ & 55 & --- & $<2$ \\
\hline 2011BAE028A & 10 & $<1$ & 15 & 33 & $<2$ & $<4$ & $<1$ & 286 & $<2$ \\
\hline 2011BAE036A & 20 & $<1$ & 1 & 74 & $<2$ & $<4$ & $<1$ & 195 & $<2$ \\
\hline 2011BAE037A & 18 & $<1$ & $<1$ & 289 & $<2$ & $<4$ & $<1$ & 159 & $<2$ \\
\hline 2011BAE039A & 25 & $<1$ & 1 & 98 & $<2$ & $<4$ & $<1$ & 98 & $<2$ \\
\hline 2011BAE041A & 29 & $<1$ & 1 & 118 & $<2$ & $<4$ & $<1$ & 193 & $<2$ \\
\hline 2011BAE045A & 29 & $<1$ & 1 & 91 & $<2$ & $<4$ & $<1$ & 150 & $<2$ \\
\hline 2011BAE048A & $<4$ & $<1$ & 5 & 171 & $<2$ & $<4$ & $<1$ & 241 & $<2$ \\
\hline 2011BAE050A & $<4$ & $<1$ & 4 & 108 & $<2$ & $<4$ & $<1$ & 9 & $<2$ \\
\hline 2011BAE051B & $<4$ & $<1$ & 3 & $<30$ & $<2$ & $<4$ & $<1$ & 158 & $<2$ \\
\hline 2011BAE053A & 28 & $<1$ & $<1$ & 51 & $<2$ & $<4$ & $<1$ & 299 & $<2$ \\
\hline 2011BAE054A & $<4$ & $<1$ & $<1$ & 71 & $<2$ & $<4$ & $<1$ & 221 & $<2$ \\
\hline 2011BAE056A & $<4$ & $<1$ & 3 & 87 & $<2$ & $<4$ & $<1$ & 293 & $<2$ \\
\hline 2011BAE065A & $<4$ & $<1$ & 2 & 64 & $<2$ & $<4$ & $<1$ & 88 & $<2$ \\
\hline 2011BAE085A & $<4$ & $<1$ & 8 & 218 & $<2$ & $<4$ & $<1$ & 151 & $<2$ \\
\hline 2011BAE085B & $<4$ & $<1$ & 6 & 98 & $<2$ & $<4$ & $<1$ & 263 & $<2$ \\
\hline 2011BAE105A & $<4$ & $<1$ & 470 & 150 & $<2$ & $<4$ & $<1$ & 260 & $<2$ \\
\hline 2011BAE127A & 29 & 17 & 1554 & 162 & $<2$ & $<4$ & 1690 & 6 & $<2$ \\
\hline 2011BAE235A & 31 & $<1$ & 11 & 121 & $<2$ & 35 & $<1$ & 216 & $<2$ \\
\hline 2011BAE264A & 63 & $<1$ & 284 & 140 & $<2$ & 6 & $<1$ & 66 & 33 \\
\hline 2011BAE264B & 57 & $<1$ & 274 & 77 & $<2$ & $<4$ & $<1$ & 37 & $<2$ \\
\hline 2011BAE287B & 48 & $<1$ & 509 & 275 & $<2$ & 15.7 & $<1$ & 20 & 8.2 \\
\hline 2011BAE292A & 19 & $<1$ & 106 & 125 & $<2$ & $<4$ & $<1$ & 470 & $<2$ \\
\hline 2011BAE292A & 36 & $<1$ & 106 & 123 & $<2$ & $<4$ & $<1$ & 497 & $<2$ \\
\hline 2011BAE297C & 52 & $<1$ & 125 & 100 & $<2$ & $<4$ & $<1$ & 253 & $<2$ \\
\hline 2011BAE297C & 32 & $<1$ & 106 & 187 & $<2$ & $<4$ & $<1$ & 263 & $<2$ \\
\hline 2011BAE298C & $<4$ & $<1$ & 40 & 1930 & $<2$ & $<4$ & $<1$ & 279 & $<2$ \\
\hline 2011BAE305A & 44 & $<1$ & 9 & 175 & $<2$ & $<4$ & $<1$ & 103 & $<2$ \\
\hline
\end{tabular}


Table 8. (continued)

\begin{tabular}{|c|c|c|c|c|c|c|c|c|}
\hline Sample_ID & U_ppm & V_ppm & W_ppm & Y_ppm & Y_ppm_2 & Zn_ppm & Zr_ppm & Zr_ppm_2 \\
\hline 2011BAE010A & $\overline{c 1}$ & 465 & $<<8$ & $\overline{<1}$ & 27 & 80 & $\overline{<9}$ & 129 \\
\hline 2011BAE013A & $<1$ & 353 & $<8$ & $<1$ & 26 & 76 & $<9$ & 150 \\
\hline 2011BAE013B & $<1$ & 294 & $<8$ & $<1$ & 21 & 59 & $<9$ & 103 \\
\hline 2011BAE014A & $<1$ & 367 & $<8$ & $<1$ & 23 & 71 & $<9$ & 116 \\
\hline 2011BAE027A & $<1$ & 309 & $<8$ & 20 & --- & 50 & 200 & --- \\
\hline 2011BAE028A & $<1$ & 321 & $<8$ & $<1$ & 22 & 79 & $<9$ & 96 \\
\hline 2011BAE036A & $<1$ & 478 & $<8$ & $<1$ & 38 & 110 & $<9$ & 152 \\
\hline 2011BAE037A & $<1$ & 327 & $<8$ & $<1$ & 41 & 258 & $<9$ & 96 \\
\hline 2011BAE039A & $<1$ & 561 & $<8$ & $<1$ & 35 & 293 & $<9$ & 151 \\
\hline 2011BAE041A & $<1$ & 257 & $<8$ & $<1$ & 23 & 114 & $<9$ & 34 \\
\hline 2011BAE045A & $<1$ & 229 & $<8$ & $<1$ & 14 & 54 & $<9$ & 26 \\
\hline 2011BAE048A & $<1$ & 297 & $<8$ & $<1$ & 32 & 113 & $<9$ & 55 \\
\hline 2011BAE050A & $<1$ & 474 & $<8$ & $<1$ & 27 & 71 & $<9$ & 102 \\
\hline 2011BAE051B & $<1$ & 394 & $<8$ & $<1$ & 25 & 88 & $<9$ & 83 \\
\hline 2011BAE053A & $<1$ & 496 & $<8$ & $<1$ & 28 & 139 & $<9$ & 131 \\
\hline 2011BAE054A & $<1$ & 339 & $<8$ & $<1$ & 28 & 105 & $<9$ & 140 \\
\hline 2011BAE056A & $<1$ & 517 & $<8$ & $<1$ & 36 & 123 & $<9$ & 219 \\
\hline 2011BAE065A & $<1$ & 154 & $<8$ & $<1$ & 11 & 76 & $<9$ & 29 \\
\hline 2011BAE085A & $<1$ & 222 & $<8$ & $<1$ & 36 & 64 & $<9$ & 650 \\
\hline 2011BAE085B & $<1$ & 496 & $<8$ & $<1$ & 27 & 89 & $<9$ & 140 \\
\hline 2011BAE105A & $<1$ & 405 & $<8$ & $<1$ & 95 & 188 & $<9$ & 270 \\
\hline 2011BAE127A & $<1$ & $<25$ & $<8$ & 29 & 3 & 24 & 45 & $<9$ \\
\hline 2011BAE235A & $<1$ & 450 & $<8$ & $<1$ & 26 & 105 & $<9$ & 143 \\
\hline 2011BAE264A & 6 & $<25$ & $<8$ & $<1$ & 59 & 97 & $<9$ & 81 \\
\hline 2011BAE264B & $<1$ & $<25$ & $<8$ & $<1$ & 95 & 141 & $<9$ & 72 \\
\hline 2011BAE287B & 25.8 & $<8$ & 32 & $<1$ & 24 & 14 & $<9$ & 26 \\
\hline 2011BAE292A & $<1$ & 245 & $<8$ & $<1$ & 31 & 76 & $<9$ & 194 \\
\hline 2011BAE292A & $<1$ & 240 & $<8$ & $<1$ & 36 & 78 & $<9$ & 202 \\
\hline 2011BAE297C & $<1$ & 222 & $<8$ & $<1$ & 15 & 122 & $<9$ & 34 \\
\hline 2011BAE297C & $<1$ & 254 & $<8$ & $<1$ & 12 & 95 & $<9$ & 35 \\
\hline 2011BAE298C & $<1$ & 366 & $<8$ & $<1$ & 27 & 150 & $<9$ & 81 \\
\hline 2011BAE305A & $<1$ & 440 & $<8$ & $<1$ & 27 & 196 & $<9$ & 141 \\
\hline
\end{tabular}


Table 8. (continued)

\begin{tabular}{|c|c|c|c|c|c|c|c|}
\hline Sample_ID & Latitude & Longitude & Easting & Northing & $\begin{array}{l}\text { UTM_ } \\
\text { Zone }\end{array}$ & Root_name & Comments \\
\hline 2011BAE306A & 65.183106 & $\mid-153.797316$ & 462658 & 7228905 & $5 \mathrm{~N}$ & Albite-calcite-chlorite schist & Porous \\
\hline 2011BAE307A & 65.180993 & -153.794733 & 462776 & 7228668 & $5 \mathrm{~N}$ & Garnet-plagioclase-chlorite schist & Inhomogenous \\
\hline 2011BAE308A & 65.178605 & -153.793317 & 462839 & 7228401 & $5 \mathrm{~N}$ & Calcite-chlorite-muscovite schist & Inhomogenous \\
\hline 2011BAE309A & 65.176060 & -153.790892 & 462949 & 7228116 & $5 \mathrm{~N}$ & Chlorite-muscovite quartz schist & Inhomogenous \\
\hline 2011BAE329B & 65.331525 & -153.086573 & 495968 & 7245214 & $5 \mathrm{~N}$ & Metamafic & Porous \\
\hline 2011BAE330A & 65.331175 & -153.087151 & 495941 & 7245175 & $5 \mathrm{~N}$ & Metagabbro & --- \\
\hline 2011BAE331C & 65.330474 & -153.088931 & 495858 & 7245097 & $5 \mathrm{~N}$ & Metagabbro & --- \\
\hline 2011BAE331D & 65.330474 & -153.088931 & 495858 & 7245097 & $5 \mathrm{~N}$ & Metagabbro & -- \\
\hline 2011BAE332B & 65.328973 & -153.092619 & 495686 & 7244930 & $5 \mathrm{~N}$ & Metadiorite(?) & --- \\
\hline 2011BAE333A & 65.327913 & -153.094783 & 495585 & 7244812 & $5 \mathrm{~N}$ & Metagabbro & --- \\
\hline 2011BAE334A & 65.327320 & -153.096606 & 495500 & 7244746 & $5 \mathrm{~N}$ & Metagabbro & Porous \\
\hline 2011BAE336A & 65.325370 & -153.100720 & 495308 & 7244529 & $5 \mathrm{~N}$ & Metagabbro & --- \\
\hline 2011BAE337A & 65.325190 & -153.102222 & 495238 & 7244509 & $5 \mathrm{~N}$ & Metagabbro & --- \\
\hline 2011BAE351C & 65.462650 & -152.805055 & 509034 & 7259840 & $5 \mathrm{~N}$ & Metagabbro & Poor polish \\
\hline 2011BAE355B & 65.462752 & -152.794351 & 509530 & 7259853 & $5 \mathrm{~N}$ & Metamafic & Poor polish \\
\hline 2011BAE359B & 65.462807 & -152.782460 & 510081 & 7259861 & $5 \mathrm{~N}$ & Granite porphyry & --- \\
\hline 2011BAE359C & 65.462807 & -152.782460 & 510081 & 7259861 & $5 \mathrm{~N}$ & Granite porphyry & Altered \\
\hline 2011BAE366B & 65.468469 & -152.764693 & 510902 & 7260495 & $5 \mathrm{~N}$ & Porphyritic granite & --- \\
\hline 2011BAE371B & 65.472522 & -152.751639 & 511505 & 7260949 & $5 \mathrm{~N}$ & Porphyritic granodiorite & --- \\
\hline 2011BAE375B & 65.477623 & -152.738528 & 512110 & 7261520 & $5 \mathrm{~N}$ & Porphyritic granite & Inhomogenous \\
\hline 2011BAE376A & 65.408933 & -152.554657 & 520680 & 7253912 & $5 \mathrm{~N}$ & Gabbro & Altered \\
\hline 2011BAE379A & 65.405898 & -152.547775 & 521002 & 7253576 & $5 \mathrm{~N}$ & Gabbro & Altered, poor polish \\
\hline 2011BAE381A & 65.402899 & -152.547052 & 521038 & 7253242 & $5 \mathrm{~N}$ & Wherlite & Altered \\
\hline 2011BAE381B & 65.402899 & -152.547052 & 521038 & 7253242 & $5 \mathrm{~N}$ & Gabbro & Altered \\
\hline 2011BAE384A & 65.397391 & \begin{tabular}{|l|}
-152.541184 \\
\end{tabular} & 521315 & 7252630 & $5 \mathrm{~N}$ & Olivine clinopyroxenite & Inhomogenous \\
\hline 2011BAE385A & 65.396895 & -152.540396 & 521352 & 7252575 & $5 \mathrm{~N}$ & Gabbro & Altered \\
\hline 2011BAE387A & 65.393392 & -152.539166 & 521412 & 7252185 & $5 \mathrm{~N}$ & Gabbro & Altered, poor polish \\
\hline 2011BAE416B & 65.371679 & -152.840696 & 507408 & 7249696 & $5 \mathrm{~N}$ & Gabbro & Altered \\
\hline 2011BAE418A & 65.373642 & -152.830511 & 507881 & 7249916 & $5 \mathrm{~N}$ & Mafic schist & Inhomogenous, porous \\
\hline 2011BAE420A & 65.374886 & -152.820180 & 508361 & 7250056 & $5 \mathrm{~N}$ & Quartzite & Inhomogenous, porous \\
\hline 2011BAE422A & 65.414876 & -152.821888 & 508269 & 7254513 & $5 \mathrm{~N}$ & Metagabbro(?) & Inhomogenous \\
\hline 2011BAE422B & 65.414876 & -152.821888 & 508269 & 7254513 & $5 \mathrm{~N}$ & Mafic schist & Inhomogenous, porous \\
\hline
\end{tabular}


Table 8. (continued)

\begin{tabular}{|c|c|c|c|c|c|c|c|c|c|}
\hline Sample_ID & $\mathrm{SiO}_{2 \_} \mathrm{pct}$ & Al203_pct & BaO_pct & CaO_pct & FeO_pct & K2O_pct & MgO_pct & MnO_pct & $\mathrm{Na} 2 \mathrm{O} \_p c t$ \\
\hline 2011BAE306A & 64.5 & 11.9 & $<<0.03$ & 2.6 & 11.5 & 0.0656 & 5.79 & 0.21 & 1.86 \\
\hline 2011BAE307A & 61.3 & 14.8 & 0.0461 & 5.84 & 10.2 & 0.315 & 4.24 & 0.287 & 1.72 \\
\hline 2011BAE308A & 58.4 & 21.1 & 0.325 & 3.34 & 6.47 & 4.92 & 2.66 & 0.0939 & 1.23 \\
\hline 2011BAE309A & 78.4 & 11.7 & 0.098 & 0.74 & 3.86 & 2.13 & 1.41 & 0.0484 & 0.875 \\
\hline 2011BAE329B & 54.8 & 15 & 0.03 & 6.29 & 11.4 & 0.18 & 4.65 & 0.17 & 5.48 \\
\hline 2011BAE330A & 53.3 & 15.9 & 0.0386 & 9.05 & 10.1 & 0.424 & 4.62 & 0.166 & 4.74 \\
\hline 2011BAE331C & 51.6 & 16.5 & 0.14 & 5.73 & 13.6 & 1.11 & 4.65 & 0.23 & 4.27 \\
\hline 2011BAE331D & 54.7 & 15.6 & 0.0626 & 9.03 & 9.63 & 0.477 & 4.95 & 0.158 & 4.2 \\
\hline 2011BAE332B & 59.6 & 11.2 & $<0.03$ & 6.63 & 13.5 & 0.02 & 4.13 & 0.16 & 2.67 \\
\hline 2011BAE333A & 50.6 & 16.4 & 0.03 & 11.4 & 9.37 & 0.03 & 8.58 & 0.2 & 2.5 \\
\hline 2011BAE334A & 58.8 & 13.8 & $<0.03$ & 3.72 & 14.1 & 0.09 & 3.73 & 0.25 & 2.98 \\
\hline 2011BAE336A & 51.5 & 15.4 & 0.06 & 10.1 & 11.5 & 0.26 & 4.35 & 0.2 & 4.18 \\
\hline 2011BAE337A & 52.7 & 17.2 & 0.06 & 10.3 & 8.18 & 0.35 & 6.36 & 0.15 & 3.68 \\
\hline 2011BAE351C & 53.3 & 16.8 & 0.05 & 9.29 & 9.94 & 0.53 & 4.38 & 0.19 & 3.3 \\
\hline 2011BAE355B & 54.6 & 16.7 & 0.04 & 9.53 & 10.6 & 0.09 & 5.15 & 0.21 & 0.818 \\
\hline 2011BAE359B & 76.6 & 13.2 & $<0.03$ & 0.673 & 0.634 & 4.38 & 0.184 & 0.072 & 4.01 \\
\hline 2011BAE359C & 80 & 14.4 & $<0.03$ & 0.0673 & 0.699 & 4.21 & 0.145 & 0.151 & 0.0893 \\
\hline 2011BAE366B & 76.6 & 13.2 & $<0.03$ & 0.424 & 0.96 & 4.32 & 0.173 & 0.0386 & 4.05 \\
\hline 2011BAE371B & 63.3 & 14 & $<0.03$ & 2.66 & 7.85 & 3.14 & 2.65 & 0.2 & 3.73 \\
\hline 2011BAE375B & 77.8 & 12.5 & $<0.03$ & 0.159 & 0.835 & 4.2 & 0.155 & $<0.03$ & 4.18 \\
\hline 2011BAE376A & 49.8 & 15.3 & 0.05 & 12.4 & 10.4 & 0.17 & 6.56 & 0.19 & 3.24 \\
\hline 2011BAE379A & 50.3 & 15.6 & 0.12 & 7.39 & 11.8 & 0.88 & 6.85 & 0.49 & 4.58 \\
\hline 2011BAE381A & 46.6 & 5.1 & $<0.03$ & 8.5 & 13 & 0.0288 & 25.7 & 0.18 & 0.144 \\
\hline 2011BAE381B & 49.2 & 9.6 & 0.1 & 14.1 & 10.5 & 0.73 & 12.5 & 0.23 & 1.58 \\
\hline 2011BAE384A & 49.3 & 5.7 & 0.03 & 10 & 11.1 & 0.11 & 21.9 & 0.2 & 0.425 \\
\hline 2011BAE385A & 50.5 & 16.7 & 0.0362 & 12.4 & 7.25 & 0.692 & 7.3 & 0.152 & 3.81 \\
\hline 2011BAE387A & 52.2 & 16.4 & 0.03 & 7.31 & 11.1 & 0.11 & 5.15 & 0.18 & 5.87 \\
\hline 2011BAE416B & 53.9 & 15.4 & $<0.03$ & 3.8 & 16 & 0.0354 & 6.32 & 0.251 & 1.78 \\
\hline 2011BAE418A & 48.6 & 17 & $<0.03$ & 11.2 & 10.8 & 0.0541 & 10.4 & 0.219 & 1.11 \\
\hline 2011BAE420A & 81.9 & 8.3 & 0.0478 & 0.148 & 5.1 & 1.55 & 1.4 & $<0.03$ & 0.356 \\
\hline 2011BAE422A & 52.3 & 14 & $<0.03$ & 11.9 & 11.2 & 0.04 & 7.6 & 0.31 & 1.48 \\
\hline 2011BAE422B & 55.9 & 14.4 & $<0.03$ & 5.73 & 11.4 & 0.23 & 7.1 & 0.23 & 3.46 \\
\hline
\end{tabular}


Table 8. (continued)

\begin{tabular}{|c|c|c|c|c|c|c|c|c|c|}
\hline Sample_ID & P2O5_pct & TiO2_pct & CO2_pct & Total_pct & As_ppm & Bi_ppm & Br_ppm & Ce_ppm & Cl_ppm \\
\hline 2011BAE306A & 0.375 & 1.09 & $<0.05$ & 99.8906 & 68 & $<2$ & $<<$ & $<60$ & 136 \\
\hline 2011BAE307A & 0.161 & 0.533 & $<0.05$ & 99.4421 & 12 & $<2$ & $<5$ & $<60$ & 137 \\
\hline 2011BAE308A & 0.0301 & 1.15 & $<0.05$ & 99.719 & 59 & $<2$ & $<5$ & $<60$ & 199 \\
\hline 2011BAE309A & 0.18 & 0.454 & $<0.05$ & 99.8954 & $<4$ & $<2$ & $<5$ & $<60$ & 129 \\
\hline 2011BAE329B & 0.3 & 1.36 & $<0.05$ & 99.6588 & $<4$ & $<2$ & $<5$ & $<60$ & 259 \\
\hline 2011BAE330A & 0.25 & 1.31 & $<0.05$ & 99.8986 & 10 & $<2$ & $<5$ & $<60$ & 104 \\
\hline 2011BAE331C & 0.3 & 1.64 & $<0.05$ & 99.77 & $<4$ & $<2$ & $<5$ & $<60$ & 130 \\
\hline 2011BAE331D & 0.303 & 0.847 & $<0.05$ & 99.9576 & $<4$ & $<2$ & $<5$ & $<60$ & 212 \\
\hline 2011BAE332B & 0.23 & 1.68 & $<0.05$ & 99.8476 & $<4$ & $<2$ & $<5$ & $<60$ & $<50$ \\
\hline 2011BAE333A & 0.06 & 0.69 & $<0.05$ & 99.8685 & 11 & $<2$ & $<5$ & $<60$ & 106 \\
\hline 2011BAE334A & 0.44 & 1.77 & $<0.05$ & 99.7041 & 5 & $<2$ & $<5$ & $<60$ & 575 \\
\hline 2011BAE336A & 0.33 & 1.91 & $<0.05$ & 99.7927 & $<4$ & $<2$ & $<5$ & $<60$ & 92 \\
\hline 2011BAE337A & 0.12 & 0.717 & $<0.05$ & 99.817 & 8 & $<2$ & $<5$ & $<60$ & 146 \\
\hline 2011BAE351C & 0.31 & 1.7 & $<0.05$ & 99.7946 & 13 & $<2$ & $<5$ & $<60$ & 440 \\
\hline 2011BAE355B & 0.26 & 1.61 & $<0.05$ & 99.6034 & 29 & $<2$ & $<5$ & $<60$ & 296 \\
\hline 2011BAE359B & $<0.03$ & 0.0234 & $<0.05$ & 99.8225 & 4 & $<2$ & $<5$ & $<60$ & 207 \\
\hline 2011BAE359C & $<0.03$ & $<0.02$ & $<0.05$ & 99.8312 & $<4$ & $<2$ & $<5$ & $<60$ & 63 \\
\hline 2011BAE366B & $<0.03$ & $<0.02$ & $<0.05$ & 99.8173 & 4 & $<2$ & $<5$ & $<60$ & 80 \\
\hline 2011BAE371B & 0.321 & 0.881 & $<0.05$ & 98.7613 & $<4$ & $<2$ & $<5$ & $<60$ & 550 \\
\hline 2011BAE375B & $<0.03$ & $<0.02$ & $<0.05$ & 99.8847 & $<4$ & $<2$ & $<5$ & $<60$ & 177 \\
\hline 2011BAE376A & 0.19 & 1.43 & $<0.05$ & 99.7222 & $<4$ & $<2$ & $<5$ & $<60$ & 131 \\
\hline 2011BAE379A & 0.24 & 1.56 & $<0.05$ & 99.806 & 6 & $<2$ & $<5$ & $<60$ & 155 \\
\hline 2011BAE381A & 0.0305 & 0.3 & $<0.05$ & 99.598 & $<4$ & $<2$ & $<5$ & $<60$ & 197 \\
\hline 2011BAE381B & 0.13 & 1.05 & $<0.05$ & 99.7126 & $<4$ & $<2$ & $<5$ & $<60$ & 242 \\
\hline 2011BAE384A & 0.08 & 0.641 & $<0.05$ & 99.4513 & $<4$ & $<2$ & $<5$ & $<60$ & 293 \\
\hline 2011BAE385A & 0.145 & 0.827 & $<0.05$ & 99.8122 & 43 & $<2$ & $<5$ & $<60$ & 214 \\
\hline 2011BAE387A & 0.27 & 1.2 & $<0.05$ & 99.8219 & 10 & $<2$ & $<5$ & $<60$ & 164 \\
\hline 2011BAE416B & 0.265 & 2.1 & $<0.05$ & 99.8387 & $<4$ & $<2$ & $<5$ & $<60$ & 237 \\
\hline 2011BAE418A & 0.0773 & 0.344 & $<0.05$ & 99.8249 & $<4$ & $<2$ & $<5$ & $<60$ & 266 \\
\hline 2011BAE420A & 0.108 & 0.99 & $<0.05$ & 99.8874 & 4 & $<2$ & $<5$ & $<60$ & 299 \\
\hline 2011BAE422A & 0.18 & 0.65 & $<0.05$ & 99.6819 & 12 & $<2$ & $<5$ & $<60$ & 616 \\
\hline 2011BAE422B & 0.26 & 0.98 & $<0.05$ & 99.7037 & 27 & $<2$ & $<5$ & $<60$ & 149 \\
\hline
\end{tabular}


Table 8. (continued)

\begin{tabular}{|c|c|c|c|c|c|c|c|c|c|}
\hline Sample_ID & Co_ppm & Cr_ppm & Cu_ppm & F_ppm & La_ppm & Mo_ppm & Nb_ppm & Nb_ppm_2 & Ni_ppm \\
\hline 2011BAE306A & 37 & 157 & 32 & $<<110$ & $<<50$ & $2<1$ & $<<1$ & 11 & 65 \\
\hline 2011BAE307A & $<10$ & 96 & 32 & 3890 & $<50$ & $<1$ & $<1$ & 7 & 100 \\
\hline 2011BAE308A & 43 & 284 & 86 & $<110$ & $<50$ & $<1$ & $<1$ & 17 & 93 \\
\hline 2011BAE309A & 18 & 108 & 32 & $<110$ & $<50$ & $<1$ & $<1$ & 8 & 28 \\
\hline 2011BAE329B & 63 & 77 & 23 & $<110$ & $<50$ & $<1$ & $<1$ & 10 & 60 \\
\hline 2011BAE330A & 49 & 63 & 103 & $<110$ & $<50$ & $<1$ & $<1$ & 10 & 49 \\
\hline 2011BAE331C & 46 & 40 & 7 & $<110$ & $<50$ & $<1$ & $<1$ & 13 & $<7$ \\
\hline 2011BAE331D & 12 & 116 & 18 & $<110$ & $<50$ & $<1$ & $<1$ & 6 & 37 \\
\hline 2011BAE332B & 13 & 77 & 105 & $<110$ & $<50$ & $<1$ & $<1$ & 16 & 105 \\
\hline 2011BAE333A & 99 & 347 & 72 & $<110$ & $<50$ & $<1$ & $<1$ & 1 & 140 \\
\hline 2011BAE334A & 123 & 125 & 123 & $<110$ & $<50$ & $<1$ & $<1$ & 13 & 15 \\
\hline 2011BAE336A & 45 & 250 & 66 & $<110$ & $<50$ & $<1$ & $<1$ & 12 & 100 \\
\hline 2011BAE337A & 71 & 126 & 61 & $<110$ & $<50$ & $<1$ & $<1$ & 5 & 82 \\
\hline 2011BAE351C & 61 & 76 & 38 & $<110$ & $<50$ & $<1$ & $<1$ & 8 & 54 \\
\hline 2011BAE355B & 75 & 156 & 11 & 2180 & $<50$ & $<1$ & $<1$ & 9 & 69 \\
\hline 2011BAE359B & $<10$ & $<30$ & 26 & $<110$ & $<50$ & $<1$ & $<1$ & 53 & 27 \\
\hline 2011BAE359C & $<10$ & $<30$ & $<7$ & 116 & $<50$ & $<1$ & $<1$ & 48 & $<7$ \\
\hline 2011BAE366B & $<10$ & $<30$ & 15 & $<110$ & $<50$ & $<1$ & $<1$ & 43 & 12 \\
\hline 2011BAE371B & 36 & 65 & 12 & 9900 & $<50$ & $<1$ & $<1$ & 55 & $<7$ \\
\hline 2011BAE375B & $<10$ & $<30$ & 14 & $<110$ & $<50$ & $<1$ & $<1$ & 35 & 27 \\
\hline 2011BAE376A & 104 & 372 & 71 & $<110$ & $<50$ & $<1$ & $<1$ & 5 & 168 \\
\hline 2011BAE379A & 131 & 319 & 107 & $<110$ & $<50$ & $<1$ & $<1$ & 9 & 98 \\
\hline 2011BAE381A & 197 & 1770 & 445 & $<110$ & $<50$ & $<1$ & $<1$ & 1 & 909 \\
\hline 2011BAE381B & 110 & 2360 & 129 & $<110$ & $<50$ & $<1$ & $<1$ & 4 & 327 \\
\hline 2011BAE384A & 121 & 1980 & 160 & $<110$ & $<50$ & $<1$ & $<1$ & 3 & 831 \\
\hline 2011BAE385A & 35 & 273 & 97 & $<110$ & $<50$ & $<1$ & $<1$ & 4 & 124 \\
\hline 2011BAE387A & 54 & 52 & $<7$ & 131 & $<50$ & $<1$ & $<1$ & 8 & 46 \\
\hline 2011BAE416B & 24 & $<30$ & 23 & $<110$ & $<50$ & $<1$ & $<1$ & 14 & 113 \\
\hline 2011BAE418A & 72 & 434 & 42 & $<110$ & $<50$ & $<1$ & $<1$ & 2 & 273 \\
\hline 2011BAE420A & $<10$ & 118 & 27 & $<110$ & $<50$ & $<1$ & $<1$ & 11 & 17 \\
\hline 2011BAE422A & 64 & 241 & 187 & $<110$ & $<50$ & $<1$ & $<1$ & 1 & 181 \\
\hline 2011BAE422B & 90 & 167 & 89 & 1080 & $<50$ & $<1$ & $<1$ & 2 & 135 \\
\hline
\end{tabular}


Table 8. (continued)

\begin{tabular}{|c|c|c|c|c|c|c|c|c|c|}
\hline Sample_ID & Pb_ppm & Rb_ppm & Rb_ppm_2 & S_ppm & Sb_ppm & Sn_ppm & Sr_ppm & Sr_ppm_2 & Th_ppm \\
\hline 2011BAE306A & 43 & $2<1$ & 3 & 194 & $<2$ & $<4$ & $<<1$ & 111 & $2<2$ \\
\hline 2011BAE307A & 42 & $<1$ & 15 & 153 & $<2$ & $<4$ & $<1$ & 266 & $<2$ \\
\hline 2011BAE308A & 64 & $<1$ & 165 & 272 & $<2$ & $<4$ & $<1$ & 234 & $<2$ \\
\hline 2011BAE309A & 21 & $<1$ & 89 & 138 & $<2$ & $<4$ & $<1$ & 44 & $<2$ \\
\hline 2011BAE329B & $<4$ & $<1$ & 10 & 1300 & $<2$ & 21 & $<1$ & 285 & $<2$ \\
\hline 2011BAE330A & $<4$ & $<1$ & 15 & 217 & $<2$ & $<4$ & $<1$ & 274 & $<2$ \\
\hline 2011BAE331C & 21 & $<1$ & 36 & 117 & $<2$ & $<4$ & $<1$ & 312 & $<2$ \\
\hline 2011BAE331D & 25 & $<1$ & 16 & 123 & $<2$ & $<4$ & $<1$ & 273 & $<2$ \\
\hline 2011BAE332B & 29 & $<1$ & $<1$ & 225 & $<2$ & $<4$ & $<1$ & 233 & $<2$ \\
\hline 2011BAE333A & $<4$ & $<1$ & 1 & 94 & $<2$ & $<4$ & $<1$ & 132 & $<2$ \\
\hline 2011BAE334A & 53 & $<1$ & 4 & 321 & $<2$ & 23 & $<1$ & 266 & $<2$ \\
\hline 2011BAE336A & $<4$ & $<1$ & 8 & 84 & $<2$ & 21 & $<1$ & 302 & $<2$ \\
\hline 2011BAE337A & 21 & $<1$ & 11 & 46 & $<2$ & $<4$ & $<1$ & 295 & $<2$ \\
\hline 2011BAE351C & 18 & $<1$ & 84 & $<30$ & $<2$ & $<4$ & $<1$ & 298 & $<2$ \\
\hline 2011BAE355B & $<4$ & $<1$ & 9 & 136 & $<2$ & $<4$ & $<1$ & 210 & $<2$ \\
\hline 2011BAE359B & 23 & $<1$ & 559 & 90 & $<2$ & 42 & $<1$ & 62 & 20 \\
\hline 2011BAE359C & 38 & $<1$ & 833.6 & 56 & $<2$ & 101.5 & $<1$ & 22 & 18.8 \\
\hline 2011BAE366B & 13 & $<1$ & 582 & 47 & $<2$ & 108 & $<1$ & 22 & 21 \\
\hline 2011BAE371B & 10.5 & $<1$ & 484 & 78 & $<2$ & 22.6 & $<1$ & 43 & 24.4 \\
\hline 2011BAE375B & 23 & $<1$ & 495 & 97 & $<2$ & 26 & $<1$ & 16 & 16 \\
\hline 2011BAE376A & $<4$ & $<1$ & 8 & 63 & $<2$ & 25 & $<1$ & 69 & $<2$ \\
\hline 2011BAE379A & 21 & $<1$ & 17 & 72 & $<2$ & $<4$ & $<1$ & 105 & $<2$ \\
\hline 2011BAE381A & $<4$ & $<1$ & 3 & 257 & $<2$ & 21 & $<1$ & 41 & $<2$ \\
\hline 2011BAE381B & 25 & $<1$ & 25 & 102 & $<2$ & 25 & $<1$ & 56 & $<2$ \\
\hline 2011BAE384A & $<4$ & $<1$ & 5 & 800 & $<2$ & $<4$ & $<1$ & 76 & 2 \\
\hline 2011BAE385A & 20 & $<1$ & 19 & 75 & $<2$ & $<4$ & $<1$ & 72 & $<2$ \\
\hline 2011BAE387A & $<4$ & $<1$ & 3 & 58 & $<2$ & $<4$ & $<1$ & 169 & $<2$ \\
\hline 2011BAE416B & $<4$ & $<1$ & 1 & 166 & $<2$ & 23 & $<1$ & 182 & $<2$ \\
\hline 2011BAE418A & 53 & $<1$ & 2 & 87 & $<2$ & $<4$ & $<1$ & 115 & $<2$ \\
\hline 2011BAE420A & 24 & $<1$ & 65 & 171 & $<2$ & 18 & $<1$ & 17 & $<2$ \\
\hline 2011BAE422A & 21 & $<1$ & 1 & 427 & $<2$ & $<4$ & $<1$ & 138 & $<2$ \\
\hline 2011BAE422B & 39 & $<1$ & 14 & 120 & $<2$ & $<4$ & $<1$ & 58 & $<2$ \\
\hline
\end{tabular}




\begin{tabular}{|c|c|c|c|c|c|c|c|c|}
\hline Sample_ID & U_ppm & V_ppm & W_ppm & Y_ppm & Y_ppm_2 & Zn_ppm & Zr_ppm & Zr_ppm_2 \\
\hline 2011BAE306A & $\overline{c<1}$ & 4661 & $<<8$ & $\angle 1$ & 27 & 132 & $<9$ & 124 \\
\hline 2011BAE307A & $<1$ & 406 & $<8$ & $<1$ & 27 & 122 & $<9$ & 163 \\
\hline 2011BAE308A & $<1$ & 365 & $<8$ & $<1$ & 38 & 162 & $<9$ & 235 \\
\hline 2011BAE309A & $<1$ & 189 & $<8$ & $<1$ & 23 & 111 & $<9$ & 133 \\
\hline 2011BAE329B & $<1$ & 466 & $<8$ & $<1$ & 29 & 122 & $<9$ & 130 \\
\hline 2011BAE330A & $<1$ & 338 & $<8$ & $<1$ & 25 & 80 & $<9$ & 119 \\
\hline 2011BAE331C & $<1$ & 558 & $<8$ & $<1$ & 33 & 141 & $<9$ & 179 \\
\hline 2011BAE331D & $<1$ & 307 & $<8$ & $<1$ & 21 & 77 & $<9$ & 109 \\
\hline 2011BAE332B & $<1$ & 444 & $<8$ & $<1$ & 31 & 148 & $<9$ & 177 \\
\hline 2011BAE333A & $<1$ & 308 & $<8$ & $<1$ & 26 & 54 & $<9$ & 45 \\
\hline 2011BAE334A & $<1$ & 528 & $<8$ & $<1$ & 35 & 167 & $<9$ & 184 \\
\hline 2011BAE336A & $<1$ & 487 & $<8$ & $<1$ & 30 & 86 & $<9$ & 154 \\
\hline 2011BAE337A & $<1$ & 270 & $<8$ & $<1$ & 14 & 80 & $<9$ & 54 \\
\hline 2011BAE351C & $<1$ & 452 & $<8$ & $<1$ & 30 & 118 & $<9$ & 117 \\
\hline 2011BAE355B & $<1$ & 458 & $<8$ & $<1$ & 27 & 127 & $<9$ & 116 \\
\hline 2011BAE359B & 31 & 28 & $<8$ & $<1$ & 156 & 14 & $<9$ & 66 \\
\hline 2011BAE359C & 6 & $<8$ & 56 & $<1$ & 146 & 38 & $<9$ & 69 \\
\hline 2011BAE366B & 27 & $<25$ & $<8$ & $<1$ & 146 & 17 & $<9$ & 67 \\
\hline 2011BAE371B & 5.3 & 186 & $<8$ & $<1$ & 107 & 84 & $<9$ & 176 \\
\hline 2011BAE375B & 12 & $<25$ & $<8$ & $<1$ & 85 & 19 & $<9$ & 59 \\
\hline 2011BAE376A & $<1$ & 340 & $<8$ & $<1$ & 23 & 88 & $<9$ & 85 \\
\hline 2011BAE379A & $<1$ & 449 & $<8$ & $<1$ & 30 & 53 & $<9$ & 116 \\
\hline 2011BAE381A & $<1$ & 115 & $<8$ & $<1$ & 5 & 78 & $<9$ & 18 \\
\hline 2011BAE381B & $<1$ & 454 & $<8$ & $<1$ & 16 & 108 & $<9$ & 62 \\
\hline 2011BAE384A & 2 & 245 & $<8$ & $<1$ & 9 & 79 & $<9$ & 36 \\
\hline 2011BAE385A & $<1$ & 268 & $<8$ & $<1$ & 15 & 64 & $<9$ & 68 \\
\hline 2011BAE387A & $<1$ & 468 & $<8$ & $<1$ & 30 & 73 & $<9$ & 143 \\
\hline 2011BAE416B & $<1$ & 523 & $<8$ & $<1$ & 26 & 199 & $<9$ & 165 \\
\hline 2011BAE418A & $<1$ & 244 & $<8$ & $<1$ & 11 & 62 & $<9$ & 22 \\
\hline 2011BAE420A & $<1$ & 171 & $<8$ & $<1$ & 17 & 52 & $<9$ & 140 \\
\hline 2011BAE422A & $<1$ & 338 & $<8$ & $<1$ & 24 & 132 & $<9$ & 52 \\
\hline 2011BAE422B & $<1$ & 391 & $<8$ & $<1$ & 36 & 135 & $<9$ & 59 \\
\hline
\end{tabular}


Table 8. (continued)

\begin{tabular}{|c|c|c|c|c|c|c|c|}
\hline Sample_ID & Latitude & Longitude & Easting & Northing & $\begin{array}{l}\text { UTM_- } \\
\text { Zone }\end{array}$ & Root_name & Comments \\
\hline 2011BAE465A & 65.197497 & -152.873745 & 505910 & 7230279 & $5 \mathrm{~N}$ & MetaQuartz grit & Porous \\
\hline 2011BAE465B & 65.197497 & -152.873745 & 505910 & 7230279 & $5 \mathrm{~N}$ & MetaQuartz wacke & --- \\
\hline 2011BAE466A & 65.198134 & -152.863936 & 506369 & 7230351 & $5 \mathrm{~N}$ & Metaquartz grit & --- \\
\hline 2011BAE466B & 65.198134 & -152.863936 & 506369 & 7230351 & $5 \mathrm{~N}$ & Chloritoid(?) quartzite & --- \\
\hline 2011BAE472A & 65.392679 & -153.402733 & 481287 & 7252087 & $5 \mathrm{~N}$ & Metamafic & Poor polish \\
\hline 2011BAE473A & 65.394414 & -153.408183 & 481035 & 7252282 & $5 \mathrm{~N}$ & Diabase(?) & Altered \\
\hline 2011BAE476A & 65.396951 & -153.422064 & 480392 & 7252569 & $5 \mathrm{~N}$ & Fine-grained gabbro & --- \\
\hline 2011BAE522A & 65.355243 & -152.749007 & 511679 & 7247878 & $5 \mathrm{~N}$ & Gabbro(?) & Altered \\
\hline 2011BAE565A & 65.314624 & -152.518849 & 522423 & 7243413 & $5 \mathrm{~N}$ & Metagabbro & --- \\
\hline 2011BAE566A & 65.316238 & -152.518755 & 522426 & 7243593 & $5 \mathrm{~N}$ & Fine-grained Metagabbro & --- \\
\hline 2011BAE604A & 65.244152 & -153.484683 & 477352 & 7235560 & $5 \mathrm{~N}$ & Muscovite quartz schist & Inhomogenous \\
\hline 2011GG005A & 65.389712 & -153.081083 & 496232 & 7251699 & $5 N$ & $\begin{array}{l}\text { Plagioclase-sillimanite(?)-muscovite-biotite } \\
\text { quartz schist }\end{array}$ & --- \\
\hline 2011GG006A & 65.299475 & -152.862470 & 506413 & 7241646 & $5 \mathrm{~N}$ & Mafic schist & --- \\
\hline 2011GG007A & 65.297564 & -152.862566 & 506409 & 7241433 & $5 \mathrm{~N}$ & Mafic schist & --- \\
\hline 2011GG028A & 65.303019 & -152.853186 & 506845 & 7242042 & $5 \mathrm{~N}$ & Metagabbro & --- \\
\hline 2011GG035B & 65.331724 & -152.801107 & 509263 & 7245248 & $5 \mathrm{~N}$ & Mafic schist & --- \\
\hline 2011GG037A & 65.335465 & -152.794508 & 509569 & 7245666 & $5 \mathrm{~N}$ & Mafic schist & --- \\
\hline 2011GG037D & 65.335465 & -152.794508 & 509569 & 7245666 & $5 \mathrm{~N}$ & Granite & Altered \\
\hline 2011GG055A & 65.319703 & -152.824484 & 508178 & 7243905 & $5 \mathrm{~N}$ & Mafic schist & --- \\
\hline 2011GG061A & 65.409254 & -152.753681 & 511438 & 7253897 & $5 \mathrm{~N}$ & Mafic schist & --- \\
\hline 2011GG080A & 65.419883 & -152.702307 & 513818 & 7255092 & $5 \mathrm{~N}$ & Mafic schist & --- \\
\hline 2011GG086B & 65.459559 & -152.823224 & 508193 & 7259493 & $5 \mathrm{~N}$ & Metamafic & --- \\
\hline 2011GG087A & 65.458152 & -152.825067 & 508108 & 7259336 & $5 \mathrm{~N}$ & Metagabbro & --- \\
\hline 2011GG112A & 65.446281 & -152.867889 & 506126 & 7258008 & $5 \mathrm{~N}$ & Scapolite-quartz rock & --- \\
\hline 2011GG168A & 65.295792 & -152.727509 & 512708 & 7241256 & $5 \mathrm{~N}$ & Gabbro & --- \\
\hline 2011GG175A & 65.461738 & -152.607878 & 518172 & 7259781 & $5 \mathrm{~N}$ & Gabbro & Altered, inhomogenous \\
\hline 2011GG176A & 65.462220 & -152.606965 & 518214 & 7259835 & $5 \mathrm{~N}$ & Gabbro & --- \\
\hline 2011GG179B & 65.462444 & -152.599689 & 518551 & 7259862 & $5 \mathrm{~N}$ & Mafic schist & --- \\
\hline 2011GG181A & 65.462415 & -152.595698 & 518736 & 7259860 & $5 \mathrm{~N}$ & Green quartzite & --- \\
\hline 2011GG181C & 65.462415 & -152.595698 & 518736 & 7259860 & $5 \mathrm{~N}$ & Fine-grained gabbro & Altered \\
\hline 2011GG183A & 65.461072 & -152.593431 & 518842 & 7259711 & $5 \mathrm{~N}$ & Gabbro & Altered \\
\hline
\end{tabular}


Table 8. (continued)

\begin{tabular}{|c|c|c|c|c|c|c|c|c|c|}
\hline Sample_ID & $\mathrm{SiO}_{2 \_} \mathrm{pct}$ & Al203_pct & BaO_pct & CaO_pct & FeO_pct & K2O_pct & MgO_pct & MnO_pct & Na2O_pct \\
\hline 2011BAE465A & 92.8 & 3.9 & 0.0405 & 0.149 & 0.89 & 0.796 & 0.533 & $<0.03$ & 0.427 \\
\hline 2011BAE465B & 88 & 6.2 & 0.0599 & 0.172 & 2.46 & 1.54 & 0.749 & $<0.03$ & 0.31 \\
\hline 2011BAE466A & 96.5 & 1.8 & $<0.03$ & 0.16 & 0.485 & 0.334 & 0.269 & $<0.03$ & 0.156 \\
\hline 2011BAE466B & 86.5 & 7.6 & 0.0663 & 0.143 & 2.28 & 1.79 & 0.651 & $<0.03$ & 0.284 \\
\hline 2011BAE472A & 45.9 & 16.9 & 0.03 & 11 & 13.8 & 0.4 & 6.3 & 0.28 & 2.63 \\
\hline 2011BAE473A & 47.4 & 15.4 & $<0.03$ & 10.7 & 14.7 & 0.236 & 5.85 & 0.247 & 2.69 \\
\hline 2011BAE476A & 48.3 & 18.4 & $<0.03$ & 12.6 & 9.79 & 0.425 & 5.39 & 0.158 & 2.89 \\
\hline 2011BAE522A & 47.4 & 16.7 & $<0.03$ & 10.9 & 9.98 & 0.0305 & 13.6 & 0.198 & 0.521 \\
\hline 2011BAE565A & 44.1 & 12.5 & 0.0451 & 18.3 & 10 & 0.163 & 6.03 & 0.147 & 5.67 \\
\hline 2011BAE566A & 54 & 14.1 & 0.0339 & 4.59 & 11.8 & 0.295 & 4.49 & 0.136 & 6.05 \\
\hline 2011BAE604A & 83.3 & 8.01 & 0.0719 & 0.464 & 3.85 & 1.71 & 1.17 & 0.0451 & 0.511 \\
\hline 2011GG005A & 67.5 & 18.7 & 0.0805 & 0.455 & 6.9 & 2.67 & 1.49 & 0.099 & 0.96 \\
\hline 2011GG006A & 48.6 & 14 & $<0.03$ & 10.2 & 14.4 & 0.0793 & 6.24 & 0.222 & 3.17 \\
\hline 2011GG007A & 52.4 & 15.4 & 0.0372 & 9.57 & 10.4 & 0.336 & 5.67 & 0.182 & 4.23 \\
\hline 2011GG028A & 48.3 & 16.9 & $<0.03$ & 13.9 & 9.32 & 0.0342 & 7.39 & 0.167 & 2.95 \\
\hline 2011GG035B & 46.1 & 15.2 & $<0.03$ & 14.9 & 11 & 0.155 & 9.61 & 0.226 & 2.25 \\
\hline 2011GG037A & 53.3 & 17.4 & 0.0341 & 6.24 & 9.08 & 0.368 & 3.52 & 0.142 & 7.27 \\
\hline 2011GG037D & 76.1 & 9.13 & $<0.03$ & 2.93 & 3.84 & 2.129 & 2.02 & 0.052 & 2.89 \\
\hline 2011GG055A & 53.1 & 13.2 & $<0.03$ & 8.97 & 11.2 & 0.051 & 6.58 & 0.227 & 5.25 \\
\hline 2011GG061A & 58.5 & 14.2 & $<0.03$ & 4.9 & 12.6 & 0.0247 & 5.55 & 0.144 & 2.13 \\
\hline 2011GG080A & 50.1 & 14.8 & 0.355 & 9.62 & 12.9 & 0.334 & 5.9 & 0.213 & 3.51 \\
\hline 2011GG086B & 48.1 & 18 & $<0.03$ & 13.2 & 10.3 & 0.56 & 7.77 & 0.223 & 0.53 \\
\hline 2011GG087A & 53 & 16.6 & 0.0396 & 9.47 & 9.97 & 0.713 & 5.07 & 0.184 & 2.95 \\
\hline 2011GG112A & 79.9 & 8.26 & $<0.03$ & 7.17 & 0.778 & 0.364 & 1 & 0.0488 & 1.65 \\
\hline 2011GG168A & 52.1 & 14 & 0.388 & 10.8 & 10.5 & 0.866 & 6.91 & 0.192 & 2.66 \\
\hline 2011GG175A & 51.1 & 15.8 & 0.03 & 11.6 & 10.6 & 0.13 & 5.32 & 0.32 & 3.25 \\
\hline 2011GG176A & 50 & 10.1 & 0.0671 & 12.3 & 10.2 & 0.532 & 14.1 & 0.183 & 1.39 \\
\hline 2011GG179B & 51.4 & 16.6 & 0.059 & 11.9 & 7.42 & 0.657 & 6.46 & 0.15 & 3.96 \\
\hline 2011GG181A & 94.1 & 1.71 & $<0.03$ & 1.17 & 1.42 & 0.0392 & 0.97 & $<0.03$ & 0.462 \\
\hline 2011GG181C & 50.2 & 15.6 & 0.04 & 12.3 & 10.8 & 0.19 & 5.95 & 0.21 & 3.2 \\
\hline 2011GG183A & 50.6 & 8.1 & 0.04 & 14 & 11.3 & 0.17 & 13.3 & 0.23 & 0.734 \\
\hline
\end{tabular}


Table 8. (continued)

\begin{tabular}{|c|c|c|c|c|c|c|c|c|c|}
\hline Sample_ID & P2O5_pct & TiO2_pct & CO2_pct & Total_pct & As_ppm & Bi_ppm & Br_ppm & Ce_ppm & Cl_ppm \\
\hline 2011BAE465A & 0.0657 & 0.247 & $<0.05$ & 99.8796 & $<4$ & $<2$ & $<<$ & $<60$ & 112 \\
\hline 2011BAE465B & 0.0747 & 0.327 & $<0.05$ & 99.9583 & $<4$ & $<2$ & $<5$ & $<60$ & 119 \\
\hline 2011BAE466A & 0.0836 & 0.127 & $<0.05$ & 99.9001 & $<4$ & $<2$ & $<5$ & $<60$ & 340 \\
\hline 2011BAE466B & 0.113 & 0.452 & $<0.05$ & 99.8816 & $<4$ & $<2$ & $<5$ & $<60$ & 200 \\
\hline 2011BAE472A & 0.2 & 2.2 & $<0.05$ & 99.6353 & $<4$ & $<2$ & $<5$ & $<60$ & 1600 \\
\hline 2011BAE473A & 0.287 & 2.22 & $<0.05$ & 99.746 & 80 & $<2$ & $<5$ & $<60$ & 1150 \\
\hline 2011BAE476A & 0.146 & 1.54 & $<0.05$ & 99.6605 & $<4$ & $<2$ & $<5$ & $<60$ & 1430 \\
\hline 2011BAE522A & 0.0441 & 0.283 & $<0.05$ & 99.6748 & $<4$ & $<2$ & $<5$ & $<60$ & 681 \\
\hline 2011BAE565A & 0.422 & 2.21 & $<0.05$ & 99.5871 & $<4$ & $<2$ & $<5$ & $<60$ & 158 \\
\hline 2011BAE566A & 0.621 & 3.54 & $<0.05$ & 99.6559 & 4 & $<2$ & $<5$ & $<60$ & 144 \\
\hline 2011BAE604A & 0.214 & 0.559 & $<0.05$ & 99.905 & 7 & $<2$ & $<5$ & $<60$ & 434 \\
\hline 2011GG005A & 0.146 & 0.828 & $<0.05$ & 99.8285 & 49 & $<2$ & $<5$ & $<60$ & 369 \\
\hline 2011GG006A & 0.372 & 2.45 & $<0.05$ & 99.7462 & $<4$ & $<2$ & $<5$ & $<60$ & $<50$ \\
\hline 2011GG007A & 0.253 & 1.32 & $<0.05$ & 99.7982 & $<4$ & $<2$ & $<5$ & $<60$ & 179 \\
\hline 2011GG028A & 0.353 & 0.485 & $<0.05$ & 99.817 & 49 & $<2$ & $<5$ & $<60$ & 225 \\
\hline 2011GG035B & $<0.03$ & 0.342 & $<0.05$ & 99.827 & 63 & $<2$ & $<5$ & $<60$ & 274 \\
\hline 2011GG037A & 0.488 & 1.99 & $<0.05$ & 99.8321 & 48 & $<2$ & $<5$ & $<60$ & 227 \\
\hline 2011GG037D & $<0.03$ & 0.44 & $<0.05$ & 99.5825 & $<4$ & $<2$ & $<5$ & $<60$ & 350 \\
\hline 2011GG055A & 0.179 & 1.08 & $<0.05$ & 99.8499 & $<4$ & $<2$ & $<5$ & $<60$ & 129 \\
\hline 2011GG061A & 0.298 & 1.42 & $<0.05$ & 99.782 & 54 & $<2$ & $<5$ & $<60$ & 86 \\
\hline 2011GG080A & 0.306 & 1.81 & $<0.05$ & 99.848 & $<4$ & $<2$ & $<5$ & $<60$ & 123 \\
\hline 2011GG086B & 0.124 & 0.96 & $<0.05$ & 99.7909 & 53 & $<2$ & $<5$ & $<60$ & 610 \\
\hline 2011GG087A & 0.238 & 1.54 & $<0.05$ & 99.7746 & 100 & $<2$ & $<5$ & $<60$ & 385 \\
\hline 2011GG112A & 0.101 & 0.188 & $<0.05$ & 99.4598 & $<4$ & $<2$ & 14 & $<60$ & 4900 \\
\hline 2011GG168A & 0.192 & 1.18 & $<0.05$ & 99.788 & $<4$ & $<2$ & $<5$ & $<60$ & 311 \\
\hline 2011GG175A & 0.17 & 1.45 & $<0.05$ & 99.7615 & $<4$ & $<2$ & $<5$ & $<60$ & 265 \\
\hline 2011GG176A & 0.112 & 0.761 & $<0.05$ & 99.7451 & $<4$ & $<2$ & $<5$ & $<60$ & 249 \\
\hline 2011GG179B & 0.188 & 1.13 & $<0.05$ & 99.924 & $<4$ & $<2$ & $<5$ & $<60$ & 54 \\
\hline 2011GG181A & 0.0838 & 0.0543 & $<0.05$ & 100.0249 & $<4$ & $<2$ & $<5$ & $<60$ & 76 \\
\hline 2011GG181C & 0.14 & 1.27 & $<0.05$ & 99.8913 & $<4$ & $<2$ & $<5$ & $<60$ & 124 \\
\hline 2011GG183A & 0.17 & 0.954 & $<0.05$ & 99.5653 & $<4$ & $<2$ & $<5$ & $<60$ & 515 \\
\hline
\end{tabular}


Table 8. (continued)

\begin{tabular}{|c|c|c|c|c|c|c|c|c|c|}
\hline Sample_ID & Co_ppm & Cr_ppm & Cu_ppm & F_ppm & La_ppm & Mo_ppm & Nb_ppm & Nb_ppm_2 & Ni_ppm \\
\hline 2011BAE465A & 27 & $<30$ & 14 & $<110$ & $<50$ & $<1$ & $<1$ & 5 & 25 \\
\hline 2011BAE465B & $<10$ & 41 & 22 & $<110$ & $<50$ & $<1$ & $<1$ & 6 & 33 \\
\hline 2011BAE466A & 24 & 65 & 14 & 111 & $<50$ & $<1$ & $<1$ & 3 & 37 \\
\hline 2011BAE466B & $<10$ & 67 & 27 & $<110$ & $<50$ & $<1$ & $<1$ & 9 & 17 \\
\hline 2011BAE472A & 96 & 178 & 162 & 233 & $<50$ & $<1$ & $<1$ & 6 & 67 \\
\hline 2011BAE473A & 57 & 234 & 175 & $<110$ & $<50$ & $<1$ & $<1$ & 7 & 147 \\
\hline 2011BAE476A & 70 & 274 & 10 & $<110$ & $<50$ & $<1$ & $<1$ & 4 & 97 \\
\hline 2011BAE522A & 133 & 569 & 48 & $<110$ & $<50$ & $<1$ & $<1$ & 1 & 410 \\
\hline 2011BAE565A & 59 & 127 & 48 & 752 & $<50$ & $<1$ & $<1$ & 22 & 89 \\
\hline 2011BAE566A & 42 & 268 & 17 & 364 & $<50$ & $<1$ & $<1$ & 47 & 73 \\
\hline 2011BAE604A & $<10$ & 78 & 30 & $<110$ & $<50$ & $<1$ & $<1$ & 8 & 47 \\
\hline 2011GG005A & 13 & 122 & 13 & $<110$ & $<50$ & $<1$ & 19 & 18 & 46 \\
\hline 2011GG006A & 96 & 133 & 165 & $<110$ & $<50$ & $<1$ & $<1$ & 15 & 50 \\
\hline 2011GG007A & 65 & 196 & 39 & $<110$ & $<50$ & $<1$ & $<1$ & 8 & 37 \\
\hline 2011GG028A & 71 & 613 & 87 & $<110$ & $<50$ & $<1$ & $<1$ & 1 & 161 \\
\hline 2011GG035B & 68 & 513 & 9 & $<110$ & $<50$ & $<1$ & $<1$ & 2 & 188 \\
\hline 2011GG037A & 52 & 125 & 71 & $<110$ & $<50$ & $<1$ & $<1$ & 12 & 85 \\
\hline 2011GG037D & $<10$ & 69 & 61 & $<110$ & $<50$ & $<1$ & 7 & --- & 62 \\
\hline 2011GG055A & 72 & 351 & 42 & $<110$ & $<50$ & $<1$ & $<1$ & 4 & 66 \\
\hline 2011GG061A & 41 & 89 & 46 & $<110$ & $<50$ & $<1$ & $<1$ & 10 & 102 \\
\hline 2011GG080A & $<10$ & 145 & 91 & $<110$ & $<50$ & $<1$ & $<1$ & 14 & 43 \\
\hline 2011GG086B & 50 & 198 & $<7$ & $<110$ & $<50$ & $<1$ & $<1$ & 1 & 169 \\
\hline 2011GG087A & 38 & $<30$ & 151 & $<110$ & $<50$ & $<1$ & $<1$ & 7 & 59 \\
\hline 2011GG112A & $<10$ & 51 & $<7$ & $<110$ & $<50$ & $<1$ & $<1$ & --- & 38 \\
\hline 2011GG168A & 68 & 307 & 87 & $<110$ & $<50$ & $<1$ & $<1$ & 7 & 121 \\
\hline 2011GG175A & 43 & 388 & 57 & $<110$ & $<50$ & $<1$ & $<1$ & 5 & 112 \\
\hline 2011GG176A & 102 & 1250 & 169 & $<110$ & $<50$ & $<1$ & $<1$ & 4 & 475 \\
\hline 2011GG179B & 37 & 339 & 55 & $<110$ & $<50$ & $<1$ & $<1$ & 6 & 91 \\
\hline 2011GG181A & $<10$ & $<30$ & 20 & $<110$ & $<50$ & $<1$ & $<1$ & 1 & $<7$ \\
\hline 2011GG181C & 77 & 232 & 159 & $<110$ & $<50$ & $<1$ & $<1$ & 5 & 51 \\
\hline 2011GG183A & 50 & 1270 & 136 & $<110$ & $<50$ & $<1$ & $<1$ & 5 & 386 \\
\hline
\end{tabular}


Table 8. (continued)

\begin{tabular}{|c|c|c|c|c|c|c|c|c|c|}
\hline Sample_ID & Pb_ppm & Rb_ppm & Rb_ppm_2 & S_ppm & Sb_ppm & Sn_ppm & Sr_ppm & Sr_ppm_2 & Th_ppm \\
\hline 2011BAE465A & 15 & $<1$ & 30 & 63 & $<2$ & $<4$ & $<1$ & 25 & $<2$ \\
\hline 2011BAE465B & 35 & $<1$ & 56 & $<30$ & $<2$ & $<4$ & $<1$ & 21 & $<2$ \\
\hline 2011BAE466A & 20 & $<1$ & 18 & 64 & $<2$ & $<4$ & $<1$ & 15 & $<2$ \\
\hline 2011BAE466B & 25 & $<1$ & 69 & 88 & $<2$ & $<4$ & $<1$ & 25 & $<2$ \\
\hline 2011BAE472A & $<4$ & $<1$ & 22 & 650 & $<2$ & $<4$ & $<1$ & 252 & $<2$ \\
\hline 2011BAE473A & 21 & $<1$ & 13 & $<30$ & $<2$ & 242 & $<1$ & 226 & $<2$ \\
\hline 2011BAE476A & $<4$ & $<1$ & 8 & 137 & $<2$ & $<4$ & $<1$ & 444 & $<2$ \\
\hline 2011BAE522A & 25 & $<1$ & $<1$ & 339 & $<2$ & $<4$ & $<1$ & 92 & $<2$ \\
\hline 2011BAE565A & $<4$ & $<1$ & 3 & 182 & $<2$ & $<4$ & $<1$ & 281 & $<2$ \\
\hline 2011BAE566A & $<4$ & $<1$ & 8 & 85 & $<2$ & $<4$ & $<1$ & 285 & $<2$ \\
\hline 2011BAE604A & 30 & $<1$ & 98 & 151 & $<2$ & $<4$ & $<1$ & 50 & $<2$ \\
\hline 2011GG005A & 63 & 125 & 155 & 158 & $<2$ & $<4$ & 155 & 155 & $<2$ \\
\hline 2011 GG006A & $<4$ & $<1$ & 2 & 47 & $<2$ & $<4$ & $<1$ & 256 & $<2$ \\
\hline 2011GG007A & 30 & $<1$ & 14 & 66 & $<2$ & $<4$ & $<1$ & 347 & $<2$ \\
\hline 2011GG028A & $<4$ & $<1$ & 1 & 62 & $<2$ & $<4$ & $<1$ & 280 & $<2$ \\
\hline 2011GG035B & $<4$ & $<1$ & 11 & 94 & $<2$ & $<4$ & $<1$ & 182 & $<2$ \\
\hline 2011GG037A & 35 & $<1$ & 7 & 266 & $<2$ & $<4$ & $<1$ & 201 & $<2$ \\
\hline 2011GG037D & 31 & 14 & --- & 78 & $<2$ & $<4$ & 173 & --- & $<2$ \\
\hline 2011GG055A & 22 & $<1$ & $<1$ & 256 & $<2$ & $<4$ & $<1$ & 221 & $<2$ \\
\hline 2011GG061A & 52 & $<1$ & 1 & 90 & $<2$ & $<4$ & $<1$ & 205 & $<2$ \\
\hline 2011GG080A & $<4$ & $<1$ & 7 & 254 & $<2$ & $<4$ & $<1$ & 241 & $<2$ \\
\hline 2011GG086B & $<4$ & $<1$ & 83 & 229 & $<2$ & $<4$ & $<1$ & 248 & $<2$ \\
\hline 2011GG087A & 30 & $<1$ & 80 & 115 & $<2$ & $<4$ & $<1$ & 282 & $<2$ \\
\hline 2011GG112A & $<4$ & 28 & --- & 218 & $<2$ & $<4$ & 221 & --- & $<2$ \\
\hline 2011GG168A & $<4$ & $<1$ & 25 & 379 & $<2$ & $<4$ & $<1$ & 85 & $<2$ \\
\hline 2011GG175A & $<4$ & $<1$ & 3 & 161 & $<2$ & $<4$ & $<1$ & 142 & $<2$ \\
\hline 2011GG176A & $<4$ & $<1$ & 17 & 64 & $<2$ & $<4$ & $<1$ & 50 & $<2$ \\
\hline 2011GG179B & $<4$ & $<1$ & 22 & $<30$ & $<2$ & $<4$ & $<1$ & 117 & $<2$ \\
\hline 2011GG181A & $<4$ & $<1$ & 2 & 86 & $<2$ & $<4$ & 29 & 29 & $<2$ \\
\hline 2011GG181C & $<4$ & $<1$ & 5 & 126 & $<2$ & 25 & $<1$ & 138 & $<2$ \\
\hline 2011GG183A & $<4$ & $<1$ & 3 & 585 & $<2$ & 22 & $<1$ & 60 & $<2$ \\
\hline
\end{tabular}


Table 8. (continued)

\begin{tabular}{|c|c|c|c|c|c|c|c|c|}
\hline Sample_ID & U_ppm & V_ppm & W_ppm & Y_ppm & Y_ppm_2 & Zn_ppm & Zr_ppm & Zr_ppm_2 \\
\hline 2011BAE465A & $<<1$ & 52 & $<<8$ & $2<1$ & 14 & 18 & $<9$ & 172 \\
\hline 2011BAE465B & $<1$ & 93 & $<8$ & $<1$ & 7 & 41 & $<9$ & 187 \\
\hline 2011BAE466A & $<1$ & 32 & $<8$ & $<1$ & 4 & 18 & $<9$ & 116 \\
\hline 2011BAE466B & $<1$ & 73 & $<8$ & $<1$ & 14 & 35 & $<9$ & 215 \\
\hline 2011BAE472A & $<1$ & 679 & $<8$ & $<1$ & 38 & 157 & $<9$ & 141 \\
\hline 2011BAE473A & $<1$ & 543 & $<8$ & $<1$ & 35 & 121 & $<9$ & 149 \\
\hline 2011BAE476A & $<1$ & 428 & $<8$ & $<1$ & 26 & 146 & $<9$ & 83 \\
\hline 2011BAE522A & $<1$ & 191 & $<8$ & $<1$ & 8 & 57 & $<9$ & 16 \\
\hline 2011BAE565A & $<1$ & 388 & $<8$ & $<1$ & 26 & 95 & $<9$ & 152 \\
\hline 2011BAE566A & $<1$ & 516 & $<8$ & $<1$ & 27 & 127 & $<9$ & 268 \\
\hline 2011BAE604A & $<1$ & 154 & $<8$ & $<1$ & 18 & 55 & $<9$ & 125 \\
\hline 2011GG005A & $<1$ & 150 & $<8$ & 3 & 21 & 132 & 148 & 178 \\
\hline 2011GG006A & $<1$ & 577 & $<8$ & $<1$ & 42 & 117 & $<9$ & 210 \\
\hline 2011GG007A & $<1$ & 316 & $<8$ & $<1$ & 20 & 59 & $<9$ & 99 \\
\hline 2011GG028A & $<1$ & 292 & $<8$ & $<1$ & 19 & 51 & $<9$ & 32 \\
\hline 2011GG035B & $<1$ & 336 & $<8$ & $<1$ & 8 & 78 & $<9$ & 18 \\
\hline 2011GG037A & $<1$ & 385 & $<8$ & $<1$ & 36 & 78 & $<9$ & 165 \\
\hline 2011GG037D & $<1$ & 191 & $<8$ & 26 & --- & 34 & 134 & --- \\
\hline 2011GG055A & $<1$ & 323 & $<8$ & $<1$ & 20 & 97 & $<9$ & 83 \\
\hline 2011GG061A & $<1$ & 424 & $<8$ & $<1$ & 34 & 189 & $<9$ & 98 \\
\hline 2011GG080A & $<1$ & 473 & $<8$ & $<1$ & 31 & 114 & $<9$ & 141 \\
\hline 2011GG086B & $<1$ & 276 & $<8$ & $<1$ & 27 & 89 & $<9$ & 55 \\
\hline \begin{tabular}{|l|}
$2011 G G 087 A$ \\
\end{tabular} & $<1$ & 447 & $<8$ & $<1$ & 29 & 130 & $<9$ & 114 \\
\hline 2011GG112A & $<1$ & 52 & $<8$ & 21 & --- & 29 & 71 & $<9$ \\
\hline 2011GG168A & $<1$ & 342 & $<8$ & $<1$ & 23 & 104 & $<9$ & 108 \\
\hline 2011GG175A & $<1$ & 362 & $<8$ & $<1$ & 25 & 108 & $<9$ & 93 \\
\hline 2011GG176A & $<1$ & 244 & $<8$ & $<1$ & 13 & 84 & $<9$ & 55 \\
\hline 2011GG179B & $<1$ & 295 & $<8$ & $<1$ & 19 & 44 & $<9$ & 99 \\
\hline 2011GG181A & $<1$ & $<25$ & $<8$ & $<1$ & 2 & 11 & 9 & 11 \\
\hline 2011GG181C & $<1$ & 413 & $<8$ & $<1$ & 24 & 76 & $<9$ & 82 \\
\hline 2011GG183A & $<1$ & 316 & $<8$ & $<1$ & 16 & 88 & $<9$ & 67 \\
\hline
\end{tabular}


Table 8. (continued)

\begin{tabular}{|c|c|c|c|c|c|c|c|}
\hline Sample_ID & Latitude & Longitude & Easting & Northing & $\begin{array}{l}\text { UTM_ } \\
\text { Zone }\end{array}$ & Root_name & Comments \\
\hline 2011GG186A & 65.461044 & $\mid-152.589440$ & $\mid 519027$ & 7259709 & $5 \mathrm{~N}$ & Plagioclase ultramafic & |-- \\
\hline 2011GG190A & 65.455068 & -152.573267 & 519781 & 7259048 & $5 \mathrm{~N}$ & Gabbro & Altered \\
\hline 2011GG191A & 65.443588 & -152.591093 & 518963 & 7257763 & $5 \mathrm{~N}$ & Fine-grained gabbro & Altered \\
\hline 2011GG193A & 65.442618 & -152.593847 & 518836 & 7257654 & $5 \mathrm{~N}$ & Gabbro with chlorite vein & Altered, inhomogenous, small \\
\hline 2011GG193B & 65.442618 & -152.593847 & 518836 & 7257654 & $5 \mathrm{~N}$ & Fine-grained gabbro & Altered \\
\hline 2011GG205A & 65.458975 & -152.604273 & 518341 & 7259474 & $5 \mathrm{~N}$ & Mafic rock & --- \\
\hline 2011GG224A & 65.305539 & -152.852678 & 506868 & 7242323 & $5 \mathrm{~N}$ & Metagabbro & --- \\
\hline 2011GG225A & 65.305469 & -152.854352 & 506790 & 7242315 & $5 \mathrm{~N}$ & Metagabbro & Small \\
\hline 2011GG226A & 65.306024 & -152.853233 & 506842 & 7242377 & $5 \mathrm{~N}$ & Metagabbro & --- \\
\hline 2011GG229A & 65.308341 & -152.854615 & 506777 & 7242635 & $5 \mathrm{~N}$ & Metamafic & --- \\
\hline 2011GG271A & 65.180909 & -153.836685 & 460811 & 7228684 & $5 \mathrm{~N}$ & Paragneiss & Inhomogenous, poor polish \\
\hline 2011GG275A & 65.177322 & -153.836231 & 460827 & 7228284 & $5 \mathrm{~N}$ & Qlbite-biotite quartz schist & --- \\
\hline 2011GG319A & 65.440663 & -152.769643 & 510684 & 7257395 & $5 \mathrm{~N}$ & Metamafic & Small \\
\hline 2011GG322A & 65.438689 & -152.775115 & 510431 & 7257174 & $5 \mathrm{~N}$ & Metagabbro & Inhomogenous \\
\hline 2011GG339A & 65.471433 & -152.819021 & 508384 & 7260817 & $5 \mathrm{~N}$ & Tourmaline-veined granite & Inhomogenous \\
\hline 2011GG342A & 65.484676 & -152.812018 & 508704 & 7262294 & $5 \mathrm{~N}$ & Metagabbro & --- \\
\hline 2011GG343B & 65.487836 & -152.813334 & 508642 & 7262646 & $5 \mathrm{~N}$ & Tourmaline-veined granitic aplite & Inhomogenous \\
\hline 2011GG346A & 65.491707 & -152.809958 & 508797 & 7263078 & $5 \mathrm{~N}$ & Biotite-muscovite quartz schist & --- \\
\hline 2011GG347A & 65.492887 & -152.806644 & 508950 & 7263210 & $5 \mathrm{~N}$ & Mafic schist & --- \\
\hline 2011GG350A & 65.497877 & -152.793513 & 509556 & 7263768 & $5 \mathrm{~N}$ & Muscovite quartz schist & --- \\
\hline 2011GG382A & 65.379733 & -152.887110 & 505248 & 7250589 & $5 \mathrm{~N}$ & Metagabbro & --- \\
\hline 2011GG383B & 65.381078 & -152.886373 & 505282 & 7250739 & $5 \mathrm{~N}$ & Metagabbro & Poor polish \\
\hline 2011GG385A & 65.298471 & -152.912827 & 504065 & 7241530 & $5 \mathrm{~N}$ & Metadiorite(?) & --- \\
\hline 2011GG392A & 65.286661 & -152.923712 & 503559 & 7240213 & $5 \mathrm{~N}$ & Metagabbro & Porous \\
\hline 2011GG395A & 65.280794 & \begin{tabular}{|l|}
-152.924865 \\
\end{tabular} & 503506 & 7239559 & $5 \mathrm{~N}$ & Paragneiss schist(?) & Altered, porous, small \\
\hline 2011GG448A & 65.231115 & -152.988214 & 500551 & 7234020 & $5 \mathrm{~N}$ & Quartz schist & Porous \\
\hline 2011GG450A & 65.229283 & -152.979745 & 500947 & 7233816 & $5 \mathrm{~N}$ & Chloritoid(?) quartzite & Inhomogenous \\
\hline 2011GG458A & 65.225386 & -152.936506 & 502969 & 7233383 & $5 \mathrm{~N}$ & Leached quartzite & Porous \\
\hline 2011GG472A & 65.439655 & -152.613685 & 517918 & 7257318 & $5 \mathrm{~N}$ & Mafic tuff & --- \\
\hline 2011GG485A & 65.377892 & -152.929234 & 503290 & 7250381 & $5 \mathrm{~N}$ & Metagabbro & --- \\
\hline 2011GG494A & 65.385712 & -152.940272 & 502776 & 7251252 & $5 \mathrm{~N}$ & Metagabbro & $\mid--$ \\
\hline 2011GG495A & 65.386296 & -152.944186 & 502594 & 7251317 & $5 \mathrm{~N}$ & Metagabbro & Porous \\
\hline
\end{tabular}


Table 8. (continued)

\begin{tabular}{|c|c|c|c|c|c|c|c|c|c|}
\hline Sample_ID & $\mathrm{SiO}_{2 \_} \mathrm{pct}$ & Al203_pct & BaO_pct & CaO_pct & FeO_pct & K2O_pct & MgO_pct & MnO_pct & $\mathrm{Na} 2 \mathrm{O} \_p c t$ \\
\hline 2011GG186A & 47.9 & 6.09 & 0.069 & 6.7 & 14.1 & 0.0791 & 23.2 & 0.253 & 0.506 \\
\hline 2011GG190A & 51.5 & 14.8 & 0.04 & 11.2 & 10.6 & 0.03 & 6.55 & 0.2 & 3.63 \\
\hline 2011GG191A & 50.9 & 14.6 & 0.07 & 10.5 & 11.8 & 0.25 & 5.64 & 0.2 & 4.01 \\
\hline 2011GG193A & 45.5 & 15.5 & 0.04 & 16.7 & 13.4 & 0.02 & 6.88 & 0.24 & 0.295 \\
\hline 2011GG193B & 47.2 & 11.7 & 0.06 & 10.4 & 16.6 & 0.26 & 6.32 & 0.22 & 4.67 \\
\hline 2011GG205A & 49.8 & 7.04 & $<0.03$ & 14.4 & 11 & 0.1 & 14.8 & 0.229 & 1.01 \\
\hline 2011GG224A & 53.1 & 15.4 & 0.04 & 8.02 & 10.6 & 0.46 & 5.05 & 0.2 & 5.67 \\
\hline 2011GG225A & 56.6 & 13.5 & $<0.03$ & 8.01 & 10.1 & 0.1 & 5.08 & 0.19 & 4.49 \\
\hline 2011GG226A & 52.7 & 16.4 & $<0.03$ & 8.75 & 10.5 & 0.1 & 4.44 & 0.2 & 5.1 \\
\hline 2011GG229A & 49.5 & 9.6 & $<0.03$ & 11.8 & 16.1 & $<.02$ & 11.2 & 0.25 & 0.0931 \\
\hline 2011GG271A & 80.2 & 9.12 & 0.0614 & 1.19 & 2.95 & 2.76 & 1.14 & 0.0656 & 1.86 \\
\hline 2011GG275A & 66.8 & 14.7 & 0.114 & 1.35 & 7.29 & 3.36 & 2.96 & 0.156 & 1.72 \\
\hline 2011GG319A & 52.8 & 16.2 & $<0.03$ & 4.71 & 11.7 & 0.2 & 5.24 & 0.22 & 7.45 \\
\hline 2011GG322A & 49.7 & 14.2 & $<0.03$ & 14.5 & 11.5 & 0.34 & 5.65 & 0.23 & 2.29 \\
\hline 2011GG339A & 77.1 & 12.7 & $<0.03$ & 0.202 & 0.939 & 4.86 & 0.0696 & 0.0328 & 3.87 \\
\hline 2011GG342A & 53.2 & 16.2 & 0.03 & 13.3 & 5.85 & 0.26 & 7.21 & 0.14 & 3.21 \\
\hline 2011GG343B & 77.3 & 12.9 & $<0.03$ & 0.286 & 1.07 & 3.55 & 0.0531 & 0.0423 & 4.57 \\
\hline 2011GG346A & 80.4 & 10.5 & 0.119 & 0.304 & 3.92 & 1.99 & 1.12 & 0.0471 & 0.721 \\
\hline 2011GG347A & 49.4 & 19.5 & $<0.03$ & 11.2 & 8.47 & 0.0286 & 8.67 & 0.169 & 1.88 \\
\hline 2011GG350A & 69.6 & 17.5 & 0.242 & 0.614 & 4.63 & 3.37 & 1.34 & 0.0577 & 0.867 \\
\hline 2011GG382A & 58.6 & 12 & $<0.03$ & 0.0868 & 21.9 & 0.12 & 5.18 & 0.4 & 0.0373 \\
\hline 2011GG383B & 50.8 & 15.9 & $<0.03$ & 9.8 & 10.8 & 0.07 & 6.14 & 0.2 & 4.8 \\
\hline 2011GG385A & 61.5 & 13.9 & $<0.03$ & 5.99 & 8.64 & 0.0363 & 2.83 & 0.147 & 5.26 \\
\hline 2011GG392A & 56.5 & 14.1 & 0.03 & 6.07 & 10.8 & 0.11 & 6.09 & 0.16 & 4.4 \\
\hline 2011GG395A & 55.8 & 28 & 0.06 & 0.226 & 7.38 & 1.29 & 0.778 & 0.98 & 4.3 \\
\hline 2011GG448A & 75.5 & 14.1 & 0.0871 & 0.107 & 4.62 & 2.17 & 0.943 & 0.0438 & 1.38 \\
\hline 2011GG450A & 90.3 & 5 & 0.0837 & 0.102 & 2.12 & 1.17 & 0.516 & $<0.03$ & 0.336 \\
\hline 2011GG458A & 94.2 & 2.1 & $<0.03$ & 0.181 & 1.9 & 0.472 & 0.314 & $<0.03$ & 0.179 \\
\hline 2011GG472A & 51.1 & 14.4 & $<0.03$ & 11 & 12.8 & 0.03 & 5.1 & 0.26 & 3.31 \\
\hline 2011GG485A & 51.4 & 15.9 & 0.03 & 11.6 & 9.57 & 0.32 & 6.63 & 0.18 & 2.85 \\
\hline 2011GG494A & 51.3 & 15.8 & $<0.03$ & 10.7 & 11.1 & 0.03 & 7.24 & 0.19 & 2.67 \\
\hline 2011GG495A & 51 & 13.9 & $<0.03$ & 9.14 & 14.4 & 0.04 & 5.07 & 0.23 & 4.22 \\
\hline
\end{tabular}


Table 8. (continued)

\begin{tabular}{|c|c|c|c|c|c|c|c|c|c|}
\hline Sample_ID & P2O5_pct & TiO2_pct & CO2_pct & Total_pct & As_ppm & Bi_ppm & Br_ppm & Ce_ppm & Cl_ppm \\
\hline 2011GG186A & 0.0854 & 0.662 & $<<0.05$ & 99.6445 & $2<4$ & $<<2$ & $<<5$ & $<60$ & 260 \\
\hline 2011GG190A & 0.16 & 1.14 & $<0.05$ & 99.8538 & $<4$ & $<2$ & $<5$ & $<60$ & 149 \\
\hline 2011GG191A & 0.25 & 1.74 & $<0.05$ & 99.9607 & $<4$ & $<2$ & $<5$ & $<60$ & 164 \\
\hline 2011GG193A & 0.13 & 1.24 & $<0.05$ & 99.9384 & $<4$ & $<2$ & $<5$ & $<60$ & 156 \\
\hline 2011GG193B & 0.35 & 2.15 & $<0.05$ & 99.9297 & $<4$ & $<2$ & $<5$ & $<60$ & $<50$ \\
\hline 2011GG205A & 0.162 & 1.06 & $<0.05$ & 99.6246 & $<4$ & $<2$ & $<5$ & $<60$ & 233 \\
\hline 2011GG224A & 0.23 & 1.09 & $<0.05$ & 99.8524 & $<4$ & $<2$ & $<5$ & $<60$ & 551 \\
\hline 2011GG225A & 0.29 & 1.26 & $<0.05$ & 99.6452 & 8 & $<2$ & $<5$ & $<60$ & 127 \\
\hline 2011GG226A & 0.33 & 1.34 & $<0.05$ & 99.8785 & 13 & $<2$ & $<5$ & $<60$ & 114 \\
\hline 2011GG229A & 0.13 & 0.908 & $<0.05$ & 99.54851 & 123 & $<2$ & $<5$ & $<60$ & 841 \\
\hline 2011GG271A & 0.0571 & 0.5 & $<0.05$ & 99.9041 & $<4$ & $<2$ & $<5$ & $<60$ & 371 \\
\hline 2011GG275A & 0.121 & 1.15 & $<0.05$ & 99.721 & 37 & $<2$ & $<5$ & $<60$ & 473 \\
\hline 2011GG319A & 0.2 & 1.04 & $<0.05$ & 99.7786 & $<4$ & $<2$ & $<5$ & $<60$ & 447 \\
\hline 2011GG322A & 0.18 & 1.19 & $<0.05$ & 99.7961 & $<4$ & $<2$ & $<5$ & $<60$ & 206 \\
\hline 2011GG339A & $<0.03$ & 0.0546 & $<0.05$ & 99.8661 & $<4$ & $<2$ & $<5$ & $<60$ & 254 \\
\hline 2011GG342A & 0.04 & 0.368 & $<0.05$ & 99.8082 & $<4$ & $<2$ & $<5$ & $<60$ & 183 \\
\hline 2011GG343B & $<0.03$ & 0.0209 & $<0.05$ & 99.8414 & $<4$ & $<2$ & $<5$ & $<60$ & 200 \\
\hline 2011GG346A & 0.121 & 0.625 & $<0.05$ & 99.8671 & 38 & $<2$ & $<5$ & $<60$ & 222 \\
\hline 2011GG347A & $<0.03$ & 0.466 & $<0.05$ & 99.8112 & 15 & $<2$ & $<5$ & $<60$ & 276 \\
\hline 2011GG350A & 0.34 & 1.02 & $<0.05$ & 99.5807 & $<4$ & $<2$ & $<5$ & $<60$ & 653 \\
\hline 2011GG382A & 0.11 & 1.31 & $<0.05$ & 99.7693 & 42 & $<2$ & $<5$ & $<60$ & 153 \\
\hline 2011GG383B & 0.2 & 1.04 & $<0.05$ & 99.7722 & $<4$ & $<2$ & $<5$ & $<60$ & 364 \\
\hline 2011GG385A & 0.384 & 1.18 & $<0.05$ & 99.8794 & $<4$ & $<2$ & $<5$ & $<60$ & 113 \\
\hline 2011GG392A & 0.48 & 1.09 & $<0.05$ & 99.8193 & $<4$ & $<2$ & $<5$ & $<60$ & 279 \\
\hline 2011GG395A & 0.15 & 0.79 & $<0.05$ & 99.5398 & 4 & $<2$ & $<5$ & $<60$ & 109 \\
\hline 2011GG448A & 0.126 & 0.782 & $<0.05$ & 99.8589 & $<4$ & $<2$ & $<5$ & $<60$ & 140 \\
\hline 2011GG450A & 0.0428 & 0.234 & $<0.05$ & 99.9018 & $<4$ & $<2$ & $<5$ & $<60$ & 395 \\
\hline 2011GG458A & 0.203 & 0.0655 & $<0.05$ & 99.6809 & $<4$ & $<2$ & $<5$ & $<60$ & 696 \\
\hline 2011GG472A & 0.21 & 1.67 & $<0.05$ & 99.905 & 9 & $<2$ & $<5$ & $<60$ & 138 \\
\hline 2011GG485A & 0.17 & 1.02 & $<0.05$ & 99.6781 & $<4$ & $<2$ & $<5$ & $<60$ & 586 \\
\hline 2011GG494A & 0.13 & 0.745 & $<0.05$ & 99.9199 & $<4$ & $<2$ & $<5$ & $<60$ & $<50$ \\
\hline 2011GG495A & 0.21 & 1.37 & $<0.05$ & 99.5998 & $<4$ & $<2$ & $<5$ & $<60$ & 1140 \\
\hline
\end{tabular}


Table 8. (continued)

\begin{tabular}{|c|c|c|c|c|c|c|c|c|c|}
\hline Sample_ID & Co_ppm & Cr_ppm & Cu_ppm & F_ppm & La_ppm & Mo_ppm & Nb_ppm & Nb_ppm_2 & Ni_ppm \\
\hline 2011GG186A & 204 & 1730 & 201 & $<110$ & $<50$ & $<<1$ & $<<1$ & \begin{tabular}{c|}
-- \\
-
\end{tabular} & 1040 \\
\hline 2011GG190A & 27 & 241 & 108 & $<110$ & $<50$ & $<1$ & $<1$ & 6 & 110 \\
\hline 2011GG191A & 74 & 120 & 34 & $<110$ & $<50$ & $<1$ & $<1$ & 19 & 38 \\
\hline 2011GG193A & 70 & 228 & 84 & $<110$ & $<50$ & $<1$ & $<1$ & 10 & 79 \\
\hline 2011GG193B & 206 & 164 & $<7$ & $<110$ & $<50$ & $<1$ & $<1$ & 21 & 72 \\
\hline 2011GG205A & 99 & 1490 & 164 & $<110$ & $<50$ & $<1$ & $<1$ & 6 & 561 \\
\hline 2011GG224A & 36 & 52 & 114 & $<110$ & $<50$ & $<1$ & $<1$ & 8 & 19 \\
\hline 2011GG225A & 78 & 47 & 23 & 1470 & $<50$ & $<1$ & $<1$ & 9 & 36 \\
\hline 2011GG226A & 49 & 107 & 30 & $<110$ & $<50$ & $<1$ & $<1$ & 9 & 41 \\
\hline 2011GG229A & 120 & 1260 & 76 & 842 & $<50$ & $<1$ & $<1$ & 2 & 483 \\
\hline 2011GG271A & $<10$ & 82 & $<7$ & $<110$ & $<50$ & $<1$ & $<1$ & 9 & 32 \\
\hline 2011GG275A & 34 & 115 & $<7$ & 1300 & $<50$ & $<1$ & $<1$ & 13 & 43 \\
\hline 2011GG319A & 25 & 98 & 222 & $<110$ & $<50$ & $<1$ & $<1$ & 1 & 7 \\
\hline 2011GG322A & 33 & 225 & 826 & $<110$ & $<50$ & $<1$ & $<1$ & 2 & 61 \\
\hline 2011GG339A & 20 & $<30$ & 38 & $<110$ & $<50$ & $<1$ & $<1$ & 68 & $<7$ \\
\hline 2011GG342A & 37 & 604 & 37 & $<110$ & $<50$ & $<1$ & $<1$ & 1 & 105 \\
\hline 2011GG343B & $<10$ & $<30$ & 40 & $<110$ & $<50$ & $<1$ & $<1$ & 34 & $<7$ \\
\hline 2011GG346A & 32 & 108 & 29 & $<110$ & $<50$ & $<1$ & $<1$ & 9 & 54 \\
\hline 2011GG347A & 68 & 429 & 54 & 834 & $<50$ & $<1$ & $<1$ & 1 & 290 \\
\hline 2011GG350A & $<10$ & 159 & 92.2 & $<110$ & $<50$ & $<1$ & $<1$ & 16 & 53.7 \\
\hline 2011GG382A & 62 & 79 & 102 & $<110$ & $<50$ & $<1$ & $<1$ & 8 & 102 \\
\hline 2011GG383B & 57 & 446 & 70 & 151 & $<50$ & $<1$ & $<1$ & 3 & 116 \\
\hline 2011GG385A & 23 & $<30$ & 153 & 488 & $<50$ & $<1$ & $<1$ & 7 & 9 \\
\hline 2011GG392A & 24 & 226 & 32 & $<110$ & $<50$ & $<1$ & $<1$ & 9 & 60 \\
\hline 2011GG395A & 59 & 125 & 136 & 1900 & $<50$ & $<1$ & $<1$ & 14 & 123 \\
\hline 2011GG448A & 29 & 82 & 17 & $<110$ & $<50$ & $<1$ & $<1$ & 13 & 46 \\
\hline 2011GG450A & $<10$ & 39 & 62 & $<110$ & $<50$ & $<1$ & $<1$ & 4 & 31 \\
\hline 2011GG458A & 27 & 42 & 46 & 1030 & $<50$ & $<1$ & $<1$ & 2 & 32 \\
\hline 2011GG472A & 13 & 184 & 30 & $<110$ & $<50$ & $<1$ & $<1$ & 6 & 75 \\
\hline 2011GG485A & 11 & 426 & 102 & $<110$ & $<50$ & $<1$ & $<1$ & 6 & 108 \\
\hline 2011GG494A & 82 & 362 & 55 & $<110$ & $<50$ & $<1$ & $<1$ & 2 & 136 \\
\hline 2011GG495A & 76 & 121 & 124 & $<110$ & $<50$ & $<1$ & $<1$ & 3 & 20 \\
\hline
\end{tabular}


Table 8. (continued)

\begin{tabular}{|c|c|c|c|c|c|c|c|c|c|}
\hline Sample_ID & Pb_ppm & Rb_ppm & Rb_ppm_2 & S_ppm & Sb_ppm & Sn_ppm & Sr_ppm & Sr_ppm_2 & Th_ppm \\
\hline 2011GG186A & $<4$ & $<1$ & --- & 67 & $<2$ & $<4$ & $<1$ & --- & $<2$ \\
\hline 2011GG190A & 29 & $<1$ & 1 & 86 & $<2$ & 19 & $<1$ & 155 & $<2$ \\
\hline 2011GG191A & 23 & $<1$ & 5 & 85 & $<2$ & $<4$ & $<1$ & 227 & $<2$ \\
\hline 2011GG193A & $<4$ & $<1$ & 1 & 140 & $<2$ & $<4$ & $<1$ & 53 & $<2$ \\
\hline 2011GG193B & $<4$ & $<1$ & 5 & 67 & $<2$ & 13 & $<1$ & 288 & $<2$ \\
\hline 2011GG205A & 33 & $<1$ & 1 & 80 & $<2$ & $<4$ & $<1$ & 45 & $<2$ \\
\hline 2011GG224A & 25 & $<1$ & 18 & 218 & $<2$ & 21 & $<1$ & 200 & $<2$ \\
\hline $2011 G G 225 A$ & 19 & $<1$ & 4 & 145 & $<2$ & $<4$ & $<1$ & 166 & $<2$ \\
\hline $2011 G G 226 \mathrm{~A}$ & $<4$ & $<1$ & 5 & 44 & $<2$ & $<4$ & $<1$ & 259 & $<2$ \\
\hline 2011GG229A & $<4$ & $<1$ & 1 & 266 & $<2$ & $<4$ & $<1$ & 66 & $<2$ \\
\hline $2011 G G 271 \mathrm{~A}$ & 38 & $<1$ & 99 & 49 & $<2$ & $<4$ & $<1$ & 152 & $<2$ \\
\hline $2011 G G 275 \mathrm{~A}$ & 46 & $<1$ & 128 & 336 & $<2$ & $<4$ & $<1$ & 198 & $<2$ \\
\hline 2011GG319A & 25 & $<1$ & 3 & 284 & $<2$ & $<4$ & $<1$ & 179 & $<2$ \\
\hline 2011GG322A & $<4$ & $<1$ & 6 & 135 & $<2$ & $<4$ & $<1$ & 301 & $<2$ \\
\hline 2011GG339A & 23.7 & $<1$ & 618 & 239 & $<2$ & 25.9 & $<1$ & 17 & 32.7 \\
\hline 2011GG342A & $<4$ & $<1$ & 4 & 207 & $<2$ & $<4$ & $<1$ & 308 & $<2$ \\
\hline 2011GG343B & 35.6 & $<1$ & 677.7 & 151 & $<2$ & 29 & $<1$ & 5 & 22.5 \\
\hline 2011GG346A & 25 & $<1$ & 85 & 51 & $<2$ & $<4$ & $<1$ & 74 & $<2$ \\
\hline 2011GG347A & 20 & $<1$ & 2 & 67 & $<2$ & $<4$ & $<1$ & 144 & $<2$ \\
\hline 2011GG350A & 46.5 & $<1$ & 133.8 & 1410 & $<2$ & $<4$ & $<1$ & 108.5 & $<2$ \\
\hline 2011GG382A & 660 & $<1$ & 8 & 696 & $<2$ & $<4$ & $<1$ & 5 & $<2$ \\
\hline 2011GG383B & 25 & $<1$ & 1 & 389 & $<2$ & $<4$ & $<1$ & 147 & $<2$ \\
\hline $2011 G G 385 A$ & $<4$ & $<1$ & 1 & 211 & $<2$ & $<4$ & $<1$ & 197 & $<2$ \\
\hline 2011GG392A & 47 & $<1$ & 4 & 131 & $<2$ & $<4$ & $<1$ & 182 & $<2$ \\
\hline 2011GG395A & 112 & $<1$ & 40 & 98 & $<2$ & $<4$ & $<1$ & 583 & $<2$ \\
\hline 2011GG448A & 31 & $<1$ & 78 & 103 & $<2$ & $<4$ & $<1$ & 92 & $<2$ \\
\hline 2011GG450A & 31 & $<1$ & 40 & 156 & $<2$ & $<4$ & $<1$ & 15 & $<2$ \\
\hline 2011GG458A & $<4$ & $<1$ & 11 & 605 & $<2$ & 19 & $<1$ & 17 & $<2$ \\
\hline 2011GG472A & $<4$ & $<1$ & $<1$ & 37 & $<2$ & $<4$ & $<1$ & 259 & $<2$ \\
\hline 2011GG485A & 21 & $<1$ & 10 & 148 & $<2$ & $<4$ & $<1$ & 247 & $<2$ \\
\hline 2011GG494A & 29 & $<1$ & 1 & 108 & $<2$ & $<4$ & $<1$ & 125 & $<2$ \\
\hline 2011GG495A & $<4$ & $<1$ & 1 & 858 & $<2$ & $<4$ & $<1$ & 81 & $<2$ \\
\hline
\end{tabular}


Table 8. (continued)

\begin{tabular}{|c|c|c|c|c|c|c|c|c|}
\hline Sample_ID & U_ppm & V_ppm & W_ppm & Y_ppm & Y_ppm_2 & Zn_ppm & Zr_ppm & Zr_ppm_2 \\
\hline 2011GG186A & $<1$ & 210 & $<8$ & $2<1$ & $\begin{array}{c}-- \\
\end{array}$ & 93 & $<9$ & $<9$ \\
\hline 2011GG190A & $<1$ & 441 & $<8$ & $<1$ & 22 & 74 & $<9$ & 85 \\
\hline 2011GG191A & $<1$ & 414 & $<8$ & $<1$ & 25 & 65 & $<9$ & 133 \\
\hline 2011GG193A & $<1$ & 367 & $<8$ & $<1$ & 22 & 126 & $<9$ & 92 \\
\hline 2011GG193B & $<1$ & 485 & $<8$ & $<1$ & 31 & 75 & $<9$ & 169 \\
\hline 2011GG205A & $<1$ & 378 & $<8$ & $<1$ & 17 & 76 & $<9$ & 74 \\
\hline 2011GG224A & $<1$ & 303 & $<8$ & $<1$ & 28 & 102 & $<9$ & 127 \\
\hline 2011GG225A & $<1$ & 221 & $<8$ & $<1$ & 24 & 113 & $<9$ & 119 \\
\hline 2011GG226A & $<1$ & 395 & $<8$ & $<1$ & 26 & 85 & $<9$ & 130 \\
\hline 2011GG229A & $<1$ & 469 & $<8$ & $<1$ & 24 & 189 & $<9$ & 49 \\
\hline 2011GG271A & $<1$ & 114 & $<8$ & $<1$ & 22 & 45 & $<9$ & 134 \\
\hline 2011GG275A & $<1$ & 190 & $<8$ & $<1$ & 30 & 111 & $<9$ & 222 \\
\hline 2011GG319A & $<1$ & 405 & $<8$ & $<1$ & 31 & 104 & $<9$ & 60 \\
\hline 2011GG322A & $<1$ & 460 & $<8$ & $<1$ & 32 & 74 & $<9$ & 66 \\
\hline 2011GG339A & 30.7 & $<25$ & $<8$ & $<1$ & 28 & 22 & $<9$ & 81 \\
\hline 2011GG342A & $<1$ & 157 & $<8$ & $<1$ & 11 & 43 & $<9$ & 14 \\
\hline 2011GG343B & 22.3 & $<8$ & 31.3 & $<1$ & 144 & 28 & $<9$ & 92 \\
\hline 2011GG346A & $<1$ & 187 & $<8$ & $<1$ & 15 & 52 & $<9$ & 134 \\
\hline 2011GG347A & $<1$ & 229 & $<8$ & $<1$ & 17 & 64 & $<9$ & 26 \\
\hline 2011GG350A & $<1$ & 288 & $<8$ & $<1$ & 26.6 & 125 & $<9$ & 202.7 \\
\hline 2011GG382A & $<1$ & 485 & $<8$ & $<1$ & 11 & 545 & $<9$ & 97 \\
\hline 2011GG383B & $<1$ & 464 & $<8$ & $<1$ & 37 & 112 & $<9$ & 96 \\
\hline 2011GG385A & $<1$ & 288 & $<8$ & $<1$ & 34 & 81 & $<9$ & 153 \\
\hline 2011GG392A & $<1$ & 351 & $<8$ & $<1$ & 22 & 79 & $<9$ & 106 \\
\hline 2011GG395A & $<1$ & 226 & $<8$ & $<1$ & 22 & 79 & $<9$ & 110 \\
\hline 2011GG448A & $<1$ & 77 & $<8$ & $<1$ & 13 & 79 & $<9$ & 239 \\
\hline 2011GG450A & $<1$ & 39 & $<8$ & $<1$ & 9 & 24 & $<9$ & 127 \\
\hline 2011GG458A & $<1$ & 118 & $<8$ & $<1$ & 3 & 17 & $<9$ & 15 \\
\hline 2011GG472A & $<1$ & 515 & $<8$ & $<1$ & 35 & 95 & $<9$ & 111 \\
\hline 2011GG485A & $<1$ & 310 & $<8$ & $<1$ & 19 & 72 & $<9$ & 79 \\
\hline 2011GG494A & $<1$ & 305 & $<8$ & $<1$ & 28 & 77 & $<9$ & 63 \\
\hline 2011GG495A & $<1$ & 551 & $<8$ & $<1$ & 47 & 142 & $<9$ & 114 \\
\hline
\end{tabular}


Table 8. (continued)

\begin{tabular}{|c|c|c|c|c|c|c|c|}
\hline Sample_ID & Latitude & Longitude & Easting & Northing & $\begin{array}{l}\text { UTM_ } \\
\text { Zone }\end{array}$ & Root_name & Comments \\
\hline 2011GG497A & 65.386666 & $\mid-152.950124$ & $\mid 502318$ & 7251358 & $5 \mathrm{~N}$ & Quartz muscovite schist & Porous, small \\
\hline 2011GG518A & 65.376954 & -152.578923 & 519577 & 7250340 & $5 \mathrm{~N}$ & Fine-grained gabbro & --- \\
\hline 2011GG519A & 65.377805 & -152.578329 & 519604 & 7250435 & $5 \mathrm{~N}$ & Gabbro & Altered \\
\hline 2011GG520A & 65.469542 & -152.603466 & 518371 & 7260652 & $5 \mathrm{~N}$ & Fine-grained gabbro & Altered \\
\hline 2011GG522A & 65.471235 & -152.602426 & 518418 & 7260841 & $5 \mathrm{~N}$ & Greenstone & Inhomogenous \\
\hline 2011GG523A & 65.474621 & -152.604058 & 518340 & 7261218 & $5 \mathrm{~N}$ & Gabbro & Altered \\
\hline 2011GG523B & 65.474621 & -152.604058 & 518340 & 7261218 & $5 \mathrm{~N}$ & Albite quartz schist & Small \\
\hline 2011GG524A & 65.477345 & -152.602419 & 518414 & 7261522 & $5 \mathrm{~N}$ & Mafic rock & --- \\
\hline 2011GG547B & 65.315870 & -152.518719 & 522428 & 7243552 & $5 \mathrm{~N}$ & Metamafic & Porous \\
\hline 2011GG564A & 65.388016 & -152.962559 & 501740 & 7251508 & $5 \mathrm{~N}$ & Schist & Inhomogenous \\
\hline 2011GG564B & 65.388016 & -152.962559 & 501740 & 7251508 & $5 \mathrm{~N}$ & Muscovite quartz schist & --- \\
\hline 2011LF006A & 65.246146 & -152.864845 & 506315 & 7235702 & $5 \mathrm{~N}$ & Mafic schist & --- \\
\hline 2011LF007B & 65.421377 & -152.585924 & 519219 & 7255289 & $5 \mathrm{~N}$ & Mafic schist & --- \\
\hline 2011LF012A & 65.291305 & -152.857496 & 506647 & 7240736 & $5 \mathrm{~N}$ & Mafic schist & --- \\
\hline 2011LF032A & 65.324191 & -152.978427 & 501005 & 7244394 & $5 \mathrm{~N}$ & Mafic schist & Small \\
\hline 2011LF051B & 65.358815 & -152.811563 & 508767 & 7248266 & $5 \mathrm{~N}$ & Quartz-chlorite-muscovite schist & --- \\
\hline 2011LF052A & 65.359721 & -152.804184 & 509110 & 7248368 & $5 \mathrm{~N}$ & Chlorite-muscovite quartz schist & -- \\
\hline 2011LF055A & 65.361074 & -152.796178 & 509482 & 7248520 & $5 \mathrm{~N}$ & Chlorite-plagioclase quartz schist & --- \\
\hline 2011LF057A & 65.361833 & -152.793442 & 509609 & 7248605 & $5 \mathrm{~N}$ & Mafic schist & --- \\
\hline 2011LF063B & 65.366198 & \begin{tabular}{|l|}
-152.777734 \\
\end{tabular} & 510338 & 7249094 & $5 \mathrm{~N}$ & Mafic schist & Small \\
\hline 2011LF069C & 65.452126 & -152.729205 & 512554 & 7258680 & $5 \mathrm{~N}$ & Mafic schist & --- \\
\hline 2011LF069C & 65.452126 & -152.729205 & 512554 & 7258680 & $5 \mathrm{~N}$ & Albite-muscovite quartz schist & --- \\
\hline 2011LF073A & 65.447917 & -152.713915 & 513265 & 7258214 & $5 \mathrm{~N}$ & Gabbro (?) & --- \\
\hline 2011LF073B & 65.447917 & -152.713915 & 513265 & 7258214 & $5 \mathrm{~N}$ & Gabbro(?) & --- \\
\hline 2011LF076A & 65.443728 & \begin{tabular}{|l|}
-152.710079 \\
\end{tabular} & 513445 & 7257748 & $5 \mathrm{~N}$ & Mafic schist & --- \\
\hline 2011LF079A & 65.441167 & -152.698982 & 513961 & 7257465 & $5 \mathrm{~N}$ & Mafic schist & --- \\
\hline 2011LF099B & 65.500417 & -152.866859 & 506161 & 7264042 & $5 \mathrm{~N}$ & Orthogneiss(?) & Small \\
\hline 2011LF099C & 65.500417 & \begin{tabular}{|c|}
-152.866859 \\
\end{tabular} & 506161 & 7264042 & $5 \mathrm{~N}$ & Amphibolite & --- \\
\hline 2011LF100B & 65.500642 & -152.868155 & 506101 & 7264067 & $5 \mathrm{~N}$ & Mafic schist & --- \\
\hline 2011LF105A & 65.507034 & -152.883146 & 505406 & 7264778 & $5 \mathrm{~N}$ & Ultramafic & --- \\
\hline 2011LF113A & 65.430340 & -152.825511 & 508096 & 7256236 & $5 \mathrm{~N}$ & Quartz-chlorite-albite schist & --- \\
\hline 2011LF114A & 65.430806 & -152.833569 & 507722 & 7256287 & $5 \mathrm{~N}$ & Chlorite-muscovite quartz schist & --- \\
\hline
\end{tabular}


Table 8. (continued)

\begin{tabular}{|c|c|c|c|c|c|c|c|c|c|}
\hline Sample_ID & $\mathrm{SiO}_{2 \_} \mathrm{pct}$ & Al203_pct & BaO_pct & CaO_pct & FeO_pct & K2O_pct & MgO_pct & MnO_pct & $\mathrm{Na} 2 \mathrm{O} \_p c t$ \\
\hline 2011GG497A & 68.1 & 18.1 & 0.163 & 0.25 & 5.62 & 3.52 & 1.59 & 0.0385 & 0.734 \\
\hline 2011GG518A & 57.8 & 11.5 & $<0.03$ & 9 & 11.9 & 0.07 & 5.69 & 0.21 & 2.08 \\
\hline 2011GG519A & 49.3 & 16.1 & 0.07 & 11.1 & 10.9 & 0.69 & 5.79 & 0.17 & 3.98 \\
\hline 2011GG520A & 51.1 & 15.1 & 0.05 & 10.8 & 12.4 & 0.12 & 4.89 & 0.23 & 3.41 \\
\hline 2011GG522A & 46.6 & 12.5 & 0.07 & 10.3 & 17.6 & 0.63 & 6.32 & 0.21 & 2.73 \\
\hline 2011GG523A & 50.1 & 15.7 & 0.06 & 11.1 & 11.5 & 0.13 & 5.86 & 0.19 & 3.61 \\
\hline 2011GG523B & 81.5 & 7.18 & 0.0347 & 1.11 & 3.29 & 0.0944 & 1.06 & 0.0639 & 5.15 \\
\hline 2011GG524A & 51.3 & 15.4 & 0.126 & 11.1 & 9.62 & 0.204 & 5.7 & 0.18 & 4.22 \\
\hline 2011GG547B & 57.9 & 16.2 & 0.03 & 2.09 & 10.2 & 0.18 & 4.87 & 0.12 & 5.52 \\
\hline 2011GG564A & 71.2 & 16.3 & 0.0692 & 0.367 & 4.55 & 3.01 & 1.5 & 0.0377 & 2.09 \\
\hline 2011GG564B & 68.9 & 18.2 & 0.0851 & 0.26 & 4.95 & 4.44 & 1.49 & 0.0383 & 0.641 \\
\hline 2011LF006A & 51.1 & 12.4 & 0.0313 & 3.21 & 19.8 & 0.0337 & 7.54 & 0.187 & 3.23 \\
\hline 2011LF007B & 46.7 & 9.49 & $<0.03$ & 10.4 & 12.8 & $<.02$ & 19.5 & 0.221 & 0.271 \\
\hline 2011LF012A & 55 & 17.6 & $<0.03$ & 1.87 & 13.9 & 0.0262 & 4.02 & 0.151 & 4.58 \\
\hline 2011LF032A & 49.1 & 16.5 & $<0.03$ & 12.7 & 9.81 & 0.0269 & 10.8 & 0.193 & 0.286 \\
\hline 2011LF051B & 46.3 & 30.3 & 0.212 & 0.0547 & 11.5 & 5.83 & 3.09 & 0.057 & 0.944 \\
\hline 2011LF052A & 76.1 & 12.9 & 0.0903 & 0.364 & 4.49 & 2.53 & 1.61 & 0.0477 & 0.803 \\
\hline 2011LF055A & 73.1 & 10.6 & $<0.03$ & 4.55 & 5.67 & 0.312 & 2.62 & 0.102 & 1.6 \\
\hline 2011LF057A & 49.8 & 14.5 & 0.0582 & 16.3 & 10.9 & 1.04 & 4.62 & 0.2 & 1.15 \\
\hline 2011LF063B & 56.4 & 19.9 & 0.135 & 8.98 & 6.46 & 2.82 & 2.99 & 0.0905 & 1.25 \\
\hline 2011LF069C & 53.7 & 16.6 & 0.191 & 0.55 & 15.9 & 2.39 & 6.68 & 0.125 & 0.0342 \\
\hline 2011LF069C & 87.9 & 7.5 & 0.0786 & 0.116 & 0.364 & 1.65 & 0.559 & $<0.03$ & 0.529 \\
\hline 2011LF073A & 53.7 & 14.3 & $<0.03$ & 12.8 & 6.61 & 0.0595 & 7.87 & 0.132 & 3.62 \\
\hline 2011LF073B & 51.2 & 18 & $<0.03$ & 9.13 & 8.68 & 0.104 & 7.3 & 0.256 & 4.68 \\
\hline 2011LF076A & 53.9 & 16.1 & $<0.03$ & 7.35 & 9.8 & 0.0503 & 4.71 & 0.168 & 6.68 \\
\hline 2011LF079A & 54.9 & 15.7 & $<0.03$ & 3.06 & 10.5 & 0.255 & 5.67 & 0.202 & 7.91 \\
\hline 2011LF099B & 70.1 & 13.6 & 0.355 & 6.31 & 2.91 & 2.35 & 2.1 & 0.0783 & 1.39 \\
\hline 2011LF099C & 48.5 & 19.3 & $<0.03$ & 13.2 & 8.33 & 0.098 & 7.71 & 0.183 & 1.51 \\
\hline 2011LF100B & 49.6 & 15.3 & 0.0761 & 9.64 & 11.3 & 0.426 & 7.54 & 0.25 & 4.18 \\
\hline 2011LF105A & 45.9 & 7.35 & $<0.03$ & 3.76 & 8.95 & $<.02$ & 33 & 0.168 & 0.0688 \\
\hline 2011LF113A & 60.1 & 13.3 & $<0.03$ & 2.42 & 13.4 & 0.0306 & 4.07 & 0.261 & 4.11 \\
\hline 2011LF114A & 67.4 & 17.7 & 0.098 & 0.311 & 7.89 & 3.32 & 1.62 & 0.169 & 0.501 \\
\hline
\end{tabular}


Table 8. (continued)

\begin{tabular}{|c|c|c|c|c|c|c|c|c|c|}
\hline Sample_ID & P2O5_pct & TiO2_pct & CO2_pct & Total_pct & As_ppm & Bi_ppm & Br_ppm & Ce_ppm & Cl_ppm \\
\hline 2011GG497A & 0.566 & 0.99 & $<0.05$ & 99.6715 & 15 & $<2$ & $<<$ & $<60$ & 122 \\
\hline 2011GG518A & 0.19 & 1.36 & $<0.05$ & 99.80199 & $<4$ & $<2$ & $<5$ & $<60$ & 108 \\
\hline 2011GG519A & 0.14 & 1.58 & $<0.05$ & 99.8205 & $<4$ & $<2$ & $<5$ & $<60$ & 289 \\
\hline 2011GG520A & 0.22 & 1.58 & $<0.05$ & 99.889 & $<4$ & $<2$ & $<5$ & $<60$ & 90 \\
\hline 2011GG522A & 0.23 & 2.57 & $<0.05$ & 99.7653 & $<4$ & $<2$ & $<5$ & $<60$ & 108 \\
\hline 2011GG523A & 0.2 & 1.3 & $<0.05$ & 99.7485 & $<4$ & $<2$ & $<5$ & $<60$ & 91 \\
\hline 2011GG523B & 0.13 & 0.262 & $<0.05$ & 99.875 & $<4$ & $<2$ & $<5$ & $<60$ & 722 \\
\hline 2011GG524A & 0.184 & 1.78 & $<0.05$ & 99.814 & $<4$ & $<2$ & $<5$ & $<60$ & 197 \\
\hline 2011GG547B & 0.46 & 2.28 & $<0.05$ & 99.8539 & 12 & $<2$ & $<5$ & $<60$ & 143 \\
\hline 2011GG564A & 0.105 & 0.599 & $<0.05$ & 99.8279 & $<4$ & $<2$ & $<5$ & $<60$ & 201 \\
\hline 2011GG564B & 0.0904 & 0.755 & $<0.05$ & 99.8498 & $<4$ & $<2$ & $<5$ & $<60$ & 204 \\
\hline 2011LF006A & 0.212 & 2.15 & $<0.05$ & 99.894 & $<4$ & $<2$ & $<5$ & $<60$ & 53 \\
\hline 2011LF007B & $<0.03$ & 0.381 & $<0.05$ & 99.81018 & $<4$ & $<2$ & $<5$ & $<60$ & 81 \\
\hline 2011LF012A & 0.332 & 2.23 & $<0.05$ & 99.7344 & 50 & $<2$ & $<5$ & $<60$ & $<50$ \\
\hline 2011LF032A & 0.0494 & 0.313 & $<0.05$ & 99.7924 & $<4$ & $<2$ & $<5$ & $<60$ & 89 \\
\hline 2011LF051B & $<0.03$ & 1.52 & $<0.05$ & 99.8207 & $<4$ & $<2$ & $<5$ & $<60$ & 156 \\
\hline 2011LF052A & 0.274 & 0.557 & $<0.05$ & 99.766 & $<4$ & $<2$ & $<5$ & 363 & 196 \\
\hline 2011LF055A & 0.441 & 0.739 & $<0.05$ & 99.7606 & $<4$ & $<2$ & $<5$ & $<60$ & 295 \\
\hline 2011LF057A & 0.161 & 1.01 & $<0.05$ & 99.7392 & 63 & $<2$ & $<5$ & $<60$ & 201 \\
\hline 2011LF063B & $<0.03$ & 0.763 & $<0.05$ & 99.8038 & 5 & $<2$ & $<5$ & $<60$ & 460 \\
\hline 2011LF069C & 0.669 & 2.42 & $<0.05$ & 99.2592 & $<4$ & $<2$ & $<5$ & $<60$ & 259 \\
\hline 2011LF069C & 0.0458 & 1.14 & $<0.05$ & 99.8989 & $<4$ & $<2$ & $<5$ & $<60$ & 133 \\
\hline 2011LF073A & $<0.03$ & 0.779 & $<0.05$ & 99.9285 & $<4$ & $<2$ & $<5$ & $<60$ & $<50$ \\
\hline 2011LF073B & 0.0753 & 0.454 & $<0.05$ & 97.8793 & $<4$ & $<2$ & $<5$ & $<60$ & 100 \\
\hline 2011LF076A & 0.19 & 0.906 & $<0.05$ & 99.8721 & $<4$ & $<2$ & $<5$ & $<60$ & $<50$ \\
\hline 2011LF079A & 0.15 & 0.735 & $<0.05$ & 99.0996 & $<4$ & $<2$ & $<5$ & $<60$ & 258 \\
\hline 2011LF099B & 0.249 & 0.406 & $<0.05$ & 99.8483 & $<4$ & $<2$ & $<5$ & $<60$ & 156 \\
\hline 2011LF099C & 0.163 & 0.739 & $<0.05$ & 99.7498 & 86 & $<2$ & $<5$ & $<60$ & 388 \\
\hline 2011LF100B & 0.244 & 1.32 & $<0.05$ & 99.8761 & $<4$ & $<2$ & $<5$ & $<60$ & 165 \\
\hline 2011LF105A & $<0.03$ & 0.149 & $<0.05$ & 99.38599 & $<4$ & $<2$ & $<5$ & $<60$ & 171 \\
\hline 2011LF113A & 0.584 & 1.63 & $<0.05$ & 99.9185 & $<4$ & $<2$ & $<5$ & $<60$ & 150 \\
\hline 2011LF114A & 0.156 & 0.763 & $<0.05$ & 99.928 & 59 & $<2$ & $<5$ & $<60$ & 119 \\
\hline
\end{tabular}


Table 8. (continued)

\begin{tabular}{|c|c|c|c|c|c|c|c|c|c|}
\hline Sample_ID & Co_ppm & Cr_ppm & Cu_ppm & F_ppm & La_ppm & Mo_ppm & Nb_ppm & Nb_ppm_2 & Ni_ppm \\
\hline 2011GG497A & 52 & 183 & 16 & 1300 & $<50$ & $<<1$ & $<<1$ & 16 & 16 \\
\hline 2011GG518A & 117 & 189 & 31 & $<110$ & $<50$ & $<1$ & $<1$ & 8 & 75 \\
\hline 2011GG519A & 57 & 184 & 93 & $<110$ & $<50$ & $<1$ & $<1$ & 5 & 114 \\
\hline 2011GG520A & 77 & 180 & 165 & 199 & $<50$ & $<1$ & $<1$ & 7 & 52 \\
\hline 2011GG522A & 121 & 306 & 87 & $<110$ & $<50$ & $<1$ & $<1$ & 7 & 135 \\
\hline 2011GG523A & 64 & 165 & 85 & $<110$ & $<50$ & $<1$ & $<1$ & 6 & 69 \\
\hline 2011GG523B & $<10$ & 30 & $<7$ & $<110$ & $<50$ & $<1$ & $<1$ & 8 & 58 \\
\hline 2011GG524A & 83 & 156 & 177 & $<110$ & $<50$ & $<1$ & $<1$ & 8 & 67 \\
\hline 2011GG547B & 54 & 225 & 49 & $<110$ & $<50$ & $<1$ & $<1$ & 26 & 69 \\
\hline 2011GG564A & 106 & 124 & 91 & $<110$ & $<50$ & $<1$ & $<1$ & 12 & 55 \\
\hline 2011GG564B & 30 & 100 & 45 & $<110$ & $<50$ & $<1$ & $<1$ & 13 & 42 \\
\hline 2011LF006A & 10 & 157 & 16 & $<110$ & $<50$ & $<1$ & $<1$ & 11 & 46 \\
\hline 2011LF007B & 107 & 1600 & 170 & $<110$ & $<50$ & $<1$ & $<1$ & 1 & 640 \\
\hline 2011LF012A & 48 & 68 & 54 & $<110$ & $<50$ & $<1$ & $<1$ & 14 & 20 \\
\hline 2011LF032A & 119 & 413 & 56 & $<110$ & $<50$ & $<1$ & $<1$ & 4 & 347 \\
\hline 2011LF051B & 121 & 231 & 90 & 121 & $<50$ & $<1$ & 26 & 22 & 73 \\
\hline 2011LF052A & 35 & 83 & 18 & $<110$ & $<50$ & $<1$ & 5 & --- & 31 \\
\hline 2011LF055A & $<10$ & 103 & 48 & 1390 & $<50$ & $<1$ & 9 & --- & 24 \\
\hline 2011LF057A & 51 & 504 & 49 & $<110$ & $<50$ & $<1$ & $<1$ & 7 & 180 \\
\hline 2011LF063B & 23 & 165 & $<7$ & $<110$ & $<50$ & $<1$ & $<1$ & 8 & 30 \\
\hline 2011LF069C & 116 & 227 & 70 & $<110$ & $<50$ & $<1$ & $<1$ & 14 & 259 \\
\hline 2011LF069C & $<10$ & 89 & 43 & 114 & $<50$ & $<1$ & 24 & --- & 22 \\
\hline 2011LF073A & 67 & 457 & 12 & $<110$ & $<50$ & $<1$ & $<1$ & 1 & 93 \\
\hline 2011LF073B & 38 & 169 & 45 & $<110$ & $<50$ & $<1$ & $<1$ & 1 & 130 \\
\hline 2011LF076A & 103 & 82 & 139 & $<110$ & $<50$ & $<1$ & $<1$ & 2 & 85 \\
\hline 2011LF079A & 49 & 57 & 74 & $<110$ & $<50$ & $<1$ & $<1$ & 1 & 49 \\
\hline 2011LF099B & 26 & 51 & 22 & 592 & $<50$ & $<1$ & 10 & --- & 30 \\
\hline 2011LF099C & 78 & 337 & $<7$ & $<110$ & $<50$ & $<1$ & $<1$ & 1 & 158 \\
\hline 2011LF100B & 63 & 383 & 16 & $<110$ & $<50$ & $<1$ & $<1$ & 9 & 67 \\
\hline 2011LF105A & 125 & 3910 & $<7$ & $<110$ & $<50$ & $<1$ & $<1$ & --- & 2400 \\
\hline 2011LF113A & 53 & 45 & $<7$ & $<110$ & $<50$ & $<1$ & 11 & 13 & 68 \\
\hline 2011LF114A & 101 & 128 & 27 & $<110$ & $<50$ & $<1$ & 15 & 16 & 45 \\
\hline
\end{tabular}


Table 8. (continued)

\begin{tabular}{|c|c|c|c|c|c|c|c|c|c|}
\hline Sample_ID & Pb_ppm & Rb_ppm & Rb_ppm_2 & S_ppm & Sb_ppm & Sn_ppm & Sr_ppm & Sr_ppm_2 & Th_ppm \\
\hline 2011GG497A & 39 & $2<1$ & 165 & 672 & $<2$ & $<4$ & $<<1$ & 67 & $2<2$ \\
\hline 2011GG518A & $<4$ & $<1$ & 1 & 100 & $<2$ & 366 & $<1$ & 142 & $<2$ \\
\hline 2011GG519A & $<4$ & $<1$ & 20 & 126 & $<2$ & $<4$ & $<1$ & 192 & $<2$ \\
\hline 2011GG520A & 27 & $<1$ & 2 & 181 & $<2$ & $<4$ & $<1$ & 161 & $<2$ \\
\hline 2011GG522A & $<4$ & $<1$ & 18 & 70 & $<2$ & $<4$ & $<1$ & 215 & $<2$ \\
\hline 2011GG523A & $<4$ & $<1$ & 2 & 362 & $<2$ & $<4$ & $<1$ & 137 & $<2$ \\
\hline 2011GG523B & $<4$ & $<1$ & 2 & 189 & $<2$ & $<4$ & $<1$ & 37 & $<2$ \\
\hline 2011GG524A & $<4$ & $<1$ & 2 & 108 & $<2$ & $<4$ & $<1$ & 204 & $<2$ \\
\hline 2011GG547B & $<4$ & $<1$ & 3 & 432 & $<2$ & $<4$ & $<1$ & 34 & $<2$ \\
\hline 2011GG564A & 39 & $<1$ & 123 & 259 & $<2$ & $<4$ & $<1$ & 120 & $<2$ \\
\hline 2011GG564B & 45 & $<1$ & 150 & 72 & $<2$ & $<4$ & $<1$ & 78 & $<2$ \\
\hline 2011LF006A & $<4$ & $<1$ & 1 & 133 & $<2$ & $<4$ & $<1$ & 59 & $<2$ \\
\hline 2011LF007B & $<4$ & $<1$ & 1 & 80 & $<2$ & $<4$ & $<1$ & 27 & $<2$ \\
\hline 2011LF012A & 22 & $<1$ & 1 & 83 & $<2$ & $<4$ & $<1$ & 77 & $<2$ \\
\hline 2011LF032A & $<4$ & $<1$ & 5 & 101 & $<2$ & $<4$ & $<1$ & 100 & $<2$ \\
\hline 2011LF051B & 34 & 317 & 258 & 104 & $<2$ & $<4$ & 93 & 77 & $<2$ \\
\hline 2011LF052A & 34 & 105 & --- & 123 & $<2$ & $<4$ & 52 & --- & $<2$ \\
\hline 2011LF055A & 45 & 20 & --- & 114 & $<2$ & $<4$ & 163 & --- & $<2$ \\
\hline 2011LF057A & 29 & $<1$ & 56 & 88 & $<2$ & $<4$ & $<1$ & 94 & $<2$ \\
\hline 2011LF063B & 36 & $<1$ & 173 & 111 & $<2$ & $<4$ & $<1$ & 178 & $<2$ \\
\hline 2011LF069C & $<4$ & $<1$ & 47 & 4460 & $<2$ & $<4$ & $<1$ & 10 & $<2$ \\
\hline 2011LF069C & 17 & 40 & --- & 196 & $<2$ & $<4$ & 68 & --- & $<2$ \\
\hline 2011LF073A & $<4$ & $<1$ & 2 & 37 & $<2$ & $<4$ & $<1$ & 127 & $<2$ \\
\hline 2011LF073B & $<4$ & $<1$ & 2 & 64 & $<2$ & $<4$ & $<1$ & 88 & $<2$ \\
\hline 2011LF076A & $<4$ & $<1$ & 1 & 37 & $<2$ & $<4$ & $<1$ & 98 & $<2$ \\
\hline 2011LF079A & $<4$ & $<1$ & 5 & 8040 & $<2$ & $<4$ & $<1$ & 50 & $<2$ \\
\hline 2011LF099B & 44 & 72 & --- & 36 & $<2$ & $<4$ & 391 & --- & $<2$ \\
\hline 2011LF099C & 28 & $<1$ & 3 & 1310 & $<2$ & $<4$ & $<1$ & 321 & $<2$ \\
\hline 2011LF100B & $<4$ & $<1$ & 13 & $<30$ & $<2$ & $<4$ & $<1$ & 312 & $<2$ \\
\hline 2011LF105A & $<4$ & $<1$ & --- & 158 & $<2$ & $<4$ & $<1$ & --- & $<2$ \\
\hline 2011LF113A & 26 & $<1$ & $<1$ & 159 & $<2$ & $<4$ & 143 & 128 & $<2$ \\
\hline 2011LF114A & $<4$ & 154 & 148 & 31 & $<2$ & $<4$ & 37 & 42 & $<2$ \\
\hline
\end{tabular}




\begin{tabular}{|c|c|c|c|c|c|c|c|c|}
\hline Sample_ID & U_ppm & V_ppm & W_ppm & Y_ppm & Y_ppm_2 & Zn_ppm & Zr_ppm & Zr_ppm_2 \\
\hline 2011GG497A & $2<1$ & 247 & $<<8$ & $<1$ & 23 & 134 & $<9$ & 211 \\
\hline 2011GG518A & $<1$ & 397 & $<8$ & $<1$ & 28 & 76 & $<9$ & 98 \\
\hline 2011GG519A & $<1$ & 465 & $<8$ & $<1$ & 27 & 84 & $<9$ & 98 \\
\hline 2011GG520A & $<1$ & 441 & $<8$ & $<1$ & 32 & 123 & $<9$ & 109 \\
\hline 2011GG522A & $<1$ & 622 & $<8$ & $<1$ & 29 & 126 & $<9$ & 131 \\
\hline 2011GG523A & $<1$ & 412 & $<8$ & $<1$ & 25 & 110 & $<9$ & 97 \\
\hline 2011GG523B & $<1$ & 89 & $<8$ & $<1$ & 17 & 26 & $<9$ & 62 \\
\hline 2011GG524A & $<1$ & 447 & $<8$ & $<1$ & 28 & 76 & $<9$ & 110 \\
\hline 2011GG547B & $<1$ & 434 & $<8$ & $<1$ & 19 & 97 & $<9$ & 187 \\
\hline 2011GG564A & $<1$ & 113 & $<8$ & $<1$ & 13 & 108 & $<9$ & 121 \\
\hline 2011GG564B & $<1$ & 141 & $<8$ & $<1$ & 19 & 79 & $<9$ & 139 \\
\hline 2011LF006A & $<1$ & 441 & $<8$ & $<1$ & 31 & 390 & $<9$ & 179 \\
\hline 2011LF007B & $<1$ & 195 & $<8$ & $<1$ & 6 & 87 & $<9$ & 21 \\
\hline 2011LF012A & $<1$ & 689 & $<8$ & $<1$ & 37 & 228 & $<9$ & 191 \\
\hline 2011LF032A & $<1$ & 171 & $<8$ & $<1$ & 23 & 68 & $<9$ & 36 \\
\hline 2011LF051B & $<1$ & 413 & $<8$ & 61 & 35 & 140 & 319 & 352 \\
\hline 2011LF052A & $<1$ & 145 & $<8$ & 34 & --- & 87 & 76 & $<9$ \\
\hline 2011LF055A & $<1$ & 146 & $<8$ & 39 & --- & 55 & 100 & --- \\
\hline 2011LF057A & $<1$ & 335 & $<8$ & $<1$ & 41 & 83 & $<9$ & 60 \\
\hline 2011LF063B & $<1$ & 410 & $<8$ & $<1$ & 18 & 66 & $<9$ & 220 \\
\hline 2011LF069C & $<1$ & 439 & $<8$ & $<1$ & 25 & 440 & $<9$ & 129 \\
\hline 2011LF069C & $<1$ & 219 & $<8$ & 48 & $\begin{array}{ll}--- \\
\end{array}$ & 26 & 196 & --- \\
\hline 2011LF073A & $<1$ & 299 & $<8$ & $<1$ & 14 & 38 & $<9$ & 22 \\
\hline 2011LF073B & $<1$ & 154 & $<8$ & $<1$ & 11 & 76 & $<9$ & 29 \\
\hline 2011LF076A & $<1$ & 343 & $<8$ & $<1$ & 29 & 77 & $<9$ & 89 \\
\hline 2011LF079A & $<1$ & 417 & $<8$ & $<1$ & 22 & 90 & $<9$ & 52 \\
\hline 2011LF099B & $<1$ & 70 & $<8$ & 35 & --- & 89 & 151 & --- \\
\hline 2011LF099C & $<1$ & 250 & $<8$ & $<1$ & 28 & 180 & $<9$ & 36 \\
\hline 2011LF100B & $<1$ & 389 & $<8$ & $<1$ & 25 & 84 & $<9$ & 112 \\
\hline 2011LF105A & $<1$ & 120 & $<8$ & $<1$ & $\overline{---}$ & 59 & $<9$ & $<9$ \\
\hline 2011LF113A & $<1$ & 477 & $<8$ & 46 & 48 & 236 & 140 & 182 \\
\hline 2011LF114A & $<1$ & 147 & $<8$ & 24 & 19 & 77 & 107 & 156 \\
\hline
\end{tabular}


Table 8. (continued)

\begin{tabular}{|c|c|c|c|c|c|c|c|}
\hline Sample_ID & Latitude & Longitude & Easting & Northing & $\begin{array}{l}\text { UTM_ } \\
\text { Zone }\end{array}$ & Root_name & Comments \\
\hline 2011LF127A & 65.427332 & -152.879083 & $\mid 505611$ & 7255895 & $5 \mathrm{~N}$ & Albite-muscovite quartz schist & |-- \\
\hline 2011LF130C & 65.254708 & -152.737909 & 512242 & 7236675 & $5 \mathrm{~N}$ & Chlorite-quartz-muscovite schist & --- \\
\hline 2011LF137A & 65.254653 & -152.764307 & 511009 & 7236664 & $5 \mathrm{~N}$ & $\begin{array}{l}\text { Albite-chlorite-muscovite- } \\
\text { Quartz schist }\end{array}$ & --- \\
\hline 2011LF141A & 65.253371 & -152.783008 & 510136 & 7236518 & $5 \mathrm{~N}$ & Albite-muscovite quartzite & --- \\
\hline 2011LF147A & 65.249292 & -152.805002 & 509110 & 7236060 & $5 \mathrm{~N}$ & Muscovite quartz schist & --- \\
\hline 2011LF149A & 65.248561 & -152.808604 & 508942 & 7235978 & $5 \mathrm{~N}$ & Albite-muscovite quartz schist & --- \\
\hline 2011LF153A & 65.312968 & -152.679140 & 514954 & 7243181 & $5 \mathrm{~N}$ & Muscovite-albite-chlorite-quartz schist & --- \\
\hline 2011LF164A & 65.310504 & -152.640766 & 516744 & 7242916 & $5 \mathrm{~N}$ & Mafic schist & --- \\
\hline 2011LF165A & 65.310589 & -152.638877 & 516832 & 7242926 & $5 \mathrm{~N}$ & ?-high magnesium, chromium, silicon & --- \\
\hline 2011LF167A & 65.309965 & -152.633157 & 517099 & 7242858 & $5 \mathrm{~N}$ & Mafic schist & --- \\
\hline 2011LF177A & 65.345649 & -152.707830 & 513600 & 7246817 & $5 \mathrm{~N}$ & Albite-muscovite-quartz schist & --- \\
\hline 2011LF181B & 65.345225 & -152.701712 & 513885 & 7246771 & $5 \mathrm{~N}$ & Mafic schist & --- \\
\hline 2011LF184A & 65.347249 & -152.691054 & 514380 & 7246999 & $5 \mathrm{~N}$ & Mafic schist & --- \\
\hline 2011LF186A & 65.348474 & -152.684465 & 514686 & 7247137 & $5 \mathrm{~N}$ & Mafic schist & --- \\
\hline 2011LF196A & 65.451681 & -152.498387 & 523255 & 7258696 & $5 \mathrm{~N}$ & Gabbro & Altered \\
\hline 2011LF200A & 65.446464 & -152.497279 & 523311 & 7258115 & $5 \mathrm{~N}$ & Mafic schist & --- \\
\hline 2011LF206A & 65.440355 & -152.503174 & 523043 & 7257432 & $5 \mathrm{~N}$ & Gabbro & --- \\
\hline 2011LF207C & 65.439035 & -152.505593 & 522932 & 7257284 & $5 \mathrm{~N}$ & Chlorite-quartz-albite schist & --- \\
\hline 2011LF209A & 65.435021 & -152.504461 & 522988 & 7256837 & $5 \mathrm{~N}$ & Gabbro & -- \\
\hline 2011LF218A & 65.428223 & -152.516572 & 522432 & 7256075 & $5 \mathrm{~N}$ & Gabbro & --- \\
\hline 2011LF219A & 65.420283 & -152.519455 & 522305 & 7255989 & $5 \mathrm{~N}$ & Mafic schist & -- \\
\hline 2011LF221A & 65.426475 & -152.522617 & 522153 & 7255878 & $5 \mathrm{~N}$ & Metagabbro & --- \\
\hline 2011LF232A & 65.412532 & -152.528212 & 521905 & 7254322 & $5 \mathrm{~N}$ & Mafic schist & --- \\
\hline 2011LF235A & 65.406861 & -152.528184 & 521911 & 7253690 & $5 \mathrm{~N}$ & Metagabbro & --- \\
\hline 2011LF238A & 65.401984 & -152.523514 & 522132 & 7253148 & $5 \mathrm{~N}$ & Gabbro & --- \\
\hline 2011LF239A & 65.324108 & -152.963787 & 501687 & 7244385 & $5 \mathrm{~N}$ & Mafic schist & --- \\
\hline 2011LF268B & 65.235238 & -152.765122 & 510979 & 7234500 & $5 \mathrm{~N}$ & Igneous(?) with feldspar(?) & Altered \\
\hline 2011LF276A & 65.341591 & -153.136439 & 493648 & 7246340 & $5 \mathrm{~N}$ & Gabbro & Altered \\
\hline 2011LF281A & 65.336915 & -153.147389 & 493137 & 7245820 & $5 \mathrm{~N}$ & Chlorite-albite schist & --- \\
\hline 2011LF285A & 65.334052 & -153.157573 & 492662 & 7245502 & $5 \mathrm{~N}$ & Metamafic & --- \\
\hline 2011LF288B & 65.330755 & -153.169555 & 492103 & 7245136 & $5 \mathrm{~N}$ & Mafic schist & Inhomogenous, altered \\
\hline
\end{tabular}


Table 8. (continued)

\begin{tabular}{|c|c|c|c|c|c|c|c|c|c|}
\hline Sample_ID & $\mathrm{SiO}_{2 \_} \mathrm{pct}$ & Al203_pct & BaO_pct & CaO_pct & FeO_pct & K2O_pct & MgO_pct & MnO_pct & Na2O_pct \\
\hline 2011LF127A & 87.2 & 5.16 & 0.0523 & 2.31 & 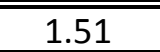 & 1.42 & 1.28 & 0.0498 & 0.671 \\
\hline 2011LF130C & 58 & 22.2 & 0.192 & 0.139 & 11 & 4.89 & 1.43 & 0.0769 & 0.772 \\
\hline 2011LF137A & 77.7 & 11.6 & 0.0708 & 0.169 & 3.64 & 2.46 & 1.78 & 0.0838 & 1.18 \\
\hline 2011LF141A & 93.3 & 4.06 & 0.0342 & 0.11 & 0.79 & 0.573 & 0.25 & $<0.03$ & 0.466 \\
\hline 2011LF147A & 87.2 & 7.04 & 0.121 & 0.0764 & 1.61 & 2.28 & 0.546 & $<0.03$ & 0.233 \\
\hline 2011LF149A & 69.9 & 16.1 & 0.118 & 0.115 & 5.71 & 3.99 & 1.89 & 0.182 & 1.19 \\
\hline 2011LF153A & 85.6 & 5.68 & 0.0544 & 0.216 & 3.53 & 0.832 & 2.47 & 0.0521 & 0.921 \\
\hline 2011LF164A & 57.5 & 13.1 & $<0.03$ & 3.49 & 11.8 & 0.0584 & 5.45 & 0.123 & 4.4 \\
\hline 2011LF165A & 63.1 & 11 & $<0.03$ & 1.54 & 12.4 & 0.0233 & 7.86 & 0.153 & 2.09 \\
\hline 2011LF167A & 54.4 & 14.7 & 0.0409 & 0.538 & 16.1 & 0.116 & 4.71 & 0.295 & 4.31 \\
\hline 2011LF177A & 72.5 & 15.1 & 0.0682 & 0.145 & 6.28 & 1.83 & 1.15 & 0.274 & 1.71 \\
\hline 2011LF181B & 51.1 & 13.6 & 0.0484 & 9.31 & 12 & 0.423 & 5.59 & 0.233 & 5.33 \\
\hline 2011LF184A & 52.6 & 15.8 & 0.0956 & 9.41 & 10.1 & 0.556 & 5.46 & 0.159 & 4.32 \\
\hline 2011LF186A & 53.3 & 16.6 & 0.0549 & 9.01 & 10.1 & 0.357 & 4.63 & 0.18 & 3.56 \\
\hline 2011LF196A & 49.5 & 15.2 & 0.0585 & 11.6 & 9.56 & 0.536 & 10 & 0.164 & 2.32 \\
\hline 2011LF200A & 50.8 & 11.2 & 0.0549 & 10.3 & 13 & 0.366 & 10.7 & 0.235 & 1.61 \\
\hline 2011LF206A & 48.7 & 15.7 & 0.0443 & 13.4 & 7.89 & 0.254 & 9.77 & 0.141 & 3 \\
\hline 2011LF207C & 68.9 & 14.8 & $<0.03$ & 0.791 & 3.77 & 0.0466 & 2.05 & 0.115 & 8.86 \\
\hline 2011LF209A & 47.7 & 15.1 & $<0.03$ & 11.9 & 12.9 & 0.0235 & 7 & 0.197 & 3.13 \\
\hline 2011LF218A & 50.1 & 15.2 & $<0.03$ & 12.6 & 9.82 & 0.0815 & 8 & 0.184 & 2.63 \\
\hline 2011LF219A & 55.6 & 15.2 & 0.0775 & 6.98 & 9.44 & 1.66 & 5 & 0.175 & 4.39 \\
\hline 2011LF221A & 52.1 & 14.9 & 0.13 & 8.43 & 10.4 & 0.884 & 6.82 & 0.246 & 4.36 \\
\hline 2011LF232A & 47.2 & 15.5 & 0.0646 & 13.5 & 11.3 & 0.929 & 7.44 & 0.193 & 1.87 \\
\hline 2011LF235A & 51.4 & 14.8 & 0.396 & 7.45 & 11.6 & 1.89 & 6.03 & 0.249 & 4.04 \\
\hline 2011LF238A & 45.3 & 16.2 & $<0.03$ & 19.5 & 6.81 & $<.02$ & 11 & 0.136 & 0.281 \\
\hline 2011LF239A & 46.5 & 18.9 & $<0.03$ & 17.1 & 8.19 & 0.0233 & 8.36 & 0.173 & 0.189 \\
\hline 2011LF268B & 89.7 & 5.45 & 0.0353 & 0.28 & 1.94 & 0.755 & 0.76 & $<0.03$ & 0.576 \\
\hline 2011LF276A & 55.5 & 12 & $<0.03$ & 11.9 & 10.5 & 0.0483 & 6.77 & 0.233 & 1.46 \\
\hline 2011LF281A & 62 & 14.3 & 0.149 & 4.6 & 8.59 & 0.543 & 1.82 & 0.15 & 5.86 \\
\hline 2011LF285A & 52.9 & 14.5 & 0.0302 & 7.47 & 12.3 & 0.0918 & 4.58 & 0.208 & 5.92 \\
\hline 2011LF288B & 51.8 & 16.6 & 0.219 & 8.83 & 10.9 & 1.21 & 4.75 & 0.174 & 4.14 \\
\hline
\end{tabular}


Table 8. (continued)

\begin{tabular}{|c|c|c|c|c|c|c|c|c|c|}
\hline Sample_ID & P2O5_pct & TiO2_pct & CO2_pct & Total_pct & As_ppm & Bi_ppm & Br_ppm & Ce_ppm & Cl_ppm \\
\hline 2011LF127A & 0.0917 & 0.233 & $<0.05$ & 99.9778 & $<4$ & $<2$ & $<5$ & $<60$ & 188 \\
\hline 2011LF130C & 0.139 & 0.97 & $<0.05$ & 99.8089 & $<4$ & $<2$ & $<5$ & $<60$ & 123 \\
\hline 2011LF137A & 0.285 & 0.711 & $<0.05$ & 99.6796 & 47 & $<2$ & $<5$ & $<60$ & 235 \\
\hline 2011LF141A & 0.07 & 0.25 & $<0.05$ & 99.91125 & 33 & $<2$ & $<5$ & $<60$ & 109 \\
\hline 2011LF147A & 0.0328 & 0.355 & $<0.05$ & 99.50323 & $<4$ & $<2$ & $<5$ & $<60$ & 186 \\
\hline 2011LF149A & 0.0497 & 0.613 & $<0.05$ & 99.8577 & $<4$ & $<2$ & $<5$ & $<60$ & 268 \\
\hline 2011LF153A & 0.203 & 0.361 & $<0.05$ & 99.9195 & $<4$ & $<2$ & $<5$ & $<60$ & 148 \\
\hline 2011LF164A & 0.881 & 2.88 & $<0.05$ & 99.6995 & 67 & $<2$ & $<5$ & $<60$ & 144 \\
\hline 2011LF165A & 0.27 & 1.28 & $<0.05$ & 99.7163 & 48 & $<2$ & $<5$ & $<60$ & 91 \\
\hline 2011LF167A & 0.623 & 3.93 & $<0.05$ & 99.7629 & 9 & $<2$ & $<5$ & $<60$ & 277 \\
\hline 2011LF177A & 0.101 & 0.673 & $<0.05$ & 99.8312 & 53 & $<2$ & $<5$ & $<60$ & 128 \\
\hline 2011LF181B & 0.163 & 1.52 & $<0.05$ & 99.3174 & $<4$ & $<2$ & $<5$ & $<60$ & 219 \\
\hline 2011LF184A & 0.292 & 1.05 & $<0.05$ & 99.8426 & 28 & $<2$ & $<5$ & $<60$ & 50 \\
\hline 2011LF186A & 0.319 & 1.67 & $<0.05$ & 99.7809 & 5 & $<2$ & $<5$ & $<60$ & 123 \\
\hline 2011LF196A & 0.138 & 0.681 & $<0.05$ & 99.7575 & $<4$ & $<2$ & $<5$ & $<60$ & 199 \\
\hline 2011LF200A & 0.097 & 1.39 & $<0.05$ & 99.7529 & $<4$ & $<2$ & $<5$ & $<60$ & 161 \\
\hline 2011LF206A & 0.104 & 0.771 & $<0.05$ & 99.7743 & $<4$ & $<2$ & $<5$ & $<60$ & 112 \\
\hline 2011LF207C & 0.0629 & 0.493 & $<0.05$ & 99.9102 & $<4$ & $<2$ & $<5$ & $<60$ & 70 \\
\hline 2011LF209A & 0.339 & 1.57 & $<0.05$ & 99.8889 & $<4$ & $<2$ & $<5$ & $<60$ & 149 \\
\hline 2011LF218A & 0.165 & 1.04 & $<0.05$ & 99.8446 & 5 & $<2$ & $<5$ & $<60$ & 61 \\
\hline 2011LF219A & 0.381 & 0.877 & $<0.05$ & 99.7805 & $<4$ & $<2$ & $<5$ & $<60$ & 104 \\
\hline 2011LF221A & 0.245 & 1.3 & $<0.05$ & 99.815 & $<4$ & $<2$ & $<5$ & $<60$ & 471 \\
\hline 2011LF232A & 0.252 & 1.5 & $<0.05$ & 99.7486 & 49 & $<2$ & $<5$ & $<60$ & 123 \\
\hline 2011LF235A & 0.22 & 1.68 & $<0.05$ & 99.755 & $<4$ & $<2$ & $<5$ & $<60$ & 292 \\
\hline 2011LF238A & 0.0608 & 0.511 & $<0.05$ & 99.82442 & $<4$ & $<2$ & $<5$ & $<60$ & 91 \\
\hline 2011LF239A & 0.0389 & 0.339 & $<0.05$ & 99.8292 & $<4$ & $<2$ & $<5$ & $<60$ & 110 \\
\hline 2011LF268B & 0.0691 & 0.302 & $<0.05$ & 99.87465 & 43 & $<2$ & $<5$ & $<60$ & 311 \\
\hline 2011LF276A & 0.195 & 1.09 & $<0.05$ & 99.7131 & $<4$ & $<2$ & $<5$ & $<60$ & 156 \\
\hline 2011LF281A & 0.473 & 1.39 & $<0.05$ & 99.875 & 4 & $<2$ & $<5$ & $<60$ & $<50$ \\
\hline 2011LF285A & 0.529 & 1.36 & $<0.05$ & 99.889 & 41 & $<2$ & $<5$ & $<60$ & 231 \\
\hline 2011LF288B & 0.214 & 1.1 & $<0.05$ & 99.937 & 56 & $<2$ & $<5$ & $<60$ & 210 \\
\hline
\end{tabular}


Table 8. (continued)

\begin{tabular}{|c|c|c|c|c|c|c|c|c|c|}
\hline Sample_ID & Co_ppm & Cr_ppm & Cu_ppm & F_ppm & La_ppm & Mo_ppm & Nb_ppm & Nb_ppm_2 & Ni_ppm \\
\hline 2011LF127A & $<10$ & 56 & $<7$ & $<110$ & $<50$ & $<1$ & $<1$ & $\begin{array}{c}-- \\
-\end{array}$ & 26 \\
\hline 2011LF130C & $<10$ & 122 & 17 & $<110$ & $<50$ & $<1$ & 21 & 18 & 51 \\
\hline 2011LF137A & $<10$ & 88 & 48 & 1430 & $<50$ & $<1$ & $<1$ & 8 & 72 \\
\hline 2011LF141A & $<10$ & 50 & $<7$ & $<110$ & $<50$ & $<1$ & 6 & --- & 22 \\
\hline 2011LF147A & $<10$ & 53 & $<7$ & $<110$ & $<50$ & $<1$ & 8 & 7 & 30 \\
\hline 2011LF149A & $<10$ & 97 & $<7$ & $<110$ & $<50$ & $<1$ & 20 & $\overline{---}$ & 61 \\
\hline 2011LF153A & $<10$ & 230 & 31 & $<110$ & $<50$ & $<1$ & 6 & 3 & 102 \\
\hline 2011LF164A & 52 & 219 & 126 & $<110$ & $<50$ & $<1$ & $<1$ & 76 & 56 \\
\hline 2011LF165A & 94 & 1150 & 75 & $<110$ & $<50$ & $<1$ & 16 & --- & 273 \\
\hline 2011LF167A & 43 & 116 & 59 & $<110$ & $<50$ & $<1$ & $<1$ & 30 & 20 \\
\hline 2011LF177A & 11 & 91 & 49 & $<110$ & $<50$ & $<1$ & 12 & --- & 42 \\
\hline 2011LF181B & 16 & 189 & 229 & $<110$ & $<50$ & $<1$ & $<1$ & 5 & 69 \\
\hline 2011LF184A & 28 & 146 & 42 & $<110$ & $<50$ & $<1$ & $<1$ & 7 & 49 \\
\hline 2011LF186A & 37 & 84 & 65 & $<110$ & $<50$ & $<1$ & $<1$ & 8 & 50 \\
\hline 2011LF196A & 94 & 189 & 111 & $<110$ & $<50$ & $<1$ & $<1$ & 4 & 194 \\
\hline 2011LF200A & 65 & 572 & 109 & $<110$ & $<50$ & $<1$ & $<1$ & 5 & 353 \\
\hline 2011LF206A & 85 & 716 & 166 & $<110$ & $<50$ & $<1$ & $<1$ & 4 & 299 \\
\hline 2011LF207C & $<10$ & 51 & 11 & $<110$ & $<50$ & $<1$ & 14 & 12 & 68 \\
\hline 2011LF209A & 71 & 291 & 111 & $<110$ & $<50$ & $<1$ & $<1$ & 12 & 85 \\
\hline 2011LF218A & 48 & 116 & 16 & $<110$ & $<50$ & $<1$ & $<1$ & 5 & 62 \\
\hline 2011LF219A & 34 & 118 & 50 & $<110$ & $<50$ & $<1$ & $<1$ & 9 & 57 \\
\hline 2011LF221A & 63 & 312 & 19 & $<110$ & $<50$ & $<1$ & $<1$ & 8 & 59 \\
\hline 2011LF232A & 123 & 753 & 127 & $<110$ & $<50$ & $<1$ & $<1$ & 8 & 153 \\
\hline 2011LF235A & 73 & 130 & 190 & $<110$ & $<50$ & $<1$ & $<1$ & 8 & 20 \\
\hline 2011LF238A & 40 & 582 & 153 & $<110$ & $<50$ & $<1$ & $<1$ & 2 & 218 \\
\hline 2011LF239A & 54 & 246 & 48 & $<110$ & $<50$ & $<1$ & $<1$ & 2 & 206 \\
\hline 2011LF268B & $<10$ & 80 & $<7$ & $<110$ & $<50$ & $<1$ & 6 & 5 & $<7$ \\
\hline 2011LF276A & 89 & 506 & $<7$ & 1520 & $<50$ & $<1$ & $<1$ & 7 & 109 \\
\hline 2011LF281A & 54 & $<30$ & 19 & $<110$ & $<50$ & $<1$ & $<1$ & 21 & $<7$ \\
\hline 2011LF285A & 95 & 82 & $<7$ & $<110$ & $<50$ & $<1$ & $<1$ & 10 & 23 \\
\hline 2011LF288B & $<10$ & 54 & 31.2 & $<110$ & $<50$ & $<1$ & $<1$ & 8.1 & 41 \\
\hline
\end{tabular}


Table 8. (continued)

\begin{tabular}{|c|c|c|c|c|c|c|c|c|c|}
\hline Sample_ID & Pb_ppm & Rb_ppm & Rb_ppm_2 & S_ppm & Sb_ppm & Sn_ppm & Sr_ppm & Sr_ppm_2 & Th_ppm \\
\hline 2011LF127A & $<4$ & 59 & $\begin{array}{l}-- \\
-\end{array}$ & 47 & $<2$ & $<4$ & 116 & $\begin{array}{c}-- \\
-\end{array}$ & $<2$ \\
\hline 2011LF130C & $<4$ & 217 & 205 & 56 & $<2$ & $<4$ & 51 & 54 & $<2$ \\
\hline 2011LF137A & 58 & 101 & 79 & 109 & $<2$ & $<4$ & 18 & 11 & $<2$ \\
\hline 2011LF141A & 14 & 27 & --- & 64 & $<2$ & $<4$ & 30 & --- & $<2$ \\
\hline 2011LF147A & $<4$ & 94 & 88 & 4260 & $<2$ & $<4$ & 12 & 13 & $<2$ \\
\hline 2011LF149A & 22 & 164 & --- & 85 & $<2$ & $<4$ & 61 & $\overline{---}$ & $<2$ \\
\hline 2011LF153A & 24 & 26 & 24 & 77 & $<2$ & $<4$ & 12 & 9 & $<2$ \\
\hline 2011LF164A & 27 & $<1$ & 2 & 905 & $<2$ & $<4$ & $<1$ & 49 & $<2$ \\
\hline 2011LF165A & 29 & 6 & --- & 83 & $<2$ & $<4$ & 16 & --- & $<2$ \\
\hline 2011LF167A & 28 & $<1$ & 4 & 131 & $<2$ & $<4$ & $<1$ & 50 & $<2$ \\
\hline 2011LF177A & 66 & 87 & --- & 54 & $<2$ & $<4$ & 110 & --- & $<2$ \\
\hline 2011LF181B & $<4$ & $<1$ & 14 & 5170 & $<2$ & $<4$ & $<1$ & 207 & $<2$ \\
\hline 2011LF184A & 22 & $<1$ & 21 & 34 & $<2$ & $<4$ & $<1$ & 355 & $<2$ \\
\hline 2011LF186A & 30 & $<1$ & 46 & 30 & $<2$ & $<4$ & $<1$ & 279 & $<2$ \\
\hline 2011LF196A & $<4$ & $<1$ & 17 & 40 & $<2$ & $<4$ & $<1$ & 181 & $<2$ \\
\hline 2011LF200A & $<4$ & $<1$ & 11 & 69 & $<2$ & $<4$ & $<1$ & 197 & $<2$ \\
\hline 2011LF206A & $<4$ & $<1$ & 7 & 82 & $<2$ & $<4$ & $<1$ & 124 & $<2$ \\
\hline 2011LF207C & $<4$ & $<1$ & 1 & 43 & $<2$ & $<4$ & 48 & 44 & $<2$ \\
\hline 2011LF209A & $<4$ & $<1$ & 1 & 53 & $<2$ & $<4$ & $<1$ & 101 & $<2$ \\
\hline 2011LF218A & 21 & $<1$ & 2 & 43 & $<2$ & $<4$ & $<1$ & 314 & $<2$ \\
\hline 2011LF219A & $<4$ & $<1$ & 42 & 103 & $<2$ & $<4$ & $<1$ & 1240 & $<2$ \\
\hline 2011LF221A & $<4$ & $<1$ & 30 & 69 & $<2$ & $<4$ & $<1$ & 144 & $<2$ \\
\hline 2011LF232A & 15 & $<1$ & 31 & 40 & $<2$ & $<4$ & $<1$ & 78 & $<2$ \\
\hline 2011LF235A & $<4$ & $<1$ & 59 & $<30$ & $<2$ & $<4$ & $<1$ & 262 & $<2$ \\
\hline 2011LF238A & $<4$ & $<1$ & 1 & 43 & $<2$ & $<4$ & $<1$ & 271 & $<2$ \\
\hline 2011LF239A & 38 & $<1$ & 1 & $<30$ & $<2$ & $<4$ & $<1$ & 119 & $<2$ \\
\hline 2011LF268B & 39 & 28 & 23 & 68 & $<2$ & $<4$ & 43 & 34 & $<2$ \\
\hline 2011LF276A & $<4$ & $<1$ & $<1$ & 38 & $<2$ & $<4$ & $<1$ & 330 & $<2$ \\
\hline 2011LF281A & $<4$ & $<1$ & 20 & 54 & $<2$ & $<4$ & $<1$ & 309 & $<2$ \\
\hline 2011LF285A & 19 & $<1$ & 2 & 86 & $<2$ & 20 & $<1$ & 300 & $<2$ \\
\hline 2011LF288B & 25.7 & $<1$ & 39.5 & 213 & $<2$ & $<4$ & $<1$ & 286.8 & $<2$ \\
\hline
\end{tabular}


Table 8. (continued)

\begin{tabular}{|c|c|c|c|c|c|c|c|c|}
\hline Sample_ID & U_ppm & V_ppm & W_ppm & Y_ppm & Y_ppm_2 & Zn_ppm & Zr_ppm & Zr_ppm_2 \\
\hline 2011LF127A & $<1$ & 38 & $<8$ & 18 & 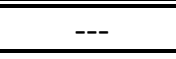 & 12 & 75 & $<9$ \\
\hline 2011LF130C & $<1$ & 169 & $<8$ & 31 & 32 & 105 & 119 & 173 \\
\hline 2011LF137A & $<1$ & 135 & $<8$ & 25 & 20 & 68 & 214 & 193 \\
\hline 2011LF141A & $<1$ & 46 & $<8$ & 6 & --- & 15 & 208 & $\overline{---}$ \\
\hline 2011LF147A & $<1$ & 62 & $<8$ & 7 & 9 & 25 & 120 & 144 \\
\hline 2011LF149A & $<1$ & 145 & $<8$ & 31 & --- & 72 & 163 & --- \\
\hline 2011LF153A & $<1$ & 96 & $<8$ & 9 & 5 & 61 & 60 & 63 \\
\hline 2011LF164A & $<1$ & 490 & $<8$ & $<1$ & 33 & 268 & $<9$ & 386 \\
\hline 2011LF165A & $<1$ & 379 & $<8$ & 15 & --- & 143 & 118 & --- \\
\hline 2011LF167A & $<1$ & 844 & $<8$ & $<1$ & 42 & 153 & $<9$ & 305 \\
\hline 2011LF177A & $<1$ & 97 & $<8$ & 20 & --- & 78 & 93 & --- \\
\hline 2011LF181B & $<1$ & 367 & $<8$ & $<1$ & 28 & 120 & $<9$ & 89 \\
\hline 2011LF184A & $<1$ & 350 & $<8$ & $<1$ & 24 & 96 & $<9$ & 119 \\
\hline 2011LF186A & $<1$ & 434 & $<8$ & $<1$ & 30 & 110 & $<9$ & 121 \\
\hline 2011LF196A & $<1$ & 173 & $<8$ & $<1$ & 13 & 67 & $<9$ & 45 \\
\hline 2011LF200A & $<1$ & 456 & $<8$ & $<1$ & 16 & 134 & $<9$ & 64 \\
\hline 2011LF206A & $<1$ & 264 & $<8$ & $<1$ & 11 & 56 & $<9$ & 43 \\
\hline 2011LF207C & $<1$ & 145 & $<8$ & 21 & 23 & 23 & 281 & 319 \\
\hline 2011LF209A & $<1$ & 411 & $<8$ & $<1$ & 29 & 113 & $<9$ & 128 \\
\hline 2011LF218A & $<1$ & 191 & $<8$ & $<1$ & 15 & 69 & $<9$ & 45 \\
\hline 2011LF219A & $<1$ & 366 & $<8$ & $<1$ & 20 & 96 & $<9$ & 60 \\
\hline 2011LF221A & $<1$ & 442 & $<8$ & $<1$ & 24 & 97 & $<9$ & 107 \\
\hline 2011LF232A & $<1$ & 397 & $<8$ & $<1$ & 16 & 103 & $<9$ & 96 \\
\hline 2011LF235A & $<1$ & 529 & $<8$ & $<1$ & 25 & 106 & $<9$ & 105 \\
\hline 2011LF238A & $<1$ & 194 & $<8$ & $<1$ & 7 & 51 & $<9$ & 23 \\
\hline 2011LF239A & $<1$ & 190 & $<8$ & $<1$ & 6 & 57 & $<9$ & 18 \\
\hline 2011LF268B & $<1$ & 75 & $<8$ & 11 & 11 & 16 & 129 & 139 \\
\hline 2011LF276A & $<1$ & 322 & $<8$ & $<1$ & 22 & 117 & $<9$ & 95 \\
\hline 2011LF281A & $<1$ & 244 & $<8$ & $<1$ & 50 & 65 & $<9$ & 332 \\
\hline 2011LF285A & $<1$ & 379 & $<8$ & $<1$ & 34 & 92 & $<9$ & 119 \\
\hline 2011LF288B & $<1$ & 333 & $<8$ & $<1$ & 22.7 & 77.1 & $<9$ & 116.5 \\
\hline
\end{tabular}


Table 8. (continued)

\begin{tabular}{|c|c|c|c|c|c|c|c|}
\hline Sample_ID & Latitude & Longitude & Easting & Northing & $\begin{array}{l}\text { UTM_ } \\
\text { Zone }\end{array}$ & Root_name & Comments \\
\hline 2011LF301A & 65.457771 & $\mid-152.751456$ & 511520 & 7259305 & $5 \mathrm{~N}$ & Granite aplite & 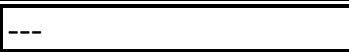 \\
\hline 2011LF307A & 65.451363 & -152.819224 & 508381 & 7258580 & $5 \mathrm{~N}$ & Metagabbro & --- \\
\hline 2011LF309A & 65.449574 & -152.816389 & 508513 & 7258381 & $5 \mathrm{~N}$ & Metamafic & --- \\
\hline 2011LF310A & 65.449250 & -152.815766 & 508542 & 7258345 & $5 \mathrm{~N}$ & Calc-silicate honrfels(?) & Small \\
\hline 2011LF312A & 65.447452 & -152.812759 & 508682 & 7258145 & $5 \mathrm{~N}$ & Metagabbro(?) & --- \\
\hline 2011LF317A & 65.469164 & -152.666391 & 515456 & 7260593 & $5 \mathrm{~N}$ & Quartzite & Inhomogenous \\
\hline 2011LF319A & 65.504161 & -152.748940 & 511616 & 7264476 & $5 \mathrm{~N}$ & Granite aplite & --- \\
\hline 2011LF343A & 65.454553 & -152.597955 & 518637 & 7258983 & $5 \mathrm{~N}$ & Fine-grained gabbro(?) & --- \\
\hline 2011LF344A & 65.453967 & -152.596950 & 518684 & 7258918 & $5 \mathrm{~N}$ & Fine-grained gabbro(?) & Poor polish \\
\hline 2011LF347A & 65.450608 & -152.595599 & 518749 & 7258544 & $5 \mathrm{~N}$ & Fine-grained Metagabbro(?) & --- \\
\hline 2011LF350A & 65.445784 & -152.610683 & 518053 & 7258002 & $5 \mathrm{~N}$ & Gabbro & Altered, porous \\
\hline 2011LF355B & 65.378361 & -152.887805 & 505216 & 7250436 & $5 \mathrm{~N}$ & Quartz-chlorite schist & Inhomogenous \\
\hline 2011LF357B & 65.378394 & -152.884556 & 505367 & 7250440 & $5 \mathrm{~N}$ & Coarse-grained Metamafic & Inhomogenous \\
\hline 2011LF359A & 65.378419 & -152.881567 & 505506 & 7250443 & $5 \mathrm{~N}$ & Ankerite(?)-chlorite schist & Inhomogenous \\
\hline 2011LF359B & 65.378419 & -152.881567 & 505506 & 7250443 & $5 \mathrm{~N}$ & Ankerite(?)-chlorite schist & Inhomogenous \\
\hline 2011LF366B & 65.301056 & -153.112723 & 494744 & 7241820 & $5 \mathrm{~N}$ & Chloritoid quartz schist & Inhomogenous \\
\hline 2011LF372B & 65.378942 & -152.863969 & 506324 & 7250503 & $5 \mathrm{~N}$ & Garnet amphibolite(?) & -- \\
\hline 2011LF384A & 65.265173 & -152.988413 & 500541 & 7237816 & $5 \mathrm{~N}$ & Glaucophane Metamafic & --- \\
\hline 2011LF384B & 65.265173 & -152.988413 & 500541 & 7237816 & $5 \mathrm{~N}$ & Coarse-grained Metamafic & --- \\
\hline 2011LF388B & 65.262868 & -153.001991 & 499907 & 7237559 & $5 \mathrm{~N}$ & Clinopyroxenite(?) & Altered \\
\hline 2011LF389A & 65.260634 & -153.004218 & 499803 & 7237310 & $5 \mathrm{~N}$ & Glaucophane Metamafic & --- \\
\hline 2011LF418A & 65.261546 & -152.832922 & 507802 & 7237422 & $5 \mathrm{~N}$ & Metagabbro(?) & Porous \\
\hline 2011LF419B & 65.261664 & -152.834506 & 507728 & 7237435 & $5 \mathrm{~N}$ & Metagabbro(?) & Porous \\
\hline 2011LF441B & 65.306625 & -152.835254 & 507680 & 7242446 & $5 \mathrm{~N}$ & Calcareous greenschist(?) & Porous \\
\hline 2011LF450B & 65.298556 & -152.816990 & 508534 & 7241549 & $5 \mathrm{~N}$ & Muscovite-chloritoid quartz schist & -- \\
\hline 2011LF450B & 65.298556 & -152.816990 & 508534 & 7241549 & $5 \mathrm{~N}$ & Muscovite-chloritoid quartz schist & --- \\
\hline 2011LF466A & 65.384373 & -152.598103 & 518680 & 7251161 & $5 \mathrm{~N}$ & Quartzite & Porous \\
\hline 2011LF474A & 65.390861 & -152.622968 & 517520 & 7251877 & $5 \mathrm{~N}$ & Quartzite & Porous \\
\hline 2011LF477B & 65.388742 & -152.633219 & 517045 & 7251638 & $5 \mathrm{~N}$ & Greenstone & Porous \\
\hline 2011LF484B & 65.425908 & -152.983752 & 500754 & 7255731 & $5 \mathrm{~N}$ & Quartzite & Inhomogenous \\
\hline 2011LF485B & 65.397168 & -152.633898 & 517008 & 7252577 & $5 \mathrm{~N}$ & Quartzite & --- \\
\hline 2011LF486A & 65.398017 & -152.635953 & 516912 & 7252671 & $5 \mathrm{~N}$ & Plagioclase-biotite-quartz paragneiss & --- \\
\hline
\end{tabular}


Table 8. (continued)

\begin{tabular}{|c|c|c|c|c|c|c|c|c|c|}
\hline Sample_ID & $\mathrm{SiO}_{2 \_} \mathrm{pct}$ & Al203_pct & BaO_pct & CaO_pct & FeO_pct & K2O_pct & MgO_pct & MnO_pct & $\mathrm{Na} 2 \mathrm{O} \_p c t$ \\
\hline 2011LF301A & 76.6 & 13.4 & $<<0.03$ & 0.539 & 0.172 & 4.3 & 0.317 & $<0.03$ & 4.43 \\
\hline 2011LF307A & 52.1 & 16.6 & 0.0333 & 8.52 & 10.1 & 0.345 & 5.57 & 0.166 & 4.63 \\
\hline 2011LF309A & 51 & 13.8 & 0.0378 & 11.7 & 11.9 & 0.301 & 6.87 & 0.258 & 2.46 \\
\hline 2011LF310A & 66.1 & 16.1 & 0.0648 & 6.98 & 4.34 & 1.73 & 2.18 & 0.0729 & 1.38 \\
\hline 2011LF312A & 51.2 & 17.9 & $<0.03$ & 9.43 & 10.2 & 0.0821 & 5.17 & 0.174 & 3.74 \\
\hline 2011LF317A & 94.8 & 2.9 & 0.0772 & 0.0684 & 0.425 & 0.727 & 0.369 & $<0.03$ & 0.0365 \\
\hline 2011LF319A & 76.7 & 13 & 0.245 & 1.14 & 0.179 & 5.24 & 0.15 & $<0.03$ & 3.23 \\
\hline 2011LF343A & 51.8 & 14.6 & $<0.03$ & 9.32 & 11.6 & 0.132 & 5.19 & 0.148 & 4.3 \\
\hline 2011LF344A & 54.9 & 14 & 0.0567 & 7.59 & 10.3 & 0.219 & 4.93 & 0.163 & 5.4 \\
\hline 2011LF347A & 50.8 & 14.8 & 0.198 & 9 & 12.3 & 0.343 & 5.19 & 0.203 & 4.76 \\
\hline 2011LF350A & 52.7 & 16 & 0.0602 & 5.62 & 12.1 & 0.0291 & 5.03 & 0.458 & 6.09 \\
\hline 2011LF355B & 61.6 & 13.5 & $<0.03$ & 3.81 & 12.6 & 0.0403 & 3.99 & 0.187 & 1.77 \\
\hline 2011LF357B & 50.4 & 15.2 & $<0.03$ & 9.5 & 11.1 & 0.0758 & 8.85 & 0.209 & 3.48 \\
\hline 2011LF359A & 57.8 & 13 & 0.0436 & 8.75 & 8.59 & 1.25 & 8.92 & 0.119 & 0.124 \\
\hline 2011LF359B & 57.6 & 15.3 & 0.0391 & 5.19 & 10.4 & 0.217 & 6.84 & 0.104 & 3.13 \\
\hline 2011LF366B & 88.8 & 6.1 & 0.0525 & 0.096 & 2.35 & 1 & 0.35 & $<0.03$ & 0.673 \\
\hline 2011LF372B & 50.4 & 14 & $<0.03$ & 9.13 & 14 & 0.129 & 5.1 & 0.181 & 4.39 \\
\hline 2011LF384A & 49.3 & 14.6 & $<0.03$ & 11.5 & 12.4 & 0.0582 & 6.1 & 0.161 & 3.75 \\
\hline 2011LF384B & 50.3 & 15.4 & 0.047 & 12.6 & 9.11 & 0.226 & 7.96 & 0.159 & 3.03 \\
\hline 2011LF388B & 51.5 & 6.6 & 0.0455 & 16.4 & 10 & 0.209 & 12 & 0.179 & 1.35 \\
\hline 2011LF389A & 52 & 16.1 & 0.137 & 10.3 & 8.74 & 0.91 & 6.44 & 0.146 & 3.96 \\
\hline 2011LF418A & 55.9 & 12.3 & $<0.03$ & 5.32 & 11.7 & 0.104 & 8.84 & 0.191 & 3.69 \\
\hline 2011LF419B & 50.48716 & 14.5 & 0.04069 & 6.13931 & 13.17882 & 0.20243 & 6.74498 & 0.26417 & 4.89164 \\
\hline 2011LF441B & 51.2 & 18 & $<0.03$ & 0.36 & 21.4 & 0.0707 & 3.63 & 0.362 & 0.552 \\
\hline 2011LF450B & 77.2 & 12.6 & 0.115 & 0.475 & 4.46 & 2.22 & 1.38 & 0.0386 & 0.233 \\
\hline 2011LF450B & 77.1 & 12.6 & 0.102 & 0.472 & 4.38 & 2.18 & 1.47 & 0.0474 & 0.25 \\
\hline 2011LF466A & 98.2 & 0.9 & 0.0666 & 0.0942 & 0.133 & 0.106 & 0.271 & $<0.03$ & 0.138 \\
\hline 2011LF474A & 98.1 & 0.9 & $<0.03$ & 0.0747 & 0.331 & 0.0809 & 0.184 & $<0.03$ & 0.117 \\
\hline 2011LF477B & 61.1 & 11.4 & $<0.03$ & 2.56 & 15.3 & 0.151 & 4.3 & 0.213 & 2.37 \\
\hline 2011LF484B & 78.8 & 9.58 & 0.0492 & 1.37 & 3.59 & 2.49 & 1.51 & 0.0448 & 1.59 \\
\hline 2011LF485B & 94.8 & 2.4 & $<0.03$ & 0.111 & 1.13 & 0.603 & 0.613 & $<0.03$ & 0.0579 \\
\hline 2011LF486A & 97.4 & 1.2 & 0.032 & 0.0355 & 0.659 & 0.237 & 0.153 & $<0.03$ & 0.098 \\
\hline
\end{tabular}


Table 8. (continued)

\begin{tabular}{|c|c|c|c|c|c|c|c|c|c|}
\hline Sample_ID & P2O5_pct & TiO2_pct & CO2_pct & Total_pct & As_ppm & Bi_ppm & Br_ppm & Ce_ppm & Cl_ppm \\
\hline 2011LF301A & $<<0.03$ & 0.0235 & $<<0.05$ & 99.7981 & 11 & $<<2$ & $<<$ & $<60$ & 188 \\
\hline 2011LF307A & 0.25 & 1.46 & $<0.05$ & 99.7743 & $<4$ & $<2$ & $<5$ & $<60$ & 576 \\
\hline 2011LF309A & 0.113 & 1.19 & $<0.05$ & 99.6298 & $<4$ & $<2$ & $<5$ & $<60$ & 1250 \\
\hline 2011LF310A & 0.294 & 0.564 & $<0.05$ & 99.8057 & $<4$ & $<2$ & $<5$ & $<60$ & 744 \\
\hline 2011LF312A & 0.261 & 1.61 & $<0.05$ & 99.7866 & 6 & $<2$ & $<5$ & $<60$ & 441 \\
\hline 2011LF317A & 0.36 & 0.187 & $<0.05$ & 99.9052 & $<4$ & $<2$ & $<5$ & $<60$ & 202 \\
\hline 2011LF319A & $<0.03$ & 0.0412 & $<0.05$ & 99.94215 & $<4$ & $<2$ & $<5$ & $<60$ & 63 \\
\hline 2011LF343A & 0.316 & 2.36 & $<0.05$ & 99.7948 & $<4$ & $<2$ & $<5$ & $<60$ & 199 \\
\hline 2011LF344A & 0.305 & 1.95 & $<0.05$ & 99.8137 & $<4$ & $<2$ & $<5$ & $<60$ & 81 \\
\hline 2011LF347A & 0.288 & 1.87 & $<0.05$ & 99.752 & $<4$ & $<2$ & $<5$ & $<60$ & 119 \\
\hline 2011LF350A & 0.395 & 1.33 & $<0.05$ & 99.8123 & $<4$ & $<2$ & $<5$ & $<60$ & 180 \\
\hline 2011LF355B & 0.113 & 2.14 & $<0.05$ & 99.7704 & 73 & $<2$ & $<5$ & $<60$ & 156 \\
\hline 2011LF357B & 0.099 & 0.804 & $<0.05$ & 99.737 & 5 & $<2$ & $<5$ & $<60$ & 430 \\
\hline 2011LF359A & 0.385 & 0.722 & $<0.05$ & 99.7036 & $<4$ & $<2$ & $<5$ & $<60$ & 548 \\
\hline 2011LF359B & 0.0367 & 0.887 & $<0.05$ & 99.7438 & $<4$ & $<2$ & $<5$ & $<60$ & 198 \\
\hline 2011LF366B & 0.0616 & 0.329 & $<0.05$ & 99.8703 & $<4$ & $<2$ & $<5$ & $<60$ & 81 \\
\hline 2011LF372B & 0.406 & 2.01 & $<0.05$ & 99.7678 & 8 & $<2$ & $<5$ & $<60$ & 126 \\
\hline 2011LF384A & 0.0915 & 1.86 & $<0.05$ & 99.8441 & 59 & $<2$ & $<5$ & $<60$ & 158 \\
\hline 2011LF384B & 0.354 & 0.72 & $<0.05$ & 99.906 & $<4$ & $<2$ & $<5$ & $<60$ & 146 \\
\hline 2011LF388B & 0.162 & 1.06 & $<0.05$ & 99.5455 & $<4$ & $<2$ & $<5$ & $<60$ & 613 \\
\hline 2011LF389A & 0.205 & 0.955 & $<0.05$ & 99.893 & 7 & $<2$ & $<5$ & $<60$ & 229 \\
\hline 2011LF418A & 0.313 & 1.37 & $<0.05$ & 99.7558 & $<4$ & $<2$ & $<5$ & $<60$ & 133 \\
\hline 2011LF419B & 2 & 1.27611 & $<0.05$ & 99.78723 & $<4$ & $<2$ & $<5$ & $<60$ & 148 \\
\hline 2011LF441B & 0.538 & 3.36 & $<0.05$ & 99.4905 & 13 & $<2$ & $<5$ & $<60$ & 129 \\
\hline 2011LF450B & 0.53 & 0.675 & $<0.05$ & 99.9266 & $<4$ & $<2$ & $<5$ & $<60$ & 144 \\
\hline \begin{tabular}{|l|} 
2011LF450B \\
\end{tabular} & 0.529 & 0.668 & $<0.05$ & 99.7984 & $<4$ & $<2$ & $<5$ & $<60$ & 156 \\
\hline 2011LF466A & $<0.03$ & 0.0285 & $<0.05$ & 99.9838 & $<4$ & $<2$ & $<5$ & $<60$ & 295 \\
\hline 2011LF474A & $<0.03$ & 0.0344 & $<0.05$ & 99.83412 & $<4$ & $<2$ & $<5$ & $<60$ & 391 \\
\hline \begin{tabular}{|l|} 
2011LF477B \\
\end{tabular} & 0.789 & 1.63 & $<0.05$ & 99.841 & $<4$ & $<2$ & $<5$ & $<60$ & 141 \\
\hline 2011LF484B & 0.131 & 0.691 & $<0.05$ & 99.846 & $<4$ & $<2$ & $<5$ & 204 & 525 \\
\hline 2011LF485B & 0.0869 & 0.0939 & $<0.05$ & 99.9377 & $<4$ & $<2$ & $<5$ & $<60$ & 257 \\
\hline 2011LF486A & 0.0473 & 0.0467 & $<0.05$ & 99.9185 & $<4$ & $<2$ & $<5$ & $<60$ & $<50$ \\
\hline
\end{tabular}


Table 8. (continued)

\begin{tabular}{|c|c|c|c|c|c|c|c|c|c|}
\hline Sample_ID & Co_ppm & Cr_ppm & Cu_ppm & F_ppm & La_ppm & Mo_ppm & Nb_ppm & Nb_ppm_2 & Ni_ppm \\
\hline 2011LF301A & $<<10$ & $<<30$ & 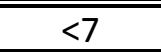 & $<110$ & $<50$ & $2<1$ & $<<1$ & 50 & 33 \\
\hline 2011LF307A & 43 & 40 & 57 & $<110$ & $<50$ & $<1$ & $<1$ & 7 & 37 \\
\hline 2011LF309A & 10 & 660 & 25 & $<110$ & $<50$ & $<1$ & $<1$ & 6 & 70 \\
\hline 2011LF310A & 25 & 117 & 7 & $<110$ & $<50$ & $<1$ & $<1$ & 12 & 27 \\
\hline 2011LF312A & 75 & 39 & 34 & $<110$ & $<50$ & $<1$ & $<1$ & 9 & 46 \\
\hline 2011LF317A & $<10$ & 63 & $<7$ & $<110$ & $<50$ & $<1$ & $<1$ & 3 & 19 \\
\hline 2011LF319A & $<10$ & $<30$ & $<7$ & $<110$ & $<50$ & $<1$ & $<1$ & 57 & $<7$ \\
\hline 2011LF343A & 51 & 201 & 29 & $<110$ & $<50$ & $<1$ & $<1$ & 22 & 74 \\
\hline 2011LF344A & 88 & 67 & 69 & $<110$ & $<50$ & $<1$ & $<1$ & 22 & 43 \\
\hline 2011LF347A & 93 & 73 & 56 & $<110$ & $<50$ & $<1$ & $<1$ & 18 & 47 \\
\hline 2011LF350A & 53 & 117 & 104 & $<110$ & $<50$ & $<1$ & $<1$ & 8 & 54 \\
\hline 2011LF355B & 40 & 109 & 56 & $<110$ & $<50$ & $<1$ & $<1$ & 15 & 19 \\
\hline 2011LF357B & 79 & 151 & 11 & $<110$ & $<50$ & $<1$ & $<1$ & 1 & 229 \\
\hline 2011LF359A & 58 & 743 & $<7$ & $<110$ & $<50$ & $<1$ & $<1$ & 4 & 372 \\
\hline 2011LF359B & 35 & 960 & 38 & $<110$ & $<50$ & $<1$ & $<1$ & 5 & 240 \\
\hline 2011LF366B & $<10$ & 57 & $<7$ & $<110$ & $<50$ & $<1$ & $<1$ & 7 & 15 \\
\hline 2011LF372B & 101 & 98 & 303 & $<110$ & $<50$ & $<1$ & $<1$ & 14 & 35 \\
\hline 2011LF384A & 70 & 75 & 51 & $<110$ & $<50$ & $<1$ & $<1$ & 5 & 45 \\
\hline 2011LF384B & 57 & 313 & 42 & $<110$ & $<50$ & $<1$ & $<1$ & 4 & 61 \\
\hline 2011LF388B & 49.6 & 2450 & 93.4 & $<110$ & $<50$ & $<1$ & $<1$ & 4.8 & 376 \\
\hline 2011LF389A & 44 & 120 & 44 & $<110$ & $<50$ & $<1$ & $<1$ & 6 & 80 \\
\hline 2011LF418A & 87 & 420 & 64 & 310 & $<50$ & $<1$ & $<1$ & 10 & 257 \\
\hline 2011LF419B & 57 & 525 & 77 & $<110$ & $<50$ & $<1$ & $<1$ & 10 & 348 \\
\hline 2011LF441B & 102 & 91 & 177 & 3130 & $<50$ & $<1$ & $<1$ & 19 & 51 \\
\hline 2011LF450B & 14 & 94 & 9 & $<110$ & $<50$ & $<1$ & $<1$ & --- & 85 \\
\hline 2011LF450B & $<10$ & 104 & $<7$ & 726 & $<50$ & $<1$ & $<1$ & 8 & 56 \\
\hline 2011LF466A & $<10$ & $<30$ & 19 & $<110$ & $<50$ & $<1$ & $<1$ & 1 & 16 \\
\hline 2011LF474A & 19 & $<30$ & 39 & $<110$ & $<50$ & $<1$ & $<1$ & 1 & 10 \\
\hline 2011LF477B & 20 & $<30$ & 28 & $<110$ & $<50$ & $<1$ & $<1$ & 31 & 24 \\
\hline 2011LF484B & 11 & 81 & 43 & $<110$ & $<50$ & $<1$ & $<1$ & 10 & 37 \\
\hline 2011LF485B & 38 & 46 & 18 & 111 & $<50$ & $<1$ & $<1$ & 3 & 19 \\
\hline 2011LF486A & 21 & $<30$ & 22 & $<110$ & $<50$ & $<1$ & $<1$ & 2 & 29 \\
\hline
\end{tabular}


Table 8. (continued)

\begin{tabular}{|c|c|c|c|c|c|c|c|c|c|}
\hline Sample_ID & Pb_ppm & Rb_ppm & Rb_ppm_2 & S_ppm & Sb_ppm & Sn_ppm & Sr_ppm & Sr_ppm_2 & Th_ppm \\
\hline 2011LF301A & 56 & $<1$ & 406 & 93 & $<2$ & $<4$ & $<1$ & 7 & 23 \\
\hline 2011LF307A & $<4$ & $<1$ & 37 & 432 & $<2$ & $<4$ & $<1$ & 340 & $<2$ \\
\hline 2011LF309A & $<4$ & $<1$ & 20 & 207 & $<2$ & $<4$ & $<1$ & 313 & $<2$ \\
\hline 2011LF310A & 18 & $<1$ & 178 & 250 & $<2$ & $<4$ & $<1$ & 222 & $<2$ \\
\hline 2011LF312A & 27 & $<1$ & 4 & 326 & $<2$ & $<4$ & $<1$ & 339 & $<2$ \\
\hline 2011LF317A & 19 & $<1$ & 25 & 150 & $<2$ & $<4$ & $<1$ & 6 & $<2$ \\
\hline 2011LF319A & 22 & $<1$ & 299 & $<30$ & $<2$ & $<4$ & $<1$ & 57 & 22 \\
\hline 2011LF343A & $<4$ & $<1$ & 2 & 649 & $<2$ & $<4$ & $<1$ & 250 & $<2$ \\
\hline 2011LF344A & 19 & $<1$ & 4 & 267 & $<2$ & $<4$ & $<1$ & 194 & $<2$ \\
\hline 2011LF347A & $<4$ & $<1$ & 8 & 176 & $<2$ & 22 & $<1$ & 371 & $<2$ \\
\hline 2011LF350A & 35 & $<1$ & $<1$ & 131 & $<2$ & $<4$ & $<1$ & 319 & $<2$ \\
\hline 2011LF355B & 69 & $<1$ & 2 & 177 & $<2$ & $<4$ & $<1$ & 191 & $<2$ \\
\hline 2011LF357B & $<4$ & $<1$ & 2 & 238 & $<2$ & $<4$ & $<1$ & 189 & $<2$ \\
\hline 2011LF359A & $<4$ & $<1$ & 77 & 213 & $<2$ & $<4$ & $<1$ & 91 & $<2$ \\
\hline 2011LF359B & $<4$ & $<1$ & 14 & 110 & $<2$ & $<4$ & $<1$ & 81 & $<2$ \\
\hline 2011LF366B & $<4$ & $<1$ & 38 & 133 & $<2$ & $<4$ & $<1$ & 28 & $<2$ \\
\hline 2011LF372B & 24 & $<1$ & 2 & 125 & $<2$ & $<4$ & $<1$ & 223 & $<2$ \\
\hline 2011LF384A & $<4$ & $<1$ & 1 & 119 & $<2$ & 34 & $<1$ & 294 & $<2$ \\
\hline 2011LF384B & 25 & $<1$ & 7 & 43 & $<2$ & 22 & $<1$ & 320 & $<2$ \\
\hline 2011LF388B & $<4$ & $<1$ & 6 & 286 & $<2$ & $<4$ & $<1$ & 118.4 & $<2$ \\
\hline 2011LF389A & $<4$ & $<1$ & 34 & 51 & $<2$ & $<4$ & $<1$ & 240 & $<2$ \\
\hline 2011LF418A & 19 & $<1$ & 5 & 164 & $<2$ & $<4$ & $<1$ & 50 & $<2$ \\
\hline 2011LF419B & 32 & $<1$ & 4 & 157 & $<2$ & $<4$ & $<1$ & 125 & $<2$ \\
\hline 2011LF441B & $<4$ & $<1$ & 3 & 77 & $<2$ & 25 & $<1$ & 87 & $<2$ \\
\hline 2011LF450B & 27 & $<1$ & --- & 45 & $<2$ & 18 & $<1$ & --- & $<2$ \\
\hline 2011LF450B & 26 & $<1$ & 68 & 30 & $<2$ & $<4$ & $<1$ & 72 & $<2$ \\
\hline 2011LF466A & $<4$ & $<1$ & 5 & 89 & $<2$ & $<4$ & $<1$ & 6 & $<2$ \\
\hline 2011LF474A & 35 & $<1$ & 4 & 332 & $<2$ & 13 & $<1$ & 8 & $<2$ \\
\hline 2011LF477B & $<4$ & $<1$ & 4 & 559 & $<2$ & $<4$ & $<1$ & 163 & $<2$ \\
\hline 2011LF484B & $<4$ & $<1$ & 207 & 81 & $<2$ & $<4$ & $<1$ & 115 & 37 \\
\hline 2011LF485B & 25 & $<1$ & 22 & 211 & $<2$ & $<4$ & $<1$ & 8 & $<2$ \\
\hline 2011LF486A & 29 & $<1$ & 10 & 41 & $<2$ & $<4$ & $<1$ & 7 & $<2$ \\
\hline
\end{tabular}




\begin{tabular}{|c|c|c|c|c|c|c|c|c|}
\hline Sample_ID & U_ppm & V_ppm & W_ppm & Y_ppm & Y_ppm_2 & Zn_ppm & Zr_ppm & Zr_ppm_2 \\
\hline 2011LF301A & 16.5 & $<25$ & $<<8$ & $<1$ & 36 & $<<8$ & $<9$ & 119 \\
\hline 2011LF307A & $<1$ & 421 & $<8$ & $<1$ & 26 & 107 & $<9$ & 118 \\
\hline 2011LF309A & $<1$ & 334 & $<8$ & $<1$ & 19 & 166 & $<9$ & 45 \\
\hline 2011LF310A & $<1$ & 119 & $<8$ & $<1$ & 37 & 94 & $<9$ & 272 \\
\hline 2011LF312A & $<1$ & 415 & $<8$ & $<1$ & 27 & 125 & $<9$ & 124 \\
\hline 2011LF317A & $<1$ & 132 & $<8$ & $<1$ & 6 & 23 & $<9$ & 41 \\
\hline 2011LF319A & 22 & $<25$ & $<8$ & $<1$ & 38 & $<8$ & $<9$ & 39 \\
\hline 2011LF343A & $<1$ & 500 & $<8$ & $<1$ & 33 & 116 & $<9$ & 184 \\
\hline 2011LF344A & $<1$ & 397 & $<8$ & $<1$ & 31 & 101 & $<9$ & 191 \\
\hline 2011LF347A & $<1$ & 469 & $<8$ & $<1$ & 32 & 122 & $<9$ & 171 \\
\hline 2011LF350A & $<1$ & 467 & $<8$ & $<1$ & 27 & 82 & $<9$ & 110 \\
\hline 2011LF355B & $<1$ & 564 & $<8$ & $<1$ & 41 & 155 & $<9$ & 215 \\
\hline 2011LF357B & $<1$ & 347 & $<8$ & $<1$ & 25 & 89 & $<9$ & 56 \\
\hline 2011LF359A & $<1$ & 309 & $<8$ & $<1$ & 28 & 112 & $<9$ & 111 \\
\hline 2011LF359B & $<1$ & 316 & $<8$ & $<1$ & 28 & 139 & $<9$ & 68 \\
\hline 2011LF366B & $<1$ & 45 & $<8$ & $<1$ & 7 & 21 & $<9$ & 230 \\
\hline 2011LF372B & $<1$ & 561 & $<8$ & $<1$ & 34 & 136 & $<9$ & 181 \\
\hline 2011LF384A & $<1$ & 644 & $<8$ & $<1$ & 14 & 66 & $<9$ & 56 \\
\hline 2011LF384B & $<1$ & 251 & $<8$ & $<1$ & 16 & 68 & $<9$ & 48 \\
\hline 2011LF388B & $<1$ & 451 & $<8$ & $<1$ & 21.4 & 84.7 & $<9$ & 65.2 \\
\hline 2011LF389A & $<1$ & 305 & $<8$ & $<1$ & 21 & 72 & $<9$ & 53 \\
\hline 2011LF418A & $<1$ & 261 & $<8$ & $<1$ & 25 & 96 & $<9$ & 99 \\
\hline 2011LF419B & $<1$ & 292 & $<8$ & $<1$ & 24 & 169 & $<9$ & 106 \\
\hline 2011LF441B & $<1$ & 799 & $<8$ & $<1$ & 45 & 461 & $<9$ & 229 \\
\hline 2011LF450B & $<1$ & 151 & $<8$ & $<1$ & --- & 78 & $<9$ & $<9$ \\
\hline 2011LF450B & $<1$ & 188 & $<8$ & $<1$ & 19 & 90 & $<9$ & 106 \\
\hline 2011LF466A & $<1$ & $<25$ & $<8$ & $<1$ & $<8$ & 9 & $<9$ & 13 \\
\hline 2011LF474A & $<1$ & $<25$ & $<8$ & $<1$ & 1 & 9 & $<9$ & 11 \\
\hline 2011LF477B & $<1$ & 193 & $<8$ & $<1$ & 25 & 94 & $<9$ & 247 \\
\hline 2011LF484B & $<1$ & 123 & $<8$ & $<1$ & 24 & 26 & $<9$ & 162 \\
\hline 2011LF485B & $<1$ & 40 & $<8$ & $<1$ & 1 & 27 & $<9$ & 28 \\
\hline 2011LF486A & $<1$ & 31 & $<8$ & $<1$ & 1 & $<8$ & $<9$ & 17 \\
\hline
\end{tabular}


Table 8. (continued)

\begin{tabular}{|c|c|c|c|c|c|c|c|}
\hline Sample_ID & Latitude & Longitude & Easting & Northing & $\begin{array}{l}\text { UTM_ } \\
\text { Zone }\end{array}$ & Root_name & Comments \\
\hline 2011LF490B & 65.424418 & $\mid-152.973970$ & $\mid 501208$ & 7255565 & $5 \mathrm{~N}$ & Potassium feldspar quartzite & Inhomogenous \\
\hline 2011LF494A & 65.419937 & -152.957300 & 501982 & 7255066 & $5 \mathrm{~N}$ & Muscovite quartz schist & Small \\
\hline 2011LF504A & 65.348596 & -152.683153 & 514747 & 7247151 & $5 \mathrm{~N}$ & Kaolinite-quartz iron oxide & Poor polish \\
\hline 2011LF509A & 65.355507 & -152.684295 & 514690 & 7247921 & $5 \mathrm{~N}$ & Fine-grained greenstone & Inhomogenous, poor polish \\
\hline 2011LF509B & 65.355507 & -152.684295 & 514690 & 7247921 & $5 \mathrm{~N}$ & Chert(?) & Porous \\
\hline 2011LF509B & 65.355507 & -152.684295 & 514690 & 7247921 & $5 \mathrm{~N}$ & Chert(?) & Porous \\
\hline 2011LF509B & 65.355507 & -152.684295 & 514690 & 7247921 & $5 \mathrm{~N}$ & Chert(?) & Porous \\
\hline 2011LF516A & 65.366145 & -152.691112 & 514367 & 7249105 & $5 \mathrm{~N}$ & Fine-grained gabbro(?) & --- \\
\hline 2011LF516A & 65.366145 & -152.691112 & 514367 & 7249105 & $5 \mathrm{~N}$ & Fine-grained gabbro(?) & --- \\
\hline 2011LF529A & 65.389773 & -152.663849 & 515621 & 7251745 & $5 \mathrm{~N}$ & Quartz-calcite-muscovite schist & Porous, small \\
\hline 2011LF533B & 65.470352 & -152.604619 & 518317 & 7260742 & $5 \mathrm{~N}$ & Fine-grained gabbro & Altered \\
\hline 2011LF535A & 65.469796 & -152.608081 & 518157 & 7260679 & $5 \mathrm{~N}$ & Gabbro & Altered \\
\hline 2011LF536A & 65.470654 & -152.610335 & 518052 & 7260774 & $5 \mathrm{~N}$ & Gabbro & Altered \\
\hline 2011LF537A & 65.471340 & -152.619089 & 517646 & 7260848 & $5 \mathrm{~N}$ & Metagabbro(?) & Inhomogenous \\
\hline 2011LF553A & 65.363273 & -152.471008 & 524607 & 7248853 & $5 \mathrm{~N}$ & Diabase(?) & Altered \\
\hline 2011LF556A & 65.357939 & -152.474941 & 524429 & 7248257 & $5 \mathrm{~N}$ & Gabbro & Altered \\
\hline 2011LF561A & 65.294159 & -152.918716 & 503791 & 7241049 & $5 \mathrm{~N}$ & Chlorite-calcite quartz schist & -- \\
\hline 2011LF562B & 65.293576 & -152.919769 & 503742 & 7240984 & $5 \mathrm{~N}$ & Chlorite-quartz-albite schist & Inhomgenous \\
\hline 2011LF567B & 65.289299 & -152.944927 & 502569 & 7240506 & $5 \mathrm{~N}$ & Quartz-chlorite-albite schist & Inhomogenous \\
\hline 2011LF570A & 65.290264 & -152.962097 & 501768 & 7240613 & $5 \mathrm{~N}$ & Garnet-amphibolite(?) & --- \\
\hline 2011LF572A & 65.291092 & -152.971743 & 501318 & 7240705 & $5 \mathrm{~N}$ & Amphibolite(?) & --- \\
\hline 2011LF572B & 65.291092 & -152.971743 & 501318 & 7240705 & $5 \mathrm{~N}$ & Pyrite gabbro(?) & Altered \\
\hline 2011LF576A & 65.293444 & -152.983405 & 500774 & 7240967 & $5 \mathrm{~N}$ & Glaucophane Metamafic & --- \\
\hline 2011LF577A & 65.293893 & -152.985291 & 500686 & 7241017 & $5 \mathrm{~N}$ & Glaucophane Metamafic & --- \\
\hline 2011LF580A & 65.214773 & \begin{tabular}{|l|}
-152.849635 \\
\end{tabular} & 507034 & 7232207 & $5 \mathrm{~N}$ & Albite metagrit(?) & Porous \\
\hline 2011LF591A & 65.436402 & -152.644560 & 516488 & 7256947 & $5 \mathrm{~N}$ & Chlorite-muscovite quartz schist & Porous \\
\hline 2011LF592B & 65.432251 & -152.642095 & 516605 & 7256485 & $5 \mathrm{~N}$ & Muscovite-chlorite quartz schist & Porous, small \\
\hline 2011LF594A & 65.424737 & -152.644223 & 516511 & 7255647 & $5 \mathrm{~N}$ & Quartzite & Porous \\
\hline 2011LF594C & 65.424737 & -152.644223 & 516511 & 7255647 & $5 \mathrm{~N}$ & Chlorite-quartz-albite schist & Porous, small \\
\hline 2011RN151B & 65.245713 & -152.926292 & 503444 & 7235649 & $5 \mathrm{~N}$ & Gabbro & Altered, inhomgenous \\
\hline 2011RN169A & 65.403679 & -152.500983 & 523177 & 7253345 & $5 \mathrm{~N}$ & Gabbro & Altered \\
\hline 2011RN171A & 65.401736 & -152.507737 & 522865 & 7253126 & $5 \mathrm{~N}$ & Gabbro & Altered \\
\hline
\end{tabular}


Table 8. (continued)

\begin{tabular}{|c|c|c|c|c|c|c|c|c|c|}
\hline Sample_ID & $\mathrm{SiO}_{2 \_p c t}$ & Al203_pct & BaO_pct & CaO_pct & FeO_pct & K2O_pct & MgO_pct & MnO_pct & $\mathrm{Na} 2 \mathrm{O} \_p c t$ \\
\hline 2011LF490B & 89.3 & 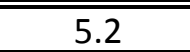 & 0.0697 & 0.888 & 0.713 & 2.1 & 0.568 & $<0.03$ & 0.795 \\
\hline 2011LF494A & 87.50587 & 7.2 & 0.03623 & 0.15284 & 2.89093 & 0.89193 & 0.47471 & $<0.03$ & 0.07978 \\
\hline 2011LF504A & 44.6 & 22 & 0.0384 & 0.0825 & 28 & 0.099 & 0.398 & 0.6 & 0.0731 \\
\hline 2011LF509A & 54.4 & 16.5 & $<0.03$ & 6.03 & 11.5 & 0.146 & 3.65 & 0.221 & 6.39 \\
\hline 2011LF509B & 94.3 & 1.3 & $<0.03$ & 0.96 & 0.959 & 0.097 & 0.221 & $<0.03$ & 0.166 \\
\hline 2011LF509B & 93.8 & 1.3 & $<0.03$ & 1.2 & 0.98 & 0.0753 & 0.153 & $<0.03$ & 0.0853 \\
\hline 2011LF509B & 94.4 & 1.33 & $<0.03$ & 0.931 & 0.941 & 0.0956 & 0.191 & $<0.03$ & 0.167 \\
\hline 2011LF516A & 51.5 & 14.4 & $<0.03$ & 7.93 & 12.5 & 0.0343 & 6.62 & 0.253 & 4.85 \\
\hline 2011LF516A & 52 & 14 & $<0.03$ & 7.73 & 12.2 & 0.0345 & 6.79 & 0.263 & 5.03 \\
\hline 2011LF529A & 46.5 & 17.5 & 0.0691 & 10 & 14.6 & 5.29 & 1.67 & 0.168 & 0.287 \\
\hline 2011LF533B & 49.8 & 15.1 & $<0.03$ & 8.57 & 12.4 & 0.106 & 5.91 & 0.3 & 5.79 \\
\hline 2011LF535A & 50.8 & 12.6 & 0.0703 & 12.1 & 10.2 & 0.634 & 9.08 & 0.203 & 2.65 \\
\hline 2011LF536A & 51.7 & 10 & 0.0404 & 13.8 & 9.72 & 0.333 & 10.3 & 0.226 & 2.46 \\
\hline 2011LF537A & 53.4 & 14.4 & 0.0326 & 10.2 & 11.8 & 0.151 & 5.26 & 0.174 & 2.87 \\
\hline 2011LF553A & 56.3 & 14.6 & 0.0622 & 6.17 & 11.5 & 0.525 & 2.83 & 0.201 & 5.47 \\
\hline 2011LF556A & 50.9 & 16.9 & 0.0456 & 12.9 & 6.82 & 0.75 & 7.93 & 0.125 & 2.55 \\
\hline 2011LF561A & 74.01196 & 5.9 & 0.04941 & 15.03889 & 2.52471 & 0.94767 & 0.57607 & 0.05162 & 0.31496 \\
\hline 2011LF562B & 74.89452 & 12 & $<0.03$ & 0.27901 & 4.03489 & 0.04194 & 1.30066 & 0.07694 & 6.71397 \\
\hline 2011LF567B & 63 & 15.6 & 0.0819 & 0.373 & 9.77 & 0.722 & 3.69 & 0.131 & 5.15 \\
\hline 2011LF570A & 51.9 & 13.3 & 0.0579 & 8.63 & 15.2 & 0.282 & 5.39 & 0.269 & 2.57 \\
\hline 2011LF572A & 50.43179 & 14.9 & 0.04317 & 11.86834 & 10.48648 & 0.39611 & 7.5933 & 0.16557 & 2.86006 \\
\hline 2011LF572B & 44.2 & 14.9 & $<0.03$ & 10.2 & 18.8 & 0.0925 & 6.64 & 0.189 & 2.42 \\
\hline 2011LF576A & 52.88923 & 16 & 0.04338 & 9.12928 & 9.5277 & 0.16359 & 5.74286 & 0.11404 & 5.09998 \\
\hline 2011LF577A & 51.42263 & 14.1 & 0.05171 & 10.01802 & 11.26653 & 0.30076 & 7.0013 & 0.15735 & 4.02804 \\
\hline 2011LF580A & 85.9 & 9.2 & 0.0331 & 0.103 & 1.22 & 0.338 & 0.369 & 0.159 & 1.89 \\
\hline 2011LF591A & 83.6 & 7.6 & 0.0599 & 0.136 & 5.1 & 1.14 & 0.72 & 0.0917 & 0.406 \\
\hline 2011LF592B & 79.2 & 7.9 & 0.0745 & 2.1 & 5.35 & 1.47 & 2.45 & 0.0704 & 0.236 \\
\hline 2011LF594A & 79.04791 & 11.2 & 0.05101 & 0.36158 & 3.2172 & 1.3328 & 1.29711 & 0.22574 & 2.11262 \\
\hline 2011LF594C & 64.10873 & 16.2 & 0.04717 & 0.22252 & 8.25732 & 0.79253 & 3.57922 & 0.18275 & 5.26568 \\
\hline 2011RN151B & 53.3 & 15.5 & $<0.03$ & 8.24 & 10.9 & 0.109 & 7.94 & 0.179 & 2.54 \\
\hline 2011RN169A & 48 & 15.3 & 0.0391 & 12.3 & 11.6 & 0.573 & 6.32 & 0.322 & 3.17 \\
\hline 2011RN171A & 48.9 & 16.2 & 0.0489 & 13.5 & 8.4 & 0.267 & 8.56 & 0.244 & 2.86 \\
\hline
\end{tabular}


Table 8. (continued)

\begin{tabular}{|c|c|c|c|c|c|c|c|c|c|}
\hline Sample_ID & P2O5_pct & TiO2_pct & CO2_pct & Total_pct & As_ppm & Bi_ppm & Br_ppm & Ce_ppm & Cl_ppm \\
\hline 2011LF490B & 0.0682 & 0.155 & $<<0.05$ & 99.90642 & $<4$ & $<<2$ & $<<$ & $<60$ & 347 \\
\hline 2011LF494A & 0.16963 & 0.47643 & $<0.05$ & 99.91544 & $<4$ & $<2$ & $<5$ & $<60$ & $<50$ \\
\hline 2011LF504A & 0.585 & 3.18 & $<0.05$ & 99.656 & 13 & $<2$ & $<5$ & $<60$ & 284 \\
\hline 2011LF509A & 0.0517 & 0.943 & $<0.05$ & 99.8517 & $<4$ & $<2$ & $<5$ & $<60$ & 177 \\
\hline 2011LF509B & 2 & 0.0485 & $<0.05$ & 100.0456 & 11 & $<2$ & $<5$ & $<60$ & 426 \\
\hline 2011LF509B & 2.1 & 0.0542 & $<0.05$ & 99.7258 & 9 & $<2$ & $<5$ & $<60$ & 85 \\
\hline 2011LF509B & 1.8 & 0.0439 & $<0.05$ & 99.9123 & 17 & $<2$ & $<5$ & $<60$ & 392 \\
\hline 2011LF516A & 0.184 & 1.61 & $<0.05$ & 99.9002 & $<4$ & $<2$ & $<5$ & $<60$ & 130 \\
\hline 2011LF516A & 0.189 & 1.61 & $<0.05$ & 99.8566 & $<4$ & $<2$ & $<5$ & $<60$ & 182 \\
\hline 2011LF529A & 0.46 & 3.2 & $<0.05$ & 99.7341 & 61 & $<2$ & $<5$ & $<60$ & 348 \\
\hline 2011LF533B & 0.195 & 1.65 & $<0.05$ & 99.8437 & $<4$ & $<2$ & $<5$ & $<60$ & $<50$ \\
\hline 2011LF535A & 0.155 & 1.1 & $<0.05$ & 99.5923 & $<4$ & $<2$ & $<5$ & $<60$ & 288 \\
\hline 2011LF536A & 0.152 & 1.07 & $<0.05$ & 99.7814 & $<4$ & $<2$ & $<5$ & $<60$ & 346 \\
\hline 2011LF537A & 0.218 & 1.29 & $<0.05$ & 99.7956 & $<4$ & $<2$ & $<5$ & $<60$ & 112 \\
\hline 2011LF553A & 0.465 & 1.72 & $<0.05$ & 99.8432 & $<4$ & $<2$ & $<5$ & $<60$ & 147 \\
\hline 2011LF556A & 0.179 & 0.764 & $<0.05$ & 99.8636 & $<4$ & $<2$ & $<5$ & $<60$ & 256 \\
\hline 2011LF561A & 0.17433 & 0.31516 & $<0.05$ & 99.90497 & $<4$ & $<2$ & $<5$ & $<60$ & 140 \\
\hline \begin{tabular}{|l|} 
2011LF562B \\
\end{tabular} & 0.19013 & 0.37544 & $<0.05$ & 99.94002 & $<4$ & $<2$ & $<5$ & $<60$ & 52 \\
\hline 2011LF567B & 0.415 & 0.919 & $<0.05$ & 99.8519 & $<4$ & $<2$ & $<5$ & $<60$ & 374 \\
\hline 2011LF570A & 0.266 & 1.86 & $<0.05$ & 99.7249 & 17 & $<2$ & $<5$ & $<60$ & 72 \\
\hline 2011LF572A & 0.21703 & 0.88631 & $<0.05$ & 99.85729 & $<4$ & $<2$ & $<5$ & $<60$ & 150 \\
\hline 2011LF572B & $<0.03$ & 2.11 & $<0.05$ & 99.6005 & $<4$ & $<2$ & $<5$ & $<60$ & 212 \\
\hline 2011LF576A & 0.21273 & 0.89322 & $<0.05$ & 99.86094 & $<4$ & $<2$ & $<5$ & $<60$ & 128 \\
\hline 2011LF577A & 0.28826 & 1.20052 & $<0.05$ & 99.79614 & 6 & $<2$ & $<5$ & $<60$ & 219 \\
\hline 2011LF580A & 0.0456 & 0.568 & $<0.05$ & 99.8457 & $<4$ & $<2$ & $<5$ & $<60$ & $<50$ \\
\hline 2011LF591A & 0.244 & 0.627 & $<0.05$ & 99.7246 & $<4$ & $<2$ & $<5$ & $<60$ & 160 \\
\hline 2011LF592B & 0.238 & 0.679 & $<0.05$ & 99.7579 & $<4$ & $<2$ & $<5$ & $<60$ & 225 \\
\hline 2011LF594A & 0.36177 & 0.59788 & $<0.05$ & 99.83314 & 6 & $<2$ & $<5$ & $<60$ & 505 \\
\hline 2011LF594C & 0.24506 & 1 & $<0.05$ & 99.85639 & $<4$ & $<2$ & $<5$ & $<60$ & 148 \\
\hline 2011RN151B & 0.128 & 0.96 & $<0.05$ & 99.821 & $<4$ & $<2$ & $<5$ & $<60$ & 181 \\
\hline 2011RN169A & 0.151 & 1.41 & $<0.05$ & 99.1851 & $<4$ & $<2$ & $<5$ & $<60$ & 659 \\
\hline 2011RN171A & 0.119 & 0.702 & $<0.05$ & 99.8009 & $<4$ & $<2$ & $<5$ & $<60$ & 256 \\
\hline
\end{tabular}


Table 8. (continued)

\begin{tabular}{|c|c|c|c|c|c|c|c|c|c|}
\hline Sample_ID & Co_ppm & Cr_ppm & Cu_ppm & F_ppm & La_ppm & Mo_ppm & Nb_ppm & Nb_ppm_2 & Ni_ppm \\
\hline 2011LF490B & $<<10$ & 48 & 25 & $<<110$ & $<50$ & $2<1$ & $<1$ & 3 & 17 \\
\hline 2011LF494A & 36 & 100 & 30 & 113 & $<50$ & $<1$ & $<1$ & 7 & 32 \\
\hline 2011LF504A & 39 & 214 & 17 & $<110$ & $<50$ & $<1$ & $<1$ & 15 & 51 \\
\hline 2011LF509A & 80 & 96 & 86 & $<110$ & $<50$ & $<1$ & $<1$ & 2 & 106 \\
\hline 2011LF509B & $<10$ & 35 & 41 & 110 & $<50$ & $<1$ & $<1$ & --- & 37 \\
\hline 2011LF509B & $<10$ & $<30$ & 47 & $<110$ & $<50$ & $<1$ & $<1$ & 1 & 26 \\
\hline 2011LF509B & $<10$ & $<30$ & $<7$ & $<110$ & $<50$ & $<1$ & $<1$ & 1 & 28 \\
\hline 2011LF516A & 57 & 384 & 12 & $<110$ & $<50$ & $<1$ & $<1$ & 1 & 96 \\
\hline 2011LF516A & 34.9 & 156 & 20 & $<110$ & $<50$ & $<1$ & $<1$ & 1.1 & 51 \\
\hline 2011LF529A & 51 & 136 & 13 & 177 & $<50$ & $<1$ & $<1$ & 10 & 48 \\
\hline 2011LF533B & 33 & 231 & 110 & $<110$ & $<50$ & $<1$ & $<1$ & 5 & 56 \\
\hline 2011LF535A & 87 & 805 & 229 & $<110$ & $<50$ & $<1$ & $<1$ & 6 & 196 \\
\hline 2011LF536A & 33 & 1030 & 138 & $<110$ & $<50$ & $<1$ & $<1$ & 4 & 199 \\
\hline 2011LF537A & 33 & 211 & 34 & $<110$ & $<50$ & $<1$ & $<1$ & 6 & 71 \\
\hline 2011LF553A & 22 & $<30$ & 103 & $<110$ & $<50$ & $<1$ & $<1$ & 14 & 9 \\
\hline 2011LF556A & 31 & 311 & 47 & $<110$ & $<50$ & $<1$ & $<1$ & 4 & 151 \\
\hline 2011LF561A & $<10$ & 52 & 43 & $<110$ & $<50$ & $<1$ & $<1$ & 6 & 24 \\
\hline \begin{tabular}{|l|} 
2011LF562B \\
\end{tabular} & $<10$ & $<30$ & 30 & $<110$ & $<50$ & $<1$ & $<1$ & 8 & 23 \\
\hline 2011LF567B & 48 & 74 & 46 & $<110$ & $<50$ & $<1$ & $<1$ & 5 & 40 \\
\hline 2011LF570A & 72 & 141 & 182 & 1090 & $<50$ & $<1$ & $<1$ & 14 & 56 \\
\hline 2011LF572A & 87 & 148 & 35 & $<110$ & $<50$ & $<1$ & $<1$ & 5 & 51 \\
\hline 2011LF572B & 205 & 96 & $<7$ & $<110$ & $<50$ & $<1$ & $<1$ & 4 & 42 \\
\hline 2011LF576A & 45 & 186 & 50 & $<110$ & $<50$ & $<1$ & $<1$ & 7 & 108 \\
\hline 2011LF577A & 75 & 466 & 101 & $<110$ & $<50$ & $<1$ & $<1$ & 7 & 95 \\
\hline 2011LF580A & 28 & 81 & 244 & $<110$ & $<50$ & $<1$ & $<1$ & 10 & 29 \\
\hline \begin{tabular}{|l|}
$2011 L F 591 A$ \\
\end{tabular} & 36 & 84 & 34 & 1130 & $<50$ & $<1$ & $<1$ & 7 & 40 \\
\hline 2011LF592B & $<10$ & 290 & 39 & 1080 & $<50$ & $<1$ & $<1$ & 7 & 90 \\
\hline 2011LF594A & $<10$ & 110 & 48 & $<110$ & $<50$ & $<1$ & $<1$ & 7 & 40 \\
\hline 2011LF594C & 30 & 150 & 56 & $<110$ & $<50$ & $<1$ & $<1$ & 12 & $<7$ \\
\hline 2011RN151B & 59 & 394 & 62 & $<110$ & $<50$ & $<1$ & $<1$ & 5 & 166 \\
\hline 2011RN169A & 110 & 266 & 196 & $<110$ & $<50$ & $<1$ & $<1$ & 4 & 67 \\
\hline 2011RN171A & 37 & 272 & 64 & $<110$ & $<50$ & $<1$ & $<1$ & 3 & 196 \\
\hline
\end{tabular}


Table 8. (continued)

\begin{tabular}{|c|c|c|c|c|c|c|c|c|c|}
\hline Sample_ID & Pb_ppm & Rb_ppm & Rb_ppm_2 & S_ppm & Sb_ppm & Sn_ppm & Sr_ppm & Sr_ppm_2 & Th_ppm \\
\hline 2011LF490B & 25 & $<1$ & 61 & 110 & $<2$ & $<4$ & $<1$ & 71 & $<2$ \\
\hline 2011LF494A & 26 & $<1$ & 43 & 47 & $<2$ & $<4$ & $<1$ & 6 & $<2$ \\
\hline 2011LF504A & $<4$ & $<1$ & 3 & 245 & $<2$ & 27 & $<1$ & 7 & $<2$ \\
\hline 2011LF509A & $<4$ & $<1$ & 3 & 171 & $<2$ & $<4$ & $<1$ & 162 & $<2$ \\
\hline 2011LF509B & 35 & $<1$ & --- & 338 & $<2$ & $<4$ & $<1$ & --- & $<2$ \\
\hline 2011LF509B & 17 & $<1$ & $<1$ & 229 & $<2$ & $<4$ & $<1$ & 32 & $<2$ \\
\hline 2011LF509B & $<4$ & $<1$ & 4 & 313 & $<2$ & $<4$ & $<1$ & 30 & $<2$ \\
\hline 2011LF516A & $<4$ & $<1$ & 1 & 126 & $<2$ & 30 & $<1$ & 98 & $<2$ \\
\hline 2011LF516A & 15 & $<1$ & 1 & 62 & $<2$ & $<4$ & $<1$ & 90.3 & $<2$ \\
\hline 2011LF529A & $<4$ & $<1$ & 166 & 194 & $<2$ & $<4$ & $<1$ & 29 & $<2$ \\
\hline 2011LF533B & $<4$ & $<1$ & 3 & 49 & $<2$ & $<4$ & $<1$ & 139 & $<2$ \\
\hline 2011LF535A & $<4$ & $<1$ & 20 & 601 & $<2$ & $<4$ & $<1$ & 92 & $<2$ \\
\hline 2011LF536A & 31 & $<1$ & 9 & 106 & $<2$ & $<4$ & $<1$ & 112 & $<2$ \\
\hline 2011LF537A & $<4$ & $<1$ & 3 & 324 & $<2$ & $<4$ & $<1$ & 96 & $<2$ \\
\hline 2011LF553A & $<4$ & $<1$ & 17 & 209 & $<2$ & $<4$ & $<1$ & 149 & $<2$ \\
\hline 2011LF556A & $<4$ & $<1$ & 21 & 138 & $<2$ & $<4$ & $<1$ & 163 & $<2$ \\
\hline 2011LF561A & $<4$ & $<1$ & 47 & 61 & $<2$ & $<4$ & $<1$ & 132 & $<2$ \\
\hline 2011LF562B & $<4$ & $<1$ & 2 & 60 & $<2$ & $<4$ & $<1$ & 22 & $<2$ \\
\hline 2011LF567B & 63 & $<1$ & 22 & 113 & $<2$ & $<4$ & $<1$ & 29 & $<2$ \\
\hline 2011LF570A & $<4$ & $<1$ & 10 & 129 & $<2$ & 28 & $<1$ & 258 & $<2$ \\
\hline 2011LF572A & 20 & $<1$ & 14 & 69 & $<2$ & $<4$ & $<1$ & 314 & $<2$ \\
\hline 2011LF572B & $<4$ & $<1$ & 1 & 2010 & $<2$ & 24 & $<1$ & 246 & $<2$ \\
\hline 2011LF576A & $<4$ & $<1$ & 5 & 111 & $<2$ & $<4$ & $<1$ & 234 & $<2$ \\
\hline 2011LF577A & $<4$ & $<1$ & 9 & 250 & $<2$ & $<4$ & $<1$ & 239 & $<2$ \\
\hline 2011LF580A & 43 & $<1$ & 12 & 72 & $<2$ & $<4$ & $<1$ & 106 & $<2$ \\
\hline 2011LF591A & 22 & $<1$ & 47 & 128 & $<2$ & $<4$ & $<1$ & 35 & $<2$ \\
\hline 2011LF592B & $<4$ & $<1$ & 74 & 277 & $<2$ & $<4$ & $<1$ & 58 & $<2$ \\
\hline 2011LF594A & 43 & $<1$ & 68 & 44 & $<2$ & $<4$ & $<1$ & 74 & $<2$ \\
\hline 2011LF594C & 51 & $<1$ & 38 & $<30$ & $<2$ & $<4$ & $<1$ & 18 & $<2$ \\
\hline 2011RN151B & $<4$ & $<1$ & 1 & 68 & $<2$ & $<4$ & $<1$ & 192 & $<2$ \\
\hline 2011RN169A & $<4$ & $<1$ & 18 & 5430 & $<2$ & $<4$ & $<1$ & 72 & $<2$ \\
\hline 2011RN171A & $<4$ & $<1$ & 8 & 108 & $<2$ & $<4$ & $<1$ & 166 & $<2$ \\
\hline
\end{tabular}




\begin{tabular}{|c|c|c|c|c|c|c|c|c|}
\hline Sample_ID & U_ppm & V_ppm & W_ppm & Y_ppm & Y_ppm_2 & Zn_ppm & Zr_ppm & Zr_ppm_2 \\
\hline 2011LF490B & $2<1$ & 33 & $<<8$ & $<1$ & 11 & $\overline{13}$ & $<9$ & $\overline{56}$ \\
\hline 2011LF494A & $<1$ & 80 & $<8$ & $<1$ & 17 & 40 & $<9$ & 129 \\
\hline 2011LF504A & $<1$ & 543 & $<8$ & $<1$ & 39 & 222 & $<9$ & 215 \\
\hline 2011LF509A & $<1$ & 501 & $<8$ & $<1$ & 31 & 112 & $<9$ & 71 \\
\hline 2011LF509B & $<1$ & 72 & $<8$ & $<1$ & --- & 18 & $<9$ & $<9$ \\
\hline 2011LF509B & $<1$ & 54 & $<8$ & $<1$ & 10 & 15 & $<9$ & 13 \\
\hline 2011LF509B & $<1$ & 68 & $<8$ & $<1$ & 8 & 18 & $<9$ & 13 \\
\hline 2011LF516A & $<1$ & 344 & $<8$ & $<1$ & 45 & 239 & $<9$ & 131 \\
\hline 2011LF516A & $<1$ & 401 & $<8$ & $<1$ & 45.2 & 262 & $<9$ & 129.7 \\
\hline 2011LF529A & $<1$ & 638 & $<8$ & $<1$ & 40 & 98 & $<9$ & 195 \\
\hline 2011LF533B & $<1$ & 474 & $<8$ & $<1$ & 31 & 147 & $<9$ & 105 \\
\hline 2011LF535A & $<1$ & 374 & $<8$ & $<1$ & 18 & 123 & $<9$ & 75 \\
\hline 2011LF536A & $<1$ & 410 & $<8$ & $<1$ & 19 & 76 & $<9$ & 63 \\
\hline 2011LF537A & $<1$ & 422 & $<8$ & $<1$ & 27 & 99 & $<9$ & 93 \\
\hline 2011LF553A & $<1$ & 302 & $<8$ & $<1$ & 41 & 136 & $<9$ & 210 \\
\hline 2011LF556A & $<1$ & 259 & $<8$ & $<1$ & 14 & 37 & $<9$ & 56 \\
\hline 2011LF561A & $<1$ & 95 & $<8$ & $<1$ & 16 & 50 & $<9$ & 99 \\
\hline 2011LF562B & $<1$ & $<25$ & $<8$ & $<1$ & 38 & 48 & $<9$ & 221 \\
\hline 2011LF567B & $<1$ & 261 & $<8$ & $<1$ & 22 & 168 & $<9$ & 110 \\
\hline 2011LF570A & $<1$ & 447 & $<8$ & $<1$ & 44 & 116 & $<9$ & 179 \\
\hline 2011LF572A & $<1$ & 307 & $<8$ & $<1$ & 17 & 76 & $<9$ & 81 \\
\hline 2011LF572B & $<1$ & 980 & $<8$ & $<1$ & 12 & 126 & $<9$ & 44 \\
\hline 2011LF576A & $<1$ & 321 & $<8$ & $<1$ & 19 & 64 & $<9$ & 115 \\
\hline 2011LF577A & $<1$ & 381 & $<8$ & $<1$ & 21 & 75 & $<9$ & 83 \\
\hline 2011LF580A & $<1$ & 103 & $<8$ & $<1$ & 14 & 17 & $<9$ & 210 \\
\hline 2011LF591A & $<1$ & 223 & $<8$ & $<1$ & 12 & 78 & $<9$ & 89 \\
\hline 2011LF592B & $<1$ & 107 & $<8$ & $<1$ & 36 & 66 & $<9$ & 150 \\
\hline 2011LF594A & $<1$ & 167 & $<8$ & $<1$ & 34 & 26 & $<9$ & 107 \\
\hline 2011LF594C & $<1$ & 283 & $<8$ & $<1$ & 29 & 164 & $<9$ & 182 \\
\hline 2011RN151B & $<1$ & 238 & $<8$ & $<1$ & 21 & 83 & $<9$ & 97 \\
\hline 2011RN169A & $<1$ & 453 & $<8$ & $<1$ & 27 & 94 & $<9$ & 83 \\
\hline 2011RN171A & $<1$ & 244 & $<8$ & $<1$ & 14 & 66 & $<9$ & 47 \\
\hline
\end{tabular}


Table 8. (continued)

\begin{tabular}{|c|c|c|c|c|c|c|c|}
\hline Sample_ID & Latitude & Longitude & Easting & Northing & $\begin{array}{l}\text { UTM_ } \\
\text { Zone }\end{array}$ & Root_name & Comments \\
\hline 2011RN176B & 65.403829 & $\mid-152.998514$ & $\mid 500069$ & 7253270 & $5 \mathrm{~N}$ & Granite aplite & |-- \\
\hline 2011RN177C & 65.404843 & -152.990224 & 500454 & 7253383 & $5 \mathrm{~N}$ & Tourmaline granite aplite & --- \\
\hline 2011RN178A & 65.406125 & -152.987231 & 500593 & 7253526 & $5 \mathrm{~N}$ & Quartz-muscovite schist & --- \\
\hline 2011RN179A & 65.407650 & -152.984538 & 500718 & 7253696 & $5 \mathrm{~N}$ & Garnet orthogneiss(?) & Inhomogenous \\
\hline 2011RN179B & 65.407650 & -152.984538 & 500718 & 7253696 & $5 \mathrm{~N}$ & Tourmaline granite aplite & Inhomogenous \\
\hline 2011RN182C & 65.411103 & -152.971248 & 501335 & 7254081 & $5 \mathrm{~N}$ & Tourmaline granite aplite & --- \\
\hline 2011RN187A & 65.351343 & -152.675169 & 515117 & 7247459 & $5 \mathrm{~N}$ & Muscovite quartzite & Inhomogenous \\
\hline 2011RN216A & 65.488008 & -152.587353 & 519104 & 7262715 & $5 \mathrm{~N}$ & Gabbro & Altered \\
\hline 2011RN217A & 65.488851 & -152.587253 & 519108 & 7262809 & $5 \mathrm{~N}$ & Metagabbro & --- \\
\hline 2011RN218A & 65.490122 & -152.592591 & 518860 & 7262949 & $5 \mathrm{~N}$ & Fine-grained wacke(?) & --- \\
\hline 2011RN218B & 65.490122 & -152.592591 & 518860 & 7262949 & $5 \mathrm{~N}$ & Fine-grained gabbro & Altered \\
\hline 2011RN220A & 65.494465 & -152.596347 & 518683 & 7263432 & $5 \mathrm{~N}$ & Fine-grained gabbro & Altered \\
\hline 2011RN223A & 65.338031 & -153.860875 & 459917 & 7246210 & $5 \mathrm{~N}$ & Allanite granite(?) & Inhomogenous \\
\hline 2011RN224B & 65.336564 & -153.855308 & 460174 & 7246043 & $5 \mathrm{~N}$ & Fine-grained granite & --- \\
\hline 2011RN229B & 65.331435 & -153.832273 & 461239 & 7245457 & $5 \mathrm{~N}$ & Medium-grained greenstone & --- \\
\hline 2011RN236A & 65.322882 & -153.795940 & 462919 & 7244482 & $5 \mathrm{~N}$ & Feldspar-calc-silicate quartzite(?) & Inhomogenous \\
\hline 2011RN238A & 65.322213 & -153.786775 & 463345 & 7244402 & $5 \mathrm{~N}$ & Feldspar quartzite & Inhomogenous, poor polish \\
\hline 2011RN245A & 65.389542 & -152.518876 & 522358 & 7251763 & $5 \mathrm{~N}$ & Gabbro & Altered \\
\hline 2011RN246A & 65.388541 & -152.517130 & 522440 & 7251652 & $5 \mathrm{~N}$ & Fine-grained metawacke & --- \\
\hline 2011RN249A & 65.384742 & -152.518857 & 522363 & 7251228 & $5 \mathrm{~N}$ & Fine-grained metawacke & --- \\
\hline 2011RN250A & 65.383062 & -152.518048 & 522402 & 7251041 & $5 \mathrm{~N}$ & Fine-grained metawacke & --- \\
\hline 2011RN254A & 65.366248 & -152.887878 & 505215 & 7249086 & $5 \mathrm{~N}$ & Muscovite quartz schist & Inhomgenous \\
\hline 2011RN254B & 65.366248 & -152.887878 & 505215 & 7249086 & $5 \mathrm{~N}$ & Chlorite schist & Porous \\
\hline 2011RN254B & 65.366248 & -152.887878 & 505215 & 7249086 & $5 \mathrm{~N}$ & Chlorite schist & Porous \\
\hline 2011RN256B & 65.364778 & -152.889453 & 505142 & 7248922 & $5 \mathrm{~N}$ & Metagabbro & --- \\
\hline 2011RN257B & 65.363443 & -152.891351 & 505054 & 7248773 & $5 \mathrm{~N}$ & Metagabbro & Porous \\
\hline 2011RN258A & 65.362709 & -152.893977 & 504932 & 7248691 & $5 \mathrm{~N}$ & Mica quartz schist & Inhomogenous \\
\hline 2011RN258B & 65.362709 & -152.893977 & 504932 & 7248691 & $5 \mathrm{~N}$ & Metamafic & --- \\
\hline 2011RN259A & 65.361237 & -152.892800 & 504987 & 7248527 & $5 \mathrm{~N}$ & Metagabbro & --- \\
\hline 2011RN262A & 65.356260 & -152.897463 & 504771 & 7247972 & $5 \mathrm{~N}$ & Fine-grained gabbro & Altered \\
\hline 2011RN263B & 65.354888 & -152.898005 & 504746 & 7247819 & $5 \mathrm{~N}$ & Quartz mica schist & Inhomogenous \\
\hline 2011RN264A & 65.353094 & -152.899065 & 504697 & 7247619 & $5 \mathrm{~N}$ & Metagabbro & --- \\
\hline
\end{tabular}


Table 8. (continued)

\begin{tabular}{|c|c|c|c|c|c|c|c|c|c|}
\hline Sample_ID & $\mathrm{SiO}_{2 \_} \mathrm{pct}$ & Al203_pct & BaO_pct & CaO_pct & FeO_pct & K2O_pct & MgO_pct & MnO_pct & $\mathrm{Na} 2 \mathrm{O} \_p c t$ \\
\hline 2011RN176B & 70.7 & 15 & 0.099 & 1.44 & 2.13 & 6.88 & 0.333 & $<0.03$ & 2.67 \\
\hline 2011RN177C & 75.7 & 13.5 & 0.0322 & 0.54 & 0.891 & 4.29 & 0.214 & $<0.03$ & 4.4 \\
\hline 2011RN178A & 69.1 & 17.2 & 0.108 & 0.602 & 4.68 & 4.1 & 1.75 & 0.0521 & 0.836 \\
\hline 2011RN179A & 70.5 & 17.2 & 0.11 & 0.279 & 4.59 & 3.22 & 1.4 & 0.0589 & 1.62 \\
\hline 2011RN179B & 75.4 & 14.3 & 0.0454 & 0.646 & 0.743 & 4.61 & 0.207 & $<0.03$ & 3.66 \\
\hline 2011RN182C & 77.4 & 12.8 & $<0.03$ & 0.531 & 0.702 & 4.49 & 0.164 & $<0.03$ & 3.74 \\
\hline 2011RN187A & 91.8 & 3.8 & 0.0317 & 0.0592 & 1.75 & 0.929 & 0.436 & $<0.03$ & 0.055 \\
\hline 2011RN216A & 51.7 & 13.3 & 0.0855 & 12 & 10.4 & 0.0786 & 7.59 & 0.196 & 3.01 \\
\hline 2011RN217A & 50.6 & 15.7 & 0.0563 & 11.6 & 10.4 & 0.219 & 6.39 & 0.189 & 3.31 \\
\hline 2011RN218A & 78.6 & 8.2 & $<0.03$ & 0.819 & 4.45 & 0.099 & 1.28 & 0.0477 & 5.81 \\
\hline 2011RN218B & 48 & 15.5 & 0.112 & 10.9 & 13.3 & 0.186 & 6.11 & 0.268 & 3.58 \\
\hline 2011RN220A & 51.3 & 15.5 & 0.0823 & 11.2 & 10.5 & 0.156 & 5.8 & 0.213 & 3.4 \\
\hline 2011RN223A & 77 & 10.1 & $<0.03$ & 5 & 0.434 & 4.55 & 0.159 & $<0.03$ & 2.56 \\
\hline 2011RN224B & 74.4 & 13.5 & 0.0794 & 1.34 & 1.59 & 4.97 & 0.353 & 0.0364 & 3.32 \\
\hline 2011RN229B & 79 & 11.2 & $<0.03$ & 0.675 & 1.26 & 4.74 & 0.287 & $<0.03$ & 2.51 \\
\hline 2011RN236A & 89.3 & 3.4 & $<0.03$ & 3.4 & 1.04 & 0.379 & 1.31 & $<0.03$ & 0.889 \\
\hline 2011RN238A & 87.8 & 5.4 & 0.0923 & 1.69 & 0.445 & 2.72 & 0.698 & $<0.03$ & 0.751 \\
\hline 2011RN245A & 50.6 & 17.7 & 0.0305 & 13.4 & 5.86 & 0.235 & 6.88 & 0.133 & 4.15 \\
\hline 2011RN246A & 63.7 & 18.5 & 0.0381 & 2.84 & 1.67 & 0.0943 & 1.42 & 0.0442 & 10.8 \\
\hline 2011RN249A & 59.1 & 19.5 & 0.0642 & 4.77 & 4.21 & 0.115 & 1.38 & 0.072 & 9.72 \\
\hline 2011RN250A & 58.1 & 18 & 0.0585 & 5.92 & 5.09 & 0.709 & 2.18 & 0.106 & 8.59 \\
\hline 2011RN254A & 81.5 & 8.9 & 0.0862 & 0.213 & 4.55 & 1.34 & 1.2 & 0.0493 & 0.675 \\
\hline 2011RN254B & 55.8 & 13.7 & $<0.03$ & 0.178 & 16.7 & 0.0203 & 10.9 & 0.274 & 0.309 \\
\hline 2011RN254B & 53.8 & 16.3 & 0.0613 & 0.0848 & 16.9 & 0.757 & 8.85 & 0.253 & 0.486 \\
\hline 2011RN256B & 54.3 & 14.1 & $<0.03$ & 8.69 & 11.6 & 0.0278 & 5.56 & 0.205 & 4.06 \\
\hline 2011RN257B & 60.4 & 11.9 & $<0.03$ & 4.55 & 14.3 & 0.0212 & 5.02 & 0.25 & 1.38 \\
\hline 2011RN258A & 80.7 & 9.2 & 0.112 & 0.812 & 3.71 & 1.97 & 1.99 & 0.0394 & 0.531 \\
\hline 2011RN258B & 52.5 & 15.6 & $<0.03$ & 10 & 10.1 & 0.242 & 6.06 & 0.174 & 3.79 \\
\hline 2011RN259A & 50.6 & 15.2 & $<0.03$ & 10.8 & 10.3 & 0.0243 & 8.31 & 0.241 & 3.61 \\
\hline 2011RN262A & 52.3 & 17.9 & $<0.03$ & 7.86 & 10.2 & 0.0645 & 6.91 & 0.119 & 3.64 \\
\hline 2011RN263B & 72.1 & 14.9 & 0.127 & 0.134 & 6.44 & 2.92 & 1.51 & 0.0583 & 0.456 \\
\hline 2011RN264A & 57.7 & 11.6 & $<0.03$ & 10.8 & 11.5 & $<.02$ & 3.49 & 0.191 & 2.77 \\
\hline
\end{tabular}


Table 8. (continued)

\begin{tabular}{|c|c|c|c|c|c|c|c|c|c|}
\hline Sample_ID & P2O5_pct & TiO2_pct & CO2_pct & Total_pct & As_ppm & Bi_ppm & Br_ppm & Ce_ppm & Cl_ppm \\
\hline 2011RN176B & 0.0947 & 0.395 & $<0.05$ & 99.7666 & 43 & $<2$ & $<5$ & $<60$ & 510 \\
\hline 2011RN177C & 0.186 & 0.086 & $<0.05$ & 99.8513 & $<4$ & $<2$ & $<5$ & $<60$ & 735 \\
\hline 2011RN178A & 0.329 & 0.936 & $<0.05$ & 99.6931 & $<4$ & $<2$ & $<5$ & $<60$ & 520 \\
\hline 2011RN179A & 0.127 & 0.722 & $<0.05$ & 99.8269 & 4 & $<2$ & $<5$ & $<60$ & 593 \\
\hline 2011RN179B & 0.219 & 0.0797 & $<0.05$ & 99.9236 & $<4$ & $<2$ & $<5$ & $<60$ & 118 \\
\hline 2011RN182C & 0.0581 & 0.0444 & $<0.05$ & 99.9555 & $<4$ & $<2$ & $<5$ & $<60$ & 308 \\
\hline 2011RN187A & 0.111 & 0.221 & $<0.05$ & 99.15776 & $<4$ & $<2$ & $<5$ & $<60$ & 168 \\
\hline 2011RN216A & 0.172 & 1.26 & $<0.05$ & 99.7921 & $<4$ & $<2$ & $<5$ & $<60$ & 72 \\
\hline 2011RN217A & 0.187 & 1.3 & $<0.05$ & 99.9513 & $<4$ & $<2$ & $<5$ & $<60$ & 95 \\
\hline 2011RN218A & 0.212 & 0.362 & $<0.05$ & 99.8672 & $<4$ & $<2$ & $<5$ & $<60$ & 480 \\
\hline 2011RN218B & 0.268 & 1.57 & $<0.05$ & 99.794 & $<4$ & $<2$ & $<5$ & $<60$ & 351 \\
\hline 2011RN220A & 0.201 & 1.36 & $<0.05$ & 99.7123 & $<4$ & $<2$ & $<5$ & $<60$ & 195 \\
\hline 2011RN223A & $<0.03$ & 0.0421 & $<0.05$ & 99.827 & $<4$ & $<2$ & $<5$ & $<60$ & 678 \\
\hline 2011RN224B & 0.112 & 0.192 & $<0.05$ & 99.8928 & $<4$ & $<2$ & $<5$ & $<60$ & 416 \\
\hline 2011RN229B & 0.0524 & 0.146 & $<0.05$ & 99.9095 & $<4$ & $<2$ & $<5$ & $<60$ & 181 \\
\hline 2011RN236A & 0.0737 & 0.143 & $<0.05$ & 99.9606 & $<4$ & $<2$ & $<5$ & $<60$ & 110 \\
\hline 2011RN238A & 0.197 & 0.057 & $<0.05$ & 99.8299 & $<4$ & $<2$ & $<5$ & $<60$ & 412 \\
\hline 2011RN245A & 0.114 & 0.705 & $<0.05$ & 99.8075 & $<4$ & $<2$ & $<5$ & $<60$ & 221 \\
\hline 2011RN246A & 0.266 & 0.511 & $<0.05$ & 99.8836 & $<4$ & $<2$ & $<5$ & $<60$ & 341 \\
\hline 2011RN249A & 0.349 & 0.642 & $<0.05$ & 99.9222 & $<4$ & $<2$ & $<5$ & $<60$ & 62 \\
\hline 2011RN250A & 0.435 & 0.671 & $<0.05$ & 99.8595 & $<4$ & $<2$ & $<5$ & $<60$ & 124 \\
\hline 2011RN254A & 0.204 & 0.571 & $<0.05$ & 99.3085 & 4 & $<2$ & $<5$ & 567 & 696 \\
\hline 2011RN254B & 0.092 & 1.53 & $<0.05$ & 99.5213 & $<4$ & $<2$ & $<5$ & $<60$ & 329 \\
\hline 2011RN254B & 0.0399 & 1.46 & $<0.05$ & 98.992 & 23 & $<2$ & $<5$ & $<60$ & 243 \\
\hline 2011RN256B & 0.255 & 1.03 & $<0.05$ & 99.8468 & 8 & $<2$ & $<5$ & $<60$ & 386 \\
\hline 2011RN257B & 0.135 & 1.7 & $<0.05$ & 99.6804 & 75 & $<2$ & $<5$ & $<60$ & 460 \\
\hline 2011RN258A & 0.116 & 0.761 & $<0.05$ & 99.8914 & $<4$ & $<2$ & $<5$ & $<60$ & 135 \\
\hline 2011RN258B & 0.267 & 1 & $<0.05$ & 99.73616 & 13 & $<2$ & $<5$ & $<60$ & 196 \\
\hline 2011RN259A & 0.277 & 0.5 & $<0.05$ & 99.8808 & 4 & $<2$ & $<5$ & $<60$ & 103 \\
\hline 2011RN262A & 0.135 & 0.628 & $<0.05$ & 99.7833 & 32 & $<2$ & $<5$ & $<60$ & 261 \\
\hline 2011RN263B & 0.136 & 0.97 & $<0.05$ & 99.7513 & 29 & $<2$ & $<5$ & $<60$ & 50 \\
\hline 2011RN264A & 0.405 & 1.31 & $<0.05$ & 99.7762 & 17 & $<2$ & $<5$ & $<60$ & 110 \\
\hline
\end{tabular}


Table 8. (continued)

\begin{tabular}{|c|c|c|c|c|c|c|c|c|c|}
\hline Sample_ID & Co_ppm & Cr_ppm & Cu_ppm & F_ppm & La_ppm & Mo_ppm & Nb_ppm & Nb_ppm_2 & Ni_ppm \\
\hline 2011RN176B & $<<10$ & $<<30$ & $<<7$ & $<<110$ & $<<50$ & $2<1$ & $<<1$ & 12 & $<>$ \\
\hline 2011RN177C & $<10$ & $<30$ & $<7$ & $<110$ & $<50$ & $<1$ & $<1$ & 8 & $<7$ \\
\hline 2011RN178A & 103 & 102 & 28 & 970 & $<50$ & $<1$ & $<1$ & 14 & $<7$ \\
\hline 2011RN179A & 31 & 101 & $<7$ & $<110$ & $<50$ & $<1$ & $<1$ & 13 & 44 \\
\hline 2011RN179B & $<10$ & $<30$ & 14 & $<110$ & $<50$ & $<1$ & $<1$ & 9 & 29 \\
\hline 2011RN182C & $<10$ & $<30$ & $<7$ & $<110$ & $<50$ & $<1$ & $<1$ & 11 & $<7$ \\
\hline 2011RN187A & $<10$ & 63 & 40 & $<110$ & $<50$ & $<1$ & $<1$ & 3 & 43 \\
\hline 2011RN216A & 45 & 513 & 153 & $<110$ & $<50$ & $<1$ & $<1$ & 5 & 162 \\
\hline 2011RN217A & 47 & 231 & 136 & $<110$ & $<50$ & $<1$ & $<1$ & 5 & 96 \\
\hline 2011RN218A & 14 & $<30$ & $<7$ & $<110$ & $<50$ & $<1$ & $<1$ & 4 & 42 \\
\hline 2011RN218B & 51 & 149 & 145 & $<110$ & $<50$ & $<1$ & $<1$ & 7 & 78 \\
\hline 2011RN220A & 31 & 160 & 149 & $<110$ & $<50$ & $<1$ & $<1$ & 6 & 77 \\
\hline 2011RN223A & $<10$ & $<30$ & $<7$ & $<110$ & $<50$ & $<1$ & $<1$ & 5 & $<7$ \\
\hline 2011RN224B & $<10$ & $<30$ & 104 & $<110$ & $<50$ & $<1$ & $<1$ & 12 & 20 \\
\hline 2011RN229B & $<10$ & $<30$ & $<7$ & $<110$ & $<50$ & $<1$ & $<1$ & 8 & $<7$ \\
\hline 2011RN236A & 25 & $<30$ & 17 & $<110$ & $<50$ & $<1$ & $<1$ & 3 & 19 \\
\hline 2011RN238A & $<10$ & 44 & 20 & $<110$ & $<50$ & $<1$ & $<1$ & 2 & 24 \\
\hline 2011RN245A & 74 & 304 & 86 & $<110$ & $<50$ & $<1$ & $<1$ & 5 & 114 \\
\hline 2011RN246A & $<10$ & 66 & 57 & $<110$ & $<50$ & $<1$ & $<1$ & 15 & 42 \\
\hline 2011RN249A & 36 & 63 & $<7$ & $<110$ & $<50$ & $<1$ & $<1$ & 16 & 52 \\
\hline 2011RN250A & $<10$ & 67 & $<7$ & $<110$ & $<50$ & $<1$ & $<1$ & 15 & 33 \\
\hline 2011RN254A & 27 & 124 & 44 & $<110$ & 165 & $<1$ & $<1$ & 8 & 107 \\
\hline 2011RN254B & 40 & 372 & 102 & 1430 & $<50$ & $<1$ & $<1$ & 4 & 25 \\
\hline 2011RN254B & 10 & 379 & 176 & 1620 & $<50$ & $<1$ & $<1$ & 6 & 32 \\
\hline 2011RN256B & 75 & 96 & 37 & $<110$ & $<50$ & $<1$ & $<1$ & 8 & 58 \\
\hline 2011RN257B & 29 & 76 & 26 & $<110$ & $<50$ & $<1$ & $<1$ & 15 & 72 \\
\hline 2011RN258A & 27 & 75 & 36 & $<110$ & $<50$ & $<1$ & $<1$ & 10 & 8 \\
\hline 2011RN258B & 21 & 237 & 49 & $<110$ & $<50$ & $<1$ & $<1$ & 7 & 108 \\
\hline 2011RN259A & 62 & 537 & 215 & $<110$ & $<50$ & $<1$ & $<1$ & 1 & 121 \\
\hline 2011RN262A & 11 & 388 & 78 & $<110$ & $<50$ & $<1$ & $<1$ & 2 & 202 \\
\hline 2011RN263B & $<10$ & 108 & 33 & 1170 & $<50$ & $<1$ & $<1$ & 14 & 37 \\
\hline 2011RN264A & 31 & 90 & 65 & $<110$ & $<50$ & $<1$ & $<1$ & 9 & 40 \\
\hline
\end{tabular}


Table 8. (continued)

\begin{tabular}{|c|c|c|c|c|c|c|c|c|c|}
\hline Sample_ID & Pb_ppm & Rb_ppm & Rb_ppm_2 & S_ppm & Sb_ppm & Sn_ppm & Sr_ppm & Sr_ppm_2 & Th_ppm \\
\hline 2011RN176B & 42 & $<1$ & 253 & 75 & $<2$ & $<4$ & $<1$ & 185 & 19 \\
\hline 2011RN177C & 42 & $<1$ & 210 & 192 & $<2$ & $<4$ & $<1$ & 43 & 6 \\
\hline 2011RN178A & 60 & $<1$ & 137 & 55 & $<2$ & 15 & $<1$ & 73 & $<2$ \\
\hline 2011RN179A & 21 & $<1$ & 134 & 46 & $<2$ & $<4$ & $<1$ & 189 & $<2$ \\
\hline 2011RN179B & 72 & $<1$ & 223 & $<30$ & $<2$ & $<4$ & $<1$ & 73 & 7 \\
\hline 2011RN182C & 71 & $<1$ & 306 & 71 & $<2$ & $<4$ & $<1$ & 21 & 14 \\
\hline 2011RN187A & 21 & $<1$ & 31 & 8110 & $<2$ & $<4$ & $<1$ & 6 & $<2$ \\
\hline 2011RN216A & $<4$ & $<1$ & 2 & 86 & $<2$ & $<4$ & $<1$ & 90 & $<2$ \\
\hline 2011RN217A & $<4$ & $<1$ & 4 & 48 & $<2$ & $<4$ & $<1$ & 119 & $<2$ \\
\hline 2011RN218A & $<4$ & $<1$ & 4 & 334 & $<2$ & $<4$ & $<1$ & 43 & $<2$ \\
\hline 2011RN218B & $<4$ & $<1$ & 4 & 158 & $<2$ & $<4$ & $<1$ & 176 & $<2$ \\
\hline 2011RN220A & $<4$ & $<1$ & 3 & 109 & $<2$ & $<4$ & $<1$ & 261 & $<2$ \\
\hline 2011RN223A & 47 & $<1$ & 353 & 124 & $<2$ & $<4$ & $<1$ & 23 & 24 \\
\hline 2011RN224B & 62 & $<1$ & 287 & 57 & $<2$ & $<4$ & $<1$ & 98 & 28 \\
\hline 2011RN229B & 50 & $<1$ & 243 & 104 & $<2$ & $<4$ & $<1$ & 47 & 28 \\
\hline 2011RN236A & 15 & $<1$ & 11 & 37 & $<2$ & $<4$ & $<1$ & 119 & $<2$ \\
\hline 2011RN238A & 38 & $<1$ & 60 & 133 & $<2$ & 20 & $<1$ & 192 & $<2$ \\
\hline 2011RN245A & $<4$ & $<1$ & 7 & 75 & $<2$ & $<4$ & $<1$ & 125 & $<2$ \\
\hline 2011RN246A & $<4$ & $<1$ & 2 & 145 & $<2$ & $<4$ & $<1$ & 105 & $<2$ \\
\hline 2011RN249A & 32 & $<1$ & 2 & 42 & $<2$ & $<4$ & $<1$ & 116 & $<2$ \\
\hline 2011RN250A & 26 & $<1$ & 13 & 40 & $<2$ & $<4$ & $<1$ & 196 & $<2$ \\
\hline 2011RN254A & 37 & $<1$ & 60 & 3990 & $<2$ & $<4$ & $<1$ & 35 & $<2$ \\
\hline 2011RN254B & $<4$ & $<1$ & 1 & 303 & $<2$ & 22 & $<1$ & 10 & $<2$ \\
\hline 2011RN254B & 85 & $<1$ & 43 & 5730 & $<2$ & $<4$ & $<1$ & 15 & $<2$ \\
\hline 2011RN256B & $<4$ & $<1$ & $<1$ & 290 & $<2$ & $<4$ & $<1$ & 212 & $<2$ \\
\hline 2011RN257B & 41 & $<1$ & 1 & 263 & $<2$ & $<4$ & $<1$ & 139 & $<2$ \\
\hline 2011RN258A & 34 & $<1$ & 73 & 113 & $<2$ & 20 & $<1$ & 44 & $<2$ \\
\hline 2011RN258B & $<4$ & $<1$ & 11 & 143 & $<2$ & $<4$ & $<1$ & 216 & $<2$ \\
\hline 2011RN259A & $<4$ & $<1$ & $<1$ & 159 & $<2$ & $<4$ & $<1$ & 166 & $<2$ \\
\hline 2011RN262A & 40 & $<1$ & 3 & 313 & $<2$ & $<4$ & $<1$ & 200 & $<2$ \\
\hline 2011RN263B & 52 & $<1$ & 129 & 318 & $<2$ & $<4$ & $<1$ & 35 & 20 \\
\hline 2011RN264A & 22 & $<1$ & $<1$ & 109 & $<2$ & $<4$ & $<1$ & 192 & $<2$ \\
\hline
\end{tabular}




\begin{tabular}{|c|c|c|c|c|c|c|c|c|}
\hline Sample_ID & U_ppm & V_ppm & W_ppm & Y_ppm & Y_ppm_2 & Zn_ppm & Zr_ppm & Zr_ppm_2 \\
\hline 2011RN176B & 1 & $<25$ & $<8$ & $<1$ & 35 & 27 & $<9$ & 348 \\
\hline 2011RN177C & 3 & 37 & $<8$ & $<1$ & 15 & 20 & $<9$ & 46 \\
\hline 2011RN178A & $<1$ & 187 & $<8$ & $<1$ & 27 & 62 & $<9$ & 198 \\
\hline 2011RN179A & $<1$ & 138 & $<8$ & $<1$ & 18 & 39 & $<9$ & 135 \\
\hline 2011RN179B & 4 & $<25$ & $<8$ & $<1$ & 18 & 38 & $<9$ & 44 \\
\hline 2011RN182C & 5 & $<25$ & $<8$ & $<1$ & 51 & 24 & $<9$ & 65 \\
\hline 2011RN187A & $<1$ & 79 & $<8$ & $<1$ & 3 & 32 & $<9$ & 41 \\
\hline 2011RN216A & $<1$ & 330 & $<8$ & $<1$ & 24 & 99 & $<9$ & 93 \\
\hline 2011RN217A & $<1$ & 401 & $<8$ & $<1$ & 25 & 88 & $<9$ & 91 \\
\hline 2011RN218A & $<1$ & 89 & $<8$ & $<1$ & 24 & 33 & $<9$ & 82 \\
\hline 2011RN218B & $<1$ & 420 & $<8$ & $<1$ & 31 & 140 & $<9$ & 110 \\
\hline 2011RN220A & $<1$ & 439 & $<8$ & $<1$ & 26 & 97 & $<9$ & 95 \\
\hline 2011RN223A & 9 & $<25$ & $<8$ & $<1$ & 450 & 12 & $<9$ & 56 \\
\hline 2011RN224B & 3 & 29 & $<8$ & $<1$ & 29 & 24 & $<9$ & 135 \\
\hline 2011RN229B & 1 & $<25$ & $<8$ & $<1$ & 11 & 24 & $<9$ & 63 \\
\hline 2011RN236A & $<1$ & 54 & $<8$ & $<1$ & 7 & 21 & $<9$ & 71 \\
\hline 2011RN238A & $<1$ & $<25$ & $<8$ & $<1$ & 5 & $<8$ & $<9$ & 70 \\
\hline 2011RN245A & $<1$ & 191 & $<8$ & $<1$ & 12 & 39 & $<9$ & 60 \\
\hline 2011RN246A & $<1$ & 106 & $<8$ & $<1$ & 17 & 17 & $<9$ & 139 \\
\hline 2011RN249A & $<1$ & 154 & $<8$ & $<1$ & 20 & 31 & $<9$ & 138 \\
\hline 2011RN250A & $<1$ & 215 & $<8$ & $<1$ & 20 & 41 & $<9$ & 124 \\
\hline 2011RN254A & 12 & 162 & $<8$ & $<1$ & 81 & 159 & $<9$ & 145 \\
\hline 2011RN254B & $<1$ & 449 & $<8$ & $<1$ & 12 & 705 & $<9$ & 83 \\
\hline 2011RN254B & $<1$ & 539 & $<8$ & $<1$ & 26 & 734 & $<9$ & 102 \\
\hline 2011RN256B & $<1$ & 402 & $<8$ & $<1$ & 22 & 134 & $<9$ & 107 \\
\hline 2011RN257B & $<1$ & 584 & $<8$ & $<1$ & 40 & 152 & $<9$ & 167 \\
\hline 2011RN258A & $<1$ & 138 & $<8$ & $<1$ & 15 & 101 & $<9$ & 177 \\
\hline 2011RN258B & $<1$ & 304 & $<8$ & $<1$ & 21 & 110 & $<9$ & 98 \\
\hline 2011RN259A & $<1$ & 313 & $<8$ & $<1$ & 22 & 90 & $<9$ & 28 \\
\hline 2011RN262A & $<1$ & 273 & $<8$ & $<1$ & 28 & 132 & $<9$ & 44 \\
\hline 2011RN263B & $<1$ & 221 & $<8$ & $<1$ & 23 & 113 & $<9$ & 170 \\
\hline 2011RN264A & $<1$ & 473 & $<8$ & $<1$ & 25 & 139 & $<9$ & 103 \\
\hline
\end{tabular}


Table 8. (continued)

\begin{tabular}{|c|c|c|c|c|c|c|c|}
\hline Sample_ID & Latitude & Longitude & Easting & Northing & $\begin{array}{l}\text { UTM_ } \\
\text { Zone }\end{array}$ & Root_name & Comments \\
\hline 2011RN266A & 65.351881 & -152.895933 & 504843 & 7247484 & $5 \mathrm{~N}$ & Metagabbro & --- \\
\hline 2011RN267A & 65.254064 & -152.728602 & 512677 & 7236605 & $5 \mathrm{~N}$ & Chloritoid(?) quartzite(?) & Porous \\
\hline 2011RN271A & 65.248711 & -152.716136 & 513262 & 7236011 & $5 \mathrm{~N}$ & Quartz-mica schist & --- \\
\hline 2011RN283B & 65.220456 & -152.701578 & 513957 & 7232865 & $5 \mathrm{~N}$ & Chloritoid(?) quartz schist & Inhomogenous \\
\hline 2011RN285A & 65.243169 & -153.489009 & 477149 & 7235452 & $5 \mathrm{~N}$ & Quartz-mica schist & Inhomogenous \\
\hline 2011RN300B & 65.224440 & -153.511503 & 476081 & 7233373 & $5 \mathrm{~N}$ & Metagabbro & --- \\
\hline 2011RN301A & 65.201067 & -153.730700 & 465801 & 7230869 & $5 \mathrm{~N}$ & Garnet(?)-chlorite-muscovite quartz schist & --- \\
\hline 2011RN303B & 65.202924 & -153.727183 & 465968 & 7231074 & $5 \mathrm{~N}$ & Quartz-mica schist & --- \\
\hline 2011RN303C & 65.202924 & -153.727183 & 465968 & 7231074 & $5 \mathrm{~N}$ & Mica quartz schist & Inhomogenous \\
\hline 2011RN305A & 65.204334 & -153.721217 & 466249 & 7231228 & $5 \mathrm{~N}$ & Chlorite-muscovite quartz schist & --- \\
\hline 2011RN310A & 65.204177 & -153.707023 & 466913 & 7231203 & $5 \mathrm{~N}$ & Garnet-muscovite quartz schist & Porous \\
\hline 2011RN311A & 65.203618 & -153.705769 & 466971 & 7231140 & $5 \mathrm{~N}$ & Garnet greenstone & --- \\
\hline 2011RN312A & 65.203295 & -153.701914 & 467151 & 7231102 & $5 \mathrm{~N}$ & Fine-grained greenstone & Poor polish \\
\hline 2011RN313A & 65.202826 & -153.700512 & 467216 & 7231049 & $5 \mathrm{~N}$ & Garnet greenstone & --- \\
\hline 2011RN313A & 65.202826 & -153.700512 & 467216 & 7231049 & $5 \mathrm{~N}$ & Garnet greenstone & --- \\
\hline 2011RN314A & 65.202629 & -153.698456 & 467312 & 7231026 & $5 \mathrm{~N}$ & Albite-porphyroblastic greenstone & Poor polish \\
\hline 2011RN315A & 65.201754 & -153.699416 & 467266 & 7230929 & $5 \mathrm{~N}$ & Metagabbro & --- \\
\hline 2011RN315A & 65.201754 & -153.699416 & 467266 & 7230929 & $5 \mathrm{~N}$ & Metagabbro & --- \\
\hline 2011RN321A & 65.372812 & -152.542774 & 521261 & 7249890 & $5 \mathrm{~N}$ & Gabbro & Altered \\
\hline 2011RN322B & 65.371400 & -152.538799 & 521447 & 7249734 & $5 \mathrm{~N}$ & Fine-grained wacke & --- \\
\hline 2011RN324A & 65.370870 & -152.529798 & 521866 & 7249678 & $5 \mathrm{~N}$ & Gabbro & --- \\
\hline 2011RN326A & 65.370552 & -152.522901 & 522187 & 7249645 & $5 \mathrm{~N}$ & Fine-grained gabbro & Altered \\
\hline 2011RN328A & 65.368751 & -152.517945 & 522419 & 7249446 & $5 \mathrm{~N}$ & Fine-grained gabbro & Altered \\
\hline 2011TL003A & 65.239492 & -153.597918 & 472056 & 7235086 & $5 \mathrm{~N}$ & Metagabbro & --- \\
\hline 2011TL003B & 65.239492 & -153.597918 & 472056 & 7235086 & $5 \mathrm{~N}$ & Gabbro & Altered \\
\hline $2011 Z 002 \mathrm{~A}$ & 65.419122 & -152.588049 & 519122 & 7255037 & $5 \mathrm{~N}$ & Gabbro & Altered \\
\hline $2011 Z 003 A$ & 65.444462 & -152.760208 & 511120 & 7257820 & $5 \mathrm{~N}$ & Metagabbro & --- \\
\hline 2011Z006A & 65.264940 & -152.985114 & 500695 & 7237790 & $5 \mathrm{~N}$ & Serpentinite & --- \\
\hline 2011Z006A & 65.264940 & -152.985114 & 500695 & 7237790 & $5 \mathrm{~N}$ & Metagabbro(?) & --- \\
\hline 2011Z010A & 65.269749 & -152.991045 & 500418 & 7238326 & $5 \mathrm{~N}$ & Metagabbro & --- \\
\hline 2011Z027A & 65.352841 & -152.991705 & 500386 & 7247587 & $5 \mathrm{~N}$ & Albite-biotite quartz schist & --- \\
\hline 2011Z029A & 65.348893 & -152.989815 & 500474 & 7247147 & $5 \mathrm{~N}$ & Chlorite-albite-muscovite quartz schist & --- \\
\hline
\end{tabular}


Table 8. (continued)

\begin{tabular}{|c|c|c|c|c|c|c|c|c|c|}
\hline Sample_ID & $\mathrm{SiO}_{2 \_} \mathrm{pct}$ & Al203_pct & BaO_pct & CaO_pct & FeO_pct & K2O_pct & MgO_pct & MnO_pct & $\mathrm{Na} 2 \mathrm{O} \_p c t$ \\
\hline 2011RN266A & 52.4 & 15.6 & $<<0.03$ & 10.2 & 10.8 & 0.0882 & 5.86 & 0.174 & 3.42 \\
\hline 2011RN267A & 86.3 & 7.2 & 0.0442 & 0.128 & 3.1 & 0.99 & 0.77 & $<0.03$ & 0.773 \\
\hline 2011RN271A & 64.9 & 18.3 & 0.147 & 0.109 & 8.84 & 3.74 & 1.89 & 0.121 & 0.755 \\
\hline 2011RN283B & 75.7 & 13.8 & 0.0545 & 0.114 & 5.46 & 1.32 & 0.887 & 0.0562 & 1.82 \\
\hline 2011RN285A & 72.4 & 14.6 & 0.0592 & 0.187 & 5.94 & 2.67 & 1.74 & 0.0669 & 1.05 \\
\hline 2011RN300B & 53.6 & 14.1 & 0.0717 & 10.5 & 9.62 & 0.637 & 6.77 & 0.149 & 2.71 \\
\hline 2011RN301A & 65.7 & 19.9 & 0.0667 & 0.12 & 6.08 & 3.44 & 1.77 & 0.109 & 1.29 \\
\hline 2011RN303B & 67.1 & 17.9 & 0.0755 & 0.168 & 7.47 & 3.21 & 1.32 & 0.0368 & 1.41 \\
\hline 2011RN303C & 74.8 & 12.2 & 0.05 & 0.225 & 5.55 & 2.29 & 1.34 & 0.0641 & 0.558 \\
\hline 2011RN305A & 72.2 & 15.1 & 0.258 & 0.197 & 4.62 & 3.13 & 1.22 & 0.0876 & 1.44 \\
\hline 2011RN310A & 75.2 & 16.8 & 0.112 & 0.0619 & 0.515 & 4.82 & 1.03 & $<0.03$ & 0.246 \\
\hline 2011RN311A & 51.2 & 15.9 & $<0.03$ & 13.6 & 8.84 & 0.0604 & 8.17 & 0.188 & 1.39 \\
\hline 2011RN312A & 53.6 & 13.3 & 0.0483 & 7.1 & 14.1 & 0.845 & 4.28 & 0.228 & 4.25 \\
\hline 2011RN313A & 54.5 & 14.2 & $<0.03$ & 5.26 & 13.2 & 0.0354 & 4.93 & 0.228 & 5.66 \\
\hline 2011RN313A & 54.5 & 14.2 & $<0.03$ & 5.28 & 13.3 & 0.0329 & 4.84 & 0.213 & 5.62 \\
\hline 2011RN314A & 51.8 & 12.9 & 0.0319 & 9.68 & 14.7 & 0.431 & 5.38 & 0.218 & 2.82 \\
\hline 2011RN315A & 48.6 & 16.4 & 0.163 & 10.7 & 10.5 & 0.97 & 7.28 & 0.214 & 2.93 \\
\hline 2011RN315A & 48.7 & 16.6 & 0.164 & 10.5 & 10.5 & 0.951 & 7.08 & 0.221 & 2.96 \\
\hline 2011RN321A & 50 & 16.1 & 0.0391 & 11.8 & 10.2 & 0.265 & 6.27 & 0.198 & 3.39 \\
\hline 2011RN322B & 87.1 & 5.6 & $<0.03$ & 0.476 & 1.79 & 0.0216 & 0.328 & $<0.03$ & 4.29 \\
\hline 2011RN324A & 50.6 & 15.3 & 0.0575 & 10.7 & 11.8 & 0.267 & 5.32 & 0.211 & 3.65 \\
\hline 2011RN326A & 44.9 & 13.6 & $<0.03$ & 8.58 & 17.7 & 0.096 & 7.67 & 0.375 & 4.19 \\
\hline 2011RN328A & 50.7 & 15 & 0.0308 & 11.4 & 10 & 0.17 & 6.12 & 0.201 & 4.55 \\
\hline 2011TL003A & 51.9 & 15.9 & $<0.03$ & 8.26 & 9.99 & 0.324 & 8.54 & 0.187 & 3.87 \\
\hline 2011TL003B & 50.8 & 8.2 & 0.285 & 15 & 10.2 & 0.0559 & 13.2 & 0.195 & 0.631 \\
\hline $2011 Z 002 \mathrm{~A}$ & 49.6 & 18.7 & $<0.03$ & 13.1 & 7.96 & 0.0622 & 8.08 & 0.168 & 1.5 \\
\hline $2011 Z 003 \mathrm{~A}$ & 50.3 & 18.1 & 0.056 & 13.2 & 6.95 & 0.288 & 7.45 & 0.138 & 2.8 \\
\hline $2011 Z 006 \mathrm{~A}$ & 44.7 & 4.49 & $<0.03$ & 2.82 & 12.5 & 0.0651 & 34.4 & 0.191 & 0.0809 \\
\hline $2011 Z 006 A$ & 50.3 & 14.7 & 0.0351 & 9.78 & 11.3 & 0.0311 & 6.26 & 0.201 & 5.44 \\
\hline $2011 Z 010 A$ & 50.1 & 15 & $<0.03$ & 10.1 & 11.4 & 0.113 & 8.24 & 0.208 & 3.46 \\
\hline $2011 Z 027 A$ & 86 & 6.87 & 0.0877 & 0.29 & 3.26 & 0.9 & 0.865 & $<0.03$ & 0.695 \\
\hline $2011 Z 029 A$ & 83.4 & 8.08 & 0.505 & 0.197 & 3.08 & 1.58 & 1.25 & 0.269 & 1.12 \\
\hline
\end{tabular}


Table 8. (continued)

\begin{tabular}{|c|c|c|c|c|c|c|c|c|c|}
\hline Sample_ID & P2O5_pct & TiO2_pct & CO2_pct & Total_pct & As_ppm & Bi_ppm & Br_ppm & Ce_ppm & Cl_ppm \\
\hline 2011RN266A & 0.108 & 1.18 & $<0.05$ & 99.8465 & 24 & $<2$ & $<<5$ & $<60$ & $<50$ \\
\hline 2011RN267A & 0.0908 & 0.423 & $<0.05$ & 99.8889 & $<4$ & $<2$ & $<5$ & 63 & 78 \\
\hline 2011RN271A & 0.0328 & 1.03 & $<0.05$ & 99.8648 & 23 & $<2$ & $<5$ & $<60$ & 56 \\
\hline 2011RN283B & 0.058 & 0.637 & $<0.05$ & 99.9067 & 17 & $<2$ & $<5$ & $<60$ & $<50$ \\
\hline 2011RN285A & 0.125 & 0.685 & $<0.05$ & 99.5231 & 5 & $<2$ & $<5$ & $<60$ & 468 \\
\hline 2011RN300B & 0.24 & 0.852 & $<0.05$ & 99.2497 & 15 & $<2$ & $<5$ & $<60$ & 487 \\
\hline 2011RN301A & 0.106 & 0.97 & $<0.05$ & 99.5517 & 41 & $<2$ & $<5$ & $<60$ & 233 \\
\hline 2011RN303B & 0.107 & 0.785 & $<0.05$ & 99.5823 & $<4$ & $<2$ & $<5$ & $<60$ & 676 \\
\hline 2011RN303C & 0.229 & 0.62 & $<0.05$ & 97.9261 & 5 & $<2$ & $<5$ & $<60$ & 247 \\
\hline 2011RN305A & 0.188 & 0.744 & $<0.05$ & 99.1846 & 61 & $<2$ & $<5$ & $<60$ & 642 \\
\hline 2011RN310A & $<0.03$ & 1.02 & $<0.05$ & 99.8526 & $<4$ & $<2$ & $<5$ & $<60$ & 121 \\
\hline 2011RN311A & 0.0585 & 0.468 & $<0.05$ & 99.8749 & $<4$ & $<2$ & $<5$ & $<60$ & 139 \\
\hline 2011RN312A & 0.27 & 1.82 & $<0.05$ & 99.8413 & 26 & $<2$ & $<5$ & $<60$ & 117 \\
\hline 2011RN313A & 0.252 & 1.56 & $<0.05$ & 99.8455 & 7 & $<2$ & $<5$ & $<60$ & 151 \\
\hline 2011RN313A & 0.267 & 1.56 & $<0.05$ & 99.8297 & $<4$ & $<2$ & $<5$ & $<60$ & 110 \\
\hline 2011RN314A & 0.25 & 1.69 & $<0.05$ & 99.9009 & $<4$ & $<2$ & $<5$ & $<60$ & 105 \\
\hline 2011RN315A & 0.223 & 1.91 & $<0.05$ & 99.89 & $<4$ & $<2$ & $<5$ & $<60$ & 83 \\
\hline 2011RN315A & 0.225 & 1.92 & $<0.05$ & 99.821 & $<4$ & $<2$ & $<5$ & $<60$ & 206 \\
\hline 2011RN321A & 0.228 & 1.37 & $<0.05$ & 99.8601 & $<4$ & $<2$ & $<5$ & $<60$ & 96 \\
\hline 2011RN322B & 0.0513 & 0.228 & $<0.05$ & 99.9544 & $<4$ & $<2$ & $<5$ & $<60$ & 200 \\
\hline 2011RN324A & 0.262 & 1.62 & $<0.05$ & 99.7875 & $<4$ & $<2$ & $<5$ & $<60$ & 72 \\
\hline 2011RN326A & 0.306 & 2.43 & $<0.05$ & 99.8716 & $<4$ & $<2$ & $<5$ & $<60$ & 86 \\
\hline 2011RN328A & 0.167 & 1.49 & $<0.05$ & 99.8288 & $<4$ & $<2$ & $<5$ & $<60$ & $<50$ \\
\hline 2011TL003A & 0.114 & 0.614 & $<0.05$ & 99.70281 & 43 & $<2$ & $<5$ & $<60$ & 181 \\
\hline 2011TL003B & 0.123 & 0.924 & $<0.05$ & 99.6139 & $<4$ & $<2$ & $<5$ & $<60$ & 101 \\
\hline $2011 Z 002 \mathrm{~A}$ & 0.0496 & 0.578 & $<0.05$ & 99.811 & 29 & $<2$ & $<5$ & $<60$ & 373 \\
\hline $2011 Z 003 \mathrm{~A}$ & 0.0348 & 0.598 & $<0.05$ & 99.9148 & $<4$ & $<2$ & $<5$ & $<60$ & 74 \\
\hline 2011Z006A & 0.0443 & 0.213 & $<0.05$ & 99.5208 & $<4$ & $<2$ & $<5$ & $<60$ & 201 \\
\hline 2011Z006A & 0.216 & 1.55 & $<0.05$ & 99.8132 & $<4$ & $<2$ & $<5$ & $<60$ & $<50$ \\
\hline 2011Z010A & 0.139 & 1.06 & $<0.05$ & 99.8387 & $<4$ & $<2$ & $<5$ & $<60$ & 72 \\
\hline $2011 Z 027 A$ & 0.298 & 0.593 & $<0.05$ & 99.888 & $<4$ & $<2$ & $<5$ & $<60$ & 117 \\
\hline $2011 Z 029 A$ & 0.107 & 0.367 & $<0.05$ & 99.955 & $<4$ & $<2$ & $<5$ & $<60$ & 143 \\
\hline
\end{tabular}


Table 8. (continued)

\begin{tabular}{|c|c|c|c|c|c|c|c|c|c|}
\hline Sample_ID & Co_ppm & Cr_ppm & Cu_ppm & F_ppm & La_ppm & Mo_ppm & Nb_ppm & Nb_ppm_2 & Ni_ppm \\
\hline 2011RN266A & 37 & 233 & 61 & $<<110$ & $<<50$ & $2<1$ & $<<1$ & 8 & 95 \\
\hline 2011RN267A & $<10$ & 76 & $<7$ & $<110$ & 224 & $<1$ & $<1$ & 8 & 44 \\
\hline 2011RN271A & 24 & 132 & 37 & $<110$ & $<50$ & $<1$ & $<1$ & 19 & 46 \\
\hline 2011RN283B & $<10$ & 71 & $<7$ & $<110$ & $<50$ & $<1$ & $<1$ & 12 & 23 \\
\hline 2011RN285A & 15 & 92 & 27 & $<110$ & $<50$ & $<1$ & $<1$ & -- & 44 \\
\hline 2011RN300B & 120 & 586 & 72 & 939 & $<50$ & $<1$ & $<1$ & 5 & 184 \\
\hline 2011RN301A & $<10$ & 114 & 25 & $<110$ & 279 & $<1$ & $<1$ & 20 & 47 \\
\hline 2011RN303B & $<10$ & 128 & 9 & 1080 & $<50$ & $<1$ & $<1$ & 16 & 43 \\
\hline 2011RN303C & $<10$ & 95 & $<7$ & $<110$ & $<50$ & $<1$ & $<1$ & 12 & 48 \\
\hline 2011RN305A & 17 & 98 & 32 & $<110$ & $<50$ & $<1$ & $<1$ & 13 & 49 \\
\hline 2011RN310A & $<10$ & 195 & $<7$ & $<110$ & $<50$ & $<1$ & $<1$ & 15 & 37 \\
\hline 2011RN311A & 57 & 394 & 199 & $<110$ & $<50$ & $<1$ & $<1$ & 1 & 228 \\
\hline 2011RN312A & 55 & $<30$ & 81 & $<110$ & $<50$ & $<1$ & $<1$ & 12 & 48 \\
\hline 2011RN313A & 35 & 107 & 204 & $<110$ & $<50$ & $<1$ & $<1$ & 8 & 39 \\
\hline 2011RN313A & 49 & 142 & 175 & $<110$ & $<50$ & $<1$ & $<1$ & 8 & 34 \\
\hline 2011RN314A & 23 & 114 & 108 & $<110$ & $<50$ & $<1$ & $<1$ & 10 & 21 \\
\hline 2011RN315A & 103 & 154 & 114 & $<110$ & $<50$ & $<1$ & $<1$ & 9 & 91 \\
\hline 2011RN315A & 35 & 146 & 78 & $<110$ & $<50$ & $<1$ & $<1$ & 8 & 53 \\
\hline 2011RN321A & 81 & 238 & 81 & $<110$ & $<50$ & $<1$ & $<1$ & 8 & 142 \\
\hline 2011RN322B & $<10$ & $<30$ & 7 & $<110$ & $<50$ & $<1$ & $<1$ & 2 & 17 \\
\hline 2011RN324A & 37 & 168 & 178 & $<110$ & $<50$ & $<1$ & $<1$ & 8 & 72 \\
\hline 2011RN326A & 48 & 145 & 88 & $<110$ & $<50$ & $<1$ & $<1$ & 10 & 46 \\
\hline 2011RN328A & $<10$ & 260 & 166 & $<110$ & $<50$ & $<1$ & $<1$ & 6 & 100 \\
\hline 2011TL003A & 85 & 816 & 91 & $<110$ & $<50$ & $<1$ & $<1$ & 3 & 190 \\
\hline 2011TL003B & 128 & 1890 & 217 & $<110$ & $<50$ & $<1$ & $<1$ & 4 & 429 \\
\hline \begin{tabular}{|l|l}
$2011 Z 002 A$ \\
\end{tabular} & 77 & 419 & 19 & $<110$ & $<50$ & $<1$ & $<1$ & 1 & 184 \\
\hline $2011 Z 003 \mathrm{~A}$ & 35 & 335 & 47 & $<110$ & $<50$ & $<1$ & $<1$ & 3 & 98 \\
\hline $2011 Z 006 \mathrm{~A}$ & 231 & 2210 & 31 & $<110$ & $<50$ & $<1$ & $<1$ & --- & 1690 \\
\hline $2011 Z 006 \mathrm{~A}$ & 10 & 285 & 21 & $<110$ & $<50$ & $<1$ & $<1$ & 1 & 57 \\
\hline $2011 Z 010 A$ & 49 & 521 & 75 & $<110$ & $<50$ & $<1$ & $<1$ & 4 & 131 \\
\hline $2011 Z 027 A$ & $<10$ & 97 & $<7$ & $<110$ & $<50$ & $<1$ & 8 & --- & $<7$ \\
\hline $2011 Z 029 A$ & $<10$ & 61 & 61 & $<110$ & $<50$ & $<1$ & 7 & 6 & 61 \\
\hline
\end{tabular}


Table 8. (continued)

\begin{tabular}{|c|c|c|c|c|c|c|c|c|c|}
\hline Sample_ID & Pb_ppm & Rb_ppm & Rb_ppm_2 & S_ppm & Sb_ppm & Sn_ppm & Sr_ppm & Sr_ppm_2 & Th_ppm \\
\hline 2011RN266A & 34 & $<1$ & 3 & 50 & $<2$ & $<4$ & 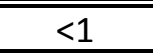 & 228 & $2<2$ \\
\hline 2011RN267A & 26 & $<1$ & 49 & 37 & $<2$ & $<4$ & $<1$ & 64 & $<2$ \\
\hline 2011RN271A & 21 & $<1$ & 150 & 67 & $<2$ & $<4$ & $<1$ & 62 & $<2$ \\
\hline 2011RN283B & 19 & $<1$ & 56 & 604 & $<2$ & $<4$ & $<1$ & 143 & $<2$ \\
\hline 2011RN285A & 54 & $<1$ & --- & 4370 & $<2$ & $<4$ & $<1$ & --- & $<2$ \\
\hline 2011RN300B & $<4$ & $<1$ & 38 & 5030 & $<2$ & $<4$ & $<1$ & 151 & $<2$ \\
\hline 2011RN301A & 17 & $<1$ & 146 & 2860 & $<2$ & $<4$ & $<1$ & 86 & $<2$ \\
\hline 2011RN303B & 28 & $<1$ & 139 & 2020 & $<2$ & $<4$ & $<1$ & 55 & $<2$ \\
\hline 2011RN303C & $<4$ & $<1$ & 100 & 165 & $<2$ & $<4$ & $<1$ & 34 & $<2$ \\
\hline 2011RN305A & 29 & $<1$ & 123 & 7040 & $<2$ & $<4$ & $<1$ & 43 & $<2$ \\
\hline 2011RN310A & 18 & $<1$ & 117 & 102 & $<2$ & $<4$ & $<1$ & 55 & $<2$ \\
\hline 2011RN311A & $<4$ & $<1$ & 2 & 80 & $<2$ & $<4$ & $<1$ & 92 & $<2$ \\
\hline 2011RN312A & 13 & $<1$ & 38 & $<30$ & $<2$ & $<4$ & $<1$ & 140 & $<2$ \\
\hline 2011RN313A & $<4$ & $<1$ & 1 & 177 & $<2$ & $<4$ & $<1$ & 80 & $<2$ \\
\hline 2011RN313A & $<4$ & $<1$ & 1 & 167 & $<2$ & $<4$ & $<1$ & 80 & $<2$ \\
\hline 2011RN314A & 34 & $<1$ & 16 & 118 & $<2$ & $<4$ & $<1$ & 101 & $<2$ \\
\hline 2011RN315A & $<4$ & $<1$ & 20 & 75 & $<2$ & $<4$ & $<1$ & 86 & $<2$ \\
\hline 2011RN315A & $<4$ & $<1$ & 20 & 93 & $<2$ & $<4$ & $<1$ & 87 & $<2$ \\
\hline 2011RN321A & $<4$ & $<1$ & 4 & 76 & $<2$ & $<4$ & $<1$ & 151 & $<2$ \\
\hline 2011RN322B & $<4$ & $<1$ & 1 & 131 & $<2$ & $<4$ & $<1$ & 11 & $<2$ \\
\hline 2011RN324A & 37 & $<1$ & 5 & 40 & $<2$ & $<4$ & $<1$ & 291 & $<2$ \\
\hline 2011RN326A & $<4$ & $<1$ & 3 & 48 & $<2$ & $<4$ & $<1$ & 104 & $<2$ \\
\hline 2011RN328A & $<4$ & $<1$ & 3 & 55 & $<2$ & $<4$ & $<1$ & 320 & $<2$ \\
\hline 2011TL003A & $<4$ & $<1$ & 22 & 148 & $<2$ & $<4$ & $<1$ & 171 & $<2$ \\
\hline 2011TL003B & 28 & $<1$ & 1 & 304 & $<2$ & $<4$ & $<1$ & 187 & $<2$ \\
\hline $2011 Z 002 \mathrm{~A}$ & 19 & $<1$ & 7 & 103 & $<2$ & $<4$ & $<1$ & 215 & $<2$ \\
\hline $2011 Z 003 \mathrm{~A}$ & $<4$ & $<1$ & 10 & $<30$ & $<2$ & $<4$ & $<1$ & 141 & $<2$ \\
\hline 2011Z006A & $<4$ & $<1$ & --- & 137 & $<2$ & $<4$ & $<1$ & --- & $<2$ \\
\hline 2011Z006A & $<4$ & $<1$ & 1 & 105 & $<2$ & $<4$ & $<1$ & 124 & $<2$ \\
\hline 2011Z010A & $<4$ & $<1$ & 2 & 51 & $<2$ & $<4$ & $<1$ & 174 & $<2$ \\
\hline $2011 Z 027 A$ & 23 & 37 & --- & 50 & $<2$ & $<4$ & 43 & --- & $<2$ \\
\hline $2011 Z 029 \mathrm{~A}$ & $<4$ & 71 & 68 & 45 & $<2$ & $<4$ & 47 & 43 & $<2$ \\
\hline
\end{tabular}




\begin{tabular}{|c|c|c|c|c|c|c|c|c|}
\hline Sample_ID & U_ppm & V_ppm & W_ppm & Y_ppm & Y_ppm_2 & Zn_ppm & Zr_ppm & Zr_ppm_2 \\
\hline 2011RN266A & $\overline{c<1}$ & 372 & $<<8$ & $\angle 1$ & 23 & 159 & $<9$ & 108 \\
\hline 2011RN267A & $<1$ & 89 & $<8$ & $<1$ & 14 & 44 & $<9$ & 202 \\
\hline 2011RN271A & $<1$ & 176 & $<8$ & $<1$ & 25 & 103 & $<9$ & 244 \\
\hline 2011RN283B & $<1$ & 92 & $<8$ & $<1$ & 9 & 56 & $<9$ & 208 \\
\hline 2011RN285A & $<1$ & 137 & $<8$ & $<1$ & --- & 85 & $<9$ & $<9$ \\
\hline 2011RN300B & $<1$ & 302 & $<8$ & $<1$ & 17 & 63 & $<9$ & 75 \\
\hline 2011RN301A & $<1$ & 163 & $<8$ & $<1$ & 12 & 85 & $<9$ & 195 \\
\hline 2011RN303B & $<1$ & 156 & $<8$ & $<1$ & 18 & 85 & $<9$ & 147 \\
\hline 2011RN303C & $<1$ & 130 & $<8$ & $<1$ & 17 & 70 & $<9$ & 122 \\
\hline 2011RN305A & $<1$ & 138 & $<8$ & $<1$ & 11 & 75 & $<9$ & 140 \\
\hline 2011RN310A & $<1$ & 477 & $<8$ & $<1$ & 32 & 26 & $<9$ & 181 \\
\hline 2011RN311A & $<1$ & 217 & $<8$ & $<1$ & 19 & 51 & $<9$ & 34 \\
\hline 2011RN312A & $<1$ & 411 & $<8$ & $<1$ & 42 & 133 & $<9$ & 192 \\
\hline 2011RN313A & $<1$ & 503 & $<8$ & $<1$ & 36 & 145 & $<9$ & 158 \\
\hline 2011RN313A & $<1$ & 551 & $<8$ & $<1$ & 36 & 160 & $<9$ & 161 \\
\hline 2011RN314A & $<1$ & 543 & $<8$ & $<1$ & 41 & 137 & $<9$ & 167 \\
\hline 2011RN315A & $<1$ & 514 & $<8$ & $<1$ & 32 & 97 & $<9$ & 129 \\
\hline 2011RN315A & $<1$ & 576 & $<8$ & $<1$ & 32 & 102 & $<9$ & 130 \\
\hline 2011RN321A & $<1$ & 408 & $<8$ & $<1$ & 27 & 82 & $<9$ & 103 \\
\hline 2011RN322B & $<1$ & 55 & $<8$ & $<1$ & 15 & $<8$ & $<9$ & 66 \\
\hline 2011RN324A & $<1$ & 463 & $<8$ & $<1$ & 32 & 89 & $<9$ & 123 \\
\hline 2011RN326A & $<1$ & 482 & $<8$ & $<1$ & 39 & 193 & $<9$ & 180 \\
\hline 2011RN328A & $<1$ & 428 & $<8$ & $<1$ & 26 & 87 & $<9$ & 88 \\
\hline 2011TL003A & $<1$ & 292 & $<8$ & $<1$ & 19 & 86 & $<9$ & 55 \\
\hline 2011TL003B & $<1$ & 307 & $<8$ & $<1$ & 17 & 75 & $<9$ & 55 \\
\hline $2011 Z 002 \mathrm{~A}$ & $<1$ & 174 & $<8$ & $<1$ & 21 & 68 & $<9$ & 31 \\
\hline $2011 Z 003 A$ & $<1$ & 79 & $<8$ & $<1$ & 10 & 50 & $<9$ & 32 \\
\hline $2011 Z 006 \mathrm{~A}$ & $<1$ & 140 & $<8$ & $<1$ & --- & 101 & $<9$ & $<9$ \\
\hline 2011Z006A & $<1$ & 399 & $<8$ & $<1$ & 47 & 89 & $<9$ & 147 \\
\hline 2011Z010A & $<1$ & 312 & $<8$ & $<1$ & 21 & 81 & $<9$ & 79 \\
\hline $2011 Z 027 A$ & $<1$ & 128 & $<8$ & 10 & --- & 46 & 179 & --- \\
\hline 2011Z029A & $<1$ & 127 & $<8$ & 28 & 11 & 60 & 58 & 84 \\
\hline
\end{tabular}




\begin{tabular}{|c|c|c|c|c|c|c|c|}
\hline Sample_ID & Latitude & Longitude & Easting & Northing & $\begin{array}{l}\text { UTM_- } \\
\text { Zone }\end{array}$ & Root_name & Comments \\
\hline 2011Z030A & 65.324865 & -152.804615 & 509102 & 7244483 & $5 \mathrm{~N}$ & Calcite-muscovite-albite quartz schist & --- \\
\hline 2011Z031A & 65.324737 & -152.803092 & 509173 & 7244469 & $5 \mathrm{~N}$ & Mafic schist & Small \\
\hline 2011Z034B & 65.322654 & -152.794908 & 509555 & 7244238 & $5 \mathrm{~N}$ & Metagabbro & --- \\
\hline 2011Z038B & 65.319398 & -152.776562 & 510411 & 7243878 & $5 \mathrm{~N}$ & Mafic schist & Altered \\
\hline $2011 Z 042 A$ & 65.318147 & -152.756872 & 511329 & 7243742 & $5 \mathrm{~N}$ & Metagabbro & --- \\
\hline 2011Z045A & 65.322647 & -152.748674 & 511709 & 7244245 & $5 \mathrm{~N}$ & Metagabbro(?) & --- \\
\hline 2011Z057B & 65.351089 & -152.825478 & 508122 & 7247403 & $5 \mathrm{~N}$ & Metagabbro(?) & --- \\
\hline 2011Z080A & 65.456443 & -152.725364 & 512730 & 7259162 & $5 \mathrm{~N}$ & Metagabbro(?) & --- \\
\hline 2011Z081A & 65.458486 & -152.723897 & 512797 & 7259390 & $5 \mathrm{~N}$ & Gabbro & Altered \\
\hline 2011Z088A & 65.462192 & -152.805253 & 509025 & 7259789 & $5 \mathrm{~N}$ & Quartz-muscovite schist & --- \\
\hline 2011Z088A & 65.462192 & -152.805253 & 509025 & 7259789 & $5 \mathrm{~N}$ & Quartz-muscovite schist & --- \\
\hline 2011Z088A & 65.462192 & -152.805253 & 509025 & 7259789 & $5 \mathrm{~N}$ & Quartz-muscovite schist & --- \\
\hline 2011Z088A & 65.462192 & -152.805253 & 509025 & 7259789 & $5 \mathrm{~N}$ & Quartz-muscovite schist & --- \\
\hline 2011Z089A & 65.462686 & -152.805270 & 509024 & 7259844 & $5 \mathrm{~N}$ & Metamafic & --- \\
\hline $2011 Z 092 A$ & 65.465143 & -152.811640 & 508728 & 7260117 & $5 \mathrm{~N}$ & Mafic schist & --- \\
\hline 2011Z105A & 65.396639 & -152.939020 & 502833 & 7252470 & $5 \mathrm{~N}$ & $\begin{array}{l}\text { Garnet-sillimanite-muscovite- } \\
\text { biotite quartz schist }\end{array}$ & --- \\
\hline 2011Z110A & 65.405328 & -152.928385 & 503326 & 7253439 & $5 \mathrm{~N}$ & Plagioclase-biotite quartz schist & --- \\
\hline 2011Z110B & 65.405328 & -152.928385 & 503326 & 7253439 & $5 \mathrm{~N}$ & Biotite quartzite & --- \\
\hline 2011Z112A & 65.405613 & -152.924013 & 503529 & 7253471 & $5 \mathrm{~N}$ & Fine-grained, feldspar-rich orthogneiss(?) & Inhomogenous \\
\hline $2011 Z 113 A$ & 65.406141 & -152.920415 & 503696 & 7253530 & $5 \mathrm{~N}$ & Muscovite-albite-biotite quartz schist & --- \\
\hline 2011Z116A & 65.413436 & -152.921772 & 503632 & 7254343 & $5 N$ & $\begin{array}{l}\text { Biotite-plagioclase-Quartz- } \\
\text { paragneiss }\end{array}$ & --- \\
\hline $2011 Z 120 B$ & 65.258635 & -152.752088 & 511578 & 7237110 & $5 \mathrm{~N}$ & Muscovite-albite quartz schist & Porous \\
\hline 2011Z123A & 65.264890 & -152.752586 & 511552 & 7237807 & $5 \mathrm{~N}$ & $\begin{array}{l}\text { Calcite-albite-chlorite- } \\
\text { muscovite quartz schist }\end{array}$ & --- \\
\hline 2011Z135A & 65.250036 & -152.610378 & 518202 & 7236185 & $5 \mathrm{~N}$ & Albite-chlorite-muscovite & --- \\
\hline 2011Z149A & 65.298478 & -152.586974 & 519260 & 7241591 & $5 \mathrm{~N}$ & Albite-chlorite-quartz schist & --- \\
\hline $2011 Z 160 A$ & 65.243216 & \begin{tabular}{|l|}
-153.588693 \\
\end{tabular} & 472491 & 7235497 & $5 \mathrm{~N}$ & orthogneiss(?) & Inhomogenous \\
\hline 2011Z161A & 65.244856 & -153.598211 & 472048 & 7235684 & $5 \mathrm{~N}$ & $\begin{array}{l}\text { Chlorite-albite-muscovite } \\
\text { Quartz schist }\end{array}$ & --- \\
\hline
\end{tabular}


Table 8. (continued)

\begin{tabular}{|c|c|c|c|c|c|c|c|c|c|}
\hline Sample_ID & $\mathrm{SiO}_{2 \_} \mathrm{pct}$ & Al203_pct & BaO_pct & CaO_pct & FeO_pct & K2O_pct & MgO_pct & MnO_pct & $\mathrm{Na} 2 \mathrm{O} \_p c t$ \\
\hline $2011 Z 030 A$ & 67.6 & 12.9 & 0.383 & 8.58 & 2.25 & 2.16 & 1.49 & 0.0596 & 3.76 \\
\hline 2011Z031A & 51 & 14.6 & $<0.03$ & 7.54 & 14.2 & 0.0778 & 5.13 & 0.189 & 5.71 \\
\hline 2011Z034B & 51 & 13.4 & $<0.03$ & 11.7 & 13.2 & $<.02$ & 6.57 & 0.246 & 2.52 \\
\hline $2011 Z 038 \mathrm{~B}$ & 58.3 & 14.1 & $<0.03$ & 3 & 14 & 0.0414 & 5.7 & 0.136 & 2.63 \\
\hline $2011 Z 042 \mathrm{~A}$ & 51.5 & 15.1 & $<0.03$ & 9.74 & 11.6 & 0.139 & 4.98 & 0.194 & 4.45 \\
\hline $2011 Z 045 A$ & 50.2 & 15.8 & $<0.03$ & 10.2 & 10.8 & 0.0211 & 7.72 & 0.193 & 3.99 \\
\hline 2011Z057B & 47.2 & 18.5 & $<0.03$ & 13 & 10.4 & 0.034 & 8.06 & 0.184 & 2.15 \\
\hline 2011Z080A & 54.3 & 18.5 & 0.0464 & 6.87 & 8.52 & 0.399 & 4.03 & 0.171 & 6 \\
\hline $2011 Z 081 A$ & 55.5 & 18.9 & $<0.03$ & 8.01 & 6.34 & 0.279 & 3.53 & 0.133 & 6.18 \\
\hline 2011Z088A & 69.8 & 15.4 & 0.193 & 0.194 & 8.01 & 3.38 & 1.16 & 0.221 & 0.21 \\
\hline $2011 Z 088 A$ & 69.7 & 15.7 & 0.186 & 0.187 & 7.66 & 3.43 & 1.13 & 0.212 & 0.22 \\
\hline $2011 Z 088 \mathrm{~A}$ & 70.7 & 15.4 & 0.175 & 0.252 & 7.24 & 3.36 & 1.09 & 0.219 & 0.189 \\
\hline 2011Z088A & 70.2 & 15.6 & 0.181 & 0.23 & 7.37 & 3.52 & 1.06 & 0.217 & 0.226 \\
\hline 2011Z089A & 53.3 & 15.5 & $<0.03$ & 7.06 & 10.9 & 0.0502 & 8.92 & 0.24 & 3.52 \\
\hline $2011 Z 092 \mathrm{~A}$ & 50.7 & 15.5 & $<0.03$ & 10.4 & 11.4 & 0.134 & 6.46 & 0.223 & 3.78 \\
\hline 2011Z105A & 68.2 & 20.1 & 0.0908 & 0.157 & 4.91 & 3.11 & 1.34 & 0.0823 & 0.96 \\
\hline $2011 Z 110 A$ & 70 & 16 & 0.0794 & 0.944 & 6.38 & 2.94 & 1.29 & 0.054 & 0.922 \\
\hline 2011Z110B & 92.1 & 3.59 & 0.0385 & 1.18 & 0.95 & 0.761 & 0.799 & $<0.03$ & 0.231 \\
\hline $2011 Z 112 \mathrm{~A}$ & 67.4 & 17.7 & 0.0834 & 0.614 & 6.17 & 3.63 & 1.14 & 0.102 & 1.8 \\
\hline $2011 Z 113 \mathrm{~A}$ & 75 & 11.3 & 0.0782 & 1.36 & 4.27 & 3.16 & 1.92 & 0.0687 & 1.84 \\
\hline 2011Z116A & 72.5 & 12.5 & 0.0541 & 2.1 & 4.24 & 2.35 & 2.08 & 0.0745 & 3.08 \\
\hline 2011Z120B & 89.1 & 4.37 & 0.0374 & 0.31 & 2.94 & 0.93 & 0.393 & $<0.03$ & 1.13 \\
\hline $2011 Z 123 A$ & 77.5 & 9.57 & 0.097 & 3.4 & 3.94 & 1.55 & 1.93 & 0.0329 & 1.24 \\
\hline $2011 Z 135 A$ & 74.6 & 13.8 & 0.098 & 0.143 & 5.64 & 3.08 & 1.15 & 0.0303 & 0.609 \\
\hline 2011Z149A & 74.2 & 9.24 & $<0.03$ & 0.292 & 6.09 & 0.106 & 6.14 & 0.0449 & 2.71 \\
\hline $2011 Z 160 A$ & 72.1 & 15.1 & 0.0866 & 1.19 & 2.4 & 3.86 & 0.856 & 0.0366 & 3.83 \\
\hline 2011Z161A & 71.8 & 13.7 & 0.0931 & 1.31 & 5.02 & 3.07 & 2.29 & 0.0691 & 2 \\
\hline
\end{tabular}


Table 8. (continued)

\begin{tabular}{|c|c|c|c|c|c|c|c|c|c|}
\hline Sample_ID & P2O5_pct & TiO2_pct & CO2_pct & Total_pct & As_ppm & Bi_ppm & Br_ppm & Ce_ppm & Cl_ppm \\
\hline 2011Z030A & 0.208 & 0.506 & $<<0.05$ & 99.8966 & $\overline{51}$ & $<<2$ & $<<$ & $<60$ & 335 \\
\hline $2011 Z 031 \mathrm{~A}$ & 0.218 & 1.2 & $<0.05$ & 99.8648 & $<4$ & $<2$ & $<5$ & $<60$ & $<50$ \\
\hline 2011Z034B & 0.161 & 1.13 & $<0.05$ & 99.9493 & 49 & $<2$ & $<5$ & $<60$ & 140 \\
\hline $2011 Z 038 B$ & 0.199 & 1.68 & $<0.05$ & 99.8004 & 82 & $<2$ & $<5$ & $<60$ & 174 \\
\hline $2011 Z 042 A$ & 0.294 & 1.78 & $<0.05$ & 99.8046 & 14 & $<2$ & $<5$ & $<60$ & 95 \\
\hline $2011 Z 045 A$ & 0.0881 & 0.772 & $<0.05$ & 99.7963 & $<4$ & $<2$ & $<5$ & $<60$ & $<50$ \\
\hline 2011Z057B & $<0.03$ & 0.435 & $<0.05$ & 99.9947 & 18 & $<2$ & $<5$ & $<60$ & 400 \\
\hline $2011 Z 080 A$ & 0.227 & 0.826 & $<0.05$ & 99.8894 & 24 & $<2$ & $<5$ & $<60$ & 597 \\
\hline $2011 Z 081 A$ & 0.218 & 0.663 & $<0.05$ & 99.7812 & 36 & $<2$ & $<5$ & $<60$ & 888 \\
\hline $2011 Z 088 \mathrm{~A}$ & 0.266 & 0.767 & $<0.05$ & 99.601 & 37 & $<2$ & $<5$ & $<60$ & 324 \\
\hline $2011 Z 088 A$ & 0.232 & 0.786 & $<0.05$ & 99.443 & 5 & $<2$ & $<5$ & $<60$ & 338 \\
\hline $2011 Z 088 \mathrm{~A}$ & 0.287 & 0.765 & $<0.05$ & 99.677 & 45 & $<2$ & $<5$ & $<60$ & 106 \\
\hline 2011Z088A & 0.284 & 0.776 & $<0.05$ & 99.664 & 37 & $<2$ & $<5$ & $<60$ & 341 \\
\hline 2011Z089A & $<0.03$ & 0.322 & $<0.05$ & 99.8449 & 62 & $<2$ & $<5$ & $<60$ & 182 \\
\hline $2011 Z 092 \mathrm{~A}$ & 0.1 & 1.11 & $<0.05$ & 99.807 & $<4$ & $<2$ & $<5$ & $<60$ & 821 \\
\hline $2011 Z 105 A$ & 0.0889 & 0.726 & $<0.05$ & 99.765 & $<4$ & $<2$ & $<5$ & $<60$ & $<50$ \\
\hline $2011 Z 110 A$ & 0.162 & 0.98 & $<0.05$ & 99.7514 & 39 & $<2$ & $<5$ & $<60$ & 131 \\
\hline $2011 Z 110 B$ & 0.0679 & 0.191 & $<0.05$ & 99.9288 & $<4$ & $<2$ & $<5$ & $<60$ & 236 \\
\hline $2011 Z 112 \mathrm{~A}$ & 0.105 & 0.801 & $<0.05$ & 99.5454 & 207 & $<2$ & $<5$ & $<60$ & 511 \\
\hline $2011 Z 113 \mathrm{~A}$ & 0.172 & 0.714 & $<0.05$ & 99.8829 & $<4$ & $<2$ & $<5$ & $<60$ & 229 \\
\hline $2011 Z 116 A$ & 0.168 & 0.756 & $<0.05$ & 99.9026 & 30 & $<2$ & $<5$ & $<60$ & 322 \\
\hline $2011 Z 120 B$ & 0.44 & 0.277 & $<0.05$ & 99.9539 & $<4$ & $<2$ & $<5$ & $<60$ & 76 \\
\hline 2011Z123A & 0.193 & 0.504 & $<0.05$ & 99.9569 & 48 & $<2$ & $<5$ & $<60$ & $<50$ \\
\hline $2011 Z 135 A$ & 0.0798 & 0.733 & $<0.05$ & 99.9631 & $<4$ & $<2$ & $<5$ & $<60$ & $<50$ \\
\hline $2011 Z 149 A$ & 0.274 & 0.61 & $<0.05$ & 99.7304 & $<4$ & $<2$ & $<5$ & $<60$ & 119 \\
\hline $2011 Z 160 \mathrm{~A}$ & 0.157 & 0.196 & $<0.05$ & 99.8122 & $<4$ & $<2$ & $<5$ & $<60$ & 174 \\
\hline 2011Z161A & 0.0954 & 0.453 & $<0.05$ & 99.9006 & 47 & $<2$ & $<5$ & $<60$ & 196 \\
\hline
\end{tabular}


Table 8. (continued)

\begin{tabular}{|c|c|c|c|c|c|c|c|c|c|}
\hline Sample_ID & Co_ppm & Cr_ppm & Cu_ppm & F_ppm & La_ppm & Mo_ppm & Nb_ppm & Nb_ppm_2 & Ni_ppm \\
\hline 2011Z030A & $<<10$ & 42 & 33 & $<<110$ & $<50$ & $2<1$ & 7 & 8 & 22 \\
\hline 2011Z031A & 89 & 77 & 41 & $<110$ & $<50$ & $<1$ & $<1$ & 2 & 14 \\
\hline 2011Z034B & 117 & 210 & 59 & $<110$ & $<50$ & $<1$ & $<1$ & $<1$ & 54 \\
\hline $2011 Z 038 B$ & 52 & 102 & 31 & $<110$ & $<50$ & $<1$ & $<1$ & 14 & 7 \\
\hline $2011 Z 042 A$ & 94 & 189 & 97 & $<110$ & $<50$ & $<1$ & $<1$ & 10 & 62 \\
\hline $2011 Z 045 A$ & 52 & 519 & 23 & $<110$ & $<50$ & $<1$ & $<1$ & 1 & 62 \\
\hline 2011Z057B & 63 & 522 & 55 & $<110$ & $<50$ & $<1$ & $<1$ & 2 & 170 \\
\hline $2011 Z 080 A$ & 57 & 74 & 44 & $<110$ & $<50$ & $<1$ & $<1$ & 1 & 39 \\
\hline $2011 Z 081 A$ & 40 & 145 & 91 & $<110$ & $<50$ & $<1$ & $<1$ & 2 & 49 \\
\hline $2011 Z 088 \mathrm{~A}$ & $<10$ & 116 & $<7$ & 1840 & $<50$ & $<1$ & $<1$ & 12 & 57 \\
\hline $2011 Z 088 A$ & $<10$ & 106 & 16 & 2780 & $<50$ & $<1$ & $<1$ & 12 & 95 \\
\hline $2011 Z 088 \mathrm{~A}$ & $<10$ & 117 & $<7$ & 1720 & $<50$ & $<1$ & $<1$ & 12 & 52 \\
\hline 2011Z088A & $<10$ & 115 & 23 & 1815 & $<50$ & $<1$ & $<1$ & 12 & 74 \\
\hline 2011Z089A & 37 & 420 & 17 & $<110$ & $<50$ & $<1$ & $<1$ & 3 & 160 \\
\hline 2011Z092A & 49 & 103 & 16 & $<110$ & $<50$ & $<1$ & $<1$ & 1 & 36 \\
\hline 2011Z105A & $<10$ & 112 & $<7$ & $<110$ & $<50$ & $<1$ & 11 & 13 & 38 \\
\hline $2011 Z 110 \mathrm{~A}$ & 33 & 72 & 55 & 1250 & $<50$ & $<1$ & 18 & 15 & 41 \\
\hline $2011 Z 110 B$ & $<10$ & $<30$ & $<7$ & $<110$ & $<50$ & $<1$ & $<1$ & 3 & 60 \\
\hline $2011 Z 112 \mathrm{~A}$ & $<10$ & 117 & 33 & 2610 & $<50$ & $<1$ & $<1$ & 15 & 39 \\
\hline $2011 Z 113 \mathrm{~A}$ & $<10$ & 77 & $<7$ & $<110$ & $<50$ & $<1$ & 10 & --- & $<7$ \\
\hline 2011Z116A & $<10$ & 88 & 27 & 402 & $<50$ & $<1$ & 6 & 11 & 25 \\
\hline $2011 Z 120 B$ & $<10$ & 76 & 63 & $<110$ & $<50$ & $<1$ & $<1$ & --- & 39 \\
\hline 2011Z123A & $<10$ & 77 & 25 & $<110$ & $<50$ & $<1$ & $<1$ & 8 & 29 \\
\hline $2011 Z 135 A$ & $<10$ & 94 & 14 & $<110$ & $<50$ & $<1$ & 15 & --- & 29 \\
\hline $2011 Z 149 A$ & $<10$ & 822 & 27 & 859 & $<50$ & $<1$ & 8 & 4 & 249 \\
\hline $2011 Z 160 \mathrm{~A}$ & 81 & 40 & $<7$ & $<110$ & $<50$ & $<1$ & $<1$ & 9 & 16 \\
\hline 2011Z161A & $<10$ & 82 & $<7$ & $<110$ & $<50$ & $<1$ & 9 & 10 & 27 \\
\hline
\end{tabular}




\begin{tabular}{|c|c|c|c|c|c|c|c|c|c|}
\hline Sample_ID & Pb_ppm & Rb_ppm & Rb_ppm_2 & S_ppm & Sb_ppm & Sn_ppm & Sr_ppm & Sr_ppm_2 & Th_ppm \\
\hline 2011Z030A & 32 & 70 & 63 & 193 & $<2$ & $<4$ & 219 & 195 & $<2$ \\
\hline 2011Z031A & $<4$ & $<1$ & 1 & $<30$ & $<2$ & $<4$ & $<1$ & 153 & $<2$ \\
\hline 2011Z034B & 21 & $<1$ & $<1$ & 66 & $<2$ & $<4$ & $<1$ & 148 & $<2$ \\
\hline 2011Z038B & 24 & $<1$ & $<1$ & 69 & $<2$ & $<4$ & $<1$ & 201 & $<2$ \\
\hline $2011 Z 042 \mathrm{~A}$ & $<4$ & $<1$ & 5 & 51 & $<2$ & $<4$ & $<1$ & 274 & $<2$ \\
\hline $2011 Z 045 A$ & 27 & $<1$ & $<1$ & 50 & $<2$ & $<4$ & $<1$ & 184 & $<2$ \\
\hline 2011Z057B & $<4$ & $<1$ & $<1$ & 80 & $<2$ & $<4$ & $<1$ & 132 & $<2$ \\
\hline $2011 Z 080 \mathrm{~A}$ & 21 & $<1$ & 61 & 37 & $<2$ & $<4$ & $<1$ & 287 & $<2$ \\
\hline 2011Z081A & 19 & $<1$ & 20 & 68 & $<2$ & $<4$ & $<1$ & 300 & $<2$ \\
\hline $2011 Z 088 A$ & 22 & $<1$ & 282 & 324 & $<2$ & 58.3 & $<1$ & 11 & 7.5 \\
\hline $2011 Z 088 \mathrm{~A}$ & 38 & $<1$ & 284 & 316 & $<2$ & 59.2 & $<1$ & 11 & 9.8 \\
\hline $2011 Z 088 \mathrm{~A}$ & $<4$ & $<1$ & 289 & 145 & $<2$ & 50 & $<1$ & 12 & $<2$ \\
\hline $2011 Z 088 \mathrm{~A}$ & 26 & $<1$ & 291 & 267 & $<2$ & 59.9 & $<1$ & 12 & 9.6 \\
\hline $2011 Z 089 \mathrm{~A}$ & 25 & $<1$ & 2 & 157 & $<2$ & $<4$ & $<1$ & 131 & $<2$ \\
\hline $2011 Z 092 A$ & $<4$ & $<1$ & 10 & 349 & $<2$ & $<4$ & $<1$ & 177 & $<2$ \\
\hline $2011 Z 105 A$ & 40 & 118 & 128 & 1060 & $<2$ & $<4$ & 112 & 118 & $<2$ \\
\hline $2011 Z 110 \mathrm{~A}$ & 20 & 130 & 135 & 53 & $<2$ & $<4$ & 82 & 86 & $<2$ \\
\hline $2011 Z 110 B$ & $<4$ & 60 & 56 & 91 & $<2$ & $<4$ & 32 & 34 & $<2$ \\
\hline $2011 Z 112 \mathrm{~A}$ & 47 & $<1$ & 223 & 55 & $<2$ & $<4$ & $<1$ & 177 & $<2$ \\
\hline $2011 Z 113 \mathrm{~A}$ & 35 & 112 & --- & 243 & $<2$ & $<4$ & 106 & --- & $<2$ \\
\hline 2011Z116A & 44 & 97 & 99 & 39 & $<2$ & $<4$ & 172 & 178 & $<2$ \\
\hline $2011 Z 120 \mathrm{~B}$ & 30 & 39 & --- & 118 & $<2$ & $<4$ & 21 & --- & $<2$ \\
\hline 2011Z123A & 19 & 62 & 60 & 77 & $<2$ & $<4$ & 183 & 160 & $<2$ \\
\hline $2011 Z 135 A$ & $<4$ & 126 & --- & $<30$ & $<2$ & $<4$ & 44 & --- & $<2$ \\
\hline $2011 Z 149 A$ & 22 & 12 & 4 & 54 & $<2$ & $<4$ & 17 & 14 & $<2$ \\
\hline $2011 Z 160 A$ & 31 & $<1$ & 154 & 417 & $<2$ & $<4$ & $<1$ & 68 & $<2$ \\
\hline 2011Z161A & 22 & 138 & 141 & 68 & $<2$ & $<4$ & 33 & 38 & $<2$ \\
\hline
\end{tabular}




\begin{tabular}{|c|c|c|c|c|c|c|c|c|}
\hline Sample_ID & U_ppm & V_ppm & W_ppm & Y_ppm & Y_ppm_2 & Zn_ppm & Zr_ppm & Zr_ppm_2 \\
\hline 2011Z030A & $<1$ & 121 & $<8$ & 34 & 20 & 51 & 201 & 209 \\
\hline 2011Z031A & $<1$ & 473 & $<8$ & $<1$ & 51 & 106 & $<9$ & 114 \\
\hline 2011Z034B & $<1$ & 299 & $<8$ & $<1$ & 49 & 116 & $<9$ & 56 \\
\hline 2011Z038B & $<1$ & 550 & $<8$ & $<1$ & 25 & 109 & $<9$ & 97 \\
\hline $2011 Z 042 A$ & $<1$ & 400 & $<8$ & $<1$ & 30 & 100 & $<9$ & 159 \\
\hline $2011 Z 045 \mathrm{~A}$ & $<1$ & 307 & $<8$ & $<1$ & 28 & 55 & $<9$ & 57 \\
\hline 2011Z057B & $<1$ & 240 & $<8$ & $<1$ & 12 & 61 & $<9$ & 22 \\
\hline 2011Z080A & $<1$ & 242 & $<8$ & $<1$ & 22 & 107 & $<9$ & 59 \\
\hline 2011Z081A & $<1$ & 307 & $<8$ & $<1$ & 25 & 76 & $<9$ & 75 \\
\hline $2011 Z 088 \mathrm{~A}$ & 3.4 & 225 & $<8$ & $<1$ & 30 & 137 & $<9$ & 144 \\
\hline $2011 Z 088 \mathrm{~A}$ & 2.6 & 302 & $<8$ & $<1$ & 30 & 133 & $<9$ & 145 \\
\hline 2011Z088A & $<1$ & 232 & $<8$ & $<1$ & 31 & 124 & $<9$ & 146 \\
\hline $2011 Z 088 \mathrm{~A}$ & 2.4 & 228 & $<8$ & $<1$ & 33 & 131 & $<9$ & 146 \\
\hline 2011Z089A & $<1$ & 257 & $<8$ & $<1$ & 32 & 56 & $<9$ & 23 \\
\hline 2011Z092A & $<1$ & 452 & $<8$ & $<1$ & 29 & 116 & $<9$ & 45 \\
\hline $2011 Z 105 A$ & $<1$ & 126 & $<8$ & 15 & 21 & 79 & 125 & 156 \\
\hline $2011 Z 110 \mathrm{~A}$ & $<1$ & 184 & $<8$ & 27 & 32 & 70 & 149 & 167 \\
\hline 2011Z110B & $<1$ & 44 & $<8$ & 5 & 8 & 15 & 85 & 102 \\
\hline $2011 Z 112 A$ & $<1$ & 147 & $<8$ & $<1$ & 43 & 91 & $<9$ & 171 \\
\hline $2011 Z 113 A$ & $<1$ & 102 & $<8$ & 19 & --- & 68 & 132 & --- \\
\hline 2011Z116A & $<1$ & 134 & $<8$ & 12 & 20 & 68 & 127 & 176 \\
\hline 2011Z120B & $<1$ & 219 & $<8$ & 18 & --- & 43 & 50 & $<9$ \\
\hline $2011 Z 123 A$ & $<1$ & 156 & $<8$ & 14 & 18 & 75 & 133 & 156 \\
\hline $2011 Z 135 \mathrm{~A}$ & $<1$ & 109 & $<8$ & 24 & --- & 55 & 205 & --- \\
\hline 2011Z149A & $<1$ & 227 & $<8$ & 22 & 14 & 76 & 62 & 92 \\
\hline 2011Z160A & $<1$ & $<25$ & $<8$ & $<1$ & 20 & 40 & $<9$ & 133 \\
\hline 2011Z161A & $<1$ & 154 & $<8$ & 21 & 30 & 66 & 111 & 148 \\
\hline
\end{tabular}


Table 8. (continued)

\begin{tabular}{|c|c|c|c|c|c|c|c|}
\hline Sample_ID & Latitude & Longitude & Easting & Northing & $\begin{array}{l}\text { UTM_ } \\
\text { Zone }\end{array}$ & Root_name & Comments \\
\hline 2011Z165A & 65.324838 & $\mid-152.985124$ & $\mid 500693$ & 7244466 & $5 \mathrm{~N}$ & Biotite-albite-muscovite quartz schist & |-- \\
\hline $2011 Z 165 A$ & 65.220532 & -153.507728 & 476254 & 7232936 & $5 \mathrm{~N}$ & chloritoid-quartz-mica schist & Inhomogenous \\
\hline 2011Z166A & 65.452720 & -152.571084 & 519884 & 7258787 & $5 \mathrm{~N}$ & Metagabbro(?) & --- \\
\hline 2011Z168B & 65.448997 & -152.558592 & 520466 & 7258376 & $5 \mathrm{~N}$ & Cherty slate & --- \\
\hline 2011Z169A & 65.447038 & -152.557482 & 520519 & 7258158 & $5 \mathrm{~N}$ & Metagabbro & --- \\
\hline 2011Z173A & 65.441840 & -152.565785 & 520138 & 7257576 & $5 \mathrm{~N}$ & Quartzite & --- \\
\hline $2011 Z 175 \mathrm{~A}$ & 65.440109 & -152.569263 & 519978 & 7257382 & $5 \mathrm{~N}$ & Mafic schist & --- \\
\hline 2011Z178B & 65.435810 & -152.578323 & 519561 & 7256900 & $5 \mathrm{~N}$ & Pyrite slate & Small \\
\hline $2011 Z 180 A$ & 65.432751 & -152.581519 & 519415 & 7256558 & $5 \mathrm{~N}$ & Albite quartzite & --- \\
\hline 2011Z183A & 65.428024 & -152.588750 & 519083 & 7256029 & $5 \mathrm{~N}$ & Mafic schist & --- \\
\hline $2011 Z 186 \mathrm{~A}$ & 65.418086 & -152.589746 & 519044 & 7254921 & $5 \mathrm{~N}$ & Metagabbro & --- \\
\hline 2011Z187A & 65.416791 & -152.592028 & 518939 & 7254776 & $5 \mathrm{~N}$ & Metagabbro(?) & --- \\
\hline 2011Z188A & 65.415834 & -152.593227 & 518884 & 7254669 & $5 \mathrm{~N}$ & Metadacite(?) & --- \\
\hline 2011Z189A & 65.414851 & -152.594686 & 518817 & 7254559 & $5 \mathrm{~N}$ & Serpentinite & --- \\
\hline 2011Z190B & 65.413194 & -152.595616 & 518775 & 7254374 & $5 \mathrm{~N}$ & Biotite-chlorite-albite schist(?) & --- \\
\hline 2011Z194A & 65.406854 & -152.597006 & 518715 & 7253667 & $5 \mathrm{~N}$ & Metagabbro & --- \\
\hline 2011Z207A & 65.338726 & -152.978458 & 501003 & 7246014 & $5 \mathrm{~N}$ & Mafic schist & --- \\
\hline 2011Z209A & 65.337819 & -152.975602 & 501136 & 7245913 & $5 \mathrm{~N}$ & Mafic schist(?) & --- \\
\hline 2011Z220B & 65.324838 & -152.985124 & 500693 & 7244466 & $5 \mathrm{~N}$ & Muscovite-chlorite quartz schist & --- \\
\hline 2011Z224A & 65.321169 & -152.996780 & 500150 & 7244057 & $5 \mathrm{~N}$ & Albite-muscovite quartz schist & --- \\
\hline $2011 Z 256 B$ & 65.180341 & -153.888678 & 458375 & 7228654 & $5 \mathrm{~N}$ & Granitic orthogneiss & --- \\
\hline $2011 Z 269 A$ & 65.318163 & -153.522849 & 475637 & 7243823 & $5 \mathrm{~N}$ & Metadiorite(?) & Inhomogenous \\
\hline $2011 Z 272 A$ & 65.320170 & -153.516064 & 475955 & 7244044 & $5 \mathrm{~N}$ & Feldspar-mica schist(?) & --- \\
\hline $2011 Z 275 A$ & 65.318974 & -153.503378 & 476545 & 7243906 & $5 \mathrm{~N}$ & Chloritoid-quartz-mica schist & --- \\
\hline 2011Z277A & 65.318827 & -153.493653 & 476998 & 7243886 & $5 \mathrm{~N}$ & Fine-grained gabbro & --- \\
\hline 2011Z279A & 65.319484 & -153.487463 & 477287 & 7243957 & $5 \mathrm{~N}$ & Metadiorite(?) & --- \\
\hline 2011Z281A & 65.319561 & -153.477506 & 477751 & 7243962 & $5 \mathrm{~N}$ & Mica quartz schist & Inhomogenous \\
\hline $2011 Z 286 A$ & 65.315219 & -152.980194 & 500923 & 7243394 & $5 \mathrm{~N}$ & Chlorite-muscovite quartz schist & Inhomogenous \\
\hline $2011 Z 286 \mathrm{~A}$ & 65.315219 & -152.980194 & 500923 & 7243394 & $5 \mathrm{~N}$ & Chlorite-muscovite quartz schist & Inhomogenous \\
\hline $2011 Z 288 \mathrm{~A}$ & 65.311782 & -152.976613 & 501090 & 7243011 & $5 \mathrm{~N}$ & Garnet amphibolitite(?) & Inhomogenous \\
\hline $2011 Z 293 A$ & 65.303132 & -152.968663 & 501461 & 7242047 & $5 \mathrm{~N}$ & Metadiorite(?) & Porous \\
\hline 2011Z294B & 65.301391 & -152.967657 & 501508 & 7241853 & $5 \mathrm{~N}$ & Metagabbro & Porous \\
\hline
\end{tabular}


Table 8. (continued)

\begin{tabular}{|c|c|c|c|c|c|c|c|c|c|}
\hline Sample_ID & $\mathrm{SiO}_{2 \_} \mathrm{pct}$ & Al203_pct & BaO_pct & CaO_pct & FeO_pct & K2O_pct & MgO_pct & MnO_pct & $\mathrm{Na} 2 \mathrm{O} \_p c t$ \\
\hline $2011 Z 165 A$ & 69.4 & 16.9 & 0.0758 & 0.15 & "6.55 & 3.03 & 1.35 & 0.0841 & 1.5 \\
\hline $2011 Z 165 A$ & 68.9 & 16.9 & 0.0668 & 0.271 & 6.78 & 2.9 & 1.27 & 0.109 & 1.42 \\
\hline $2011 Z 166 A$ & 49.4 & 16.5 & 0.0589 & 12.3 & 10.2 & 0.459 & 6.7 & 0.212 & 2.68 \\
\hline 2011Z168B & 81.8 & 7.13 & 0.54 & 2.37 & 3.6 & 0.864 & 0.866 & 0.828 & 1.52 \\
\hline 2011Z169A & 49.3 & 18.1 & 0.0956 & 11.7 & 8.09 & 1.21 & 7.03 & 0.166 & 3.09 \\
\hline $2011 Z 173 A$ & 95 & 2.4 & 0.217 & 0.108 & 0.548 & 0.504 & 0.675 & $<0.03$ & 0.359 \\
\hline $2011 Z 175 A$ & 49.7 & 14.9 & 0.087 & 11.2 & 12.5 & 0.116 & 6.03 & 0.211 & 3.28 \\
\hline 2011Z178B & 57.1 & 11 & 0.166 & 2.98 & 15 & 0.97 & 5.55 & 0.102 & 6 \\
\hline $2011 Z 180 A$ & 95 & 2.01 & 0.0494 & 0.781 & 0.6 & 0.276 & 0.56 & $<0.03$ & 0.54 \\
\hline 2011Z183A & 49 & 13.9 & 0.304 & 7.53 & 15.7 & 0.359 & 4.59 & 0.273 & 4.45 \\
\hline $2011 Z 186 A$ & 51.4 & 14.3 & $<0.03$ & 10.3 & 12.9 & 0.098 & 6.48 & 0.179 & 2.61 \\
\hline $2011 Z 187 A$ & 50.8 & 16.3 & $<0.03$ & 10.9 & 9.29 & 0.442 & 8.51 & 0.186 & 2.62 \\
\hline 2011Z188A & 68.1 & 13.9 & 0.171 & 3.36 & 5.79 & 1.12 & 2.03 & 0.148 & 4.46 \\
\hline 2011Z189A & 44.7 & 4.5 & $<0.03$ & 2.75 & 12.6 & 0.0622 & 34.4 & 0.209 & 0.0778 \\
\hline 2011Z190B & 57.3 & 16.4 & 0.118 & 5.38 & 7.48 & 1.67 & 2.95 & 0.165 & 7.2 \\
\hline 2011Z194A & 49.9 & 15.7 & 0.0675 & 9.58 & 12 & 0.918 & 5.38 & 0.189 & 4.24 \\
\hline $2011 Z 207 A$ & 53.2 & 17.4 & $<0.03$ & 5.57 & 9.3 & 0.0284 & 6.92 & 0.124 & 6.76 \\
\hline $2011 Z 209 A$ & 53.1 & 17.6 & $<0.03$ & 5.48 & 9.21 & 0.0256 & 6.96 & 0.134 & 6.8 \\
\hline 2011Z220B & 76.8 & 9.91 & 0.0561 & 1.55 & 6.28 & 1.51 & 2.23 & 0.0508 & 0.564 \\
\hline $2011 Z 224 A$ & 86 & 6.98 & 0.114 & 0.272 & 2.85 & 1.03 & 1.14 & $<0.03$ & 0.859 \\
\hline $2011 Z 256 B$ & 75.3 & 13.5 & $<0.03$ & 0.715 & 0.825 & 5.04 & 0.14 & $<0.03$ & 4.06 \\
\hline $2011 Z 269 A$ & 66.7 & 9.8 & 0.0433 & 5.85 & 8.26 & 0.341 & 2.82 & 0.264 & 3.64 \\
\hline $2011 Z 272 A$ & 67.7 & 11.9 & 0.0709 & 8.12 & 4.56 & 2 & 2.49 & 0.0452 & 2.27 \\
\hline $2011 Z 275 A$ & 68.7 & 16.9 & 0.12 & 0.229 & 7.93 & 2.98 & 1.24 & 0.135 & 0.687 \\
\hline $2011 Z 277 A$ & 49.9 & 14.5 & 0.0669 & 8.99 & 13.3 & 0.218 & 4.84 & 0.237 & 5.17 \\
\hline $2011 Z 279 A$ & 60 & 17.2 & 0.0318 & 6.45 & 9.28 & 0.804 & 2.91 & 0.0955 & 2.17 \\
\hline $2011 Z 281 A$ & 83.4 & 7.2 & 0.096 & 2.43 & 2.81 & 0.676 & 1.24 & 0.0819 & 1.49 \\
\hline $2011 Z 286 \mathrm{~A}$ & 75.5 & 12.3 & 0.137 & 0.188 & 6.35 & 2.31 & 1.35 & 0.0459 & 0.59 \\
\hline $2011 Z 286 A$ & 75.5 & 12.5 & 0.119 & 0.173 & 6.36 & 2.28 & 1.3 & 0.0323 & 0.454 \\
\hline $2011 Z 288 \mathrm{~A}$ & 51.6 & 14 & 0.0365 & 8.52 & 13.2 & 0.214 & 4.93 & 0.263 & 4.43 \\
\hline $2011 Z 293 A$ & 60.9 & 13.9 & $<0.03$ & 2.7 & 12.1 & 0.0326 & 5.03 & 0.182 & 3.56 \\
\hline 2011Z294B & 51.4 & 16.9 & 0.0401 & 9.42 & 9.86 & 0.364 & 4.31 & 0.2 & 5.55 \\
\hline
\end{tabular}


Table 8. (continued)

\begin{tabular}{|c|c|c|c|c|c|c|c|c|c|}
\hline Sample_ID & P2O5_pct & TiO2_pct & CO2_pct & Total_pct & As_ppm & Bi_ppm & Br_ppm & Ce_ppm & Cl_ppm \\
\hline $2011 Z 165 A$ & 0.155 & 0.737 & $<0.05$ & 99.9319 & $<4$ & $2<2$ & $<<5$ & $<60$ & 135 \\
\hline $2011 Z 165 A$ & 0.212 & 0.809 & $<0.05$ & 99.6378 & 4 & $<2$ & $<5$ & $<60$ & 431 \\
\hline 2011Z166A & 0.2 & 1.24 & $<0.05$ & 99.9499 & $<4$ & $<2$ & $<5$ & $<60$ & $<50$ \\
\hline 2011Z168B & $<0.03$ & 0.369 & $<0.05$ & 99.9148 & $<4$ & $<2$ & $<5$ & $<60$ & 107 \\
\hline 2011Z169A & 0.109 & 0.921 & $<0.05$ & 99.8116 & $<4$ & $<2$ & $<5$ & $<60$ & 72 \\
\hline $2011 Z 173 A$ & 0.0362 & 0.103 & $<0.05$ & 99.9724 & $<4$ & $<2$ & $<5$ & $<60$ & 73 \\
\hline $2011 Z 175 A$ & 0.241 & 1.55 & $<0.05$ & 99.815 & $<4$ & $<2$ & $<5$ & $<60$ & $<50$ \\
\hline 2011Z178B & 0.113 & 0.647 & $<0.05$ & 99.628 & $<4$ & $<2$ & $<5$ & $<60$ & 251 \\
\hline $2011 Z 180 A$ & 0.0384 & 0.0842 & $<0.05$ & 99.9522 & $<4$ & $<2$ & $<5$ & 247 & 131 \\
\hline $2011 Z 183 A$ & 0.637 & 3.11 & $<0.05$ & 99.853 & $<4$ & $<2$ & $<5$ & $<60$ & 185 \\
\hline $2011 Z 186 \mathrm{~A}$ & 0.214 & 1.31 & $<0.05$ & 99.817 & $<4$ & $<2$ & $<5$ & $<60$ & 68 \\
\hline $2011 Z 187 A$ & 0.101 & 0.601 & $<0.05$ & 99.75393 & 101 & $<2$ & $<5$ & $<60$ & 113 \\
\hline 2011Z188A & 0.306 & 0.541 & $<0.05$ & 99.926 & $<4$ & $<2$ & $<5$ & $<60$ & 106 \\
\hline 2011Z189A & 0.0501 & 0.223 & $<0.05$ & 99.5847 & $<4$ & $<2$ & $<5$ & $<60$ & 157 \\
\hline 2011Z190B & 0.45 & 0.796 & $<0.05$ & 99.909 & $<4$ & $<2$ & $<5$ & $<60$ & 178 \\
\hline 2011Z194A & 0.246 & 1.58 & $<0.05$ & 99.8005 & $<4$ & $<2$ & $<5$ & $<60$ & 101 \\
\hline 2011Z207A & 0.171 & 0.396 & $<0.05$ & 99.8858 & 15 & $<2$ & $<5$ & $<60$ & 90 \\
\hline $2011 Z 209 \mathrm{~A}$ & 0.179 & 0.387 & $<0.05$ & 99.8867 & 19 & $<2$ & $<5$ & $<60$ & 94 \\
\hline $2011 Z 220 B$ & 0.0917 & 0.704 & $<0.05$ & 99.7466 & 43 & $<2$ & $<5$ & $<60$ & 139 \\
\hline 2011Z224A & 0.142 & 0.504 & $<0.05$ & 99.9107 & 31 & $<2$ & $<5$ & $<60$ & 107 \\
\hline $2011 Z 256 \mathrm{~B}$ & 0.21 & 0.0866 & $<0.05$ & 99.9038 & $<4$ & $<2$ & $<5$ & $<60$ & 312 \\
\hline $2011 Z 269 A$ & 0.33 & 1.75 & $<0.05$ & 99.8383 & 11 & $<2$ & $<5$ & $<60$ & 112 \\
\hline $2011 Z 272 \mathrm{~A}$ & 0.142 & 0.609 & $<0.05$ & 99.9071 & 8 & $<2$ & $<5$ & $<60$ & 299 \\
\hline $2011 Z 275 A$ & 0.202 & 0.808 & $<0.05$ & 99.931 & $<4$ & $<2$ & $<5$ & $<60$ & $<50$ \\
\hline $2011 Z 277 A$ & 0.351 & 2.21 & $<0.05$ & 99.7829 & $<4$ & $<2$ & $<5$ & $<60$ & 101 \\
\hline $2011 Z 279 A$ & 0.16 & 0.743 & $<0.05$ & 99.8443 & 10 & $<2$ & $<5$ & $<60$ & 123 \\
\hline $2011 Z 281 \mathrm{~A}$ & 0.257 & 0.196 & $<0.05$ & 99.8869 & 4 & $<2$ & $<5$ & $<60$ & 255 \\
\hline $2011 Z 286 \mathrm{~A}$ & 0.135 & 0.889 & $<0.05$ & 99.7949 & 63 & $<2$ & $<5$ & $<60$ & 465 \\
\hline $2011 Z 286 \mathrm{~A}$ & 0.138 & 0.97 & $<0.05$ & 99.8263 & 68 & $<2$ & $<5$ & $<60$ & 271 \\
\hline $2011 Z 288 A$ & 0.413 & 2.17 & $<0.05$ & 99.7765 & 19 & $<2$ & $<5$ & $<60$ & 515 \\
\hline $2011 Z 293 A$ & 0.427 & 0.924 & $<0.05$ & 99.7758 & $<4$ & $<2$ & $<5$ & $<60$ & 243 \\
\hline $2011 Z 294 B$ & 0.319 & 1.54 & $<0.05$ & 99.9031 & $<4$ & $<2$ & $<5$ & $<60$ & 103 \\
\hline
\end{tabular}


Table 8. (continued)

\begin{tabular}{|c|c|c|c|c|c|c|c|c|c|}
\hline Sample_ID & Co_ppm & Cr_ppm & Cu_ppm & F_ppm & La_ppm & Mo_ppm & Nb_ppm & Nb_ppm_2 & Ni_ppm \\
\hline $2011 Z 165 A$ & $2<10$ & 116 & $<>$ & $<110$ & $<50$ & $<<1$ & 12 & 16 & 46 \\
\hline $2011 Z 165 A$ & $<10$ & 89 & $<7$ & 1700 & $<50$ & $<1$ & $<1$ & 16 & 31 \\
\hline 2011Z166A & 87 & 288 & 65 & $<110$ & $<50$ & $<1$ & $<1$ & 6 & 162 \\
\hline 2011Z168B & 27 & $<30$ & 63 & $<110$ & $<50$ & $<1$ & 7 & --- & 80 \\
\hline 2011Z169A & 74 & 243 & 94 & $<110$ & $<50$ & $<1$ & $<1$ & 4 & 113 \\
\hline $2011 Z 173 A$ & $<10$ & 59 & $<7$ & $<110$ & $<50$ & $<1$ & $<1$ & --- & 46 \\
\hline $2011 Z 175 A$ & 91 & 225 & 91 & $<110$ & $<50$ & $<1$ & $<1$ & 6 & 70 \\
\hline 2011Z178B & 95 & 62 & 65 & $<110$ & $<50$ & $<1$ & 7 & --- & 23 \\
\hline $2011 Z 180 A$ & $<10$ & 39 & 57 & $<110$ & $<50$ & $<1$ & $<1$ & --- & 48 \\
\hline $2011 Z 183 A$ & 103 & 97 & 11 & $<110$ & $<50$ & $<1$ & $<1$ & 37 & 24 \\
\hline $2011 Z 186 \mathrm{~A}$ & 24 & 266 & 157 & $<110$ & $<50$ & $<1$ & $<1$ & 5 & 76 \\
\hline $2011 Z 187 A$ & 35 & 727 & 154 & $<110$ & $<50$ & $<1$ & $<1$ & 3 & 303 \\
\hline 2011Z188A & 28 & 77 & 60 & $<110$ & $<50$ & $<1$ & 13 & 10 & 79 \\
\hline 2011Z189A & 178 & 2250 & 32 & $<110$ & $<50$ & $<1$ & $<1$ & $<1$ & 1790 \\
\hline 2011Z190B & $<10$ & 57 & 7 & $<110$ & $<50$ & $<1$ & 24 & 16 & 57 \\
\hline 2011Z194A & 107 & 71 & 9 & $<110$ & $<50$ & $<1$ & $<1$ & 7 & 41 \\
\hline $2011 Z 207 A$ & 99 & 399 & 64 & $<110$ & $<50$ & $<1$ & $<1$ & 3 & 165 \\
\hline $2011 Z 209 \mathrm{~A}$ & 45 & 422 & 33 & $<110$ & $<50$ & $<1$ & $<1$ & 3 & 120 \\
\hline $2011 Z 220 B$ & 34 & 49 & 29 & 1060 & $<50$ & $<1$ & 9 & --- & 42 \\
\hline 2011Z224A & $<10$ & 68 & 29 & $<110$ & $<50$ & $<1$ & 9 & 7 & 39 \\
\hline $2011 Z 256 \mathrm{~B}$ & $<10$ & $<30$ & 45 & $<110$ & $<50$ & $<1$ & $<1$ & 9 & $<7$ \\
\hline $2011 Z 269 A$ & 68 & 319 & 72 & $<110$ & $<50$ & $<1$ & $<1$ & 16 & 122 \\
\hline $2011 Z 272 \mathrm{~A}$ & $<10$ & 90 & $<7$ & $<110$ & $<50$ & $<1$ & $<1$ & 11 & 40 \\
\hline $2011 Z 275 A$ & $<10$ & 113 & $<7$ & $<110$ & $<50$ & $<1$ & $<1$ & 15 & 23 \\
\hline $2011 Z 277 A$ & $<10$ & 160 & 44 & $<110$ & $<50$ & $<1$ & $<1$ & 12 & 72 \\
\hline $2011 Z 279 A$ & $<10$ & $<30$ & $<7$ & 749 & $<50$ & $<1$ & $<1$ & 2 & $<7$ \\
\hline $2011 Z 281 \mathrm{~A}$ & $<10$ & 94 & 44 & $<110$ & $<50$ & $<1$ & $<1$ & 4 & 29 \\
\hline $2011 Z 286 \mathrm{~A}$ & 19.2 & 122 & 26.2 & $<110$ & $<50$ & $<1$ & $<1$ & 15.6 & 45 \\
\hline $2011 Z 286 \mathrm{~A}$ & 53 & 110 & 15 & $<110$ & $<50$ & $<1$ & $<1$ & 14 & 39 \\
\hline $2011 Z 288 A$ & 54 & 121 & 83 & $<110$ & $<50$ & $<1$ & $<1$ & 10.4 & $<7$ \\
\hline $2011 Z 293 A$ & $<10$ & 148 & $<7$ & $<110$ & $<50$ & $<1$ & $<1$ & 8 & 55 \\
\hline $2011 Z 294 B$ & $<10$ & 101 & 45 & $<110$ & $<50$ & $<1$ & $<1$ & 11 & 53 \\
\hline
\end{tabular}


Table 8. (continued)

\begin{tabular}{|c|c|c|c|c|c|c|c|c|c|}
\hline Sample_ID & Pb_ppm & Rb_ppm & Rb_ppm_2 & S_ppm & Sb_ppm & Sn_ppm & Sr_ppm & Sr_ppm_2 & Th_ppm \\
\hline $2011 Z 165 A$ & 36 & 142 & 144 & 53 & $<2$ & $<4$ & 46 & 44 & $2<2$ \\
\hline $2011 Z 165 A$ & 30 & $<1$ & 137 & 207 & $<2$ & $<4$ & $<1$ & 46 & $<2$ \\
\hline 2011Z166A & $<4$ & $<1$ & 9 & 57 & $<2$ & $<4$ & $<1$ & 222 & $<2$ \\
\hline 2011Z168B & 39 & 23 & --- & 56 & $<2$ & $<4$ & 25 & --- & $<2$ \\
\hline 2011Z169A & $<4$ & $<1$ & 33 & $<30$ & $<2$ & $<4$ & $<1$ & 192 & $<2$ \\
\hline $2011 Z 173 A$ & $<4$ & 22 & --- & 31 & $<2$ & $<4$ & 5 & --- & $<2$ \\
\hline $2011 Z 175 A$ & $<4$ & $<1$ & 3 & 103 & $<2$ & $<4$ & $<1$ & 244 & $<2$ \\
\hline 2011Z178B & 13 & 28 & --- & 2580 & $<2$ & $<4$ & 24 & --- & $<2$ \\
\hline $2011 Z 180 A$ & $<4$ & 11 & & 218 & $<2$ & $<4$ & 11 & --- & $<2$ \\
\hline $2011 Z 183 A$ & $<4$ & $<1$ & 13 & 135 & $<2$ & $<4$ & $<1$ & 209 & $<2$ \\
\hline $2011 Z 186 \mathrm{~A}$ & $<4$ & $<1$ & 2 & 38 & $<2$ & $<4$ & $<1$ & 105 & $<2$ \\
\hline $2011 Z 187 A$ & $<4$ & $<1$ & 28 & 80 & $<2$ & $<4$ & $<1$ & 212 & $<2$ \\
\hline 2011Z188A & $<4$ & 29 & 28 & 47 & $<2$ & $<4$ & 288 & 283 & $<2$ \\
\hline 2011Z189A & $<4$ & $<1$ & 11 & 137 & $<2$ & $<4$ & $<1$ & 12 & $<2$ \\
\hline 2011Z190B & $<4$ & 34 & 30 & 35 & $<2$ & $<4$ & 299 & 280 & $<2$ \\
\hline 2011Z194A & $<4$ & $<1$ & 32 & $<30$ & $<2$ & $<4$ & $<1$ & 421 & $<2$ \\
\hline $2011 Z 207 A$ & $<4$ & $<1$ & 1 & 137 & $<2$ & $<4$ & $<1$ & 80 & $<2$ \\
\hline $2011 Z 209 A$ & $<4$ & $<1$ & 1 & 44 & $<2$ & $<4$ & $<1$ & 79 & $<2$ \\
\hline $2011 Z 220 B$ & 22 & 91 & --- & 112 & $<2$ & $<4$ & 18 & --- & $<2$ \\
\hline 2011Z224A & 16 & 49 & 48 & 105 & $<2$ & $<4$ & 51 & 45 & $<2$ \\
\hline $2011 Z 256 \mathrm{~B}$ & 61 & $<1$ & 200 & 128 & $<2$ & $<4$ & $<1$ & 55 & $<2$ \\
\hline $2011 Z 269 A$ & $<4$ & $<1$ & 10 & 117 & $<2$ & $<4$ & $<1$ & 68 & $<2$ \\
\hline $2011 Z 272 \mathrm{~A}$ & 17 & $<1$ & 81 & 164 & $<2$ & $<4$ & $<1$ & 86 & $<2$ \\
\hline $2011 Z 275 A$ & 33 & $<1$ & 122 & 157 & $<2$ & $<4$ & $<1$ & 60 & $<2$ \\
\hline $2011 Z 277 A$ & 42 & $<1$ & 3 & 132 & $<2$ & $<4$ & $<1$ & 308 & $<2$ \\
\hline $2011 Z 279 A$ & 24 & $<1$ & 37 & 105 & $<2$ & $<4$ & $<1$ & 338 & $<2$ \\
\hline $2011 Z 281 \mathrm{~A}$ & 18 & $<1$ & 24 & 75 & $<2$ & $<4$ & $<1$ & 134 & $<2$ \\
\hline $2011 Z 286 \mathrm{~A}$ & 30.1 & $<1$ & 104.5 & 236 & $<2$ & $<4$ & $<1$ & 41.4 & 12.3 \\
\hline $2011 Z 286 \mathrm{~A}$ & 17 & $<1$ & 100 & 162 & $<2$ & $<4$ & $<1$ & 42 & $<2$ \\
\hline $2011 Z 288 A$ & $<4$ & $<1$ & 6.8 & 291 & $<2$ & $<4$ & $<1$ & 251.7 & $<2$ \\
\hline $2011 Z 293 A$ & $<4$ & $<1$ & 1 & 256 & $<2$ & $<4$ & $<1$ & 107 & $<2$ \\
\hline $2011 Z 294 B$ & $<4$ & $<1$ & 12 & 86 & $<2$ & $<4$ & $<1$ & 240 & $<2$ \\
\hline
\end{tabular}




\begin{tabular}{|c|c|c|c|c|c|c|c|c|}
\hline Sample_ID & U_ppm & V_ppm & W_ppm & Y_ppm & Y_ppm_2 & Zn_ppm & Zr_ppm & Zr_ppm_2 \\
\hline $2011 Z 165 A$ & $2<1$ & 135 & $<<8$ & 14 & 24 & 100 & 130 & 148 \\
\hline $2011 Z 165 A$ & $<1$ & 162 & $<8$ & $<1$ & 21 & 75 & $<9$ & 152 \\
\hline 2011Z166A & $<1$ & 354 & $<8$ & $<1$ & 25 & 78 & $<9$ & 97 \\
\hline 2011Z168B & $<1$ & 98 & $<8$ & 16 & --- & 82 & 63 & $<9$ \\
\hline 2011Z169A & $<1$ & 348 & $<8$ & $<1$ & 14 & 60 & $<9$ & 64 \\
\hline $2011 Z 173 A$ & $<1$ & 48 & $<8$ & 2 & --- & 24 & 36 & $<9$ \\
\hline $2011 Z 175 A$ & $<1$ & 500 & $<8$ & $<1$ & 35 & 95 & $<9$ & 113 \\
\hline 2011Z178B & $<1$ & 175 & $<8$ & 21 & --- & 152 & 111 & --- \\
\hline 2011Z180A & $<1$ & 41 & $<8$ & $<1$ & --- & 22 & 34 & $<9$ \\
\hline 2011Z183A & $<1$ & 575 & $<8$ & $<1$ & 62 & 205 & $<9$ & 323 \\
\hline $2011 Z 186 A$ & $<1$ & 374 & $<8$ & $<1$ & 28 & 119 & $<9$ & 101 \\
\hline $2011 Z 187 A$ & $<1$ & 355 & $<8$ & $<1$ & 18 & 87 & $<9$ & 52 \\
\hline $2011 Z 188 \mathrm{~A}$ & $<1$ & 151 & $<8$ & 16 & 21 & 165 & 104 & 130 \\
\hline 2011Z189A & $<1$ & 110 & $<8$ & $<1$ & 8 & 100 & $<9$ & 42 \\
\hline 2011Z190B & $<1$ & 204 & $<8$ & 105 & 20 & 44 & 101 & 116 \\
\hline 2011Z194A & $<1$ & 433 & $<8$ & $<1$ & 22 & 71 & $<9$ & 86 \\
\hline $2011 Z 207 A$ & $<1$ & 236 & $<8$ & $<1$ & 14 & 72 & $<9$ & 28 \\
\hline $2011 Z 209 A$ & $<1$ & 224 & $<8$ & $<1$ & 15 & 57 & $<9$ & 28 \\
\hline $2011 Z 220 B$ & $<1$ & 163 & $<8$ & 47 & --- & 87 & 166 & --- \\
\hline $2011 Z 224 \mathrm{~A}$ & $<1$ & 119 & $<8$ & 9 & 10 & 53 & 147 & 149 \\
\hline 2011Z256B & $<1$ & 126 & $<8$ & $<1$ & 18 & $<8$ & $<9$ & 45 \\
\hline 2011Z269A & $<1$ & 267 & $<8$ & $<1$ & 19 & 81 & $<9$ & 137 \\
\hline $2011 Z 272 A$ & $<1$ & 134 & $<8$ & $<1$ & 18 & 81 & $<9$ & 151 \\
\hline $2011 Z 275 A$ & $<1$ & 141 & $<8$ & $<1$ & 27 & 84 & $<9$ & 151 \\
\hline $2011 Z 277 A$ & $<1$ & 516 & $<8$ & $<1$ & 46 & 110 & $<9$ & 192 \\
\hline $2011 Z 279 A$ & $<1$ & 354 & $<8$ & $<1$ & 19 & 78 & $<9$ & 61 \\
\hline $2011 Z 281 \mathrm{~A}$ & $<1$ & 116 & $<8$ & $<1$ & 18 & 40 & $<9$ & 70 \\
\hline $2011 Z 286 \mathrm{~A}$ & 2.2 & 219 & $<8$ & $<1$ & 26.4 & 128 & $<9$ & 204 \\
\hline $2011 Z 286 \mathrm{~A}$ & $<1$ & 195 & $<8$ & $<1$ & 23 & 141 & $<9$ & 210 \\
\hline 2011Z288A & $<1$ & 596 & $<8$ & $<1$ & 22 & 117 & $<9$ & 115.8 \\
\hline $2011 Z 293 \mathrm{~A}$ & $<1$ & 411 & $<8$ & $<1$ & 25 & 142 & $<9$ & 108 \\
\hline 2011Z294B & $<1$ & 513 & $<8$ & $<1$ & 28 & 90 & $<9$ & 141 \\
\hline
\end{tabular}


Table 8. (continued)

\begin{tabular}{|c|c|c|c|c|c|c|c|}
\hline Sample_ID & Latitude & Longitude & Easting & Northing & $\begin{array}{l}\text { UTM_ } \\
\text { Zone }\end{array}$ & Root_name & Comments \\
\hline 2011Z296B & 65.298412 & \begin{tabular}{|l|}
-152.965281 \\
\end{tabular} & $\mid 501619$ & 7241521 & $5 \mathrm{~N}$ & Metamafic & --- \\
\hline 2011Z311A & 65.484214 & -152.668359 & 515356 & 7262270 & $5 \mathrm{~N}$ & Mica quartz schist & Porous \\
\hline $2011 Z 320 \mathrm{~A}$ & 65.498338 & -152.653270 & 516046 & 7263848 & $5 \mathrm{~N}$ & Metamafic & Porous \\
\hline 2011Z326A & 65.400974 & -152.521896 & 522208 & 7253036 & $5 \mathrm{~N}$ & Gabbro & Altered \\
\hline $2011 Z 326 B$ & 65.400974 & -152.521896 & 522208 & 7253036 & $5 \mathrm{~N}$ & Gabbro & --- \\
\hline 2011Z327A & 65.399515 & -152.520244 & 522286 & 7252874 & $5 \mathrm{~N}$ & Wherlite & Altered \\
\hline $2011 Z 328 \mathrm{~A}$ & 65.397579 & -152.520946 & 522255 & 7252658 & $5 \mathrm{~N}$ & Metagabbro & --- \\
\hline $2011 Z 330 \mathrm{~A}$ & 65.395194 & -152.521442 & 522234 & 7252392 & $5 \mathrm{~N}$ & Metaandesite & --- \\
\hline $2011 Z 331 \mathrm{~A}$ & 65.394189 & -152.521266 & 522243 & 7252280 & $5 \mathrm{~N}$ & Metagabbro & --- \\
\hline $2011 Z 336 A$ & 65.389356 & -152.513801 & 522594 & 7251744 & $5 \mathrm{~N}$ & Wherlite & Altered \\
\hline 2011Z345B & 65.358387 & -152.853866 & 506799 & 7248213 & $5 \mathrm{~N}$ & Metabreccia & Inhomogenous \\
\hline 2011Z349A & 65.360504 & -152.844933 & 507214 & 7248450 & $5 \mathrm{~N}$ & Metagabbro & --- \\
\hline 2011Z350A & 65.361426 & -152.842606 & 507322 & 7248553 & $5 \mathrm{~N}$ & Metagabbro & --- \\
\hline $2011 Z 355 A$ & 65.370191 & -152.825437 & 508118 & 7249532 & $5 \mathrm{~N}$ & Metaandesite(?) & Porous \\
\hline 2011Z368B & 65.371615 & -152.889876 & 505121 & 7249684 & $5 \mathrm{~N}$ & Metagabbro(?) & Inhomogenous \\
\hline $2011 Z 371 \mathrm{~A}$ & 65.264114 & -152.984494 & 500724 & 7237698 & $5 \mathrm{~N}$ & Gabbro & Altered \\
\hline $2011 Z 371 \mathrm{~A}$ & 65.264114 & -152.984494 & 500724 & 7237698 & $5 \mathrm{~N}$ & Metagabbro & Altered \\
\hline $2011 Z 372 A$ & 65.263038 & -152.984452 & 500726 & 7237578 & $5 \mathrm{~N}$ & Metagabbro & --- \\
\hline 2011Z373A & 65.261709 & -152.981754 & 500852 & 7237430 & $5 \mathrm{~N}$ & Metagabbro & --- \\
\hline $2011 Z 374 A$ & 65.260489 & -152.982719 & 500807 & 7237294 & $5 \mathrm{~N}$ & Metagabbro & --- \\
\hline $2011 Z 376 A$ & 65.258927 & -152.976317 & 501106 & 7237120 & $5 \mathrm{~N}$ & Metagabbro & --- \\
\hline $2011 Z 378 \mathrm{~A}$ & 65.258979 & -152.967410 & 501522 & 7237126 & $5 \mathrm{~N}$ & Metagabbro & --- \\
\hline $2011 Z 379 A$ & 65.259095 & -152.962142 & 501768 & 7237139 & $5 \mathrm{~N}$ & Metagabbro & -- \\
\hline $2011 Z 380 A$ & 65.258591 & -152.957411 & 501989 & 7237083 & $5 \mathrm{~N}$ & Calcite-quartz mica schist & Porous \\
\hline $2011 Z 383 B$ & 65.254238 & -152.952279 & 502229 & 7236598 & $5 \mathrm{~N}$ & Mica quartz schist & Inhomogenous, porous \\
\hline $2011 Z 385 A$ & 65.254046 & -152.942860 & 502669 & 7236577 & $5 \mathrm{~N}$ & Serpentinite & --- \\
\hline 2011Z397A & 65.245132 & -152.855647 & 506745 & 7235590 & $5 \mathrm{~N}$ & Quartz-mica schist & Inhomogenous \\
\hline 2011Z399A & 65.243818 & -152.860855 & 506502 & 7235443 & $5 \mathrm{~N}$ & Calcite-mica quartz schist & Porous \\
\hline $2011 Z 402 A$ & 65.246905 & -152.870598 & 506046 & 7235786 & $5 \mathrm{~N}$ & Quartzite & Inhomogenous \\
\hline $2011 Z 403 \mathrm{~A}$ & 65.246659 & -152.866383 & 506243 & 7235759 & $5 \mathrm{~N}$ & Quartzite & Inhomogenous \\
\hline $2011 Z 412 \mathrm{~A}$ & 65.236747 & -152.859394 & 506572 & 7234655 & $5 \mathrm{~N}$ & Mica quartzite & Inhomogenous, porous \\
\hline $2011 Z 412 A$ & 65.236747 & -152.859394 & 506572 & 7234655 & $5 \mathrm{~N}$ & Muscovite-quartz schist & Porous \\
\hline
\end{tabular}


Table 8. (continued)

\begin{tabular}{|c|c|c|c|c|c|c|c|c|c|}
\hline Sample_ID & $\mathrm{SiO}_{2 \_} \mathrm{pct}$ & Al203_pct & BaO_pct & CaO_pct & FeO_pct & K2O_pct & MgO_pct & MnO_pct & $\mathrm{Na} 2 \mathrm{O} \_p c t$ \\
\hline $2011 Z 296 \mathrm{~B}$ & 38.7 & 22.2 & $<<0.03$ & 7.79 & 19.3 & 0.0239 & 9.77 & 0.296 & 0.092 \\
\hline $2011 Z 311 \mathrm{~A}$ & 82.5 & 8.3 & 0.239 & 0.0546 & 4.81 & 2.62 & 0.756 & $<0.03$ & 0.0613 \\
\hline $2011 Z 320 A$ & 52.5 & 16 & 0.0441 & 2.31 & 12.9 & 1.42 & 6.41 & 0.345 & 6.13 \\
\hline $2011 Z 326 A$ & 47.3 & 16.4 & 0.0305 & 21 & 4.66 & 0.0655 & 8.53 & 0.099 & 1.2 \\
\hline 2011Z326B & 55.4 & 16.5 & $<0.03$ & 11.1 & 4.01 & 0.0438 & 2.79 & 0.0915 & 7.56 \\
\hline $2011 Z 327 A$ & 44.1 & 5.1 & $<0.03$ & 3.68 & 12.9 & 0.0708 & 33 & 0.19 & 0.142 \\
\hline 2011Z328A & 51.1 & 16.8 & 0.146 & 10.1 & 9.97 & 1.33 & 5.26 & 0.193 & 3.6 \\
\hline 2011Z330A & 59.3 & 17.6 & 0.186 & 7.97 & 5.85 & 1.88 & 1.65 & 0.181 & 3.96 \\
\hline $2011 Z 331 A$ & 52.4 & 16.3 & 0.106 & 6.8 & 10.6 & 1.71 & 4.9 & 0.274 & 5.4 \\
\hline 2011Z336A & 46.5 & 4.6 & $<0.03$ & 6.42 & 12 & 0.112 & 29 & 0.197 & 0.222 \\
\hline 2011Z345B & 48.1 & 10.5 & 0.102 & 0.0568 & 37.5 & 1.59 & $<0.05$ & $<0.03$ & 0.377 \\
\hline $20112349 \mathrm{~A}$ & 51.9 & 14.1 & $<0.03$ & 9.88 & 11.6 & 0.0717 & 6.49 & 0.223 & 4.55 \\
\hline $2011 Z 350 \mathrm{~A}$ & 49.9 & 16.1 & $<0.03$ & 10.1 & 11 & 0.0319 & 8.18 & 0.193 & 3.45 \\
\hline $2011 Z 355 A$ & 62.6 & 20.8 & 0.102 & 0.159 & 7.87 & 2.1 & 2.91 & 0.237 & 2 \\
\hline 2011Z368B & 46.9 & 14.2 & $<0.03$ & 16.9 & 11.3 & 0.485 & 8.2 & 0.227 & 0.611 \\
\hline $2011 Z 371 \mathrm{~A}$ & 49.9 & 15.5 & 0.0379 & 14.5 & 8.46 & 0.154 & 8.49 & 0.164 & 1.96 \\
\hline $2011 Z 371 A$ & 49.9 & 16 & 0.042 & 14.2 & 8.05 & 0.18 & 8.3 & 0.151 & 2.2 \\
\hline $2011 Z 372 A$ & 50.8 & 15.5 & 0.124 & 13.2 & 10.4 & 0.82 & 6.36 & 0.2 & 1.05 \\
\hline $2011 Z 373 \mathrm{~A}$ & 51.1 & 16.1 & 0.0785 & 10.9 & 9.3 & 0.296 & 6.7 & 0.16 & 4.02 \\
\hline $2011 Z 374 A$ & 50.4 & 17.3 & 0.0376 & 13.4 & 7.34 & 0.195 & 7.02 & 0.129 & 3.1 \\
\hline $2011 Z 376 A$ & 49.8 & 16.5 & 0.047 & 13.5 & 8.52 & 0.217 & 7.36 & 0.149 & 2.93 \\
\hline $2011 Z 378 A$ & 50.5 & 15.1 & 0.0695 & 13.2 & 8.44 & 0.298 & 8.3 & 0.145 & 2.72 \\
\hline $2011 Z 379 A$ & 50.5 & 15.8 & $<0.03$ & 12.6 & 9.45 & 0.169 & 7.54 & 0.168 & 2.67 \\
\hline $2011 Z 380 A$ & 69 & 18.7 & 0.0557 & 0.146 & 5.47 & 1.01 & 1.65 & 0.0441 & 3.13 \\
\hline 2011Z383B & 70.8 & 16 & 0.071 & 0.201 & 6.66 & 0.754 & 1.69 & 0.0411 & 2.47 \\
\hline $2011 Z 385 A$ & 49.5 & 2.4 & $<0.03$ & 0.0342 & 8.07 & $<.02$ & 39.3 & 0.094 & 0.0234 \\
\hline 2011Z397A & 56.9 & 22.5 & 0.175 & 0.167 & 11.1 & 5.05 & 2.1 & 0.104 & 0.488 \\
\hline $20112399 A$ & 84.9 & 7.3 & 0.0849 & 0.157 & 4.73 & 1.37 & 0.619 & 0.0337 & 0.28 \\
\hline $2011 Z 402 \mathrm{~A}$ & 96.2 & 0.9 & 0.0494 & 0.0548 & 2.07 & 0.163 & 0.162 & $<0.03$ & 0.0343 \\
\hline $2011 Z 403 A$ & 94.9 & 2.9 & $<0.03$ & 0.156 & 0.554 & 0.499 & 0.26 & $<0.03$ & 0.351 \\
\hline $2011 Z 412 \mathrm{~A}$ & 93.6 & 3.14 & 0.0431 & 0.0671 & 1.8 & 0.528 & 0.29 & $<0.03$ & 0.208 \\
\hline $2011 Z 412 A$ & 85.1 & 8.73 & 0.0626 & 0.096 & 2.74 & 1.28 & 0.537 & $<0.03$ & 0.928 \\
\hline
\end{tabular}


Table 8. (continued)

\begin{tabular}{|c|c|c|c|c|c|c|c|c|c|}
\hline Sample_ID & P2O5_pct & TiO2_pct & CO2_pct & Total_pct & As_ppm & Bi_ppm & Br_ppm & Ce_ppm & Cl_ppm \\
\hline $2011 Z 296 B$ & 0.0818 & 1.55 & $<0.05$ & 99.8231 & $<4$ & $2<2$ & $<<5$ & $<60$ & 133 \\
\hline $2011 Z 311 \mathrm{~A}$ & 0.109 & 0.532 & $<0.05$ & 99.9562 & 4 & $<2$ & $<5$ & $<60$ & 117 \\
\hline $2011 Z 320 A$ & 0.216 & 1.62 & $<0.05$ & 99.8951 & $<4$ & $<2$ & $<5$ & $<60$ & 233 \\
\hline $2011 Z 326 \mathrm{~A}$ & $<0.03$ & 0.54 & $<0.05$ & 99.8543 & 9 & $<2$ & $<5$ & $<60$ & 73 \\
\hline $2011 Z 326 B$ & 0.537 & 1.75 & $<0.05$ & 99.8017 & $<4$ & $<2$ & $<5$ & $<60$ & 126 \\
\hline $2011 Z 327 A$ & 0.0382 & 0.3 & $<0.05$ & 99.5719 & $<4$ & $<2$ & $<5$ & $<60$ & 299 \\
\hline $2011 Z 328 \mathrm{~A}$ & 0.241 & 1.09 & $<0.05$ & 99.83 & $<4$ & $<2$ & $<5$ & $<60$ & 153 \\
\hline $2011 Z 330 A$ & 0.45 & 0.785 & $<0.05$ & 99.812 & $<4$ & $<2$ & $<5$ & $<60$ & 405 \\
\hline 2011Z331A & 0.362 & 0.913 & $<0.05$ & 99.765 & $<4$ & $<2$ & $<5$ & $<60$ & 130 \\
\hline $2011 Z 336 A$ & 0.0663 & 0.428 & $<0.05$ & 99.581 & 7 & $<2$ & $<5$ & $<60$ & 283 \\
\hline 2011Z345B & 0.538 & 0.598 & $<0.05$ & 99.3728 & 3510 & 3.1 & $<5$ & $<60$ & 113 \\
\hline 2011Z349A & 0.129 & 0.928 & $<0.05$ & 99.8901 & $<4$ & $<2$ & $<5$ & $<60$ & 121 \\
\hline $2011 Z 350 A$ & 0.0898 & 0.863 & $<0.05$ & 99.9243 & $<4$ & $<2$ & $<5$ & $<60$ & 133 \\
\hline $2011 Z 355 A$ & $<0.03$ & 0.98 & $<0.05$ & 99.7818 & 18 & $<2$ & $<5$ & $<60$ & 212 \\
\hline $2011 Z 368 B$ & 0.0432 & 0.86 & $<0.05$ & 99.7558 & $<4$ & $<2$ & $<5$ & $<60$ & 89 \\
\hline $2011 Z 371 \mathrm{~A}$ & 0.064 & 0.615 & $<0.05$ & 99.8449 & $<4$ & $<2$ & $<5$ & $<60$ & 228 \\
\hline $2011 Z 371 \mathrm{~A}$ & 0.067 & 0.75 & $<0.05$ & 99.84 & $<4$ & $<2$ & $<5$ & $<60$ & 235 \\
\hline $2011 Z 372 A$ & 0.223 & 1.27 & $<0.05$ & 99.947 & $<4$ & $<2$ & $<5$ & $<60$ & 107 \\
\hline $2011 Z 373 A$ & 0.228 & 0.98 & $<0.05$ & 99.8625 & $<4$ & $<2$ & $<5$ & $<60$ & 275 \\
\hline $2011 Z 374 A$ & 0.0885 & 0.782 & $<0.05$ & 99.7921 & $<4$ & $<2$ & $<5$ & $<60$ & 179 \\
\hline 2011Z376A & 0.0684 & 0.767 & $<0.05$ & 99.8584 & $<4$ & $<2$ & $<5$ & $<60$ & 166 \\
\hline 2011Z378A & 0.117 & 0.81 & $<0.05$ & 99.6995 & 6 & $<2$ & $<5$ & $<60$ & 200 \\
\hline $2011 Z 379 A$ & 0.0609 & 0.936 & $<0.05$ & 99.9218 & $<4$ & $<2$ & $<5$ & $<60$ & 482 \\
\hline $2011 Z 380 A$ & 0.0445 & 0.581 & $<0.05$ & 99.8313 & 35 & $<2$ & $<5$ & $<60$ & 307 \\
\hline $2011 Z 383 B$ & 0.203 & 0.98 & $<0.05$ & 99.8701 & 5 & $<2$ & $<5$ & $<60$ & 320 \\
\hline $2011 Z 385 A$ & $<0.03$ & $<0.02$ & $<0.05$ & 99.46306 & 13 & $<2$ & $<5$ & $<60$ & 63 \\
\hline $2011 Z 397 A$ & 0.16 & 0.957 & $<0.05$ & 99.701 & $<4$ & $<2$ & $<5$ & $<60$ & 73 \\
\hline 2011Z399A & 0.106 & 0.403 & $<0.05$ & 99.9336 & 108 & $<2$ & $<5$ & $<60$ & 178 \\
\hline $2011 Z 402 \mathrm{~A}$ & 0.308 & 0.0276 & $<0.05$ & 99.9351 & 12 & $<2$ & $<5$ & $<60$ & 100 \\
\hline $2011 Z 403 A$ & 0.0627 & 0.204 & $<0.05$ & 99.90576 & 8 & $<2$ & $<5$ & $<60$ & 229 \\
\hline $2011 Z 412 \mathrm{~A}$ & 0.0352 & 0.172 & $<0.05$ & 99.9039 & $<4$ & $<2$ & $<5$ & $<60$ & 424 \\
\hline $2011 Z 412 \mathrm{~A}$ & 0.0309 & 0.388 & $<0.05$ & 99.9176 & 77 & $<2$ & $<5$ & 286 & 134 \\
\hline
\end{tabular}


Table 8. (continued)

\begin{tabular}{|c|c|c|c|c|c|c|c|c|c|}
\hline Sample_ID & Co_ppm & Cr_ppm & Cu_ppm & F_ppm & La_ppm & Mo_ppm & Nb_ppm & Nb_ppm_2 & Ni_ppm \\
\hline $2011 Z 296 B$ & $2<10$ & 648 & 106 & $<110$ & $<50$ & $2<1$ & $2<1$ & 2 & 135 \\
\hline $2011 Z 311 \mathrm{~A}$ & $<10$ & 91 & 74 & $<110$ & $<50$ & $<1$ & $<1$ & 8 & 36 \\
\hline $2011 Z 320 A$ & 71 & 508 & $<7$ & $<110$ & $<50$ & $<1$ & $<1$ & 6 & 180 \\
\hline $2011 Z 326 \mathrm{~A}$ & 52 & 639 & 163 & $<110$ & $<50$ & $<1$ & $<1$ & 2 & 139 \\
\hline $2011 Z 326 B$ & $<10$ & $<30$ & 25 & $<110$ & $<50$ & $<1$ & $<1$ & 20 & 40 \\
\hline $2011 Z 327 A$ & 197 & 2370 & $<7$ & $<110$ & $<50$ & $<1$ & $<1$ & 2 & 1610 \\
\hline $2011 Z 328 \mathrm{~A}$ & 70 & 147 & 109 & $<110$ & $<50$ & $<1$ & $<1$ & 7 & 32 \\
\hline $2011 Z 330 A$ & 26.5 & 77.3 & 51.7 & $<110$ & $<50$ & $<1$ & $<1$ & 19.3 & 8 \\
\hline 2011Z331A & $<10$ & 108 & 60 & $<110$ & $<50$ & $<1$ & $<1$ & 8 & 68 \\
\hline $2011 Z 336 A$ & 184 & 1980 & 48 & $<110$ & $<50$ & $<1$ & $<1$ & 3 & 1120 \\
\hline 2011Z345B & $<10$ & 103 & 242 & $<110$ & $<50$ & 4 & $<1$ & 8 & 110 \\
\hline $2011 Z 349 A$ & 95 & 214 & 49 & $<110$ & $<50$ & $<1$ & $<1$ & 1 & 47 \\
\hline $2011 Z 350 A$ & 50 & 77 & 40 & $<110$ & $<50$ & $<1$ & $<1$ & 1 & 128 \\
\hline $2011 Z 355 A$ & 79 & 142 & 43 & $<110$ & $<50$ & $<1$ & $<1$ & 13 & 122 \\
\hline $2011 Z 368 B$ & 57 & 370 & 62 & $<110$ & $<50$ & $<1$ & $<1$ & 1 & 246 \\
\hline $2011 Z 371 \mathrm{~A}$ & 36 & 314 & 85 & $<110$ & $<50$ & $<1$ & $<1$ & 3 & 104 \\
\hline $2011 Z 371 \mathrm{~A}$ & $<10$ & 348 & 51 & $<110$ & $<50$ & $<1$ & $<1$ & 4 & 144 \\
\hline $2011 Z 372 A$ & 43 & 245 & 18 & $<110$ & $<50$ & $<1$ & $<1$ & 7 & 86 \\
\hline $2011 Z 373 A$ & $<10$ & 477 & 74 & $<110$ & $<50$ & $<1$ & $<1$ & 6 & 76 \\
\hline $2011 Z 374 A$ & $<10$ & 580 & 56 & $<110$ & $<50$ & $<1$ & $<1$ & 4 & 134 \\
\hline $2011 Z 376 A$ & $<10$ & 701 & 69 & $<110$ & $<50$ & $<1$ & $<1$ & 5 & 98 \\
\hline 2011Z378A & 63 & 1020 & 134 & $<110$ & $<50$ & $<1$ & $<1$ & 4 & 175 \\
\hline $2011 Z 379 A$ & 39 & 280 & 45 & $<110$ & $<50$ & $<1$ & $<1$ & 4 & 101 \\
\hline $2011 Z 380 A$ & $<10$ & $<30$ & 27 & $<110$ & $<50$ & $<1$ & $<1$ & 2 & $<7$ \\
\hline $2011 Z 383 B$ & 25 & 44 & 44 & $<110$ & $<50$ & $<1$ & $<1$ & 9 & 10 \\
\hline $2011 Z 385 A$ & 167 & 2400 & 7 & $<110$ & $<50$ & $<1$ & $<1$ & 1 & 3020 \\
\hline $2011 Z 397 A$ & 50 & 137 & 48 & 2280 & $<50$ & $<1$ & $<1$ & 17 & $<7$ \\
\hline 2011Z399A & $<10$ & 59 & 32 & $<110$ & $<50$ & $<1$ & $<1$ & 7 & 39 \\
\hline $2011 Z 402 \mathrm{~A}$ & $<10$ & 31 & 56 & $<110$ & $<50$ & $<1$ & $<1$ & 1 & 23 \\
\hline $2011 Z 403 A$ & $<10$ & $<30$ & 67 & $<110$ & $<50$ & $<1$ & $<1$ & 4 & 38 \\
\hline $2011 Z 412 \mathrm{~A}$ & $<10$ & 50 & $<7$ & 116 & $<50$ & $<1$ & $<1$ & 3 & 38 \\
\hline $2011 Z 412 \mathrm{~A}$ & 11 & 72 & 38 & $<110$ & $<50$ & $<1$ & $<1$ & 7 & 48 \\
\hline
\end{tabular}


Table 8. (continued)

\begin{tabular}{|c|c|c|c|c|c|c|c|c|c|}
\hline Sample_ID & Pb_ppm & Rb_ppm & Rb_ppm_2 & S_ppm & Sb_ppm & Sn_ppm & Sr_ppm & Sr_ppm_2 & Th_ppm \\
\hline $2011 Z 296 B$ & $2<4$ & $2<1$ & 2 & 134 & $<2$ & $<4$ & $2<1$ & 179 & $2<2$ \\
\hline $2011 Z 311 \mathrm{~A}$ & 20 & $<1$ & 73 & 61 & $<2$ & $<4$ & $<1$ & 6 & $<2$ \\
\hline $2011 Z 320 A$ & $<4$ & $<1$ & 20 & 135 & $<2$ & $<4$ & $<1$ & 45 & $<2$ \\
\hline $2011 Z 326 \mathrm{~A}$ & $<4$ & $<1$ & 1 & 125 & $<2$ & $<4$ & $<1$ & 174 & $<2$ \\
\hline $2011 Z 326 B$ & $<4$ & $<1$ & $<1$ & 85 & $<2$ & $<4$ & $<1$ & 285 & $<2$ \\
\hline $2011 Z 327 A$ & $<4$ & $<1$ & 2 & 356 & $<2$ & $<4$ & $<1$ & 72 & $<2$ \\
\hline $2011 Z 328 \mathrm{~A}$ & $<4$ & $<1$ & 39 & 79 & $<2$ & $<4$ & $<1$ & 100 & $<2$ \\
\hline $2011 Z 330 A$ & $<4$ & $<1$ & 35.3 & 229 & $<2$ & $<4$ & $<1$ & 619.9 & $<2$ \\
\hline 2011Z331A & 32 & $<1$ & 40 & 102 & $<2$ & $<4$ & $<1$ & 777 & $<2$ \\
\hline $2011 Z 336 A$ & $<4$ & $<1$ & 4 & 332 & $<2$ & $<4$ & $<1$ & 76 & $<2$ \\
\hline 2011Z345B & 45 & $<1$ & 76 & 651 & 3 & $<4$ & $<1$ & 36 & $<2$ \\
\hline $2011 Z 349 A$ & 32 & $<1$ & 2 & 184 & $<2$ & $<4$ & $<1$ & 134 & $<2$ \\
\hline $2011 Z 350 A$ & $<4$ & $<1$ & 1 & 58 & $<2$ & $<4$ & $<1$ & 110 & $<2$ \\
\hline $2011 Z 355 A$ & 51 & $<1$ & 80 & 105 & $<2$ & $<4$ & $<1$ & 117 & $<2$ \\
\hline $2011 Z 368 B$ & $<4$ & $<1$ & 32 & 100 & $<2$ & $<4$ & $<1$ & 203 & $<2$ \\
\hline $2011 Z 371 \mathrm{~A}$ & 24 & $<1$ & 3 & 43 & $<2$ & $<4$ & $<1$ & 306 & $<2$ \\
\hline $2011 Z 371 \mathrm{~A}$ & 25 & $<1$ & 4 & 144 & $<2$ & $<4$ & $<1$ & 303 & $<2$ \\
\hline $2011 Z 372 A$ & 21 & $<1$ & 31 & 50 & $<2$ & $<4$ & $<1$ & 254 & $<2$ \\
\hline $2011 Z 373 A$ & $<4$ & $<1$ & 10 & 58 & $<2$ & $<4$ & $<1$ & 367 & $<2$ \\
\hline $2011 Z 374 A$ & $<4$ & $<1$ & 5 & 58 & $<2$ & $<4$ & $<1$ & 304 & $<2$ \\
\hline $2011 Z 376 A$ & $<4$ & $<1$ & 8 & 70 & $<2$ & $<4$ & $<1$ & 308 & $<2$ \\
\hline 2011Z378A & $<4$ & $<1$ & 10 & 54 & $<2$ & $<4$ & $<1$ & 284 & $<2$ \\
\hline $2011 Z 379 A$ & $<4$ & $<1$ & 5 & 107 & $<2$ & $<4$ & $<1$ & 285 & $<2$ \\
\hline $2011 Z 380 A$ & 30 & $<1$ & 31 & 142 & $<2$ & $<4$ & $<1$ & 66 & $<2$ \\
\hline $2011 Z 383 B$ & $<4$ & $<1$ & 25 & 134 & $<2$ & $<4$ & $<1$ & 116 & $<2$ \\
\hline $2011 Z 385 A$ & $<4$ & $<1$ & 1 & 41 & $<2$ & $<4$ & $<1$ & 2 & $<2$ \\
\hline $2011 Z 397 A$ & $<4$ & $<1$ & 196 & 40 & $<2$ & $<4$ & $<1$ & 43 & $<2$ \\
\hline 2011Z399A & 33 & $<1$ & 46 & 162 & $<2$ & $<4$ & $<1$ & 19 & $<2$ \\
\hline $2011 Z 402 \mathrm{~A}$ & 32 & $<1$ & 6 & $<30$ & $<2$ & $<4$ & $<1$ & 34 & $<2$ \\
\hline $2011 Z 403 A$ & 20 & $<1$ & 16 & 129 & $<2$ & $<4$ & $<1$ & 24 & $<2$ \\
\hline $2011 Z 412 \mathrm{~A}$ & 18 & $<1$ & 19 & 414 & $<2$ & $<4$ & $<1$ & 17 & $<2$ \\
\hline $2011 Z 412 \mathrm{~A}$ & 37 & $<1$ & 51 & 81 & $<2$ & $<4$ & $<1$ & 57 & $<2$ \\
\hline
\end{tabular}




\begin{tabular}{|c|c|c|c|c|c|c|c|c|}
\hline Sample_ID & U_ppm & V_ppm & W_ppm & Y_ppm & Y_ppm_2 & Zn_ppm & Zr_ppm & Zr_ppm_2 \\
\hline $2011 Z 296 B$ & $<1$ & 459 & $<8$ & $<1$ & 45 & 313 & $<9$ & 94 \\
\hline $2011 Z 311 \mathrm{~A}$ & $<1$ & 117 & $<8$ & $<1$ & 6 & 76 & $<9$ & 80 \\
\hline 2011Z320A & $<1$ & 438 & $<8$ & $<1$ & 26 & 97 & $<9$ & 117 \\
\hline $2011 Z 326 A$ & $<1$ & 227 & $<8$ & $<1$ & 8 & 34 & $<9$ & 26 \\
\hline 2011Z326B & $<1$ & 295 & $<8$ & $<1$ & 50 & 30 & $<9$ & 319 \\
\hline $2011 Z 327 A$ & $<1$ & 99 & $<8$ & $<1$ & 3 & 109 & $<9$ & 19 \\
\hline $2011 Z 328 \mathrm{~A}$ & $<1$ & 397 & $<8$ & $<1$ & 24 & 86 & $<9$ & 119 \\
\hline $2011 Z 330 A$ & $<1$ & 214 & $<8$ & $<1$ & 21.7 & 86.6 & $<9$ & 131.3 \\
\hline 2011Z331A & $<1$ & 375 & $<8$ & $<1$ & 20 & 100 & $<9$ & 61 \\
\hline $2011 Z 336 \mathrm{~A}$ & $<1$ & 145 & $<8$ & $<1$ & 5 & 102 & $<9$ & 26 \\
\hline 2011Z345B & $<1$ & 223 & 15.6 & $<1$ & 19 & 488 & $<9$ & 109 \\
\hline 2011Z349A & $<1$ & 289 & $<8$ & $<1$ & 27 & 82 & $<9$ & 51 \\
\hline $2011 Z 350 A$ & $<1$ & 257 & $<8$ & $<1$ & 24 & 74 & $<9$ & 68 \\
\hline $2011 Z 355 A$ & $<1$ & 249 & $<8$ & $<1$ & 35 & 249 & $<9$ & 190 \\
\hline 2011Z368B & $<1$ & 385 & $<8$ & $<1$ & 25 & 88 & $<9$ & 44 \\
\hline $2011 Z 371 \mathrm{~A}$ & $<1$ & 204 & $<8$ & $<1$ & 12 & 81 & $<9$ & 37 \\
\hline $2011 Z 371 A$ & $<1$ & 200 & $<8$ & $<1$ & 12 & 59 & $<9$ & 38 \\
\hline $2011 Z 372 \mathrm{~A}$ & $<1$ & 380 & $<8$ & $<1$ & 21 & 92 & $<9$ & 76 \\
\hline $2011 Z 373 A$ & $<1$ & 313 & $<8$ & $<1$ & 18 & 77 & $<9$ & 60 \\
\hline $2011 Z 374 A$ & $<1$ & 242 & $<8$ & $<1$ & 12 & 63 & $<9$ & 44 \\
\hline $2011 Z 376 \mathrm{~A}$ & $<1$ & 242 & $<8$ & $<1$ & 11 & 53 & $<9$ & 43 \\
\hline 2011Z378A & $<1$ & 250 & $<8$ & $<1$ & 12 & 46 & $<9$ & 49 \\
\hline $2011 Z 379 A$ & $<1$ & 256 & $<8$ & $<1$ & 14 & 82 & $<9$ & 41 \\
\hline 2011Z380A & $<1$ & 102 & $<8$ & $<1$ & 9 & 199 & $<9$ & 103 \\
\hline 2011Z383B & $<1$ & 200 & $<8$ & $<1$ & 11 & 91 & $<9$ & 108 \\
\hline $2011 Z 385 A$ & $<1$ & 72 & $<8$ & $<1$ & $<8$ & 47 & $<9$ & $<9$ \\
\hline $2011 Z 397 A$ & $<1$ & 215 & $<8$ & $<1$ & 41 & 116 & $<9$ & 153 \\
\hline 2011Z399A & $<1$ & 68 & $<8$ & $<1$ & 12 & 47 & $<9$ & 162 \\
\hline $2011 Z 402 \mathrm{~A}$ & $<1$ & 147 & $<8$ & $<1$ & 8 & 19 & $<9$ & 13 \\
\hline $2011 Z 403 \mathrm{~A}$ & $<1$ & $<25$ & $<8$ & $<1$ & 5 & 57 & $<9$ & 184 \\
\hline $2011 Z 412 A$ & $<1$ & $<25$ & $<8$ & $<1$ & 5 & 35 & $<9$ & 124 \\
\hline 2011Z412A & $<1$ & 69 & $<8$ & $<1$ & 9 & 44 & $<9$ & 168 \\
\hline
\end{tabular}


Table 8. (continued)

\begin{tabular}{|c|c|c|c|c|c|c|c|}
\hline Sample_ID & Latitude & Longitude & Easting & Northing & $\begin{array}{l}\text { UTM_ } \\
\text { Zone }\end{array}$ & Root_name & Comments \\
\hline 2011Z417B & 65.462086 & -152.610657 & 518043 & 7259819 & $5 \mathrm{~N}$ & Quartzite & --- \\
\hline 2011Z418A & 65.462414 & -152.612249 & 517969 & 7259855 & $5 \mathrm{~N}$ & Metamafic & --- \\
\hline $2011 Z 420 \mathrm{~A}$ & 65.462153 & -152.619201 & 517647 & 7259824 & $5 \mathrm{~N}$ & Metagabbro & --- \\
\hline 2011Z421A & 65.461099 & -152.621050 & 517562 & 7259706 & $5 \mathrm{~N}$ & Metamafic & --- \\
\hline $2011 Z 423 A$ & 65.460564 & -152.629819 & 517156 & 7259644 & $5 \mathrm{~N}$ & Metamafic & --- \\
\hline $2011 Z 426 B$ & 65.456474 & -152.637535 & 516801 & 7259186 & $5 \mathrm{~N}$ & Quartzite & Inhomogenous \\
\hline $2011 Z 433 A$ & 65.200643 & -152.784358 & 510093 & 7230641 & $5 \mathrm{~N}$ & Chloritoid(?)-mica schist & Inhomogenous \\
\hline $2011 Z 433 C$ & 65.200643 & -152.784358 & 510093 & 7230641 & $5 \mathrm{~N}$ & Chloritoid(?)-mica schist & Inhomogenous \\
\hline 2011Z435A & 65.201826 & -152.765225 & 510988 & 7230776 & $5 \mathrm{~N}$ & Muscovite quartzite & Porous \\
\hline 2011Z437A & 65.376905 & -152.928828 & 503309 & 7250271 & $5 \mathrm{~N}$ & Metagabbro & --- \\
\hline 2011Z438A & 65.376072 & -152.931949 & 503164 & 7250178 & $5 \mathrm{~N}$ & Mica quartz schist & Porous \\
\hline 2011Z440A & 65.374496 & -152.938039 & 502881 & 7250002 & $5 \mathrm{~N}$ & Metagabbro & Inhomogenous, porous \\
\hline $2011 Z 443 A$ & 65.370425 & -152.945510 & 502534 & 7249548 & $5 \mathrm{~N}$ & Metagabbro & --- \\
\hline 2011Z444B & 65.369510 & -152.946286 & 502498 & 7249446 & $5 \mathrm{~N}$ & Metagabbro & Inhomogenous \\
\hline $2011 Z 445 A$ & 65.368372 & -152.949750 & 502337 & 7249319 & $5 \mathrm{~N}$ & Garnet(?) Metagabbro(?) & --- \\
\hline $2011 Z 454 A$ & 65.342515 & -152.655280 & 516048 & 7246480 & $5 \mathrm{~N}$ & Fine-grained gabbro & Porous \\
\hline 2011Z469A & 65.483993 & -152.582471 & 519333 & 7262269 & $5 \mathrm{~N}$ & Metagabbro & --- \\
\hline 2011Z469A & 65.483993 & -152.582471 & 519333 & 7262269 & $5 \mathrm{~N}$ & Metagabbro & --- \\
\hline 2011Z471A & 65.486810 & -152.575903 & 519635 & 7262585 & $5 \mathrm{~N}$ & Fine-grained Metagabbro & --- \\
\hline $2011 Z 472 A$ & 65.488246 & -152.572834 & 519776 & 7262746 & $5 \mathrm{~N}$ & Greenstone & --- \\
\hline $2011 Z 472 A$ & 65.488246 & -152.572834 & 519776 & 7262746 & $5 \mathrm{~N}$ & Greenstone & |-- \\
\hline $2011 Z 483 A$ & 65.355460 & -152.988910 & 500516 & 7247879 & $5 \mathrm{~N}$ & Quartz muscovite schist & Inhomogenous, porous \\
\hline 2011Z489A & 65.360177 & -152.969240 & 501431 & 7248405 & $5 \mathrm{~N}$ & Garnet-amphibole schist(?) & Inhomogenous \\
\hline 2011Z491A & 65.360921 & -152.965241 & 501617 & 7248488 & $5 \mathrm{~N}$ & Garnet amphibolite(?) & Porous \\
\hline 2011Z492B & 65.360866 & -152.960899 & 501819 & 7248482 & $5 \mathrm{~N}$ & Garnet amphibolite(?) & --- \\
\hline $2011 Z 500 \mathrm{~A}$ & 65.296247 & -152.800646 & 509297 & 7241294 & $5 \mathrm{~N}$ & Metamafic & Porous \\
\hline $2011 Z 510 A$ & 65.283250 & -152.771703 & 510652 & 7239850 & $5 \mathrm{~N}$ & Garnet amphibolite(?) & Porous \\
\hline $2011 Z 511 \mathrm{~A}$ & 65.281883 & -152.769143 & 510772 & 7239698 & $5 \mathrm{~N}$ & Mica quartz schist & --- \\
\hline 2011Z511B & 65.281883 & -152.769143 & 510772 & 7239698 & $5 \mathrm{~N}$ & Metagabbro(?) & Porous \\
\hline 2011Z519E & 65.218391 & -154.574853 & 426345 & 7233521 & $5 \mathrm{~N}$ & Garnet orthogneiss(?) & Inhomogenous \\
\hline
\end{tabular}


Table 8. (continued)

\begin{tabular}{|c|c|c|c|c|c|c|c|c|c|}
\hline Sample_ID & $\mathrm{SiO}_{2} \_\mathrm{pct}$ & Al203_pct & BaO_pct & CaO_pct & FeO_pct & K2O_pct & MgO_pct & MnO_pct & Na2O_pct \\
\hline $2011 Z 417 B$ & 96 & 0.9 & $<<0.03$ & 0.436 & 1.45 & 0.0606 & 0.633 & $<0.03$ & 0.36 \\
\hline 2011Z418A & 48.4 & 15.4 & 0.0882 & 9.49 & 13.7 & 0.0914 & 6.44 & 0.487 & 4.36 \\
\hline $2011 Z 420 A$ & 49.6 & 7.2 & $<0.03$ & 12.6 & 11.9 & 0.199 & 16.1 & 0.206 & 0.584 \\
\hline $2011 Z 421 \mathrm{~A}$ & 48.6 & 15.2 & 0.034 & 12.3 & 12.1 & 0.0799 & 5.81 & 0.285 & 3.46 \\
\hline $2011 Z 423 A$ & 49.1 & 15 & 0.0685 & 12.1 & 12.2 & 0.096 & 5.83 & 0.243 & 3.3 \\
\hline $2011 Z 426 \mathrm{~B}$ & 96.1 & 1.4 & 0.599 & 0.0847 & 0.929 & 0.398 & 0.228 & $<0.03$ & 0.0725 \\
\hline $2011 Z 433 A$ & 65.3 & 17 & 0.13 & 0.676 & 9.02 & 4.43 & 1.48 & 0.09 & 0.241 \\
\hline $2011 Z 433 C$ & 62.5 & 21 & 0.165 & 0.208 & 6.48 & 5.62 & 1.81 & 0.0675 & 0.738 \\
\hline $2011 Z 435 A$ & 91.9 & 4 & 0.0395 & 0.136 & 2.18 & 0.734 & 0.377 & 0.0698 & 0.283 \\
\hline $2011 Z 437 A$ & 54.9 & 13.9 & $<0.03$ & 8.33 & 11.6 & 0.226 & 5.08 & 0.164 & 3.94 \\
\hline $2011 Z 438 \mathrm{~A}$ & 87 & 6.5 & 0.046 & 0.184 & 3.17 & 0.601 & 0.82 & $<0.03$ & 0.824 \\
\hline $2011 Z 440 A$ & 53.6 & 13.5 & 0.0813 & 4.89 & 16.6 & 0.0584 & 4.39 & 0.335 & 4.55 \\
\hline 2011Z443A & 51.9 & 15.1 & $<0.03$ & 9.22 & 10.7 & 0.0245 & 6.04 & 0.176 & 5.63 \\
\hline 2011Z444B & 54 & 13 & $<0.03$ & 10.3 & 11.7 & 0.0555 & 5.64 & 0.259 & 3.25 \\
\hline $2011 Z 445 A$ & 58.1 & 16 & $<0.03$ & 4.61 & 7.67 & 0.0402 & 7.31 & 0.117 & 5.51 \\
\hline $2011 Z 454 A$ & 51.3 & 17.2 & 0.343 & 7.14 & 10.2 & 1.86 & 4.64 & 0.154 & 4.05 \\
\hline $2011 Z 469 A$ & 51.5 & 14.3 & 0.0485 & 10.7 & 11.5 & 0.23 & 7.09 & 0.195 & 2.93 \\
\hline $2011 Z 469 A$ & 50.8 & 14.4 & 0.0473 & 10.9 & 11.6 & 0.242 & 7.26 & 0.194 & 2.89 \\
\hline $2011 Z 471 \mathrm{~A}$ & 49.6 & 14.9 & 0.0377 & 11.3 & 11.2 & 0.131 & 7.78 & 0.212 & 3.12 \\
\hline $2011 Z 472 A$ & 50 & 14.6 & $<0.03$ & 11.6 & 12.9 & 0.0352 & 6.08 & 0.23 & 2.44 \\
\hline $2011 Z 472 \mathrm{~A}$ & 50 & 14.6 & $<0.03$ & 11.6 & 12.9 & 0.0352 & 6.08 & 0.23 & 2.44 \\
\hline $2011 Z 483 A$ & 65.9 & 17.5 & 0.09 & 2.36 & 6.67 & 3.1 & 1.78 & 0.099 & 1.28 \\
\hline 2011Z489A & 40.3 & 13.6 & $<0.03$ & 14.1 & 18 & 0.0834 & 9.47 & 0.322 & 1.32 \\
\hline 2011Z491A & 58.4 & 13.4 & $<0.03$ & 3.99 & 14.5 & 0.0263 & 5.62 & 0.181 & 2.26 \\
\hline 2011Z492B & 52 & 14.7 & 0.0315 & 8.22 & 12.9 & 0.223 & 5.69 & 0.213 & 4.48 \\
\hline $2011 Z 500 A$ & 58.2 & 14.5 & 0.0467 & 0.385 & 11.6 & 0.27 & 3.58 & 0.064 & 5.78 \\
\hline $2011 Z 510 A$ & 49.5 & 15.6 & 0.0738 & 0.744 & 17 & 0.291 & 7.27 & 0.172 & 3.33 \\
\hline $2011 Z 511 \mathrm{~A}$ & 73.7 & 7.9 & 0.149 & 8.12 & 3.91 & 1.67 & 2.74 & 0.106 & 0.712 \\
\hline 2011Z511B & 54.9 & 11.9 & $<0.03$ & 3.95 & 13.4 & 0.051 & 9.54 & 0.195 & 3.43 \\
\hline 2011Z519E & 66.7 & 17.2 & 0.132 & 1.05 & 4.98 & 4.9 & 1.37 & 0.0803 & 2.21 \\
\hline
\end{tabular}


Table 8. (continued)

\begin{tabular}{|c|c|c|c|c|c|c|c|c|c|}
\hline Sample_ID & P2O5_pct & TiO2_pct & CO2_pct & Total_pct & As_ppm & Bi_ppm & Br_ppm & Ce_ppm & Cl_ppm \\
\hline 2011Z417B & "<0.03 & 0.0495 & $<<0.05$ & 999.9617 & $2<4$ & $<2$ & $2<5$ & $<<60$ & $<<50$ \\
\hline $2011 Z 418 A$ & 0.118 & 1.36 & $<0.05$ & 99.9346 & $<4$ & $<2$ & $<5$ & $<60$ & 88 \\
\hline $2011 Z 420 A$ & 0.13 & 1.05 & $<0.05$ & 99.5879 & $<4$ & $<2$ & $<5$ & $<60$ & 200 \\
\hline $2011 Z 421 \mathrm{~A}$ & 0.175 & 1.89 & $<0.05$ & 99.9339 & 4 & $<2$ & $<5$ & $<60$ & 87 \\
\hline $2011 Z 423 A$ & 0.216 & 1.71 & $<0.05$ & 99.8635 & $<4$ & $<2$ & $<5$ & $<60$ & 86 \\
\hline $2011 Z 426 B$ & $<0.03$ & 0.0702 & $<0.05$ & 99.9075 & $<4$ & $<2$ & $<5$ & $<60$ & 182 \\
\hline $2011 Z 433 A$ & 0.711 & 0.698 & $<0.05$ & 99.776 & $<4$ & $<2$ & $<5$ & $<60$ & 673 \\
\hline $2011 Z 433 C$ & 0.158 & 1.08 & $<0.05$ & 99.8265 & $<4$ & $<2$ & $<5$ & $<60$ & 155 \\
\hline $2011 Z 435 A$ & 0.0403 & 0.169 & $<0.05$ & 99.9086 & $<4$ & $<2$ & $<5$ & $<60$ & 445 \\
\hline $2011 Z 437 A$ & 0.265 & 1.34 & $<0.05$ & 99.7647 & 13 & $<2$ & $<5$ & $<60$ & 191 \\
\hline $2011 Z 438 A$ & 0.155 & 0.555 & $<0.05$ & 99.8609 & $<4$ & $<2$ & $<5$ & $<60$ & 288 \\
\hline $2011 Z 440 A$ & 0.348 & 1.48 & $<0.05$ & 99.8327 & $<4$ & $<2$ & $<5$ & $<60$ & 161 \\
\hline $2011 Z 443 A$ & 0.119 & 0.88 & $<0.05$ & 99.8 & 14 & $<2$ & $<5$ & $<60$ & 100 \\
\hline 2011Z444B & 0.307 & 1.2 & $<0.05$ & 99.7274 & 71 & $<2$ & $<5$ & $<60$ & 543 \\
\hline $2011 Z 445 A$ & 0.0374 & 0.523 & $<0.05$ & 99.9352 & $<4$ & $<2$ & $<5$ & $<60$ & 282 \\
\hline 2011Z454A & 0.372 & 2.58 & $<0.05$ & 99.839 & 7 & $<2$ & $<5$ & $<60$ & 117 \\
\hline $2011 Z 469 A$ & 0.214 & 1.2 & $<0.05$ & 99.9075 & $<4$ & $<2$ & $<5$ & $<60$ & 117 \\
\hline $2011 Z 469 A$ & 0.204 & 1.24 & $<0.05$ & 99.7773 & $<4$ & $<2$ & $<5$ & $<60$ & 187 \\
\hline $2011 Z 471 A$ & 0.173 & 1.37 & $<0.05$ & 99.8237 & $<4$ & $<2$ & $<5$ & $<60$ & 140 \\
\hline $2011 Z 472 A$ & 0.198 & 1.74 & $<0.05$ & 99.8423 & $<4$ & $<2$ & $<5$ & $<60$ & 112 \\
\hline $2011 Z 472 A$ & 0.198 & 1.74 & $<0.05$ & 99.8423 & $<4$ & $<2$ & $<5$ & $<60$ & 112 \\
\hline $2011 Z 483 A$ & 0.175 & 0.786 & $<0.05$ & 99.74 & 11 & $<2$ & $<5$ & $<60$ & 485 \\
\hline $2011 Z 489 A$ & 1.9 & 0.596 & $<0.05$ & 99.7454 & $<4$ & $<2$ & $<5$ & $<60$ & 240 \\
\hline $2011 Z 491 \mathrm{~A}$ & 0.261 & 1.17 & $<0.05$ & 99.8249 & $<4$ & $<2$ & $<5$ & $<60$ & 191 \\
\hline 2011Z492B & 0.231 & 1.21 & $<0.05$ & 99.8985 & 4 & $<2$ & $<5$ & $<60$ & 146 \\
\hline $2011 Z 500 A$ & 0.491 & 4.81 & $<0.05$ & 99.7267 & 11 & $<2$ & $<5$ & $<60$ & 321 \\
\hline $2011 Z 510 A$ & 0.857 & 4.76 & $<0.05$ & 99.5978 & 235 & $<2$ & $<5$ & $<60$ & 467 \\
\hline $2011 Z 511 \mathrm{~A}$ & 0.329 & 0.438 & $<0.05$ & 99.784 & 59 & $<2$ & $<5$ & $<60$ & 716 \\
\hline 2011Z511B & 0.444 & 1.82 & $<0.05$ & 99.6491 & $<4$ & $<2$ & $<5$ & $<60$ & 230 \\
\hline $2011 Z 519 E$ & 0.148 & 0.649 & $<0.05$ & 99.4193 & $<4$ & $<2$ & $<5$ & $<60$ & 559 \\
\hline
\end{tabular}


Table 8. (continued)

\begin{tabular}{|c|c|c|c|c|c|c|c|c|c|}
\hline Sample_ID & Co_ppm & Cr_ppm & Cu_ppm & F_ppm & La_ppm & Mo_ppm & Nb_ppm & Nb_ppm_2 & Ni_ppm \\
\hline 2011Z417B & $2<10$ & $<30$ & 24 & $<110$ & $<50$ & $<<1$ & $<<1$ & 2 & 27 \\
\hline $2011 Z 418 \mathrm{~A}$ & 120 & 142 & 21 & $<110$ & $<50$ & $<1$ & $<1$ & 5 & 60 \\
\hline $2011 Z 420 A$ & 116 & 2100 & 301 & $<110$ & $<50$ & $<1$ & $<1$ & 5 & 586 \\
\hline $2011 Z 421 \mathrm{~A}$ & 102 & 176 & 214 & $<110$ & $<50$ & $<1$ & $<1$ & 7 & 33 \\
\hline $2011 Z 423 A$ & 79 & 136 & 255 & $<110$ & $<50$ & $<1$ & $<1$ & 8 & 94 \\
\hline $2011 Z 426 B$ & $<10$ & $<30$ & 30 & $<110$ & $<50$ & $<1$ & $<1$ & 2 & 21 \\
\hline 2011Z433A & $<10$ & 83 & 114 & $<110$ & $<50$ & $<1$ & $<1$ & 13 & $<7$ \\
\hline $2011 Z 433 C$ & 13 & 98 & 24 & $<110$ & $<50$ & $<1$ & $<1$ & 17 & 54 \\
\hline $2011 Z 435 A$ & $<10$ & 41 & 16 & $<110$ & $<50$ & $<1$ & $<1$ & 4 & 27 \\
\hline $2011 Z 437 A$ & 67 & 156 & 98 & $<110$ & $<50$ & $<1$ & $<1$ & 10 & 42 \\
\hline $2011 Z 438 \mathrm{~A}$ & $<10$ & 118 & $<7$ & $<110$ & $<50$ & $<1$ & $<1$ & 8 & 32 \\
\hline $2011 Z 440 A$ & 91 & 89 & 141 & $<110$ & $<50$ & $<1$ & $<1$ & 11 & 24 \\
\hline $2011 Z 443 A$ & 47 & 540 & 123 & $<110$ & $<50$ & $<1$ & $<1$ & 1 & 40 \\
\hline 2011Z444B & 33 & 365 & 208 & $<110$ & $<50$ & $<1$ & $<1$ & 1 & 109 \\
\hline $2011 Z 445 A$ & 66 & 152 & 32 & $<110$ & $<50$ & $<1$ & $<1$ & 5 & 61 \\
\hline $2011 Z 454 \mathrm{~A}$ & $<10$ & 254 & 65 & $<110$ & $<50$ & $<1$ & $<1$ & 12 & 63 \\
\hline $2011 Z 469 A$ & 118 & 371 & 120 & $<110$ & $<50$ & $<1$ & $<1$ & 7 & 181 \\
\hline $2011 Z 469 A$ & 45 & 414 & 113 & $<110$ & $<50$ & $<1$ & $<1$ & 7 & 188 \\
\hline $2011 Z 471 \mathrm{~A}$ & 60 & 458 & 260 & $<110$ & $<50$ & $<1$ & $<1$ & 6 & 151 \\
\hline $2011 Z 472 A$ & 91 & 202 & 127 & $<110$ & $<50$ & $<1$ & $<1$ & 7 & 70 \\
\hline $2011 Z 472 A$ & 91 & 202 & 127 & $<110$ & $<50$ & $<1$ & $<1$ & 6 & 70 \\
\hline $2011 Z 483 A$ & $<10$ & 102 & 43 & $<110$ & $<50$ & $<1$ & $<1$ & 12 & 51 \\
\hline $2011 Z 489 A$ & 49 & 312 & 69 & $<110$ & $<50$ & $<1$ & $<1$ & 2 & 161 \\
\hline $2011 Z 491 \mathrm{~A}$ & 16 & 153 & 53 & $<110$ & $<50$ & $<1$ & $<1$ & 9 & 36 \\
\hline 2011Z492B & $<10$ & 97 & 33 & $<110$ & $<50$ & $<1$ & $<1$ & 8 & 47 \\
\hline $2011 Z 500 A$ & $<10$ & 200 & 65 & $<110$ & $<50$ & $<1$ & $<1$ & 38 & 46 \\
\hline $2011 Z 510 A$ & 146 & 1390 & 120 & $<110$ & $<50$ & $<1$ & $<1$ & 69 & 618 \\
\hline $2011 Z 511 \mathrm{~A}$ & 18 & 83 & 106 & $<110$ & $<50$ & $<1$ & $<1$ & 8 & 35 \\
\hline $2011 Z 511 B$ & 64 & 1020 & $<7$ & $<110$ & $<50$ & $<1$ & $<1$ & 19 & 429 \\
\hline 2011Z519E & $<10$ & 105 & 43 & $<110$ & $<50$ & $<1$ & $<1$ & 12 & 22 \\
\hline
\end{tabular}


Table 8. (continued)

\begin{tabular}{|c|c|c|c|c|c|c|c|c|c|}
\hline Sample_ID & Pb_ppm & Rb_ppm & Rb_ppm_2 & S_ppm & Sb_ppm & Sn_ppm & Sr_ppm & Sr_ppm_2 & Th_ppm \\
\hline 2011Z417B & $2<4$ & $2<1$ & 3 & $<30$ & $<2$ & $<4$ & $<<1$ & 23 & $<2$ \\
\hline 2011Z418A & $<4$ & $<1$ & 1 & 60 & $<2$ & $<4$ & $<1$ & 194 & $<2$ \\
\hline $2011 Z 420 A$ & $<4$ & $<1$ & 5 & 54 & $<2$ & $<4$ & $<1$ & 39 & $<2$ \\
\hline $2011 Z 421 \mathrm{~A}$ & $<4$ & $<1$ & 2 & 53 & $<2$ & $<4$ & $<1$ & 108 & $<2$ \\
\hline $2011 Z 423 \mathrm{~A}$ & $<4$ & $<1$ & 2 & 57 & $<2$ & $<4$ & $<1$ & 213 & $<2$ \\
\hline $2011 Z 426 B$ & 30 & $<1$ & 21 & 558 & $<2$ & $<4$ & $<1$ & 7 & $<2$ \\
\hline $2011 Z 433 A$ & $<4$ & $<1$ & 163 & 545 & $<2$ & $<4$ & $<1$ & 73 & $<2$ \\
\hline $2011 Z 433 C$ & 28 & $<1$ & 187 & 168 & $<2$ & $<4$ & $<1$ & 56 & $<2$ \\
\hline $2011 Z 435 A$ & 35 & $<1$ & 26 & 219 & $<2$ & $<4$ & $<1$ & 33 & $<2$ \\
\hline $2011 Z 437 A$ & $<4$ & $<1$ & 11 & 109 & $<2$ & $<4$ & $<1$ & 218 & $<2$ \\
\hline 2011Z438A & 17 & $<1$ & 26 & 120 & $<2$ & $<4$ & $<1$ & 65 & $<2$ \\
\hline $2011 Z 440 A$ & $<4$ & $<1$ & 3 & 377 & $<2$ & $<4$ & $<1$ & 137 & $<2$ \\
\hline $2011 Z 443 A$ & $<4$ & $<1$ & $<1$ & 161 & $<2$ & $<4$ & $<1$ & 307 & $<2$ \\
\hline 2011Z444B & $<4$ & $<1$ & 2 & 417 & $<2$ & $<4$ & $<1$ & 86 & $<2$ \\
\hline $2011 Z 445 A$ & $<4$ & $<1$ & 1 & 132 & $<2$ & $<4$ & $<1$ & 188 & $<2$ \\
\hline $2011 Z 454 A$ & $<4$ & $<1$ & 21 & 169 & $<2$ & $<4$ & $<1$ & 257 & $<2$ \\
\hline 2011Z469A & $<4$ & $<1$ & 5 & 68 & $<2$ & $<4$ & $<1$ & 39 & $<2$ \\
\hline $2011 Z 469 A$ & 25 & $<1$ & 6 & 160 & $<2$ & $<4$ & $<1$ & 39 & $<2$ \\
\hline $2011 Z 471 \mathrm{~A}$ & $<4$ & $<1$ & 2 & 114 & $<2$ & $<4$ & $<1$ & 62 & $<2$ \\
\hline $2011 Z 472 A$ & $<4$ & $<1$ & 1 & 110 & $<2$ & $<4$ & $<1$ & 81 & $<2$ \\
\hline $2011 Z 472 \mathrm{~A}$ & $<4$ & $<1$ & 1 & 110 & $<2$ & $<4$ & $<1$ & 82 & $<2$ \\
\hline $2011 Z 483 \mathrm{~A}$ & $<4$ & $<1$ & 154 & 334 & $<2$ & $<4$ & $<1$ & 52 & $<2$ \\
\hline 2011Z489A & $<4$ & $<1$ & 5 & 227 & $<2$ & 26 & $<1$ & 221 & $<2$ \\
\hline 2011Z491A & 46 & $<1$ & 2 & 554 & $<2$ & 41 & $<1$ & 164 & $<2$ \\
\hline 2011Z492B & $<4$ & $<1$ & 10 & 103 & $<2$ & $<4$ & $<1$ & 167 & $<2$ \\
\hline $2011 Z 500 A$ & $<4$ & $<1$ & 6 & 393 & $<2$ & $<4$ & $<1$ & 25 & $<2$ \\
\hline $2011 Z 510 \mathrm{~A}$ & $<4$ & $<1$ & 13 & 263 & $<2$ & 26 & $<1$ & 30 & $<2$ \\
\hline $2011 Z 511 \mathrm{~A}$ & 31 & $<1$ & 59 & 291 & $<2$ & $<4$ & $<1$ & 240 & $<2$ \\
\hline $2011 Z 511 B$ & $<4$ & $<1$ & 2 & 381 & $<2$ & $<4$ & $<1$ & 34 & $<2$ \\
\hline $2011 Z 519 E$ & 39 & $<1$ & 190 & 4145 & $<2$ & $<4$ & $<1$ & 196 & $<2$ \\
\hline
\end{tabular}




\begin{tabular}{|c|c|c|c|c|c|c|c|c|}
\hline Sample_ID & U_ppm & V_ppm & W_ppm & Y_ppm & Y_ppm_2 & Zn_ppm & Zr_ppm & Zr_ppm_2 \\
\hline 2011Z417B & $<<1$ & $<25$ & $<8$ & $<<1$ & 5 & 12 & $<9$ & 17 \\
\hline 2011Z418A & $<1$ & 428 & $<8$ & $<1$ & 28 & 94 & $<9$ & 83 \\
\hline $2011 Z 420 \mathrm{~A}$ & $<1$ & 297 & $<8$ & $<1$ & 15 & 100 & $<9$ & 59 \\
\hline $2011 Z 421 \mathrm{~A}$ & $<1$ & 479 & $<8$ & $<1$ & 31 & 91 & $<9$ & 117 \\
\hline $2011 Z 423 \mathrm{~A}$ & $<1$ & 498 & $<8$ & $<1$ & 33 & 108 & $<9$ & 124 \\
\hline 2011Z426B & $<1$ & 68 & $<8$ & $<1$ & 2 & $<8$ & $<9$ & 29 \\
\hline $2011 Z 433 \mathrm{~A}$ & $<1$ & 121 & $<8$ & $<1$ & 36 & 69 & $<9$ & 128 \\
\hline $2011 Z 433 C$ & $<1$ & 180 & $<8$ & $<1$ & 33 & 109 & $<9$ & 504 \\
\hline $2011 Z 435 \mathrm{~A}$ & $<1$ & 56 & $<8$ & $<1$ & 4 & 21 & $<9$ & 126 \\
\hline $2011 Z 437 \mathrm{~A}$ & $<1$ & 464 & $<8$ & $<1$ & 27 & 158 & $<9$ & 123 \\
\hline $2011 Z 438 \mathrm{~A}$ & $<1$ & 146 & $<8$ & $<1$ & 17 & 47 & $<9$ & 215 \\
\hline 2011Z440A & $<1$ & 500 & $<8$ & $<1$ & 41 & 271 & $<9$ & 161 \\
\hline $2011 Z 443 A$ & $<1$ & 454 & $<8$ & $<1$ & 33 & 72 & $<9$ & 49 \\
\hline 2011Z444B & $<1$ & 481 & $<8$ & $<1$ & 32 & 93 & $<9$ & 63 \\
\hline $2011 Z 445 \mathrm{~A}$ & $<1$ & 182 & $<8$ & $<1$ & 21 & 89 & $<9$ & 76 \\
\hline $2011 Z 454 \mathrm{~A}$ & $<1$ & 566 & $<8$ & $<1$ & 37 & 150 & $<9$ & 234 \\
\hline 2011Z469A & $<1$ & 398 & $<8$ & $<1$ & 24 & 79 & $<9$ & 91 \\
\hline $2011 Z 469 \mathrm{~A}$ & $<1$ & 386 & $<8$ & $<1$ & 24 & 91 & $<9$ & 94 \\
\hline 2011Z471A & $<1$ & 418 & $<8$ & $<1$ & 26 & 74 & $<9$ & 95 \\
\hline $2011 Z 472 \mathrm{~A}$ & $<1$ & 423 & $<8$ & $<1$ & 30 & 125 & $<9$ & 108 \\
\hline $2011 Z 472 A$ & $<1$ & 423 & $<8$ & $<1$ & 30 & 125 & $<9$ & 109 \\
\hline $2011 Z 483 \mathrm{~A}$ & $<1$ & 218 & $<8$ & $<1$ & 24 & 93 & $<9$ & 177 \\
\hline 2011Z489A & $<1$ & 390 & $<8$ & $<1$ & 30 & 223 & $<9$ & 25 \\
\hline 2011Z491A & $<1$ & 423 & $<8$ & $<1$ & 24 & 218 & $<9$ & 124 \\
\hline 2011Z492B & $<1$ & 396 & $<8$ & $<1$ & 26 & 119 & $<9$ & 133 \\
\hline $2011 \mathrm{Z} 500 \mathrm{~A}$ & $<1$ & 717 & $<8$ & $<1$ & 17 & 101 & $<9$ & 298 \\
\hline $2011 \mathrm{Z} 510 \mathrm{~A}$ & $<1$ & 678 & $<8$ & $<1$ & 27 & 196 & $<9$ & 336 \\
\hline $2011 Z 511 \mathrm{~A}$ & $<1$ & 118 & $<8$ & $<1$ & 22 & 57 & $<9$ & 189 \\
\hline 2011Z511B & $<1$ & 436 & $<8$ & $<1$ & 20 & 131 & $<9$ & 149 \\
\hline $20117519 \mathrm{E}$ & $<1$ & 161 & $<8$ & $<1$ & 25 & 54 & $<9$ & 179 \\
\hline
\end{tabular}


Table 9. Detection limits and analytical methods for major-oxide, minor-oxide, and trace-element analyses of polished rock slab samples.

\begin{tabular}{|c|c|c|c|c|c|}
\hline \multirow[b]{2}{*}{$\begin{array}{c}\text { Chemical } \\
\text { formula }\end{array}$} & \multirow[b]{2}{*}{ Oxide/Element } & \multirow[b]{2}{*}{ Units } & \multicolumn{3}{|c|}{ UAF } \\
\hline & & & $\begin{array}{c}\text { Lower Detection } \\
\text { Limit }\end{array}$ & $\begin{array}{c}\text { Upper detection } \\
\text { Limit }\end{array}$ & Analytical Method \\
\hline 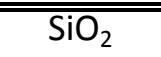 & Silicon dioxide & \% & 0.1 & 100 & $\overline{\mathrm{XRF}}$ \\
\hline $\mathrm{Al}_{2} \mathrm{O}_{3}$ & Aluminum(III) oxide & $\%$ & 0.05 & 75 & $\mathrm{XRF}$ \\
\hline $\mathrm{BaO}$ & Barium oxide & $\%$ & 0.03 & 75 & XRF \\
\hline $\mathrm{CaO}$ & Calcium oxide & $\%$ & 0.02 & 75 & $\mathrm{XRF}$ \\
\hline $\mathrm{FeO}$ & Iron oxide & $\%$ & 0.03 & 75 & XRF \\
\hline $\mathrm{K}_{2} \mathrm{O}$ & Potassium oxide & $\%$ & 0.02 & 75 & XRF \\
\hline $\mathrm{MgO}$ & Magnesium oxide & $\%$ & 0.05 & 75 & XRF \\
\hline $\mathrm{MnO}$ & Manganese(II) oxide & $\%$ & 0.03 & 75 & XRF \\
\hline $\mathrm{Na}_{2} \mathrm{O}$ & Sodium oxide & $\%$ & 0.02 & 75 & $\mathrm{XRF}$ \\
\hline $\mathrm{P}_{2} \mathrm{O}_{5}$ & Posphorous pentoxide & $\%$ & 0.03 & 75 & $\mathrm{XRF}$ \\
\hline $\mathrm{TiO}_{2}$ & Titanium(IV) oxide & $\%$ & 0.02 & 75 & $\overline{X R F}$ \\
\hline $\mathrm{CO}_{2}$ & Carbon dioxide & $\%$ & 0.05 & 75 & $\mathrm{XRF}$ \\
\hline Total & -- & $\%$ & --- & --- & Calculation \\
\hline As & Arsenic & $\mathrm{ppm}$ & 4 & 750,000 & $\mathrm{XRF}$ \\
\hline $\mathrm{Bi}$ & Bismuth & $\mathrm{ppm}$ & 2 & 750,000 & $\mathrm{XRF}$ \\
\hline $\mathrm{Br}$ & Bromine & $\mathrm{ppm}$ & 5 & 750,000 & $\mathrm{XRF}$ \\
\hline $\mathrm{Ce}$ & Cerium & $\mathrm{ppm}$ & 60 & 750,000 & XRF \\
\hline $\mathrm{Cl}$ & Chlorine & $\mathrm{ppm}$ & 50 & 750,000 & $\mathrm{XRF}$ \\
\hline Co & Cobalt & $\mathrm{ppm}$ & 10 & 750,000 & $\mathrm{XRF}$ \\
\hline $\mathrm{Cr}$ & Chromium & $\mathrm{ppm}$ & 30 & 750,000 & $\mathrm{XRF}$ \\
\hline $\mathrm{Cu}$ & Copper & $\mathrm{ppm}$ & 7 & 750,000 & $\mathrm{XRF}$ \\
\hline $\mathrm{F}$ & Fluorine & $\mathrm{ppm}$ & 110 & 750,000 & $\mathrm{XRF}$ \\
\hline La & Lanthanum & $\mathrm{ppm}$ & 50 & 750,000 & $\mathrm{XRF}$ \\
\hline Mo & Molybdenum & $\mathrm{ppm}$ & 1 & 750,000 & $\mathrm{XRF}$ \\
\hline $\mathrm{Nb}$ & Niobium & $\mathrm{ppm}$ & 1 & 750,000 & $\mathrm{XRF}$ \\
\hline $\mathrm{Ni}$ & Nickle & $\mathrm{ppm}$ & 7 & 750,000 & $\mathrm{XRF}$ \\
\hline $\mathrm{Pb}$ & Lead & $\mathrm{ppm}$ & 4 & 750,000 & $\mathrm{XRF}$ \\
\hline $\mathrm{Rb}$ & Rubidium & $\mathrm{ppm}$ & 1 & 750,000 & XRF \\
\hline$S$ & Sulfur & $\mathrm{ppm}$ & 30 & 750,000 & $\mathrm{XRF}$ \\
\hline $\mathrm{Sb}$ & Antimony & $\mathrm{ppm}$ & 2 & 750,000 & $\mathrm{XRF}$ \\
\hline $\mathrm{Sn}$ & Tin & $\mathrm{ppm}$ & 4 & 750,000 & XRF \\
\hline $\mathrm{Sr}$ & Strontium & $\mathrm{ppm}$ & 1 & 750,000 & $\mathrm{XRF}$ \\
\hline Th & Thorium & $\mathrm{ppm}$ & 2 & 750,000 & $\mathrm{XRF}$ \\
\hline$U$ & Unranium & $\mathrm{ppm}$ & 1 & 750,000 & $\mathrm{XRF}$ \\
\hline $\mathrm{V}$ & Vanadium & $\mathrm{ppm}$ & 25 & 750,000 & XRF \\
\hline$W$ & Tungston & $\mathrm{ppm}$ & 8 & 750,000 & $\mathrm{XRF}$ \\
\hline$Y$ & Yttrium & $\mathrm{ppm}$ & 1 & 750,000 & $\mathrm{XRF}$ \\
\hline $\mathrm{Zn}$ & Zinc & $\mathrm{ppm}$ & 8 & 750,000 & XRF \\
\hline $\mathrm{Zr}$ & Zirconium & $\mathrm{ppm}$ & 9 & 750,000 & $\mathrm{XRF}$ \\
\hline
\end{tabular}


Table 10. Location and description of rocks collected for rare-earth- and trace-element analyses in the Moran area, Tanana and Melozitna quadrangles, Alaska.

NOTE: Coordinates are based on NAD 27

\begin{tabular}{|c|c|c|c|c|c|c|}
\hline Sample_ID & Latitude & Longitude & Easting & Northing & $\begin{array}{l}\text { UTM_- } \\
\text { Zone }\end{array}$ & Lithology_Description \\
\hline 2011BAE100A & 65.487364 & -152.831978 & 507779 & 7262591 & $5 \mathrm{~N}$ & $\begin{array}{l}\text { Biotite-muscovite monzogranite (Syenogranite); medium grained, equigranular, and } \\
\text { massive. Minerals include minor muscovite, } 10-12 \% 1-3 \mathrm{~cm} \text { clean biotite books, occasional } \\
\text { euhedral potassium feldspar megacrysts that are up to } 1.5 \mathrm{~cm} \text { long and have clean, } \\
\text { straight matrix contacts, and smoky, subhedral quartz phenocrysts that can be rounded. } \\
100 \% \text { of exposure. }\end{array}$ \\
\hline 2011BAE107C & 65.484924 & -152.858386 & 506557 & 7262316 & $5 \mathrm{~N}$ & $\begin{array}{l}\text { Aplite dike (Syenogranitic aplite dike); fine grained, pink and aphanitic. Occasional visible } \\
\text { quartz or quartz and feldspar crystals near granite margins. } 5 \% \text { of exposure. }\end{array}$ \\
\hline 2011BAE202A & 65.456879 & -152.502041 & 523081 & 7259274 & $5 \mathrm{~N}$ & $\begin{array}{l}\text { Metagabbro (Metagabbro); rock is medium grained (1-2 mm), pale green (epidotized } \\
\text { feldspar?) to dark, olive green, massive, homogenous, and mafic. } 100 \% \text { of exposure. }\end{array}$ \\
\hline 2011BAE247A & 65.325527 & -152.905396 & 504407 & 7244546 & $5 \mathrm{~N}$ & $\begin{array}{l}\text { Metagabbro (Metagabbro); medium grained and crumbly. Fresh rock color is a mottled } \\
\text { gray with green, unoriented actinolite crystals and possibly some lighter colors and/or } \\
\text { spots of iron oxide. Rock weathers brown. } 100 \% \text { of exposure. }\end{array}$ \\
\hline 2011BAE337A & 65.325190 & -153.102222 & 495238 & 7244509 & $5 \mathrm{~N}$ & $\begin{array}{l}\text { Mica-feldspar-quartz porphyroclastic schist (Metagabbro); gray, fine to coarse grained, } \\
\text { and granular with platy schistosity. Minerals include quartz or feldspar in foliation planes } \\
\text { and a gray mica (biotite/chlorite?). } 100 \% \text { of exposure. }\end{array}$ \\
\hline 2011BAE351C & 65.462650 & -152.805055 & 509034 & 7259840 & $5 \mathrm{~N}$ & $\begin{array}{l}\text { Basalt (Basalt); fine grained, black, massive, homogenous, and undeformed. Rock has } \\
\text { blocky, angular parting and sooty surfaces. Visible minerals include amphibole and } \\
\text { plagioclase. } 49 \% \text { of exposure. Trace. }\end{array}$ \\
\hline 2011BAE357A & 65.463469 & -152.787267 & 509858 & 7259934 & $5 \mathrm{~N}$ & $\begin{array}{l}\text { Biotite-muscovite granite (Alkali-feldspar granite); fine to medium grained (1-2 } \mathrm{mm} \text { ), } \\
\text { massive, homogenous, equigranular, and pink. Minerals include } 5 \% \text { muscovite, } 5-10 \% \\
\text { biotite, and occasional 3-5 mm potassium feldspar megacrysts. 50\% of exposure. }\end{array}$ \\
\hline 2011BAE359B & 65.462807 & -152.782460 & 510081 & 7259861 & $5 \mathrm{~N}$ & $\begin{array}{l}\text { Dacite dike (Rhyolite dike); very fine to fine grained, light gray, massive, homogenous, and } \\
\text { porphyritic. Minerals include euhedral feldspars and quartz phenocrysts as well spots of } \\
\text { iron oxide. } 10 \% \text { of exposure. }\end{array}$ \\
\hline
\end{tabular}


Table 10. (continued)

\begin{tabular}{|c|c|c|c|c|c|c|}
\hline 2011BAE359C & 65.462807 & -152.782460 & 510081 & 7259861 & $5 \mathrm{~N}$ & $\begin{array}{l}\text { Dacite dike (Rhyolite dike); very fine grained, light gray, massive, homogenous, and } \\
\text { aphanitic. Rock contains small, 1-2 mm brown spots (biotite clots after clinopyroxene?) } \\
\text { and occasional small, euhedral quartz or light feldspar. } 10 \% \text { of exposure. }\end{array}$ \\
\hline 2011GG426A & 65.392661 & -153.402776 & 481285 & 7252085 & $5 \mathrm{~N}$ & $\begin{array}{l}\text { Microgabbro (Microgabbro); fine-grained, black, slightly foliated. Minerals include } \\
\text { approximately equal amounts of plagioclase, amphibole, and pyroxene. Plagioclase and } \\
\text { amphibole crystals are locally aligned. } 100 \% \text { of exposure. }\end{array}$ \\
\hline 2011GG527A & 65.384860 & -153.830285 & 461410 & 7251410 & $5 N$ & $\begin{array}{l}\text { Granite (Monzogranite); black and white rock. } 20 \% 1-3 \mathrm{~mm} \text { anhedral clear and smokey } \\
\text { quartz, } 10-15 \% \text { subhedral } 2-3 \mathrm{~mm} \text { biotite, and } 65-70 \% \text { feldspars, mostly } 2-5 \mathrm{~mm} \text { anhedral } \\
\text { to subhedral crystals with minor } 5-7 \mathrm{~mm} \text { subhedral crystals. } 100 \% \text { of exposure. }\end{array}$ \\
\hline 2011LF078A & 65.441124 & -152.704480 & 513706 & 7257459 & $5 \mathrm{~N}$ & $\begin{array}{l}\text { Greenstone (Metabasalt); greenish gray. Rock contains } 2 \% 0.5 \mathrm{~mm} \text { relict amphiboles and } \\
3 \% \text { white to gray mica in a fine-grained, sugary groundmass. } 100 \% \text { of exposure. }\end{array}$ \\
\hline 2011LF282A & 65.336063 & -153.146976 & 493156 & 7245725 & $5 \mathrm{~N}$ & $\begin{array}{l}\text { Biotite amphibolite (Metagabbro); dark green color. Rock contains } 25 \% 5 \mathrm{~mm} \text { brown } \\
\text { biotite, } 75 \% 1 \mathrm{~mm} \text { prismatic amphibole. } 100 \% \text { of exposure. }\end{array}$ \\
\hline 2011LF326A & 65.495858 & -152.741003 & 511987 & 7263552 & $5 \mathrm{~N}$ & $\begin{array}{l}\text { Syenogranite (Syenogranite); coarse-grained seriate texture. Rock contains } 5 \% 3 \mathrm{~cm} \\
\text { potassium feldspar megacrystals, } 30 \% 1 \mathrm{~cm} \text { quartz, } 20 \% 1 \mathrm{~cm} \text { potassium feldspar, and } 15 \% \\
2 \mathrm{~mm} \text { biotite in a } 2 \mathrm{~mm} \text { feldspar groundmass. } 100 \% \text { of exposure. }\end{array}$ \\
\hline 2011LF486C & 65.425908 & -152.983752 & 500754 & 7255731 & $5 \mathrm{~N}$ & $\begin{array}{l}\text { Monzogranite (Syenogranite); fine grained. Rock contains } 10 \% 0.5 \mathrm{~mm} \text { biotite, } 3 \% 5 \mathrm{~mm} \\
\text { clots of biotite, and } 5 \% 1 \mathrm{~mm} \text { quartz in a } 0.1 \mathrm{~mm} \text {, mixed-feldspar groundmass ( } 82 \% \text { of } \\
\text { rock). } 25 \% \text { of exposure. }\end{array}$ \\
\hline 2011LF536A & 65.470654 & -152.610335 & 518052 & 7260774 & $5 N$ & $\begin{array}{l}\text { Hornblende metagabbro (Metagabbro); } 20 \% 4 \mathrm{~mm} \text { relict hornblende phenocrysts, } 45 \% \\
\text { clinozoisite after } 1 \mathrm{~mm} \text { plagioclase laths, and } 2 \% 0.5 \mathrm{~mm} \text { sphene in } 35 \% \text { green matrix. } \\
100 \% \text { of exposure. }\end{array}$ \\
\hline 2011LF573A & 65.291505 & -152.973351 & 501243 & 7240751 & $5 N$ & $\begin{array}{l}\text { Metadiorite (Metagabbro); } 60 \% 1 \mathrm{~mm} \text { feldspars, } 35 \% \text { chlorite, and } 0.5 \mathrm{~mm} \text { hornblende } \\
\text { pseudomorphs in an interlocking, subhedral texture. } 100 \% \text { of exposure. }\end{array}$ \\
\hline 2011RN221A & 65.495344 & -152.599618 & 518531 & 7263529 & $5 \mathrm{~N}$ & Greenstone (Metabasalt); fine-grained, green, massive and blocky. $100 \%$ of exposure. \\
\hline
\end{tabular}


Table 10. (continued)

\begin{tabular}{|c|c|c|c|c|c|c|}
\hline 2011RN260A & 65.359713 & -152.894805 & 504894 & 7248357 & $5 N$ & $\begin{array}{l}\text { Greenstone (Metabasalt); fine-grained }(1-2 \mathrm{~mm}) \text {, massive, blocky, and foliated metamafic } \\
\text { rock. Contains } 40-60 \% \text { albite } \pm \text { clinozoisite (?) and } 40-60 \% \text { chlorite. } 100 \% \text { of exposure. }\end{array}$ \\
\hline 2011RN325A & 65.370436 & -152.525978 & 522044 & 7249631 & $5 N$ & $\begin{array}{l}\text { Greenstone (Metabasalt); massive, unfoliated, blocky }\left(<3 \mathrm{~m}^{3}\right) \text {, and locally derived Tozitna } \\
\text { greenstone. } 100 \% \text { of exposure. }\end{array}$ \\
\hline 2011Z004A & 65.400751 & -153.010419 & 499516 & 7252927 & $5 N$ & $\begin{array}{l}\text { Biotite granite (Metamonzogranite); coarse to very coarse grained. Contains } 35 \% \\
\text { porphyritic, pinkish potassium feldspar (=3 cm in diameter), } 20 \% \text { gray, interstitial quartz, } \\
10 \% \text { fine-grained ( } 1-2 \mathrm{~mm} \text { in diameter), brownish to brownish-black biotite, and } 35 \% \\
\text { white plagioclase. } 100 \% \text { of exposure. }\end{array}$ \\
\hline 2011Z083A & 65.462248 & -152.725044 & 512742 & 7259809 & $5 N$ & $\begin{array}{l}\text { Biotite granite (Syenogranite); medium to very coarse grained. Rock contains porphyritic } \\
\text { pink feldspar phenocrysts }=3 \mathrm{~cm} \text { long (average maximum dimensions are } 5 \mathrm{~cm} \times 2 \mathrm{~cm} \text { ), } \\
30 \% \text { biotite crystals }=2 \mathrm{~mm} \text { in diameter, and } 20 \% 2 \mathrm{~mm} \text {, rounded, gray quartz phenocrysts } \\
\text { in amedium-grained granitic matrix. } 100 \% \text { of exposure. }\end{array}$ \\
\hline 2011Z256A & 65.180341 & -153.888678 & 458375 & 7228654 & $5 N$ & $\begin{array}{l}\text { Biotite-quartz monzonite (Monzogranite); extremely coarse-grained and porphyritic. } \\
\text { Contains } 40-45 \%=5 \times 2 \mathrm{~cm} \text { porphyritic white feldspar (potassium?) , } 15 \%=1 \mathrm{~cm} \text { gray, } \\
\text { irregular quartz, } 15-25 \% \text { black, irregular masses of biotite in an orange and black matrix } \\
\text { with cream feldspars. Many feldspar phenocrysts are aligned parallel to long axis. } 100 \% \text { of } \\
\text { exposure. }\end{array}$ \\
\hline 2011Z265B & 65.185046 & -153.865046 & 459489 & 7229163 & $5 N$ & $\begin{array}{l}\text { Aplite dike (Alkali-feldspar-granitic aplite dike); fine grained and creamy white to light } \\
\text { gray. Contains biotite grains that are aligned parallel to dike strike. The immediate area } \\
\text { contains several dikes and dikelets; the widest piece is } 25 \mathrm{~cm} \text { in float and } 20 \mathrm{~cm} \text { in outcrop. }\end{array}$ \\
\hline 2011Z323A & 65.504351 & -152.646101 & 516374 & 7264520 & $5 N$ & $\begin{array}{l}\text { Biotite granite (Syenogranite); porphyritic, with medium-grained matrix with light-orange } \\
\text { to buff weathering and equigranular plagioclase and potassium feldspar. Broken rock is } \\
\text { pink and white. Contains }=2 \mathrm{~cm} \text { long, pink potassium feldspar phenocrysts , } 20 \% 2-3 \mathrm{~mm} \\
\text { gray quartz phenocrysts, and } 15-20 \% \text { black biotite in a medium-grained ( } 1-2 \mathrm{~mm} \text { ) matrix. } \\
100 \% \text { of exposure. }\end{array}$ \\
\hline $2011 Z 336 A$ & 65.389356 & -152.513801 & 522594 & 7251744 & $5 N$ & $\begin{array}{l}\text { Metagabbro (Serpentinite); fine-grained with light brown to dull green weathering. Broken } \\
\text { rock looks very fresh and is black and green with possible relict olivine. } 100 \% \text { of exposure. }\end{array}$ \\
\hline
\end{tabular}


Table 11. Rare-earth- and trace-element analyses results for rocks collected in the Moran area, Tanana and Melozitna quadrangles, Alaska

NOTE: ppm = parts per million

\begin{tabular}{|c|c|c|c|c|c|c|c|c|c|c|c|c|}
\hline SAMPLE_ID & Ba_ppm & Ce_ppm & Co_ppm & Cr_ppm & Cs_ppm & Dy_ppm & Er_ppm & Eu_ppm & Ga_ppm & Gd_ppm & Hf_ppm & Ho_ppm \\
\hline 2011BAE100A & 60.8 & 76.9 & 0.9 & 10 & 10 & 10.85 & $\overline{77}$ & 0.31 & 20.7 & 9.05 & "5.7 & 2.12 \\
\hline 2011BAE107C & 10.8 & 27.5 & 0.5 & 10 & 5.54 & 11.95 & 8.89 & 0.08 & 24.8 & 8.89 & 7.3 & 2.46 \\
\hline 2011BAE202A & 171 & 12.8 & 52.3 & 1740 & 0.07 & 3.11 & 1.76 & 0.93 & 11 & 3.1 & 1.5 & 0.58 \\
\hline 2011BAE247A & 28.7 & 8.5 & 72.3 & 1230 & 0.11 & 1.96 & 1.07 & 0.7 & 10 & 1.89 & 1 & 0.38 \\
\hline 2011BAE337A & 266 & 16.2 & 37 & 110 & 0.27 & 2.93 & 1.62 & 1.2 & 18.4 & 2.93 & 1.8 & 0.56 \\
\hline 2011BAE351C & 291 & 28.3 & 41.6 & 60 & 4.88 & 5.45 & 3.01 & 1.74 & 21.3 & 5.55 & 3.2 & 1.03 \\
\hline 2011BAE357A & 212 & 36.1 & 1.1 & 10 & 30.7 & 5.94 & 3.27 & 0.51 & 21.3 & 5.03 & 2.8 & 1.07 \\
\hline 2011BAE359B & 111.5 & 46.6 & $<0.5$ & 10 & 27.7 & 11.55 & 8.47 & 0.05 & 27.2 & 7.5 & 5.4 & 2.34 \\
\hline 2011BAE359C & 134 & 35.6 & $<0.5$ & $<10$ & 26.3 & 11.3 & 8.5 & $<0.03$ & 33.8 & 6.45 & 6.6 & 2.27 \\
\hline 2011GG426A & 62.2 & 21 & 52 & 160 & 0.93 & 7.09 & 4.47 & 1.8 & 21.5 & 6.41 & 3.5 & 1.44 \\
\hline 2011GG527A & 895 & 166 & 7 & 20 & 11.95 & 4.75 & 2.34 & 1.44 & 22.2 & 6.88 & 6.5 & 0.82 \\
\hline 2011LF078A & 53.8 & 23 & 32.9 & 120 & 0.06 & 11.25 & 6.65 & 1.74 & 18.2 & 10.1 & 5.8 & 2.28 \\
\hline 2011LF282A & 200 & 22.7 & 61.4 & 10 & 14.95 & 4.27 & 2.34 & 1.6 & 21.3 & 4.4 & 2.7 & 0.81 \\
\hline 2011LF326A & 239 & 124 & 1.6 & 10 & 8.62 & 5.09 & 2.79 & 0.68 & 19.2 & 6.08 & 5.4 & 0.89 \\
\hline 2011LF486C & 416 & 131.5 & 2.5 & 20 & 25 & 5.43 & 2.83 & 0.9 & 17.7 & 6.38 & 4.7 & 0.98 \\
\hline 2011LF536A & 130.5 & 14.5 & 46.7 & 1220 & 0.1 & 3.65 & 2 & 1.03 & 12.9 & 3.51 & 1.7 & 0.69 \\
\hline 2011LF573A & 570 & 24.6 & 36.8 & 140 & 1.13 & 4.35 & 2.31 & 1.49 & 19.3 & 4.52 & 2.8 & 0.82 \\
\hline 2011RN221A & 365 & 14.5 & 46.4 & 200 & 1.2 & 5.06 & 2.91 & 1.35 & 18.4 & 4.56 & 2.6 & 0.98 \\
\hline 2011RN260A & 89.8 & 4.6 & 45.6 & 180 & 0.37 & 4.52 & 3 & 0.94 & 16.7 & 3.53 & 1.5 & 0.96 \\
\hline 2011RN325A & 73.9 & 16.4 & 45.3 & 140 & 0.23 & 5.68 & 3.33 & 1.58 & 21.3 & 5.33 & 3 & 1.11 \\
\hline 2011Z004A & 724 & 180.5 & 3.7 & 10 & 4.08 & 4.29 & 2.13 & 1.35 & 19.1 & 6.21 & 6.4 & 0.77 \\
\hline $2011 Z 083 A$ & 374 & 150 & 2.8 & 10 & 11.9 & 4 & 2.34 & 0.82 & 19.5 & 4.53 & 6.1 & 0.75 \\
\hline $2011 Z 256 \mathrm{~A}$ & 1370 & 200 & 5.5 & 10 & 7.28 & 6.27 & 3.11 & 2.08 & 21 & 8.88 & 9.8 & 1.12 \\
\hline 2011Z265B & 61.9 & 8.9 & 0.7 & 10 & 6.05 & 2.21 & 0.84 & 0.21 & 17.5 & 1.71 & 1.3 & 0.32 \\
\hline $2011 Z 323 A$ & 122 & 96.3 & 1.2 & 10 & 4.22 & 8.38 & 4.93 & 0.43 & 18.2 & 7.78 & 4.2 & 1.62 \\
\hline $2011 Z 336 \mathrm{~A}$ & 65.3 & 5.3 & 104.5 & 2610 & 0.09 & 1.36 & 0.72 & 0.43 & 5.9 & 1.3 & 0.7 & 0.26 \\
\hline
\end{tabular}


Table 11. (continued)

\begin{tabular}{|c|c|c|c|c|c|c|c|c|c|c|c|c|}
\hline SAMPLE_ID & La_ppm & Lu_ppm & Mo_ppm & Nb_ppm & Nd_ppm & Pr_ppm & Rb_ppm & Sm_ppm & Sn_ppm & Sr_ppm & Ta_ppm & Tb_ppm \\
\hline 2011BAE100A & 35.4 & 1.23 & $<2$ & 63.6 & 34.5 & 9.48 & 561 & 9.54 & 7 & 25.3 & 9.6 & 1.6 \\
\hline 2011BAE107C & 10.8 & 2.08 & 83 & 48.2 & 16.7 & 3.69 & 596 & 7.4 & 1 & 6.7 & 28.5 & 1.63 \\
\hline 2011BAE202A & 5.5 & 0.21 & $<2$ & 3.7 & 9.2 & 1.89 & 3.1 & 2.64 & $<1$ & 105.5 & 0.2 & 0.48 \\
\hline 2011BAE247A & 3.5 & 0.14 & $<2$ & 2.3 & 5.9 & 1.21 & 0.6 & 1.69 & $<1$ & 69.9 & 0.2 & 0.3 \\
\hline 2011BAE337A & 7.2 & 0.21 & $<2$ & 5.3 & 10.4 & 2.25 & 6.8 & 2.9 & $<1$ & 320 & 0.3 & 0.45 \\
\hline 2011BAE351C & 12.3 & 0.38 & $<2$ & 10.6 & 18.8 & 4.02 & 44.4 & 4.99 & 2 & 249 & 0.7 & 0.84 \\
\hline 2011BAE357A & 16.7 & 0.48 & $<2$ & 38.2 & 17.2 & 4.5 & 524 & 5.11 & 13 & 71.1 & 5.8 & 0.92 \\
\hline 2011BAE359B & 18.2 & 1.8 & $<2$ & 64.2 & 21.6 & 5.82 & 1000 & 7.31 & 31 & 61.8 & 20.2 & 1.54 \\
\hline 2011BAE359C & 19.9 & 2.4 & $<2$ & 50.1 & 16.4 & 4.64 & 827 & 5.67 & 65 & 22.2 & 37.5 & 1.49 \\
\hline 2011GG426A & 7.5 & 0.61 & $<2$ & 7.3 & 16.1 & 3.12 & 6.5 & 5.1 & 2 & 205 & 0.4 & 1.03 \\
\hline 2011GG527A & 81.9 & 0.33 & $<2$ & 16.2 & 69.8 & 19.35 & 274 & 10.95 & 5 & 350 & 1.1 & 0.83 \\
\hline 2011LF078A & 6.6 & 0.87 & $<2$ & 3.4 & 23.5 & 4.2 & 1.3 & 7.9 & 1 & 124.5 & 0.2 & 1.63 \\
\hline 2011LF282A & 9.7 & 0.28 & $<2$ & 7.7 & 14.8 & 3.13 & 10 & 3.99 & 1 & 368 & 0.5 & 0.66 \\
\hline 2011LF326A & 58.1 & 0.44 & $<2$ & 38 & 48.1 & 13.8 & 456 & 9.36 & 6 & 89.3 & 3.5 & 0.87 \\
\hline 2011LF486C & 65.6 & 0.38 & $<2$ & 14.6 & 51 & 14.85 & 318 & 9.07 & 3 & 139 & 1.9 & 0.91 \\
\hline 2011LF536A & 5.9 & 0.25 & $<2$ & 4.4 & 10.5 & 2.18 & 4.5 & 3.09 & $<1$ & 116.5 & 0.3 & 0.56 \\
\hline 2011LF573A & 10.6 & 0.29 & $<2$ & 8.4 & 15.9 & 3.45 & 15.3 & 4.12 & $<1$ & 338 & 0.5 & 0.66 \\
\hline 2011RN221A & 5.7 & 0.37 & $<2$ & 6.2 & 11.8 & 2.31 & 2.3 & 3.66 & 1 & 122 & 0.4 & 0.74 \\
\hline 2011RN260A & 1.9 & 0.43 & $<2$ & 0.7 & 5.6 & 0.95 & 1.4 & 2.43 & $<1$ & 182.5 & 0.1 & 0.63 \\
\hline 2011RN325A & 5.7 & 0.42 & $<2$ & 7.1 & 13.4 & 2.62 & 10.4 & 4.24 & 1 & 236 & 0.5 & 0.86 \\
\hline 2011Z004A & 99.7 & 0.26 & $<2$ & 12.5 & 67.4 & 19.9 & 245 & 9.77 & 2 & 214 & 0.7 & 0.76 \\
\hline 2011Z083A & 57.8 & 0.41 & $<2$ & 43.6 & 40.4 & 12.1 & 416 & 6.78 & 7 & 147 & 3.3 & 0.62 \\
\hline $2011 Z 256 A$ & 102.5 & 0.38 & $<2$ & 17.5 & 80.9 & 22.6 & 233 & 12.9 & 3 & 320 & 1 & 1.11 \\
\hline 2011Z265B & 4 & 0.09 & $<2$ & 11.2 & 3.8 & 1.07 & 268 & 1.43 & 4 & 41.2 & 2.4 & 0.36 \\
\hline 2011Z323A & 46.9 & 0.77 & $<2$ & 41.8 & 37.5 & 10.95 & 398 & 8.65 & 2 & 36.9 & 4.8 & 1.29 \\
\hline $2011 Z 336 A$ & 2.2 & 0.09 & $<2$ & 1.8 & 3.8 & 0.8 & 2.6 & 1.15 & $<1$ & 69 & 0.1 & 0.21 \\
\hline
\end{tabular}




\begin{tabular}{|l|c|c|c|c|c|c|c|c|c|}
\hline SAMPLE_ID & Th_ppm & TI_ppm & Tm_ppm & U_ppm & V_ppm & W_ppm & Y_ppm & Yb_ppm & Zr_ppm \\
\hline \hline 2011BAE100A & 57.7 & 1.9 & 1.13 & 31.3 & $<5$ & 3 & 71 & 8.42 & 110 \\
\hline 2011BAE107C & 22.5 & 2.1 & 1.55 & 13.85 & $<5$ & 2 & 141 & 12.95 & 54 \\
\hline 2011BAE202A & 0.78 & $<0.5$ & 0.22 & 0.24 & 302 & 1 & 15.7 & 1.4 & 50 \\
\hline 2011BAE247A & 0.48 & $<0.5$ & 0.14 & 0.16 & 206 & 1 & 10.3 & 0.94 & 32 \\
\hline 2011BAE337A & 1.09 & $<0.5$ & 0.21 & 0.34 & 348 & 1 & 15.4 & 1.39 & 67 \\
\hline 2011BAE351C & 1.69 & $<0.5$ & 0.4 & 0.59 & 405 & 1 & 27.9 & 2.53 & 118 \\
\hline 2011BAE357A & 18.55 & 2 & 0.49 & 5.4 & 5 & 9 & 35.3 & 3.36 & 65 \\
\hline 2011BAE359B & 27.4 & 4.8 & 1.46 & 27.8 & $<5$ & 9 & 96 & 11.65 & 67 \\
\hline 2011BAE359C & 19.25 & 3.9 & 1.64 & 3.93 & $<5$ & 9 & 86.7 & 14.9 & 57 \\
\hline 2011GG426A & 0.49 & $<0.5$ & 0.58 & 0.23 & 457 & 6 & 39.3 & 4.11 & 124 \\
\hline 2011GG527A & 35.8 & 1 & 0.33 & 3.54 & 54 & 1 & 23.7 & 2.19 & 235 \\
\hline 2011LF078A & 0.13 & $<0.5$ & 0.9 & 0.05 & 212 & 1 & 60.6 & 5.99 & 225 \\
\hline 2011LF282A & 1.42 & $<0.5$ & 0.3 & 0.49 & 1020 & 1 & 21.6 & 1.97 & 95 \\
\hline 2011LF326A & 65.9 & 1.5 & 0.4 & 15.05 & 10 & 18 & 28.1 & 2.97 & 157 \\
\hline 2011LF486C & 32.9 & 0.9 & 0.4 & 5.35 & 12 & 5 & 28.6 & 2.56 & 152 \\
\hline 2011LF536A & 0.82 & $<0.5$ & 0.25 & 0.28 & 345 & 1 & 18.4 & 1.71 & 58 \\
\hline 2011LF573A & 1.4 & $<0.5$ & 0.31 & 0.46 & 344 & 1 & 21.6 & 2.04 & 101 \\
\hline 2011RN221A & 0.38 & $<0.5$ & 0.39 & 0.12 & 379 & $<1$ & 26.5 & 2.54 & 91 \\
\hline 2011RN260A & 0.06 & $<0.5$ & 0.42 & 0.05 & 324 & $<1$ & 26.7 & 2.86 & 48 \\
\hline 2011RN325A & 0.48 & $<0.5$ & 0.44 & 0.16 & 427 & 1 & 29.8 & 2.89 & 110 \\
\hline 2011Z004A & 22.3 & 0.9 & 0.27 & 1.98 & 23 & 1 & 21.6 & 1.81 & 244 \\
\hline 2011Z083A & 70.3 & 1.3 & 0.36 & 10.3 & 22 & 11 & 22.2 & 2.67 & 201 \\
\hline 2011Z256A & 21.2 & 0.6 & 0.39 & 4.02 & 41 & 3 & 30.3 & 2.6 & 412 \\
\hline 2011Z265B & 1.9 & 0.8 & 0.11 & 2.51 & $<5$ & 4 & 10.5 & 0.67 & 31 \\
\hline 2011Z323A & 50.3 & 1.1 & 0.73 & 11.35 & 6 & 2 & 51.6 & 5.18 & 115 \\
\hline 2011Z336A & 0.29 & $<0.5$ & 0.1 & 0.1 & 129 & 1 & 6.8 & 0.63 & 23 \\
\hline
\end{tabular}


Table 12. Detection limits and geochemical methods for rare-earth- and trace-element analyses of rock samples.

\section{Chemex codes include:}

ME-MS81 = Lithium borate fusion and X-ray fluorescence spectroscopy

\section{Analytical methods and digestions include:}

ICP-MS = Inductively coupled plasma - mass spectroscopy

LBF $=$ Lithium borate fusion

Note: ${ }^{*}=$ Base metal oxides and sulfides that may not be completely decomposed by the lithium borate fusion.

\begin{tabular}{|c|c|c|c|c|c|c|}
\hline \multirow[b]{2}{*}{ Header } & \multirow[b]{2}{*}{ Element } & \multicolumn{5}{|c|}{ ALS Chemex } \\
\hline & & $\begin{array}{c}\text { Lower } \\
\text { Detection } \\
\text { Limit } \\
\end{array}$ & $\begin{array}{c}\text { Upper } \\
\text { Detection } \\
\text { Limit } \\
\end{array}$ & $\begin{array}{c}\text { Chemex } \\
\text { Code }\end{array}$ & $\begin{array}{c}\text { Analytical } \\
\text { Method }\end{array}$ & Digestion \\
\hline Ba_ppm & Barium & 0.5 & 10,000 & "ME-MS81 & ICP-MS & LBF \\
\hline Ce_ppm & Cerium & 0.5 & 10,000 & ME-MS81 & ICP-MS & LBF \\
\hline Co_ppm & Cobalt* & 0.5 & 10,000 & ME-MS81 & ICP-MS & LBF \\
\hline Cr_ppm & Chromium & 10 & 10,000 & ME-MS81 & ICP-MS & LBF \\
\hline Cs_ppm & Cesium & 0.01 & 10,000 & ME-MS81 & ICP-MS & LBF \\
\hline Dy_ppm & Dysprosium & 0.05 & 10,000 & ME-MS81 & ICP-MS & LBF \\
\hline Er_ppm & Erbium & 0.03 & 1,000 & ME-MS81 & ICP-MS & LBF \\
\hline Eu_ppm & Europium & 0.03 & 1,000 & ME-MS81 & ICP-MS & LBF \\
\hline Ga_ppm & Gallium & 0.1 & 1,000 & ME-MS81 & ICP-MS & LBF \\
\hline Gd_ppm & Gadolinium & 0.05 & 1,000 & ME-MS81 & ICP-MS & LBF \\
\hline Hf_ppm & Hafnium & 0.2 & 10,000 & ME-MS81 & ICP-MS & LBF \\
\hline Ho_ppm & Holmium & 0.01 & 1,000 & ME-MS81 & ICP-MS & LBF \\
\hline La_ppm & Lanthanum & 0.5 & 10,000 & ME-MS81 & ICP-MS & LBF \\
\hline Lu_ppm & Lutetium & 0.01 & 1,000 & ME-MS81 & ICP-MS & LBF \\
\hline Mo_ppm & Molybdenum* & 2 & 10,000 & ME-MS81 & ICP-MS & LBF \\
\hline Nb_ppm & Niobium & 0.2 & 10,000 & ME-MS81 & ICP-MS & LBF \\
\hline Nd_ppm & Neodymium & 0.1 & 10,000 & ME-MS81 & ICP-MS & LBF \\
\hline Pr_ppm & Praseodymium & 0.03 & 1,000 & ME-MS81 & ICP-MS & LBF \\
\hline Rb_ppm & Rubidium & 0.2 & 10,000 & ME-MS81 & ICP-MS & LBF \\
\hline Sm_ppm & Samarium & 0.03 & 1,000 & ME-MS81 & ICP-MS & LBF \\
\hline Sn_ppm & Tin & 1 & 10,000 & ME-MS81 & ICP-MS & LBF \\
\hline Sr_ppm & Strontium & 0.1 & 10,000 & ME-MS81 & ICP-MS & LBF \\
\hline Ta_ppm & Tantalum & 0.1 & 10,000 & ME-MS81 & ICP-MS & LBF \\
\hline Tb_ppm & Terbium & 0.01 & 1,000 & ME-MS81 & ICP-MS & LBF \\
\hline Th_ppm & Thorium & 0.05 & 1,000 & ME-MS81 & ICP-MS & LBF \\
\hline TI_ppm & Thallium & 0.5 & 1,000 & ME-MS81 & ICP-MS & LBF \\
\hline Tm_ppm & Thulium & 0.01 & 1,000 & ME-MS81 & ICP-MS & LBF \\
\hline U_ppm & Uranium & 0.05 & 1,000 & ME-MS81 & ICP-MS & LBF \\
\hline V_ppm & Vanadium & 5 & 10,000 & ME-MS81 & ICP-MS & LBF \\
\hline W_ppm & Tungsten & 1 & 10,000 & ME-MS81 & ICP-MS & LBF \\
\hline Y_ppm & Yttrium & 0.5 & 10,000 & ME-MS81 & ICP-MS & LBF \\
\hline Yb_ppm & Ytterbium & 0.03 & 1,000 & ME-MS81 & ICP-MS & LBF \\
\hline Zr_ppm & Zirconium & 2 & 10,000 & ME-MS81 & ICP-MS & LBF \\
\hline
\end{tabular}

